\title{
2010 Program of Study: Swirling and Swimming in Turbulence
}

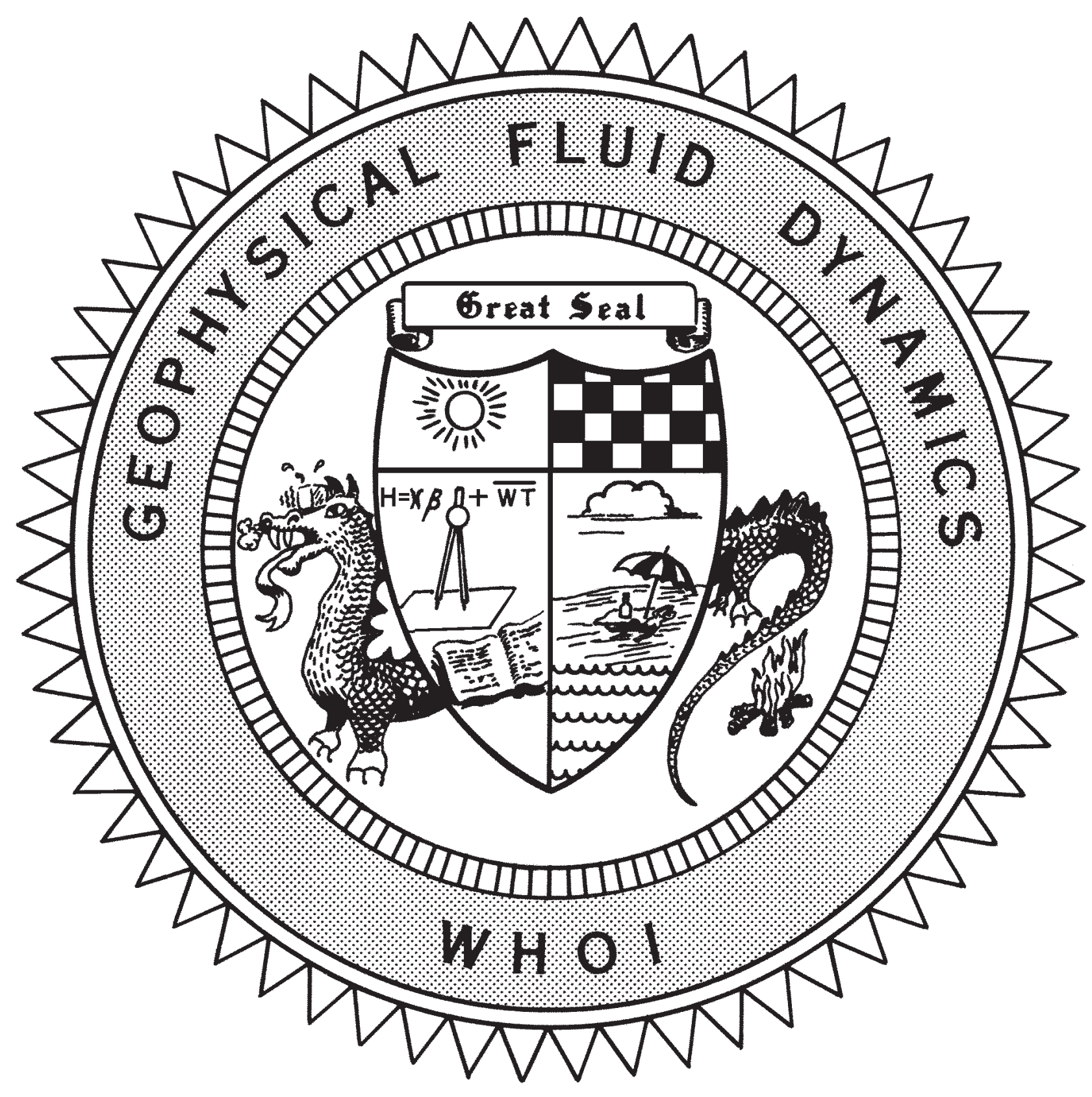

Course Lectures

Fellows Project Reports 


\title{
WHOI 2011-03
}

\section{Program of Study: Swirling and Swimming in Turbulence}

\author{
by
}

Neil Balmforth and Jean-Luc Thiffeault, Co-Directors

Woods Hole Oceanographic Institution
Woods Hole, Massachusetts 02543

June 2011

\section{Technical Report}

Funding was provided by the Office of Naval Research under Contract No. N000-14-09-10844 and the National Science Foundation through Grant No. OCE 0824636

Reproduction in whole or in part is permitted for any purpose of the United States Government. This report should be cited as Woods Hole Oceanographic Institution Technical Report, WHOI-2011-03.

Approved for public release; distribution unlimited.

Approved for Distribution:

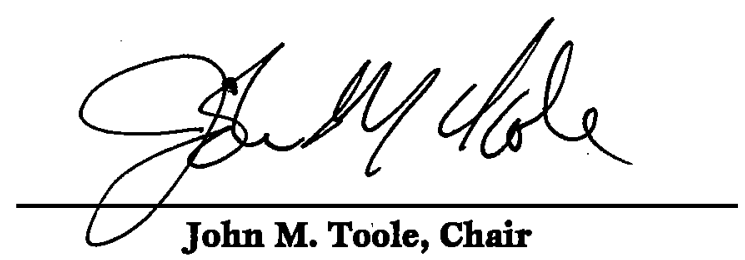

Department of Physical Oceanography 


\section{Preface}

Swirling and Swimming in Turbulence was the theme at the 2010 GFD Program. Professors Glenn Flierl (M.I.T.), Antonello Provenzale (ISAC-CNR, Turin) and Jean-Luc Thiffeault (University of Wisconsin) were the principal lecturers. Together they navigated an elegant path through topics ranging from mixing protocols and efficiencies to ecological strategies, schooling and genetic development. The first ten chapters of this volume document these lectures, each prepared by pairs of this summer's GFD fellows. Following on are the written reports of the fellows' own research projects.

This summer's fellows were:

- Michael Allshouse, MIT

- Emma Boland-Thompson, University of Cambridge

- Renske Gelderloos, KNMI, Utrecht

- David Goluskin, Columbia University

- Georgy Manucharyan, Yale University

- Woosok Moon, Yale University

- Kiori Obuse, Kyoto University

- Amanda O'Rourke, Princeton University

- Sam Pegler, University of Cambridge

- Anubhab Roy, Jawaharlal Nehru Centre for Advanced Scientific Research

In 2010 the Sears Public Lecture was delivered by Professor Jim Murray, of Oxford University and the University of Washington. Jim challenged us to use mathematics in real world problems, citing examples from biology and sociology. Specifically, Jim argued how one could exploit mathematical modelling to assist in the prediction of tumour growth, and to gauge the successfulness of marriage partnership. Over a hundred listeners filed into Redfield for the occasion, and we enjoyed refreshments in the evening air afterwards at the back of Redfield.

Neil Balmforth and Jean-Luc Thiffeault acted as the co-directors for the summer (once Jean-Luc's Lectures were over). New GFD Faculty member Colm Caulfield (University of Cambridge) nimbly acted as assistant, as well as putting in a stout performance on the Staff by supervising a good fraction of the fellows. Anders Jensen worked his usual magic in the Lab, and Janet Fields, Jeanne Fleming and Kathy Ponti smoothly ran the program behind the scenes. 


\section{TABLE OF CONTENTS}

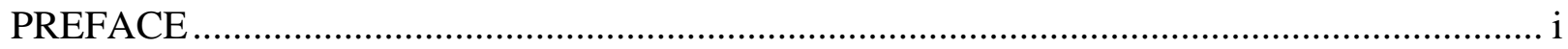

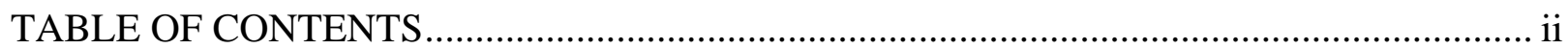

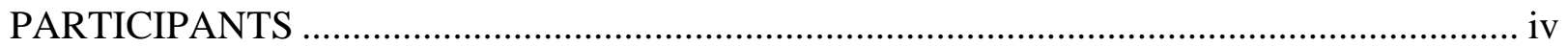

LECTURE SCHEDULE.............................................................................................. vii

PRINCIPAL LECTURES

Glenn Flierl, Massachusetts Institute of Technology, Antonello Provenzale, Istituto di Scienze Dell'Atmosfera and Jean-Luc Thiffeault, University of Wisconsin, Madison

Lecture 1

Stirring and Mixing

Jean-Luc Thiffeault....

Lecture 2

Introduction to Biological Models

Glenn Flierl

Lecture 3

Stirring by Swimming Organisms

Jean-Luc Thiffeault....

Lecture 4

Local Stretching Theories

Jean-Luc Thiffeault.....

Lecture 5

Social Behavior, Mixing and the Evolution of Schooling

Glenn Flierl

Lecture 6

Mixing in the Presence of Sources and Sinks

Jean-Luc Thiffeault

Lecture 7

Plankton Dynamics and Mesoscale Turbulence

Antonello Provenzale.

Lecture 8

Plankton Cells in Turmoil and the Dynamics of Heavy Impurities with Finite Size

Antonello Provenzale.. 
Lecture 9

Plankton Sinking and Turbulence

Antonello Provenzale....

Lecture 10

Evolutionary Models: Movement and Mixing in Trait and Physical Space

Glenn Flierl

\section{FELLOWS’ REPORTS}

Finding Lagrangian Structures via the Application of Braid Theory

Michael Allshouse, M.I.T.

Cave Rings

Emma Boland, University of Cambridge.

Dynamics of Air-Blown Dimples

Renske Gelderloos, Utrecht University

Who Ate Whom? Population Dynamics with Age-Structured Predation

David Goluskin, Columbia University

Dynamics of the Mixed Layers in Stratified Shear Flows

Georgy Manucharyan, Yale University

On-Off Intermittency in Locally Coupled Maps

Woosok Moon, Yale University

Trajectories of a Low Reynolds Number Treadmilling Organism near a Half-Finite No-Slip Wall

Kiori Obuse, Kyoto University

Optimal Mixing on the Sphere

Amanda O’Rourke, Princeton University

Snail Locomotion

Sam Pegler, University of Cambridge

Dynamics of Vorticity Defects in Stratified Shear

Anubhab Roy, Jawaharlal Nehru Centre for Advanced Scientific Research (JNCASR) .360 


\section{GFD FELLOWS, STAFF AND VISITORS}

\section{Fellows}

Michael Allshouse

Emma Boland

Renske Gelderloos

David Goluskin

Georgy Manucharyan

Woosok Moon

Kiori Obuse

Amanda O’Rourke

Sam Pegler

Anubhab Roy
Massachusetts Institute of Technology

University of Cambridge

Utrecht University

Columbia University

Yale University

Yale University

Kyoto University

Princeton University

University of Cambridge

Jawaharlal Nehru Centre for Advanced Scientific

Research (JNCASR)

\section{Staff and Visitors}

James Anderson

Gualtiero Badin

Neil Balmforth

Andrew Belmonte

Guido Bofetta

Annalisa Bracco

Ken Breuer

Greg Buck

John Bush

Colm-cille Caufield

Stephen Childress

Greg Chini

Predrag Cvitanovic

Paul Dellar

Navid Dianati Maleki

Charles Doering

Raffaele Ferrari

Matthew Finn

Glenn Flierl

Andrew Fowler

Baylor Fox-Kemper

Anthony Fragoso

Michael Graham

George Hagstrom

Karl Helfrich

Ian Hewitt

Edward Johnson

Daniel Jones
Stevens Institute of Technology

Boston University

University of British Columbia

Pennsylvania State University

INFN ICTR

Georgia Institute of Technology

Brown University

St. Anselm College

Massachusetts Institute of Technology

University of Cambridge

New York University

University of New Hampshire

Georgia Institute of Technology

Oxford Centre for Industrial and Applied Mathematics

University of Michigan

University of Michigan

Massachusetts Institute of Technology

University of Adelaide

Massachusetts Institute of Technology

University of Limerick

University of Colorado at Boulder

Yale University

University of Wisconsin, Madison

University of Texas, Austin

Woods Hole Oceanographic Institution

University of British Columbia

University College London

Colorado State University 


\author{
Shane Keating \\ Joseph Keller \\ Eric Kunze \\ Joseph LaCasce \\ Norman Lebovitz \\ Marina Levy \\ Zhi Lin \\ Yiping Ma \\ Amala Mahadevan \\ Lakshminarayanan Mahadevan \\ Willem Malkus \\ Sarah Matz \\ James McElwaine \\ Meredith Metzger \\ Stephen Morris \\ Philip Morrison \\ James Murray \\ Shavarsh Nurijanyan \\ Francesco Paparella \\ Thomas Peacock \\ Thomas Powers \\ Antonello Provenzale \\ Tiffany Shaw \\ Vitalli Sheremet \\ Alexander Soloviev \\ Andew Stewart \\ Roman Stocker \\ Bruce Sutherland \\ John Taylor \\ Jean-Luc Thiffeault \\ Yue-Kin Tsang \\ George Veronis \\ Jeffrey Weiss \\ John Whitehead
}

Courant Institute for Mathematical Science

Stanford University

University of Victoria

University of Oslo

University of Chicago

LOCEAN/IPSL

University of Minnesota

University of California, Berkeley

Boston University

Harvard University

Massachusetts Institute of Technology

University of Wisconsin, Madison

University of Cambridge

University of Utah

University of Toronto

University of Texas, Austin

University of Washington/University of Oxford

University of Twente

University of Salento

Massachusetts Institute of Technology

Brown University

Istituto di Scienze Dell'Atmosfera

Courant Institute for Mathematical Science

University of Rhode Island

NOVA Southeastern University

University of Oxford

Massachusetts Institute of Technology

University of Alberta

Massachusetts Institute of Technology

University of Wisconsin, Madison

Scripps Institution of Oceanography

Yale University

University of Colorado, Boulder

Woods Hole Oceanographic Institution 


\section{Principal Lecturers}

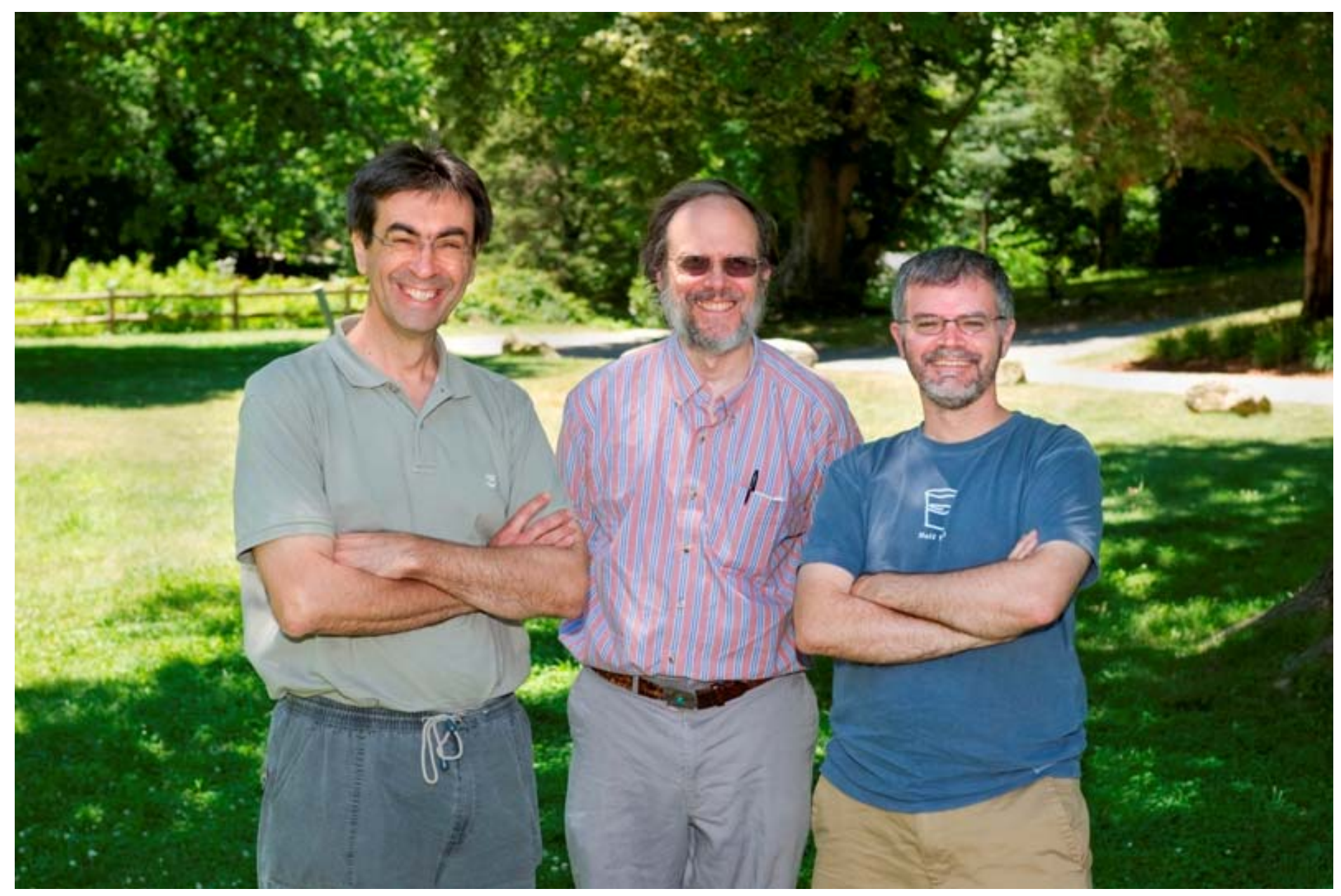

From left to right - Antonello Provenzale, Glenn Flierl, Jean-Luc Thiffeault 


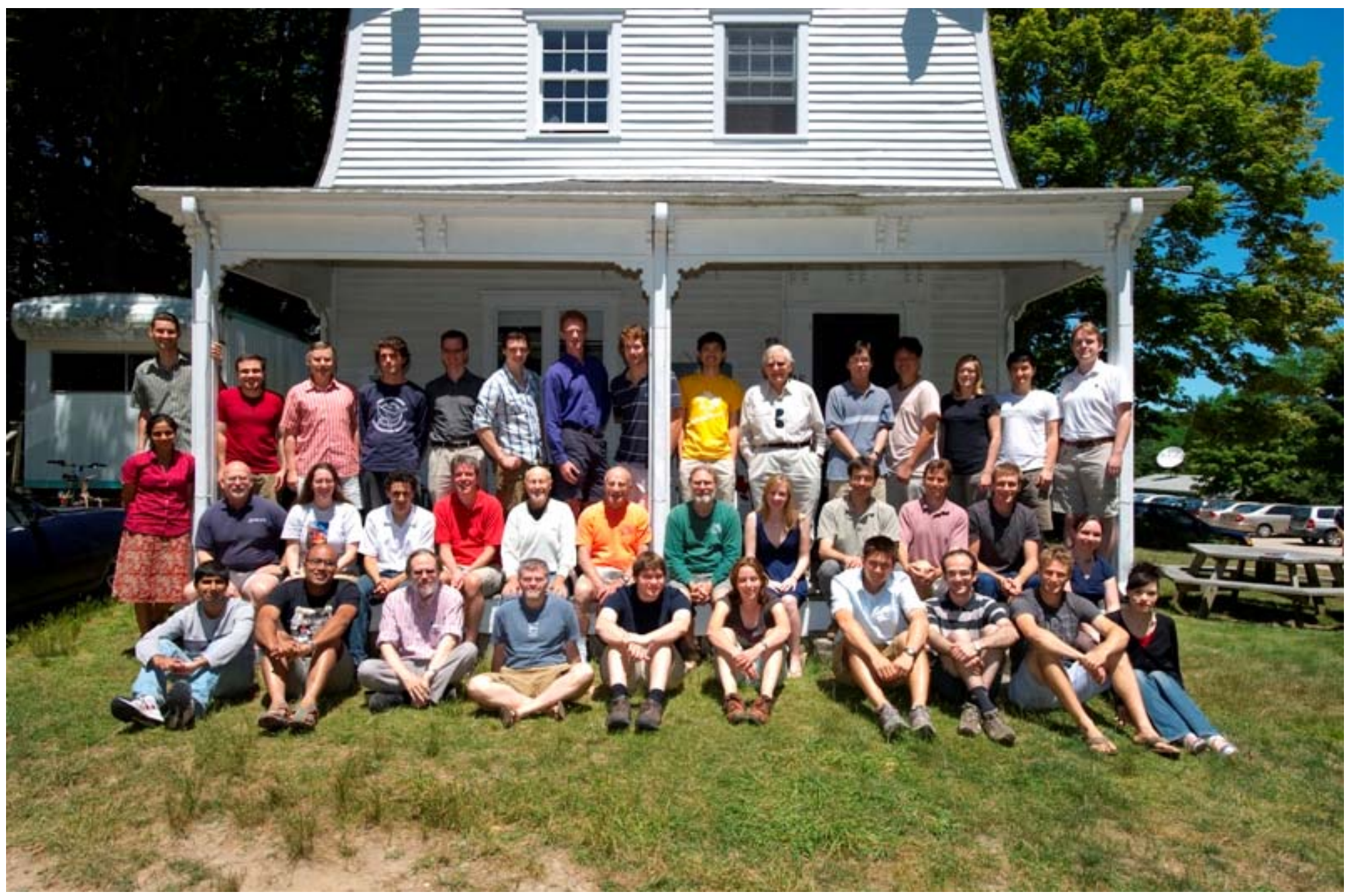

First row, seated (L/R): Shreyas Mandre, Anubhab Roy, Glenn Flierl, Jean-Luc Thiffeault, Sam Pegler, Renske Gelderloos, Michael Allshouse, Navid Dianati Malekhi, David Goluskin, Amanda O’Rourke, Kiori Obuse

Second row (L/R): Amala Mahadevan (standing), Charles Doering, Sarah Matz, George Hagstrom, Joseph LaCasce, Joseph Keller, George Veronis, Philip Morrison, Emma Boland, Antonello Provenzale, Neil Balmforth, Jim McElwaine

Back row (L/R): John Taylor, Greg Chini, Edward Johnson, Gualtiero Badin, Colm-cille Caulfield, Andrew Stewart, Onno Bokhove, Anthony Fragoso, George Lin, James Anderson, Yue-Kin Tsang, Woosok Moon, Tiffany Shaw, Georgy Manucharyan, Daniel Jones

Not pictured: Andrew Belmonte, Guido Bofetta, Annalisa Bracco, Kenneth Breuer, Greg Buck, Predrag Cvitanovic, Paul Dellar, Raffaele Ferrari, Matt Finn, Andrew Fowler, Baylor Fox-Kemper, Michael Graham, Karl Helfrich, Ian Hewitt, Annette Hosoi, Shane Keating, Eric Kunze, Norman Lebovitz, Marina Levy, Yiping Ma, Lakshminarayanan Mahedevan, Willem Malkus, Meredith Metzger, Stephen Morris, Shavarsh Nurijanyan, Francesco Paparella, Tom Peacock, Tom Powers, Amy Shen, Alexander Soloviev, Ed Spiegel, Roman Stocker, Bruce Sutherland, Jeffrey Weiss, Jack Whitehead 


\section{GFD Lecture Schedule}

Week 1

Monday, June 21

Lecture 1 - 10:00 AM

Stirring and Mixing

Jean-Luc Thiffeault, University of Wisconsin, Madison

Tuesday, June 22

Lecture 2 - 10:00 AM

Introduction to Biological Models

Glenn Flierl, MIT

Wednesday, June 23

Lecture 3 - 10:00 AM

Stirring by Swimming Organisms

Jean-Luc Thiffeault, University of Wisconsin, Madison

2:30 PM

Tutorial: Eddy Diffusivity and Active Scalers

Glenn Flierl, MIT

Thursday, June 24

Lecture 4 - 10:00 AM

Local Stretching Theories

Jean-Luc Thiffeault, University of Wisconsin, Madison

Friday, June 25

Lecture 5 - 10:00 AM

Social Behavior, Mixing, and the Evolution of Schooling Glenn Flierl, MIT 
Week 2

Monday, June 28

Lecture 6 - 10:00 AM

Mixing in the Presence of Sources and Sinks

Jean-Luc Thiffeault, University of Wisconsin, Madison

2:30 PM

Tutorial: How to Write an Ecological Model

Antonello Provenzale, Istituto di Scienze Dell'Atmosfera

Tuesday, June 29

Lecture 8 - 10:00 AM

Plankton Dynamics and Mesoscale Turbulence

Antonello Provenzale, Istituto di Scienze Dell'Atmosfera

$3: 30$

Lab Tour

Wednesday, June 30

Lecture 9 - 10:00 AM

Plankton Cells in Turmoil and the Dynamics of Heavy Impurities with Finite Size Antonello Provenzale, Istituto di Scienze Dell'Atmosfera

$4: 30$

Project Discussions

Thursday, July 1

Lecture 10 - 10:00 AM

Plankton Sinking and Turbulence

Antonello Provenzale, Istituto di Scienze Dell'Atmosfera 
Friday, July 2

Lecture 12

Evolutionary Models: Movement and Mixing in Trait and Physical Space Glenn Flierl, MIT

Week 3

Monday, July 5

HOLIDAY - INSTITUTION CLOSED

Tuesday, July 6

$10: 30$

Two Particle Separations in 2-D Turbulence

Joe LaCasce, University of Oslo

Wednesday, July 7

$10: 30$

Sub-Mesoscale Turbulence in the Ocean: How Does it Affect the Oceanic Carbon Cycle Marina Levy, LOCEAN

$1: 30$

Modelling Turbulent Mixing in Two-Dimensions using Stochastic Differential Equations Lennon O’Naraigh, University College Dublin

Thursday, July 8

$10: 30$

Biologically Generated Turbulence in the Ocean

Eric Kunze, University of Victoria

$2: 30$

Modification of the Air-Sea Interaction and Near-Surface Turbulence by Surfactants Alexander Soloviev, NOVA Southeastern University 
Friday, July 9

$10: 30$

Inertial Effects in G.I. Taylor's Swimming Sheet

Steve Childress, New York University

2:30

Beach Creation by Breaking Waves

Onno Bokhove, University of Twente

Week 4

Monday, July 12

10:30

Double Feature: Reactive Mixing and Turbulent Energy Injection

Yue-Kin Tsang, Scripps Institution of Oceanography

Tuesday, July 13

$10: 30$

Three Experiments: On the morphology of Icicles, Patterns in Meandering Syrup and Electroconvection in Thin Films

Steve Morris, University of Toronto

Wednesday, July 14

$10: 30$

Upper Bounds Yield Lower Order Models

Greg Chini, University of New Hampshire

$2: 30$

Demographic Stochasticity versus Spatial Variation in the Competition between Fast and Slow Dispersion

Charlie Doering, University of Michigan 
Thursday, July 15

$10: 30$

Mixing in Thermal Convection: The Case of Rayleigh-Taylor Turbulence Guido Boffetta, INFN ICTR

Friday, July 16

$10: 30$

Title to be announced

Tom Powers, Brown University

$2: 30$

Mesoscale Diagnosis

Baylor Fox-Kemper, University of Colorado at Boulder

Week 5

Monday, July 19

$10: 30$

Tip Growth of a Walled Cell

Lakshminarayanan Mahadevan, Harvard University

2:30

Drumlins

Andrew Fowler, University of Limerick

Tuesday, July 20

10:30

From Bioturbation to Channelization

Amala Mahadevan, Boston University

Wednesday, July 21

$10: 30$

Particle Diffusion in a Bubbly Liquid

Andrew Belmonte, Penn State University 
Thursday, July 22

10:30

Vertical Velocities and the Ocean Mesoscale

Annalisa Bracco, Georgia Institute of Technology

Friday, July 23

$10: 30$

Magma Transport in the Mantle

Ian Hewitt, University of British Columbia

Week 6

Monday, July 26

10:30

Onset and Cessation of Convection in Porous Media Anja Slim, Harvard University

Tuesday, July 27

10:30

Flagellated Bacteria, Motility and Mixing

Ken Breuer, Brown University

Wednesday, July 28

$10: 30$

Nonequilibrium Fluctuations and Climate Variability

Jeff Weiss, University of Colorado, Boulder

$2: 30$

Lateral Mixing by Submesoscale Processes

Gualtiero Badin, Boston University 
Thursday, July 29

10:30

Some Doubly Diffusive Memories of Melvin Stern

George Veronis, Yale University

Friday, July 30

10:30

Bacteria Foraging in Turbulent Waters

John Taylor, MIT

Week 7

Monday, August 2

10:30

Transport by River Plumes and Collapsing Mixed Regions Bruce Sutherland, University of Alberta

$2: 30$

Boundary Layers in Braginsky Magneto Hydro Dynamics Paul Dellar, University of Oxford

$\underline{\text { Tuesday, August } 3}$

10:30

Non-gaussian Buoyancy Statistics in Fingering Convection Francesco Paparella, University of Salento

Wednesday, August 4

10:30

Mechanism of a Splash

Shreyas Mandre, Harvard University

2:30

Hotspots

Matt Finn, University of Adelaide 
Thursday, August 5

10:30

Convection and Biological Productivity at Fronts

Raffaele Ferrari, MIT

2:30

On the Efficiency and Stability of a Size Dependent Ecosystem Model

Amit Tandon, University of Massachusetts, Dartmouth

Friday, August 6

$10: 30$

Title to be announced

Roman Stocker, MIT

$2: 30$

Optimal Solute Fluxes in Solidification: Variational Principles, Hysteresis and Chimney Spacing

Andrew Wells, Yale University

Week 8

Monday, August 9

10:30

Transforming the Mean Meridional Circulation of the Atmosphere Tiffany Shaw, Courant Institute of Mathematical Science

2:30

Feeding and Swimming Currents and Jumping Vortices in Planktonic Copepods Houshuo Jiang, Woods Hole Oceanographic Institution

$\underline{\text { Tuesday, August } 10}$

$10: 30$

Reducing Continuous Symmetries of Flows between Planes and Down Pipes Predrag Cvitanovic, Georgia Institute of Technology 
Wednesday, August 11

$10: 30$

Transport and Collective Dynamics in Bacterial Swimming Michael Graham, University of Wisconsin, Madison

$5: 00$

Public Lecture - Redfield Auditorium - Reception to follow

Mathematics in the Real World: from Brain Tumors to Saving Marriages James Murray, University of Washington and University of Oxford

Thursday, August 12

10:30

Tibetan Singing Bowls (and the nature of reality)

John Bush, MIT

2:30

Title to be announced

Tom Peacock, MIT

Friday, August 13

$10: 30$

The Teapot Effect and Free Boundary Flows Joe Keller, Stanford University

Week 9 - August 16-20 - No lectures scheduled this week

Week 10 - FELLOWS’ PRESENTATIONS

Tuesday, August 24

10:00

Finding Lagrangian Structures via the Application of Braid Theory Michael Allshouse, MIT 
$\underline{\text { Tuesday, August } 24 \text { (continued) }}$

11:00

Dynamics of Vorticity Defects in Stratified Shear

Anubhab Roy, Jawaharlal Nehru Centre for Advanced Scientific Research (JNCASR)

2:30

On-Off Intermittency in Locally Connected Maps

Woosok Moon, Yale University

$3: 30$

Optimal Mixing on the Sphere

Amanda O’Rourke, Princeton University

Wednesday, August 25

10:00

Dynamics of the Mixed Layers in Stratified Shear Flows

Georgy Manucharyan, Yale University

11:00

Cave Rings

Emma Boland, University of Cambridge

$2: 30$

Trajectories of a Low Reynolds Number Treadmilling Organisim near a Half-Finite No-Slip Wall

Kiori Obuse, Kyoto University

$3: 30$

Who Ate Whom? Population Dynamics with Age-Structured Predation

David Goluskin, Columbia University

Thursday, August 26

$10: 30$

Snail Locomotion

Sam Pegler, University of Cambridge 


\title{
Lecture 1: Stirring and mixing
}

\author{
Jean-Luc Thiffeault
}

Notes by Sam Pegler and Amanda O’Rourke

21 June 2010

\section{Introduction}

Consider a patch of dye immersed in a flowing fluid, confined within a spatial domain $\Omega$. The concentration of the dye $\theta(\boldsymbol{x}, t)$ can be modelled by the advection-diffusion equation

$$
\frac{\partial \theta}{\partial t}+\boldsymbol{u} \cdot \nabla \theta=\kappa \nabla^{2} \theta
$$

where $\kappa$ is a coefficient of diffusivity and $\boldsymbol{u}(\boldsymbol{x}, t)$ is a prescribed velocity field. By using equation (1), it is assumed that the concentration is advected precisely with the flow, and is also subject to molecular diffusion by which the concentration is allowed to gradually spread to adjacent fluid elements. The velocity field in equation (1) is assumed to have been determined a priori and satisfies the incompressibility condition

$$
\boldsymbol{\nabla} \cdot \boldsymbol{u}=0
$$

We also assume that the boundary conditions

$$
\begin{aligned}
\boldsymbol{n} \cdot \boldsymbol{u} & =0 \\
\boldsymbol{n} \cdot \boldsymbol{\nabla} \theta & =0
\end{aligned}
$$

apply on the boundary of the domain $\partial \Omega$, where $\boldsymbol{n}$ is the unit outward normal to the boundary. These conditions ensure that no fluid penetrates the boundary of the domain and that no concentration is allowed to diffuse into or out of the domain, respectively. (We can also use spatially-periodic boundary conditions, which ensure that there is no net flux in or out of the domain.)

We proceed to demonstrate some general properties of the system defined by equations (1)-(4) by deriving evolution equations for the spatial mean and variance of $\theta$. To do this we begin by multiplying equation (1) by $m \theta^{m-1}$ and reordering the derivatives to give

$$
\frac{\partial}{\partial t}\left(\theta^{m}\right)+\boldsymbol{\nabla} \cdot\left(\theta^{m} \boldsymbol{u}\right)=m \kappa\left[\boldsymbol{\nabla} \cdot\left(\theta^{m-1} \boldsymbol{\nabla} \theta\right)-(m-1) \theta^{m-2}|\boldsymbol{\nabla} \theta|^{2}\right] .
$$

Now we take the volume integral over the domain, defined by

$$
\langle\phi\rangle \equiv \int_{\Omega} \phi \mathrm{d} V
$$


where $\phi(\boldsymbol{x}, t)$ is any function, which gives

$$
\frac{d}{d t}\left\langle\theta^{m}\right\rangle=-m(m-1) \kappa\left\langle\theta^{m-2}|\nabla \theta|^{2}\right\rangle,
$$

on applying the divergence theorem to the second term on the left-hand side of equation (5) and the first term on the right-hand side, and applying boundary conditions (3)-(4) to make the resulting boundary terms vanish. Setting $m=1,2$ gives the two equations

$$
\begin{aligned}
\frac{d}{d t}\langle\theta\rangle & =0, \\
\frac{d}{d t}\left\langle\theta^{2}\right\rangle & =-2 \kappa\left\langle|\nabla \theta|^{2}\right\rangle,
\end{aligned}
$$

respectively. The first equation above represents conservation of total concentration, which is expected given that no concentration enters the domain as ensured by boundary conditions (3)-(4). The second equation is an evolution equation for the second moment of $\theta$ in terms of its gradient. This equation is not closed, i.e. it does not independently determine the time evolution of $\left\langle\theta^{2}\right\rangle$, because its right-hand side depends on $|\nabla \theta|$, whose time evolution must be determined from a solution of the advection-diffusion equation (1). However, noting that the right-hand side of equation (9) is negative-definite if $\theta$ is not constant in space, we can deduce that the second moment of the concentration must decrease in time. Thus, at large times the second moment converges toward a constant value in which the right-hand side of (9) vanishes. This occurs if and only if the gradient of the concentration is zero everywhere. The system is driven toward a fully homogenized state in which the second moment takes the value

$$
\left\langle\theta^{2}\right\rangle=\frac{\langle\theta\rangle^{2}}{\Omega} .
$$

Note that although $\left\langle\theta^{2}\right\rangle$ never quite reaches this value, we say the system is mixed once it has fallen below a certain prescribed threshold.

As mentioned previously, equation (9) does not independently determine the rate at which $\left\langle\theta^{2}\right\rangle$ decreases in time. In fact, the rate at which $\left\langle\theta^{2}\right\rangle$ decreases usually depends strongly on the prescribed velocity field, which does not appear in equation (9). Importantly, the introduction of a velocity field, which we refer to as stirring, usually has the effect of increasing gradients in the concentration field $|\nabla \theta|$ through advection and thus has the potential to significantly accelerate the onset of mixing.

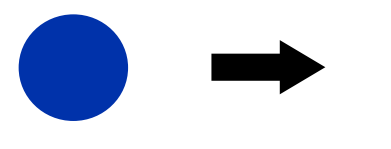

patch
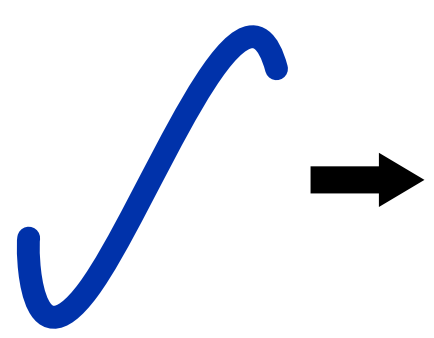

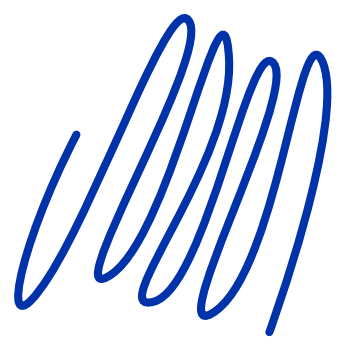

filaments

Figure 1: Typical mixing scenario in which a patch of dye is advected into filaments. 


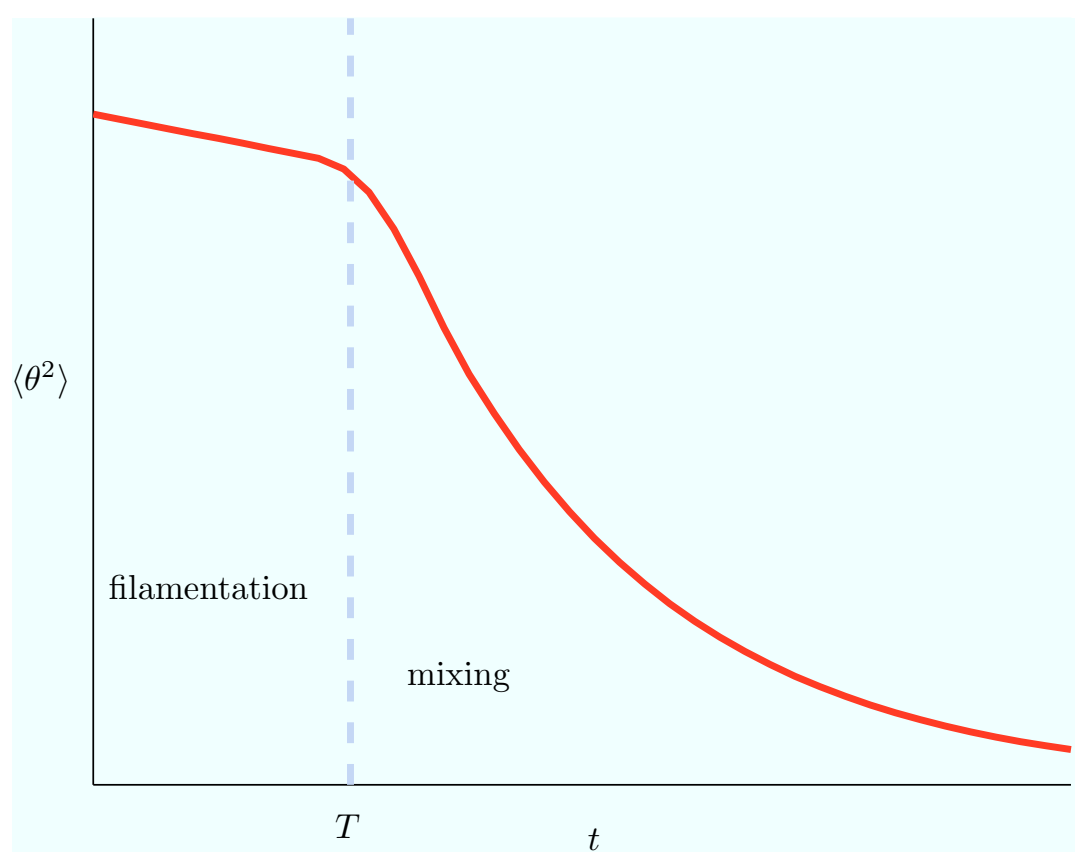

Figure 2: Decay of the second moment of the concentration field under the action of stirring.

A typical stirring scenario is illustrated in figure 1, in which an initially isolated patch of dye is advected into thin striations that exhibit a much greater concentration gradient than the initial patch. The evolution of the second moment, corresponding to this typical mixing scenario, is shown in figure 2. During the initial stirring phase, before thin filaments are formed, the concentration varies over relatively large length scales and the rate of mixing will be slow. After a short transient, by which point the filaments have become sufficiently thin, the rate of diffusion is large enough to balance the rate of thinning of the filaments and the rate of mixing $\partial\left\langle\theta^{2}\right\rangle / \partial t$ is no longer characterized by $\kappa$, but is instead characterized by the rate at which the velocity field is straining the fluid into filaments. Once this occurs the characteristic width of the filaments remains constant, and the rate of mixing reaches a maximum which persists for all time. The independence of the rate of diffusion on the parameter $\kappa$ during the mixing phase implies the scaling

$$
|\nabla \theta| \sim \kappa^{-1 / 2}
$$

which can be used as an indication of good mixing in practical applications.

The coefficient of diffusion $\kappa$ is small in most applications. Hence, the characteristic timescale over which diffusion takes place in the absence of an advective flow $(\boldsymbol{u}=\mathbf{0})$ is typically very long. For example, in the context of heat diffusing through air, $\kappa \approx$ $2.2 \times 10^{-5} \mathrm{~m}^{2} / \mathrm{s}$, so in a room with sides of length $L=10 \mathrm{~m}$, a characteristic diffusion timescale is $L^{2} / \kappa \sim 53$ days. Thus, if one is aiming to mix the concentration field, relying on diffusion in the absence of advection is typically ineffective. In the following section we present an example that can be solved analytically to demonstrate the development of the mixing phase discussed above. 


\section{The filament solution}

Consider the prescribed velocity field

$$
\boldsymbol{u}(\boldsymbol{x})=(\lambda x,-\lambda y),
$$

where $\lambda$ is a constant parameter, which drives fluid towards the $x$-axis in a straining motion with a hyperbolic point at the origin. In this case the advection-diffusion equation takes the form

$$
\frac{\partial \theta}{\partial t}+\lambda x \frac{\partial \theta}{\partial x}-\lambda y \frac{\partial \theta}{\partial y}=\kappa \nabla^{2} \theta
$$

This equation can be solved exactly, but for simplicity here we consider only $x$-independent solutions of the form

$$
\theta(y, t)=e^{-\lambda t} f(y)
$$

Substituting this into equation (13) gives the ordinary differential equation

$$
-\lambda f-\lambda y f^{\prime}=\kappa f^{\prime \prime}
$$

If we further assume that the concentration vanishes in the far field, $f \rightarrow 0$ as $y \rightarrow \pm \infty$, we can solve equation (15) and derive the solution

$$
\theta=e^{-\lambda t} e^{-y^{2} / 2 l^{2}}
$$

where

$$
l=\sqrt{\kappa / \lambda}
$$

is called the Batchelor length.

The solution (16) represents a filament that is an attractor for typical initial conditions of the system. For any reasonable initial concentration field, the compression due to the straining velocity will attempt to confine the concentration into an ever thinner region about the $x$-axis until its gradient becomes sufficiently large for diffusion to balance the rate of compression. The width of the resulting filament is characterized by the Batchelor length (17), which contains the necessary scaling for (11) to apply. Furthermore, we see from the solution (16) that the rate of mixing $\lambda$ during the mixing phase is indeed independent of $\kappa$. Note that equation (8) must be taken with a grain of salt, because the concentration does not vanish as $|x| \rightarrow \infty$. This is a consequence of the neglect of the $x$ dependence.

\section{Effective Diffusivity}

Recall that filaments undergoing advection are strained along one direction at a rate $\lambda$ and are compressed along the other to the Batchelor length $l=\sqrt{\kappa / \lambda}$, where $\kappa$ is the molecular diffusivity and $\lambda$ is the local rate of strain. In the previous section it was shown that the variance evolves according to

$$
\left\langle\theta^{2}\right\rangle \sim e^{-\lambda t}
$$

where $\lambda$ is a local rate of strain.

From simple arguments, molecular diffusivity alone is an exceedingly weak method for mixing scalar fields. We may then ask how stirring might improve tracer mixing and, more importantly, how we can quantify the effects of stirring by invoking an effective diffusivity. 


\subsection{A random walk model}

The advection-diffusion equation for a passive scalar $\theta=\theta(\boldsymbol{x}, t)$ can be written as

$$
\frac{\partial \theta}{\partial t}+\boldsymbol{u} \cdot \nabla \theta=\kappa \nabla^{2} \theta
$$

where $\boldsymbol{u}$ is a given velocity field and $\kappa$ is a constant of diffusivity. Even in the absence of stirring and the filamentation process, this diffusivity can act to mix the tracer field $\theta$ by monotonously decreasing the variance from equation (9).

The long timescales associated with diffusion alone indicates that the physical act of stirring, or the advective term $\boldsymbol{u} \cdot \nabla \theta$ of (18), is necessary to efficiently mix a tracer field. However, the advective term of (18) is more complex than the diffusivity. Ideally, one could write the effects of both the mixing of the tracer field by molecular diffusivity and by advective stirring as an effective diffusivity, such that the equations of motion can be approximated by

$$
\frac{\partial \theta}{\partial t}=\kappa \nabla^{2} \theta-\boldsymbol{u} \cdot \nabla \theta \approx \kappa_{e} \nabla^{2} \theta
$$

We consider the displacement, $x$, of an individual particle stirred by a random flow field. We would like to compare the displacemnt of the particle to a true random walk,

$$
x_{n}=x_{n-1}+\xi_{n}
$$

where $x_{n}$ is position of the particle at time $n$ and where $\xi_{n}$ is a random kick. Here we assume that all $\xi_{n}$ are independent and identically distributed random variables.

At the $n$th step, the particle position $x_{n}$ satisfies

$$
x_{n}=x_{0}+\sum_{k=1}^{n} \xi_{k}
$$

where $x_{0}$ is the initial position. If the initial position of the particle is $x_{0}=0$ and the mean individual kick $\left\langle\xi_{n}\right\rangle=0$, then the mean position of the particle at time $n$ is

$$
\left\langle x_{n}\right\rangle=\sum_{k=1}^{n}\left\langle\xi_{k}\right\rangle=0
$$

and the action of the random variations in the flow field given by $\xi$ do not change the mean position of the particle.

Consider instead the mean-squared displacement of the particle:

$$
\left\langle x_{n}^{2}\right\rangle=\sum_{k=1}^{n}=n\left\langle\xi^{2}\right\rangle=n \sigma^{2},
$$

where $\sigma^{2}=\left\langle\xi^{2}\right\rangle$. The mean-squared displacement of the particle then grows linearly with the number of steps, $n$, at a rate determined by the standard deviation of the random forcing field. 
For a process with an average length of time between kicks, $T$, the number of kicks can be converted into a time by letting $t=n T$. The squared displacement in (21) then grows linearly in time as

$$
\left\langle x^{2}(t)\right\rangle=2 \kappa t
$$

where $\kappa=\sigma^{2} / 2 T$ is a diffusivity, or a measure of how quickly the squared displacement of the particle grows in time.

The above calculations are only in one dimension. In $d$ dimensions, the mean-squared distance from the origin can be written as a sum of the mean-squared distance $\left\langle x_{k, n}^{2}\right\rangle$ along each dimension $k$ at timestep $n$,

$$
\left\langle\sum_{k=1}^{d} x_{k, n}^{2}\right\rangle=\left\langle x_{1, n}^{2}\right\rangle+\left\langle x_{2, n}^{2}\right\rangle+\ldots+\left\langle x_{d, n}^{2}\right\rangle
$$

which, assuming the random forcing is isotropic such that $\left\langle x_{k, n}^{2}\right\rangle=\left\langle x_{n}^{2}\right\rangle$, can also be written as

$$
d\left\langle x_{n}^{2}\right\rangle=d n \sigma^{2}=2 d \kappa t
$$

where $\kappa=\sigma^{2} / 2 T$ is the same as in the one dimensional case.

The diffusivity, $\kappa$, can be thought of as an effective diffusivity as it arises from the physical stirring generated by the stochastic process $\xi_{n}$. The effective diffusivity is again given by

$$
\kappa_{\text {eff }}=\frac{\sigma^{2}}{2 T}
$$

where $\sigma^{2}$ is the standard deviation of the particle displacement due to the random kicks $\xi_{n}$ and $T$ is the average interval between kicks.

\subsection{Particle concentration and diffusivity}

The random walk description of a diffusive process above applies only to a single particle with position given by $\boldsymbol{x}_{n}$. We wish to consider the evolution of a cloud of particles. Let $\theta(\boldsymbol{x}, t)$ be the density of particles within a given area (or volume in $3 \mathrm{D}$ ); then $\theta$ satisfies the diffusive equation

$$
\frac{\partial \theta}{\partial t}=\kappa_{e} \nabla^{2} \theta
$$

if each point evolves independently according to a random walk $\boldsymbol{x}_{n+1}=\boldsymbol{x}_{n}+\xi_{n}$.

The idea of a cloud of particles only works if the spatial and temporal resolution of the model are large enough such that the motion of individual particles are decorrelated. If we are zoomed in too closely, such that the grid scale is less than the standard deviation of the displacements $(L<\sigma)$, then we see only individual particles rather than a cloud of particles with a given concentration, $\theta$. If we choose sampling timescales that are too short, such that the timescale of the flow is less than the interval between kicks, $T$, then the kicks become correlated in time. 


\subsection{Numerical examples for sine flow}

Consider, for example, the advection of a cloud of particles by the famous sine flow in

a doubly-periodic domain [2]. The velocity field of the sine flow alternates between a horizontally-aligned shear flow and vertically-aligned shear flow given by

$$
\begin{array}{ll}
\boldsymbol{u}_{H}=(U \sin (2 \pi k y / L), 0) & \text { for } 0 \leq t<\frac{1}{2} \tau ; \\
\boldsymbol{u}_{V}=(0, U \sin (2 \pi k x / L)) & \text { for } \frac{1}{2} \tau \leq t<\tau,
\end{array}
$$

where here $U$ is a constant velocity, $k$ is the wavenumber of the shear flow field, $L$ is the size of the biperiodic domain, and $\tau$ is the period of the flow.

We then obtain a map of the position of the particle by solving

$$
\dot{\boldsymbol{x}}=\boldsymbol{u}, \quad \boldsymbol{x}=\boldsymbol{x}_{0}
$$

exactly as a two-step process:

$$
\begin{array}{ll}
\text { Step 1: } \quad & x(\tau / 2)=x_{0}+U \frac{\tau}{2} \sin \left(\frac{2 \pi k y_{0}}{L}\right) \\
& y(\tau / 2)=y_{0} \\
\text { Step 2: } \quad & x(\tau)=x(\tau / 2) \\
& y(\tau)=y(\tau / 2)+U \frac{\tau}{2} \sin \left(\frac{2 \pi k x(\tau / 2)}{L}\right) .
\end{array}
$$

At the end of an interval of length $\tau$, the new position $\left(x^{\prime}, y^{\prime}\right)$ of a particle that started at $(x, y)$ is then

$$
\begin{aligned}
& x^{\prime}=x+T \sin \left(\frac{2 \pi k y}{L}\right) \\
& y^{\prime}=y+T \sin \left(\frac{2 \pi k x^{\prime}}{L}\right)
\end{aligned}
$$

where $T=U \tau / 2$. For a constant velocity, $U, T$ is proportional to $\tau$ and the intensity of stirring can be manipulated by varying $T$. Note that in the second step of $(26), y^{\prime}$, is a function of $x^{\prime}$ and not $x$. This is necessary to conserve area, as can be verified by computing the Jacobian of the map (26).

\subsection{Diffusivity and the limit of small $T$}

We can vary the period of the sine flow given in (26) by varying $T$. For small $T$, the period of the stirring is less than the timestep of integration and one can expect the flow to appear nondiffusive. Indeed, in the limit of small $T$, the sine map given in (26) approaches a symplectic integrator [3]. This result can be obtained by noting that

$$
\frac{x^{\prime}-x}{T}=\sin \left(\frac{2 \pi k y}{L}\right) \quad \frac{y^{\prime}-y}{T}=\sin \left(\frac{2 \pi k x^{\prime}}{L}\right)
$$




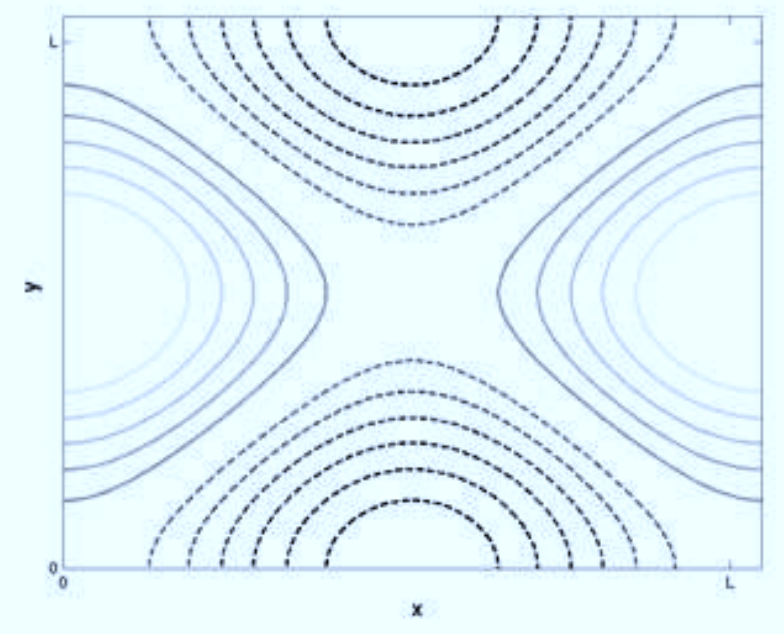

Figure 3: Streamfunction in the limit that $T \rightarrow 0$. The flow field then satisfies $\boldsymbol{u}=$ $\left(\partial_{y} \psi,-\partial_{x} \psi\right)$ so that the flow is clockwise where $\psi$ is negative (dashed) and counterclockwise where $\psi$ is positive (solid).

and by taking the limit as $T \rightarrow 0$. In this limit, $\frac{x^{\prime}-x}{T} \rightarrow \frac{d x}{d t}$ etc. such that

$$
\frac{d x}{d t}=\sin \left(\frac{2 \pi k y}{L}\right) \quad \frac{d y}{d t}=\sin \left(\frac{2 \pi k x}{L}\right) .
$$

Equation (27) is equivalent to saying the nondivergent velocity field, $\boldsymbol{u}=d \boldsymbol{x} / d t$, can be written in terms of a streamfunction $\boldsymbol{u}=\left(\partial_{y} \psi,-\partial_{x} \psi\right)$ where

$$
\psi=\frac{L}{2 \pi k}\left(\cos \left(\frac{2 \pi k x}{L}\right)-\cos \left(\frac{2 \pi k y}{L}\right)\right) .
$$

A contour plot of $\psi$ is given in figure 3 .

Consider now the advection of a cloud of particles displaced by the map (26). If we let $L=k=1$ and use a relatively small value of $T, T=0.1$, the flow follows nearly closed orbits as given in figure 4. As this flow closely approximates the streamfunction solution, the stirring given by (26) does not diffuse the concentration of particles. As a result, the use of an 'effective' diffusivity does not apply here.

While the orbit given in figure 4 is not strictly confined to the streamfunction in (28) because of the use of a finite $T$, it is not space filling. We can add noise to the sinemap though

$$
\begin{aligned}
& x^{\prime}=x+T \sin \left(\frac{2 \pi k y}{L}\right)+\sqrt{2 D} a \\
& y^{\prime}=y+T \sin \left(\frac{2 \pi k x^{\prime}}{L}\right)+\sqrt{2 D} b
\end{aligned}
$$

where $a$ and $b$ are Gaussian random variables with zero mean and unit variance. Here $D$ is a constant that controls the amplitude of the noise and can be consider a molecular diffusivity. 


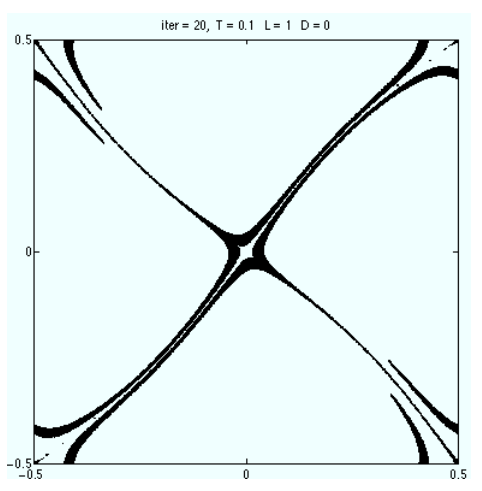

(a) $D=0, T=0.1$, and $i t=20$

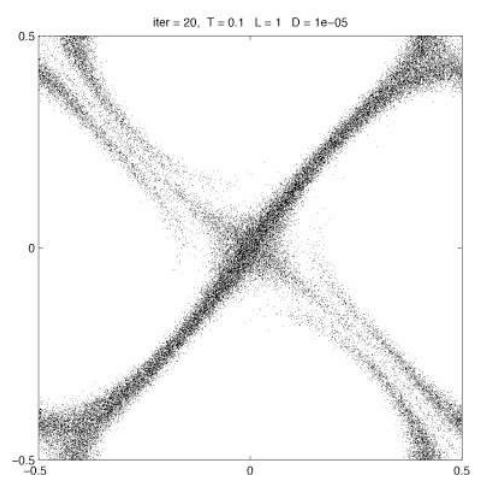

(b) $D=10^{-5}, T=0.1$, and $i t=20$

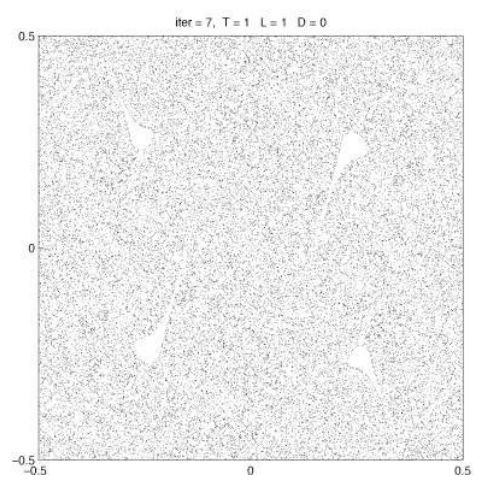

(c) $D=0, T=1$, and $i t=7$

Figure 4: Particle concentration in a box of sides $L=1$ and stirring timestep $T=1$ after 20 iterations. There is no noise in (a) and the map is confined near the streamlines given in 3. With the addition of noise, where $D=10^{-5}$, the orbit becomes diffusive. Figure 4(c) is the same as in $4(\mathrm{a})$ but with $T=1$ and is shown only at the 7th iteration.

In the absence of stirring, (29) becomes the two-dimensional version of the random walk model given in (20). As we have chosen $\left\langle a^{2}\right\rangle=\left\langle b^{2}\right\rangle=1$, the standard deviation $\sigma^{2}$ of the noise is simply $2 D$.

If we let $D=10^{-5}$ (figure $4(\mathrm{~b})$ ), the near-integrability of the map of figure 4(a) is broken, however the flow still remains constrained to near the streamlines in (28) for a moderate number of iterations. The addition of this molecular diffusivity, $D$, will cause the trajectory to eventually fill the domain. However, this process is quite slow.

The diffusive behavior captured by adding random noise in figure 4(b) can also be obtained by increasing the step size even in the absence of noise. For a box size $L=1$ and with $D=0$ and a timestep of $T=1$, ten times longer than that of the examples given in figure 4 , the flow no longer follows the streamfunction given in (28). The flow in figure $4(\mathrm{c})$ is chaotic and quite rapidly fills the entire domain with the tracer after only seven iterations.

\subsection{Filament width and the Batchelor length}

The Batchelor length, $l=\sqrt{\kappa / \lambda}$, is the approximate scale at which filaments, stretched by stirring, become so thin that the tendency for the filament to become narrower due to stirring is balanced by the diffusion of the patch. In the definition of the Batchelor length, $\lambda$ is the local rate of strain. In the sine map given in (26), modified with the diffusion equation of (29), the rate of strain is approximately $T$ and the coefficient of "molecular" diffusion is $D$. For this problem, then, the width of the stirred filaments should scale approximately as

$$
l=\sqrt{D T}
$$

such that the Batchelor length scales with $\sqrt{D}$ for fixed $T$.

Snapshot of the concentration fields for 'strong' diffusivity, with $D=10^{-4}$, and 'weak' diffusivity, with $D=10^{-6}$, are given in 5 (a) and $5(\mathrm{~b})$, respectively. The width of a typical 


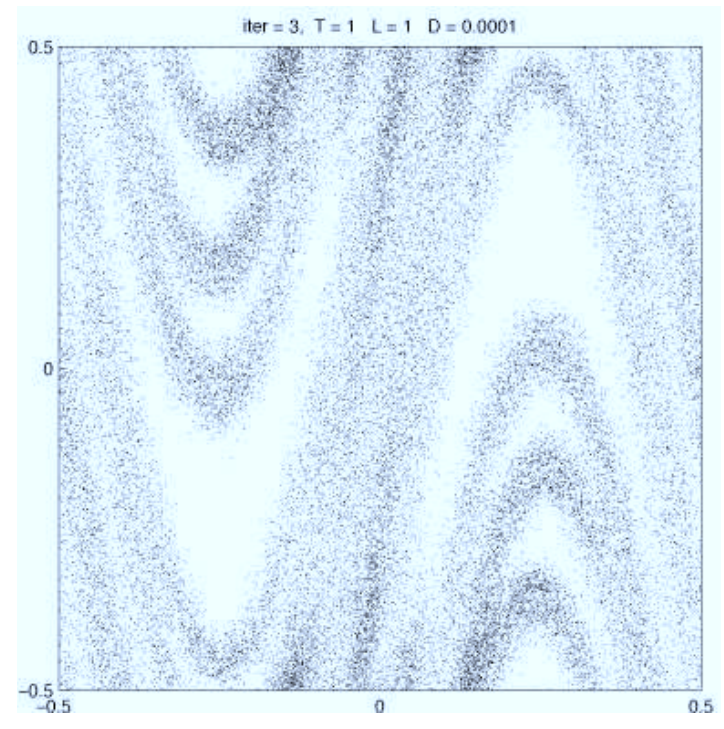

(a) $D=10^{-4}$

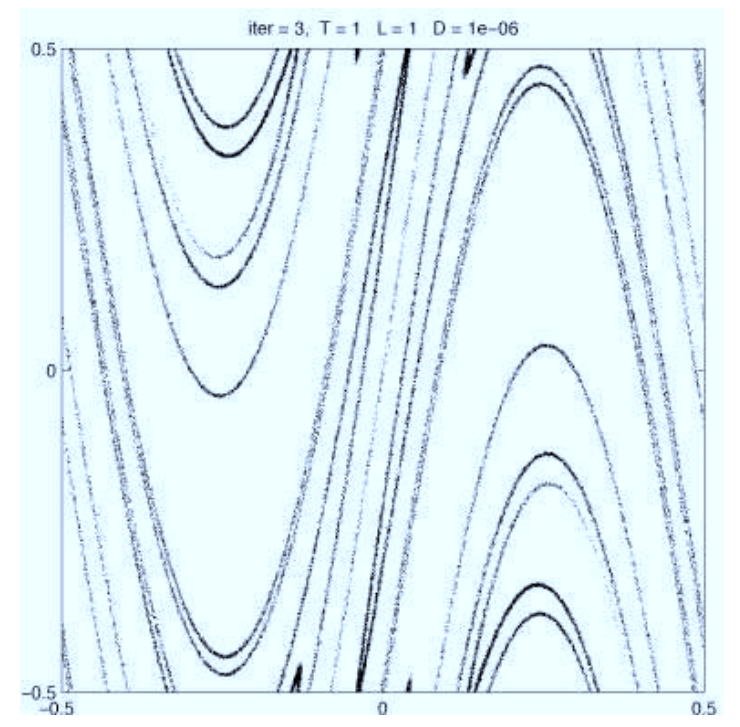

(b) $D=10^{-6}$

Figure 5: Particle concentration in a box of sides $L=1$ with $T=1$ and with varying $D$ at iteration 3. The width of the filaments scale as the Batchelor length given in (30).

filament in the 'strong' diffusivity case is 0.109 nondimensional units while a 'weakly' diffusive filament is approximately 0.006 nondimensional units. The width of the filaments for these two cases do roughly scale with $\sqrt{D}$ as suggested from (30).

\subsection{Effective diffusivity and the domain size, $L$}

For the effective diffusivity scaling given in (25) to hold for a stirred fluid, the length scale of the stirring must be less than that of the domain. In the examples above, the length scale of the stirring was the same as that of the domain. If we increase the size of the domain to $L>1$ while holding $T$ at an intermediate value, $T=1 / 2$, the concept of an 'effective' diffusivity becomes relevant.

Shown in figure 6 are snapshots of the particle concentration field after ten iterations for a domain size varying from $L=1$ to $L=25$ while the spatial correlations $\left\langle x^{2}\right\rangle,\left\langle y^{2}\right\rangle$, and $\left\langle X^{2}\right\rangle=\left\langle x^{2}+y^{2}\right\rangle$ and cross-correlations $\langle x y\rangle$ are shown in figure 7. For small domain sizes, such as in 6(a) and 6(b), the sine flow stirring efficiently fills the domain with the tracer. This homogenization of the tracer field is evident in 6(c) and 6(d) by the leveling off of the correlation values with time.

In cases with a relatively small domain size, such as $6(\mathrm{a})$ and $6(\mathrm{~b})$, the spatial variances for $\left\langle x^{2}\right\rangle,\left\langle y^{2}\right.$, and $\left\langle X^{2}\right\rangle$ do not grow linearly with time as predicted from the effective diffusivity equations in one dimension, equations (22) and (23). The concept of an effective diffusivity fails in cases where the stirring is at approximately the same scale as the domain, as predicted.

Increasing the domain size to $L=10$ and $L=25$ while holding the scale of the stirring fixed, however, shows a more promising diffusive-like behavior. The tracer concentration in 6(c) and 6(d) spreads outward from an initial patch due to the stirring with spatial variances 


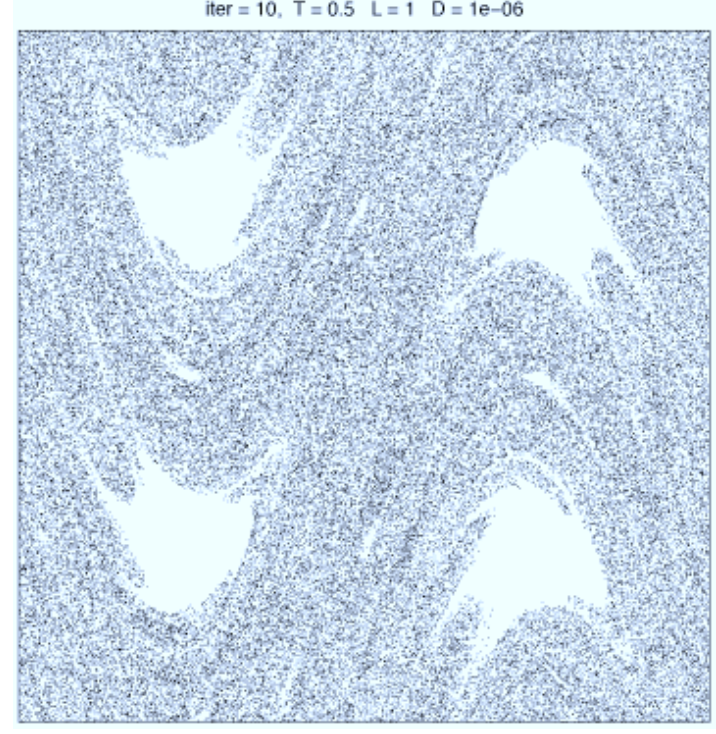

(a) $L=1$

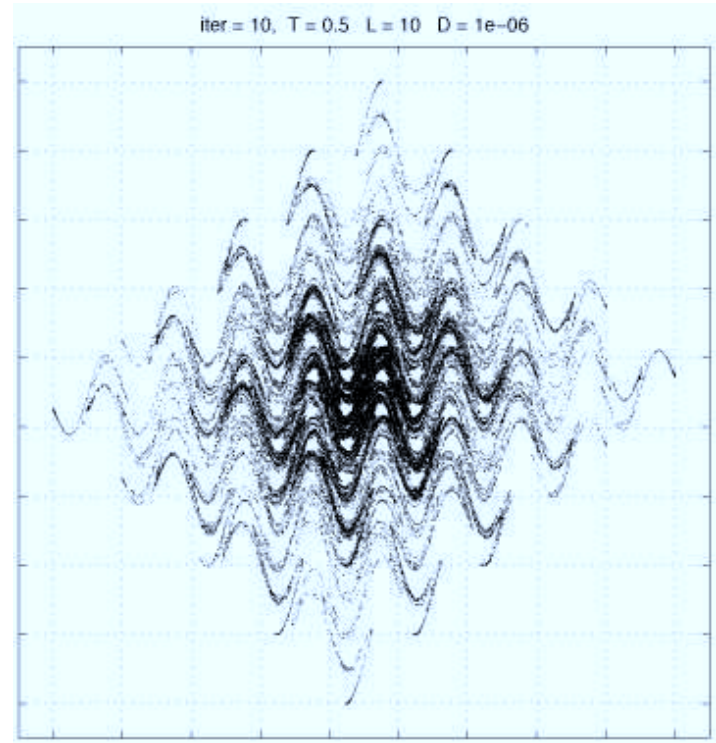

(c) $L=10$

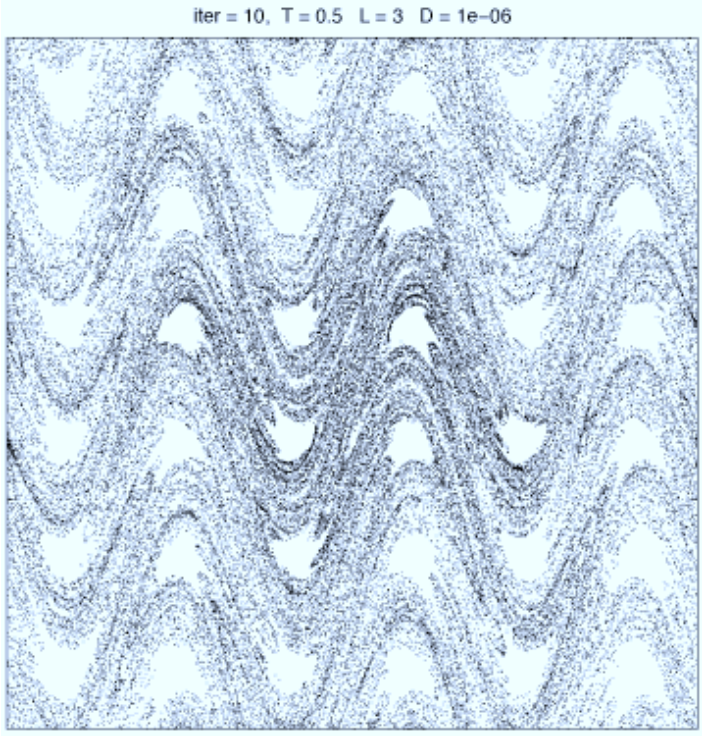

(b) $L=3$

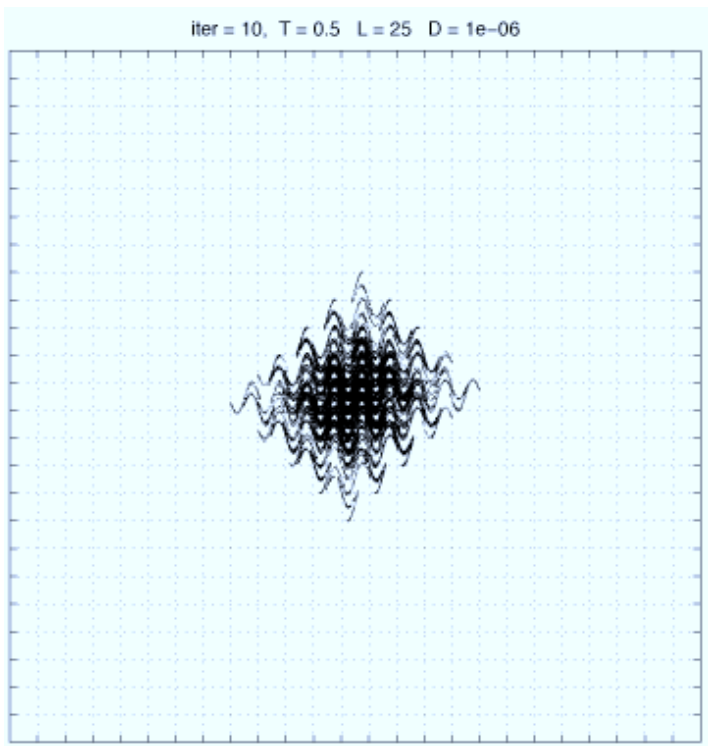

(d) $L=25$

Figure 6: Particle concentration in a box of of domain size of $L=1$ in $6(\mathrm{a}), L=3$ in 6(b), $L=10$ in $6(\mathrm{c})$, and $L=25$ in $6(\mathrm{~d})$ with $T=1$ and with $D=1 \times 10^{-6}$ at iteration 10 .

growing linearly with time, as shown in figures $7(\mathrm{c})$ and $7(\mathrm{~d})$.

Note that the variances in the $x$ and $y$ directions for all domain scales indicate isotropy, as shown by the similar behavior of $\left\langle x^{2}\right\rangle$ and $\left\langle y^{2}\right\rangle$ in figure 6 . This agrees with the assumption that the variances in multiple dimensions have the same statistics/standard deviation as we assumed in the derivation of the multi-dimensional form of the effective diffusivity equation, (23). Additionally, the cross-correlation between the $x$ and $y$ spatial dimensions, $\langle x y\rangle$, is 


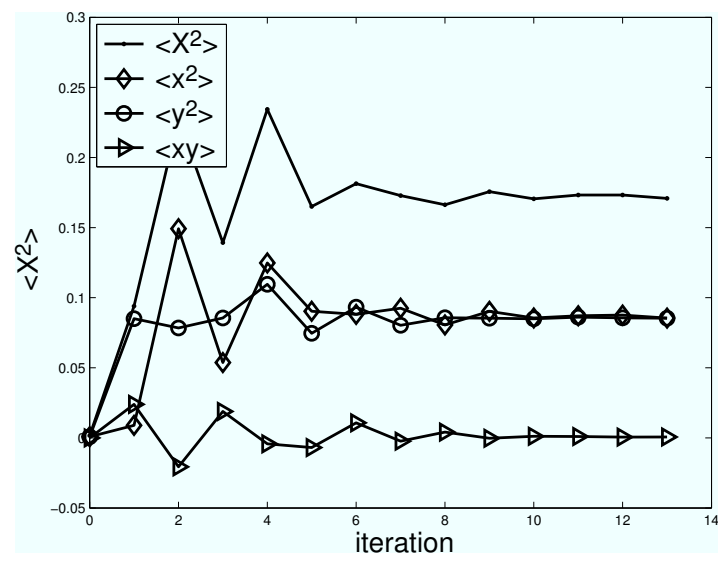

(a) $L=1$

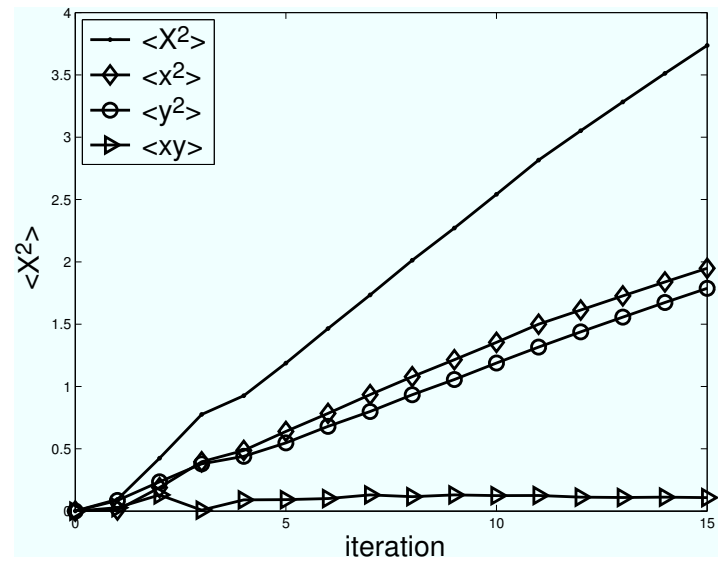

(c) $L=10$

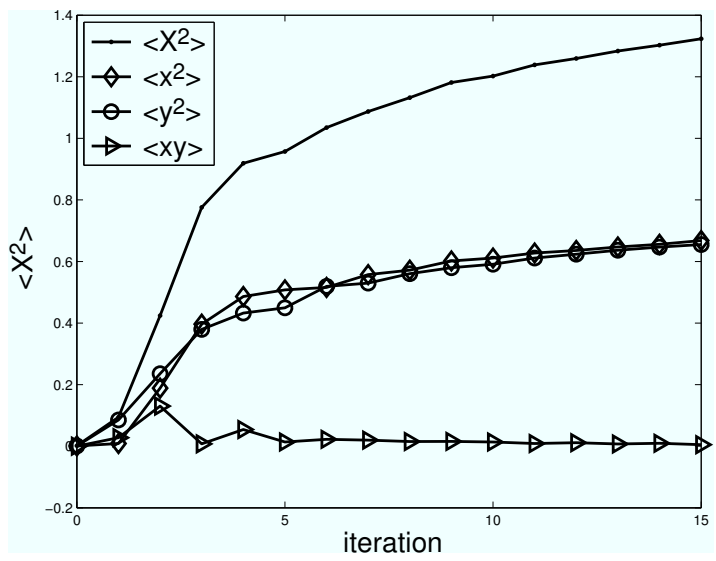

(b) $L=3$

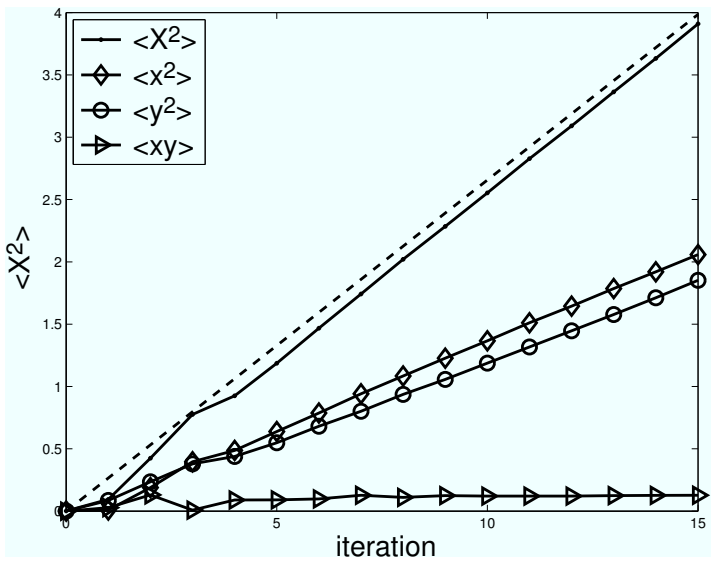

(d) $L=25$

Figure 7: Calculations of the spatial variance for the examples shown in 6 as a function of iteration and increasing domain size.

zero at long times.

From (23), we calculate the approximate effective diffusivity for the $L=25$ example as $\kappa=0.068$, four orders of magnitude larger than the molecular diffusivity, $D=10^{-6}$ the stirring in this case more efficiently disperses the tracer field than molecular diffusivity alone.

\section{Matlab Codes}

\section{1 run_sinemap.m}

Listing 1: run_sinemap.m

function [varargout] = run_sinemap $(T, L, D, N p, s q w)$

if $\mathrm{nargin}<1, \mathrm{~T}=1$; end

if nargin $<2, L=1$; end 


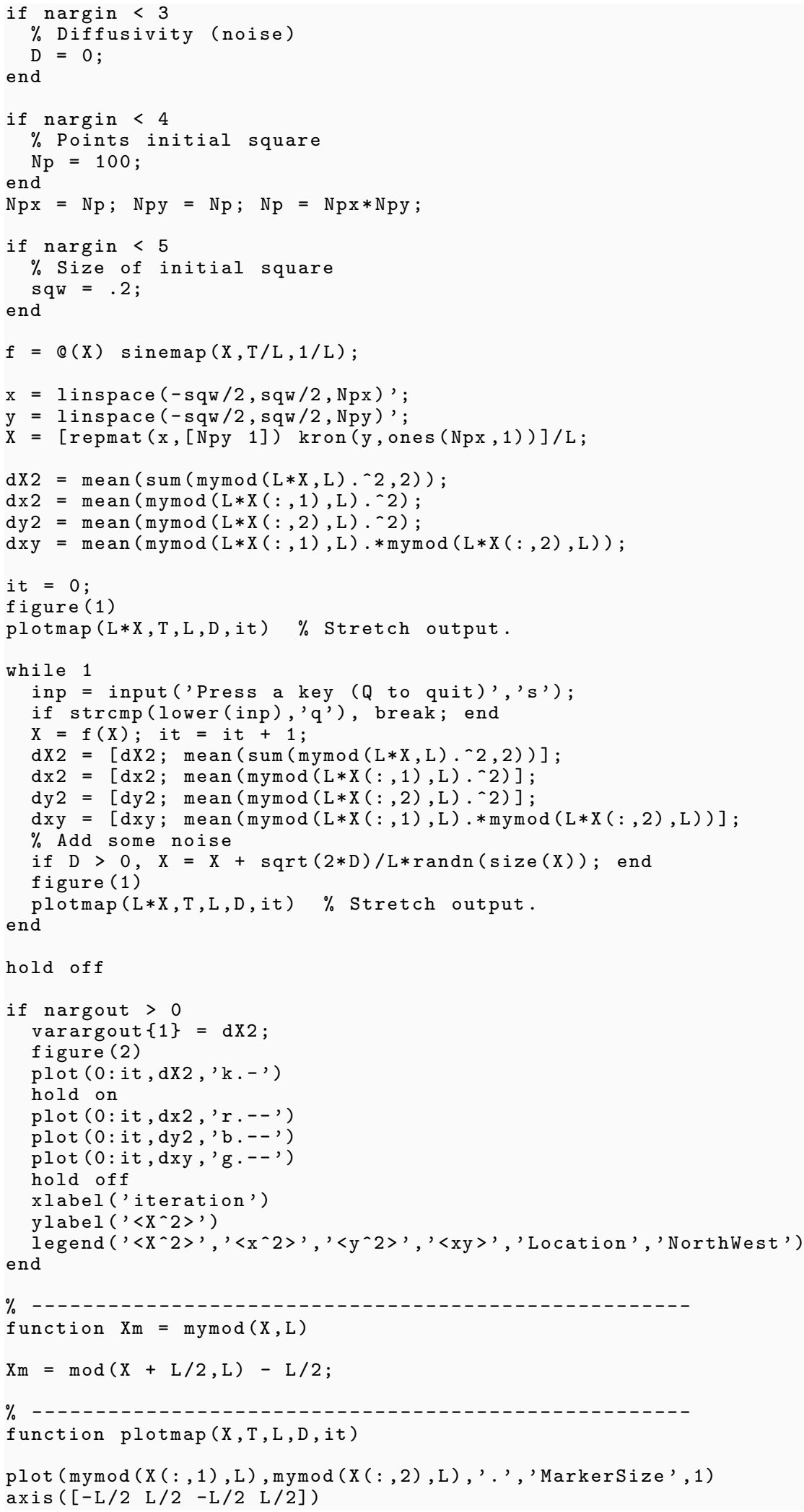




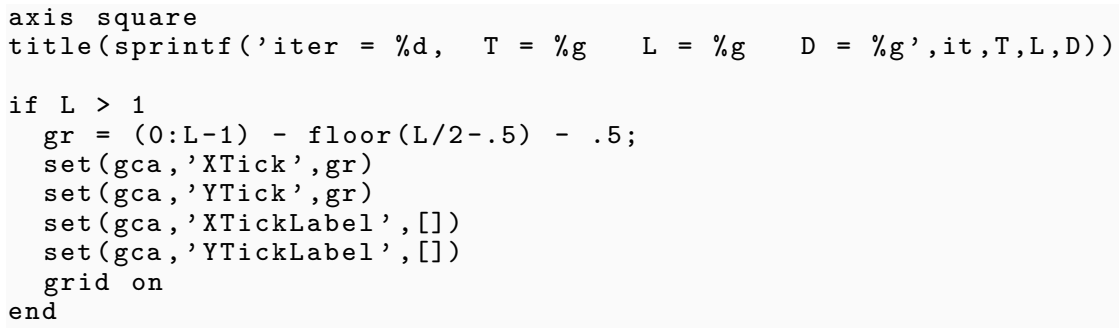

3.2 sinemap.m

Listing 2: sinemap.m

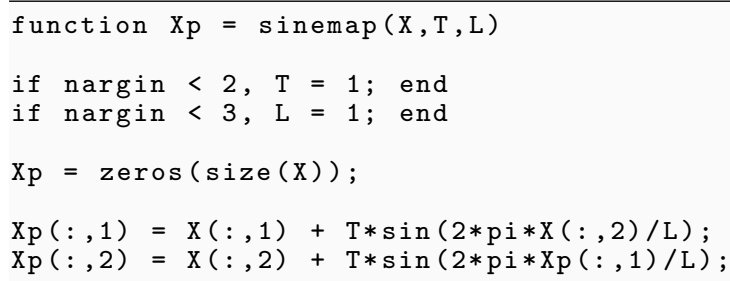

\section{References}

[1] G. K. Batchelor, Small-scale variation of convected quantities like temperature in turbulent fluid: Part 1. General discussion and the case of small conductivity, J. Fluid Mech., 5 (1959), pp. 113-133.

[2] R. T. Pierrehumbert, Tracer microstructure in the large-eddy dominated regime, Chaos Solitons Fractals, 4 (1994), pp. 1091-1110.

[3] W. R. Young, Stirring and mixing, in Proceedings of the 1999 Summer Program in Geophysical Fluid Dynamics, J.-L. Thiffeault and C. Pasquero, eds., Woods Hole, MA, 1999, Woods Hole Oceanographic Institution. http://gfd.whoi.edu/proceedings/1999/PDFvol1999.html. 


\title{
Introduction to Biological Models
}

\author{
Lecturer: Glenn Flierl
}

May 11, 2011

\section{Introduction}

The ocean is inhabited by innumerable individuals of many different genera and species, each having its own developmental/physiological state and each being immersed in its own environment. The organisms move, both because of water flow and because of their own swimming or buoyancy, and interact with their environment by gathering resources which they need and by excreting waste products. The assimilated material can be used for maintenance, growth, or reproduction. Finally, the organisms can die either from natural causes or because of attacks by another organism.

Furthermore, the processes just described must generally be regarded as stochastic. For example, the probability of a predator capturing a prey item will depend on multiple factors, each with its own probability: finding a prey item in range, the choice to attack, success in the attack, competition against others. Such a description suggests an "agent-based" or "individual-based" model (IBM), with each agent carrying information about its position, its species, its physiological state, etc. Individuals can grow, reproduce, and die. Certainly, we can build small versions of such models, but the number of individuals is necessarily limited (compared to nearly 20,000 copepods per cubic meter or to phytoplankton densities on the order of $10^{8}$ per cubic meter). However, such experiments may indeed give insight into the way in which the local, stochastic interactions translate into terms representing, for example, grazing rates in terms of average densities.

Or we could take the alternative view of attempting to predict the probability distribution for biomass in a continuous space, in this case using something like weight and species as our variables. The latter is, of course, discrete, yet different organisms can be genetically or, more importantly, functionally quite close to others. If we choose a species ordering such that the maximum growth rate varies smoothly, we may expect that other terms entering the dynamics such as the losses by predation will also fall on a fairly smooth curve. Certainly on any diagram such as figure (1) the gaps will be so small as to be negligible, and viewing the ordinate as a continuous variable is not unreasonable. We can then consider the ways that the processes described above alter the biomass distribution in this space.

As in the individual-based model, the number of variables we would have to consider is still unmanageably large. Furthermore, for each $(w, s p)$, we need to specify the sources, the sink, and the transfer rates, including possible nonlinear dependence on the local biomass $b(w, s \mid \mathbf{x}, t)$ and the density at the source/sink $b(w, s \mid \mathbf{x}, t)$.

Any attempt to construct such a model or the IBM model, will inevitably point out how little we know about most of the species inhabiting the ocean. We retreat to dealing 


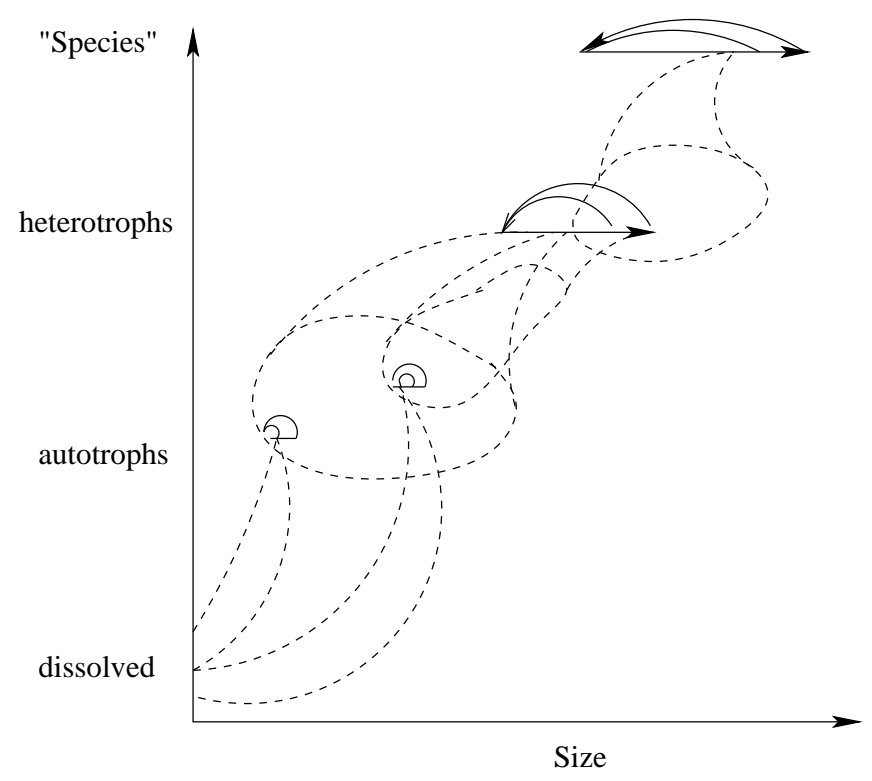

Figure 1: The range of sizes for various species

with variables such as $\mathrm{P}$ which can be viewed as the integral over some range $(s 1->s 2)$ of species and over weight. We then presume that transfers into and out of the resulting black-boxes can be represented as functions just of the integrated values and attempt to parameterize those.

\section{Basic biological models}

Exponential growth

Suppose each individual (or pair) reproduces in a time order $\tau$, then the time evolution of the population would be described as

$$
\begin{aligned}
b(t+\delta t) & =[1+g \delta t] b(t), \quad g=\ln 2 / \tau \\
\frac{\partial b}{\partial t} & =g b \Rightarrow b(t)=b_{\circ} \exp (g t) .
\end{aligned}
$$

where $b$ is the population and $g$ is its growth rate. This model predicts an unrealistic exponential growth of the population (see figure (2)). Thus, some limiting factors should exist.

Resource limitation

For example, one could write a model with the growth rate depending on a resource $R$ which itself evolves with time:

$$
\begin{aligned}
\frac{\partial b}{\partial t} & =(g R-d) b \\
\frac{\partial R}{\partial t} & =-g R b-\lambda\left(R-R_{\circ}\right)
\end{aligned}
$$




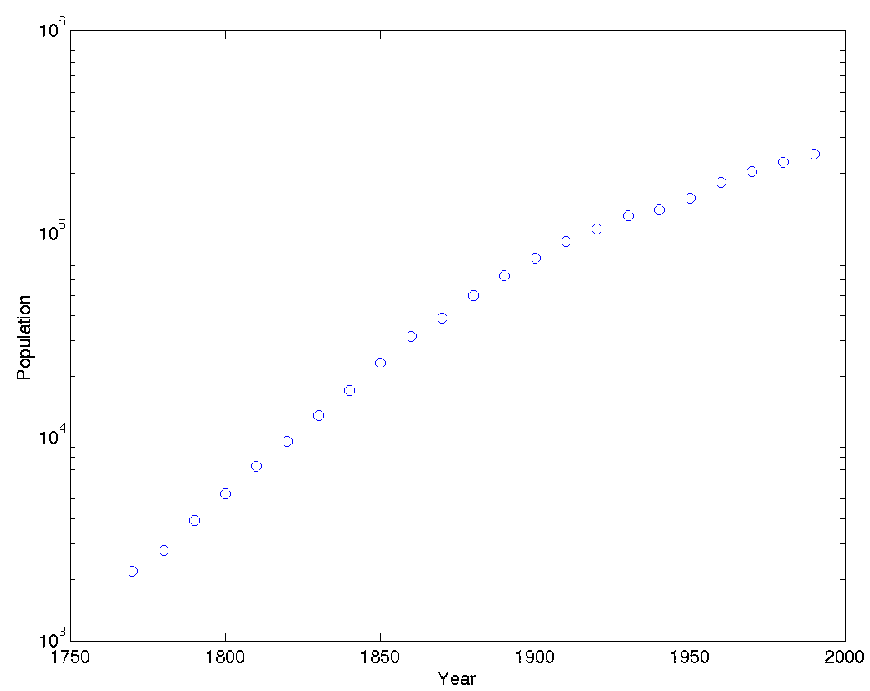

Figure 2: USA population as a function of time showing exponential growth at initial stage, but a decay of a growth rate at later stages

Here, the population evolves with a growth rate $g R$ and a death rate $d$. The resource decreases due to consumption by population and is restored to $R_{\circ}$ with a time scale $1 / \lambda$. This model allows for a steady state solution with $\bar{R}=d / g, \bar{b}=-\lambda\left(d / g-R_{\circ}\right) / d$. However, the growth rate is unconstrained with increase of the resource; a more realistic growth model would have to limit it at $R \rightarrow \infty$. One possibility is to use the form $g \frac{R}{R+k}$.

\section{Predator-pray}

Assume that zooplankton $(Z)$ grows by consuming phytoplankton $(P)$ and phytoplankton grows due to source of nutrients $(N)$ assumed to be fixed; both have natural death rates $d_{P}, d_{Z}$

$$
\begin{aligned}
& \frac{\partial P}{\partial t}=g P Z-\mu N P-d_{P} P \\
& \frac{\partial Z}{\partial t}=\alpha g P Z-d_{Z} Z
\end{aligned}
$$

This model (the Lotka-Volterra equations) gives a solution with cycles that have a conserved quantity $H=\alpha g P-d_{Z} \ln P+\mu N Z+d_{P} \ln Z$. The quadratic NPZ model assumes $N=N_{T}-P-Z$, where $N_{T}$ is a total amount of nutrients. This model has three steady states if $N_{T}>\left(d_{Z} / \alpha g\right)+\left(d_{P} / \mu\right)$, with a $P, Z$ non-zero point being a stable attractor.

$N P Z D$

The NPZ model assumes all dead organisms or excreted material is immediately remineralized to usable nutrient. In contrast, the NPZD model assumes that dead organisms and unassimilated phytoplankton would contribute to a detrital pool that eventually be- 


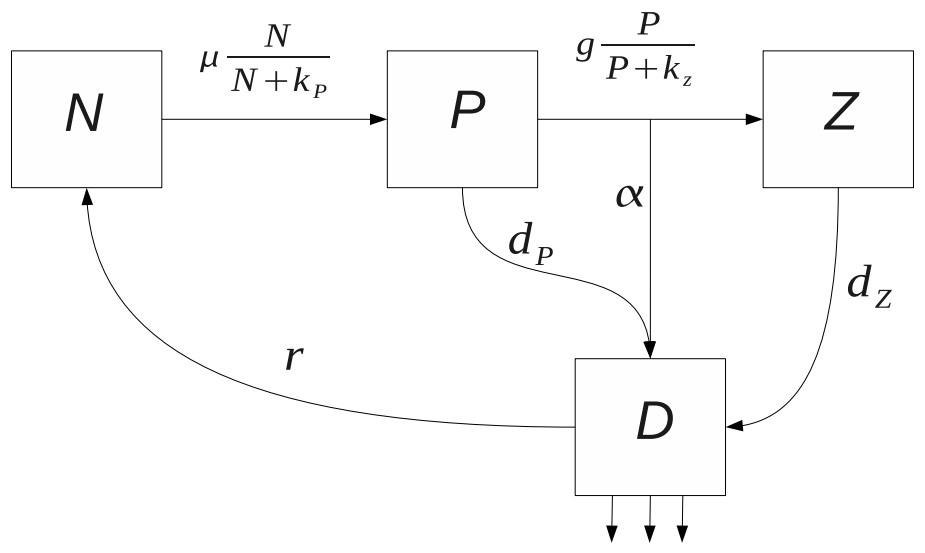

Figure 3: Schematic representation of NPZD model showing the fluxes of biomass and the parameters controling them

comes a source of nutrients. A schematic representation of this model is shown in figure (3), and a typical version of the corresponding equations (including sinking of detritus) would be:

$$
\begin{aligned}
\frac{D}{D t} P & =\mu \frac{N}{N+k_{P}} P-g \frac{P}{P+k_{Z}} Z-d_{P} P+\nabla \cdot(\kappa \nabla P) \\
\frac{D}{D t} Z & =\alpha g \frac{P}{P+k_{Z}} Z-d_{Z} Z+\nabla \cdot(\kappa \nabla Z) \\
\frac{D}{D t} D+\frac{\partial}{\partial z} w_{s} D & =(1-\alpha) g \frac{P}{P+k_{Z}} Z+d_{P} P+d_{Z} Z-r D+\nabla \cdot(\kappa \nabla D) \\
\frac{D}{D t} N & =r D-\mu \frac{N}{N+k_{P}} P+\nabla \cdot(\kappa \nabla N)
\end{aligned}
$$

where $\frac{D}{D t}=\frac{\partial}{\partial t}+\mathbf{u} \cdot \nabla$ denotes the material derivative of the advected scalar.

In general case the system equations have the form

$$
\frac{D}{D t} b_{i}+\nabla \cdot\left(\mathbf{u}_{i} b_{i}\right)=B_{i}(\mathbf{x}, t \mid \mathbf{b})+\nabla \cdot\left(\left(\kappa+\kappa_{i}\right) \nabla b_{i}\right)
$$

Here, $\mathbf{u}_{i}$ and $\kappa_{i}$ represent the motion relative to the fluid. The function $B_{i}(\mathbf{x}, t \mid \mathbf{b})$ represents interactions between different parts of the system. Some times a condition $\sum B_{i}(\mathbf{x}, t \mid \mathbf{b})=0$ is used, which assumes that the total biomass is conserved; this reduces the order of the system.

Such biological models require a large number of parameters. For example, in the NPZD model with 4 variables, the parameters are: $\mu, k_{P}, k_{Z}, g, d_{P}, d_{Z}, \alpha, r$ in addition to the 
biological movement term $w_{s}$ and physical parameters such as $\kappa$; together, they determine the fundamental behavior of the system. Thus, in addition to the mathematical question of the behavior of the equations for different parameter values, modellers need to decide on numbers which seem to give "realistic" solutions. Since the variables are not generally directly observed, comparisons with data tend to be pretty qualitative in an case.

Controlled (i.e., laboratory) experiments can be a guid for parameter choicese; however the behavior of the organisms might be affected by the laboratory constraints so that things like predation rates may be quite different. Another way is to observe the biosystem in the nature, but in reality the system is much more complicated than the model, and this can also introduce large uncertainties.

\section{Stochastic Dynamics}

An alternative method for modeling organism's population dynamics is through the use of probability density functions for the population size. The probability density functions will be written as $\mathcal{P}(n, t)$ which represents the probability of having a population of size $n$ at time $t$. In case of multiple interacting populations, the evolution of the population probability is based on the equation

$$
\mathcal{P}(\mathbf{z}, t+\delta t)=\sum \mathcal{P}_{T}(\delta \mathbf{z} \mid \mathbf{z}-\delta \mathbf{z}) \mathcal{P}(\mathbf{z}-\delta \mathbf{z}, t)
$$

where $\mathbf{z}$ is a vector representing the population of the various species considered in the model and $\mathcal{P}_{T}$ is the transition probability. This equation is essentially an application of the law of total probability. The probability of a specific population at a future time step is the sum of all possible previous populations times the probability of transitioning to the specified population during the given time step. For one of the species, the transition probability can be modeled as

$$
\mathcal{P}_{T}= \begin{cases}n d_{n} \delta t & \delta n=-1 \\ 1-n g_{n} \delta t-n d_{n} \delta t & \delta n=0 \\ n g_{n} \delta t & \delta n=1\end{cases}
$$

where $g_{n}$ and $d_{n}$ are the growth and death rates per capita depending on the population size $n$.

To make this more tractable, let us consider a case where only one population is modeled. For simplification we will change the notation from $\mathcal{P}(n, t)$ to be $\mathcal{P}_{n}(t)$. Equation 13 becomes

$$
\begin{aligned}
\mathcal{P}_{n}(t+\delta t) & =\mathcal{P}_{n-1}(t)(n-1) g_{n-1} \delta t+\mathcal{P}_{n}\left(1-n g_{n} \delta t-n d_{n} \delta t\right)+\mathcal{P}_{n+1}(n+1) d_{n+1} \delta t \\
\frac{\mathcal{P}_{n}(t+\delta t)-\mathcal{P}_{n}}{\delta t} & =\mathcal{P}_{n-1}(t)(n-1) g_{n-1}-\mathcal{P}_{n} n\left(g_{n}+d_{n}\right)+\mathcal{P}_{n+1}(n+1) d_{n+1} \\
\frac{d}{d t} \mathcal{P}_{n} & =\mathcal{P}_{n-1}(n-1) g_{n-1}-\mathcal{P}_{n} n 1\left(g_{n}+d_{n}\right)+\mathcal{P}_{n+1}(n+1) d_{n+1}
\end{aligned}
$$

From this the first few equations can be calculated to give a foundation for insight

$$
\begin{aligned}
\frac{d}{d t} \mathcal{P}_{0} & =\mathcal{P}_{1} d_{1} \\
\frac{d}{d t} \mathcal{P}_{1} & =-\mathcal{P}_{1}\left(g_{1}+d_{1}\right)+2 \mathcal{P}_{2} d_{2} \\
\frac{d}{d t} \mathcal{P}_{2} & =\mathcal{P}_{1} g_{1}-2 \mathcal{P}_{2}\left(g_{2}+d_{2}\right)+3 \mathcal{P}_{3} d_{3} \\
\frac{d}{d t} \mathcal{P}_{3} & =2 \mathcal{P}_{2} g_{2}-3 \mathcal{P}_{3}\left(g_{3}+d_{3}\right)+4 \mathcal{P}_{4} d_{4}
\end{aligned}
$$


Now we will look at the expectation of certain properties and will define the functional form

$$
<f_{m}>=\sum_{n} f_{m} \mathcal{P}_{n} v
$$

Clearly the sum of the probabilities at a given time is constant so

$$
\frac{d}{d t}<1>=0 \text {. }
$$

A more relevant calculation is the understanding of the time rate of change of the expected population change which can be shown to be

$$
\frac{d}{d t}<n>=<n\left(g_{n}-d_{n}\right)>.
$$

If however, the death and growth rates are independent of the population size than the constant can be pulled outside of the expectation calculation, so

$$
\frac{d}{d t}<n>=\left(g_{n}-d_{n}\right)<n>
$$

which has a similar form to the deterministic population model. Additionally it can be shown that given

$$
\frac{d}{d t}<n^{2}>=2<n^{2}\left(g_{n}-d_{n}\right)>+<n\left(g_{n}+d_{n}\right)>
$$

and $\sigma^{2}=<n^{2}>-<n>^{2}$, it is possible to calculate the long term trend of the ratio between the standard deviation of the population $(\sigma)$ and the expected population size with growth and death rates independent of population size

$$
\lim _{t \rightarrow \infty} \frac{\sigma}{\langle n\rangle}=\sqrt{\frac{\sigma_{0}^{2}+<n>_{0} \frac{g+d}{g-d}}{<n>_{0}^{2}}}
$$

where $\sigma_{0}$ and $\left\langle n>_{0}\right.$ are the initial standard deviations and the expected population size. If we compare a set of realizations, we would find the range of variation increases with time just as the mean size is increasing.

For populations which can double in a day, the exponential model cannot apply for long. We can examine the logistic model with $g_{n}=g_{0}\left(1-n / n_{0}\right)$, in which case the distribution can reach a nearly state. It cannot be completely steady, since a constant value of $\mathcal{P}_{1}$ implies the extinction probability, $\mathcal{P}_{0}$, is continuing to grow.

\subsection{Fokker-Planck Expansion}

when the number of individuals is very large, $\mathcal{P}(n, t)$ can be treated as a smooth function of $n$, regarded as a real number. If we start again with the Equation 13, the following Taylor expansion can be performed to approximate the dependence on the vector of population sizes

$$
\begin{aligned}
\mathcal{P}(\mathbf{z}, t)+\delta t \frac{\partial}{\partial t} \mathcal{P}(\mathbf{z}, t) & =\sum \mathcal{P}_{T}(\delta \mathbf{z} \mid \mathbf{z}, t) \mathcal{P}(\mathbf{z}, t)-\frac{\partial}{\partial z_{i}} \sum \delta z_{i} \mathcal{P}_{T}(\delta \mathbf{z} \mid \mathbf{z}, t) \mathcal{P}(\mathbf{z}, t) \\
& +\frac{1}{2} \frac{\partial^{2}}{\partial z_{i} \partial z_{j}} \sum \delta z_{i} \delta z_{j} \mathcal{P}_{T}(\delta \mathbf{z} \mid \mathbf{z}, t) \mathcal{P}(\mathbf{z}, t)
\end{aligned}
$$




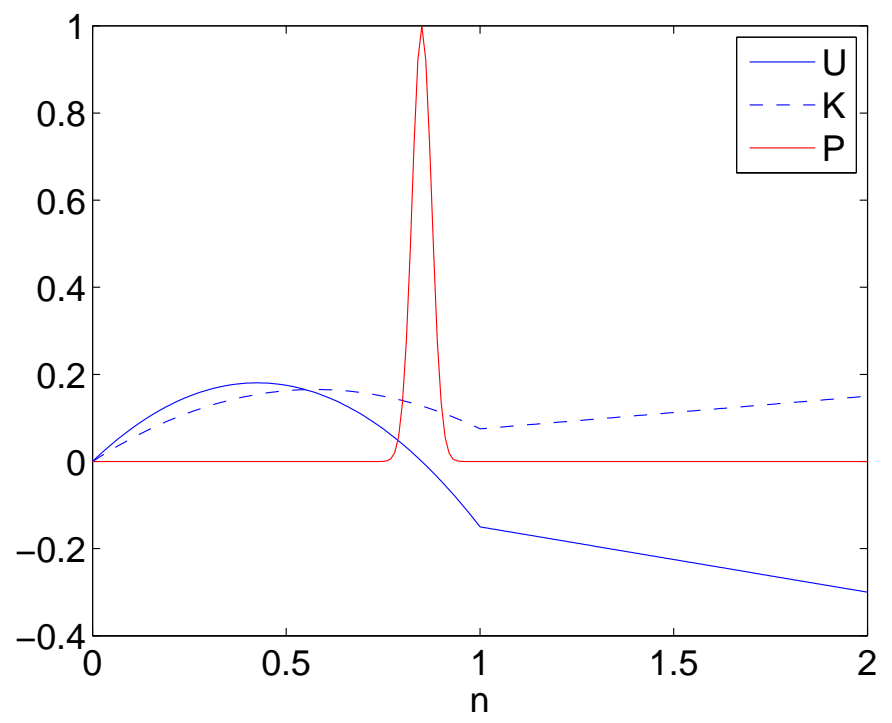

Figure 4: Plot of the $U$ and $K$ for a linearly deacreasing growth rate and a constant death rate and the resulting population density distribution.

where $\mathcal{P}(\mathbf{z}, t)$ is still the population probability function dependent on the coordinate $\mathbf{z}$ and time, $t$, and all $\delta$ 's represent a differential step in the provided coordinate. This Taylor expansion can be simplified to

$$
\frac{\partial}{\partial t} \mathcal{P}(\mathbf{z}, t)=-\frac{\partial}{\partial z_{i}} U_{i} \mathcal{P}+\frac{\partial^{2}}{\partial z_{i} \partial z_{j}} K_{i j} \mathcal{P}
$$

where the substitutions

$$
\left.U_{i}(\mathbf{z}, t)=\sum \frac{\delta z_{i}}{\delta t} \mathcal{P}_{T} \mathbf{z} \mid \mathbf{z}, t\right), \quad K_{i j}(\mathbf{z}, t)=\sum \frac{\delta z_{i} \delta z_{j}}{2 \delta t} \mathcal{P}_{T}(\delta \mathbf{z} \mid \mathbf{z}, t)
$$

have been made. For the case like 14 considering birth and death rates in one species, $U=n\left(g_{n}-d_{n}\right)$ and $K=\frac{1}{2} n\left(g_{n}+d_{n}\right)$. An example growth rate that can be used is $g_{n}=g_{0}\left(1-n / n_{0}\right)$ which corresponds to a linearly decreasing growth rate for populations under a maximum population, $n_{0}$. With a death rate at a fixed constant the function of $U$ and $K$ can be determined and are presented in Figure 4. The resulting population probability distribution function is a narrow Gaussian centered at $g_{n}=d_{n}$. 


\title{
Lecture 3: Stirring by swimming organisms
}

\author{
Jean-Luc Thiffeault \\ Notes by Woosak Moon and Renske Gelderloos
}

23 June 2010

\section{Introduction}

The question of which factors contribute to mixing in the ocean has been the subject of many studies. The idea that organisms could play a role in affecting their environment through something like mixing first appeared in Darwin's last book, called "The formation of vegetable mould through the action of earth worms, with observations on their habits." (See figure 1.) This was suggested by his uncle and future father-in-law Josiah Wedgwood II, son of the famous potter. From Darwin's book:

In the year 1837, a short paper was read by me before the Geological Society of London, "On the Formation of Mould," in which it was shown that small fragments of burnt marl, cinders \&c which had been thickly strewed over the surface of several meadows, were found after a few years lying at the depth of some inches beneath the turf, but still forming a layer. This apparent sinking of superficial bodies is due, as was first suggested to me by Mr. Wedgwood of Maer Hall in Staffordshire, to the large quantity of fine earth continually brought up to the surface by worms in the form of castings. These castings are sooner or later spread out and cover up any object left on the surface. I was thus led to conclude that all the vegetable mould over the whole country has passed many times through, and will again pass many times through, the intestinal canals of worms. [emphasis added] Hence the term "animal mould" would be in some more appropriate than that used of "vegetable mould."

The modern name for this phenomenon is 'bioturbation.' At the time, the idea that many small events could accumulate to create something much bigger was quite controversial (and still is to some people, in the case of evolution).

It was not until almost a century later that Walter Munk [8] seriously proposed marine organisms as a possible factor for mixing in the ocean. However, Munk discounted the effect as negligible. The idea lay dormant for forty years, until 2004 when Huntley \& Zhou [3] analyzed the swimming of 100 species in a wide range of sizes, and concluded that for 11 representative species the turbulent energy production is $\sim 10^{-5} \mathrm{~W} \mathrm{~kg}^{-1}$, the total of which would add up to an amount comparable to the energy dissipation by major storms. Another study on the magnitude of the mixing effect of the oceanic biosphere was performed by Dewar et al. [2], who estimated that the ocean's organisms take in a total 


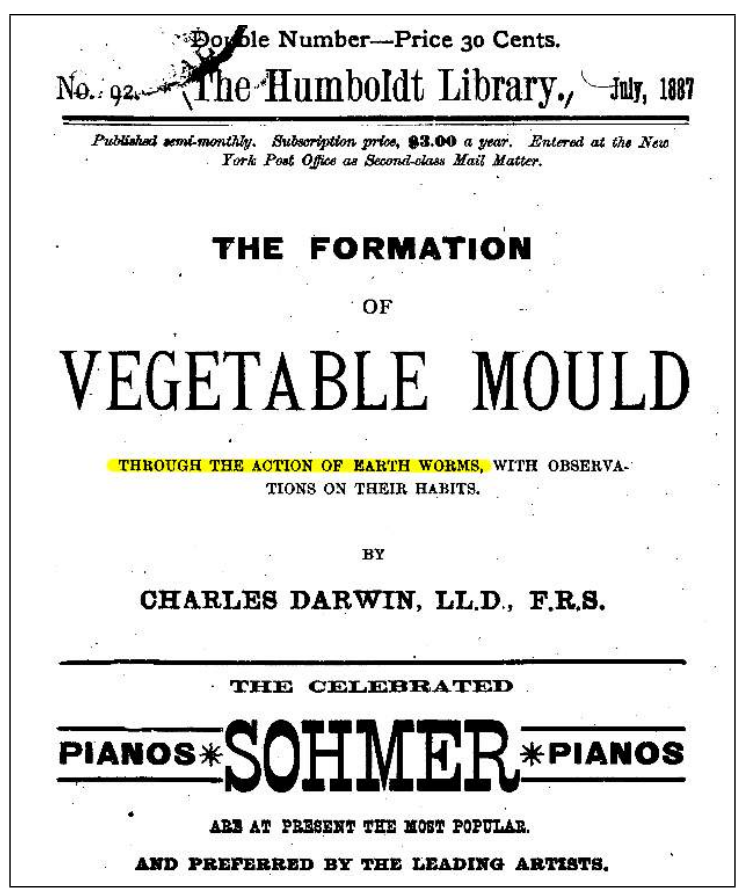

Figure 1: Darwin's last book, "The formation of vegetable mould through the action of earth worms, with observations on their habits."

power input of almost 63 TeraW. They assumed that about $1 \%$ of this amount is delivered as mechanical energy, which leads to a number comparable to the input of winds and tides [2]. An experimental study by Kunze et al. [5] showed that the level of turbulent activity in an inlet was elevated by 2 to 3 orders of magnitude during the day, due to swimming krill. (Though it must be mentioned that later studies were far less successful.)

In reaction to the papers mentioned above, Visser [10] argues that very small organisms such as zooplankton cannot produce sufficient mixing to overturn a stratified medium. The author points out that the efficiency of mixing is proportional to the ratio of the lengthscale of the organism $(L)$ and a buoyancy lengthscale, defined as $B=\left(\epsilon / N^{3}\right)^{1 / 2}$, where $\epsilon$ is the rate of turbulent energy dissipation and $N$ is the buoyancy frequency. When $L<B$, the mixing efficiency quickly drops by several orders of magnitude [10], rendering biomixing negligible. Katija and Dabiri [4] however suggest that it is not the scale of turbulence produced by the marine organisms that is the relevant quantity, but the net displacement of fluid particles they cause. This aspect will be discussed in these notes by constructing a simple model of stirring by swimming organisms, based on drift trajectories due to a moving object.

\section{Displacement of a fluid particle due to a moving body}

We will first discuss the displacement of a fluid particle due to a single moving body, and generalize this theory in the next section to multiple 'kicks' due to several moving bodies, 
and ultimately to an effective diffusivity. For simplicity we will assume the moving body to be $2 \mathrm{D}$ and symmetric with respect to its direction of motion. (Axisymmetric about its direction of motion in 3D.) (See figure 2.) We only consider potential flow. The body moves along a straight line with constant velocity $U$ for distance $\lambda$. The distance between the fluid particle and the straight line of motion is $a$, while the distance from the start of the trajectory to the point of closest approach (with respect to the initial position of the particle) is $b$. Together $a$ and $b$ are called impact parameters. Note that $a$ is positive but $b$ can have either sign, with a negative $b$ indicating a swimmer that begins its trajectory already having passed the fluid particle.

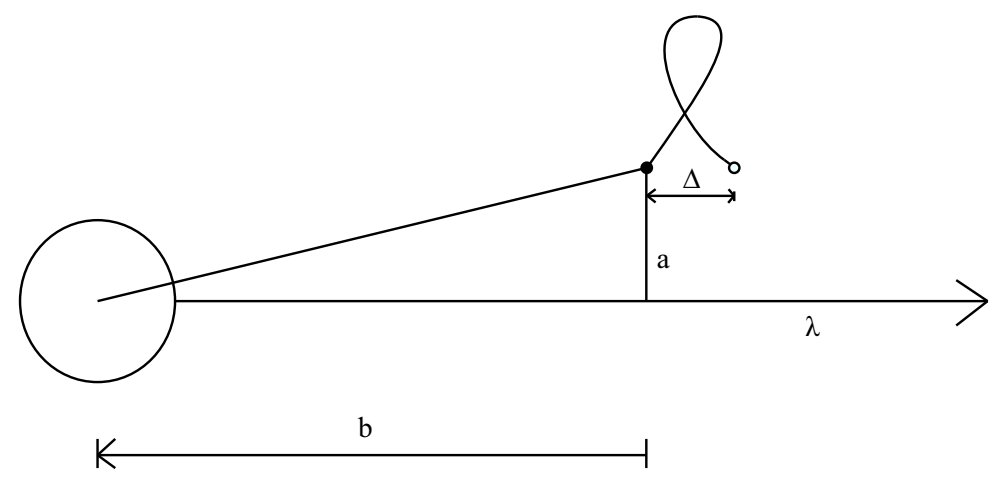

Figure 2: Displacement of a fluid particle by a swimming body.

Due to the movement of the swimming body, the fluid particle will have a net displacement $\Delta=\Delta_{\lambda}(a, b)$, known as drift. Although Maxwell [7] was the first to discuss the drift to a moving body, it is often referred as 'Darwinian drift' [1], due to Charles G. Darwin, great grandson of the other Darwin.

We first need to determine how much a fluid particle is displaced. We could solve this problem in a fixed frame centered around the moving cylinder, where the particle moves around the body (figure 3, left panel). It is however easier to work in a frame which is moving with the fluid particle (figure 3, right panel).

In the $x$ direction, the displacement of the particle solely due the flow $U$ would be $b-\lambda$ ("free-streaming"). Because of the influence of the moving body, the fluid particle ends up at $x_{\mathrm{f}}$, a distance $\Delta x$ away from $b-\lambda$. Now we can define a travel time $T$ it takes the fluid particle to go from $b$ to $x_{\mathrm{f}}$ :

$$
T=\frac{\lambda}{U}=\int_{b}^{x_{\mathrm{f}}} \frac{d x}{u(x, y)}
$$

where $u(x, y)$ is the velocity felt by the fluid particle. This assumes that the horizontal component of the velocity field never vanishes. Using $x_{\mathrm{f}}=b-\lambda+\Delta x$, this gives

$$
\begin{array}{r}
\frac{\lambda}{U}=\int_{b}^{b-\lambda+\Delta x} \frac{d x}{u}=-\int_{b}^{b-\lambda+\Delta x} \frac{d x}{|u|}=\int_{b-\lambda+\Delta x}^{b} \frac{d x}{|u|} \\
=\int_{b-\lambda}^{b} \frac{d x}{|u|}+\int_{b-\lambda+\Delta x}^{b-\lambda} \frac{d x}{|u|} .
\end{array}
$$

If the particle is only lightly displaced and $|b-\lambda|$ is large, then the velocity felt by the particle approximates the free-stream velocity $(|u| \simeq U)$ in the second integral. The second 

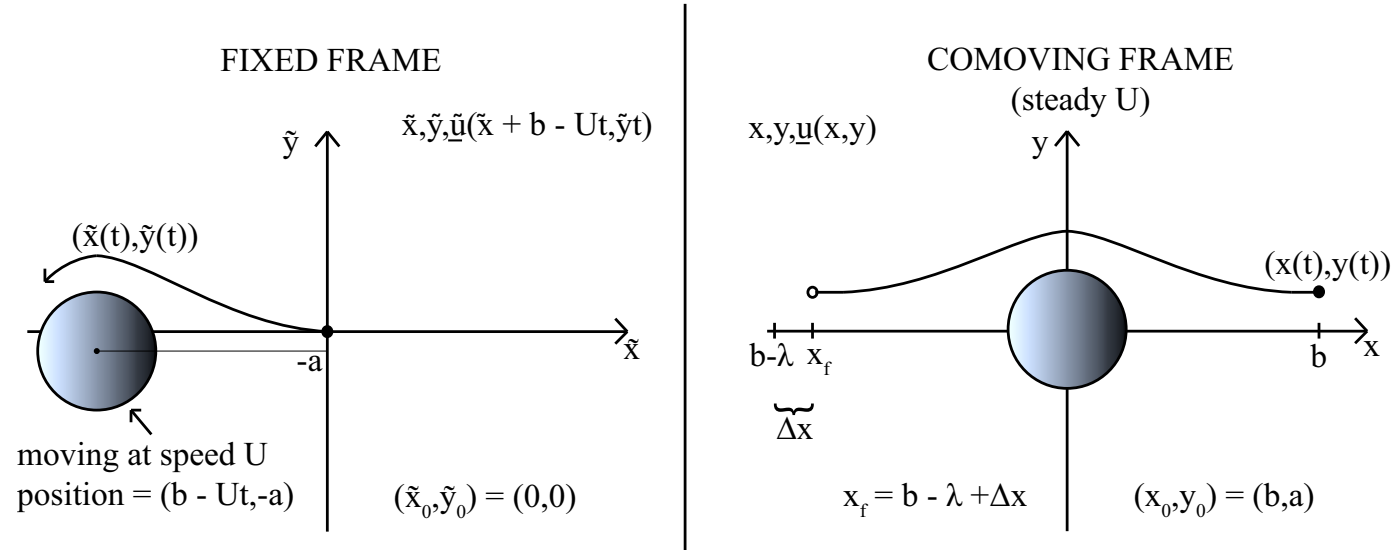

Figure 3: Left: displacement of the particle in a fixed frame. The particles starts in the origin. Right: displacement of the particle in a frame that moves with the particle. The moving cylinder is in the origin.

integral can then be approximated by $\Delta x / U$, which gives

$$
\frac{\lambda}{U} \simeq \int_{b-\lambda}^{b} \frac{d x}{|u|} \Longleftrightarrow \frac{\Delta x}{U}=\int_{b-\lambda}^{b} \frac{d x}{|u|}-\frac{\lambda}{U}
$$

From this statement we can derive an equation for the approximate horizontal displacement of the fluid particle $\Delta x$ :

$$
\Delta x \simeq U \int_{b-\lambda}^{b}\left(\frac{1}{|u|}-\frac{1}{U}\right) d x
$$

The displacement in the $y$-direction is trivial, as the fluid particle will follow a streamline of the flow in the comoving frame (assuming the flow is steady in that frame). Far away from the moving cylinder $(\lambda \rightarrow \infty, b-\lambda \rightarrow-\infty)$ the displacement in the $y$-direction must therefore be zero:

$$
\Delta y=0
$$

Note that both boxed expressions are only valid in the limit of large path length $\lambda$.

\subsection{Example: A cylinder in potential flow}

As an example, consider a cylinder in potential flow with stream function

$$
\Psi(x, y)=-U y\left(1-\frac{l^{2}}{x^{2}+y^{2}}\right)
$$

with $l$ the radius of the cylinder and

$$
u=\frac{\partial \Psi}{\partial y}, \quad v=-\frac{\partial \Psi}{\partial x} .
$$

We will choose $U=1$ and $l=1$. 


\subsubsection{Far away from the cylinder}

It can be expected that the effect of a moving cylinder on a particle far away from the body is negligible. To test this statement, one can construct a qualitative order of magnitude for the displacement of such a particle. This displacement can be represented by $\Delta x$ and $\Delta y$. We will assume the path length is infinite, so the displacement $\Delta y$ vanishes identically.

First of all, let us calculate the maximum excursion in $y, \epsilon_{\max }$. This can be calculated using the stream function in the fixed frame (figure 2). Here, the particle is located initially at $(b, a)$. Because the flow around the cylinder is steady, the particle moves along the stream line. Therefore, we can set up

$$
-y+\frac{y}{x^{2}+y^{2}}=-a+\frac{a}{a^{2}+b^{2}} \simeq-a,
$$

where $a \gg 1$ is assumed such that $\frac{a}{a^{2}+b^{2}}$ can be neglected. Let $y=a+\epsilon$, where $a \gg \epsilon$. If we input this expression into equation 5 , we obtain

$$
-a-\epsilon+\frac{a+\epsilon}{x^{2}+(a+\epsilon)^{2}} \simeq-a-\epsilon+\frac{a}{x^{2}+a^{2}} \simeq-a .
$$

Therefore,

$$
\epsilon \simeq \frac{a}{x^{2}+a^{2}}
$$

Here, $\epsilon_{\max } \simeq \max _{x}(\epsilon)$, thus

$$
\epsilon_{\max } \sim \mathcal{O}(1 / a)
$$

where we assume from now that $x \ll a$.

$\Delta x$ can be calculated from equation 2 . First, we can find $u$ using the given streamfunction in the fixed frame and then construct an approximate expression for $|u|^{-1}-1$. The velocity $u$ is

$$
u=\frac{\partial \Psi}{\partial y}=-1+\frac{x^{2}-y^{2}}{\left(x^{2}+y^{2}\right)^{2}} .
$$

Therefore, using $x \gg y \gg 1$, it follows that

$$
\frac{1}{|u|}-1=\frac{1}{1-\frac{x^{2}-y^{2}}{\left(x^{2}+y^{2}\right)^{2}}}-1 \simeq 1+\frac{x^{2}-y^{2}}{\left(x^{2}+y^{2}\right)^{2}}-1=\frac{x^{2}-y^{2}}{\left(x^{2}+y^{2}\right)^{2}} \text {. }
$$

Let $y=a+\epsilon$, this gives

$$
\frac{1}{|u|}-1 \simeq \frac{x^{2}-a^{2}}{\left(x^{2}+a^{2}\right)^{2}}-\frac{2 a \epsilon}{\left(x^{2}+a^{2}\right)^{2}}
$$

Therefore,

$$
\Delta x=\int_{-\infty}^{\infty}\left(\frac{1}{|u|}-1\right) d x \simeq \int_{-\infty}^{\infty} \frac{x^{2}-a^{2}}{\left(x^{2}+a^{2}\right)^{2}} d x-\int_{-\infty}^{\infty} \frac{2 a \epsilon}{\left(x^{2}+a^{2}\right)^{2}} d x .
$$

Here, the first integral on the right hand side is

$$
\int_{-\infty}^{\infty} \frac{x^{2}-a^{2}}{\left(x^{2}+a^{2}\right)^{2}} d x=-\frac{1}{a} \int_{-\frac{\pi}{2}}^{\frac{\pi}{2}} \cos 2 \theta d \theta=0
$$


where $x=a \tan \theta$ is used. The second integral on the right hand side is

$$
2 a \epsilon \int_{-\infty}^{\infty} \frac{1}{\left(x^{2}+a^{2}\right)^{2}} d x=\frac{2 a \epsilon}{a^{3}} \int_{-\frac{\pi}{2}}^{\frac{\pi}{2}} \cos ^{2} \theta d \theta=\frac{\epsilon \pi}{a^{2}} .
$$

Therefore,

$$
\Delta x \simeq-\frac{\epsilon \pi}{a^{2}}
$$

Previously, we showed that $\epsilon \sim O\left(a^{-1}\right)$, which leads to the conclusion that $\Delta x \sim O\left(a^{-3}\right)$. Because $a$ is much larger than 1, the displacement of the particle far away from the cylinder is negligible, which confirms our initial guess. However, note that the perturbation to the velocity field due to the moving object is of order $a^{-2}$, and the net displacement is smaller by another order of magnitude. Thus, drift trajectories far from the moving body are very nearly closed.

\subsubsection{Close to the cylinder}

A more interesting limit is the one very close to the cylinder $(a \ll 1)$. The path of the fluid particle can be subdivided into three regions (figure 4). In the first region the particle more or less follows the $x$-axis. In region 2 the fluid trajectory bends upwards and in region 3 it follows an almost circular path to the $y$-axis. On the other side of the moving body, the fluid particle follows the same path in reverse order.

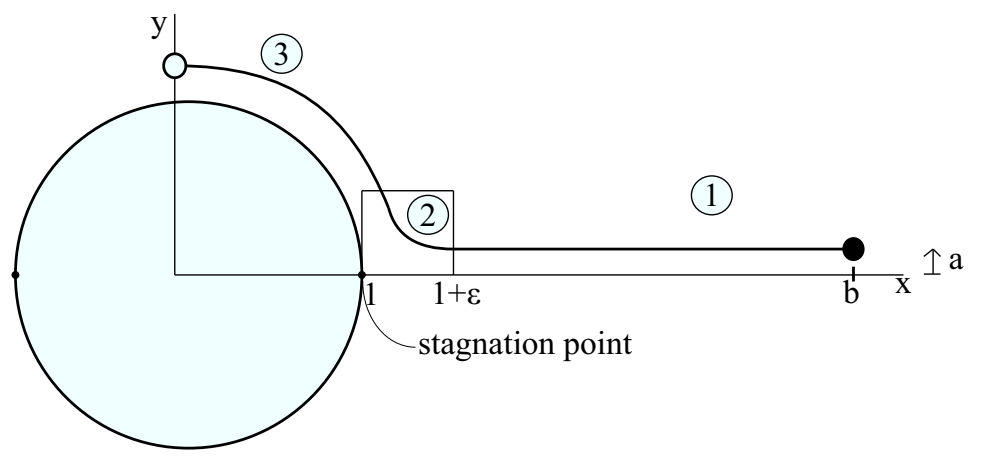

Figure 4: Path of a fluid particle close to the cylinder $(a \ll 1)$.

We want to know the displacement $\Delta x$ (equation 2). With $U=1$ the integral for $\Delta x$ becomes

$$
T \simeq \int\left(\frac{1}{u}+1\right) d x
$$

where we now use the travel time $T$ instead of distance $\Delta x$ to match the units ( $T$ has the same numerical value as $\Delta x$ for $U=1$ ). We can split the integral into the three regions. For the third region one can use the full stream function, but for the first two it is easier to 
use an approximate form. The stream function in the three region is

$$
\begin{aligned}
& \Psi_{1} \simeq-\left(1-\frac{1}{x^{2}}\right) y \quad(y \ll 1) \\
& \Psi_{2} \simeq-2(x-1) y \quad(x, y \ll 1) \\
& \Psi_{3}=-\left(1-\frac{1}{x^{2}+y^{2}}\right) y \text {. }
\end{aligned}
$$

We can now calculate the travel time in each of the three regions.

\section{Region 1}

$$
\begin{gathered}
u_{1}=\frac{\partial \Psi}{\partial y}=-\left(1-x^{-2}\right) \\
T_{1}=\int_{b}^{1+\epsilon}\left(\frac{1}{u}+1\right) d x=\int_{b}^{1+\epsilon} \frac{d x}{1-x^{2}}
\end{gathered}
$$

Using $\epsilon \ll 1$ and $b \gg 1$ this can be approximated by

$$
T_{1} \simeq \frac{1}{2} \log \left(\frac{2}{\epsilon}\right)+\frac{\epsilon}{4}-b^{-1}+\mathcal{O}\left(\epsilon^{2}, b^{-2}\right)
$$

\section{Region 2}

For region 2 we first need to specify the boundaries of the region. Let us rewrite the approximate streamfunction as

$$
\Psi_{2} \simeq-2(x-1) y=-2 X Y
$$

where $X=x-1$ and $Y=y$. The fluid particle enters region 2 at $\left(X_{0}, Y_{0}\right)$. At that point

$$
\Psi_{0}=-2 X_{0} Y_{0}=-\left(1-b^{-2}\right) a .
$$

As $X_{0}=\epsilon$ and $b \gg 1$, this is approximately

$$
-2 \epsilon Y_{0}=-a \Longrightarrow Y_{0}=\frac{a}{2 \epsilon} \text {. }
$$

Because the stream function is hyperbolic and the domain of region 2 is of size $\epsilon$ by $\epsilon$, it follows that $\left(X_{1}, Y_{1}\right)=(a / 2 \epsilon, \epsilon)$. The travel time in region 2 is thus

$$
T_{2}=\int_{X_{0}}^{X_{1}}\left(\frac{1}{u}+1\right) d x=\int_{\epsilon}^{a / 2 \epsilon}\left(\frac{1}{-2 x}+1\right) d x=-\frac{1}{2} \log \left(\frac{a}{2 \epsilon^{2}}\right)+\frac{a}{2 \epsilon}-\epsilon
$$




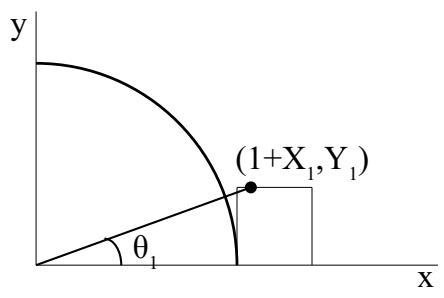

Figure 5: Close-up of the path of the fluid particle in region 3.

\section{Region 3}

From the full stream function the velocity $u$ is

$$
u(x, y)=\frac{\partial \Psi}{\partial y}=-1+\frac{x^{2}-y^{2}}{\left(x^{2}+y^{2}\right)^{2}}
$$

In polar coordinates $(x=r \cos \theta, y=r \sin \theta)$ this can be written as

$$
u(r, \theta)=-1+\frac{\cos 2 \theta}{r^{2}}
$$

Figure 5 shows a sketch of the path of fluid particle in region 3 . We define $\theta_{1}$ as the (small) angle between the $\mathrm{x}$-axis and the point $\left(1+X_{1}, Y_{1}\right)$. The travel time in region 3 is then

$$
T_{3}=\int_{\theta_{1}}^{\pi / 2}\left(\frac{1}{u}+1\right) \frac{d x}{d \theta} d \theta=\frac{1}{2} \int_{\theta_{1}}^{\pi / 2} \frac{\cos 2 \theta}{\sin \theta} d \theta=-1+\frac{1}{2} \log 2-\frac{1}{2} \log \theta_{1}+\mathcal{O}\left(\theta_{1}^{2}\right)
$$

using $d x / d \theta=-r \sin \theta$. From figure 5 one can derive

$$
\tan \theta_{1}=\frac{Y_{1}}{1+X_{1}}=\frac{\epsilon}{1+\frac{a}{\epsilon}} \simeq \epsilon\left(1-\frac{a}{\epsilon}\right)=\epsilon-a
$$

The travel time in region 3 is thus approximately

$$
T_{3} \simeq-1+\frac{1}{2} \log 2-\frac{1}{2} \log \epsilon+\frac{a}{2 \epsilon}+\mathcal{O}\left(\left(\frac{a}{\epsilon}\right)^{2}\right) .
$$

For the displacement of the fluid particle we need the total travel time, which is twice the sum of the travel time in region 1,2, and 3. Adding up the leading orders of the travel times gives

$$
T=T_{1}+T_{2}+T_{3}=\left(\frac{1}{2} \log \left(\frac{a}{\epsilon}\right)-b^{-1}\right)+\left(-\frac{1}{2} \log \left(\frac{a}{2 \epsilon^{2}}\right)\right)+\left(-1+\frac{1}{2} \log 2-\frac{1}{2} \log \epsilon\right) .
$$

The $\epsilon$ 's cancel out. To leading order, the total transit time for half the particle path is then

$$
T=-\frac{1}{2} \log a-1+\frac{3}{2} \log 2-b^{-1} .
$$

For small $a$, the first term dominates the travel time, i.e., the particle tends to get stuck near the stagnation point in region 2 for a while. As $a \rightarrow 0$, we see that $T \rightarrow \infty$. In general, 
for a moving body of any shape in 2D potential flow it turns out that the coefficient of $\log a$ is given by the sum over the linearization coefficients for each hyperbolic stagnation point encountered, as long as $a$ is small. The displacement of a fluid particle close to a moving body in potential flow due to this moving body is thus well-approximated by

$$
\Delta_{\lambda}(a, b)= \begin{cases}-\log a & 0 \leq b \leq \lambda \\ 0 & \text { otherwise }\end{cases}
$$

The cutoff for $b<0$ and $b>\lambda$ is due to the fact that since the displacement is mostly due to the stagnation points, the particle must at least reach the stagnation point. (We take the size of the body itself as negligible.)

\section{Displacement of a fluid particle due to multiple kicks}

In previous section an expression for $\Delta_{\lambda}(a, b)$ was obtained. Based on this information we can derive an effective diffusivity. Here, we have four constants and two random variables: $U$ is the mean velocity of the cylinder, $l$ is the length scale, $\lambda$ is the mean free path of the cylinder, and $n$ the number density. The position of the particle is coordinated by two random variables, $a$ and $b$, which were described in the previous section. If we pick a point, $(x, y)$, in Cartesian coordinates, the probability measure of the point is simply represented as $(1 / V) d x d y$, where $V$ is the total volume. Instead of $(x, y)$ space, we have to use $(a, b)$ space for representing the PDF of the particle distribution. Considering that $a$ is positive and neglecting the edge of the domain, we find

$$
\frac{1}{V} d x d y=\frac{2}{V} d a d b
$$

A similar relation can be found in 3D. In this case, the probability measure can be expressed as

$$
\frac{1}{V} d x d y d z=\frac{2 \pi a}{V} d a d b .
$$

Now, assume that our target particle is kicked by a swimmer. For $N$ steps or kicks,

$$
\boldsymbol{x}_{N}=\boldsymbol{x}_{0}+\sum_{k=1}^{N} \Delta_{\lambda}\left(a_{k}, b_{k}\right) \hat{\boldsymbol{r}}_{k}
$$

where $a_{k}, b_{k}$, and $\hat{\boldsymbol{r}}_{k}$ are random variables. Their choices at each step are independent. In particular, $\left\langle\hat{\boldsymbol{r}}_{k} \cdot \hat{\boldsymbol{r}}_{l}\right\rangle=\delta_{k l}$. The direction of a swimmer is chosen independently. Without loss of generality, we can assume that $\boldsymbol{x}_{0}=0$. Since $\left\langle\hat{\boldsymbol{r}}_{k}\right\rangle=0$, it follows from equation (14) that $\left\langle\boldsymbol{x}_{N}\right\rangle=0$. To calculate the effective diffusivity, the second moment must be considered:

$$
\left\langle\left|\boldsymbol{x}_{N}\right|^{2}\right\rangle=\sum_{k=1}^{N}\left\langle\Delta_{\lambda}^{2}\left(a_{k}, b_{k}\right)\right\rangle+\text { vanishing cross terms. }
$$

The cross terms vanish because $\left\langle\hat{\boldsymbol{r}}_{k} \cdot \hat{\boldsymbol{r}}_{l}\right\rangle=\delta_{k l}$. Each step of the target particle caused by the swimmers is independent such that the particle movement can be considered to be a 
random walk. Since the variables are identically distributed, we get

$$
\sum_{k=1}^{N}\left\langle\Delta_{\lambda}^{2}\left(a_{k}, b_{k}\right) \hat{r}_{k} \hat{r}_{k}\right\rangle=N\left\langle\Delta_{\lambda}^{2}(a, b)\right\rangle=\frac{N}{V} \iint \Delta_{\lambda}^{2}(a, b) 2 d a d b .
$$

$N$ is introduced as the number of time steps. Physically, $N$ can be understood as the number of collisions between the particle and the swimmers. It can be represented as $t / T$, where $t$ is an elapsed time and $T$ is the mean free time, the average time for a swimmer to collide with the particle. Hence, $t / T$ is the average number of collisions during the time $t$. Here, $T$ can be represented as $\lambda / U$, where $\lambda$ is the mean length which a swimmer proceeds before changing its direction, so that $N$ can be written as $U t / \lambda$. Hence,

$$
\left\langle|x(t)|^{2}\right\rangle=\frac{U t}{\lambda} \frac{1}{V} \iint \Delta_{\lambda}^{2}(a, b) 2 d a d b,
$$

where $x(t)$ is used instead of $x_{N}$. To eliminate $V$ from the equation, observe that the above equation is for one swimmer, so $n=1 / V$ is the number density. Therefore,

$$
\left\langle|x(t)|^{2}\right\rangle=\frac{2 U n t}{\lambda} \iint \Delta_{\lambda}^{2}(a, b) d a d b=4 \kappa t,
$$

where $\kappa$ is the effective diffusivity. $4 \kappa t$ comes from the result of random walk (see previous lecture). The above expression is for a two-dimensional system, but can be easily generalized for 3D. We obtain finally

$$
\kappa= \begin{cases}\frac{U n}{2 \lambda} \int \Delta_{\lambda}^{2}(a, b) d a d b, & 2 D \\ \frac{\pi U n}{3 \lambda} \int \Delta_{\lambda}^{2}(a, b) a d a d b, & 3 D\end{cases}
$$

Recall our approximate form for displacement due to a cylinder, including dimensions:

$$
\Delta_{\lambda}(a, b)=\left\{\begin{array}{ll}
-l \log (a / l) & 0 \leq b \leq \lambda \\
\text { negligible } & \text { otherwise }
\end{array},\right.
$$

the calculation of $\kappa$ becomes

$$
\kappa \simeq \frac{U n}{2 \lambda} \lambda \int_{0}^{l} l^{2} \log ^{2}(a / l) d a
$$

Using $\int_{0}^{1} \log ^{2} x d x=2$, we obtain

$$
\kappa \simeq U n l^{3} .
$$

Note that this result is completely independent of $\lambda$ (for $\lambda$ large).

Another relevant case is a swimmer with a 'bubble wake,' that is, a region enclosed by streamlines that follows the swimmer, often called the atmosphere of the swimmer. If there is a bubble wake behind a swimmer, a particle inside the bubble wake will follow the swimmer until the swimmer changes its direction. Therefore, the particle trapped inside the bubble moves by $\lambda$. Our approximate expression of $\Delta_{\lambda}(a, b)$ is

$$
\Delta_{\lambda}(a, b)= \begin{cases}\lambda & 0 \leq b \leq \lambda \\ 0 & \text { otherwise. }\end{cases}
$$




\begin{tabular}{lcc}
\hline \hline Swimmer & $\lambda$-dependence & far/near field dominance \\
\hline potential (slip) & none & near \\
viscous (squirmer) & none & far \\
viscous (no-slip) & $\log \lambda$ & near \\
trapped bubble & $\lambda$ & near \\
\hline
\end{tabular}

Table 1: Dependence of displacement on path length and whether the displacement is dominated by far or near field, for various swimmer models.

In this case, our calculation of $\kappa$ is

$$
6 \kappa=\frac{2 U n}{\lambda} \int_{\text {bubble interior }} \lambda^{2} d a d b=U n \lambda V_{\text {bubble }},
$$

where $V_{\text {bubble }}$ is the total volume of the bubble. Therefore,

$$
\kappa=\frac{1}{6} U n \lambda V_{\text {bubble }} .
$$

Now, unlike the potential flow case, the effective diffusivity depends explictly on path length $\lambda$. This $\kappa$ can be much larger than that for untrapped fluid.

More complexity could come in if we consider viscous swimmers with a boundary layer. In this case, some simple estimates [9] suggest $\kappa \sim \log \lambda$. For micro-organisms in the Stokes flow approximation, the transport is larger and is dominated by far-field (a few body lengths) hydrodynamics [6]. The dependence on flow characteristics and boundary conditions is summarized in Table 1, but it should be noted that the details of this table are still the object of active research.

\section{References}

[1] C. G. Darwin, Note on hydrodynamics, Proc. Camb. Phil. Soc., 49 (1953), pp. 342354.

[2] W. K. Dewar, R. J. Bingham, R. L. Iverson, D. P. Nowacek, L. C. St. Laurent, And P. H. Wiebe, Does the marine biosphere mix the ocean?, J. Mar. Res., 64 (2006), pp. 541-561.

[3] M. E. Huntley And M. Zhou, Influence of animals on turbulence in the sea, Mar. Ecol. Prog. Ser., 273 (2004), pp. 65-79.

[4] K. KatiJa And J. O. DabiRI, A viscosity-enhanced mechanism for biogenic ocean mixing, Nature, 460 (2009), pp. 624-627.

[5] E. Kunze, J. F. Dower, I. Beveridge, R. Dewey, and K. P. Bartlett, Observations of biologically generated turbulence in a coastal inlet, Science, 313 (2006), pp. $1768-1770$.

[6] Z. Lin, J.-L. Thiffeault, And S. Childress, Stirring by squirmers, J. Fluid Mech., (2010). http://arxiv.org/abs/1007.1740, in press. 
[7] J. C. Maxwell, On the displacement in a case of fluid motion, Proc. London Math. Soc., s1-3 (1869), pp. 82-87.

[8] W. H. Munk, Abyssal recipes, Deep-Sea Res., 13 (1966), pp. 707-730.

[9] J.-L. Thiffeault and S. Childress, Stirring by swimming bodies, Phys. Lett. A, 374 (2010), pp. 3487-3490.

[10] A. W. Visser, Biomixing of the oceans?, Science, 316 (2007), pp. 838-839. 


\title{
Lecture 4: Local stretching theories
}

\author{
Jean-Luc Thiffeault \\ Notes by David Goluskin and Anubhab Roy
}

24 June 2010

\section{Introduction}

In this lecture we will try to understand local or Lagrangian theories involved in mixing of passive scalars. This involves solving the advection-diffusion (AD) equation along fluid trajectories. The origins of these local theories can be traced to Batchelor's idea of describing the flow via spatially-constant strain-rate matrices with prescribed time dependence [2]. Kraichnan addressed the problem next by considering the velocity field in the AD equation to be a stochastic Gaussian field with a time correlation that decays infinitely rapidly (or is white in time) and a spatial correlation that has a power-law structure [6]. This led to solving a stochastic differential equation. Zeldovich encountered the problem in the context of heat diffusion and the magnetic dynamo and adopted a random matrix theory approach [13-15]. More recently, tools from large deviation theory and path integration have aided in obtaining a complete solution of the problem, as will be discussed in this lecture.

Let us revisit the $\mathrm{AD}$ equation,

$$
\partial_{t} \theta+\boldsymbol{u} \cdot \nabla \theta=\kappa \nabla^{2} \theta, \quad \Omega=\mathbb{R}^{2} \text { or } \mathbb{R}^{3} .
$$

As mentioned before $\theta(\boldsymbol{x}, t)$ denotes a passive scalar field (say concentration of dye) and $\boldsymbol{u}(\boldsymbol{x}, t)$ is the ambient velocity field which stirs the scalar as it diffuses with a diffusivity $\kappa$.

Previously (Lecture 1) we have examined how a "patch" of dye evolves in an extensional flow, $\boldsymbol{u}=(\lambda x,-\lambda y)$. We will now address the evolution of a passive scalar when acted upon by an arbitrary linear flow. By definition, for a linear flow the velocity field depends linearly on the position. Rigid body rotation, extensional flow, and simple shear are some classic examples of linear flow. In general,

$$
\boldsymbol{u}(\boldsymbol{x}, t)=\boldsymbol{U}(t)+\boldsymbol{x} \cdot \boldsymbol{A}(t), \quad \operatorname{Tr}(\boldsymbol{A})=0,
$$

where $\boldsymbol{A}(t)$ is the velocity gradient tensor. The requirement for it to be traceless is a consequence of incompressibility of the flow field $(\nabla \cdot \boldsymbol{u}=0)$. For turbulent or Lagrangian-chaotic flows, $\boldsymbol{A}(t)$ is a random matrix, having a finite correlation time, which is the Lagrangian correlation time of the velocity. Here $\boldsymbol{U}(t)$ is a uniform flow. The advantage of using linear flows lies in making the problem analytically tractable and most importantly it serves as a nice prototype for more complicated flows. We will simplify things further by considering 


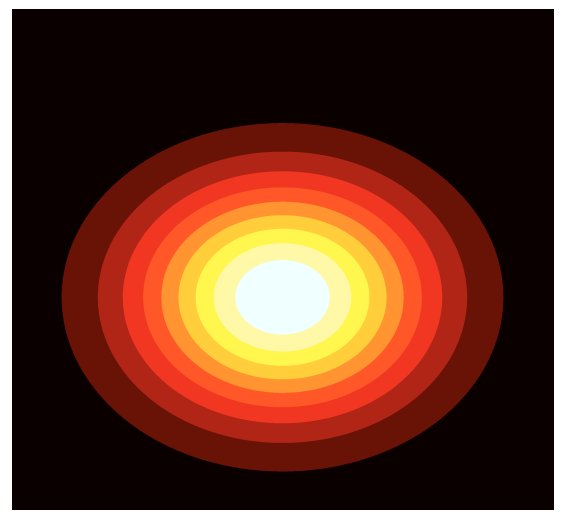

(a)

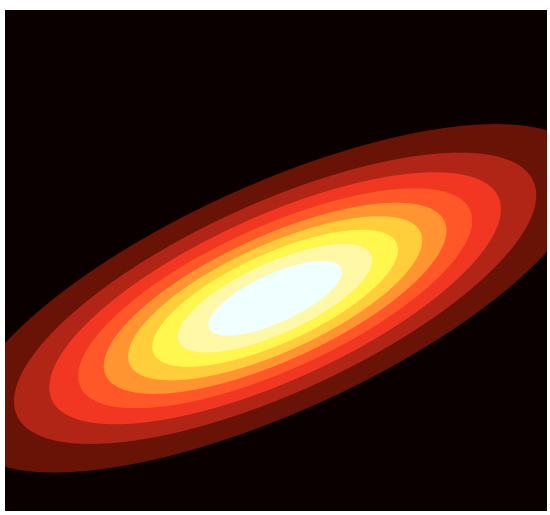

(b)

Figure 1: Advection of a concentration field by a simple shear flow, $\boldsymbol{u}=\left(U_{x}, U_{y}\right)=(\dot{\gamma} y, 0)$ (neglecting diffusion, $\dot{\gamma}$ assumed constant). An intially isotropic concentration field (a) gets both stretched and rotated by the background flow (b).

the limit of high Schmidt number, which is a dimensionless quantity defined as

$$
S c:=\nu / \kappa
$$

where $\nu$ is the kinematic viscosity of the fluid and $\kappa$ as already mentioned is the diffusivity of the scalar. (If the scalar is heat, then the Schmidt number becomes the Prandtl number.) Momentum diffuses significantly faster than the passive scalar for flows with large $S c$. This is the desired limit for chaotic advection: $S c \gg 1$ ensures that the velocity field appears much smoother than the passive scalar concentration, allowing the linear local approximation (2). This is the regime that was studied by Batchelor and leads to the celebrated Batchelor spectrum. For $S c \leq 1$ we have the Kolmogorov-Obukhov-Corrsin regime, where the scalar gets advected by the turbulent inertial range, unlike the Batchelor regime. (We will not be discussing the KOC regime in this lecture.)

\section{2 'Inertia tensor' of a patch of passive scalar}

It is of great interest to understand the response of a passive scalar to a flow with chaotic Lagrangian trajectories. As already discussed, a decent approximation to such a velocity field can be made by superposing random linear flows (in the limit $S c \gg 1$ ). In order to get a statistical description of the passive scalar field subjected to such an ensemble of linear flows, we need to understand the behaviour of the moments of $\theta(\boldsymbol{x}, t)$. We use angle brackets to denote the volume-integrated value of a quantity (similar to an average),

$$
\langle f\rangle=\int_{\Omega} f d V .
$$

We have already seen (Lecture 1) that when the domain is either periodic or is bounded by insulating walls then

$$
\partial_{t}\langle\theta\rangle=0
$$


that is, the mean value of $\theta$ is conserved. This is a consequence of the no-net-flux boundary condition imposed on the system. To track the evolution of 'a blob of dye,' one can define the 'center of mass' of $\theta$ as

$$
c_{i}=\frac{\left\langle x_{i} \theta\right\rangle}{\langle\theta\rangle} .
$$

To obtain an evolution equation for $\boldsymbol{c}$ we consider the first moment of the $\mathrm{AD}$ equation, and integrate by parts a few times:

$$
\begin{aligned}
\partial_{t}\left\langle x_{i} \theta\right\rangle+\left\langle x_{i} \nabla \cdot((\boldsymbol{U}+\boldsymbol{x} \cdot \boldsymbol{A}) \theta)\right\rangle & =\kappa\left\langle x_{i} \nabla^{2} \theta\right\rangle, \\
\Rightarrow \partial_{t}\left\langle x_{i} \theta\right\rangle-\left\langle\left(U_{j}+x_{\ell} A_{\ell j}\right) \theta \partial_{j} x_{i}\right\rangle & =0, \\
\Rightarrow \partial_{t}\left\langle x_{i} \theta\right\rangle-U_{i}\langle\theta\rangle-A_{\ell i}\langle\theta\rangle c_{\ell} & =0, \quad\left(\partial_{j} x_{i}=\delta_{i j}\right)
\end{aligned}
$$

which leads to

$$
\dot{c}=\boldsymbol{U}+\boldsymbol{c} \cdot \boldsymbol{A} .
$$

Here the overdot represents a derivative with respect to time. In the calculation the contribution due to the dissipative term disappeared due to the no-net-flux boundary conditions. Thus the center of mass of the patch gets advected by the background flow.

Proceeding to the calculation for the next moment we define the 'inertia tensor' of the patch as

$$
M_{i j}=\frac{\left\langle x_{i} x_{j} \theta\right\rangle}{\langle\theta\rangle}-c_{i} c_{j}
$$

The additional $-c_{i} c_{j}$ term makes $\boldsymbol{M}$ translationally invariant. By construction $\boldsymbol{M}$ is also a symmetric matrix. We proceed in similar fashion as above to obtain the corresponding evolution equation for $\boldsymbol{M}$ :

$$
\begin{array}{ll} 
& \partial_{t}\left\langle x_{i} x_{j} \theta\right\rangle+\left\langle x_{i} x_{j} \nabla \cdot((\boldsymbol{U}+\boldsymbol{x} \cdot \boldsymbol{A}) \theta)\right\rangle=\kappa\left\langle x_{i} x_{j} \nabla^{2} \theta\right\rangle \\
\Rightarrow & \partial_{t}\left\langle x_{i} x_{j} \theta\right\rangle-U_{j}\left\langle x_{i} \theta\right\rangle-U_{i}\left\langle x_{j} \theta\right\rangle-A_{p j}\left\langle x_{i} x_{p} \theta\right\rangle-A_{p i}\left\langle x_{j} x_{p} \theta\right\rangle=2 \kappa \delta_{i j}\langle\theta\rangle \\
\Rightarrow & \partial_{t}\left(M_{i j}+c_{i} c_{j}\right)-U_{j} c_{i}-U_{i} c_{j}-A_{p j}\left(M_{i p}+c_{i} c_{p}\right)-A_{p i}\left(M_{j p}+c_{j} c_{p}\right)=2 \kappa \delta_{i j} \\
\Rightarrow & \partial_{t} M_{i j}+c_{i}\left(\partial_{t} c_{j}-U_{j}-c_{p} A_{p j}\right)+c_{j}\left(\partial_{t} c_{i}-U_{i}-c_{p} A_{p i}\right)=M_{i p} A_{p j}+A_{p i} M_{j p}+2 \kappa \delta_{i j}
\end{array}
$$

so that finally

$$
\dot{\boldsymbol{M}}=\boldsymbol{M} \cdot \boldsymbol{A}+\boldsymbol{A}^{\dagger} \cdot \boldsymbol{M}+2 \kappa \boldsymbol{I} .
$$

Thus we have obtained an evolution equation for the 'inertia tensor' of the patch. Here $\boldsymbol{A}^{\dagger}$ denotes the transpose of the velocity gradient tensor.

The solution for $\boldsymbol{c}(t)$ and $\boldsymbol{M}(t)$ can be explicitly written as

$$
\begin{aligned}
& \boldsymbol{c}(t)=\boldsymbol{c}(0) \cdot e^{\int_{0}^{t} \boldsymbol{A}\left(\tau_{1}\right) d \tau_{1}}+\int_{0}^{t} \boldsymbol{U}(\tau) \cdot e^{\int_{\tau}^{t} \boldsymbol{A}\left(\tau_{1}\right) d \tau_{1}} d \tau \\
& \boldsymbol{M}(t)=e^{\int_{0}^{t} \boldsymbol{A}^{\dagger}\left(\tau_{1}\right) d \tau_{1}} \cdot \boldsymbol{M}(0) \cdot e^{\int_{0}^{t} \boldsymbol{A}\left(\tau_{1}\right) d \tau_{1}}+2 \kappa \int_{0}^{t} e^{\int_{\tau}^{t} \boldsymbol{A}^{\dagger}\left(\tau_{1}\right) d \tau_{1}} \cdot e^{\int_{\tau}^{t} \boldsymbol{A}\left(\tau_{1}\right) d \tau_{1}} d \tau .
\end{aligned}
$$

Here the quantity $e^{\boldsymbol{B}}$ is a matrix exponential, defined by the usual power series

$$
e^{\boldsymbol{B}}=\boldsymbol{I}+\boldsymbol{B}+\frac{1}{2} \boldsymbol{B} \cdot \boldsymbol{B}+\ldots
$$


It should be noted that in the expression (8) for $\boldsymbol{M}(t)$ the two matrix exponentials cannot be combined and written as $e_{\tau}^{\int_{\tau}^{t}\left(\boldsymbol{A}^{\dagger}+\boldsymbol{A}\right)\left(\tau_{1}\right) d \tau_{1}}$. For matrix exponentials, $e^{\boldsymbol{B}_{1}+\boldsymbol{B}_{2}}=e^{\boldsymbol{B}_{1}} \cdot e^{\boldsymbol{B}_{2}}$ only if the matrices $\boldsymbol{B}_{1}$ and $\boldsymbol{B}_{2}$ commute. The velocity gradient matrix, $\boldsymbol{A}$, is typically a non-normal matrix $\left(\boldsymbol{A} \boldsymbol{A}^{\dagger} \neq \boldsymbol{A}^{\dagger} \boldsymbol{A}\right)$.

For a simple shear flow, we have $\boldsymbol{u}=(\dot{\gamma} y, 0)$ and

$$
\boldsymbol{A}=\left(\begin{array}{cc}
0 & 0 \\
\dot{\gamma} & 0
\end{array}\right)
$$

Since $\boldsymbol{A}$ is nilpotent $\left(\boldsymbol{A}^{2}=0\right)$, the matrix exponential has only two terms in its power series representation, so that

$$
e^{\boldsymbol{A} t}=\boldsymbol{I}+\boldsymbol{A} t=\left(\begin{array}{cc}
1 & 0 \\
\dot{\gamma} t & 1
\end{array}\right)
$$

\section{Computation of Lyapunov exponents}

Since $\boldsymbol{M}$ is a symmetric matrix the eigendecomposition takes the simple form

$$
M=R D R^{\dagger}
$$

where $\boldsymbol{R}$ is an orthogonal matrix (the columns of which are eigenvectors of $\boldsymbol{M}$ ), and $\boldsymbol{D}$ is real and diagonal (having the eigenvalues of $\boldsymbol{M}$ on the diagonal). When the eigenvalues are sufficiently distinct, the radial and angular degrees of freedom decouple from each other, as we will now show. (Our derivation parallels that of [1].) Let us substitute the expression (9) in the evolution equation (8):

$$
\begin{array}{r}
\dot{R} D R^{\dagger}+R D \dot{R}^{\dagger}+R \dot{D} R^{\dagger}=R D R^{\dagger} A+A^{\dagger} R D R^{\dagger}+2 \kappa I \\
R^{\dagger} \dot{R} D+D \dot{R}^{\dagger} R+\dot{D}=D R^{\dagger} A R+R^{\dagger} A^{\dagger} R D+2 \kappa I .
\end{array}
$$

Since

$$
\frac{d}{d t}\left(\boldsymbol{R}^{\dagger} \boldsymbol{R}\right)=\dot{\boldsymbol{R}}^{\dagger} \boldsymbol{R}+\boldsymbol{R}^{\dagger} \dot{\boldsymbol{R}}=0
$$

both $\dot{\boldsymbol{R}}^{\dagger} \boldsymbol{R}$ and $\boldsymbol{R}^{\dagger} \dot{\boldsymbol{R}}$ are antisymmetric matrices. Thus in equation (10) the first two terms on the left do not have any diagonal elements. Denoting $\tilde{\boldsymbol{A}}=\boldsymbol{R}^{\dagger} \boldsymbol{A} \boldsymbol{R}$ as the rotated velocity gradient matrix we have the following equation for the diagonal elements,

$$
\dot{D}_{i i}=2 \tilde{A}_{i i} D_{i i}+2 \kappa,
$$

where repeated indices do not indicate summation. If we assume $D_{i i}=e^{2 \rho_{i}}\left(\rho_{i}>0\right.$ denotes extension whereas $\rho_{i}<0$ represents compression) then one has

$$
\dot{\rho}_{i}=\tilde{A}_{i i}+\kappa e^{-2 \rho_{i}} .
$$

Without loss of generality, we arrange the eigenvalues $e^{2 \rho_{1}}, e^{2 \rho_{2}}, \ldots, e^{2 \rho_{d}}$ in non-increasing order:

$$
\rho_{1} \geq \rho_{2} \cdots \geq \rho_{d}
$$

where $d$ is the dimension of space. 


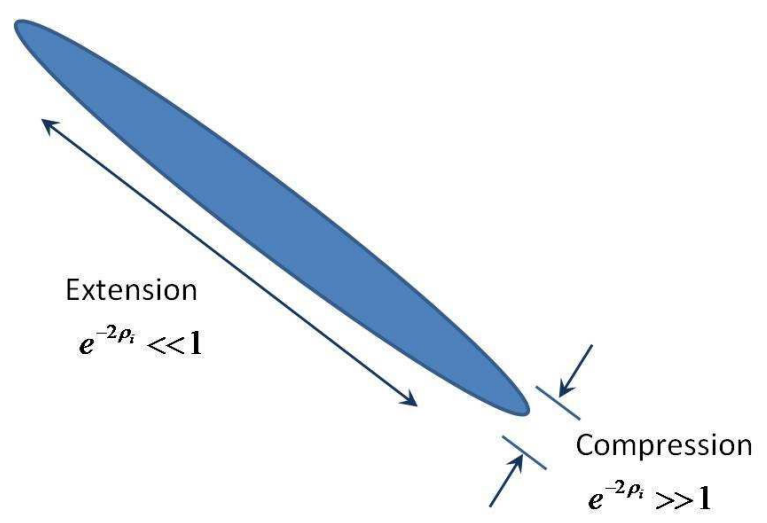

Figure 2: The diffusive term $\left(\kappa e^{-2 \rho_{i}}\right)$ in the equation for $\rho_{i}$ would be negligible along the extensional axis compared to the compressional direction.

Having worked with the diagonal matrix, $\boldsymbol{D}$, let us focus on the eigenvector matrix, $\boldsymbol{R}$. Considering the off-diagonal terms of equation (10),

$$
\begin{aligned}
& {\left[\boldsymbol{R}^{\dagger} \dot{\boldsymbol{R}} \boldsymbol{D}\right]_{i j}=\left[\boldsymbol{R}^{\dagger} \dot{\boldsymbol{R}}\right]_{i \ell} D_{\ell j}=\left[\boldsymbol{R}^{\dagger} \dot{\boldsymbol{R}}\right]_{i j} D_{j j}} \\
& {\left[\boldsymbol{D} \dot{\boldsymbol{R}}^{\dagger} \boldsymbol{R}\right]_{i j}=D_{i \ell}\left[\dot{\boldsymbol{R}}^{\dagger} \boldsymbol{R}\right]_{\ell j}=-\left[\boldsymbol{R}^{\dagger} \dot{\boldsymbol{R}}\right]_{i j} D_{i i}}
\end{aligned}
$$

where repeated indices do not indicate summation. Let us define $\boldsymbol{R}^{\dagger} \dot{\boldsymbol{R}}=\boldsymbol{\Omega}$, an antisymmetric matrix. Thus we have,

$$
\begin{aligned}
\left(D_{j j}-D_{i i}\right) \Omega_{i j} & =D_{i i} \tilde{A}_{i j}+\tilde{A}_{j i} D_{j j} \\
\Omega_{i j} & =\frac{e^{2 \rho_{i}} \tilde{A}_{i j}+e^{2 \rho_{j}} \tilde{A}_{j i}}{e^{2 \rho_{j}}-e^{2 \rho_{i}}}, \quad i \neq j
\end{aligned}
$$

Let us assume there exists scale separation in $\rho^{\prime}$ s $\left(\rho_{1} \gg \rho_{2} \gg \cdots \gg \rho_{d}\right) .{ }^{1}$ Thus if $\rho_{i} \gg \rho_{j}$, the $t \gg 1$ solution is

$$
\Omega_{i j} \approx\left\{\begin{array}{rr}
-\tilde{A}_{i j}, & i<j \\
\tilde{A}_{j i}, & i>j
\end{array}\right.
$$

Thus $\tilde{\boldsymbol{A}}$ is independent of the eigenvalues and we can solve equation (11) directly,

$$
\begin{aligned}
\rho_{i}(t) & =\rho_{i}(0)+\mathcal{A}_{i}(t)+\frac{1}{2} \log \left[1+2 \kappa e^{-2 \rho_{i}(0)} \int_{0}^{t} e^{-2 \mathcal{A}_{i}\left(t^{\prime}\right)} d t^{\prime}\right] \\
\text { where } \quad \mathcal{A}_{i}(t) & =\int_{0}^{t} \tilde{A}_{i i}(\tau) d \tau .
\end{aligned}
$$

When diffusion vanishes $(\kappa=0)$, this reduces to

$$
\rho_{i}(t)=\rho_{i}(0)+\int_{0}^{t} \tilde{A}_{i i}(\tau) d \tau .
$$

\footnotetext{
${ }^{1}$ This is almost always true for long times, but it may fail locally or due to a symmetry in the problem.
} 
The $\kappa=0$ case can be used to compute Lyapunov exponents ${ }^{2}$ from the eigenvalues of $\boldsymbol{M}$ :

$$
\lambda_{i}=\lim _{t \rightarrow \infty} \frac{\log \left(e^{\rho_{i}(t)}\right)}{t}=\lim _{t \rightarrow \infty} \frac{\rho_{i}(t)}{t}
$$

The convergence of the above limit is assured by Oseledec's multiplicative ergodic theorem [7].

A blob of initial size $L$ will become an ellipsoid with the length of its main axis changing as $\exp \left(\lambda_{i} t\right)$ along directions corresponding to positive and negative Lyapunov exponents. The rate of decrease of the smallest dimension is determined by $\lambda_{d}$. Because of diffusion, the contraction terminates after a time, $t_{\kappa} \approx\left|\lambda_{d}\right|^{-1} \log \left(L^{2}\left|\lambda_{d}\right| / \kappa\right)$, when the diffusive lengthscale, $r_{\kappa}=\sqrt{\kappa /\left|\lambda_{d}\right|}$ is attained.

\section{Large-deviation form of the central limit theorem ${ }^{3}$}

To form a probabilistic theory of mixing, we make $\boldsymbol{A}$, and therefore $\rho_{1}$ and $\rho_{2}$ (we restrict to $d=2$ dimensions from now), into random variables and deduce the approximate longtime moments of concentration. To do so, we require a central limit theorem (CLT) which is also valid for large deviations.

Let $x_{i}$ be i.i.d. (independent and identically distributed) random variables with finite mean $\bar{x}$ and variance $\sigma^{2}$. Let $x$ be the mean of $n$ such $x_{i}$. Roughly speaking, the classical CLT asserts that

$$
P(x, n) \sim \frac{1}{\sqrt{2 \pi n \sigma^{2}}} \exp \left(-\frac{n(x-\bar{x})^{2}}{2 \sigma^{2}}\right) \text { when } n \gg 1 \text { and } x-\bar{x}<\frac{\sigma}{\sqrt{n}} .
$$

The theorem applies to large $n$, but the condition that $x-\bar{x}<\frac{\sigma}{\sqrt{n}}$ means that as $n$ gets large, the region around the mean to which the theorem applies grows increasingly narrow. If one is concerned about the tails of the distribution, the theorem is not powerful enough. This is not surprising since it contains only second-moment information about the $x_{i}$ variables, and higher moments may be critical at the tails. In the theory of mixing, the tails of the $\rho$ PDF's are indeed significant, so we must use the large deviation form of the CLT, which is stronger and uses information from all moments of the $x_{i}$ variables. The significance of the tails will be evident a posteriori from the analysis using the large deviation CLT. The large deviation CLT asserts that

$$
P(x, n) \sim \exp (-n S(x-\bar{x})),
$$

where $S$ is the Cramér function (or rate function, or entropy function) determined by the PDF $p\left(x_{i}\right)$. (In these notes we typically neglect algebraic prefactors in asymptotic relations, such as the one above.) Whatever the form of $p\left(x_{i}\right), S$ is convex, and $S(0)$ and $S^{\prime}(0)$ vanish. Taylor expanding $S$ about 0 in the large deviation CLT, the first nonzero term is quadratic

\footnotetext{
${ }^{2}$ Lyapunov exponents describe the rate at which infinitesimally close trajectories in a dynamical system diverge from each other. A chaotic system is usually indicated by a positive infinite-time Lyapunov exponent.

${ }^{3}$ The 'facts' invoked in this section come from theorems which for the most part give bounds, not equalities. We are leaving out many details that are necessary to make the theory rigorous. See for example $[3,8,11]$ for more details.
} 
in $x-\bar{x}$. Truncating after this term recovers the classical form of the CLT, so the classical form simply approximates the large deviation form to the first nontrivial order. The explicit expression for the Cramér function in terms of $p\left(x_{i}\right)$ involves forward and inverse Fourier transforms, which generally cannot be explicitly evaluated. Rather than prove the largedeviation CLT or derive the general expression for the Cramér function (see e.g. $[3,8,10,11]$ ), we shall merely derive the Cramér function explicitly in the simple case where the $x_{i}$ have a Bernoulli distribution, though the general formula for $S$ may be derived by an analogous procedure.

\section{Large deviation CLT for the Bernoulli distribution}

Let $x_{i}$ have the PDF of a fair coin,

$$
p\left(x_{i}\right)=\frac{1}{2} \delta\left(x_{i}+1\right)+\frac{1}{2} \delta\left(x_{i}-1\right) .
$$

The characteristic function of a random variable is the Fourier transform of its PDF, generally written in the form $e^{-s(k)}$. We wish to derive $p(x, n)$, the PDF of the mean $x$ of $n$ random variables $x_{i}$. We are given $p\left(x_{i}\right)$, not $p(x, n)$, but we can express the latter in terms of the former. Because the $x_{i}$ are independent, the joint distribution of $x_{1}, \ldots, x_{n}$ is simply the product of their individual PDF's, so

$$
\begin{aligned}
p(x, n) & =\int p\left(x_{1}, \ldots, x_{n}\right) \delta\left(x-\frac{1}{n} \sum_{i=1}^{n} x_{i}\right) d x_{1} \cdots d x_{n} \\
& =\int p\left(x_{1}\right) \cdots p\left(x_{n}\right) \delta\left(x-\frac{1}{n} \sum_{i=1}^{n} x_{i}-x\right) d x_{1} \cdots d x_{n} .
\end{aligned}
$$

It is hard to compute the PDF directly from the PDF of $x_{i}$ by the above formula. Rather, we shall compute the generating function of $x$, and then its PDF. This is easier because integrating out $x$-dependence allows us to then integrate out the $x_{i}$ variables. The generating function of $x$ is

$$
\begin{aligned}
e^{-S(k)} & =\int p(x, n) e^{-i k x} d x \\
& =\int p\left(x_{1}\right) \cdots p\left(x_{n}\right) \delta\left(x-\frac{1}{n} \sum_{i=1}^{n} x_{i}\right) e^{-i k x} d x_{1} \cdots d x_{n} d x \\
& =\int p\left(x_{1}\right) \cdots p\left(x_{n}\right) \exp \left(-\frac{i k}{n} \sum_{i=1}^{n} x\right) d x_{1} \cdots d x_{n} \\
& =\prod_{i=1}^{n} \int p\left(x_{i}\right) e^{-\frac{i k x_{i}}{n}} d x_{i} \\
& =\left(\int p\left(x_{1}\right) e^{-\frac{i k x_{1}}{n}} d x_{1}\right)^{n} \\
& =\left(\frac{1}{2} \int\left(\delta\left(x_{1}+1\right)+\delta\left(x_{1}-1\right)\right) e^{-\frac{i k x_{1}}{n}} d x_{1}\right)^{n} \\
& =\cos ^{n}\left(\frac{k}{n}\right) .
\end{aligned}
$$


We recover the PDF of $x$ by taking the inverse Fourier transform of its generating function:

$$
\begin{aligned}
p(x, n) & =\frac{1}{2 \pi} \int \cos ^{n}\left(\frac{k}{n}\right) e^{i k x} d k \\
& =\frac{1}{2 \pi} \int \exp \left(n \ln \left[\cos \left(\frac{k}{x}\right)\right]+i k x\right) d k \\
& =\frac{n}{2 \pi} \int \exp (n[\ln (\cos K)+i K x]) d K,
\end{aligned}
$$

where $K \equiv \frac{k}{n}$. The last form of the above integral suggests the stationary phase approximation, since the frequency of the oscillatory part of the integrand goes to infinity as $n$ does. Omitting the details, the stationary phase approximation yields

$$
S(x) \sim-\frac{1}{2}(x+1) \ln \left(\frac{1-x}{x+1}\right)+\ln (1-x) .
$$

The classical form the the CLT would give only

$$
\begin{aligned}
p(x, n) & \sim \exp \left(-\frac{N}{2} S^{\prime \prime}(0)(x-\bar{x})^{2}\right) \\
& \sim \exp \left(-\frac{(x-\bar{x})^{2}}{2 / N}\right) .
\end{aligned}
$$

In this example, the classical CLT overestimates the weight of the tails of $p(x, n)$, though typically the opposite is the case. In any case, the large deviation form is needed for accuracy at the tails.

\section{$6 \quad$ Large deviation CLT applied to mixing}

Recall that $\rho_{2}$ has generally become quite small at long times. However, in a fluid with diffusion, the $\rho_{2}$ dimension eventually becomes small enough that further contraction by stretching is balanced by diffusion. This is the Batchelor width, $\sqrt{\kappa / \lambda_{1}}$, beyond which the scalar blob will contract no further. As such late times we can assume that the PDF of $\rho_{2}$ is stationary in time, so as $t \rightarrow \infty$,

$$
P\left(\rho_{1}, \rho_{2}, t\right) \sim \exp \left(-t S\left(\frac{\rho_{1}}{t}-\lambda_{1}\right)\right) P_{\text {stat }}\left(\rho_{2}\right) .
$$

The exact stationary distribution of $\rho_{2}$ is irrelevant to the heuristics of this theory. Suppose that the concentration at each point in a blob of scalar is inversely proportional to the blob's total volume, so

$$
\theta(\boldsymbol{x}, t) \sim(\operatorname{det} M)^{1 / 2}=e^{-\left(\rho_{1}+\rho_{2}\right)} .
$$

This is an accurate assumption for a Gaussian blob, for instance. We now combine the above approximations to estimate the expectation of $\theta^{\alpha}$.

$$
\begin{aligned}
\left\langle\theta^{\alpha}\right\rangle(t) & \sim C(t) \int e^{-\alpha\left(\rho_{1}+\rho_{2}\right)} \exp \left(-t S\left(\frac{\rho_{1}}{t}-\lambda_{1}\right)\right) P_{\text {stat }}\left(\rho_{2}\right) d \rho_{1} d \rho_{2} \\
& \sim \int e^{-\alpha \rho_{1}} \exp \left(-t S\left(\frac{\rho_{1}}{t}-\lambda_{1}\right)\right) d \rho_{1}
\end{aligned}
$$



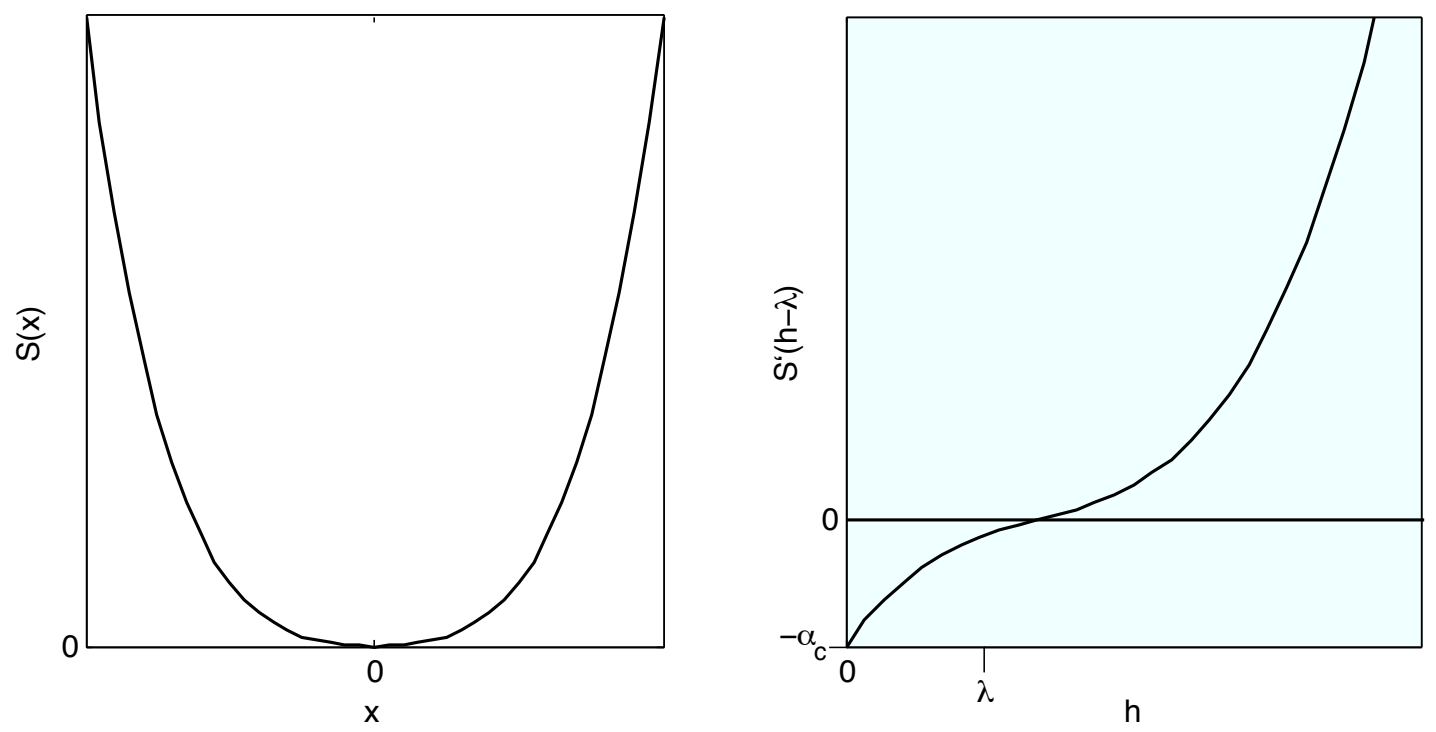

Figure 3: Generic $S(x)$ and $S\left(h-\lambda_{1}\right)$. When $\alpha>\alpha_{c}$, there is no positive $h *$ such that $S^{\prime}\left(h^{*}-\lambda\right)=-\alpha$, so we must choose $h^{*}=0$ for the saddle-point approximation.

where $C(t)$ is a normalization that is algebraic in $t$ and is therefore asymptotically dominated by the integral, so we have ignored it along with whatever constant emerges from integrating over $\rho_{2}$. Letting $h \equiv \frac{\rho_{1}}{t}$,

$$
\left\langle\theta^{\alpha}\right\rangle(t) \sim \int e^{-t\left(\alpha h+S\left(h-\lambda_{1}\right)\right)} d h=\int e^{-t H(h)} d h,
$$

where $H(h) \equiv \alpha h+S(h-\lambda)$. This integral may be approximated by the saddle-point method for large $t$.

The saddle-point method typically asserts that

$$
\left\langle\theta^{\alpha}\right\rangle(t) \sim e^{-H\left(h^{*}\right) t}
$$

where $h^{*}$ is such that $H^{\prime}\left(h^{*}\right)=0$, i.e. such that $S^{\prime}\left(h^{*}-\lambda_{1}\right)=-\alpha$. In the present case, it is not always possible to choose such an $h^{*}$ because $h$ must be nonnegative. This is clear from the generic $S$ and $S^{\prime}(h-\lambda)$ curves plotted in Figure 6. Whatever the exact form of the Cramér function, $S^{\prime}(h)$ begins at the origin and increases monotonically in $h$, so $S^{\prime}(h-\lambda)$ begins at some negative point $\left(0,-\alpha_{c}\right)$ and increases monotonically in $h$. When $\alpha<\alpha_{c}$, $H^{\prime}\left(h^{*}\right)=0$ is possible, but when $\alpha>\alpha_{c}$, the best we can do is to choose $h^{*}=0$. That is,

$$
\left\langle\theta^{\alpha}\right\rangle(t) \sim e^{-t\left(\alpha h^{*}+S^{\prime}\left(h^{*}-\lambda_{1}\right)\right)},
$$

where $h^{*}=\max \left\{0, \lambda+\left(S^{\prime}\right)^{-1}(-\alpha)\right\}$. Neglecting large deviations, the classical CLT would have given a decay rate of $-\alpha \lambda$, which is an upper bound on the decay rate we derived. In fact, the tails of the PDF of $\rho_{1}$ act significantly to slow the decay of concentration. Above $\alpha_{c},\left\langle\theta^{\alpha}\right\rangle(t)$ is constant in $\alpha$. We may understand this plateauing as high concentration blobs dominating the expectation for large $\alpha$. These are the blobs that have experienced no 
stretching, so $\alpha>\alpha_{c}$ moments are determined solely by realizations of $\theta$ without stretching. Note that the plateauing of moments also means that the $L^{\alpha}$ norm of $\theta$ vanishes as $\alpha \rightarrow \infty$, which is characteristic of intermittency.

\section{References}

[1] E. Balkovsky And A. Fouxon, Universal long-time properties of Lagrangian statistics in the Batchelor regime and their application to the passive scalar problem, Phys. Rev. E, 60 (1999), pp. 4164-4174.

[2] G. K. BAtchelor, Small-scale variation of convected quantities like temperature in turbulent fluid: Part 1. General discussion and the case of small conductivity, J. Fluid Mech., 5 (1959), pp. 113-133.

[3] R. S. ElLis, Entropy, Large Deviations, and Statistical Mechanics, Springer-Verlag, New York, 1985.

[4] G. Falkovich, K. Gawȩdzki, and M. Vergassola, Particles and fields in turbulence, Rev. Mod. Phys., 73 (2001), pp. 913-975.

[5] P. H. Haynes And J. Vanneste, What controls the decay of passive scalars in smooth flows?, Phys. Fluids, 17 (2005), p. 097103.

[6] R. H. KRAichnan, Small-scale structure of a scalar field convected by turbulence, Phys. Fluids, 11 (1968), pp. 945-953.

[7] V. I. Oseledec, A multiplicative theorem: Lyapunov characteristic numbers for dynamical systems, Trans. Moscow Math. Soc., 19 (1968), pp. 197-231.

[8] A. Schwartz And A. Weiss, Large Deviations for Performance Analysis, Chapman \& Hall, London, 1995.

[9] J. Sukhatme and R. T. Pierrehumbert, Decay of passive scalars under the action of single scale smooth velocity fields in bounded two-dimensional domains: From nonself-similar probability distribution functions to self-similar eigenmodes, Phys. Rev. E, 66 (2002), p. 056032.

[10] J.-L. Thiffeault, Scalar decay in chaotic mixing, in Transport and Mixing in Geophysical Flows, J. B. Weiss and A. Provenzale, eds., vol. 744 of Lecture Notes in Physics, Berlin, 2008, Springer, pp. 3-35.

[11] S. R. S. VARAdhan, Large deviations and applications, Society for Industrial and Applied Mathematics, Philadelphia, PA, 1984.

[12] W. R. Young, Stirring and mixing, in Proceedings of the 1999 Summer Program in Geophysical Fluid Dynamics, J.-L. Thiffeault and C. Pasquero, eds., Woods Hole, MA, 1999, Woods Hole Oceanographic Institution. http://gfd. whoi.edu/proceedings/1999/PDFvol1999.html. 
[13] Y. B. Zeldovich, The asymptotic law of heat transfer at small velocities in the finite domain problem, Zhurnal eksperimentalnoï i teoreticheskoï fiziki, 7 (1937), pp. 14661468.

[14] _ Exact solution to the problem of diffusion in a periodic velocity field and turbulent diffusion, Doklady Akademiia Nauk SSSR, 266 (1982), pp. 821-826.

[15] Y. B. Zeldovich, A. A. Ruzmaikin, S. A. Molchanov, and D. D. Sokoloff, Kinematic dynamo problem in a linear velocity field, J. Fluid Mech., 144 (1984), pp. 111. 


\title{
Social Behavior, Mixing, and the Evolution of Schooling
}

\author{
Glenn Flierl \\ Notes by Kiori Obuse and Emma Boland (née Thompson)
}

May 11, 2011

\section{Introduction}

Swimming organisms adjust their behavior in response to environmental conditions and form structures such as patches, swarms, and schools. Disadvantages of these tendencies include tougher competition for food, the attraction of predators; some hydrodynamic disadvantages - turbulent wakes disrupt movement, and more energy is required to swim, etc. There are several advantages however, such as enhanced reproduction, predator avoidance, and the easier search for prey; some hydrodynamic advantages - extra turbulence brings higher encounter rates, and coordinated swimming. Here, we study a model for such organisms' behavior.

\section{Lagrangian Dynamics}

In order to consider the model mentioned above, we start with the Lagrangian dynamics of an individual or molecule of fluid. The stochastic differential equations for the position $\mathbf{X}$ and velocity $\mathbf{U}$ are

$$
\begin{gathered}
d X_{i}=U_{i} d t, \\
d U_{i}=A_{i} d t+\beta_{i j} d W_{j},
\end{gathered}
$$

where $\mathbf{A}$ is the acceleration produced by deterministic, or large-scale, forces, $\beta_{i j} d W_{j}$ is the random acceleration, with the random increment $d W_{j}$ satisfying $\left\langle d W_{i}\right\rangle=0$ and $\left\langle d W_{i} d W_{j}\right\rangle=$ $\delta_{i j} d t$, and $\beta_{i j}$ will be a diagonal matrix. The equations (1) and (2) also hold for continuous $\mathbf{X}$ and discrete $\mathbf{U}$. As an example, consider a drag law for the acceleration

$$
\mathbf{A}=-r(\mathbf{U}-\mathbf{u})
$$

with $\mathbf{u}$ being the water velocity and $\beta_{i j}=\beta \delta_{i j}$. Then the dispersion is determined by $\beta$ and $r$; from the equations, by assuming that the statistics become time-independent for large $t$, $\left\langle U_{i}(t)\right\rangle=\left\langle U_{i}(t+d t)\right\rangle$ in the long-time limit or solving the equations (1) and (2) by utilizing Ito's lemma (see, for example, [2]), we can show that

$$
\begin{aligned}
\left\langle U_{i}\right\rangle & \rightarrow u_{i}, \\
\left\langle\left(U_{i}-u_{i}\right)\left(U_{j}-u_{j}\right)\right\rangle & \rightarrow \frac{\beta^{2}}{2 r} \delta_{i j}, \\
\left\langle X_{i}(t) X_{j}(t)\right\rangle & \rightarrow\left\langle X_{i}(0) X_{j}(0)\right\rangle+\frac{\beta^{2}}{r^{2}} \delta_{i j} t .
\end{aligned}
$$



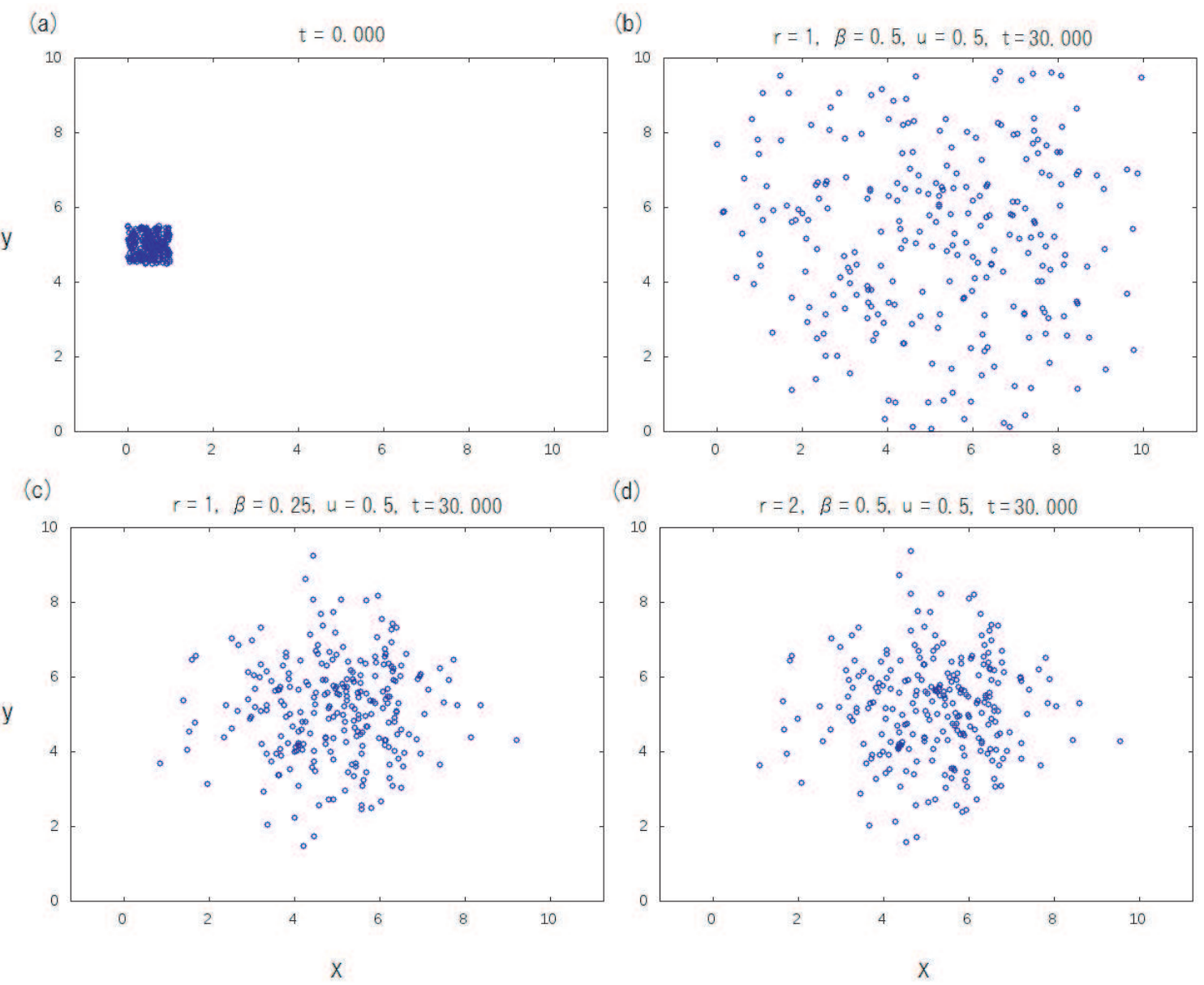

Figure 1: Distributions produced by numerical simulation of Lagrangian particles from equations (1,2), showing (a) the initial conditions for all cases, (b) $r=1, \beta=0.5, u=$ $0.5, t=30$, (c) $r=1, \beta=0.25, u=0.5, t=30$, (d) $r=2, \beta=0.5, u=0.5, t=30$.
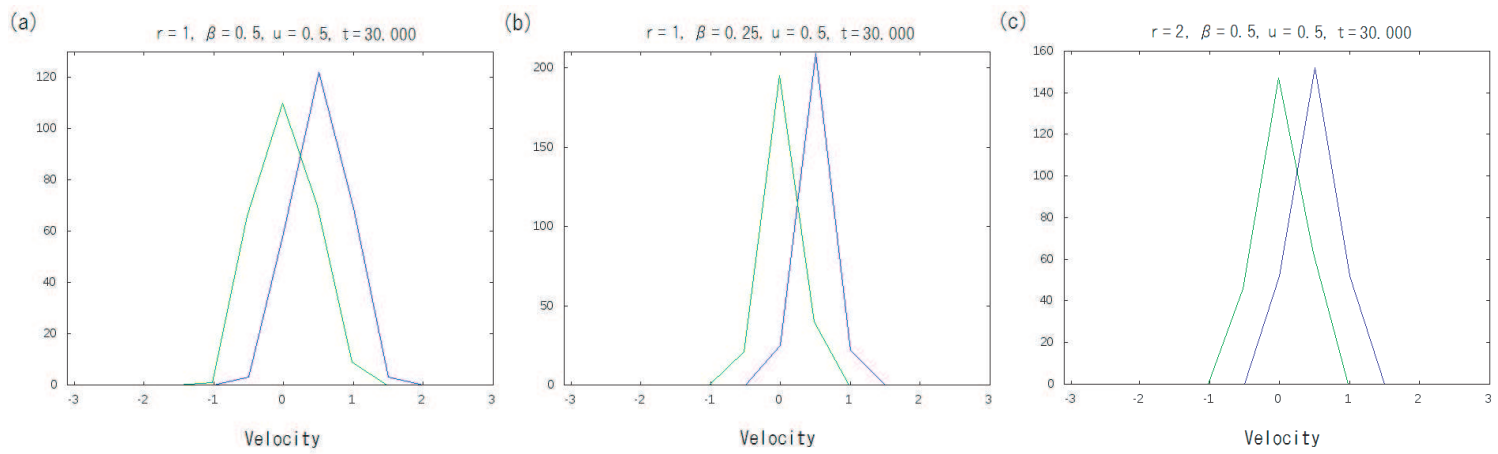

Figure 2: Histogram of velocities from numerical simulations of Lagrangian particles with the parameters (a) $r=1, \beta=0.5, u=0.5, t=30$ (b) $r=1, \beta=0.5, u=0.25, t=300$ (c) $r=2, \beta=0.5, u=0.5, t=30$. The blue and the green lines in each panel represent the velocities in the $\mathrm{x}$ - and $\mathrm{y}$-directions, respectively. 
As diffusion coefficient $\kappa$ is $\kappa \equiv\left\langle d X_{i} d X_{j}\right\rangle /(2 d t)$, the latter corresponding to a diffusivity of $\kappa=\beta^{2} / 2 r^{2}$, which means that the area grows like $4 \kappa t$ and the velocity variance is $r \kappa$.

Figure 1 contains snapshots of the distribution of particles for different combinations of the parameters $r$ and $\beta$. We can see that the area occupied by the particles grows more for larger $\beta$ (see Fig.1(b) and (c)) and smaller $r$ (see Fig.1 (b) and (d)). This can also be confirmed by the probability density functions (not normalized to one) of the $\mathrm{x}$ - and y-velocities shown in Fig.2.

\section{Grouping Mechanisms}

To consider a variety of motions, we use the form

$$
\begin{gathered}
d X_{i}=U_{i} d t \\
d U_{i}=-r\left(U_{i}-u_{i}-V_{i}\right) d t+\beta_{i j} d W_{j} .
\end{gathered}
$$

Here, $\mathbf{V}$ denotes the preferred swimming velocity, swimming towards the surface for example. Now we distinguish between various behaviors of swimming organisms. The properties described below maybe used individually or together, depending upon the organisms considered.

- taxis: $\mathbf{V}$ depends on gradient of cue field $\nabla C(\mathbf{x}, t)$ (Fig.3), where cue $C(\mathbf{x}, t)$ may be environmental (food, light, depth, etc.) or social (positions of neighbors, etc.). This describes a large-scale preferred velocity that the group tends to.

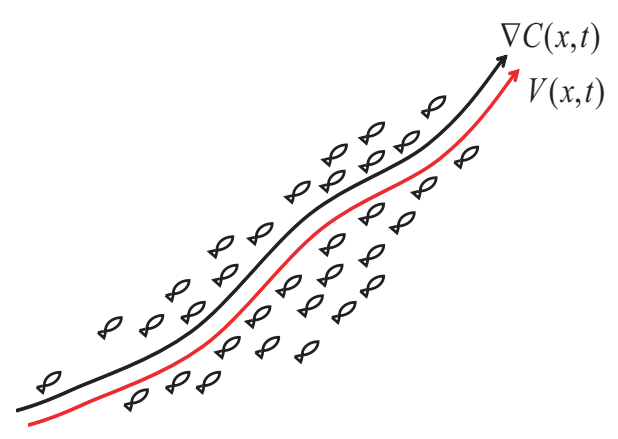

Figure 3: Representation of taxis.

Here, we define taxis as a preference for moving up the gradient of the cue field,

$$
\mathbf{V}=\alpha \nabla C(\mathbf{x}) .
$$

As an example, we consider

$$
C=C_{0}[1-\cos (k x)] / 2
$$

so that $\mathbf{V}=V_{0} \sin (k x) \hat{\mathbf{x}}$ and $\mathbf{A}=-r\left[\mathbf{U}-\mathbf{u}-V_{0} \sin (k x) \hat{\mathbf{x}}\right]$. Figures 4 and 5 show the distribution and the PDF (not normalized to one) of the organisms taken from numerical 
simulations, respectively. Taxis on a spatially and temporally fixed cue field has its center of aggregation where $\nabla \cdot \mathbf{V}_{\text {pref }}\left(\right.$ or $\nabla^{2} C$ ) is most negative when there is no advection (see Fig.4 (b)), and the advection can shift this downstream (see Fig.4 (b)-(d)), which decreases the strength of the aggregation as a result (see Fig.4 (b), (c), and (d)). We can also see that the diffusion coefficient $\kappa=\beta^{2} / 2 r^{2}$ controls the width of the aggregation (Figs.4, 5).
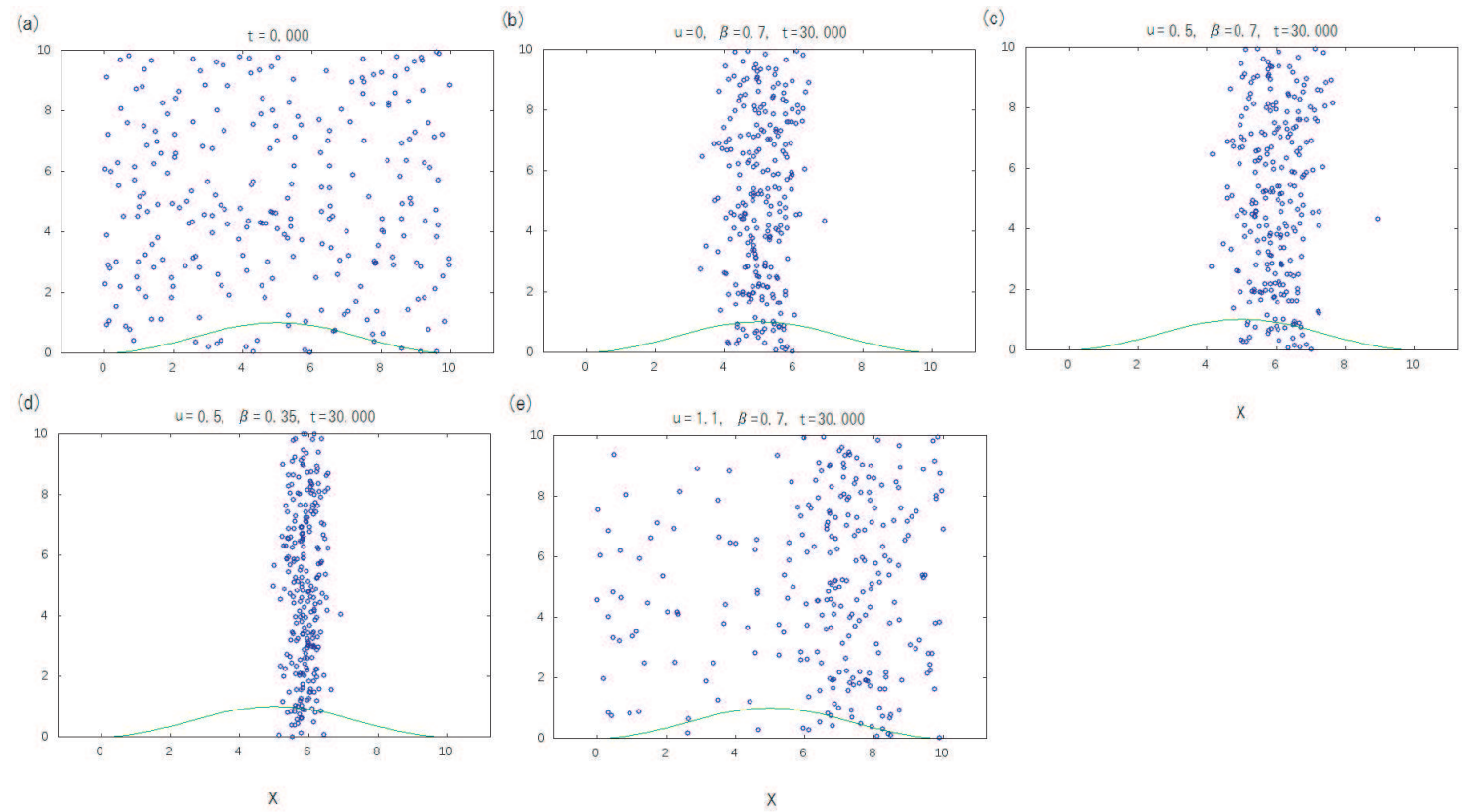

Figure 4: Taxis: Numerical simulation results of the example described in Section 3 showing organism distribution (blue dots) and the profile of $\mathrm{C}$ (green line) with (a) the initial conditions for all cases, (b) $u=0, \beta=0.7, t=30$, (c) $u=0.5, \beta=0.7, t=30$, (d) $u=0.5, \beta=0.35, t=30$, (e) $u=1.1, \beta=0.7, t=30$.

We then define social taxis as grouping when the cue field is defined in terms of the positions of other organisms;

$$
C(\mathbf{X})=\sum_{\mathbf{X}^{\prime}}\left[\frac{1}{4}\left|\mathbf{X}^{\prime}-\mathbf{X}\right|^{4}-\frac{1}{2}\left|\mathbf{X}^{\prime}-\mathbf{X}\right|^{2}\right], \quad\left|\mathbf{X}^{\prime}-\mathbf{X}\right|<1,
$$

so that the $\nabla C$ can be expressed as $\nabla C=\left(\mathbf{X}-\mathbf{X}^{\prime}\right) w\left(\left|\left(\mathbf{X}-\mathbf{X}^{\prime}\right)\right|\right)$ with weight function $w$ having the profile shown in Fig.6. Using this form of social taxis, the temporal variation of the aggregation of the considered organisms depends upon the displacement of each set of two organisms, i.e. $\left|\mathbf{X}^{\prime}-\mathbf{X}\right|$, and so groups close to each other merge as time progresses as shown in Fig.7 (note the groups in red circles).

- kinesis: $\beta$ depends on the cue field:

$$
\beta=\beta(C) .
$$

This describes an individual's tendency to move randomly, when they, for example, find some food close to them (Fig.8), and is a more primitive response in which the random accelerations increase or decrease depending on the cue field. 

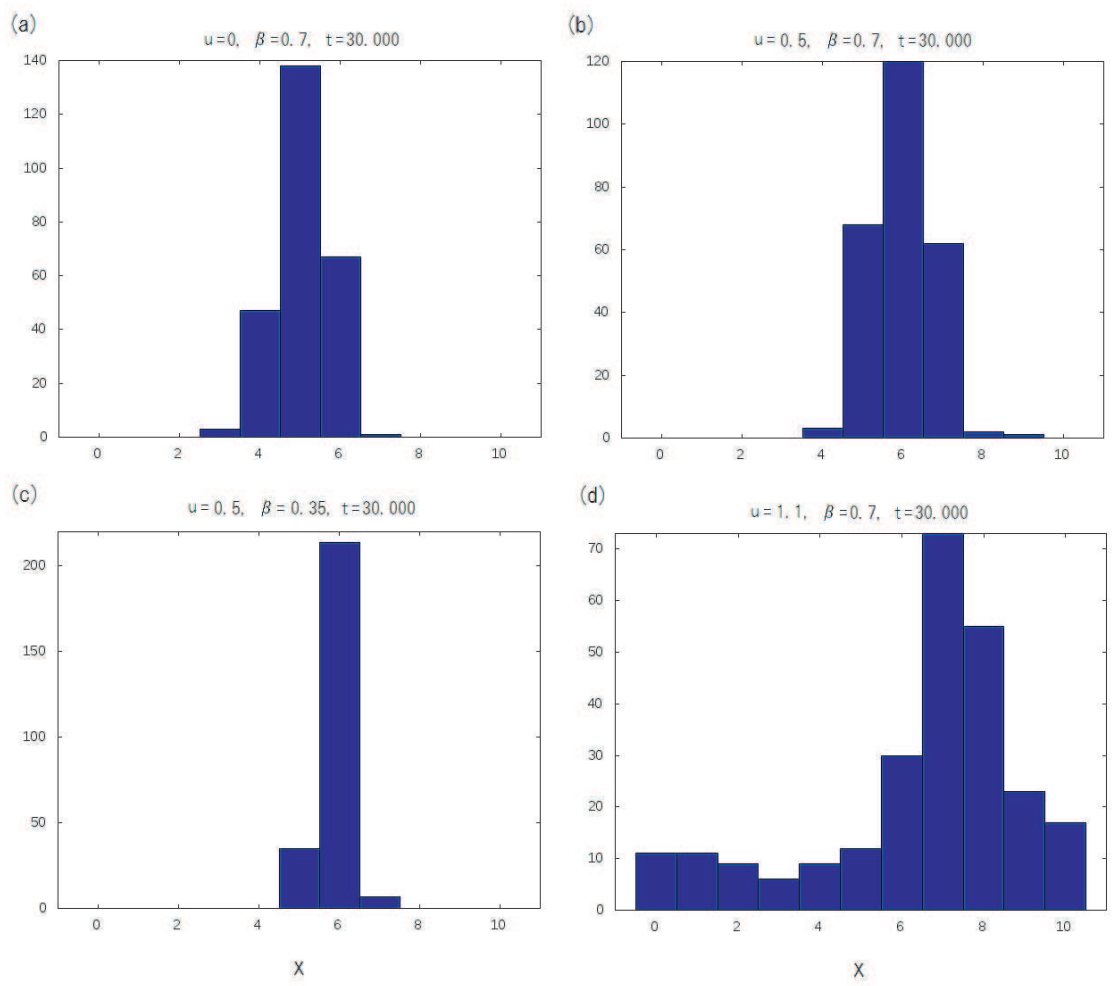

Figure 5: Taxis: Histograms from numerical simulations of organism distribution in the x-direction for (a) $u=0, \beta=0.7, t=30$, (b) $u=0.5, \beta=0.7, t=30$, (c) $u=0.5, \beta=$ $0.35, t=30$, (d) $u=1.1, \beta=0.7, t=30$.

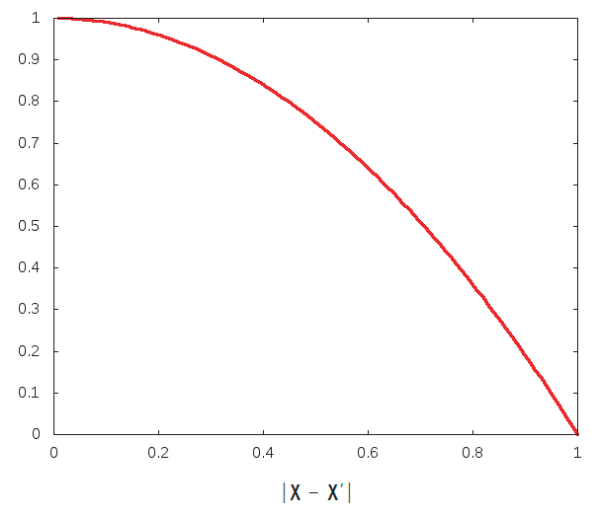

Figure 6: An example of the weight function $w\left(\left|\mathbf{X}^{\prime}-\mathbf{X}\right|\right)$ used in social taxis. Here, $w(x)=\left(|x|^{3}-|x|\right) / x$. 

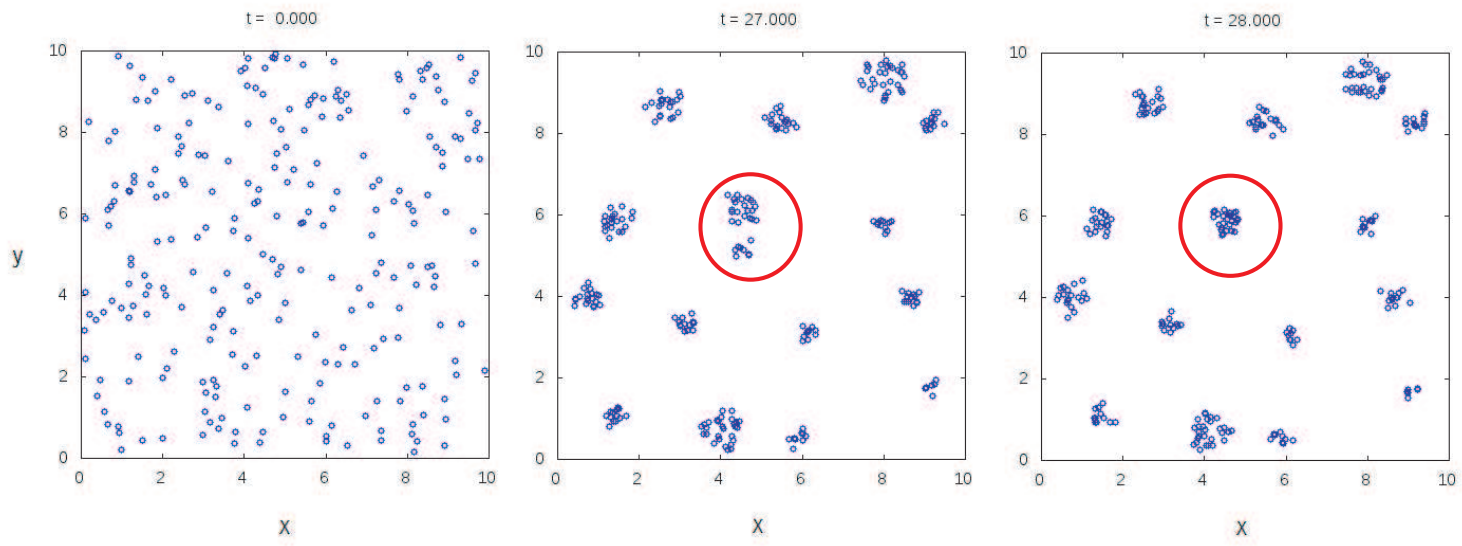

Figure 7: Social Taxis: The temporal variation of organism distributions from numerical simulations. From left to right, the panels show the organism distribution at times $t=$ $0, t=27$, and $t=28$.

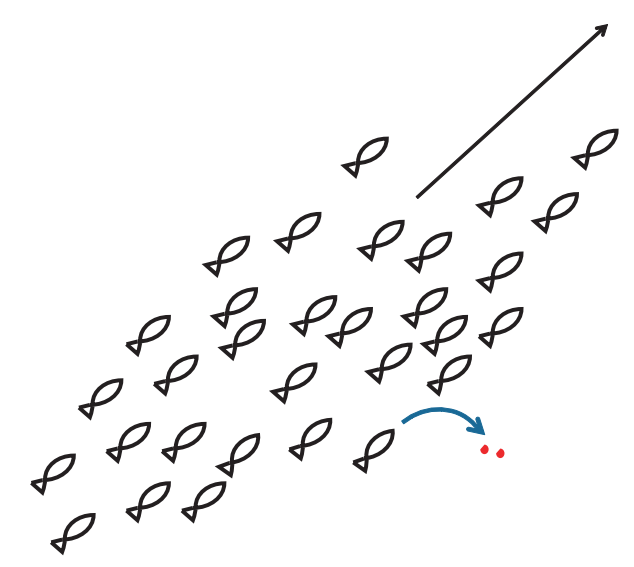

Figure 8: Representation of kinesis.

Let $\beta=\beta_{0}-\beta_{1} C / C_{0}$, for example, and consider $C$ given by (8) again. Figures 9 and 10 show example distributions of organisms from numerical simulations. Kinesis can produce aggregation since $\beta$ decreases as $C$ increases, which means $d U / d t$ decreases as $C$ increases. The groups tend to be looser however, depending upon $\beta_{\max } / \beta_{\min }$, and $\mathbf{u}$ as mentioned before.

As another example of kinesis, we assume that the organisms turn more frequently and have smaller mean free path in the presence of many neighbors. Now we consider $\beta$ in the form $\beta \propto \exp \left(-C / C_{0}\right)$, and write social kinesis as

$$
C(\mathbf{X})=\sum_{\mathbf{X}^{\prime}}\left[1-\left|\mathbf{X}-\mathbf{X}^{\prime}\right|^{2}\right], \quad\left|\mathbf{X}-\mathbf{X}^{\prime}\right|<1 .
$$

This does not include the fourth order term as in (9), since social kinesis appears in $\beta$ as a non-derivative form. The possible situations that can be produced are some random turning, more random turning, and the avoidance of others as shown in Fig.11. 

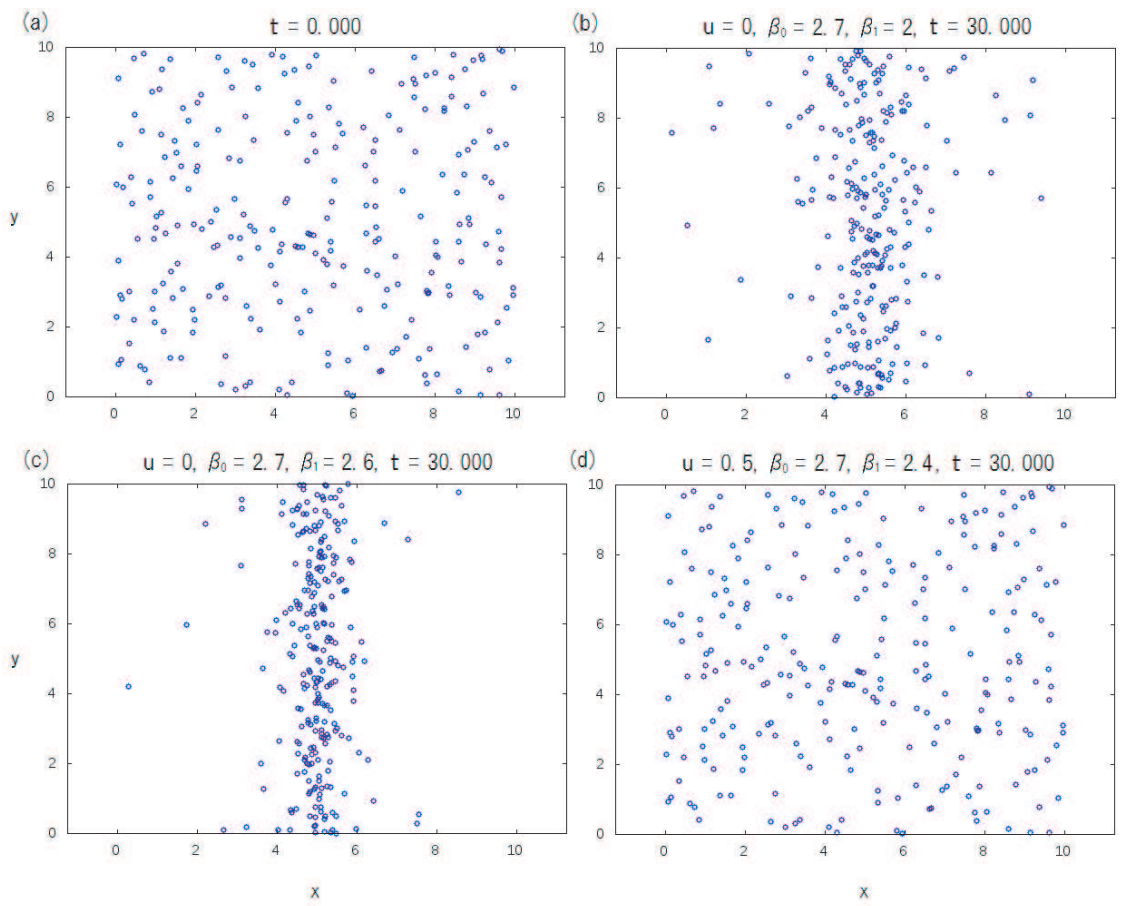

Figure 9: Kinesis: Distributions of organisms from numerical simulations with (a) initial time for all cases, (b) $u=0, \beta_{0}=2.7, \beta_{1}=2.0, t=30$, (c) $u=0, \beta_{0}=2.7, \beta_{1}=2.6, t=30$, (d) $u=0.5, \beta_{0}=2.7, \beta_{1}=2.4, t=30$.
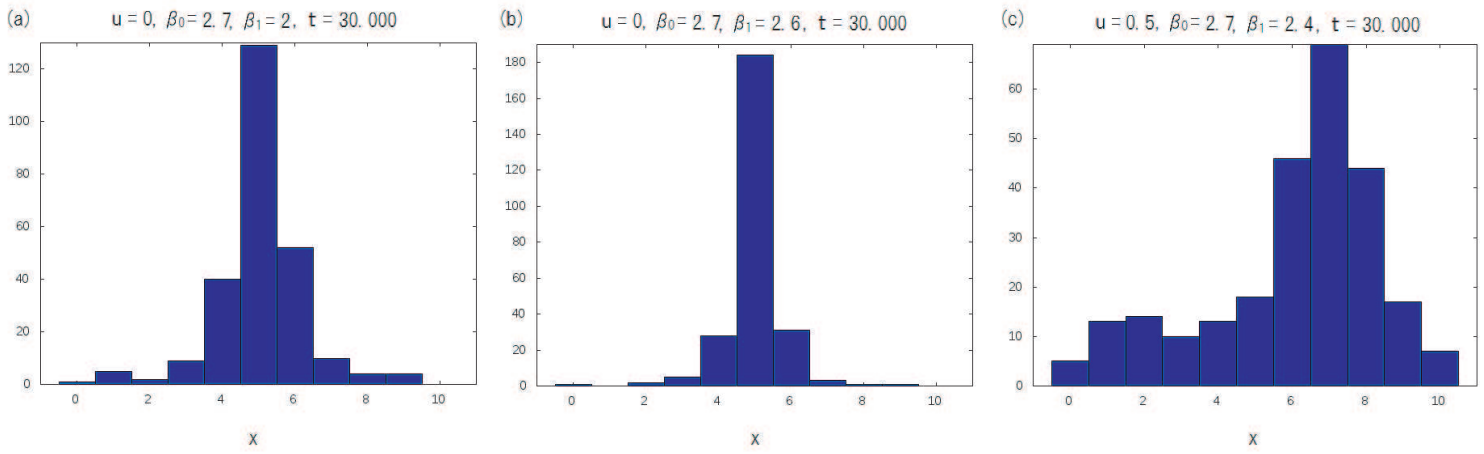

Figure 10: Kinesis: Histograms of organism distributions in the $\mathrm{x}$-direction from numerical simulations for (a) $u=0, \beta_{0}=2.7, \beta_{1}=2.0, t=30$, (b) $u=0, \beta_{0}=2.7, \beta_{1}=2.6, t=30$, (c) $u=0.5, \beta_{0}=2.7, \beta_{1}=2.4, t=30$. 

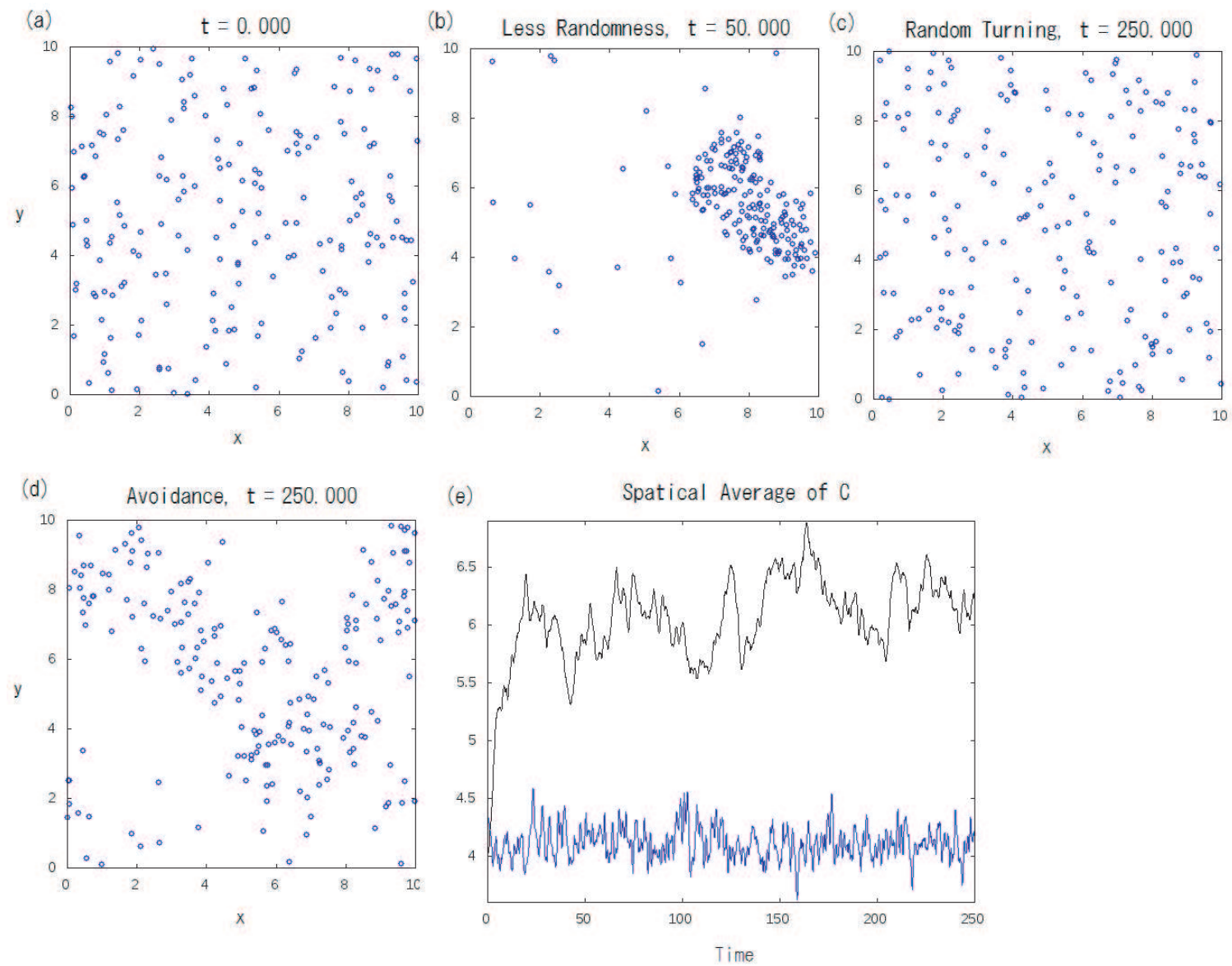

Figure 11: Social kinesis. (a)-(d) Distributions of organisms from numerical simulations showing (a) the initial distribution for all cases, distributions after the time shown in the cases (b) with some random turning, (c) more frequent random turning, and (d) organisms avoiding neighbors; and (e) the temporal variation of spatial average of $C$ for panels (c) (blue) and (d) (black). 
In the case that organisms turn randomly occasionally, the system ends up with a large group of organisms (Fig.11(b)), whilst the other two cases end up with much looser groups (Fig.11(c) and (d)). Comparing the cases of turning randomly and avoidance, the avoidance case shows stronger grouping, since the small mean free path interferes with the avoiding behaviour. This can also be verified by the temporal variation of the spatial average of the social kinesis $C$, which measures the strength of the grouping; the avoidance case shows a much larger value of average $C$ than that of the case with frequent random turning(Fig.11(e)).

- Schooling: V depends on neighbor's $\mathbf{U}$ with $|\mathbf{V}|$ having a fixed value (Fig.12): $\mathbf{V}=$ $\mathbf{V}\left(\mathbf{U}_{\text {neighbors }}\right)$. This describes the behavior of the organisms that tend to swim similarly to their neighbors.

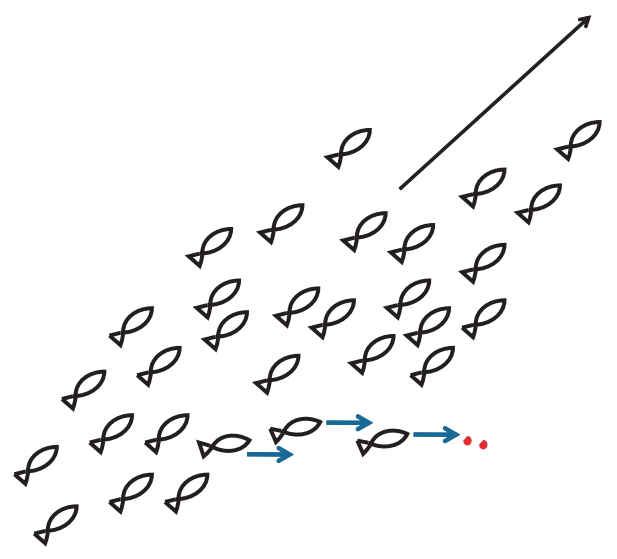

Figure 12: Representation of schooling.

The preferred direction of the swimming organisms results from a combination of attraction and alignment tendencies, and so schooling can be represented as

$$
\begin{gathered}
\mathbf{V}=V_{0} \mathbf{V}_{1} /\left|\mathbf{V}_{1}\right| \\
\mathbf{V}_{1}=\alpha \sum_{\mathbf{X}^{\prime}}\left(\mathbf{X}^{\prime}-\mathbf{X}\right) w\left(\left|\mathbf{X}^{\prime}-\mathbf{X}\right|\right)+\sum_{\mathbf{X}^{\prime}} \mathbf{U}^{\prime} w\left(\left|\mathbf{X}^{\prime}-\mathbf{X}\right|\right) .
\end{gathered}
$$

The first term represents attraction to neighbors. $\alpha$ controls the strength of the attraction; larger $\alpha$ gives stronger schooling as seen in Figure 13 which shows the temporal variation of organism distributions from numerical simulations for different $\alpha$. Paying attention to the groups surrounded by circles, we notice that the case $\alpha=0.3$ shows weak schooling and the groups tend to break apart easily, whilst the case $\alpha=0.7$ shows much stronger schooling. The second term represents the tendency of organisms to align their swimming with their neighbors. The distance between two organisms at which alignment becomes effective tends to be smaller than that for attraction, as shown in Fig.14. Here, the choice of the weighting function $w$ affects the internal structure of self-organized groups. 

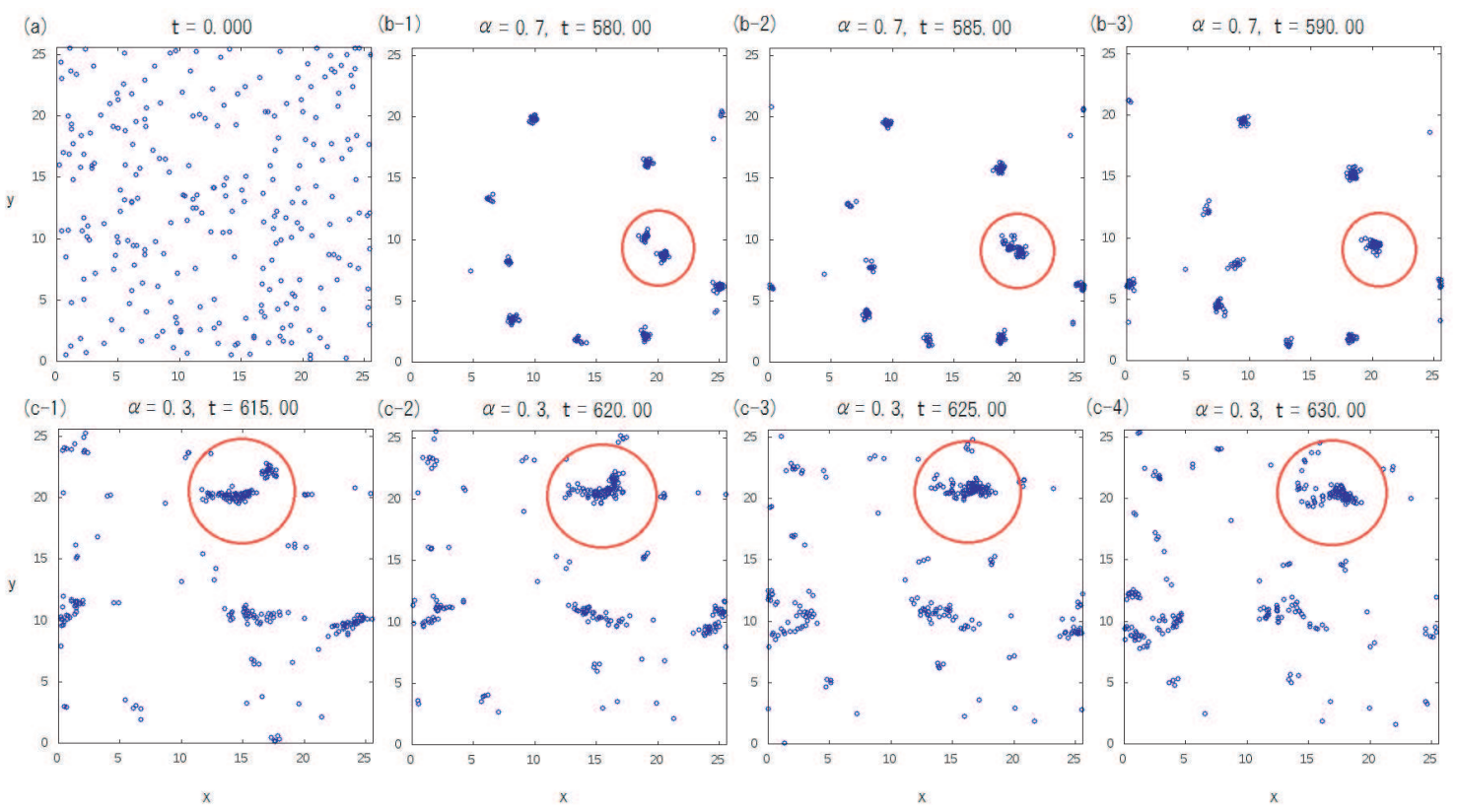

Figure 13: Schooling: Snapshots of organism distributions from numerical simulations. (a) the initial condition for all cases, (b-1) $\alpha=0.7, t=580$, (b-2) $\alpha=0.7, t=585$, (b-3) $\alpha=0.7, t=590,(\mathrm{c}-1) \alpha=0.3, t=615,(\mathrm{c}-2) \alpha=0.3, t=620,(\mathrm{c}-3) \alpha=0.3, t=625,(\mathrm{c}-4)$ $\alpha=0.3, t=630$.

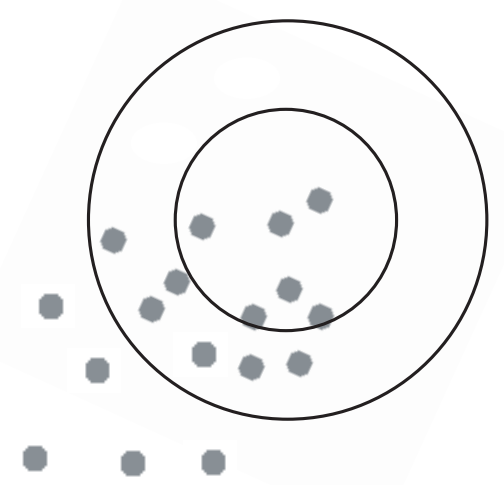

Figure 14: Schooling: Areas of attraction (outer circle) and alignment (inner circle). The gray dots represent organisms. 


\section{The Boltzmann Equation}

We are interested in finding the form of the probability density function of the system, $\mathcal{P}\left(\mathbf{X}_{1}, \mathbf{U}_{1}, \mathbf{X}_{2}, \mathbf{U}_{2}, \ldots ., t\right)$. The evolution of this quantity is described by

$$
\frac{\partial \mathcal{P}}{\partial t}=-\nabla_{\mathbf{x}_{i}} \cdot\left(\mathbf{U}_{i} \mathcal{P}\right)-\nabla_{\mathbf{u}_{i}} \cdot\left(\mathbf{A}_{i} \mathcal{P}\right)+\int d^{n} U^{\prime} T\left(U-U^{\prime} \mid x, U^{\prime}, t\right) \mathcal{P} .
$$

If $\mathcal{P}$ is sharply peaked in velocity, the last term on the RHS, which can be thought of as a 'collision' rate, can alternatively be written as

$$
\frac{\partial^{2}}{\delta \mathbf{U}_{i} \mathbf{U}_{j}}\left(\frac{\beta_{i m} \beta_{j m}}{2} \mathcal{P}\right) \text {. }
$$

If we assume independence of organisms $i$, which is not true for social behavior, the probabilities are equal for all $i$, and we can rewrite the evolution as

$$
\frac{\partial \mathcal{P}}{\partial t}=-\nabla_{\mathbf{x}} \cdot(\mathbf{U} \mathcal{P})-\nabla_{\mathbf{u}} \cdot(\mathbf{A} \mathcal{P})+\frac{1}{2} \nabla_{\mathbf{u}}^{2} \beta^{2} \mathcal{P}
$$

The first term on the RHS represents advection in the $\mathrm{x}$-direction, proportional to the velocity at that point, the second term represents advection towards the $\mathrm{x}$-axis (if $\mathbf{A} \sim-r \mathbf{U})$, and the third term represents diffusion. The first two terms are depicted in Figure 15. As can be seen, organisms will be directed towards the x-axis and parallel to it, resulting formation of a thin filament, which is restrained from reaching zero width by diffusion. In taxis, $\mathbf{A}$, the advection towards the $\mathrm{x}$-axis, depends on position $\mathbf{X}$, and in kinesis, $\beta$, the diffusive term, depends on position.

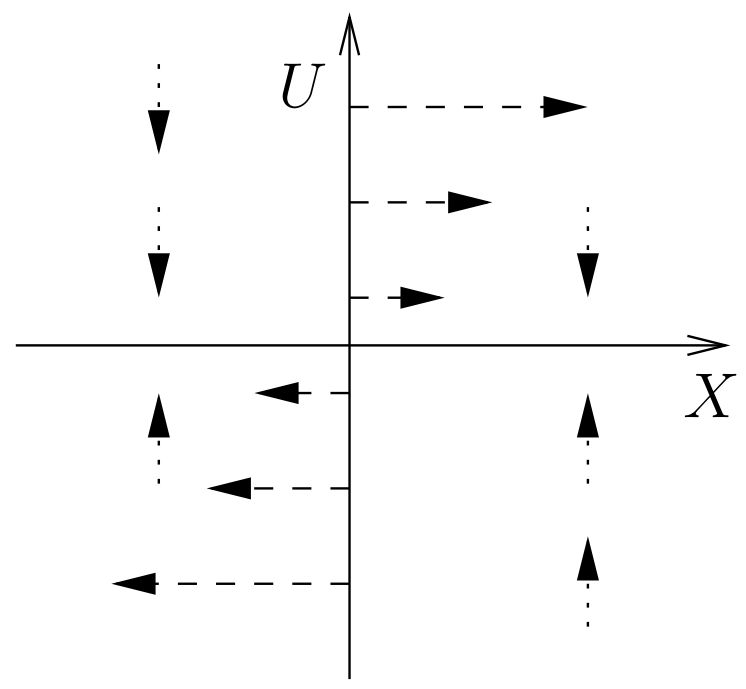

Figure 15: Representation of the directions of the first (dashed) and second (dotted) terms on the RHS of equation (12). In effect, organisms filament along the x-axis, although the diffusive term stops the width from going to zero. 


\section{Examples}

Example solutions to equation (12), if we define as before $\beta=\beta_{0}-\beta_{1} C / C_{0}$ and $\mathbf{A}=$ $-r(\mathbf{U}-\mathbf{u})$, can be seen in Figure 16. Figure 16b) shows that, with diffusion constant in space, i.e. $\beta_{1}=0$, the organisms group in velocity, but not in space. Adding a background flow, $\mathbf{u}$, or diffusion dependent on the cue field $C(x)$, causes grouping in both $|\mathbf{U}|$ and $|\mathbf{X}|$, see Figures 16d) and g).
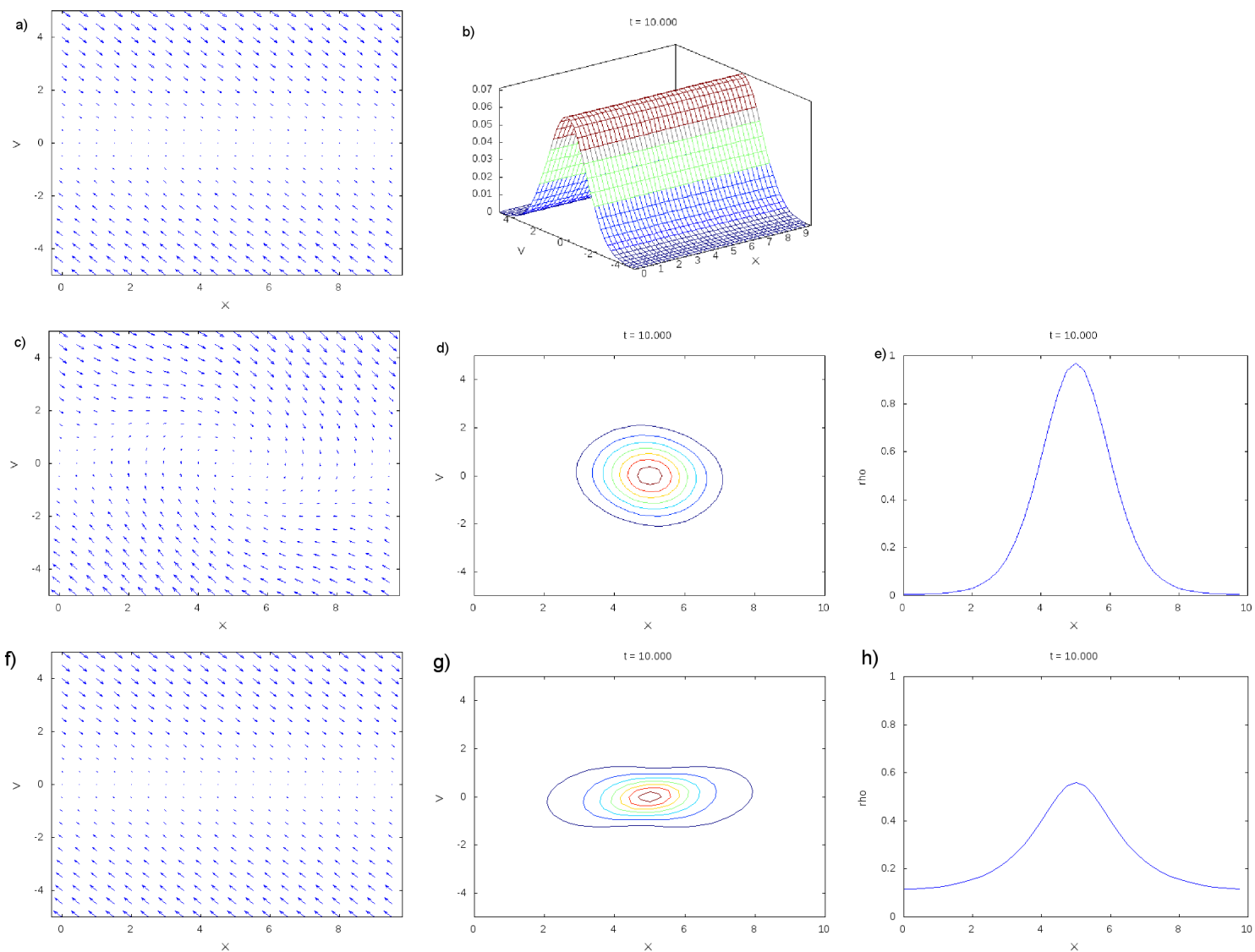

Figure 16: Plots showing the results of solving the Boltzmann equation (12). In a) c) and f), the velocity of the organisms, $\mathbf{U}$, is plotted vs. $|\mathbf{U}|$ and $|\mathbf{X}|$, in b) d) and g), the probability density function $\mathcal{P}$ at large $t$ is plotted vs. $|\mathbf{U}|$ and $|\mathbf{X}|$ and in e) and h), the density of organisms $\rho$ at large $t$ is plotted vs. $|\mathbf{X}|$. Plots a) and b) have $\beta_{0}=2, \beta_{1}=0$ and $\mathbf{u}=0$. Plots c) d) and e) have $\beta_{0}=1, \beta_{1}=0$ and $\mathbf{u}=2$. Plots f) g) and h) have $\beta_{0}=2.7, \beta_{1}=2.4$ and $\mathbf{u}=0$. All quantities have been non-dimensionalised.

\section{Density}

Let us define the density, $\rho=\int d \mathbf{U P}$, and thus the evolution is defined

$$
\frac{\partial \rho}{\partial t}=-\nabla_{\mathbf{x}} \int d \mathbf{U} \mathbf{U P}=-\nabla_{\mathbf{x}} \overline{\mathbf{U}} \rho
$$


where the bar indicates a space-mean.

\section{Velocity}

This leads to the following expression for the evolution of the quantity $\bar{\rho} \overline{\mathbf{U}_{i}}$ :

$$
\frac{\partial(\bar{\rho} \overline{\mathbf{U}})}{\partial t}=-\nabla_{\mathbf{x}_{j}}\left(\bar{\rho} \overline{\mathbf{U}_{i}} \overline{\mathbf{U}_{j}}+\bar{\rho} \overline{\mathbf{U}_{i}^{\prime} \mathbf{U}_{j}^{\prime}}\right) .
$$

We can also write $-\nabla_{\mathbf{u}}(A \mathcal{P})=-\nabla_{\mathbf{u}^{\prime}}\left(-r \overline{\mathbf{U}}-r \mathbf{U}^{\prime}\right) \mathcal{P}$, and then replace these terms back into equation (12). It can be seen that the system is not closed, but if we assume that the three terms in (12) roughly balance, i.e.

$$
\overline{\mathbf{U}_{i} \mathbf{U}_{j}} \approx \overline{\mathbf{U}}_{i} \overline{\mathbf{U}}_{j}+\frac{\beta^{2}}{2 r} \partial_{i j}
$$

then the system is closed, and $\mathcal{P}$ is now quasi-Gaussian. We can thus derive the closed mass equation

$$
\frac{\partial \rho}{\partial t}+\nabla \cdot(\mathbf{U} \bar{\rho})=0
$$

and the momentum equation

$$
\frac{\partial \overline{\mathbf{U}}_{i}}{\partial t}+\overline{\mathbf{U}}_{j} \nabla_{j} \overline{\mathbf{U}}_{i}+\frac{1}{\rho} \nabla_{i} \frac{\beta^{2}}{2 r} \rho=-r\left(\overline{\mathbf{U}}_{i}-\mathbf{u}_{i}-\mathbf{V}_{i}\right)
$$

\section{Drag Dominated Case}

If drag dominates, then we can set the velocity $\overline{\mathbf{U}}$, and so the momentum equation becomes

$$
\frac{\partial \rho}{\partial t}+\nabla \cdot(\mathbf{u} \rho+\mathbf{V} \rho)=\nabla \cdot \frac{1}{r} \nabla \frac{\beta^{2}}{2 r} \rho
$$

\section{Taxis vs. Kinesis}

Let us re-write equation (17) in terms of diffusivities:

$$
\frac{\partial \rho}{\partial t}+\nabla \cdot\left(\mathbf{u}+\mathbf{V}-\frac{1}{r} \nabla(r \kappa) \rho\right)=\nabla \cdot \kappa \nabla \rho
$$

where the diffusivity $\kappa=\beta^{2} / 2 r^{2}$ and is spatially varying. The third term on the LHS represents taxis, and the fourth kinesis. In this form it can be seen that both act similarly on the system, converging velocities to certain regions, either those with smaller velocities (taxis) or low diffusivities (kinesis). This can also be seen in Figure 17, which shows the initial and final states of organisms experiencing either (taxis) or (kinesis), as well as the final density states in both cases. However, kinesis has similar diffusivities and velocities, i.e. Peclet number is of order 1 , while the velocity in taxis can be higher. 

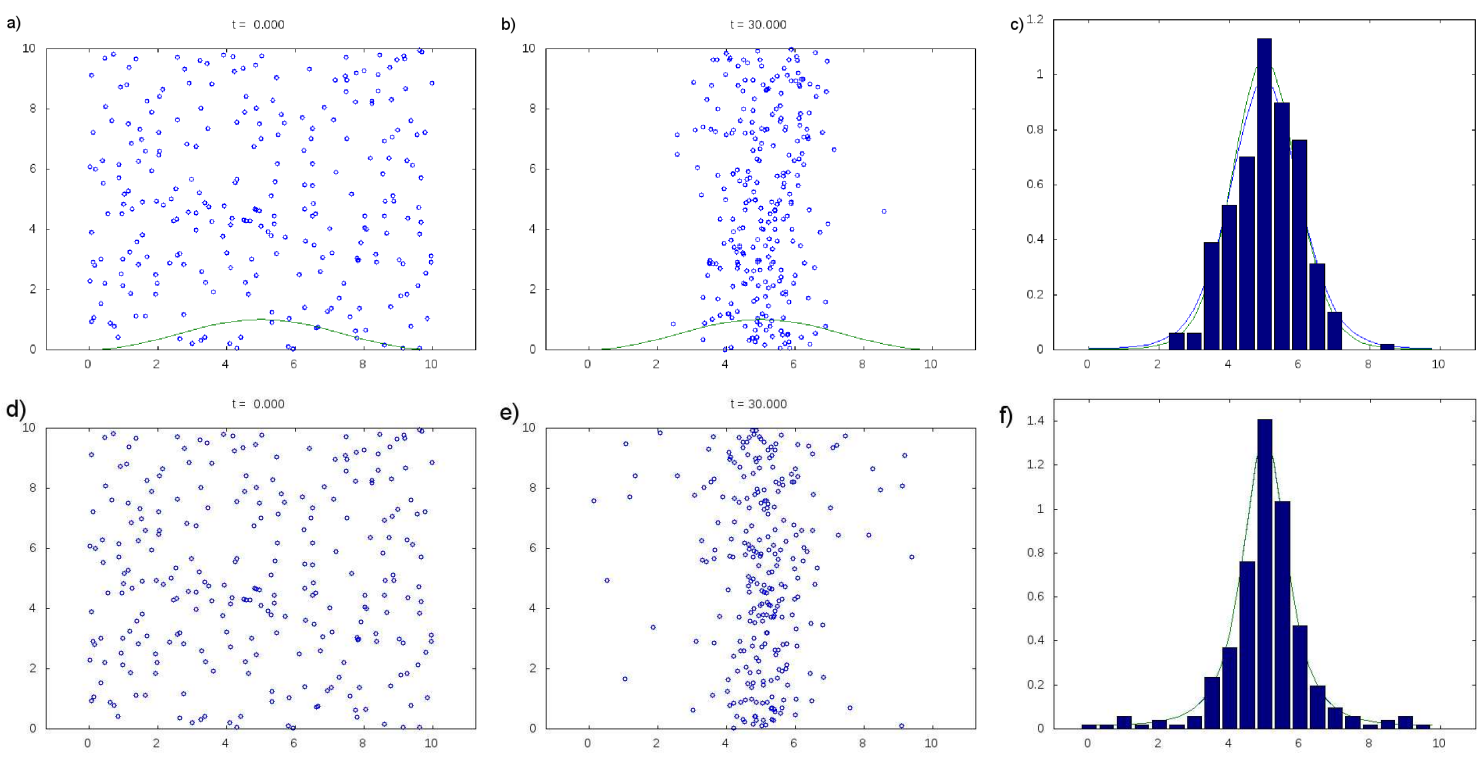

Figure 17: Numerical simulations of the system, assuming either taxis in a)-c), i.e. $\mathbf{V}=$ $\alpha \nabla C$ is spatially varying but $\beta$ is not, or kinesis in d)-f), i.e. $\beta$ is spatially varying but $\mathbf{V}$ is not. The green line in Panels a) and b) shows the distribution of the concentration field $C$. Panels a), d) and b), e) show the initial and final distributions of organisms. Panels c) and f) show the solutions of the Boltzmann equation (12), [blue line], the density equation (13) [green line] and a histogram of the density in the final snapshot of the simulation. Note that the blue and green lines lie on top of each other in Panel f). These show that, while the biological mechanisms behind them is different, both processes produce similar final density distributions. 


\section{Stability with social behavior}

In the absence of a background flow, i.e. $\mathbf{u}=0$, we redefine $\tilde{\mathbf{V}}=\mathbf{V}+\frac{1}{r} \nabla \cdot(r \kappa)$, then

$$
\frac{\partial \rho}{\partial t}+\nabla \cdot(\tilde{\mathbf{V}} \rho)=\nabla \cdot \kappa \nabla \rho
$$

The social behavior is specified by making $\kappa$ a functional of the density, defined in terms of a weighting function $w$, such that

$$
\kappa=K\left(\int d s w(\mathbf{s}) \rho(\mathbf{X}+\mathbf{s})\right)
$$

and $w$ takes the form of decreasing with $\mathbf{s}$, which represents the sensing distance of the organism, see Figure 6. If the basic state density is uniform, then $\kappa$ is constant in space, and we can rewrite $\rho$ and $\kappa$ as a sum of the means (over-bars) and small perturbations (primes), and so the density equation becomes

$$
\frac{\partial \rho^{\prime}}{\partial t}+\bar{\rho} \nabla \cdot \mathbf{V}^{\prime}=\nabla \cdot \bar{\kappa} \nabla \rho^{\prime}
$$

where

$$
\mathbf{V}^{\prime}=-\nabla \kappa^{\prime}=-K^{\prime}(\bar{\rho}) \nabla \int d \mathbf{s} w(\mathbf{s}) \rho^{\prime}(\mathbf{X}+\mathbf{s}) .
$$

Taking a Fourier transform of this equation, we can find an expression for the growth in density perturbations:

$$
\frac{\partial}{\partial t} \hat{\rho}^{\prime}=\left[\bar{\rho} K^{\prime}(\bar{\rho}) k^{2} \hat{w}(k)-\bar{\kappa} k^{2}\right] \hat{\rho} .
$$

If the bracketed term is greater than zero, the system experiences positive growth see Figure 18, which shows the relationship between growth rate and wave number $k$. In fact, the system experiences explosive (faster than exponential) growth, with the amplitude going as

$$
\frac{\partial A}{\partial t}=\sigma(k) A+\mathcal{N} A^{3},
$$

where $N>0$.

\section{Evolution of Social Behavior}

\section{I: Inheritance of Traits}

As before, we define the velocity $\mathbf{V}$ as the gradient of a scalar, i.e. $\mathbf{V}=\nabla \phi$, see [7], where

$$
\phi=w_{0} \int d \mathbf{s} w(\mathbf{s}) Z(\mathbf{X}+\mathbf{s}), \quad \int d \mathbf{s} w(\mathbf{s})=1 .
$$

In this case, the weighting function $w(\mathbf{s})$ takes the form depicted in Figure 19 with repulsion at short distances.

We define three different genetic types, or alleles, of the heterotroph $Z$ (which preys on $P$ ), each with two genes with two possible genetic traits, 0 or 1: $Z_{00}, Z_{01}, Z_{11}$. $Z_{00}$ and $Z_{11}$ 


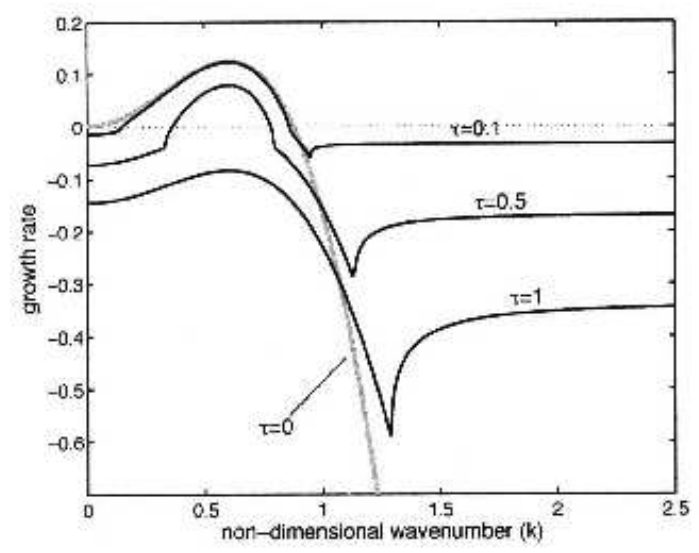

Figure 18: Figure from [7], showing "the linear growth rate of small perturbations around the equilibrium biomass versus the wave number of the perturbations, ... for different values of $\tau$ ", $\propto 1 / \kappa$, a diffusion time scale. "In a uniform distribution of particles, random variations will spontaneously lead to the formation of patches, if there is positive growth somewhere along the wave number spectrum. The gray line shows the case where particle motion is decoupled from biological activities. ... When the diffusion rate is fast compared to growth $(\tau<<1)$, population dynamics have a negligible impact on the linear growth rate of perturbations in the region of instability (positive growth). As biological processes become faster, their effect ... [is to] stabilize the equilibrium solution; the growth rate is negative for all wave numbers when $\tau \approx 1$ ".

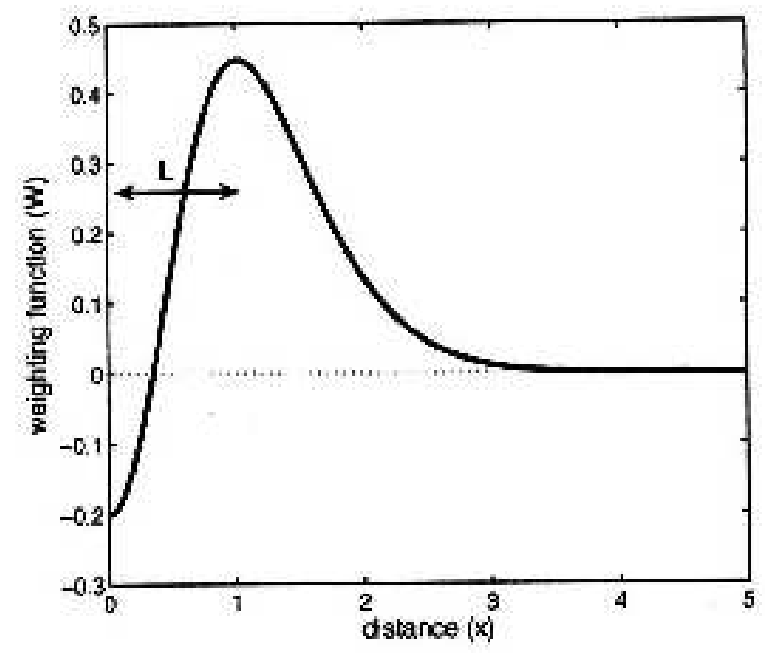

Figure 19: Figure from [7], showing the weighting function, $w(x)$, of organisms as function of their distance from neighbors located at $x=0$, as used in equation $(23)$. " $w(x)=$ $\exp \left(-x^{2} / 2\right)-\exp \left(-2 x^{2}\right)$. Distances are expressed in units of $L$, the characteristic perception length scale. The sensing radius has an approximate value of $2 L$. . Positive weighting indicates attraction to neighbors. Repulsion at short distance prevents over-crowding." 
are recessive, and only $Z_{11}$ exhibits grouping behavior, i.e. $\mathbf{V}_{00}=\mathbf{V}_{01}=0, \mathbf{V}_{11} \neq 0$. The population of each type evolves as follows:

$$
\frac{\partial}{\partial t} Z_{\alpha \beta}=a g \frac{P}{P+P_{h}} \frac{f_{\alpha \beta}}{Z+c}-d Z_{\alpha \beta}-\nabla \cdot\left(\mathbf{V}_{\alpha \beta}-\kappa \nabla\right) Z_{\alpha \beta}+M_{\alpha \beta},
$$

where $0 \leq a \leq 1$ is the conversion efficiency of $P$ into biomass by $Z, 0 \leq g \leq 1$ is a grazing rate, $d$ is the death rate, $P$ is the population of prey and $f_{\alpha \beta}$ is the inheritance fraction of the population $Z_{\alpha \beta}$. $P_{h}$ is the half-saturation density, which provides a limitation to the amount of prey that can be eaten at large $P$, i.e. $Z$ depends linearly on $P$ until the capacity of $Z$ to eat $P$ becomes reached. The $c$ term represent the "Allee" effect [1]: as the density $Z$ is small, encounters between male and female organisms are rare and depend quadratically on density, whereas for large $Z$ virtually all females have mated and reproduction depends linearly on the population gets large, virtually all females have mated. From a Mendelian table of inheritance[4] we can define the fractions $f_{\alpha \beta}$ as:

$$
\begin{aligned}
f_{00} & =Z_{00}^{2}+Z_{00} Z_{01}+\frac{1}{4} Z_{01}^{2}, \\
f_{01} & =Z_{00} Z_{01}+2 Z_{00} Z_{11}+\frac{1}{2} Z_{01}^{2}+Z_{01} Z_{11}, \\
f_{11} & =Z_{11}^{2}+Z_{11} Z_{01}+\frac{1}{4} Z_{01}^{2}, \\
Z^{2} & =f_{00}+f_{01}+f_{11}=\left(Z_{00}+Z_{01}+Z_{11}\right)^{2},
\end{aligned}
$$

where this implies that, for example, a new generation of $Z_{00}$ is produced by all interactions between two $Z_{00}$, half of the interactions between $Z_{00}$ and $Z_{01}$, and 1 in 4 of interactions between two $Z_{01}$.

We also include a mutation term, $M_{\alpha \beta}$, which allows for the possibility of a population reforming if it dies out completely:

$$
\begin{aligned}
& M_{00}=-\mu Z_{00}+\frac{1}{2} \mu Z_{01}, \\
& M_{00}=-\mu Z_{01}+\mu Z_{00}+\mu Z_{11}, \\
& M_{11}=-\mu Z_{11}+\frac{1}{2} \mu Z_{01},
\end{aligned}
$$

where $\mu$ is the fractional rate of mutation. The prey, $P$ evolve as follows;

$$
\frac{\partial P}{\partial t}=b P\left(1-\frac{P}{P_{c}}\right)-g \frac{P}{P+P_{h}} \frac{Z^{2}}{Z+c}+\nabla \kappa \nabla P,
$$

where $b$ is the growth rate, $P_{c}$ is the half-saturation density, and all other quantities are as before.

\section{Examples}

There are specific conditions under which the grouping type, 11, can win out with respect to the other two types. If the grouping strength is not too high and/or the diffusivity is not too low, then it can cause the extinction of the other two species, due to the reproduction 
advantages of grouping, see Figures 20a) and b). However, if the diffusivity is too low to bring the grouping type sufficient prey, then the other two types will dominate, while the grouping type will die out until the groups are sufficiently small to survive, see Figures 20c) and d). If the grouping species, 11, is introduced at low values to the other two types, then it will slowly take over until it reaches a critical fraction, $f_{c}$, at which the other two species become extinct and the grouping type wins, see Figure 21.

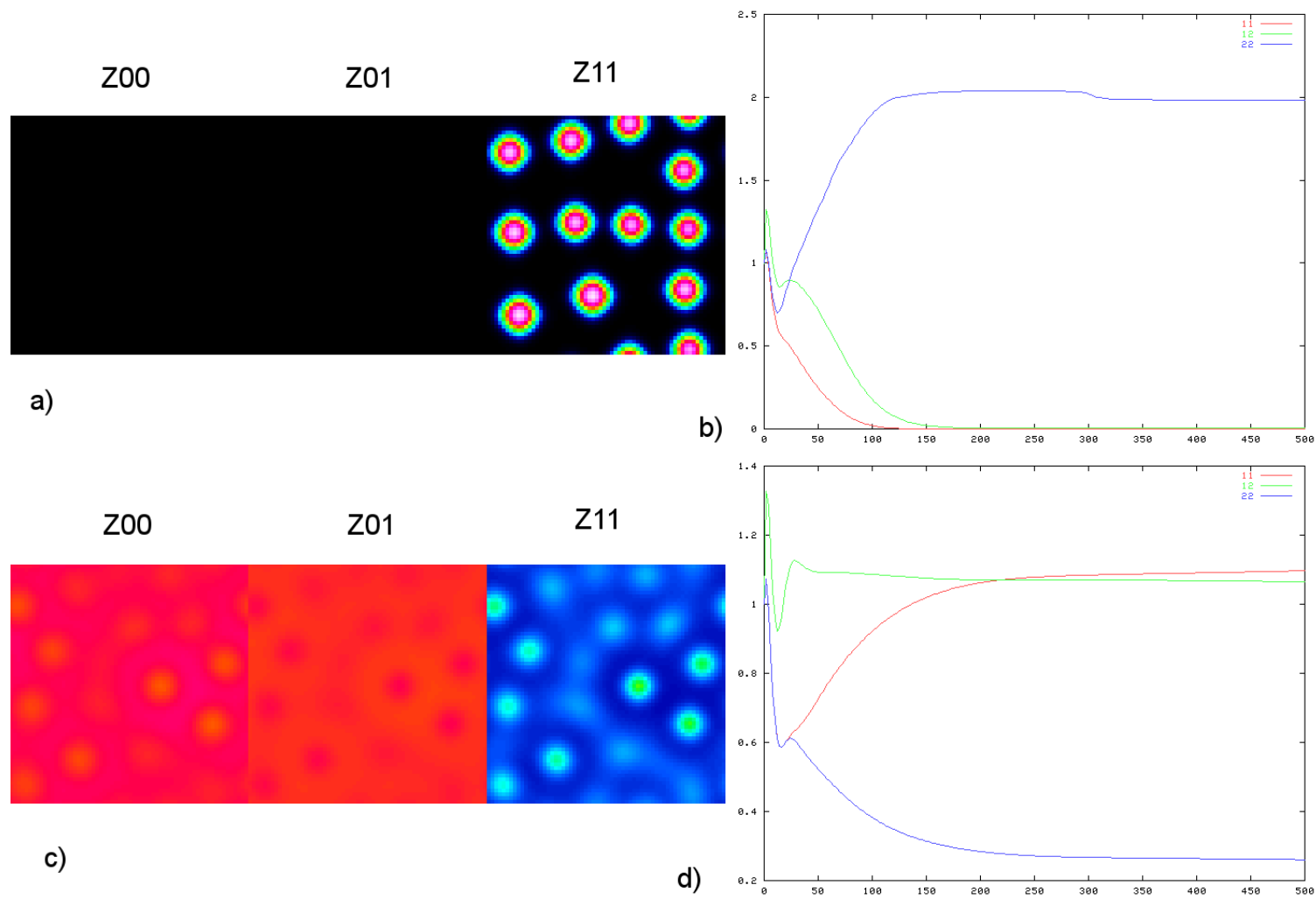

Figure 20: Final state population distributions $Z_{\alpha \beta}$ from numerical simulations (a) and c)) shown next to the fractional populations $f_{\alpha \beta}$ over time (b) and d)). In a) and b), the species $Z_{11}$ causes the other two to die out, due to the advantages of grouping for reproduction. However, if the diffusivity is low, as in c) and d), then the disadvantages of grouping, i.e. the fast depletion of prey, mean that $Z_{00}$ and $Z_{01}$ do much better than $Z_{11}$, which doesn't completely die out.

If we subject the system to background stirring motion, such as Ray Pierrehumbert's exact $\sin /$ sin stirring[5], see Figure $22(i)$, then we can test whether grouping is still advantageous or not, see Figures 22(ii)- $(v)$. It can be seen that, at low grouping parameters and low stirring, the grouping type dies out, but at large grouping parameters and large stirring, the grouping type wins. In other regimes, both types survive.

Thus, it is not only the biological but the dynamical features which can determine how a particular species will fare. 


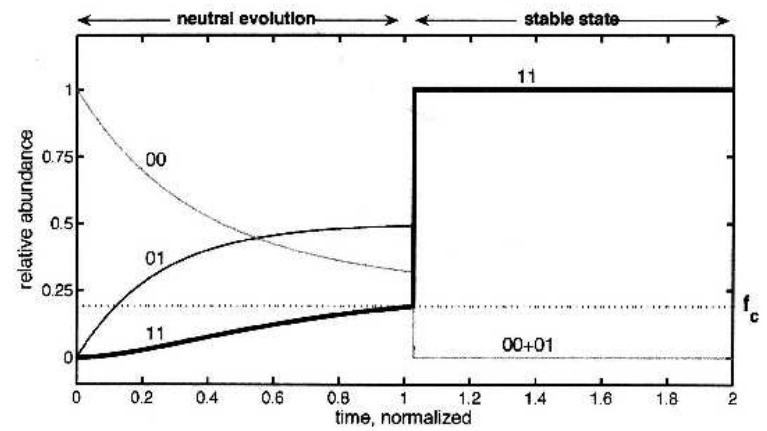

Figure 21: Frequency of $Z_{\alpha \beta}$ versus time, showing the invasion of a non-grouping population, $Z_{00}$, by the grouping type, $Z_{11}$, taken from [7]. "Time is normalized by the mutation timescale ... The new allele, introduced at low frequency at $t=0$ does not affect fitness until the frequency of grouping organisms (type 11) reaches $f_{c}$, at which part it starts to aggregate. ... In this case, social behavior is a successful strategy: the grouping type wins the competitions and the other types become extinct. The ecosystem is then stable with respect to mutations in the two-allele model."

\section{II: Grouping Parameter}

Examples of how diffusion and grouping affect the dynamics when limit cycles may be present (through setting the value of $c$ in (24)), can be seen in Figure 23.

To find optimal parameters, we let populations with different grouping strengths compete, and note whether their populations grow or decay. The effect of the half saturation ratios, $P_{h}$ and $c$ in equation (24), on the optimal grouping strength can be seen in Figure 24 .

\section{III: Schooling}

As before, we represent schooling as velocity alignment with neighbors and attraction (except very nearby). We add a food supply, confined to the mixed layer at the top of the ocean, with variability produced by upwelling which brings further nutrients. As the mixed layer is by definition assumed well-mixed, downwelling does not change the concentration of nutrients. We specify a streamfunction, $\psi$, and a vertical velocity, $w$, that depend on the depth of the thermocline, $h$ :

$$
\psi=\frac{g^{\prime}}{f} h, \quad w=-\frac{\partial h}{\partial t}
$$

where $g^{\prime}$ is the modified gravitational acceleration and $f$ is the planetary vorticity. We can then derive the evolution of the nutrient, $N$ :

$$
\frac{\partial N}{\partial t}=\frac{w(w>0)}{H}\left(N_{\text {deep }}-N\right)-\mu N P+\nabla \cdot \kappa_{\text {fluid }} \nabla N,
$$

where $H$ is the average depth of the thermocline, $N_{\text {deep }}$ is the nutrient value at depth, and $P$ is the population of the autotroph, e.g. phytoplankton. Example biomass distributions from numerical simulations can be seen in Figure 25. 


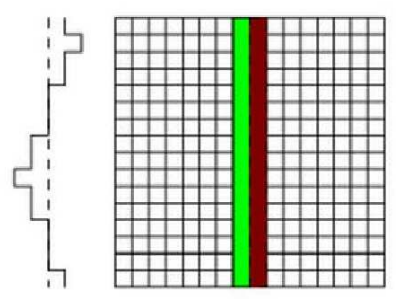

(i)

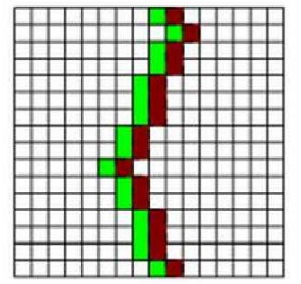

几

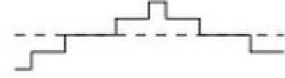

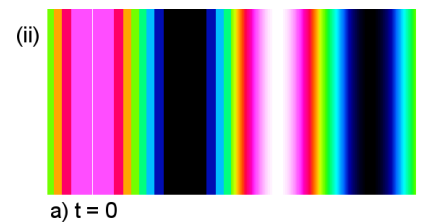

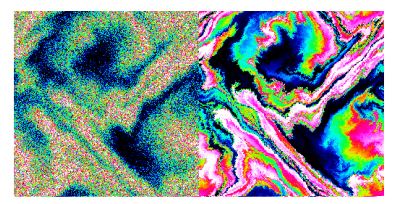

b) $\mathrm{t}=12$

(iii)

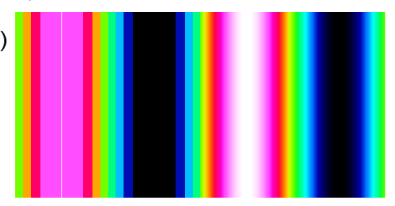

a) $t=0$

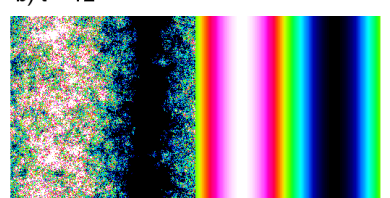

b) $t=12$

(iv)
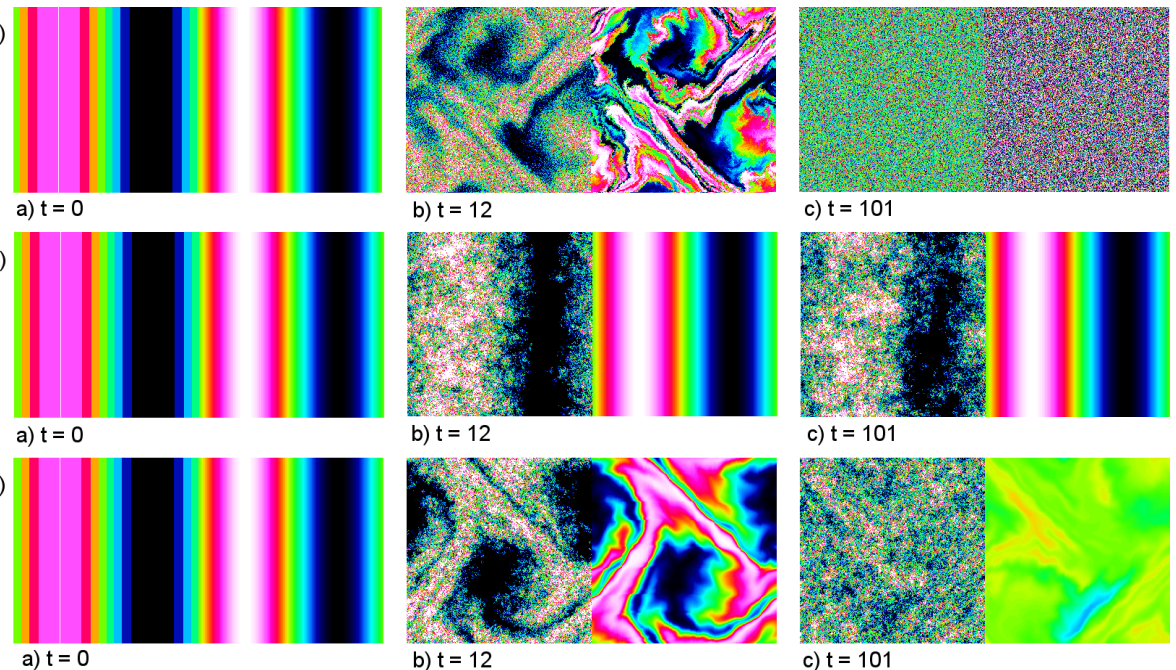

c) $t=101$

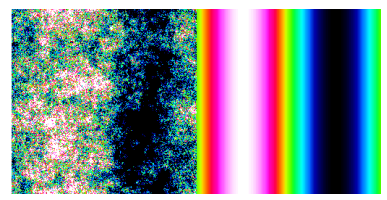

c) $t=101$
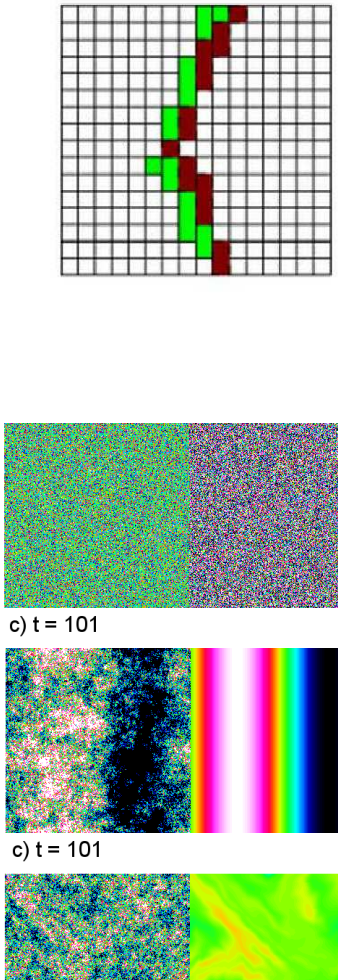

$+x+2$

(a.r.t.

c) $\mathrm{t}=101$

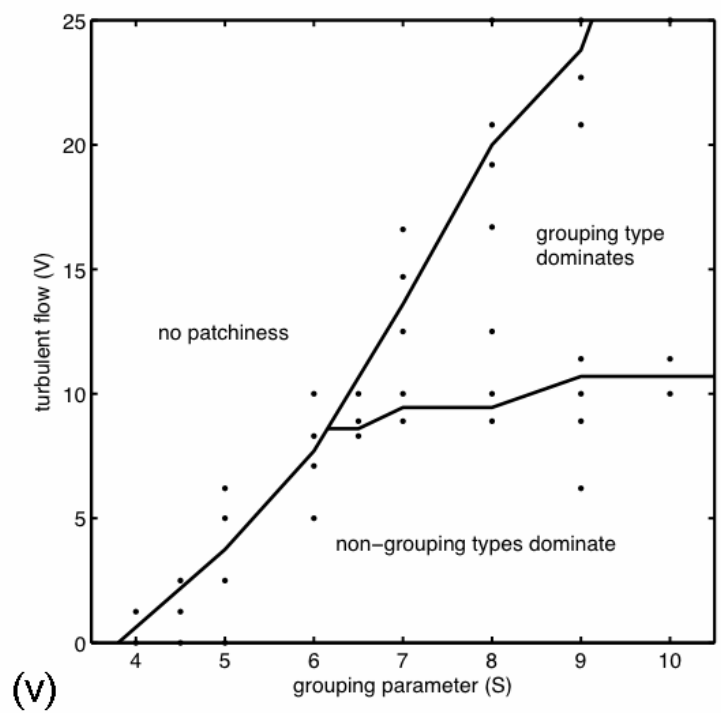

Figure 22: $(i)$ depicts the form of the Pierrehumberts exact $\sin / \sin$ stirring. $(i i)-(i v)$ show snapshots from numerical simulations of $Z_{11}$ (left panels) and a passive tracer (right panels); (ii) and (iii) have no diffusion, i.e. $\kappa=0,(i i)$ and $(i v)$ are subject to a medium rate of stirring, and (iii) is not subject to any stirring. (iv) shows that, while diffusion eventually completely removes any gradients in the tracer, $Z_{11}$ still remains grouped. $(v)$ shows a summary of the conditions where grouping and non-grouping $Z$ survive, from [7]. 
a)

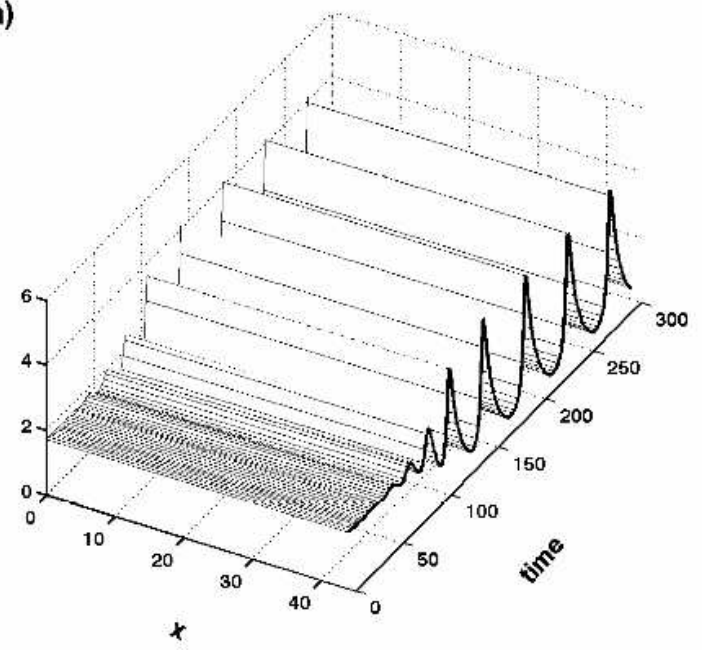

c)

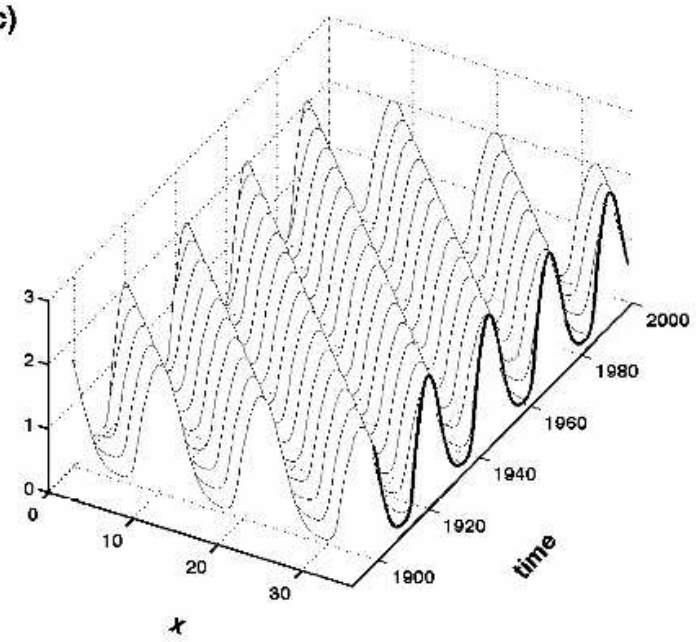

b)

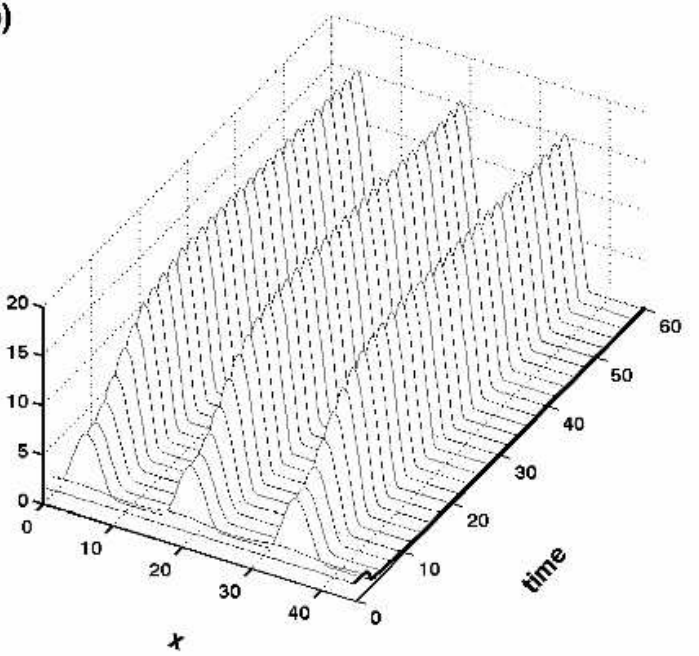

d)

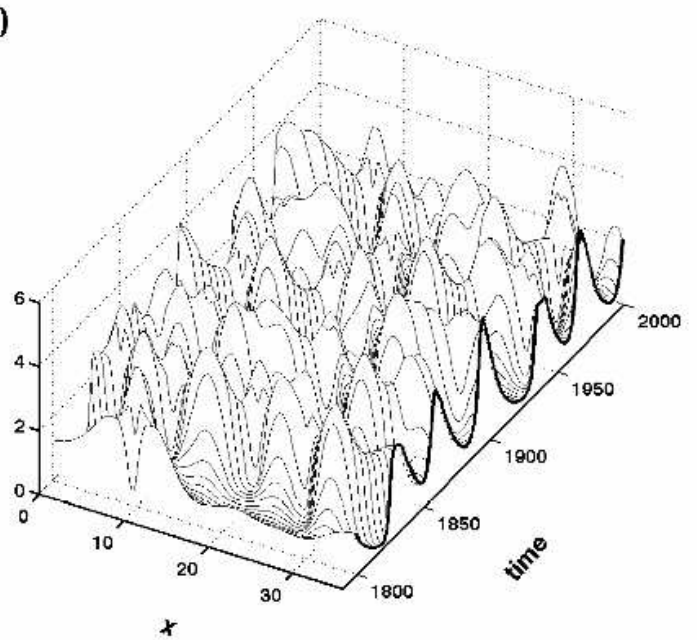

Figure 23: Numerical simulations of predator biomass, $Z$, distribution in time and space, from [6]. "Thin black contours show the instantaneous spatial distribution, at fixed intervals in time. Thick black curve shows the time series of biomass at a fixed location. a) Without aggregation behavior" $\left(w_{0}=0\right)$, "the predator-prey system reaches a limit cycle after a period of transients; the simulation shown here is initialized near the unstable coexistence fixed point. b) With social behavior" $\left(w_{0}=2\right)$, "and fast diffusion, patches form spontaneously and the system loses its oscillatory behavior. c) With social behavior" $\left(w_{0}=15\right)$, "and slow diffusion, the system supports a regular wave traveling through the domain; transients are not shown. d) With social behavior" $\left(w_{0}=5\right)$, "and slow diffusion, the density field can appear chaotic." 
a)

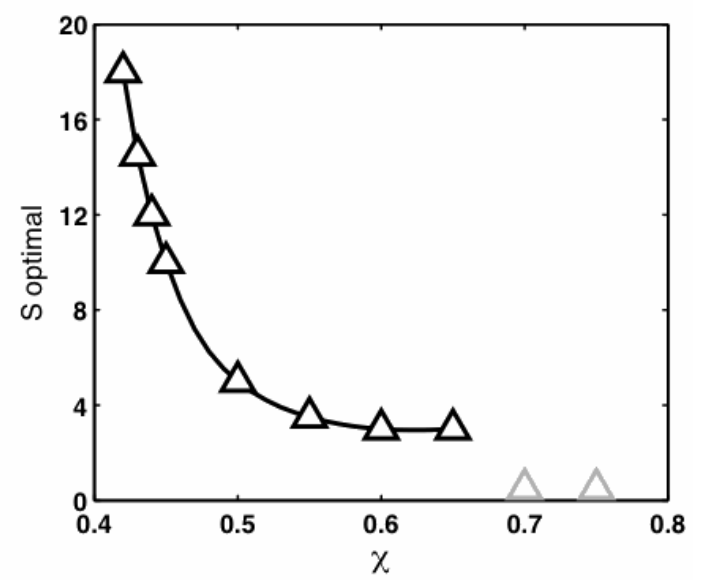

b)

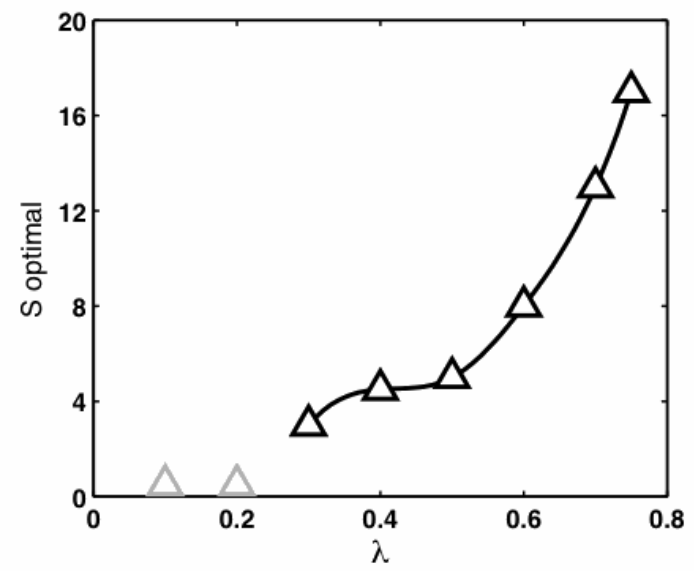

Figure 24: Optimal strategy vs $P_{h}$ and $c$, from [6]. $\chi$ is non-dimensionalised $P_{h}, \lambda$ is nondimensionalised $c$, and $S$ is our $w_{0}$. "Black symbols indicate optimal parameter values of $\left[w_{0}\right]$ from numerical simulations of the competition model, solid line is the spline interpolation. Gray symbols indicate that social behavior is not a successful strategy, in which case nongrouping types ... are the best competitors."

We model foraging by assuming that the turning rate of an organism is dependent on the difference between past feeding, $\xi$ compared to current food values. Here we consider the turning rate $\delta \theta$ of a heterotroph, $Z$, in terms of the population of its prey, $P$. The memory of food evolves as follows:

$$
\frac{\partial \xi_{i}}{\partial t}=\frac{P\left(\mathbf{X}_{i}\right)}{P\left(\mathbf{X}_{i}\right)+P_{h}}-\frac{\xi_{i}}{\tau}
$$

where $\tau$ is the memory period of the heterotroph. Then we model the turning as

$$
\delta \theta=1+\tanh \left(\alpha\left(\xi_{i}-P\left(\mathbf{X}_{i}\right)\right),\right.
$$

where $\alpha$ is a constant. Figures 26 and 27 show how the success of the foraging $Z$ depends on how fast they swim. Foragers that are too speedy over-shoot the largest concentrations of prey; slow foragers, however, don't find the prey for a long time. Figure 28 shows how successful schooling is for foragers in various levels of turbulence and resources. It can be seen that, for low levels of resources there is no clear advantage to schooling, and it can actually be a disadvantage at low levels of $\alpha$, the ratio of alignment to attraction, as defined in equation (29). However, in cases with medium or high resources, there are values of $\alpha$ that provide a clear advantage.

\section{Aggregation}

Observations of Antarctic krill swarms can be seen in Figure 29. Generally, during the day there tends to be many small swarms near the surface, and at night there tend to be a 
a) Nutrients (N)

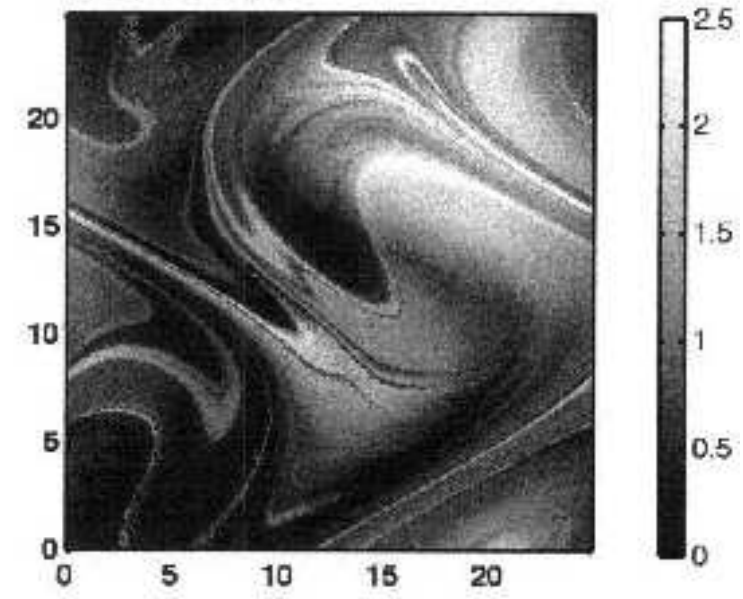

\section{b) Phytoplankton ( $\mathrm{P})$}

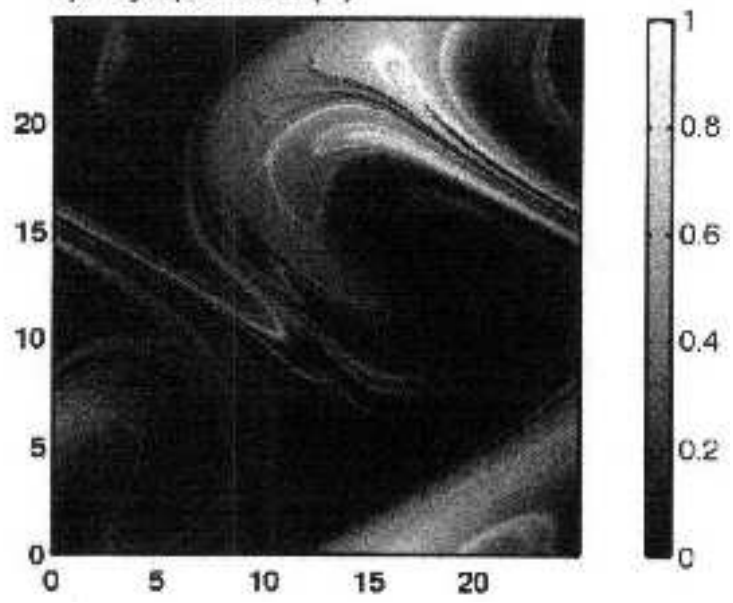

Figure 25: Figure from [6], showing results from numerical simulations with schooling and turbulent advection. a) biomass of nutrients $N$, b) biomass of phytoplankton $P$. "Lighter shades of gray indicate larger values. The two fields tend to be anti-correlated." 

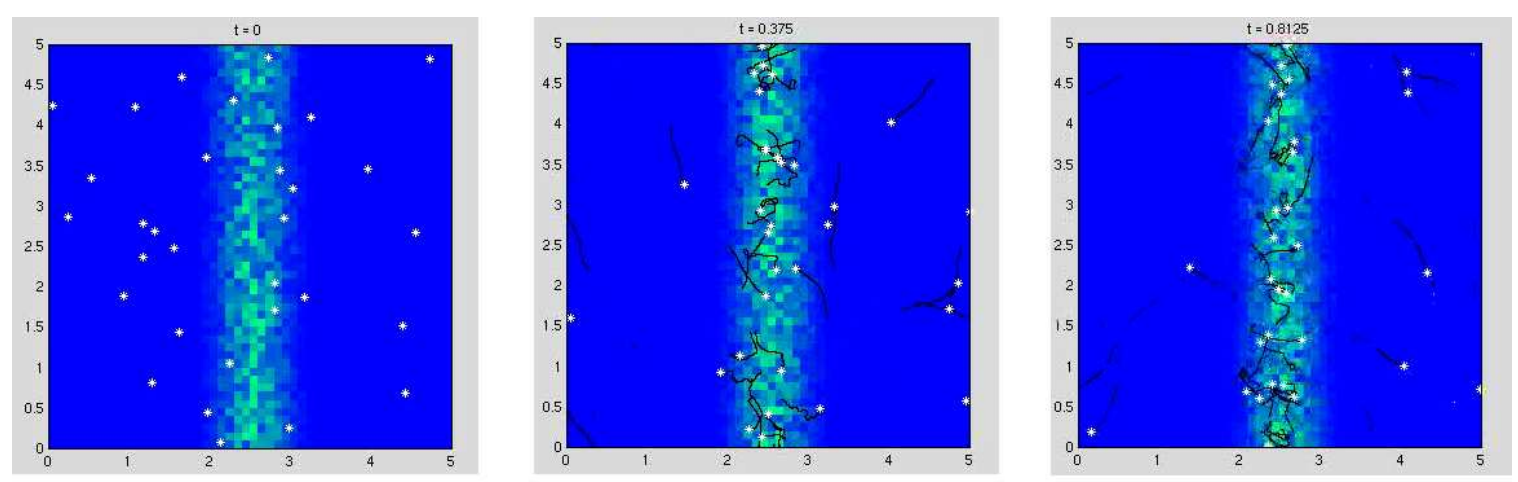

a)
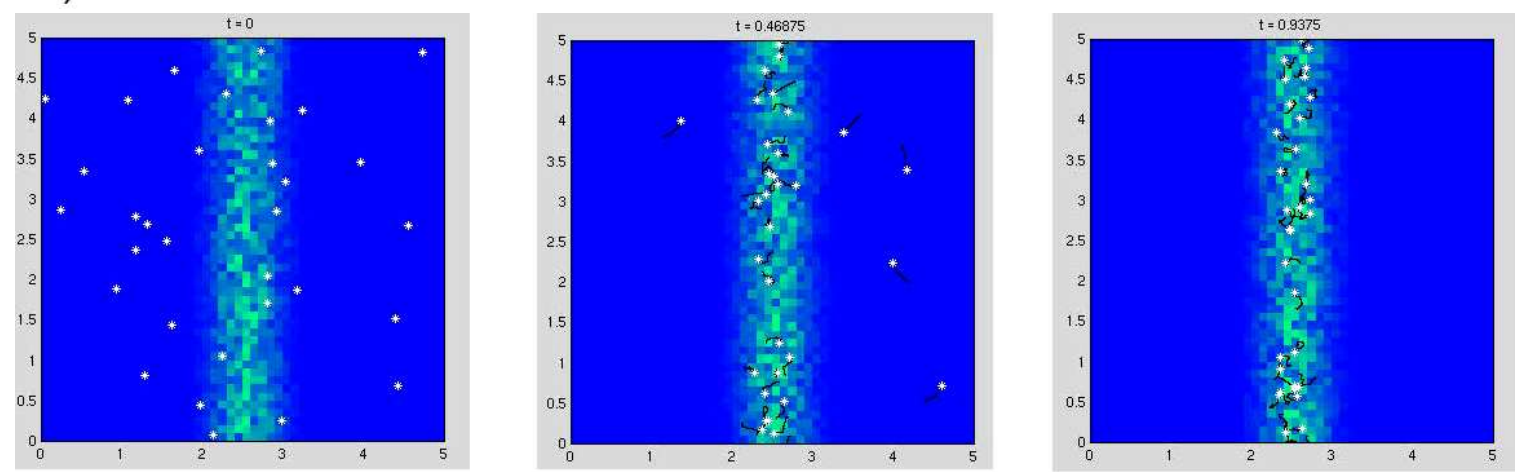

b)

Figure 26: Figures showing numerical simulations of foraging $Z$ with a) fast swimming speeds and b) slow speeds at various non-dimensionalised times. The white dots represent the individual $Z$, the black tails represent the organism's grazing memory and the color scale represents the concentration of prey $P$. It can be seen that the fast swimmers overshoot and never all end up concentrated on the prey, whereas the slow swimmers quickly group over the high concentration of prey. 


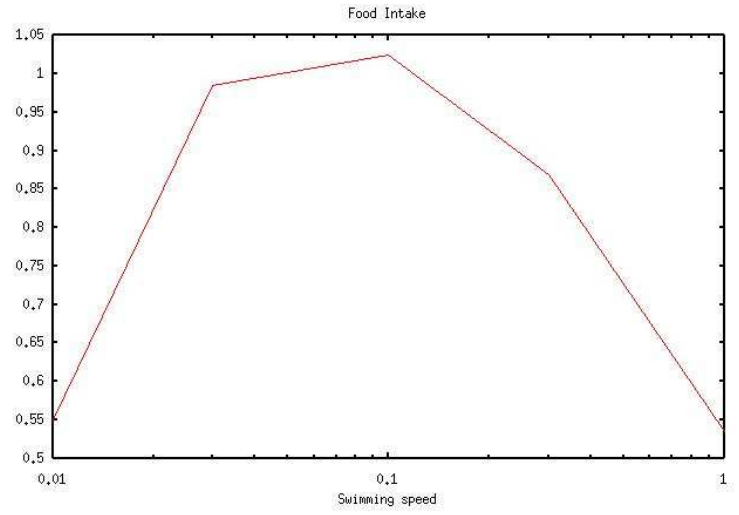

Figure 27: Figure summarizing the (non-dimensionalised) food intake of foraging $Z$ with a specific swimming speed from numerical simulations. Clearly there is an ideal swimming speed at which the swimmers find the food within a reasonable time but do not overshoot the highest concentration by swimming too fast.

few large swarms at depths $(\sim 250 \mathrm{~m})$. Different types of aggregation can be classified using a measure of polarization: the degree to which the grouping is swarm-like or school-like. Figure 30 shows results from numerical simulations, with the degree of polarization plotted against the degree of alignment, $\alpha$, and the swimming velocity, $V$. Five specific grouping phases are identified and examples shown of each.

\section{Conclusion}

It can be seen that a wide range of grouping structures in organisms can be simply modelled. We have seen that in turbulent environments it is sometimes advantageous to school or group, depending on the properties of the organisms themselves as well as how the environment affects their supply of food. In general, it seems that there is the need for a certain amount of resource availability to make it advantageous for foraging predators to school.

\section{References}

[1] W. C. Allee, Principles of animal ecology,, Philadelphia, Saunders Co., 1949.

[2] C. W. Gardiner, Handbook of Stochastic Methods for Physics, Chemistry and the Natural Sciences, Springer, 3rd ed., 2003.

[3] G. Lawson, Distribution, patchiness, and behaviour of antarcti zooplankton, assessed using multi-frequency acoustic techniques., PhD thesis, MIT-WHOI Joint Program in Oceanography, 2006.

[4] G. Mendel, Versuche ber pflanzen-hybriden, Verh. Naturforsch. Ver. Brnn, 4 (1866), pp. 3-47. 


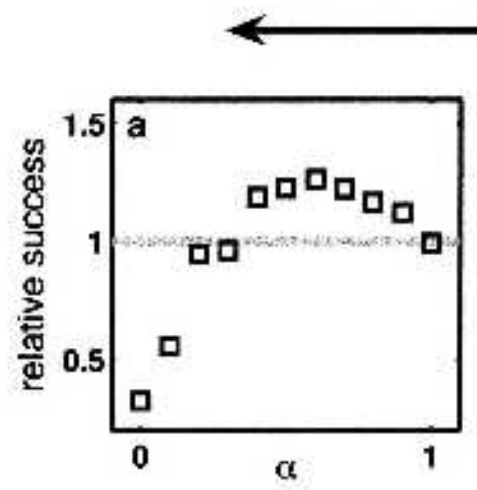

\section{more resources}
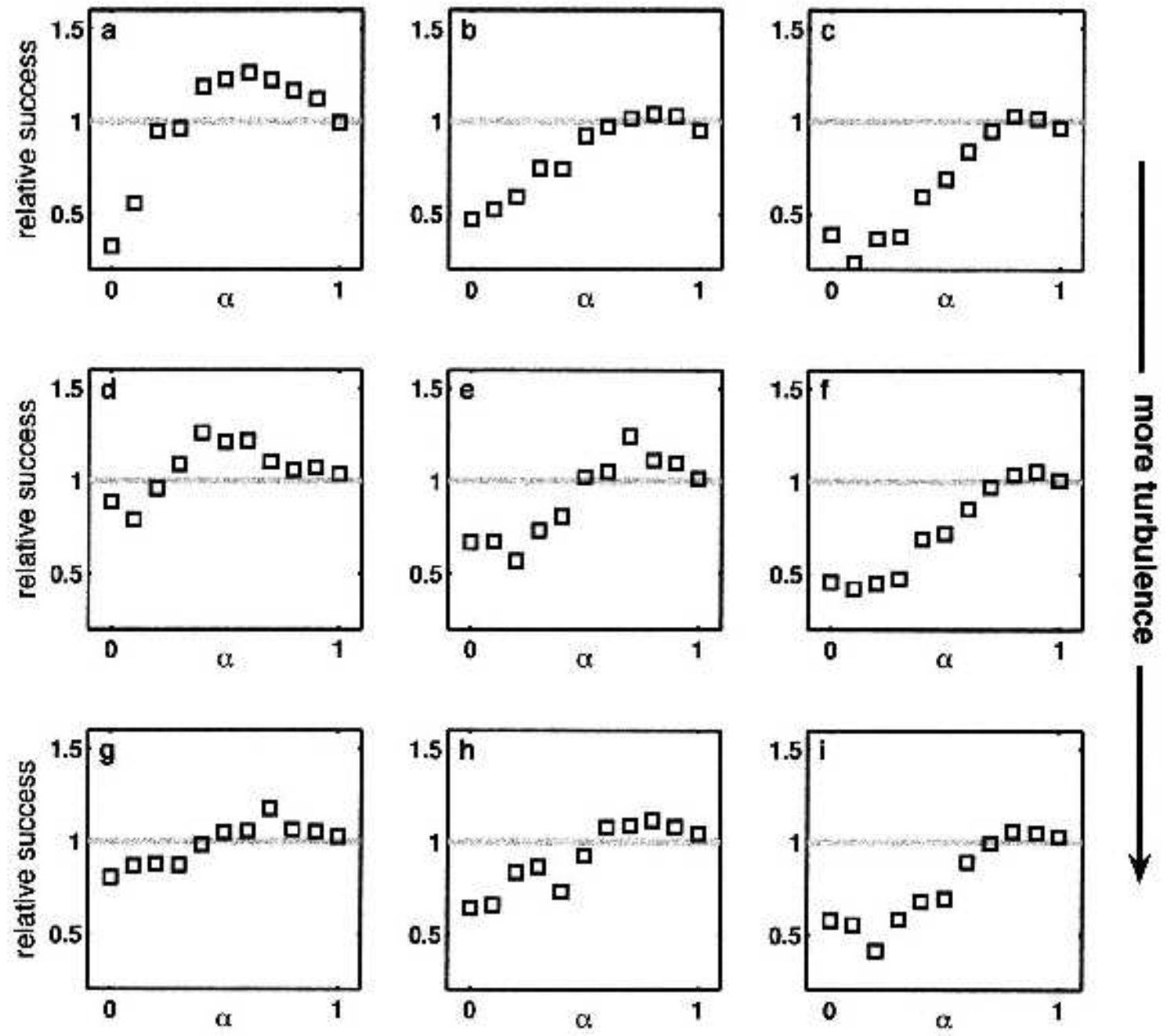

Figure 28: The foraging success of schooling $Z$, taken from [6], "normalized by the success of random walkers under the same conditions. In each panel, the relative success ... is shown for different strategies ( $\alpha$, the ratio of alignment to attraction", see equation (29) "varying between 0 and 1). Each column corresponds to a different level of resource availability; each row has different flow characteristics." In the low resource panels, there is no advantage to schooling, and in fact it can be a disadvantage at low values of $\alpha$. However, with medium to high resources, there are values of $\alpha$ that provide a clear advantage. 

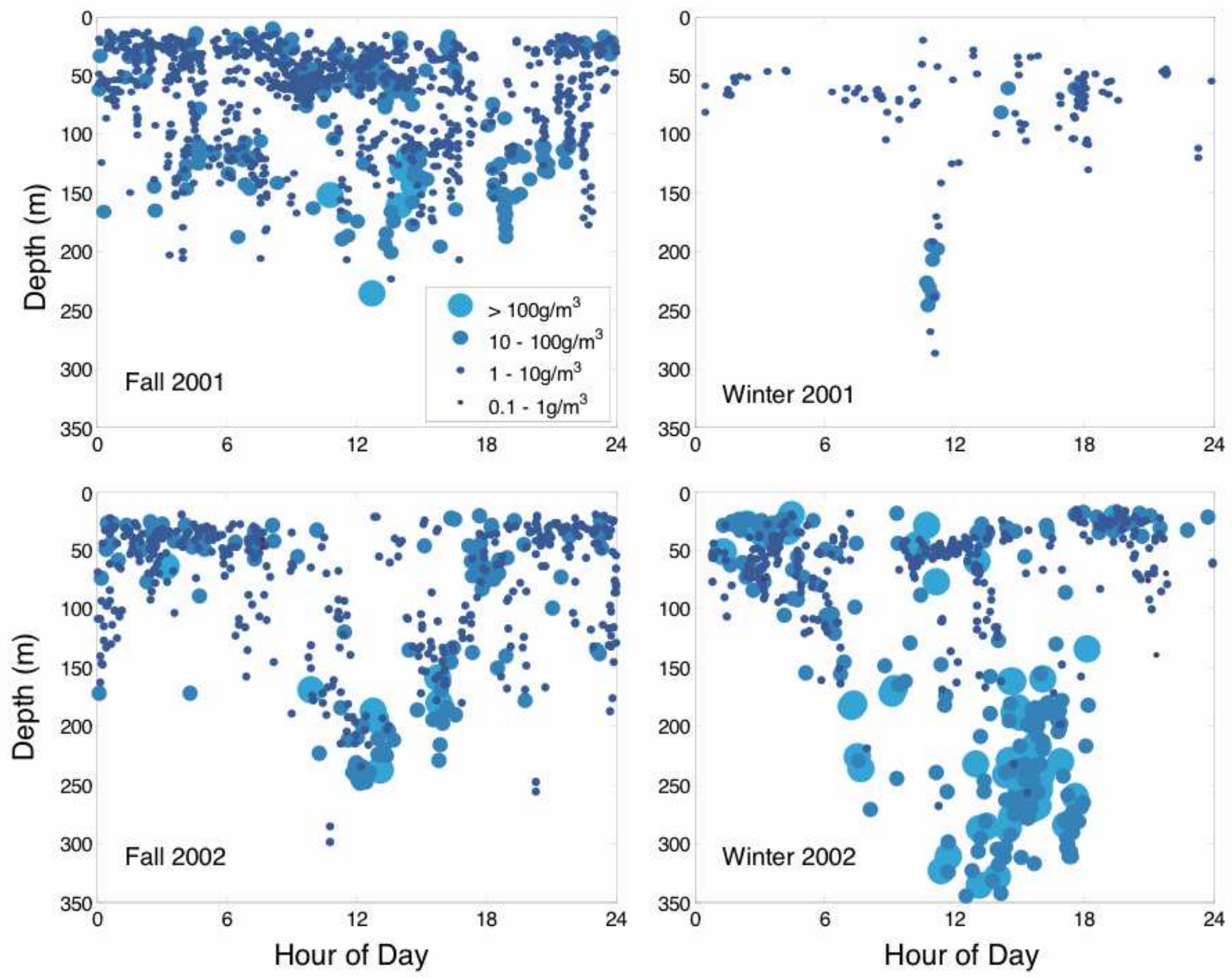

Figure 29: Observations of Antarctic krill from acoustic measurements [3]. Points represent aggregations, which are plotted as a function of the time of day of observation (x-axis) and mean depth (y-axis). The size and color represent the mean density. The general trend shows a few patches of large density at depth at night and many patches of small density near the surface during the day. 

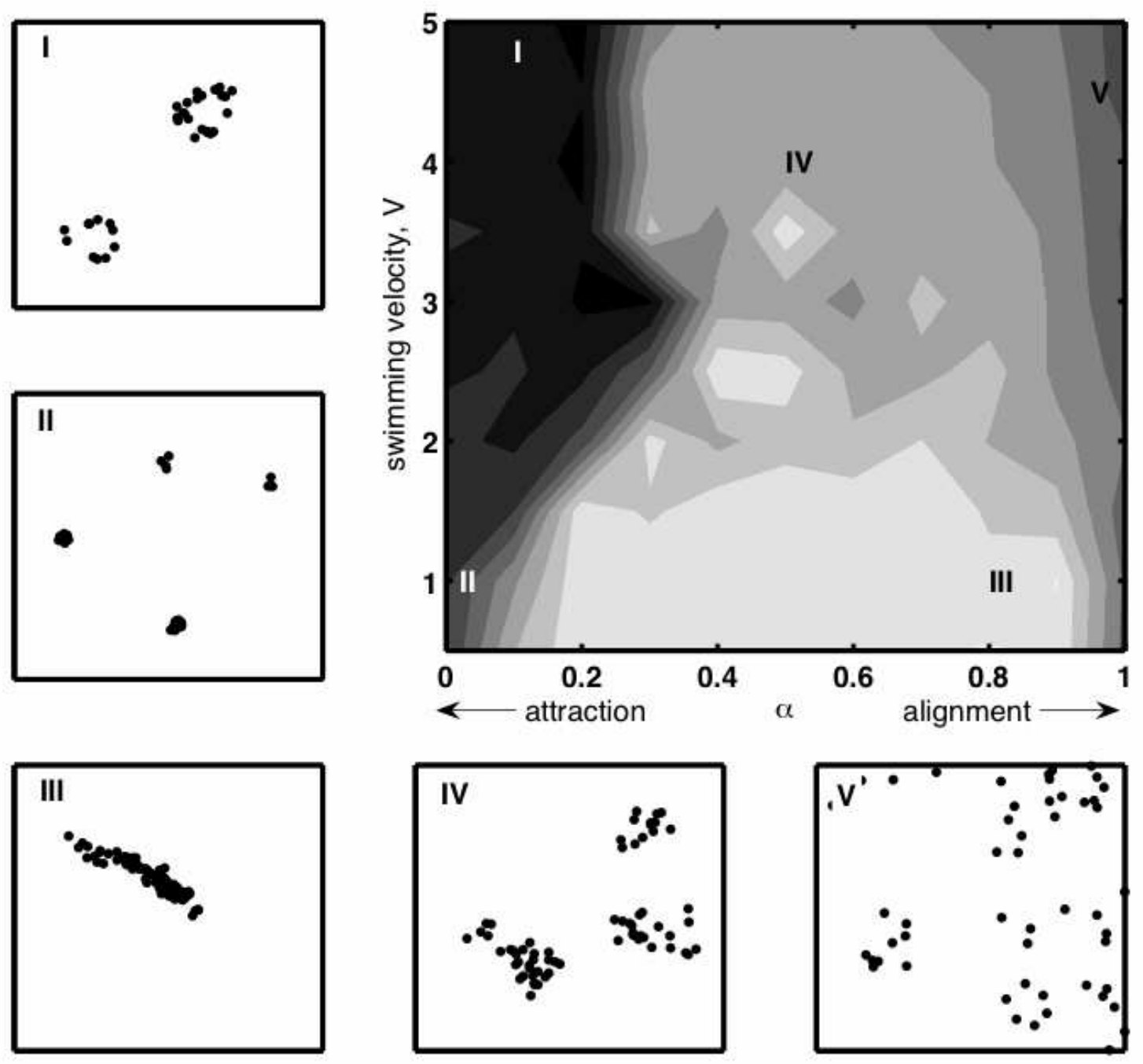

Figure 30: Simulations results from [6]. The gray shading indicates the degree of polarization; high vales (light gray) represents schooled groups, low values (black) represent swarming groups. This is plotted against $\alpha$, degree of alignment, and $V$, swimming velocity. Labels I-V indicate distinct phases: I milling; II swarms; III large schools; IV small schools; $\mathrm{V}$ transient alignment. 
[5] R. Pierrehumbert, Tracer Microstructure in the Large-Eddy Dominated Regime, Chaos Solitons \& Fractals, 4 (1994), pp. 1091-1110.

[6] A. Verdy, Dynamics of marine zooplankton: social behavior, ecological interactions, and physically-induced variability, PhD thesis, MIT-WHOI Joint Program in Oceanography, 2007.

[7] A. Verdy and G. Flierl, Evolution and social behavior in krill, Deep Sea Research Part II: Topical Studies in Oceanography, 55 (2008), pp. 472 - 484. Dynamics of Plankton, Krill, and Predators in Relation to Environmental Features of the Western Antarctic Peninsula and Related Areas: SO GLOBEC Part II. 


\title{
Lecture 6: Mixing in the presence of sources and sinks
}

\author{
Jean-Luc Thiffeault \\ Notes by Sam Pegler and Woosok Moon
}

28 June 2010

\section{Norms}

In this section we define a measure of mixing that does not necessarily require diffusion to measure the amount of homogenization that occurs during the mixing process. Recall the advection-diffusion equation

$$
\frac{\partial \theta}{\partial t}+\boldsymbol{u} \cdot \nabla \theta=\kappa \nabla^{2} \theta
$$

where $\theta$ is a concentration field in a finite domain $\Omega$, with no-net-flux boundary conditions. We assume without loss of generality that

$$
\int_{\Omega} \theta \mathrm{d} \Omega=0
$$

and define the $L^{2}$-norm, or variance, as

$$
\|\theta\|_{2}^{2}=\int_{\Omega} \theta^{2} \mathrm{~d} \Omega
$$

Recall from Lecture 1 that the variance evolves according to

$$
\frac{d}{d t}\|\theta\|_{2}^{2}=-2 \kappa\|\nabla \theta\|_{2}^{2}
$$

and decays in time as the system mixes. The variance indicates the extent to which the concentration has homogenized and is thus a good measure of the amount of mixing that has occurred. However, the variance requires knowledge of small scales in $\theta$, which we are not necessarily interested in. A measure of how well-mixed the concentration is does not necessarily require knowledge of how much homogenization has occurred due to diffusion at small scales. This is more in keeping with the definition of mixing in the sense of ergodic theory [2]. In this regard, we proceed to consider the pure advection equation

$$
\frac{\partial \theta}{\partial t}+\boldsymbol{u} \cdot \nabla \theta=0
$$

Note that in this case equation (4) predicts that the variance satisfies

$$
\frac{d}{d t}\|\theta\|_{2}^{2}=0
$$


and cannot therefore be used as a measure of mixing.

The advection equation (5) takes us closer to the ergodic sense of mixing in which we think of the advection due to the velocity field as a time-dependent operator $S^{t}: \Omega \rightarrow \Omega$ that moves an initial patch of dye according to

$$
\theta_{0}(\boldsymbol{x}) \mapsto \theta(\boldsymbol{x}, t)=S^{t} \theta_{0}(\boldsymbol{x}) .
$$

If we consider a region $A$ of uniform concentration defined by

$$
\theta_{0}(\boldsymbol{x})= \begin{cases}1 & \text { if } \boldsymbol{x} \in A \\ 0 & \text { otherwise }\end{cases}
$$

then the volume of the patch

$$
\operatorname{Vol}[\theta(\boldsymbol{x}, t)]=\operatorname{Vol}(A),
$$

remains constant in time by incompressibility. We can associate the volume of the patch with the Lebesgue measure and, because of the result (9) above, $S^{t}$ is measure-preserving.

We define mixing in the sense of ergodic theory by

$$
\lim _{t \rightarrow \infty} \operatorname{Vol}\left[A \cap S^{t}(B)\right]=\operatorname{Vol}(A) \operatorname{Vol}(B),
$$

for all patches $A, B \in \Omega$. This definition follows our intuition for what good mixing is. Referring to figure 1, when the system is well-mixed the intersection of $A$ and $S^{t} B$ is proportional to both $\operatorname{Vol}(A)$ and $\operatorname{Vol}(B)$. Thus, if the condition (10) holds then $S^{t}$ must spread any initial patch throughout the domain. This condition is referred to as strong mixing and can be shown to imply ergodicity.

The intersection of the advected patch $B$ with the reference patch $A$ is analogous to projection onto $L^{2}$ functions. This motivates the following weak convergence condition

$$
\lim _{t \rightarrow \infty}\langle\theta(\boldsymbol{x}, t), g\rangle=0
$$

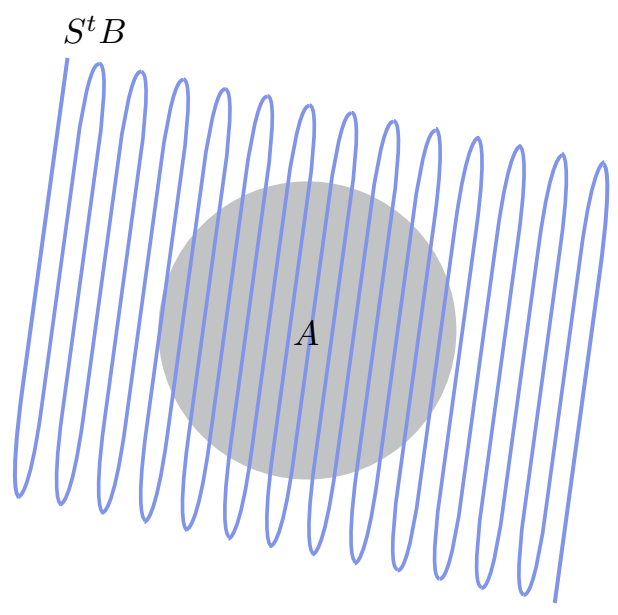

Figure 1: An advected patch $S^{t} B$ that has undergone strong mixing. At late times the patch covers an arbitrary reference patch $A$. 
for all functions $g \in L^{2}(\Omega)$, where the inner product is defined by

$$
\langle f, g\rangle=\int_{\Omega} f(\boldsymbol{x}) g(\boldsymbol{x}) \mathrm{d} \Omega
$$

and $f \in L^{2}(\Omega)$ if $\int_{\Omega}|f|^{2} \mathrm{~d} \Omega<\infty$. Weak convergence is equivalent to mixing as a consequence of the Riemann-Lebesgue lemma. The equivalent conditions (10) and (11) require computing over all patches $A$ or functions $g$, respectively. Thus, neither of these conditions is useful in practice. However, we proceed to describe a theorem that shows there is a simpler way to determine whether or not weak convergence is satisfied.

Mathew, Mezic and Petzold [5] introduced the mix-norm, which for mean-zero functions is equivalent to

$$
\|\theta\|_{\dot{H}^{-1 / 2}}:=\left\|\nabla^{-1 / 2} \theta\right\|_{2} .
$$

Doering and Thiffeault [1] and Lin, Thiffeault and Doering [3] generalized the mix-norm to

$$
\|\theta\|_{\dot{H}^{q}}:=\left\|\nabla^{q} \theta\right\|_{2}, \quad q<0,
$$

which is a negative homogeneous Sobolev norm. This norm can be interpreted for negative $q$ via eigenfunctions of the Laplacian operator. For example, in a periodic domain, we have

$$
\|\theta\|_{\dot{H}^{q}}^{2}=\sum_{\boldsymbol{k}}|\boldsymbol{k}|^{2 q}\left|\hat{\theta}_{\boldsymbol{k}}\right|^{2}
$$

from which we see that, for $q<0,\|\theta\|_{\dot{H}}^{q}$ smooths $\theta$ before taking the $L^{2}$ norm. The theorem

$$
\lim _{t \rightarrow \infty}\|\theta\|_{\dot{H}^{q}}=0, \quad q<0 \Longleftrightarrow \theta \text { converges weakly to } 0,
$$

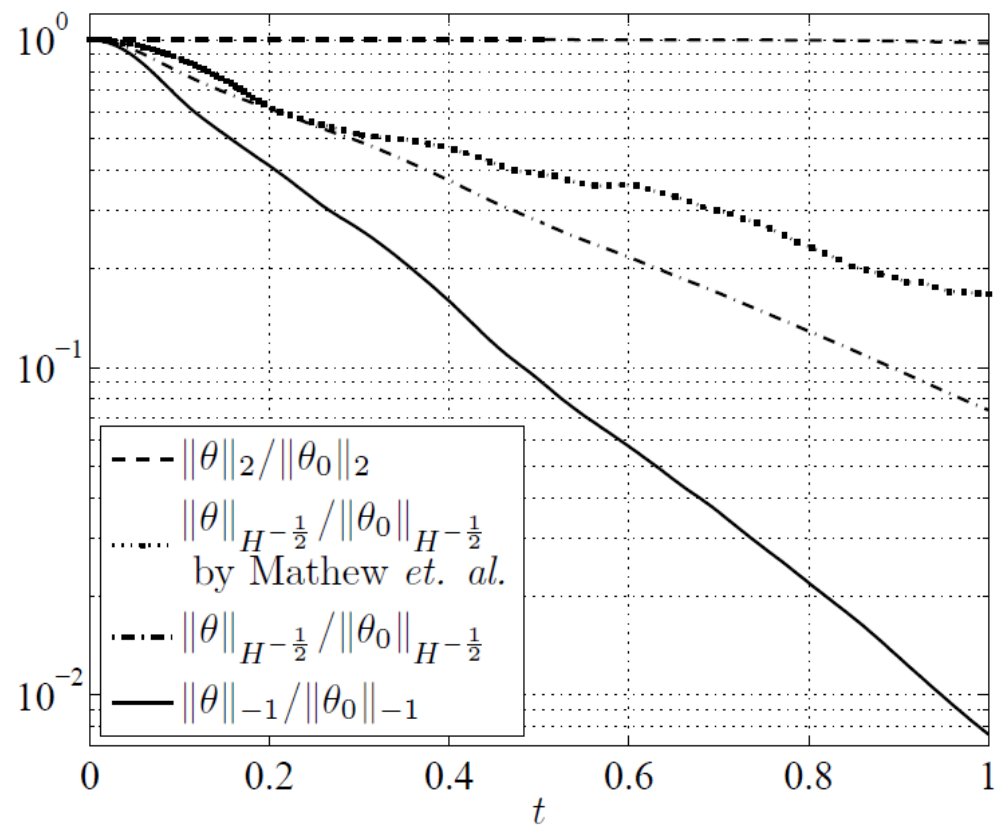

Figure 2: Comparison of the mix-norms for a flow optimized using the separate methods of optimal control and optimal instantaneous decay. Figure from Lin et al. [3]. 

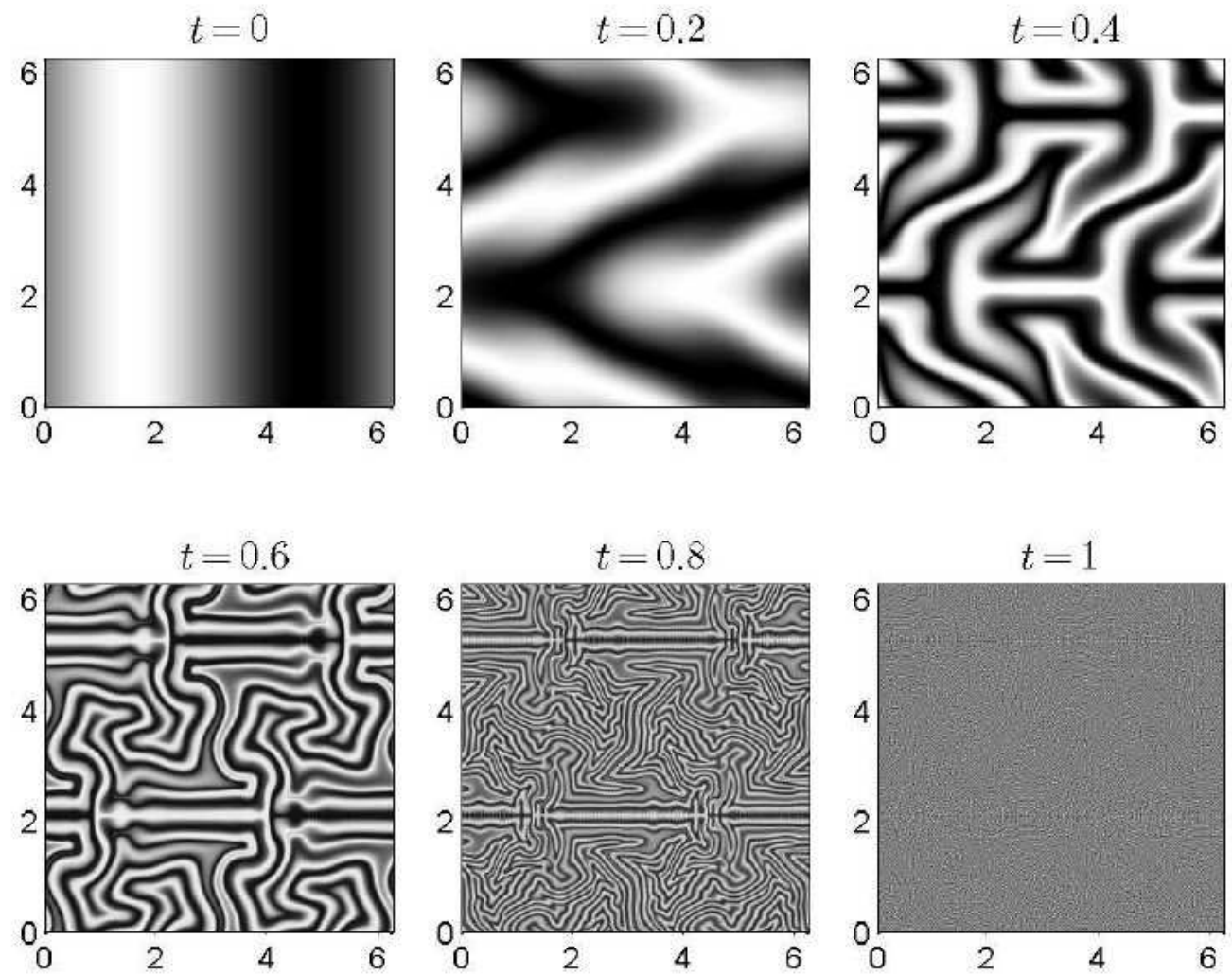

Figure 3: Evolution of the concentration field for the flow optimized in the case $q=-1$ as computed by Lin et al. [3].

due to Mathew, Mezic and Petzold [5] and Doering, Lin and Thiffeault [3] shows that we can track any mix-norm to determine whether a system is mixing (in the weak sense). The existence of this quadratic norm makes optimization of the velocity field for good mixing considerably easier. Mathew, Mezic, Grivopoulos, Vaidya and Petzold [4] have used optimal control to optimize the decay of the $q=-1 / 2$ mix-norm. Lin, Doering and Thiffeault [3] have optimized the instantaneous decay rate of the $q=-1$ norm using the method of steepest descent, which is easier to compute numerically but yields suboptimal, but nevertheless very effective, stirring velocity fields. A comparison of the methods for optimized mixing is shown in figure 2. The solid line decays faster, but this is merely because the $\dot{H}^{-1}$ cannot be compared directly with $\dot{H}^{-1 / 2}$. The corresponding evolution of the concentration field for the case $q=-1$ from Lin et al. [3] is shown in figure 3 . 


\section{Sources and Sinks}

Now let us consider the situation with a sources-sink term $s(\boldsymbol{x}, t)$,

$$
\begin{aligned}
\partial_{t} \theta+\boldsymbol{u} \cdot \nabla \theta & =\kappa \nabla^{2} \theta+s(\boldsymbol{x}, t), \\
\nabla \cdot \boldsymbol{u} & =0 .
\end{aligned}
$$

For simplicity, assume that $\int_{\Omega} s(\boldsymbol{x}, t) d \Omega=0$. Otherwise, we can subtract the mean of $\theta$. It is convenient to think of sources and sinks as hot and cold regions.

Let us also assume that our sources and sinks are time-independent. Then, the system eventually achieves a steady state $\theta(\boldsymbol{x})$ that satisfies

$$
\boldsymbol{u} \cdot \nabla \theta=\kappa \nabla^{2} \theta+s .
$$

We define the operator

$$
\mathcal{L} \equiv y \cdot \nabla-\kappa \nabla^{2}
$$

so that (18) can be written

$$
\mathcal{L} \theta=s
$$

The steady solution is then

$$
\theta=\mathcal{L}^{-1} s
$$

where the mean-zero condition on $\theta$ makes this unique. Note that $\kappa \neq 0$ is needed to achieve a steady state. So, assuming the system has reached a steady-state, we have to determine how we measure the quality of mixing. One of the possible ways is to look at the norms $\|\theta\|_{\dot{H}^{q}}$, where $q=0$ represents standard derivation. But we have to decide what we will compare to. One possibility is $\|\theta\|_{\dot{H}^{q}} /\|s\|_{\dot{H}^{q}}$. This ratio is a reasonable choice, but has units of inverse time. It is preferable to use a dimensionless quantity for measuring the quality of mixing. In this spirit, we define mixing enhancement factors:

$$
\varepsilon_{q}=\frac{\|\tilde{\theta}\|_{\dot{H}^{q}}}{\|\theta\|_{\dot{H}^{q}}}
$$

where $\tilde{\theta}$ is the purely-diffusive solution which satisfies

$$
\tilde{\mathcal{L}} \tilde{\theta}=s .
$$

Here, $\tilde{\mathcal{L}}=-\kappa \nabla^{2}$ is the pure diffusive operator, so $\tilde{\theta}$ can be interpreted as the solution in the absence of stirring. Since $\|\theta\|_{\dot{H}^{q}}$ is usually decreased by stirring, $\varepsilon_{q}$ measures the enhancement over the pure-diffusion state. Several properties are given in Doering and Thiffeault [1], Shaw, Thiffeault and Doering [7], and Thiffeault and Pavliotis [8]. We interpret a large $\varepsilon_{q}$ as 'good stirring,' since it in that case the norm is decreased by stirring.

A natural question is whether $\varepsilon_{q}$ can ever be less than unity, that is, if stirring can ever be worse than not stirring. Let's consider

$$
\varepsilon_{1}=\frac{\|\nabla \tilde{\theta}\|_{2}}{\|\nabla \theta\|_{2}}
$$


Here,

$$
\tilde{\theta}=\tilde{\mathcal{L}}^{-1} s=\left(-\kappa \nabla^{2}\right)^{-1} s=-\kappa^{-1} \nabla^{-2} s \Rightarrow \nabla \tilde{\theta}=-\kappa^{-1} \nabla^{-1} s
$$

Also, from $\mathcal{L} \theta=s$, we can multiply $\theta$ on both sides and take spatial average and then get

$$
\langle\theta \mathcal{L} \theta\rangle=\langle s \theta\rangle,
$$

where $\langle\cdot\rangle=\int_{\Omega} \cdot d \Omega$. We expand the left-hand side:

$$
\begin{aligned}
\langle\theta \mathcal{L} \theta\rangle & =\langle\theta u \cdot \nabla \theta\rangle-\kappa\left\langle\theta \nabla^{2} \theta\right\rangle \\
& =\left\langle\nabla \cdot\left(u \theta^{2} / 2\right)\right\rangle-\kappa\left\langle\theta \nabla^{2} \theta\right\rangle \\
& =-\kappa\left\langle\theta \nabla^{2} \theta\right\rangle=\kappa\left\langle|\nabla \theta|^{2}\right\rangle .
\end{aligned}
$$

As for the right-hand side,it can be written as

$$
\langle\theta s\rangle=\left\langle\theta \nabla \cdot \nabla^{-1} s\right\rangle=-\left\langle\nabla \theta \cdot \nabla^{-1} s\right\rangle=\kappa\langle\nabla \theta \cdot \nabla \tilde{\theta}\rangle,
$$

where we used

$$
\begin{aligned}
\tilde{\theta} & =\tilde{\mathcal{L}}^{-1} s=\left(-\kappa \nabla^{2}\right)^{-1} s=-\kappa^{-1} \nabla^{-2} s \\
& \Longleftrightarrow \nabla \tilde{\theta}=-\kappa^{-1} \nabla^{-1} s .
\end{aligned}
$$

Recall that $\left\langle|\nabla \theta|^{2}\right\rangle=\|\theta\|_{\dot{H}^{1}}^{2}$. Therefore,

$$
\|\theta\|_{\dot{H}^{1}}^{2}=\langle\nabla \theta \cdot \nabla \tilde{\theta}\rangle \leq\|\nabla \theta\|_{2}\|\nabla \tilde{\theta}\|_{2}=\|\theta\|_{\dot{H}^{1}}\|\tilde{\theta}\|_{\dot{H}^{1}} .
$$

We conclude that

$$
\|\theta\|_{\dot{H}^{1}} \leq\|\tilde{\theta}\|_{\dot{H}^{1}} \Longleftrightarrow \varepsilon_{1} \leq 1 .
$$

This is somewhat counter-intuitive because gradients are usually increased by stirring. However, the gradients in a steady-state have been affected by diffusion.

What about $\varepsilon_{q}$ for values of $q$ other than 1 ? We tried and failed to prove $\varepsilon_{q} \leq 1$, simply because it is not true. Following a challenge by Charlie Doering at a workshop at the IMA in 2010, Jeff Weiss came up with something like:

$$
\begin{aligned}
& u=(2 \sin x \cos 2 y,-\cos x \sin 2 y), \\
& s=\left(\cos x-\frac{1}{2}\right) \sin y .
\end{aligned}
$$

This velocity field manages to concentrate the source and sink distribution more than diffusion alone. Streamlines of $u$ and level sets of $s$ are shown in figure 4 . In this example, we could get $\varepsilon_{0} \simeq 0.978$ and $\varepsilon_{-1} \simeq 0.945$, which are slightly less than 1 . It is an open problem to characterize such 'unmixing' flows. 

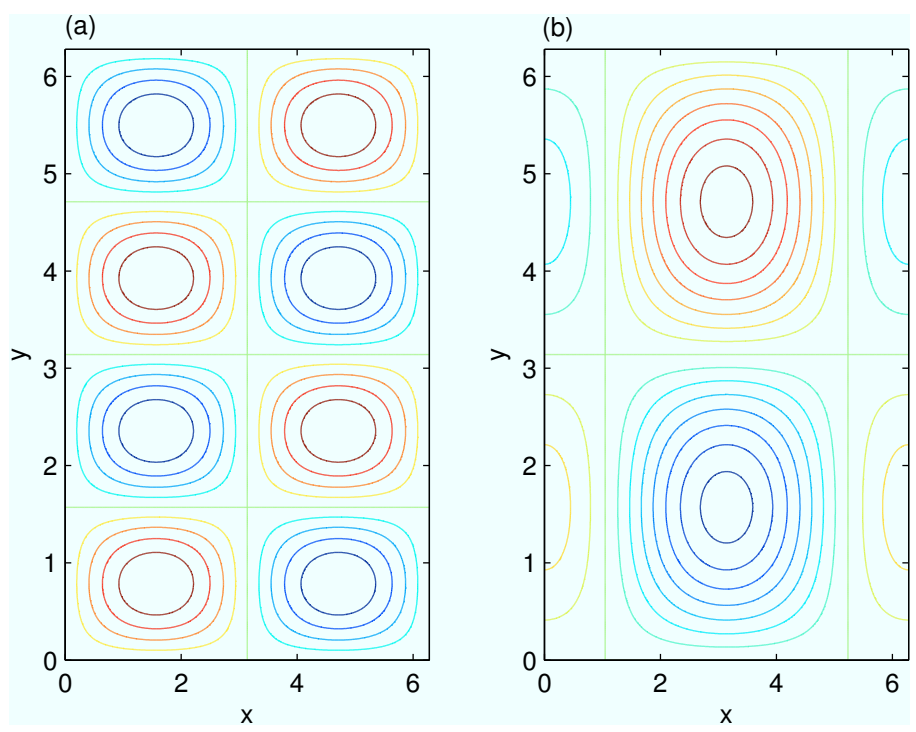

Figure 4: The pattern of velocity field and source for the 'unmixing' flow and source distribution (24).

\section{Optimization}

We defined the mixing enhancement factors based on Sobolev norms. Large mixing enhancement factor indicates good mixing for a given source and sink pattern. One of the relevant questions in this step is what kinds of flow give the largest $\varepsilon_{q}$ given source and sink distribution $s(\boldsymbol{x})$.

Here is a simple but surprising example. The source and sink distribution is given by $s(x)=\sin x$ with periodic boundary conditions on $\theta$. The optimal solution for this source and sink distribution is $\boldsymbol{u}=U \hat{x}$, which is constant flow from the hot region to the cold region [6-8] (figure 5). This example demonstrates that, with body sources, the best

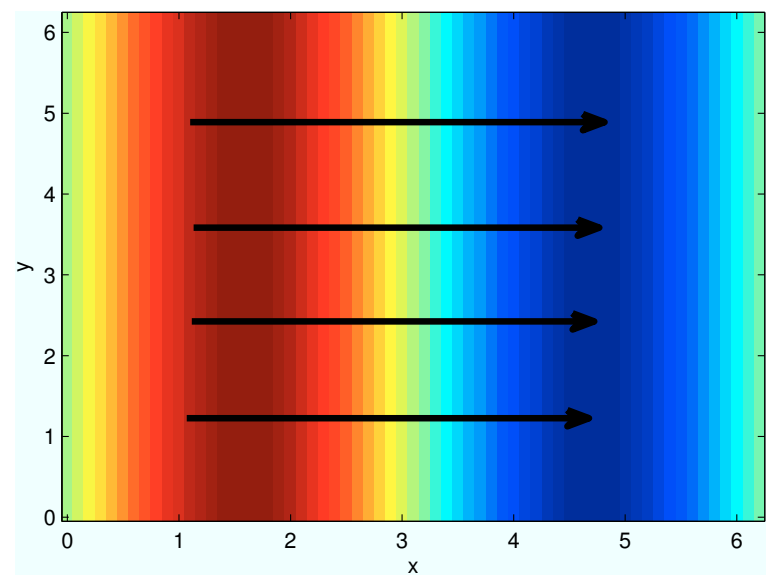

Figure 5: The optimal velocity field (solid arrows) for the source distribution $s(x)=\sin x$. 

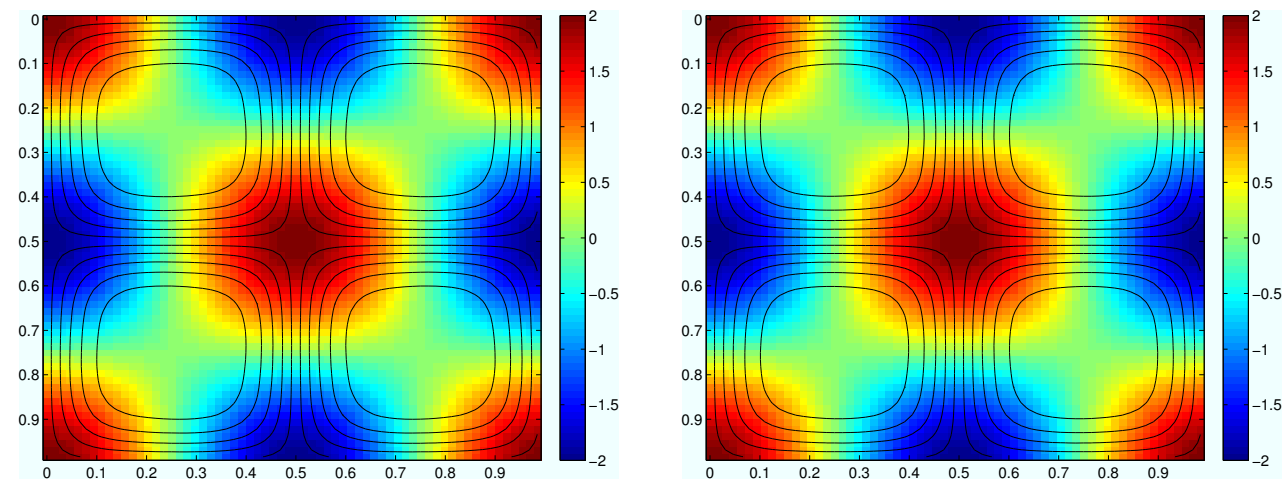

Figure 6: Optimal stirring velocity field (solid lines) for the source $s(\boldsymbol{x})=\sin x \sin y$ (colored background), for $q=0$ (left) and $q=-1$ (right). The optimal velocity is the same in both cases because the source is an eigenfunction of the Laplacian. (Matlab programs example(1) and example(2) in the Appendix.)

stirring often has more to do with transport than with creation of small scales.

More generally, we have to solve the optimization problem numerically. Figure 6 shows contours of the streamfunction for the optimal stirring velocity (lines) for a source $s(\boldsymbol{x})=$ $\sin x \sin y$, for $q=0$ and $q=-1$. The optimal velocity fields are identical for the two values of $q$, because the source is an eigenfunction of the Laplacian.

Contrast this to the optimal solutions in figure 7 , for the source distribution $s(\boldsymbol{x})=$ $\cos x \cos y+\cos 3 y+(1 / 4) \sin 3 y$. This source is not an eigenfunction of the Laplacian, and we expect optimal solutions to depend on $q$. Comparing the left $(q=0)$ and $\operatorname{right}(q=-1)$ figures, we see this is indeed the case, though the difference in this case is fairly small.

Finally, given an optimization code, it is simple to turn it around to anti-optimize, that is, find the worst stirring velocity for a given source distribution. Figure 8 shows this for the source (24b) and $q=0$. Note how the velocity field seems to work to concentrate the source sink, thereby increasing the variance. The efficiency for this anti-optimal solution is $\varepsilon_{0}=0.9736$, which is not much lower than Jeff Weiss's unoptimized flow (24a), which had $\varepsilon_{0} \simeq 0.978$.

To reproduce the 5 figures in this section run the program example $(n)$ in the Appendix, where $n$ is a number from 1 to 5 .

\section{Appendix: Matlab code}

\section{Program file example.m}

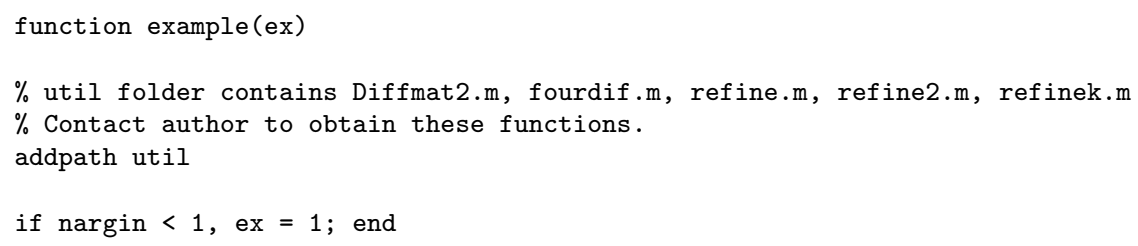



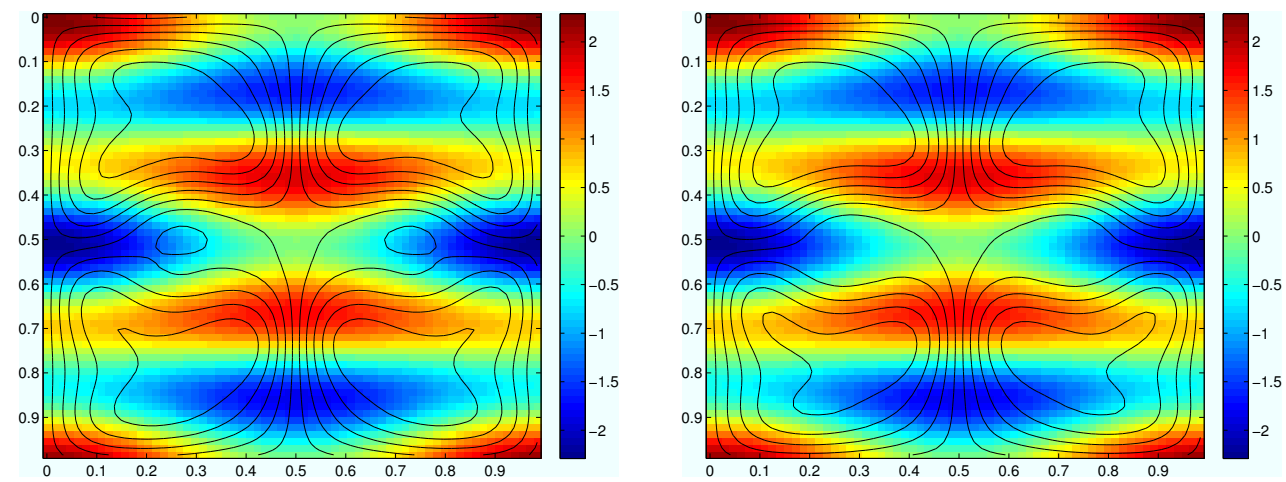

Figure 7: Optimal stirring velocity field (solid lines) for the source $\cos x \cos y+\cos 3 y+$ $(1 / 4) \sin 3 y$ (colored background), for $q=0$ (left) and $q=-1$ (right). The optimal velocities are different since the source is not an eigenfunction of the Laplacian. (Matlab programs example(3) and example(4) in the Appendix.)

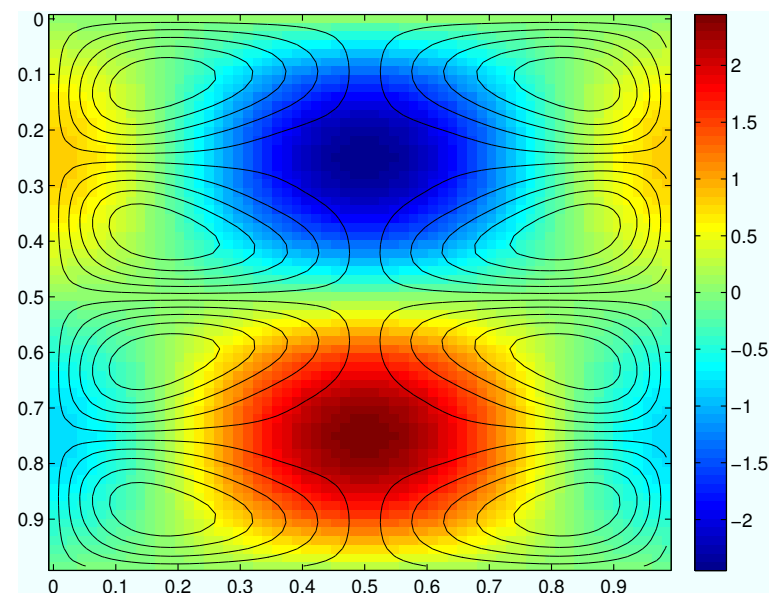

Figure 8: Optimal 'unmixing' solution for the source $\left(\cos x-\frac{1}{2}\right) \sin y$, with mixing efficiency $\varepsilon_{0}=0.9736$. (Matlab program example(5) in the Appendix.) 


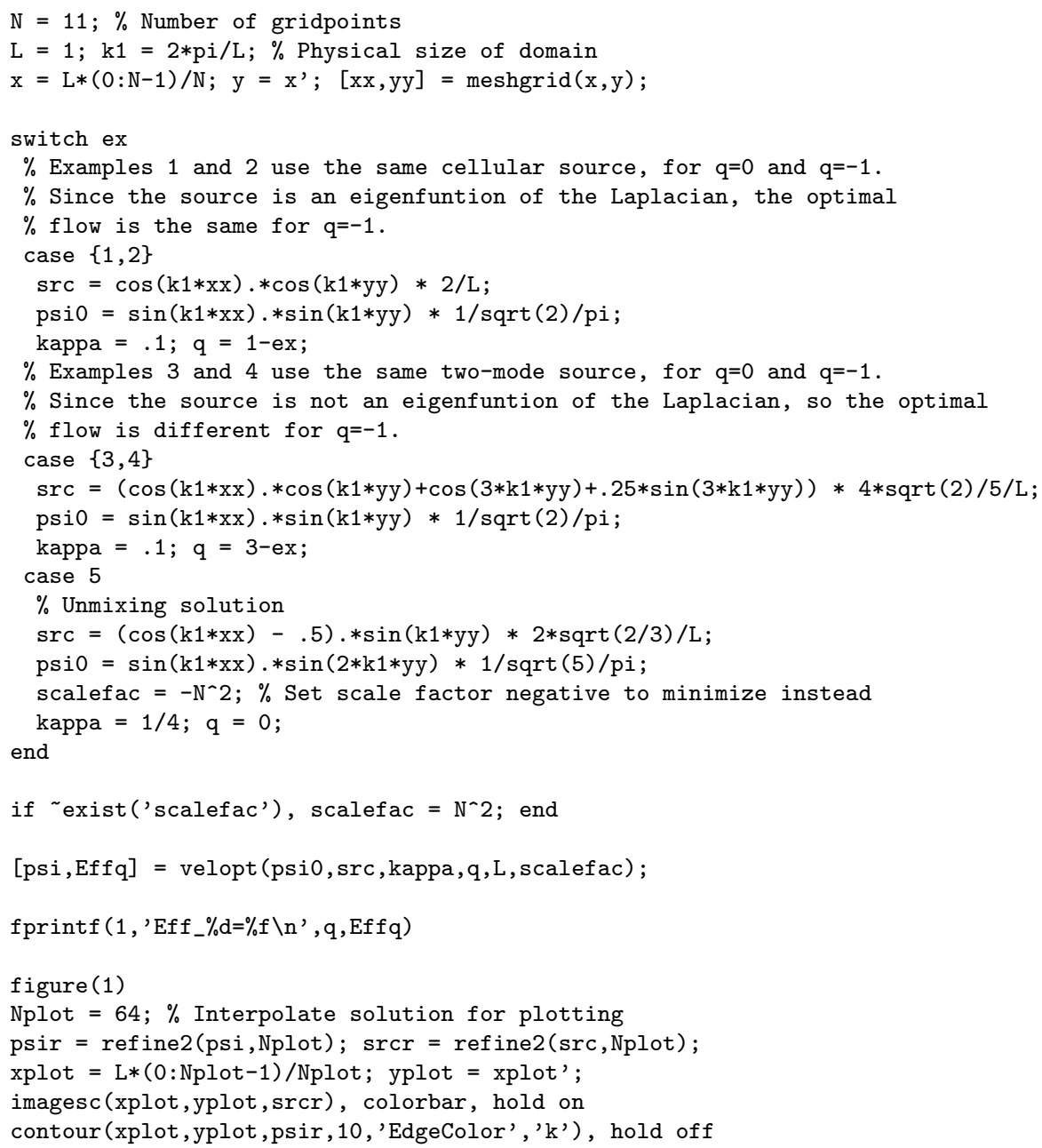

\section{Program file velopt.m}

function $[p s i, E f f q]=\operatorname{velopt}(p s i 0, \operatorname{src}, \operatorname{kappa}, q, L$, scalefac $)$

\% Problem parameters for Matlab's optimizer fmincon.

psio $=\operatorname{psi0}(:) ;$ problem $\times 0=\operatorname{psi0}(2:$ end $)$;

problem.objective $=\varrho(x)$ normHq2 $(x$, src, kappa, $q, L$, scalef ac $)$;

problem.nonlcon $=\mathbb{Q}(\mathrm{x})$ nonlcon $(\mathrm{x}, \mathrm{src}, \mathrm{kappa}, \mathrm{q}, \mathrm{L}, \mathrm{scalefac})$;

problem.solver = 'fmincon';

problem.options $=$ optimset ('Display', 'iter', 'TolFun', $1 \mathrm{e}-10, \ldots$

'GradObj' , 'on' , 'GradConstr', 'on', ... .

'algorithm', 'interior-point') ;

[psi,Hq2] = fmincon (problem);

\% Mixing efficiency: call normHq2 with no flow to get pure-conduction solution. $\mathrm{Effq}=\operatorname{sqrt}(\operatorname{normHq} 2(\operatorname{zeros}(\operatorname{size}($ psi) $), \operatorname{src}, \operatorname{kappa}, \mathrm{q}, \mathrm{L}$, scalefac $) / \mathrm{Hq} 2)$;

psi $=\operatorname{reshape}([0 ;$ psi $]$, size $(\operatorname{src})) ; \%$ Convert psi back into a square grid 


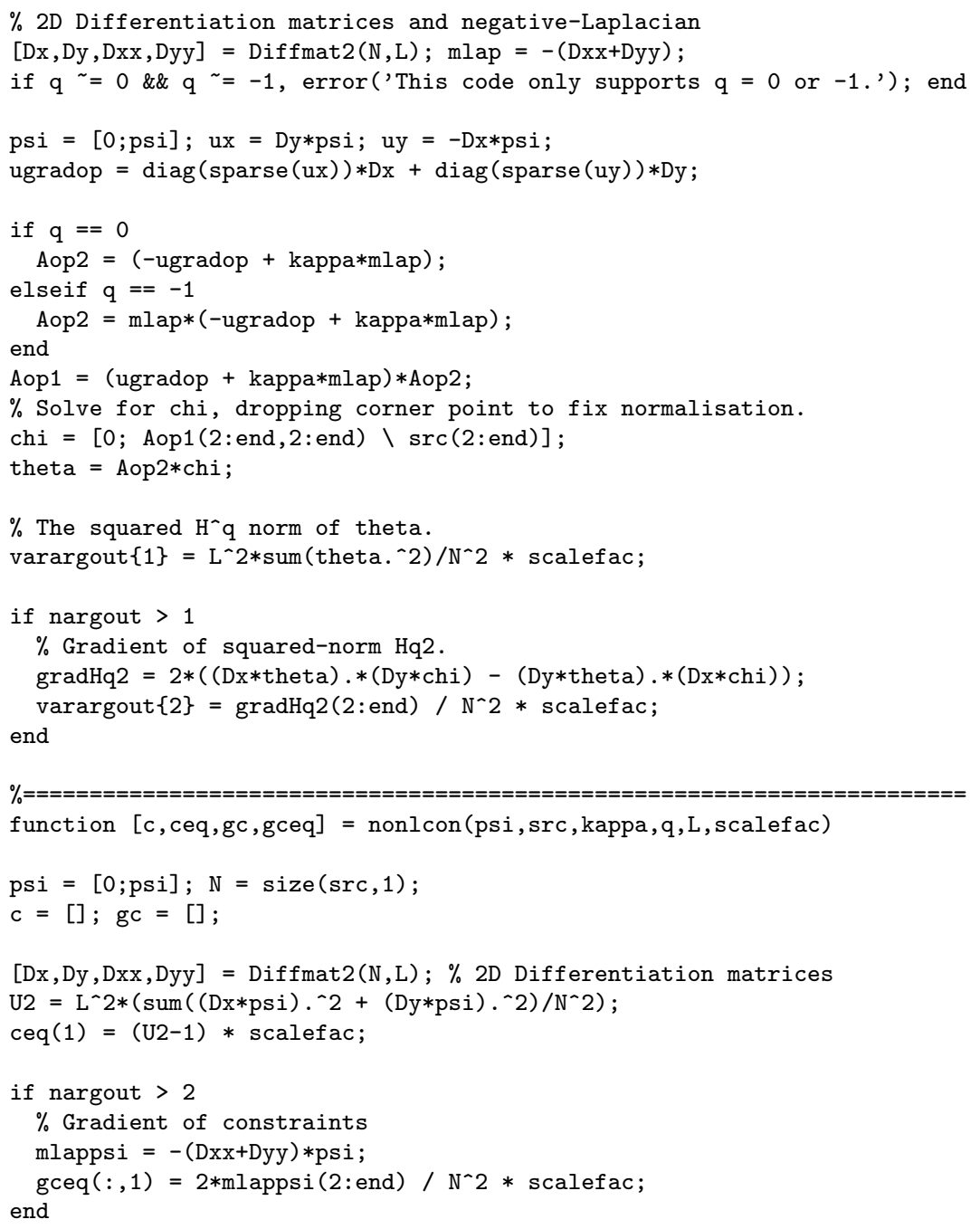

\section{References}

[1] C. R. Doering And J.-L. Thiffeault, Multiscale mixing efficiencies for steady sources, Phys. Rev. E, 74 (2006), p. 025301(R).

[2] A. Lasota And M. C. Mackey, Chaos, Fractals, and Noise, Springer-Verlag, New York, 1994.

[3] Z. Lin, C. R. Doering, And J.-L. Thiffeault, An optimal stirring strategy for passive scalar mixing, 2010. preprint.

[4] G. Mathew, I. Mezić, S. Grivopoulos, U. Vaidya, and L. Petzold, Optimal control of mixing in Stokes fluid flows, J. Fluid Mech., 580 (2007), pp. 261-281.

[5] G. Mathew, I. Mezić, And L. Petzold, A multiscale measure for mixing, Physica D, 211 (2005), pp. 23-46. 
[6] S. Plasting and W. R. Young, A bound on scalar variance for the advectiondiffusion equation, J. Fluid Mech., 552 (2006), pp. 289-298.

[7] T. A. Shaw, J.-L. Thiffeault, And C. R. Doering, Stirring up trouble: Multi-scale mixing measures for steady scalar sources, Physica D, 231 (2007), pp. 143-164.

[8] J.-L. Thiffeault And G. A. Pavliotis, Optimizing the source distribution in fluid mixing, Physica D, 237 (2008), pp. 918-929. 


\title{
Plankton dynamics and mesoscale turbulence
}

\author{
Lecture given on 29 June 2010 by A. Provenzale
}

In aquatic ecosystems, primary production (the transformation of inorganic materials and light into living matter by photosynthesis) is operated mainly by small, unicellular algae that float freely in the upper layers of oceans and lakes and are collectively called phytoplankton, see for an illustration the phytoplankters depicted in figure 1. Since phytoplankton need light, they are confined to the water layer where solar radiation can penetrate. This region is called the "euphotic layer" and it has a maximum depth of about one hundred meters. In the ocean, this roughly corresponds to the depth of the mixed layer; thus, the environment where phytoplankton live is a highly energetic fluid region characterized by intense turbulent mixing.

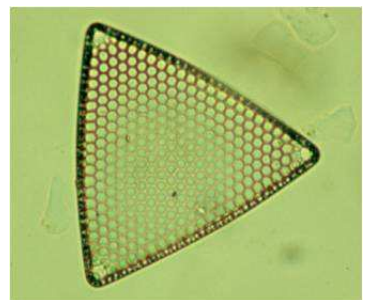

(a)

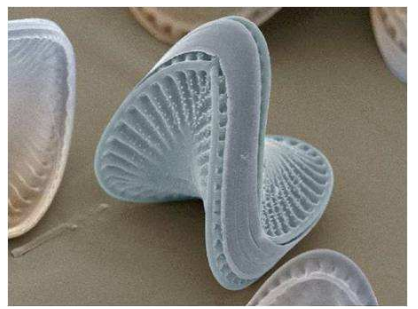

(b)

Figure 1: Some examples of phytoplankton cells

Aquatic ecosystems are characterized by the essential role played by fluid dynamics. The small organisms which compose the plankton are advected by the surrounding flow and must cope with environmental currents, turbulence, and waves. And those organisms which anchor themselves to the rocks and to the bottom must prevent being wiped away by turbulent bursts. Fluid dynamics determines the motion of the individual organisms and, on a larger scale, the distribution and concentration of entire populations. Fluid dynamics and turbulence enter marine and lacustrine Ecology at all levels, and must be properly studied and understood in order to obtain a correct description of aquatic ecosystem functioning. On the other side, the transport and advection of a biologically reactive tracer, such as the plankton distribution, or of individual small living "impurities" (as we could call them) in a turbulent flow make fluid dynamics even more intriguing, opening up new problems and challenges which can stimulate the interest of the more mathematically-oriented types. In these three lectures, we shall touch upon some of these issues, with no attempt at completeness but rather barely scratching the surface of this topic. 


\section{Introduction}

Plankton distributions in the ocean and lakes are highly inhomogeneous. One of the reasons for this inhomogeneity lies in the presence of strong horizontal advection, associated with mesoscale turbulence, coupled with the spatial inhomogeneity of the nutrient input and the nonlinear dynamics of plankton populations.

Phytoplankton are 'primary producers', which means that they take inorganic materials, such as nitrogen and carbon, and convert them into biomass via photosynthesis. The main limiting factors for phytoplankton growth are light and nutrient availability; for this reason, phytoplankton populations are confined to the upper layers of lakes and oceans, in a region known as the euphotic layer, see figure 1. In the ocean, nutrients are supplied by different mechanisms: (1) upwelling of deeper waters, the most crucial process as the majority of nutrients are generated by bacterial activity at depth; (2) direct bacterial regeneration of nutrients from dead biomass and excreted organic substances in the euphotic layer; and (3) dust deposition on the surface. As photosynthesis becomes less efficient when the solar radiation is too intense, and since most nutrients come from deeper waters, phytoplankton tend to live near the middle of the euphotic layer.

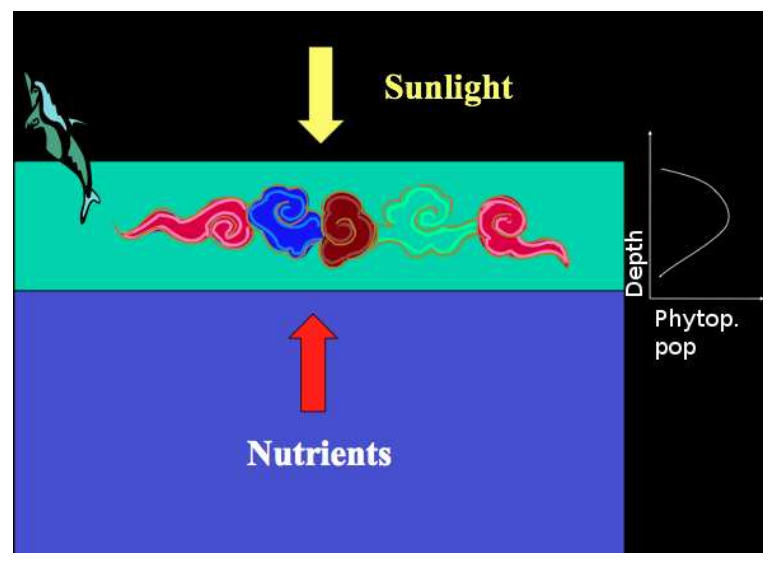

Figure 2: The structure of the upper ocean, as modeled in this lecture. Phytoplankton populations are confined to the euphotic layer (turquoise), where there is enough sunlight for photosynthesis. Nutrients are supplied mainly by upwelling of deeper waters into the euphotic layer, as the highest concentration of nutrients is at depth, where sea snow tends to settle and bacterial activity is strong. Too much sunlight is detrimental, and phytoplankton populations tend to be found near the middle of the euphotic layer.

We model meso- and large-scale interactions between fluid dynamics and biology by resorting to reaction-advection-diffusion equations. The reaction terms represent biological interactions. The advection terms here represent horizontal advection, mainly due to mesoscale circulations and flows. The diffusion terms are a parametrization of small-scale turbulence and of vertical mixing due to up- and down-welling. 


\section{Reaction-Diffusion Equations}

Reaction-diffusion equations have the form

$$
\frac{\partial \rho}{\partial t}=\mathbf{f}(\rho)+D \nabla^{2} \rho
$$

where $\rho$ is the concentration field of some (vector) quantity, and $D$ is diffusivity (assumed constant and the same for all field components). The vector quantity $\rho$ represents the concentration of different scalar fields undergoing chemical and/or biological interactions. The vector quantity $\mathbf{f}(\rho)$ represents the reactions between the various components of $\rho$.

In one spatial dimension and for a scalar field, this equation becomes

$$
\frac{\partial \rho}{\partial t}=f(\rho)+D \frac{\partial^{2} \rho}{\partial x^{2}}
$$

where $x$ is the spatial coordinate and now $\rho$ is a scalar.

The simplest biological example is the KISS model for plankton blooms [7, 17]. This includes a term describing Malthusian (exponential) growth and a diffusion term,

$$
\frac{\partial \rho}{\partial t}=\nu \rho+D \frac{\partial^{2} \rho}{\partial x^{2}}
$$

where $\nu$ is the exponential growth rate of a spatially homogeneous population. Via Fourier analysis, we can find the following expression:

$$
\rho(x, t)=\frac{1}{2 \pi} \int_{-\infty}^{\infty} \hat{\rho}(k) \exp (i k x) \exp \left[\left(\nu-D k^{2}\right) t\right] d k,
$$

and so there is instability (growth) for $k^{2}<\nu / D$ (large spatial scales), while growth is restrained at small scales. This behavior leads to the presence of patchiness in the concentration field $\rho$ with the minimum scale $2 \pi(D / \nu)^{1 / 2}$.

To saturate the exponential growth of the homogeneous population at large times, one can include an extra term in $\rho^{2}$, and obtain the celebrated Fisher equation:

$$
\frac{\partial \rho}{\partial t}=\nu \rho\left(1-\frac{\rho}{K}\right)+D \frac{\partial^{2} \rho}{\partial x^{2}}
$$

where $K$ is a constant. We can make this equation non dimensional by rescaling space, time and the concentration field:

$$
\rho \rightarrow \tilde{\rho}=\frac{\rho}{K}, \quad t \rightarrow \tilde{t}=\nu t, \quad x \rightarrow \tilde{x}=\left(\frac{\nu}{D}\right)^{1 / 2} x
$$


and we obtain, omitting the tilde for ease of notation,

$$
\frac{\partial \rho}{\partial t}=\rho(1-\rho)+\frac{\partial^{2} \rho}{\partial x^{2}}
$$

where all quantities are now non-dimensional. The homogeneous version of this equation, for $\partial \rho / \partial x=0$, is known as the logistic equation,

$$
\frac{d \rho}{d t}=\rho(1-\rho) \text {. }
$$

The Fisher equation admits a traveling wave solution of the form $U(z)=\rho(x-c t)$, which satisfies the following equation:

$$
\frac{d^{2} U}{d z^{2}}+c \frac{d U}{d z}+U(1-U)=0
$$

that is,

$$
\frac{d U}{d z}=V, \quad \frac{d V}{d z}=-c V-U(1-U)
$$

where $(U, V) \equiv(U, d U / d z)$. This system of ordinary differential equations defines a twodimensional dynamical system, which has two fixed points, namely $(U, V)=(0,0)$ and $(U, V)=(1,0)$. Linear stability analysis of the fixed points gives the eigenvalues $\lambda_{ \pm}$:

$$
\begin{array}{lll}
(0,0) & \rightarrow & \lambda_{ \pm}=\frac{1}{2}\left[-c \pm\left(c^{2}-4\right)^{1 / 2}\right], \\
(1,0) & \rightarrow & \lambda_{ \pm}=\frac{1}{2}\left[-c \pm\left(c^{2}+4\right)^{1 / 2}\right],
\end{array}
$$

Thus, $(0,0)$ is a stable node if $c^{2} \geq 4$, and a stable focus if $c^{2}<4$; the fixed point $(1,0)$ is a saddle. A stable focus would result in negative values of $U$ on some trajectories near $(0,0)$, and thus $c^{2} \geq 4$ is the only physical solution. The corresponding phase-plane picture is shown in figure 3 , along with a depiction of the form of the traveling wave solution (from $[10])$.

For general initial conditions of the form

$$
\rho(x, 0)=1, \quad x<x_{1} ; \quad \rho(x, 0)=0, \quad x \geq x_{2},
$$

where $\rho\left(x_{1} \leq x<x_{2}, 0\right)$ is an arbitrary function which joins 1 and 0 , at large time the solution will tend to the traveling wave solution, $U(x-c t)$ with $c=2$, see figure 4 .

If we replace the second initial condition as follows

$$
\rho(x, 0) \approx A \exp (-a x), \quad x \rightarrow \infty,
$$

see figure 4 , then the system tends to the solution

$$
\rho(x, t) \approx A \exp (-a[x-c t]), \quad x \rightarrow \infty,
$$

where

$$
c=a+\frac{1}{a} \quad \text { if } \quad 0<a \leq 1 ; \quad c=2 \quad \text { if } \quad a>1
$$



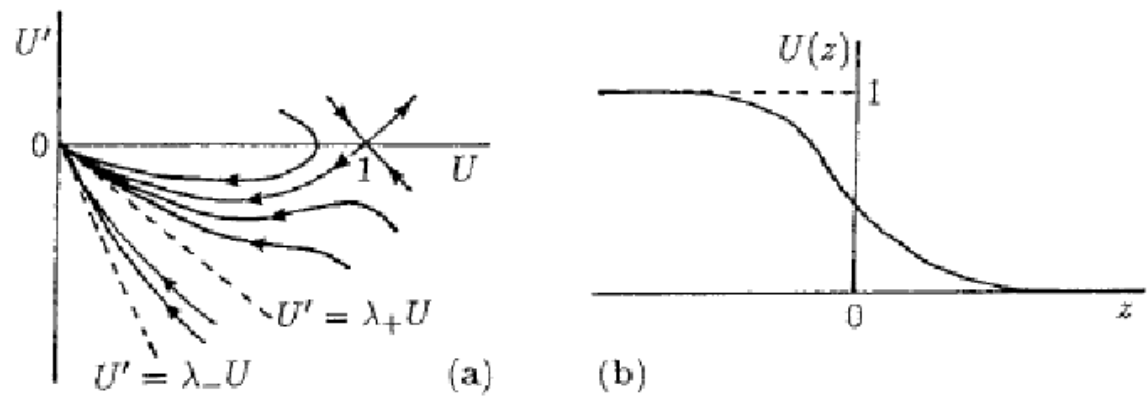

(b)

Figure 3: Panel (a) shows the phase plane trajectories for the dynamical system (9), representing the traveling wave solution of the Fisher equation (6), and panel (b) shows the traveling wave solution of equation (6), for $c \geq 2$. Both illustrations are from [10].
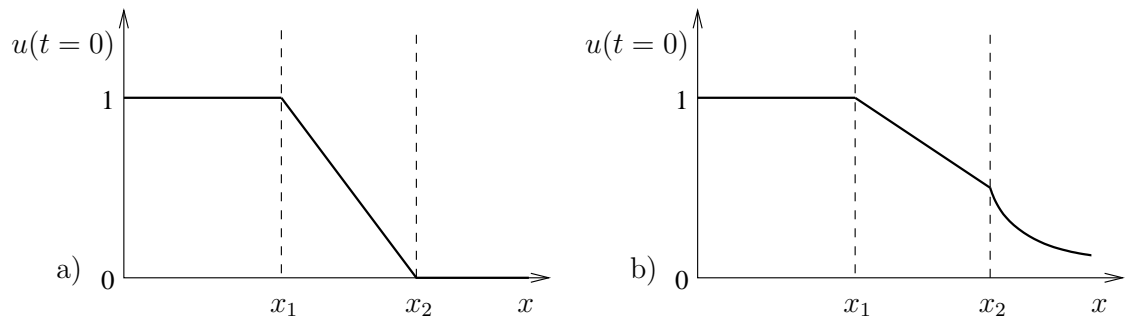

Figure 4: Qualitative description of the initial conditions which lead to a traveling wave solution, $U(x-c t)$, of the reaction-diffusion equation (6), where panel (a) shows (11) and panel (b) shows (12). The linear form of the initial conditions for $x_{1} \leq x<x_{2}$ adopted here is for illustration purposes only. 


\section{Reaction-Advection-Diffusion Equations and Plankton Dy- namics}

In a fluid flow, reactions are affected not only by diffusion, but also by advection. In such conditions, the equations for the concentrations of reactive tracers become

$$
\frac{\partial \rho}{\partial t}+\mathbf{u} \cdot \nabla \rho=\mathbf{f}(\rho)+D \nabla^{2} \rho
$$

where $\mathbf{u}$ is the advecting flow field and $D$ is the diffusivity (again assumed constant and the same for all components of the concentration field).

Often, the fluid velocity $\mathbf{u}$ is turbulent, and this is certainly true for the case of large and mesoscale ocean flows. Turbulent advection in aquatic ecosystems occurs on several scales. Some of these are:

Micro-scales: Between about $1 \mathrm{~mm}$ and a few meters, the interactions of 3-D turbulence, buoyancy and individual plankton cells are important, as will be discussed in the following lecture.

Meso- and submeso-scales: Between about $1 \mathrm{~km}$ and $200 \mathrm{~km}$, largely in the form of fronts and vortices, possibly associated with strong vertical velocities, which modulate the plankton distribution.

Large scales: Transport at ocean basin scales by large-scale gyres.

In the ocean, mesoscale advection is highly inhomogeneous, with very localized upwelling regions leading to inhomogeneous nutrient fluxes; figure 3 shows examples of mesoscale structures in the ocean dynamical properties and in the plankton distribution. In general, horizontal advection is associated with much larger velocities than vertical advection, and is responsible for transporting nutrients, phytoplankton and zooplankton as (almost) passive tracers. Advection timescales in the upper ocean are on the order of 1-7 days, which are similar to the phytoplankton and zooplankton growth timescales, which are of the order of 1-2 days and 1-2 weeks, respectively. This means that scale separation between the reaction and the advection time scales (which could allow for some simplifications) is not possible.

In keeping with the tradition of considering idealized situations, we introduce a simplified description of mesoscale turbulence in the ocean. Mesoscale ocean flows are dominated by rotation and are usually characterized by stable stratification (ignoring the localized regions of intense deep convection in some polar areas and in the Gulf of Lyons). In such conditions, one of the simplest (certainly too simple) descriptions of mesoscale ocean flows is provided by quasigeostrophic turbulence in its barotropic version, and, discarding the effects of the free surface and of differential rotation (the $\beta$-plane effect), by the formulation known as two-dimensional (2D) turbulence. Of course, this flow model cannot be taken as a true representation of mesoscale ocean flows but rather as an idealized picture that has some of the properties of the real flow (for example, the presence of intense coherent vortices). 

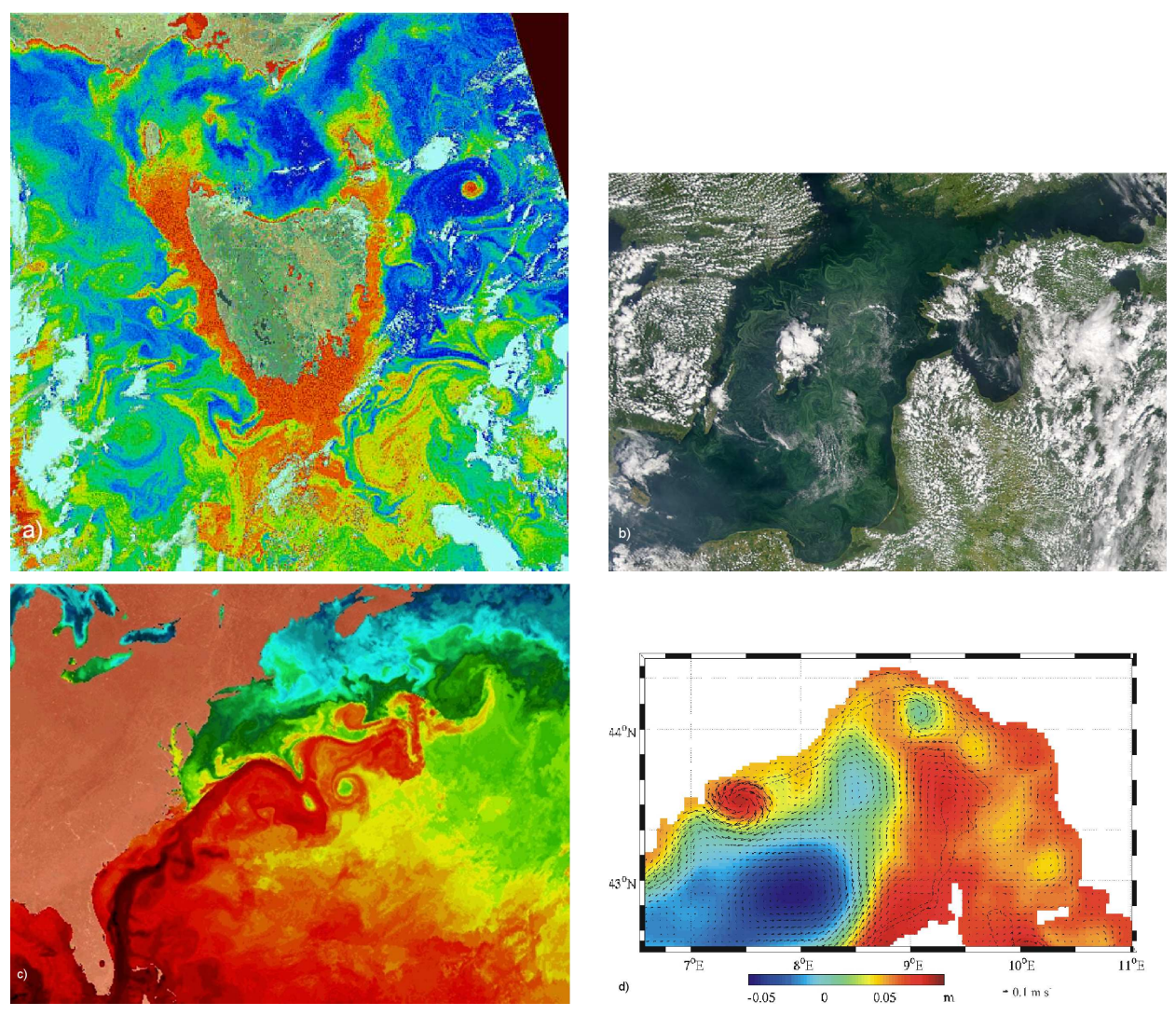

Figure 5: Examples of mesoscale structures in plankton populations and in the ocean temperature and velocity fields. Panel (a) shows the concentration of chlorophyll-a around Tasmania [11]. Panel (b) shows a phytoplankton bloom of Nodularia spumigena in the Baltic sea [12]. Panel (c) shows the intricate mesoscale structures in sea-surface temperature in the North Atlantic [13]. Panel (d) shows the velocity field (arrows) and the sea surface height variations (colorscale) in a patch of the Mediterranean Sea, near the coast of France and Italy [2]. 
The equation of motion for $2 \mathrm{D}$ turbulence is written as

$$
\frac{\partial \zeta}{\partial t}+u \frac{\partial \zeta}{\partial x}+v \frac{\partial \zeta}{\partial y}=D+F
$$

where $D$ is a (hyper)diffusion term, $F$ is forcing, $\zeta$ is vorticity,

$$
\zeta=\frac{\partial v}{\partial x}-\frac{\partial u}{\partial y}=\nabla^{2} \psi
$$

and $\psi$ is the stream function. The horizontal velocities are given by $u=-\partial \psi / \partial y$ and $v=\partial \psi / \partial x$. Many theoretical and numerical explorations of this system have shown that a random initial vorticity field organizes into a collection of coherent vortex structures which contain most of the kinetic energy and enstrophy (squared vorticity) of the flow (see [9] for a pioneering work on this subject). Figure 6 shows an example of the vorticity field produced by numerical integration, with a pseudo-spectral code, of the forced and dissipated 2D turbulence equation (15), see [14] for further details. Here we use hypeviscosity at small scales and large-scale dissipation to balance the forcing, obtained by keeping fixed the power spectrum at a given wavenumber $k_{F}$. After a transient, the flow settles into a statistically stationary state where the average energy, enstrophy and number of coherent vortices oscillate around a constant value.

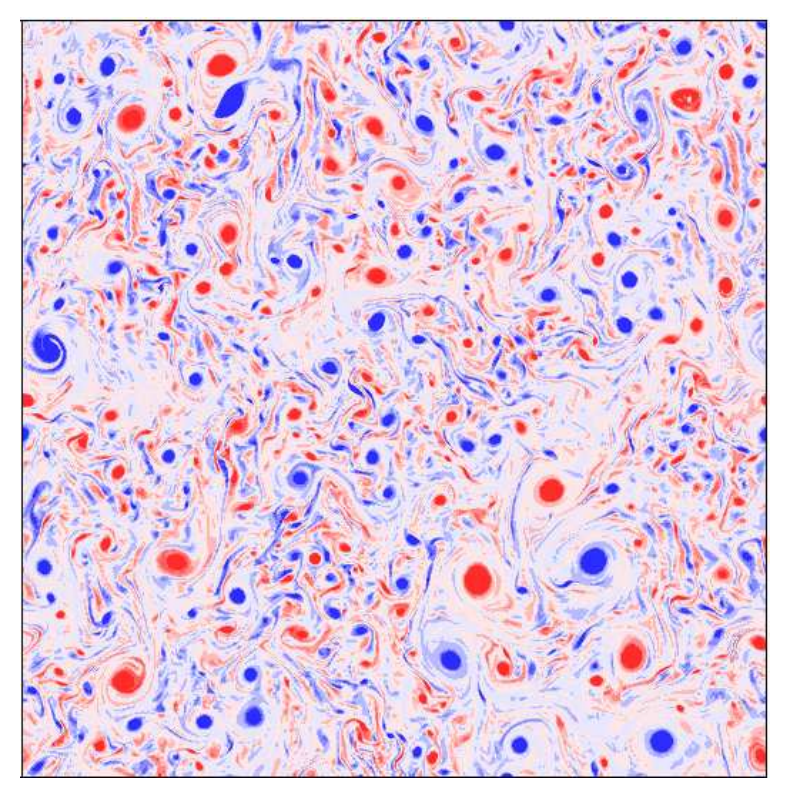

Figure 6: Vorticity field, $\zeta$, from the numerical simulation of equation (15) with forcing and dissipation, showing the presence of intense coherent vortices.

\section{The role of localized upwelling regions}

Plankton growth depends on the availability of nutrients, which have to be supplied to the oceanic mixed layer to balance biological consumption. In turn, the nutrient input fluxes 

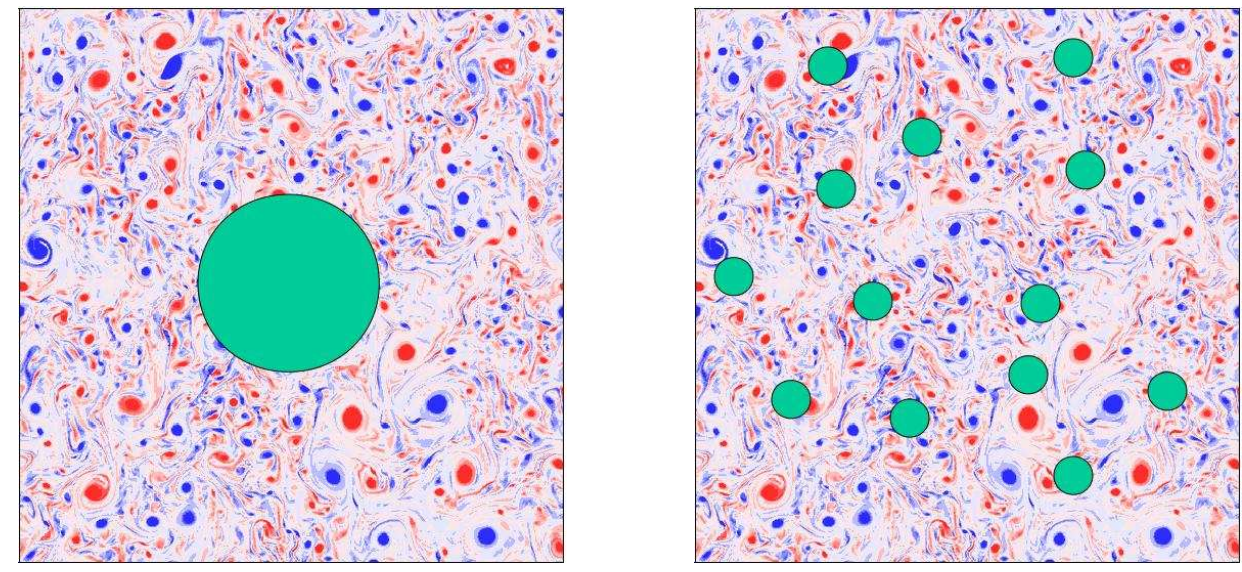

Figure 7: Green regions indicate where the intense nutrient flux takes place. The left panel refers to the case with a single region of strong upwelling (RF1), while the right panel shows a snapshot of the case where the active regions are fragmented $(R F n$, here $n=13)$. The total area of the regions with strong upwelling is approximately the same in the two cases.

are affected by different physical mechanisms such as turbulent mixing, Ekman pumping, convection, transport from the boundaries etc. Such mechanisms act on a variety of scales providing spatially and temporally localized fluxes of nutrients to the biological system. Thus, it is useful to understand the behavior of a biological model in response to such inhomogeneous and intermittent forcings.

The plankton ecosystem model that is used here includes three components, that represent nutrient, $N$, phytoplankton, $P$, and zooplankton, $Z$ (see [16] for details). The dynamics of the ecosystem is described by the reaction-advection-diffusion equations

$$
\begin{aligned}
\frac{D N}{D t}= & \Phi_{N}-\beta \frac{N}{k_{N}+N} P+ \\
& +\mu_{N}\left((1-\gamma) \frac{a \epsilon P^{2}}{a+\epsilon P^{2}} Z+\mu_{P} P+\mu_{Z} Z^{2}\right)+D \nabla^{2} N \\
\frac{D P}{D t}= & \beta \frac{N}{k_{N}+N} P-\frac{a \epsilon P^{2}}{a+\epsilon P^{2}} Z-\mu_{P} P+D \nabla^{2} P \\
\frac{D Z}{D t}= & \gamma \frac{a \epsilon P^{2}}{a+\epsilon P^{2}} Z-\mu_{Z} Z^{2}+D \nabla^{2} Z .
\end{aligned}
$$

where $D / D t=\partial / \partial t+u \partial / \partial x+v \partial / \partial y$.

The terms on the right hand side of the equation for the nutrient represent respectively vertical nutrient supply from deep water, nutrient consumption by phytoplankton, bacterial 
regeneration of the dead organic matter into nutrients in the euphotic layer, and diffusion. Bacterial processes are here assumed to be faster than the other processes; consistently, we do not model bacterial dynamics explicitly and assume that there is a large bacterial population which adjusts instantaneously to the availability of dead matter to decompose. All variables are measured in unit of nitrogen concentration.

Phytoplankton dynamics is regulated by production, dependending on available nutrients through a Holling type-II (or Michaelis-Menten) functional response, by a Holling type III grazing by zooplankton, by linear mortality and by diffusion (see for example [8] for an overview of mathematical models in Ecology and their jargon). Finally, zooplankton grows when phytoplankton is present ( $\gamma$ is the assimilation efficiency of the zooplankton), and has a quadratic mortality term used to close the system and parameterize the effects of higher trophic levels.

In the nutrient equation, the term $\mu_{N}$ is smaller than one and represents the fact that not all biological substance is immediately available as nutrient: the fraction $\left(1-\mu_{N}\right)$ is lost by sinking to deeper waters. Note, also, that nutrient enters this model by affecting the growth rate of phytoplankton. Since the formulation adopted here is two-dimensional in the horizontal and no vertical structure of the fields is allowed, vertical upwelling has to be parameterized.

Nutrient is brought to the surface from deep water by (isopycnal and diapycnal) turbulent mixing and upwelling. When these processes are sufficiently intense, it is reasonable to assume that the surface water becomes saturated in nutrients (with respect to the nutrient content in deep water) and further mixing does not change the concentration of available nutrients. Only when nutrient is removed (by phytoplankton growth and/or horizontal dispersion), vertical mixing becomes effective again. This situation can be represented by a relaxation flux, where the concentration of nutrient at the surface relaxes to a value that depends on the nutrient content in the deep reservoir. The nutrient supply can then be written in restoring form,

$$
\Phi_{N}=-s(x, y)\left(N-N_{0}\right),
$$

where $N_{0}$ is the (constant) nutrient content in deep waters and $s$ is the (spatially varying) relaxation rate of the nutrient, which is large in regions of strong vertical mixing and small in regions of weak vertical mixing. This form can also be interpreted as the finite-difference approximation to a vertical turbulent advective term that acts between two layers with nutrient concentration $N$ and $N_{0}$, and it is the standard formulation used for chemostat models when the reservoir has infinite capacity.

Advection is simulated by the action of a barotropic 2D turbulent flow - an approximation to mesoscale turbulence in the ocean. We consider the case of statistically stationary 2D turbulent field, forced at the non-dimensional wavenumber $k=40$ (that is, at a length scale which is $1 / 40$ th of the domain) and having resolution of $512^{2}$ grid points. Assuming that the forcing scale corresponds to the typical size of an eddy, of about $25 \mathrm{~km}$, then the domain size becomes $1000 \mathrm{~km}$ in dimensional units and the resolution is about $2 \mathrm{~km}$. The 
turbulent velocity field has mean eddy turnover time $T_{E}=2.8$ days.

The nutrient sources are represented by a given number of randomly distributed, circular regions with fixed radius where nutrient input is stronger than average. In our simplified view, we simulate the strong nutrient flux in these patches, which we call "active regions", by a value of the relaxation rate, $s=s_{a}$, which is 100 times larger than the relaxation rate in the rest of the domain, which we call the "passive region" and where $s=s_{p}$ with $s_{p} \ll s_{a}$.

In all the numerical simulations discussed here, the turbulent velocity field is the same. What changes from one simulation to another is the spatial arrangement of the region where the flux is strong (i.e., of the "active" region). While the total area of the active region with strong upwelling is kept constant at the $12 \%$ of the domain area, the intense upwelling is respectively confined to a circular patch at the center of the domain (RF1), or to a number of small patches randomly distributed in the domain (RFn, where $\mathrm{n}$ indicates the number of individual patches). The two types of active regions are shown in figure 7 .

To characterize the system behavior, we estimate the mean primary production, defined as $P P=\left\langle\beta N P /\left(k_{n}+N\right)\right\rangle$, where the angular brackets indicate average over the whole domain. Primary production can be taken as an indicator of the efficiency of the biological model to convert inorganic into organic matter.

The horizontal stirring induced by the turbulent velocity field displaces parcels of water that are rich in nutrient and planktonic life into areas of limited upwelling, where the supply is not sufficient to sustain the biological activity at the level present in the parcel. Viceversa, parcels with poor nutrient content and limited planktonic abundance can be displaced into active regions where the newly available nitrate can stimulate a plankton bloom. This effect depends on the intensity of turbulent stirring. The exchange rate of water parcels between active and inactive regions depends on the flow characteristics and on the amount of parcels that are close to the boundary areas between active and inactive regions, where there are strong gradients in the biogeochemical properties of water. The more fragmented are the upwelling regions, the larger are the boundary areas. Experiments RF1 and RFn are designed to illustrate the effects of a variation in the stirring rate on the biological activity when the size of the boundary areas with strong biogeochemical gradients is changed from small (RF1) to large (RFn), while the total upwelling area is kept constant.

The enhanced stirring induced by advection increases the mean flux from deep waters. The enhanced flux originates at active locations when a parcel of water that has low nutrient content is advected over them. To see how this happens, consider two nearby parcels: one is in a region with small nutrient upwelling and characterized by a steady-state nutrient concentration $N_{p}^{*}$; the other is in an active region and has a steady-state nutrient concentration $N_{a}^{*}$. In this configuration, the total nitrate flux associated with these two parcels of water is $\left(s_{p}\left(N_{0}-N_{p}^{*}\right)+s_{a}\left(N_{0}-N_{a}^{*}\right)\right)$. Suppose now that, due to advection, the two parcels switch their position: the parcel with small nutrient content gets in a strong upwelling region and viceversa. In this configuration, the vertical flux is $\left(s_{p}\left(N_{0}-N_{a}^{*}\right)+s_{a}\left(N_{0}-N_{p}^{*}\right)\right)$. The net variation of the nutrient flux is $\left(s_{a}-s_{p}\right)\left(N_{a}^{*}-N_{p}^{*}\right)$, which is proportional to $s_{a}-s_{p}$. This 

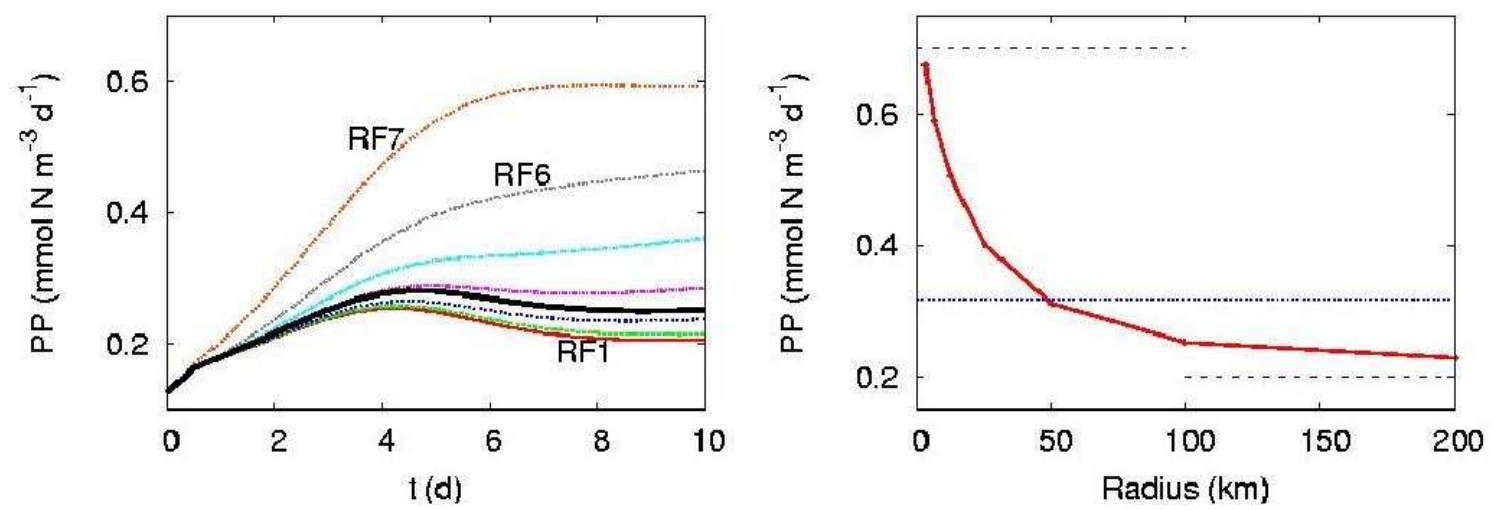

Figure 8: On the left is the time evolution of the average primary productivity. Different curves represent simulations with different number of fragmented sources (from 1 to 7 ). On the right is the dependence of the long-term primary productivity (that is, the value achieved after the transient of panel a) on the radius of the active regions. The total area of the regions with strong upwelling is approximately the same in the two cases.

term is positive as larger relaxation rates are found in regions with strong vertical mixing. The enhanced nutrient flux is thus due to the asymmetry in the relaxation times between the active and inactive regions. Note, also, that the exchange rate of water parcels between the two types of region directly affects the increased nutrient flux to the surface, determining larger values of the nutrient flux in the case RFn than in the case RF1.

The results reported here indicate that primary production has a strong dependence on the fragmentation level of the localized forcing, which is in turn associated with the presence and the properties of mesoscale structures. These results imply that the functioning of marine ecosystems is sgnificantly affected by the flow structure and by the distribution of the upwelling/downwelling regions, which is determined by the nature of mesoscale and submesoscale turbulence.

\section{Advection over topography}

As discussed above, primary productivity in the ocean depends on the availability of nutrients in the euphotic layer, which in turn depends on the presence of upwelling which brings nutrients from deeper waters.

The properties of upwelling are determined by the structure of the vertical velocities. Vertical velocities are greatly affected by the variations of topography which take place at steep continental shelves and near seamounts - areas where large abundances of plankton and fish are usually observed. In the following, we discuss the impact of a vortex propagating across a steep topographic slope on the dynamics of the plankton population [19].

The biological model is the same NPZ model used in the previous section, with the only 

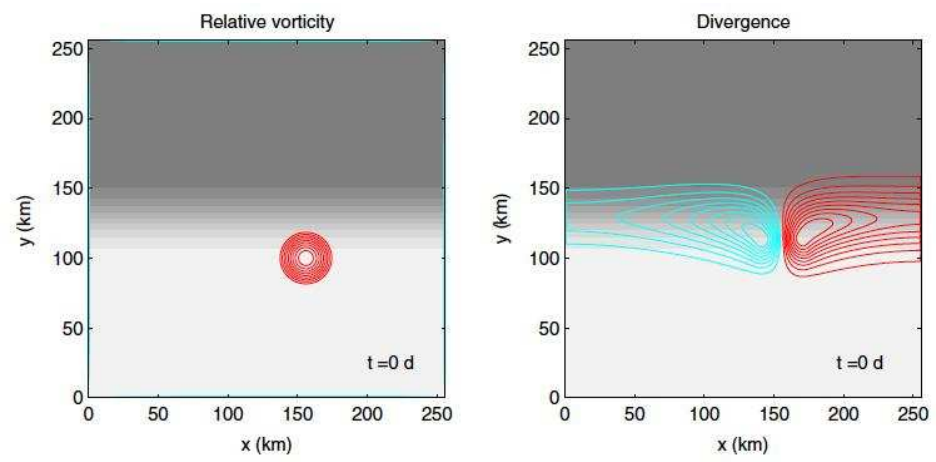

Figure 9: A cyclonic vortex on a sloping bottom topography. Vorticity is on the left; divergence on the right.
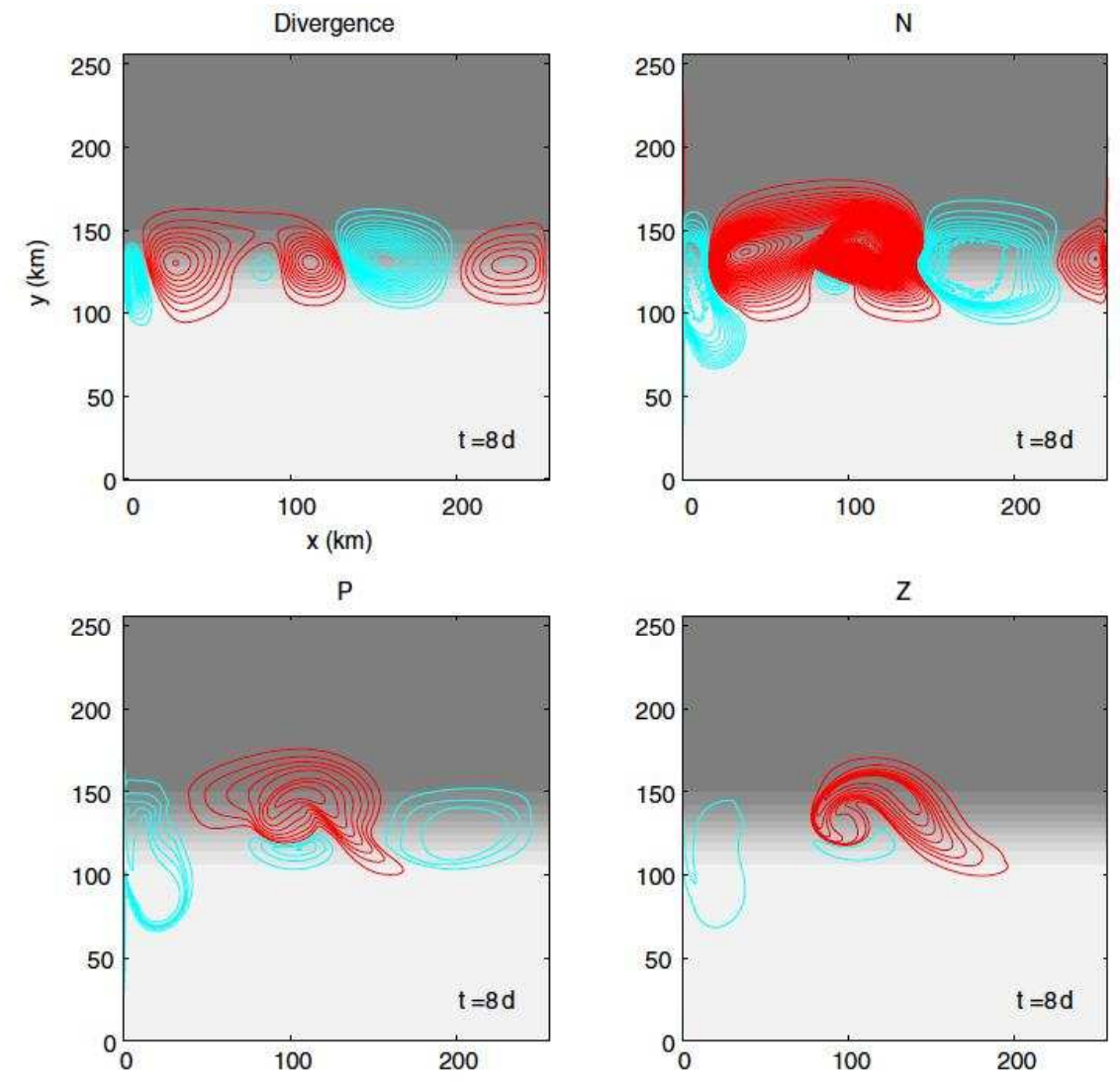

Figure 10: Contours of horizontal divergence and $N, P$ and $Z$ fields at $\mathrm{t}=8$ days. Biological fields are plotted as deviations from their equilibrium values. 

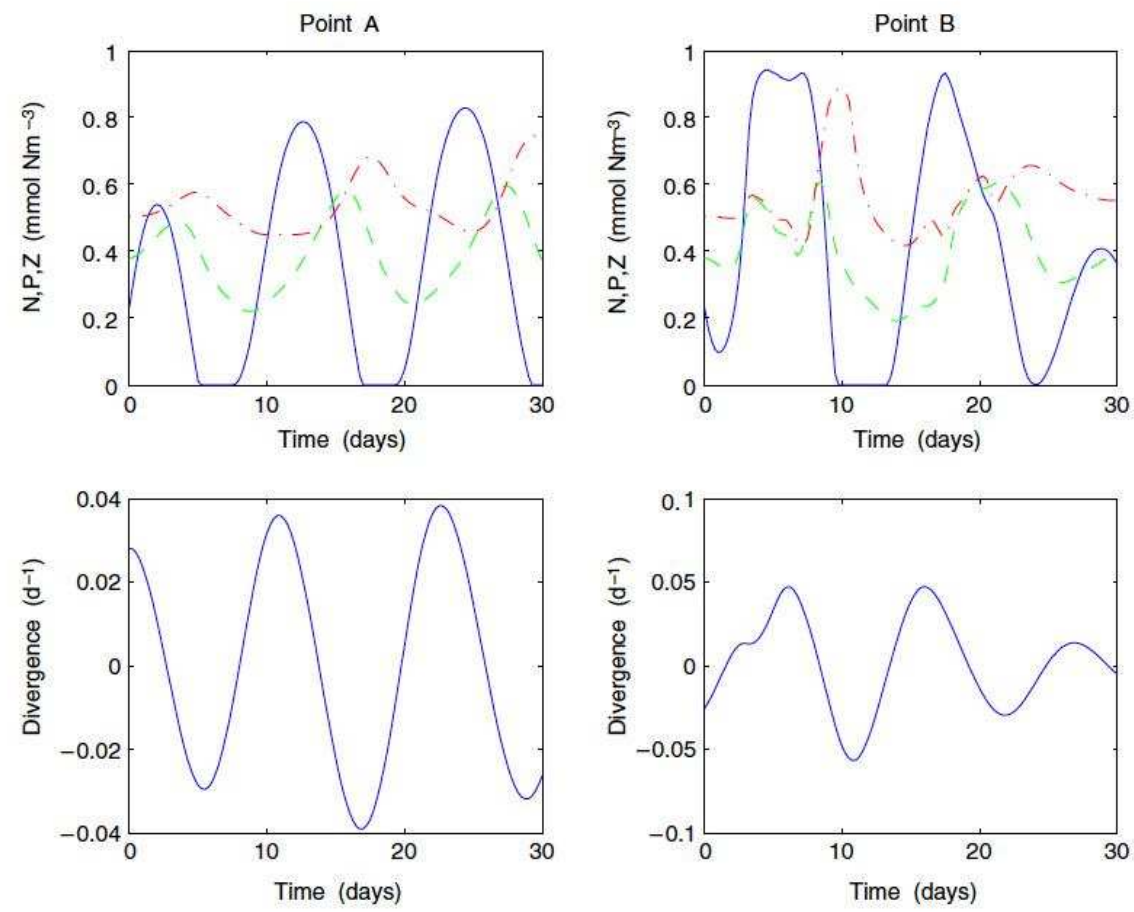

Figure 11: Time series of N,P and Z and flow divergence for two points at depth 450 meters. Solid line: $N$, dashed: $P$, dashed dotted: $Z$. 
difference that now the vertical nutrient supply is given by the term $\Phi_{N}=-\left(s_{v}+s_{n}\right)(N-$ $N_{0}$ ), where the rate $s_{v}$ is proportional to the vertical velocity of the flow and $s_{n}$ represent a weak positive nutrient supply in normal conditions that allows for sustaining a non-zero population at all times. In the proximity of a vortex, $s_{v} \gg s_{n}$.

The value of $s_{v}$ is determined by a Quasi-2D dynamical flow model in the presence of topography [4]. The flow is set in motion by initially placing a cyclonic vortex on the escarpment, see figure 9. The vortex is affected by the topography and it drifts leftward, according to a topographic $\beta$-effect, generating a set of propagating topographic Rossby waves associated with a pattern of vorticity, divergence and vertical velocity with alternating sign. Such motion provides an oscillating source of nutrients to the ecological model.

Figure 10 shows a snapshot of the flow divergence and of the $N, P$ and $Z$ fields after 8 days, when the topographic wave is travelling along the coast. The time series for $N, P$ and $Z$ and the flow divergence are shown in figure (11) for two points located in the sloping region at a depth of $450 \mathrm{~m}$ and spaced $100 \mathrm{~km}$ from each other. The divergence time series clearly shows an oscillatory pattern with a period of about 11 days. The amount of nutrients increases whenever there is positive divergence (that is, upward vertical velocities) and decreases if it is negative (that is, a downward velocity). As a response to this oscillating flux of nutrients, oscillatory behavior is observed also in the phyto- and zooplankton concentrations. This suggests that point measurements of ecological fields over steep slopes could reflect the characteristics of topographic waves rather than internal ecosystem dynamics; a proper interpretation of the measurements should thus take into account the properties of the Rossby waves.

\section{Mesoscale Turbulence and Coexistence}

As a final example, we discuss how mesoscale vortices affect species coexistence. To study this problem, we consider a system with one limiting nutrient, $N$, and two phytoplankton populations, $P_{1}$ and $P_{2}$ (see $[1,15]$ for further details). The system is assumed to be in a chemostat, i.e. there is a constantly replenishing supply of $N$ and a constant loss of $P_{i}$ :

$$
\begin{aligned}
& \frac{D N}{D t}=-s_{0}\left(N-N_{0}\right)-\frac{1}{\rho_{1}} \frac{\beta_{1} N P_{1}}{k_{1}+N}-\frac{1}{\rho_{2}} \frac{\beta_{2} N P_{2}}{k_{2}+N}+D \nabla^{2} N, \\
& \frac{D P_{i}}{D t}=\frac{\beta_{i} N P_{i}}{k_{i}+N}-\mu_{i} P_{i}+D \nabla^{2} P_{i} \quad i=1,2 .
\end{aligned}
$$

The term $-s_{0}\left(N-N_{0}\right)$ is the nutrient input, described as a relaxation to the nutrient

concentration in the deep reservoir, $N_{0}$, with a rate $s_{0}$. The terms $\frac{\beta_{i} N P_{i}}{k_{i}+N}$ represent nutrient uptake by phytoplankton, using a Michaelis-Menten (or Monod) functional form. The constants $\rho_{1}$ and $\rho_{2}$ are used to transform phytoplankton biomass into nutrient concentration if they are described by different units (for example, nitrogen concentration for nutrient and carbon or biomass for phytoplankton). The terms $\mu_{i} P_{i}$ are natural plankton mortalities (which include possible sinking of the plankton cells) and the last terms represent diffusion. Again, the total time derivative is $D / D t=\partial / \partial t+u \partial / \partial x+v \partial / \partial y$. No direct bacterial 
regeneration term has been included.

In homogeneous conditions at equilibrium, there is no co-existence of the two plankton populations, and only the most favored species survives, as determined by the values of the different parameters. In fact, the so-called Principle of Competitive Exclusion [3, 5] states that if two species are too similar, they cannot coexist in equilibrium: whenever two species compete for the same resource, the most favoured will survive and the less favoured will eventually go locally extinct. By extension, at equilibrium the number of species competing on the same resources cannot be larger than the number of resources. Phytoplankton, however, seem to escape this limitation, since a large number of species that compete for the same few resources is usually observed. This phenomenon, known as the Paradox of the Plankton, was formulated by Hutchinson about fifty years ago [6]. Competition is avoided in many instances by partitioning space and/or time: the unfavoured species may be segregated in a spatial environment forbidden to the stronger species, or the two species might perform differently at different times. Mesoscale vortices can be one of the causes of the spatial segregation of unfavoured and favoured competitors, and the sheltering effect offered by the vortices can allow unfavoured competitors to survive for prolonged periods of time.

To study this issue, we consider system (20) with initially inhonogeneous plankton distributions and consider two alternatives for the flow advection:

1. A stochastic process representing a random walk with memory; the various 'kicks' are correlated. This flow has no coherent structures.

2. $2 \mathrm{D}$ turbulence, with coherent vortices.

For the stochastic process, we use a Ornstein-Uhlenbeck (Langevin) process [18], defined as:

$$
\begin{aligned}
d X & =u d t \\
d Y & =v d t \\
d u & =-\frac{u}{T_{L}} d t+\left(\frac{2 \sigma^{2}}{T_{L}}\right) d W_{x} \\
d v & =-\frac{v}{T_{L}} d t+\left(\frac{2 \sigma^{2}}{T_{L}}\right) d W_{y},
\end{aligned}
$$

where $T_{L}$ is the velocity autocorrelation time, $\sigma$ is the standard deviation of velocities, and $W_{x}, W_{y}$ are white-noise, Gaussian Wiener processes characterized by

$$
\begin{aligned}
\langle d W\rangle & =0 \\
\left\langle d W(t) d W\left(t^{\prime}\right)\right\rangle & =\delta\left(t-t^{\prime}\right) d t
\end{aligned}
$$

such that the noise intensities at different times are not correlated. Given these assumptions, the autocorrelation of the velocity components is

$$
R(\tau)=\frac{\langle u(t) u(t+\tau)\rangle}{\left\langle u^{2}(t)\right\rangle}=\exp \left(-\tau / T_{L}\right)
$$



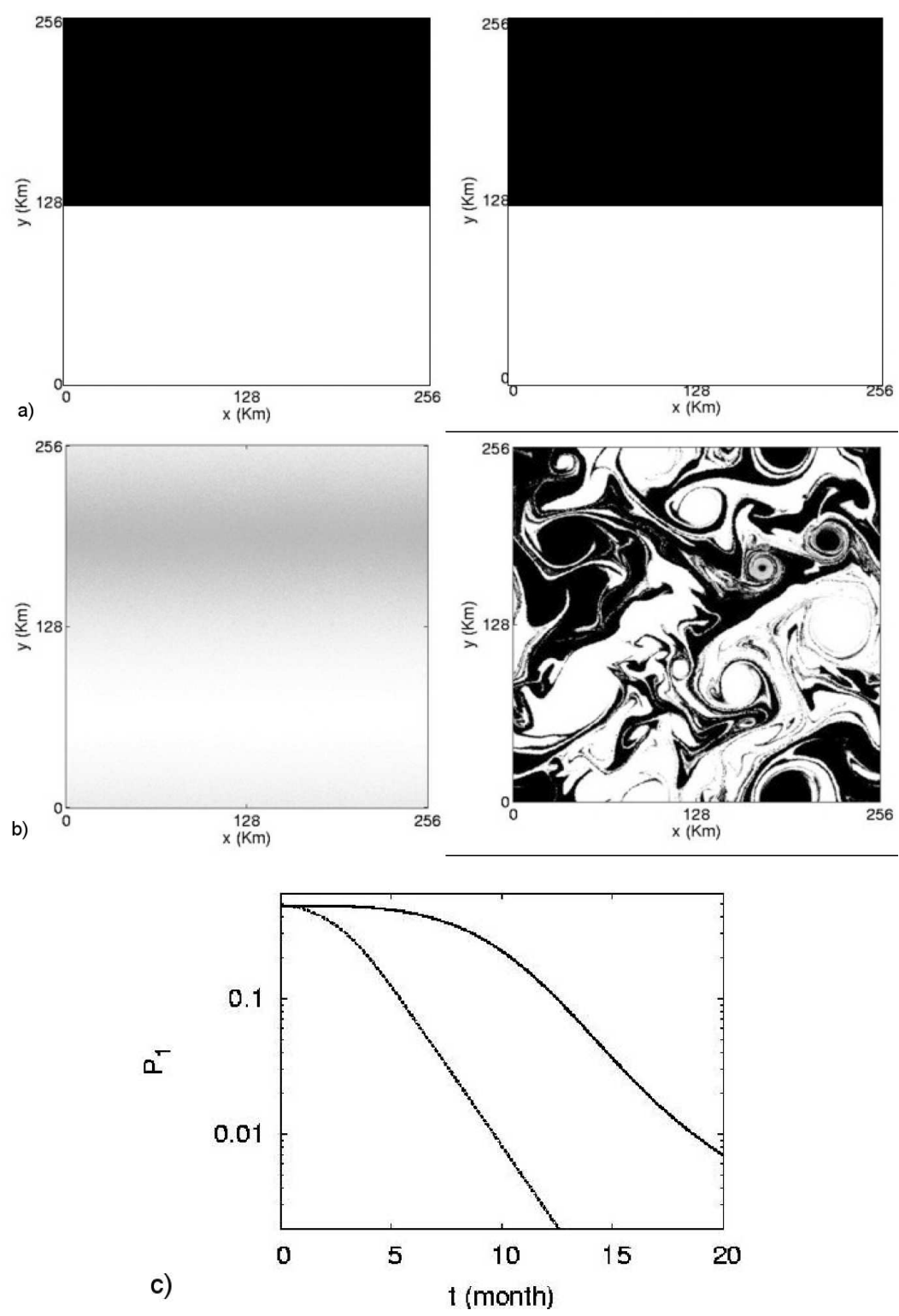

Figure 12: Panels (a) and (b) show snapshots from numerical simulations of two competing species, in white and black, described by equations $(19,20)$, at the beginning (when they are saparate in two different regions) and at a later time, when advection has transported them across the flow domain. The left panels in both a) and b) refer to advection by the OrnsteinUhlenbeck process described in equation (21), and the right panels refer to advection by 2D turbulence. Panel (c) shows the concentration of the less favoured population over time for the Ornstein-Uhlenbeck process (dotted line) and for 2D turbulence (solid line). The most favoured population 'wins' in both cases, but the vortices of $2 \mathrm{D}$ turbulence act as transport barriers and slow down the contact of the two populations and the rates of biological reactions. 
such that velocities are correlated on a timescale $T_{L}$. The probability distribution of the velocity components, $p(u)=p(v)$ since the flow is statistically isotropic, is given by

$$
p(u)=\frac{1}{\sqrt{2 \pi} \sigma} \exp \left(-\frac{u^{2}}{2 \sigma^{2}}\right) .
$$

Figure 12 shows the comparison between the plankton concentrations obtained with the two types of advecting flows, simulated in the same doubly periodic domain and with the same kinetic energy and decorrelation times, comparable with those of mesoscale turbulence in the upper layer of the ocean. The most favoured population (here represented in white) 'wins' in both cases, but it takes much longer in the case of 2D turbulence. In general, in $2 \mathrm{D}$ turbulence the vortices act to segregate the populations and allow the less favoured population to survive longer, providing a shelter area. In case the two populations have different fitnesses in different seasons, the presence of the vortices allows for the survival of the temporarily less fit population and for a long-term, seasonally oscillating coexistence between the two plankton populations. In conclusion, these results indicate that advection significantly affects biological reaction rates, and so it must be taken into account when studying the dynamics of marine ecosystems.

\section{Conclusion}

In this Lecture we saw that the spatial and temporal distribution of plankton at scales between a few $\mathrm{km}$ and a few hundred $\mathrm{km}$ is heavily affected by the properties of mesoscale (and submesoscale, but we did not explore it here) turbulence. If there is a message to be extracted from these examples, it is that the functioning of aquatic ecosystems is largely dependent on the fluid dynamics and that turbulence cannot be ignored.

\section{References}

[1] A. Bracco, A. Provenzale, and I. Scheuring, Mesoscale vortices and the paradox of the plankton, Proceedings of the Royal Society B-Biological Sciences, 267 (2000), pp. $1795-1800$.

[2] E. Casella, A. Molcard, and A. Provenzale, Mesoscale vortices in the ligurian sea and their effect on coastal upwelling processes, J. Marine Systems, (2011).

[3] G. Gause, The Struggle for Existence, Hafner Press, New York, 1934.

[4] R. Grimshaw, Y. Tang, and D. Broutman, The effect of vortex stretching on the evolution of barotropic eddies over a topographic slope, Geophys. Astrophys. Fluid Dyn., 76 (1994), pp. 43-71.

[5] G. Hardin, The competitive exclusion principle, Science, 131 (1960), pp. 1292-1297. 
[6] G. Hutchinson, The paradox of the plankton, American Naturalist, 95 (1961), pp. 137-145.

[7] H. Kierstead and L. Slobodkin, The Size of Water Masses containing Plankton Blooms, Journal of Marine Research, 12 (1953), pp. 141-147.

[8] M. Kот, Elements of Mathematical Ecology, Cambridge Un. Press, Cambridge, UK, 2001.

[9] J. McWilliams, The emergence of isolated coherent vortices in turbulent flow, J. Fluid Mechanics, 146 (1984), pp. 21-43.

[10] J. D. Murray, Mathematical Biology, Springer-Verlag, 2nd ed., 1993.

[11] NASA, November 27 1981. http://disc.sci.gsfc.nasa.gov/oceancolor/ additional/science-focus/images/tasmania_27nov81.gif.

[12] —, July 24 2003. http://disc.sci.gsfc.nasa.gov/oceancolor/additional/ science-focus/images/Baltic_bloom_July24_2003.jpg.

[13] NOOA. http://seacoos.org/Data\%20Access $\% 20$ and\%20Mapping/currents/sst.

[14] C. Pasquero, A. Babiano, and A. Provenzale, Parameterization of dispersion in two-dimensional turbulence, J. Fluid Mechanics, 439 (2001), pp. 279-303.

[15] C. Pasquero, A. Bracco, and A. Provenzale, in "Shallow Flows", edited by G.H. Jirka and W.S.J. Uijttewaal, Taylor and Francis, London, 2004.

[16] _ Impact of the spatiotemporal variability of the nutrient flux on primary productivity in the ocean, J. Geophys. Res. - Oceans, 110 (2005), p. C07005.

[17] J. Skellam, Random Dispersal in Theoretical Populations, Biometrika, 38 (1951), pp. $196-218$.

[18] G. E. Uhlenbeck And L. S. Ornstein, On the theory of the brownian motion, Phys. Rev., 36 (1930), pp. 823-841.

[19] L. Zavala Sanson and A. Provenzale, The effects of abrupt topography on plankton dynamics, Theoretical Population Biology, 76 (2009), pp. 258-267. 


\title{
Plankton cells in turmoil and the dynamics of heavy impurities with finite size
}

\author{
Lecture given on 30 June 2010 by A. Provenzale
}

\section{Introduction}

In this lecture we shall focus on the dynamics of individual phytoplankton cells and on simple models to describe their motion in the fluid flow. Phytoplankton cells are small, with dimensions up to a few hundred microns. Individual phytoplankton cells immersed in water are advected by the surrounding flow, even though some species can resist passive advection by various means, such as varying their buoyancy and/or swimming. In addition, most plankton cells are slightly heavier than water and are thus subject to gravitational sinking (i.e., they are negatively buoyant).

If we want to describe the dynamics of a phytoplankton cell advected by a flow, we need to write the equations of motion for the cell. However, real phytoplankton cells usually have a very complex shape (which can change with time), are non-homogeneous in their interior, can vary their buoyancy and can emit substances that change the local fluid viscosity. In addition, they swim, grow, reproduce (split), and eventually die or are eaten by zooplankton (small, usually multicellular organisms which feed on algae). For fluffy bodies with spines, tails and flagella and an active life such as phytoplankters, the equations of motion are rather complicated, and one should resolve the Navier-Stokes equations with the correct boundary conditions on the mobile surface of the little plankter.

In this lecture we follow a traditional path for the GFD school, and simplify - even grossly oversimplify - the problem, by considering the dynamics of infinitesimally small, spherical heavy particles, with fixed shape and density, which are passively advected by a surrounding fluid flow, and we call them "impurities." Of course, our impurities are but a far image of phytoplankton cells in the ocean.

\section{Plankton and turbulence: a matter of size}

Most fluid flows in the ocean and in lakes are turbulent, especially in the upper mixed layer where phytoplankton thrive. Thus, on large scales, the world in which plankton cells live is a turbulent environment. However, individual cells are small, usually smaller than the Kolmogorov scale - the length scale which marks the transition from laminar flow at smaller scales to turbulent dynamics at larger scales. For this reason, individual phytoplankters experience a viscous, laminar and non-turbulent flow in their immediate surroundings. 
The motion of sea or lake water under "normal" conditions (ignoring compressibility, thermodynamics, phase transitions and other complications) can be described by the incompressible Navier-Stokes equations,

$$
\rho \frac{D \mathbf{u}}{D t}=-\nabla p+\mu \nabla^{2} \mathbf{u}-\rho g \hat{\mathbf{z}}+\mathbf{F}
$$

where $\mathbf{u}(\mathbf{x}, t)$ is the fluid velocity, $\mathbf{x}$ is the spatial coordinate, $t$ is time, $\rho$ is the fluid density, $p$ is pressure, $\mu$ is the dynamic viscosity, $g$ is the gravitational acceleration, $\hat{\mathbf{z}}$ is the unit vector in the $z$ direction, $\mathbf{F}$ is a generic body force (if present) and the substantial (or Lagrangian) derivative can be expressed as $D \mathbf{u} / D t=\partial \mathbf{u} / \partial t+\mathbf{u} \cdot \nabla \mathbf{u}$. We have fixed the $z$ coordinate to point vertically upwards. If $\rho$ is assumed to be constant, the gravitation term can be absorbed in the definition of pressure.

Equation (1) is complemented by the incompressible version of the continuity equation,

$$
\nabla \cdot \mathbf{u}=0
$$

and in this way we get a complete description of the fluid flow, four equations in four variables. Of course, to (try to) solve the equations we also need boundary and initial conditions.

The Reynolds number can be defined as the ratio between the strength of the nonlinear term, $\rho|\mathbf{u} \cdot \nabla \mathbf{u}|$, and that of the viscous term, $\left|\mu \nabla^{2} \mathbf{u}\right|$. If we call $U$ and $L$ the typical scale of velocity and length, and scale time by $L / U$, we get that the Reynolds number is $\operatorname{Re}=U L / \nu$ where $\nu=\mu / \rho$ is the kinematic viscosity. When the Reynolds number is smaller than one, i.e., the velocity and/or the length scale are sufficiently small, the flow is laminar and viscosity dominates. For $\mathrm{Re} \rightarrow 0$, we can discard the inertial term in the Navier-Stokes equations and find viscosity-dominated special solutions. When Re $\gg 1$, the flow becomes turbulent and the fluid velocity varies intermittently and erratically in time and space, spanning a wide range of values.

The simplified form of the Navier Stokes equations given by eq.(1,2) with constant density describes the cleanest case of turbulence, i.e., homogeneous and isotropic threedimensional turbulence. Physically, this type of flow is a good model for turbulence for scales up to a few meters, a reasonably-sized portion of the world for a phytoplankton cell.

To quantitatively describe this type of turbulence, we assume that the energy input in the flow takes place at a certain macro length scale, $l_{0}$, and dissipation takes place mainly at a very small scale, say $l_{D}$. In the scale range between $l_{0}$ and $l_{D}$, energy gets transferred to smaller and smaller length scales by the breakdown of the larger eddies into smaller ones. Here, an "eddy" is a generic, usually short-lived, vortical structure in the three-dimensional turbulent flow, and it is characterized by a given (approximate) length scale. If $l_{0} \gg l_{D}$ and no forcing is present between these two scales, then we see the emergence of an inertial range where the dynamics is independent of dissipation and forcing. Assuming that the spectral energy transfer rate is constant in the inertial range, so to obtain a statistically stationary energy spectrum, and using simple scaling arguments, one can derive the form of 
the energy spectrum in the inertial range, $E(k)$, which obeys the celebrated Kolmogorov's $-5 / 3$ law,

$$
E(k) \sim \varepsilon^{2 / 3} k^{-5 / 3}
$$

where $k$ is the wavenumber (i.e., $2 \pi / \lambda$ where $\lambda$ is the wavelength) and $\varepsilon$ is the energy dissipation rate per unit volume.

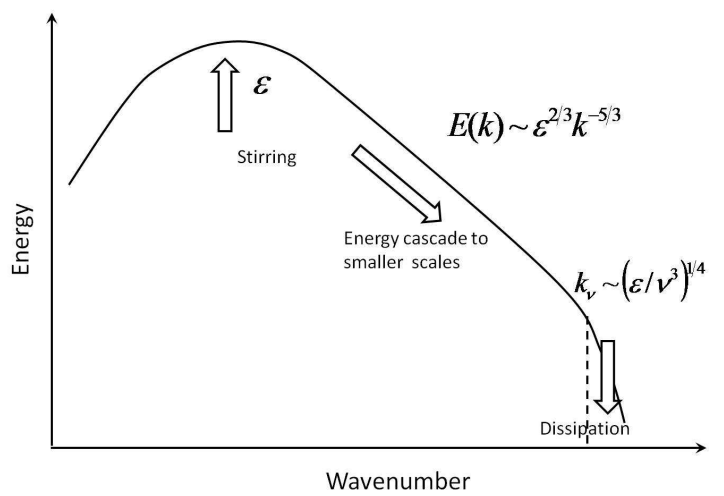

Figure 1: Energy spectrum of a three-dimensional, homogeneous and isotropic turbulent flow.

The length scale at which diffusion becomes important is called the Kolmogorov scale, $l_{\nu}$, defined as

$$
l_{\nu} \sim k_{\nu}^{-1} \sim\left(\nu^{3} / \varepsilon\right)^{1 / 4} .
$$

At scales smaller than $l_{\nu}$, the flow is laminar (but possibly unsteady). In the ocean, $l_{\nu}$ ranges between $6 \times 10^{-5} \mathrm{~m}$ and $0.01 \mathrm{~m}$. Since most phytoplankton cells are smaller than a few tens of microns in size, they do not experience a turbulent flow but are rather exposed to a viscous laminar flow.

The dominance of viscosity makes nutrient uptake by phytoplankton to be severely limited by molecular diffusion. For a plankton cell, nutrient delivery happens in two stages - nutrient delivery to the cell membrane and transport across the membrane. When the latter process is faster than the first one (as often happens), the nutrient concentration at the cell surface drops significantly below its far-field value. For a molecular diffusivity $D$ and a plankton cell of radius $a$, the concentration of nutrient in the proximity of the cell wall is governed by the equation

$$
\frac{\partial N}{\partial t}=D \nabla^{2} N
$$

whose spherically symmetric, steady state solution is

$$
N(r)=\frac{a}{r}\left(N_{0}-N_{\infty}\right)+N_{\infty}
$$

where $N_{0}$ is the concentration at the cell surface and $N_{\infty}$ is the far-field concentration. The diffusional flux to a spherical cell is then computed as

$$
Q_{D}=4 \pi D a\left(N_{\infty}-N_{0}\right)
$$


When the uptake capacity of the cell exceeds the rate of nutrient supply by diffusion, a nutrient-depleted layer is formed near the cell. The time taken by diffusion for transporting the nutrient across this layer can be estimated as

$$
T_{D}=\frac{a^{2}}{D}
$$

In water at standard upper ocean temperatures, for immotile cells with $a \leq 10 \mu m$ the nutrient limitation can be severe.

Given that diffusion is not sufficient to bring enough nutrients to the cell surface, especially for smaller organisms, phytoplankters have found ways to overcome diffusion limitation. Most of these strategies are based on the ability of phytoplankters to move along trajectories which are not fluid particle paths, thus allowing them to explore new regions of the fluid which are not nutrient depleted yet. The differences between cell trajectories and fluid parcel trajectories can be generated, for example, by

1. Swimming (as many flagellates do);

2. Sinking (as diatoms do);

3. Exploiting the fact that plankton cells have different density from the fluid and have finite size.

In the rest of this Lecture, we shall focus on the last mechanism and study how heavy impurities with finite size (our gross model for plankters) are advected by a fluid flow.

\section{Lagrangian advection}

The motion of an advected particle with the same density of the fluid and vanishingly small dimensions (i.e., a fluid parcel) is described by the standard Lagrangian equations

$$
\frac{d \mathbf{X}}{d t}=\mathbf{u}(\mathbf{X}, t)
$$

where $\mathbf{X}$ is the particle position at time $t$ and $\mathbf{u}(\mathbf{X}, t)$ is the fluid velocity at the particle position. Notice that equation (9) is non-Newtonian, in the sense that it does not obey the standard Newton's second law of motion. The reason is that the particle has no inertia with respect to the fluid, and thus we equate the Lagrangian particle velocity $d \mathbf{X} / d t$ to the Eulerian velocity $\mathbf{u}(\mathbf{X}, t)$.

Impurities with finite size and finite inertia do not behave like fluid particles. An impurity with density $\rho_{p}$ moving with velocity $\mathbf{V} \equiv d \mathbf{X} / d t$ in a flow field $\mathbf{u}$ of a fluid with density $\rho_{f}$ experiences forces due to gravity, $\mathbf{F}_{g}$, Stokes drag, $\mathbf{F}_{s}$, and the effect of the surrounding fluid (mainly pressure), $\mathbf{F}_{f}$, expressed as

$$
\mathbf{F}_{g}=-g\left(\rho_{p}-\rho_{f}\right) \hat{\mathbf{z}}, \quad \mathbf{F}_{s}=-\frac{1}{\frac{4}{3} \pi a^{3}} 6 \pi \rho_{f} \nu a(\mathbf{V}-\mathbf{u})=-\frac{9 \nu \rho_{f}}{2 a^{2}}(\mathbf{V}-\mathbf{u}), \quad \mathbf{F}_{f}=\rho_{f} \frac{D \mathbf{u}}{D t}
$$


where, as before, $a$ is the radius of the impurity.

Thus, using Newton's second law equating force to mass times acceleration, we can write for the motion of the impurity

$$
\rho_{p} \frac{d \mathbf{V}}{d t}=\mathbf{F}_{f}+\mathbf{F}_{s}+\mathbf{F}_{g}
$$

Rewriting equation (11) we have

$$
\frac{d \mathbf{V}}{d t}=\delta \frac{D \mathbf{u}}{D t}-\frac{1}{\tau_{a}}(\mathbf{V}-\mathbf{u})-(1-\delta) g \hat{\mathbf{z}}
$$

where $\delta=\rho_{f} / \rho_{p}$ and $\tau_{a}=2 a^{2} /(9 \delta \nu)$. The time scale $\tau_{a}$ measures the relaxation time of the particle velocity to the local fluid velocity and $\delta$ determines the nature of the buoyant particle $(\delta<1$ and $\delta>1$ indicate particles which are heavier or lighter than the fluid respectively).

In still fluid, where $\mathbf{u}=0$, one can define the (downward) terminal velocity of the heavy impurity as

$$
W=\frac{2}{9} g a^{2} \frac{\rho_{p}-\rho_{f}}{\rho_{f} \nu} .
$$

For example, an impurity with $\delta=0.97$ and $a=30 \mu \mathrm{m}$, as appropriate for a plankton cell, the terminal velocity $W$ is a few meters per day.

It is important to stress the difference between the two time derivatives present in equation (14). While $D / D t=\partial / \partial t+\mathbf{u} \cdot \nabla$ measures the rate of change along the path that a fluid particle would follow locally, $d / d t=\partial / \partial t+\mathbf{V} \cdot \nabla$ denotes the time derivative while tagging along the heavy impurity.

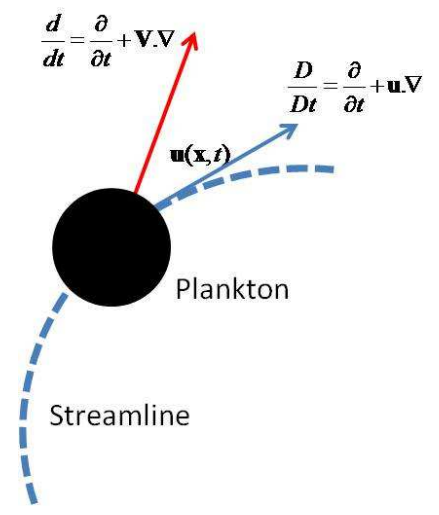

Figure 2: A graphical representation of the meanings of $d / d t$ and $D / D t$

Before proceeding further, we make eq.(12) non dimensional and write

$$
\frac{d \tilde{\mathbf{V}}}{d \tilde{t}}=\delta \frac{D \tilde{\mathbf{u}}}{D \tilde{t}}-\frac{1}{S t}(\tilde{\mathbf{V}}-\tilde{u})-(1-\delta) \frac{1}{F r^{2}} \hat{\mathbf{z}}
$$


where the tilde is used to denote dimensionless variables. In the non-dimensional equation, $S t=\tau_{a} U / L$ and $F r=U / \sqrt{g L}$ are the Stokes and Froude numbers respectively and $L$ and $U$ are typical length and velocity scales. The Stokes number $S t$ represents the ratio between the particle relaxation time scale, $\tau_{a}$, and the dynamical time scale $L / U$, whereas $\operatorname{Fr}$ quantifies the ratio of the buoyancy time scale, $1 / \sqrt{g / L}$, to the dynamical time scale. In the following we will drop the tilde and denote the non-dimensional variables by the same notation as the dimensional ones, unless a distinction needs to be made.

\section{Impurity advection in $2 \mathrm{D}$ stationary flows}

In this section we study the dynamics of an ensemble of passive impurities advected by a stationary, divergence-free, two-dimensional (2D) cellular velocity field without the effects of gravity (we can think of a flow field on a horizontal plane). This example allows for stressing the differences between the dynamics of fluid parcels and that of heavy and light impurities; note that in this simple case one can rescale the Stokes number by rescaling time.

We first consider the case of an isotropic streamfunction $\psi=2(\cos x+\cos y)$. The fluid velocity is $\mathbf{u}=(u, v)$ and the velocity components are given by $u=-\partial \psi / \partial y$ and $v=\partial \psi / \partial x$. In a 2D flow, fluid parcels obey the equations

$$
\frac{d X}{d t}=-\frac{\partial \psi}{\partial Y}, \frac{d Y}{d t}=\frac{\partial \psi}{\partial X}
$$

which is formally an Hamiltonian system with phase space $(x, y)$ where $x$ and $y$ are canonically conjugate variables and the stream function plays the role of the Hamiltonian. In this case, one has conservation of phase-space area and no preferential fluid particle concentration is possible. For a stationary flow, fluid parcels follow the streamlines; since the system is two-dimensional, there is no chaotic dynamics and the fluid parcels undergo only regular trajectories.

The situation is different for passive impurities, for which the phase space $\left(x, y, V_{x}, V_{y}\right)$ is four-dimensional and the dynamics is dissipative. In the case of particles lighter than the fluid, $\delta>1$, linear stability calculations show that the fixed points in $\left(x_{n}, y_{m}\right)=(2 n \pi, 2 m \pi)$ and $\left(x_{n}^{\prime}, y_{m}^{\prime}\right)=((2 n+1) \pi,(2 m+1) \pi)(n, m$ being integers) are stable and attracting, while the fixed points $\left(\tilde{x}_{n}, \tilde{y}_{m}\right)=(2 n \pi,(2 m+1) \pi)$ and $\left(\tilde{x}_{n}^{\prime}, \tilde{y}_{m}^{\prime}\right)=((2 n+1) \pi, 2 m \pi)$ are saddles. For heavy particles $(\delta<1)$, all these fixed points are unstable. In this case, the impurities undergo chaotic trajectories, hopping from one flow cell to another, and display non-turbulent diffusion, even in the case of a stationary velocity field [3].

Figure 4 shows how an initially homogeneous distribution of heavy and light impurities evolves in the prescribed flow. The short-time dynamics is very similar for both light and heavy particles. An interesting feature is the transformation of an initially uniform particle distribution into a distribution characterized by sharp-edged caustics; note that caustics cannot form for fluid particles, owing to the conservation of phase-plane area associated with the Hamiltonian nature of the system. This constraint does not act for impurites, 


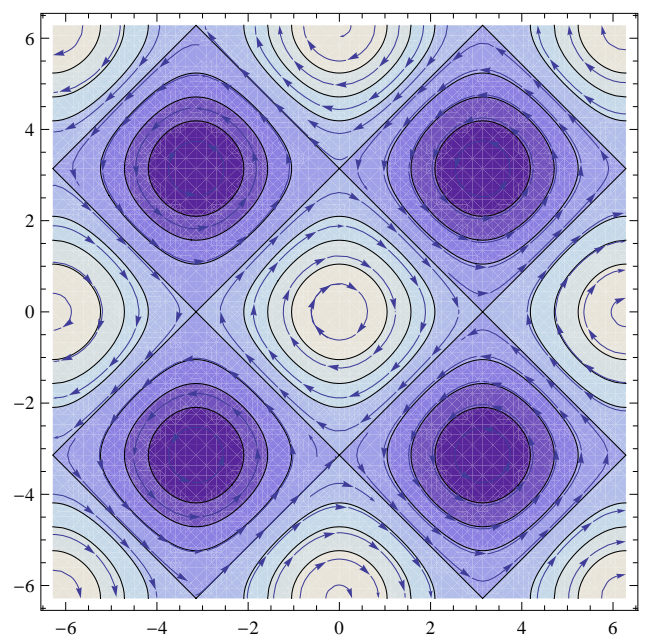

Figure 3: The stationary two-dimensional streamfunction $\psi=2(\cos x+\cos y)$ and the associated velocity field.

owing to the dissipative nature of the equations describing impurity motion. For light particles, the caustics spiral around the stable fixed points. Hence, for a stationary two dimensional flows, light particles tend to concentrate at the stationary points of the flow; for large enough times the particles are practically absent in the remaining regions of the flow. On the contrary, heavy particles exhibit a very different behavior: After the initial formation of caustics, the heavy particles undergo diffusion along the separatrices of the advecting flow, owing to the chaotic nature of the heavy particle dynamics for the parameter values adopted here.

Figure 5(a) shows the trajectory of an individual heavy impurity in the cellular flow field. The particle alternates between spiralling inward around one of the (now unstable) fixed points and escaping along one of the unstable directions, with a quasiballistic motion. Although this alternance of trapping and quasiballistic motion could be reminiscent of a Lèvy flight, for the system under study the long-time probability distribution of the particle velocity is Gaussian, and it is consistent with a standard Brownian diffusion process.

We can compute the diffusion coefficient for an ensemble of $N$ advected impurities as a function of $\epsilon=1-\delta$,

$$
\begin{gathered}
D_{x}=\lim _{t \rightarrow \infty} \frac{1}{2 t N} \sum_{i=1}^{N}\left[x_{i}(t)-x_{i}(0)\right]^{2} \\
D_{y}=\lim _{t \rightarrow \infty} \frac{1}{2 t N} \sum_{i=1}^{N}\left[y_{i}(t)-y_{i}(0)\right]^{2} .
\end{gathered}
$$

Figure 5(b) shows the dependence of $D_{x}, D_{y}$ on $\epsilon$. Since the flow is isotropic, the two diffusion coefficients are equal. In general, no anomalous (non-Brownian) diffusion has been observed for this system. 
In the case of a weakly anisotropic velocity field, one observes some new effects. In the following, we consider the flow described by the streamfunction $\psi=A \cos x+B \cos y$, where $A \neq B$. If one looks at the streamfunction (figure 6), it is evident that there are open "channels" (with our choice of parameters, in the horizontal direction) where the fluid particles undergo ballistic motion. These are analogous to the libration orbits in the phase space of a nonlinear pendulum (the closed orbits would correspond to periodic oscillations). Thus, fluid particles are either trapped inside the cellular regions or undergo ondulating ballistic motion in the channels.

A different story is observed for advected impurities. As for the isotropic flow, light particles sooner or later get trapped at the stagnation points. On the other hand, heavy impurities spend a long time in quasiballistic motion in the channels, and temporary trapping in the cellular regions is followed by jumping off to another channel. Figure 7 shows that an intially uniform particle distribution tends to become spread along the channels, with strong anisotropy.

In this flow, the diffusion coefficients along the $x$ and $y$ directions are very different. Figure 8(b) reveals that $D_{x}$, the diffusion coefficient along the channels, diverges with $\epsilon \rightarrow 0$, $D_{x} \sim \epsilon^{-0.6}$ while $D_{y} \sim \epsilon^{1.5}$. Thus a small anisotropy in the Eulerian flow $(A=2, B=2.2)$ leads to diffusion coefficients that differ by several orders in magnitude in the case of particles which are only slightly heavier than the fluid $(\delta=0.9)$. This could have interesting consequences for the motion of plankton cells in weakly anisotropic flows, as the small density difference between the plankton cells and the water could result in a strongly anisotropic diffusion of the particles.

\section{Neutrally buoyant impurities with finite size}

Often, advected impurities have a density that is rather close to that of the fluid (as happens, for example, with most plankton cells), but have a finite size, i.e., they are not fluid elements. To explore the dynamics of these particles, we consider the case of neutrally buoyant particles with $\delta=\rho_{f} / \rho_{p}=1$ and finite, albeit small, radius $a$. The equation for particle motion in this case becomes:

$$
\frac{d \mathbf{V}}{d t}=\frac{D \mathbf{u}}{D t}-\frac{1}{S t}(\mathbf{V}-\mathbf{u})
$$

where now

$$
S t=\frac{2}{9}\left(\frac{a}{L}\right)^{2} R e, \quad R e=\frac{U L}{\nu} .
$$

If we make the approximation that the time derivative along the trajectory of an impurity and that along the trajectory of a fluid parcel are the same, i.e. $d / d t=D / D t$, the problem 
becomes very simple. In this case, equation (18) can be written as

$$
\frac{d}{d t}(\mathbf{V}-\mathbf{u})=-\frac{1}{S t}(\mathbf{V}-\mathbf{u})
$$

Hence

$$
\mathbf{V}=\mathbf{u}+(\mathbf{V}-\mathbf{u})_{0} \exp \left(-\frac{1}{S t} t\right)
$$

where $(\mathbf{V}-\mathbf{u})_{0}$ denotes the initial value of $(\mathbf{V}-\mathbf{u})$. From this we could infer that even if we release a particle with an initial velocity which differs from that of the fluid, after a transient the particle velocity will tend to the fluid velocity, implying that a neutrally buoyant particle will behave, after an initial transient, as an ideal tracer.

In reality, the above conclusion is not correct, as the problem is complicated by the fact that $d / d t$ and $D / D t$ are not the same. Equation (18) should then be written as

$$
\frac{d}{d t}(\mathbf{V}-\mathbf{u})=-(\mathbf{V}-\mathbf{u}) \cdot \nabla \mathbf{u}-\frac{1}{S t}(\mathbf{V}-\mathbf{u}) .
$$

Defining $\mathbf{M} \equiv \mathbf{V}-\mathbf{u}$, equation (19) can be rewritten as

$$
\begin{aligned}
\frac{d \mathbf{M}}{d t} & =-\mathbf{M} \cdot \nabla \mathbf{u}-\frac{1}{S t} \mathbf{M} \\
& =-\left(\mathbf{J}+\frac{1}{S t} \mathbf{I}\right) \cdot \mathbf{M},
\end{aligned}
$$

where $\mathbf{I}$ is the identity matrix and $\mathbf{J}$ is the velocity Jacobian,

$$
\mathbf{J}=\left(\begin{array}{cc}
\partial_{x} u & \partial_{y} u \\
\partial_{x} v & \partial_{y} v
\end{array}\right), \quad \mathbf{u}(\mathbf{x})=\left(\begin{array}{c}
u(\mathbf{x}) \\
v(\mathbf{x})
\end{array}\right)
$$

If we diagonalise the matrix we obtain

$$
\frac{d \mathbf{M}_{d}}{d t}=\left(\begin{array}{cc}
\lambda-\frac{1}{S t} & 0 \\
0 & -\lambda-\frac{1}{S t}
\end{array}\right) \cdot \mathbf{M}_{d} .
$$

From this expression, one can obtain relevant information on the behavior of the advected impurities. For a two-dimensional stationary flow, the difference between the fluid velocity and the particle velocity evolves in time depending on the value of $\pm \lambda-1 / S t$. In particular, the particle velocity can diverge from the fluid velocity when

$$
\lambda^{2}>\left(\frac{1}{S t}\right)^{2}
$$

from which one sees that the stability of the particle trajectories (with respect to the fluid parcel trajectories) is determined by the balance between the eigenvalues of the fluid velocity field and the Stokes number. When the local value of $\lambda^{2}$ (a property of the Eulerian advecting flow) is sufficiently large, the neutrally buoyant impurity can follow a trajectory that is different from that of a fluid parcel placed at that point. 
We can get a further advancement by resorting to the Okubo-Weiss parameter $[7,11]$

$$
Q \equiv-\operatorname{det} \mathbf{J}=\frac{1}{4}\left(S^{2}-\omega^{2}\right)
$$

where $S^{2}=\left(\partial_{x} u-\partial_{y} v\right)^{2}+\left(\partial_{y} u+\partial_{x} v\right)^{2}$ is the total strain (deformation) and $\omega=\partial v / \partial x-$ $\partial u / \partial y$ is the vorticity. This parameter characterises the local shear/rotational properties of the flow and separates the fluid domain into elliptic and hyperbolic regions $[7,11,4]$ :

$$
Q \begin{cases}<0 & \text { rotation - dominated } \\ >0 & \text { shear - dominated }\end{cases}
$$

Since $\lambda$ satisfies $\operatorname{det}(\mathbf{J}-\lambda \mathbf{I})=0$, one has that

$$
\lambda^{2}-\operatorname{Tr} \mathbf{J}+\operatorname{det} \mathbf{J}=0 .
$$

In addition, as we are now considering an incompressible flow,

$$
\nabla \cdot \mathbf{u}=\partial_{x} u+\partial_{y} v=\operatorname{Tr} \mathbf{J}=0
$$

From (22) and (23), the Okubo-Weiss parameter is then $Q=\lambda^{2}$. If $Q>0, \lambda$ is real and deformation dominates, as around hyperbolic points, whereas if $Q<0, \lambda$ is imaginary and rotation dominates, as in the proximity of elliptic points. Thus, we see that hyperbolic regions can lead to a divergence between the fluid particle paths and the trajectories of the impurities, if they are "hyperbolic enough" (i.e., the value of $\lambda^{2}$ is large enough). As a result, neutrally buoyant impurities will be ejected from hyperbolic regions and will wander around till they end up in elliptic regions, were they will follow the same trajectory of a fluid parcel. This potentially leads to a preferential concentration of neutrally buoyant impurities in elliptic regions, with a biassed sampling of the advecting flow.

\subsection{Example 1: Time independent flow}

To illustrate the effects of $S t$ and $Q$ on the dynamics of a neutrally buoyant particle, we consider the simple incompressible two-dimensional model flow defined by the stream function $\psi(x, y)=A \cos x \cos y[1]$.

Figure 9(a) shows the contour lines of the stream function and of the Okubo-Weiss parameter $Q$. Figure 9 (b) shows the trajectory of a neutrally buoyant particle whose initial velocity is different from that of the fluid at the impurity position. The difference between the velocity of the impurity and the fluid velocity is amplified in highly hyperbolic regions, where impurity trajectories and fluid parcel trajectories separate exponentially. Fig.9(c) shows that the neutrally buoyant impurity has a long and complicated trajectory, wandering between different cells before it comes across the vicinity of an elliptic point and settles in one of the low- $Q$ regions. The time evolution of the difference between the impurity velocity and the fluid velocity at the impurity position is shown in Fig.9(d). Once the impurity has settled in an ellpitic region, the velocity difference between the fluid and the impurity tends to zero an no further instability is observed. However, in their wanderings impurities leave hyperbolic regions, leading to a preferential concentration of neutrally buoyant impurities in the elliptic regions of the flow. 


\subsection{Example 2: Time periodic flow}

The time-dependent flow defined by the stream function $\psi(x, y)=A \cos (x+B \sin \omega t) \cos y$ shows interesting features [1]. As in a typical non-stationary, two-dimensional non-integrable Hamiltonian system, there are regions of phase space where fluid parcels undergo chaotic motion. Figure 10(a) shows the Poincaré section of the chaotic trajectory of a fluid particle, here obtained by plotting the parcel position at times $t=0, T, 2 T, \ldots$ where $T=2 \pi / \omega$. The trajectory visits a large part of the domain but it is bounded by two separatrices parallel to the $x$ direction, which forbid fluid particle motion across them (remember that the temporal variability is only in the velocity component along $x$ ).

The trajectory of a neutrally buoyant impurity, shown in Fig.10(b), indicates that the impurity can wander on a larger portion of the domain, but in the end it converges to a non-chaotic trajectory around an elliptic point. From that moment, the impurity behaves as a fluid particle. However, the impurity has selected a specific sub-domain in space without ever coming back close to its initial conditions, at variance with what would have happened for a Hamiltonian system. The equations of motion for a neutrally buoyant impurity are dissipative, and there is no conservation of phase-space volume. For completeness, Fig.10(c) shows the difference between the velocity of the impurity and that of the fluid, as a function of time.

\subsection{An application to two-dimensional turbulence}

The results discussed above have interesting implications for two-dimensional turbulent flows $[1,9]$. We integrate the two-dimensional vorticity equation for an incompressible fluid with bi-harmonic (hyper)viscosity,

$$
\frac{\partial \omega}{\partial t}+J(\psi, \omega)=-\nu_{h} \nabla^{4} \omega
$$

where $\psi$ is the stream function, $\omega=\nabla^{2} \psi$ is the vorticity, $u=-\partial_{y} \psi$ and $v=\partial_{x} \psi$ are the velocity components and the hyperviscosity parameter is $\nu_{h}=2.5 \times 10^{-7}$. To integrate the equation, we use a pseudo-spectral method with $512^{2}$ collocation points in a doubly periodic domain; here we consider a freely-decaying case with no external forcing. In this flow, an initially homogeneous distribution of neutrally buoyant impurities evolves in time towards a distribution where the impurities are concentrated in the inner part of vortices, where $Q<0$, as shown in Fig.11. This may have interesting consequences when we design fluid experiments using neutrally buoyant tracer particles, as one should verify that the tracers really provide an unbiassed sampling of the velocity field.

\section{The role of rotation}

The presence of a rotation introduces new effects in the dynamics of the heavy impurities [9]. When the (apparent) Coriolis and centrifugal forces are acting on an impurity, the dimensional equation for the impurity motion becomes

$$
\frac{d \mathbf{V}}{d t}=\delta \frac{D \mathbf{u}}{D t}-\frac{1}{\tau_{a}}(\mathbf{V}-\mathbf{u})-(1-\delta) g \hat{\mathbf{z}}-2 \boldsymbol{\Omega} \times(\mathbf{V}-\delta \mathbf{u})+|\boldsymbol{\Omega}|^{2} \mathbf{R}(1-\delta),
$$


where $\delta$ and $\tau_{a}$ are defined as before and $\mathbf{R}$ is the distance of the particle from the axis of rotation. Notice that the term $-2 \delta \boldsymbol{\Omega} \times \mathbf{u}$ had to be subtracted from the Coriolis term acting on the impurity because it was already taken into account in the expression $\delta D \mathbf{u} / D t$. Thus, the Coriolis term acts on the density-weighted difference between the velocity of the impurity and that of the fluid. Non-dimensionalising the above equation, we obtain

$$
\frac{d \mathbf{V}}{d t}=\delta \frac{D \mathbf{u}}{D t}-\frac{1}{S t}(\mathbf{V}-\mathbf{u})-(1-\delta) \tilde{g} \hat{\mathbf{z}}-\frac{1}{R_{o}} \hat{\mathbf{z}} \times(\mathbf{V}-\delta \mathbf{u})+\frac{1}{4 R_{o}} \frac{\mathbf{R}}{L}(1-\delta),
$$

where $S t$ is defined as before and

$$
R o=\frac{U}{2 \Omega L}
$$

is the Rossby number. Fig.12 shows a distribution of 8000 heavy impurities which were initially homogeneously distributed in a statistically stationary numerical simulation of forced and dissipated 2D turbulence [9]. The numerical integration has been performed in the doubly periodic domain $[0,2 \pi] \times[0,2 \pi]$ with $128 \times 128$ collocation points. The particles have $\delta=0.8, S t=0.01$ and $R o=1 / 320$. In the integration, we have discarded the effect of the centrifugal force. The heavy impurities concentrate inside the centers of the anticyclonic vortices, leaving both the turbulent background and the cores of cyclonic vortices. This is due to the fact that the Coliolis force adds up to the inertial force to eject heavy impurities from cyclones, whereas it works against the inertia to push impurities towards the center of anticyclones. When $R o$ is small enough, the Coriolis force overcomes the centrifugal effect of inertia and the impurities concentrate in the cores of anticyclonic vortices. When the vortices are especially intense, they generate a strong centrifugal force due to the particle inertia. The strength of the centrifugal push by the vortex becomes weaker when the distance from the vortex center increases. In this case, the Coriolis force balances inertia at some distance from the vortex center and the impurities concentrate in an annular distribution inside the vortex.

As a final example, we momentarily leave the world of plankton and discuss the (again idealized!) motion of dust grains in the solar nebula [2]. In this case, $\delta \sim 10^{-8}$ so that we may may discard the term $\delta D \mathbf{V} / D t$. The impurity equation is then reduced to

$$
\frac{d \mathbf{V}}{d t}=-\frac{1}{\tau_{a}}(\mathbf{V}-\mathbf{u})-\nabla \Phi-2 \boldsymbol{\Omega} \times \mathbf{V}+|\boldsymbol{\Omega}|^{2} \mathbf{R}
$$

where $\Phi$ is a gravitational potential. In the specific case of particles spinning around the protosun in the solar nebula (a rarefied fluid composed mainly by hydrogen) the equation of motion of a dust particle becomes

$$
\frac{d \mathbf{V}}{d t}=-\frac{1}{\tau_{E}}(\mathbf{V}-\mathbf{u})-2 \boldsymbol{\Omega} \times \mathbf{V}-\frac{G M}{R^{2}} \hat{\mathbf{R}}+|\boldsymbol{\Omega}|^{2} \mathbf{R} .
$$

Here, $G$ and $M$ are the gravitational constant and the mass of the protosun, respectively. For the rarefied conditions of the protoplanetary nebula, the friction time scale $\tau_{E}$ is set to have the form $\tau_{E} \propto a / \rho_{f}$, where $a$ is the radius of the particle. This expression of the drag 
coefficient corresponds to what is called the Epstein regime for a rarefied gas.

To proceed with the study, we consider a simplified representation of the protosolar nebula by using a two-dimensional turbulent flow subject to a circularly-symmetric Keplerian shear, physically generated by the presence of a central massive object (the protosun) [2] Figure 13(a) shows the vorticity field at sufficiently large time, obtained by numerical simulation of this system. By perturbing the initial Keplerian shear (which is linearly stable in the absence of magnetic effects), both cyclonic and anticyclonic vortical perturbations tend to form. However, cyclonic vortices are rapidly destroyed by the adverse shear - or do not form at all - and only anticyclonic vortices survive in the Keplerian shear, as illustrated in Figure13(a). As already seen in the case of standard two-dimensional turbulence discussed above, the heavy impurities tend to concentrate inside the anticyclonic vortices as time progresses. Fig.13(b) shows the distribution of an ensemble of heavy impurities, initially released with a uniform distribution, in the flow field of Fig.13(a). From these results, one could speculate that dust grains in the solar nebula could be concentrated by the dynamics in the cores of anticyclonic vortices, where the subsequent evolution (collisions, gravitational attraction) could more easily ensue.

\section{Conclusion}

The dynamics of passively advected impurities is quite different from that of fluid parcels. From a mathematical point of view, the phase space of the impurity motion has twice the number of dimensions of that of the fluid parcels, since it is described by particle positions and velocities instead of just particle positions, as in the case of fluid elements. In addition, the presence of Stokes drag makes the system dissipative, with the possibility of non-homogeneous particle distributions in space and preferential particle concentrations in specific regions of the flow. Physically, this means that advected impurities may provide a non-homogeneous sampling of the fluid domain. Even neutrally buoyant impurities, when their size is large enough, can behave very differently from fluid parcels. All these results indicate that small objects immersed in a fluid - such as plankton cells in water or dust grains in the solar nebula - can undergo complicated dynamics which cannot be understood only from considering the motion of fluid particles.

Before closing this lecture, it is important to recall that so far we have simplified the description, neglecting for example the effect of unsteady forces associated with the acceleration of the impurities, such as the added mass term [6] and the Basset force [10]. These two terms become important especially when $\delta=\rho_{f} / \rho_{p} \sim 1$. Full consideration of the Basset term is especially important, but difficult from a numerical point of view owing to the need of storing the past trajectories of a large number of particles. Further complications arise from considering non-spherical particles, a fact which induces new effects, see for example [8] for experimental results on the dynamics of sinking particles with plankton-like shapes and [5] for a theoretical study of the dynamics of elongated impurities. 


\section{References}

[1] Babiano, A., J. H. E. Cartwright, O. Piro, and A. Provenzale, "Dynamics of a Small Neutrally Buoyant Sphere in a Fluid and Targeting in Hamiltonian Systems", Phys. Rev. Lett., 84, 5764-5767 (2000).

[2] Bracco, A., P. H. Chavanis, A. Provenzale, and E. A. Spiegel, "Particle aggregation in a turbulent Keplerian flow", Phys. Fluids, 11, 2280-2287 (1999).

[3] Crisanti, A., M. Falcioni, A. Provenzale, P. Tanga and A. Vulpiani, "Dynamics of passively advected impurities in simple two-dimensional flow models", Phys. Fluids A, 4, 1805-1820 (1992).

[4] Koh, T-Y. and B. Legras, "Hyperbolic lines and the stratospheric polar vortex", Chaos, 12, 382-394 (2002).

[5] Mallier, R. and M.R. Maxey, "The settling of nonspherical particles in a cellular flow field", Phys. Fluids A, 3, 1481-1494 (1991).

[6] Maxey, M. R. and J. J. Riley, "Equation of motion for a small rigid sphere in nonuniform flow", Phys. Fluids, 26, 883-889 (1983).

[7] Okubo, A. "Horizontal dispersion of floatable particles in the vicinity of velocity singularities such as convergences", Deep-Sea Res., 17, 445-454 (1970).

[8] Padisák, J., E. Soróczki-Pintér and Z. Rezner, "Sinking properties of some phytoplankton shapes and the relation of form resistance to morphological diversity of plankton an experimental study", Hydrobiologia, 500, 243-257 (2003).

[9] Provenzale, A., "Transport by coherent barotropic vortices", Annu. Rev. Fluid Mech., 31, 55-93, (1999).

[10] Reeks, M. W. and S. Mackee, "The dispersive effects of Basset history forces on particle motion in a turbulent flow",Phys. Fluids, 27, 1573-1582 (1984).

[11] Weiss, J. "The dynamics of enstrophy transfer in two-dimensional hydrodynamics", Physica D, 48, 273-294 (1991). 

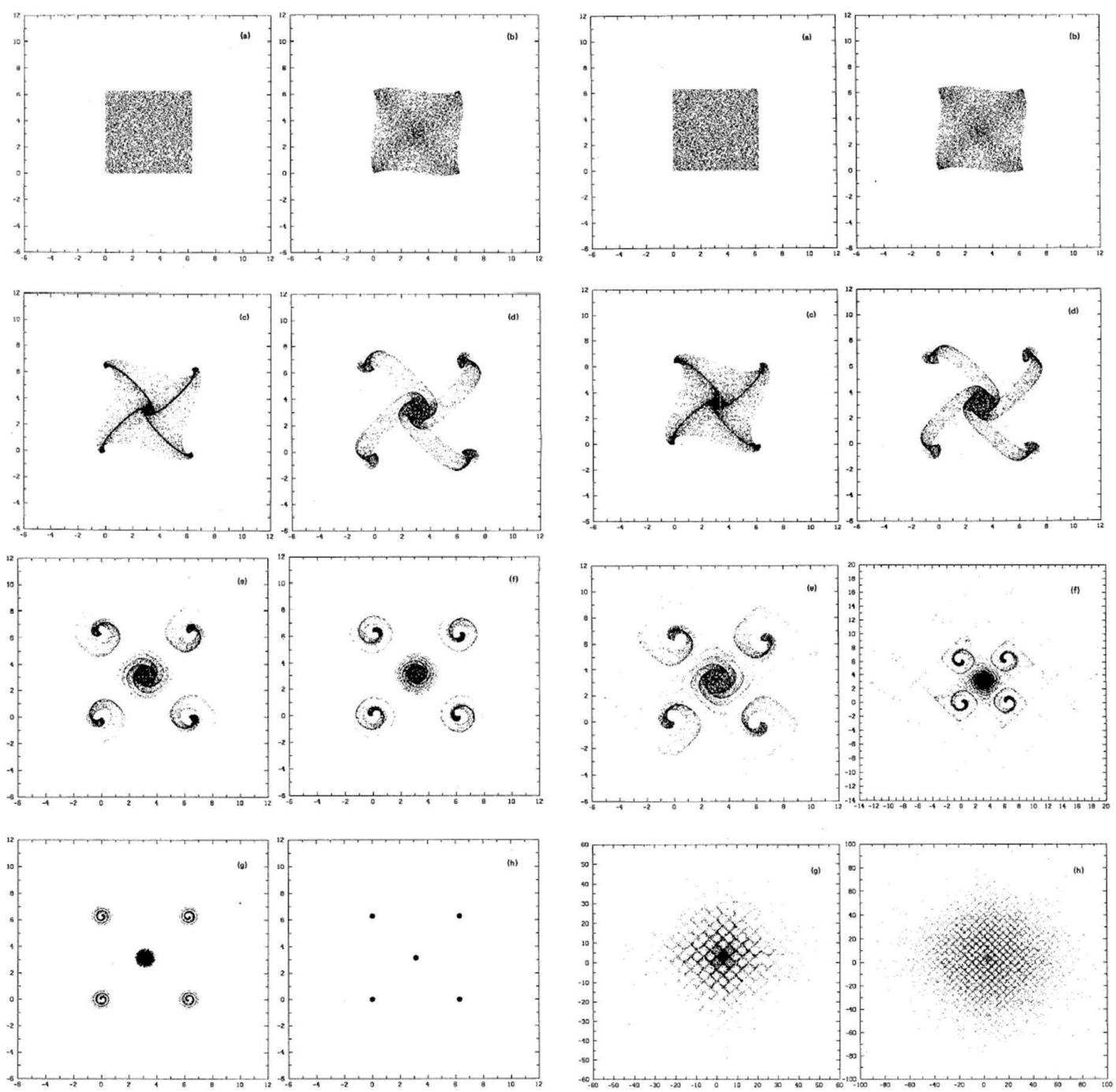

(a) $\delta=1.1$

(b) $\delta=0.9$

Figure 4: Distribution of 10,000 impurities with density ratio $\delta=1.1$ (left panels) and $\delta=0.9$ (right panels) and $S t=1$ in the advecting flow defined by the stream function $\psi=$ $2(\cos x+\cos y)$. The individual panels refer to different times, namely $t=0,0.5,1,2,5,10,40$ and 100 non-dimensional time units. 


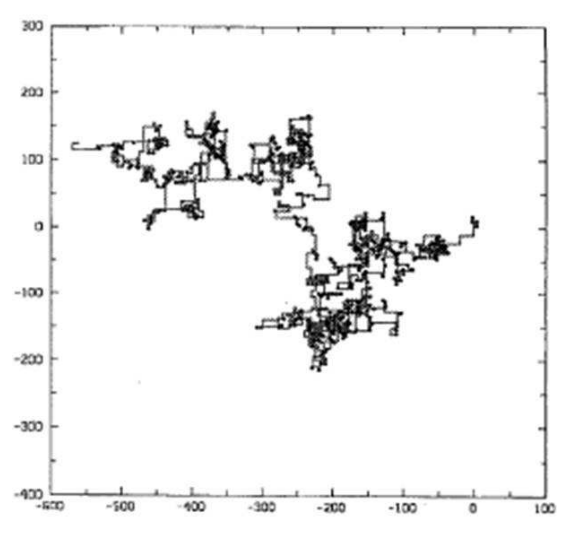

(a)

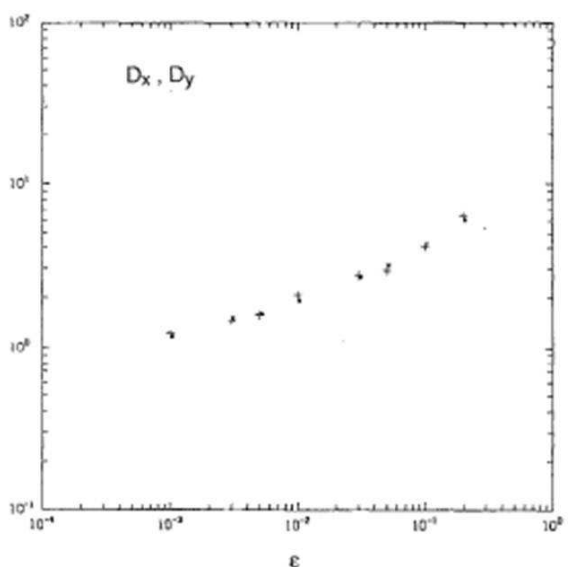

(b)

Figure 5: (a) Trajectory of an individual advected impurity with density ratio $\delta=0.9$ and $S t=1$ in the flow defined by $\psi=2(\cos x+\cos y)$, from $t=0$ to $t=10000$ non-dimensional time units. (b) Diffusion coefficients along $x$ and $y, D_{x}$ (stars) and $D_{y}$ (crosses), for an ensemble of heavy impurities as a function of $\epsilon=1-\delta$.

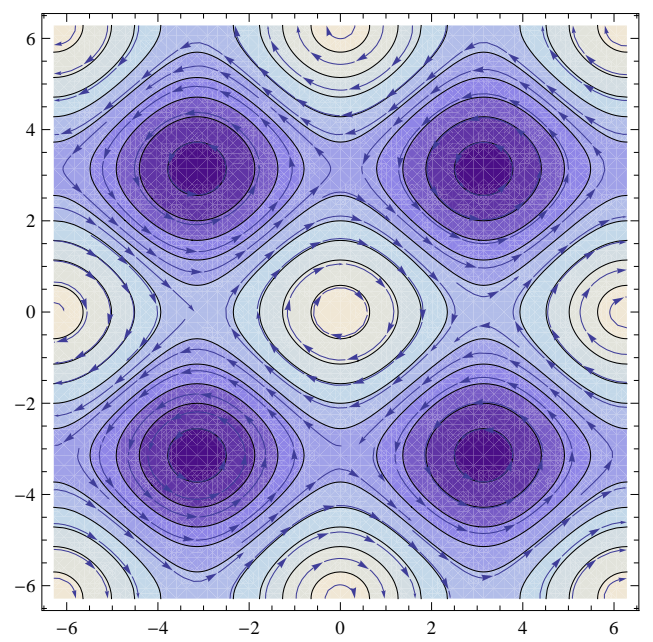

Figure 6: Streamfunction and the corresponding velocity field for the anisotropic cellular flow described by $\psi=(A \cos x+B \cos y)$ with $A=2$ and $B=2.4$. 


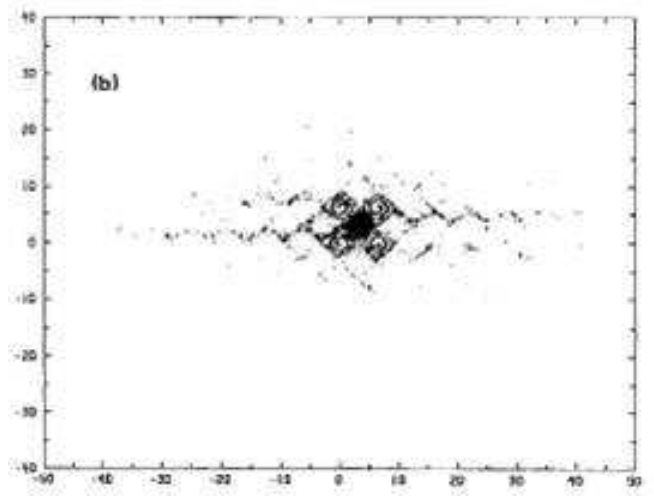

Figure 7: Distribution of 10,000 impurities of density ratio $\delta=0.9$ and $S t=1$ in the Eulerian flow defined by the anisotropic stream function $\psi=(A \cos x+B \cos y) . A=2$, $B=2.4$.

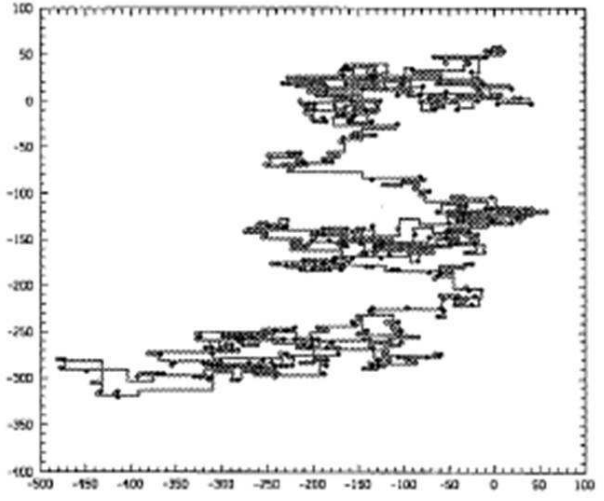

(a)

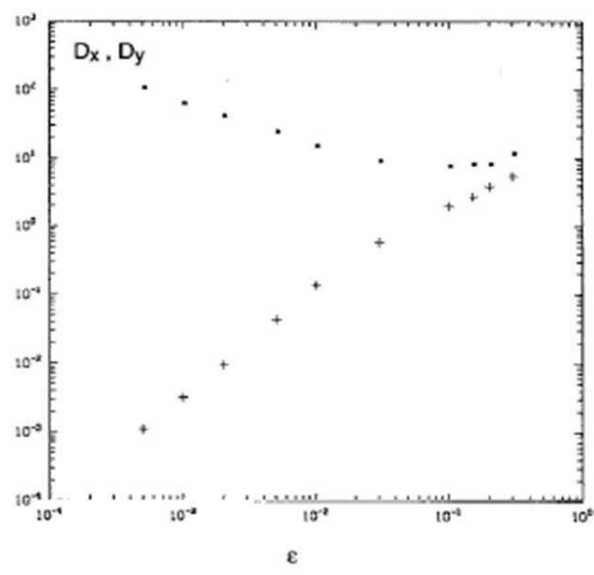

(b)

Figure 8: (a) Trajectory of an individual advected impurity with density ratio $\delta=0.9$ and $S t=1$ in the Eulerian flow defined by the anisotropic stream function $\psi=(A \cos x+B \cos y)$ with $A=2$ and $B=2.4$, from $t=0$ to $t=10000$ natural time units. (b) Diffusion coefficients, $D_{x}$ (stars) and $D_{y}$ (crosses), for heavy impurities as a function of $\epsilon=1-\delta$, for $A=2$ and $B=2.2$. 

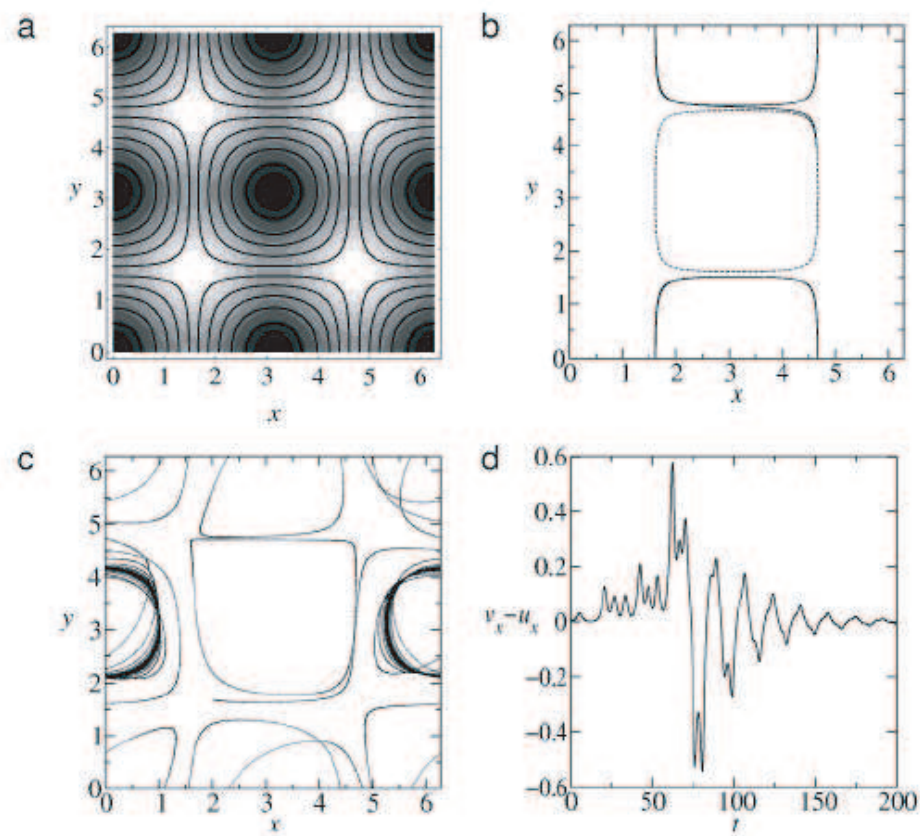

Figure 9: (a) Fluid parcel trajectories (thick lines) and magnitude of the Okubo-Weiss parameter $Q$ (background shading, lighter shading correspond to positive values of $Q$ ), for the stream function $\psi=A \cos x \cos y$. (b) Trajectory of a neutrally buoyant impurity with Stokes number $S t=0.2$ (solid line) and path of a fluid particle starting at the same initial position of the impurity (dashed line). The instability in regions of high $Q$ allows the impurity to wander between cells, diverging from the streamlines. (c) After a complicated excursion, the neutrally buoyant impurity eventually settles in a zone of low $Q$. (d) Difference between the velocity of the impurity and the fluid velocity at the instantaneous impurity position. 

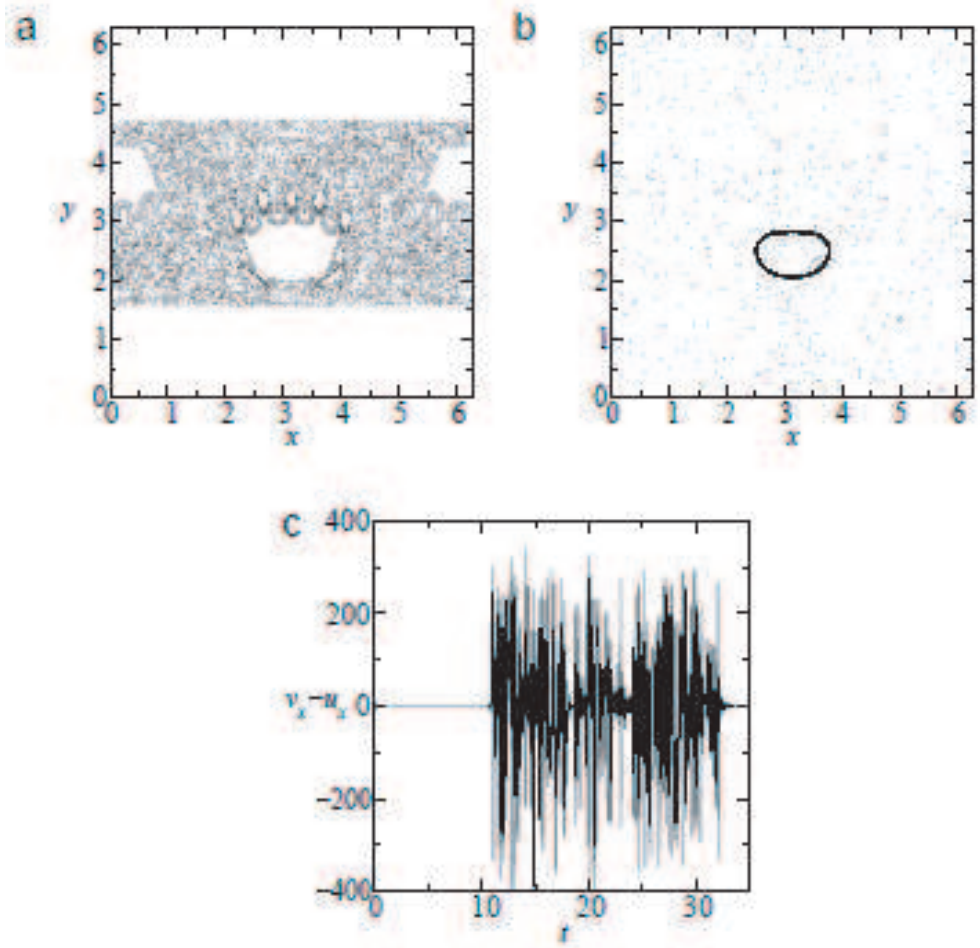

Figure 10: The upper panels show the Poincaré sections of a fluid parcel trajectory (a) and of a neutrally buoyant impurity with $S t=0.2$ (b) in a time-dependent cellular flow with $A=250, B=0.3$, and $\omega=1.0$. Panel (c) shows the time series of the difference between the velocity of the impurity and the fluid velocity at the impurity position.
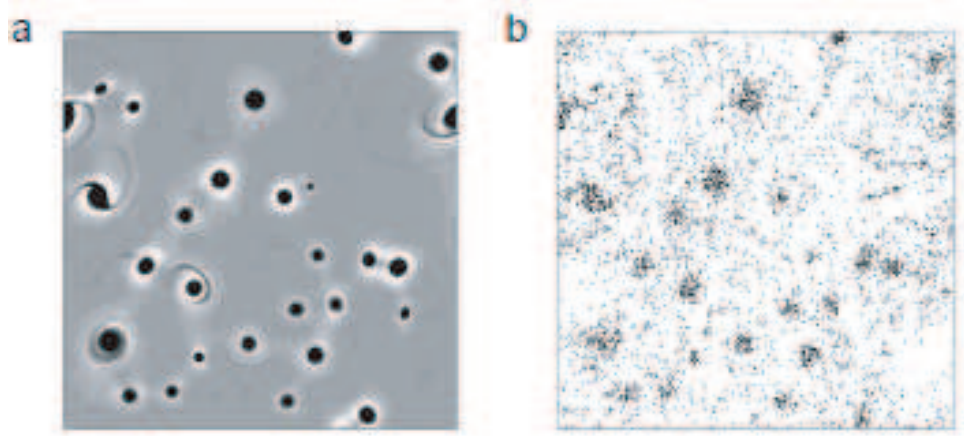

Figure 11: Small neutrally buoyant impurities with Stokes number $S t=0.2$ tend to concentrate in the centers of vortices in a two-dimensional turbulent flow. Panel (a) shows the distribution of the Okubo-Weiss parameter $Q$ at time $t=1$ non-dimensional units (lighter shading corresponds to larger $Q$, that is, to hyperbolic regions). Panel (b) shows the distribution of neutrally buoyant impurities which were initially uniformly distributed in the flow domain. 


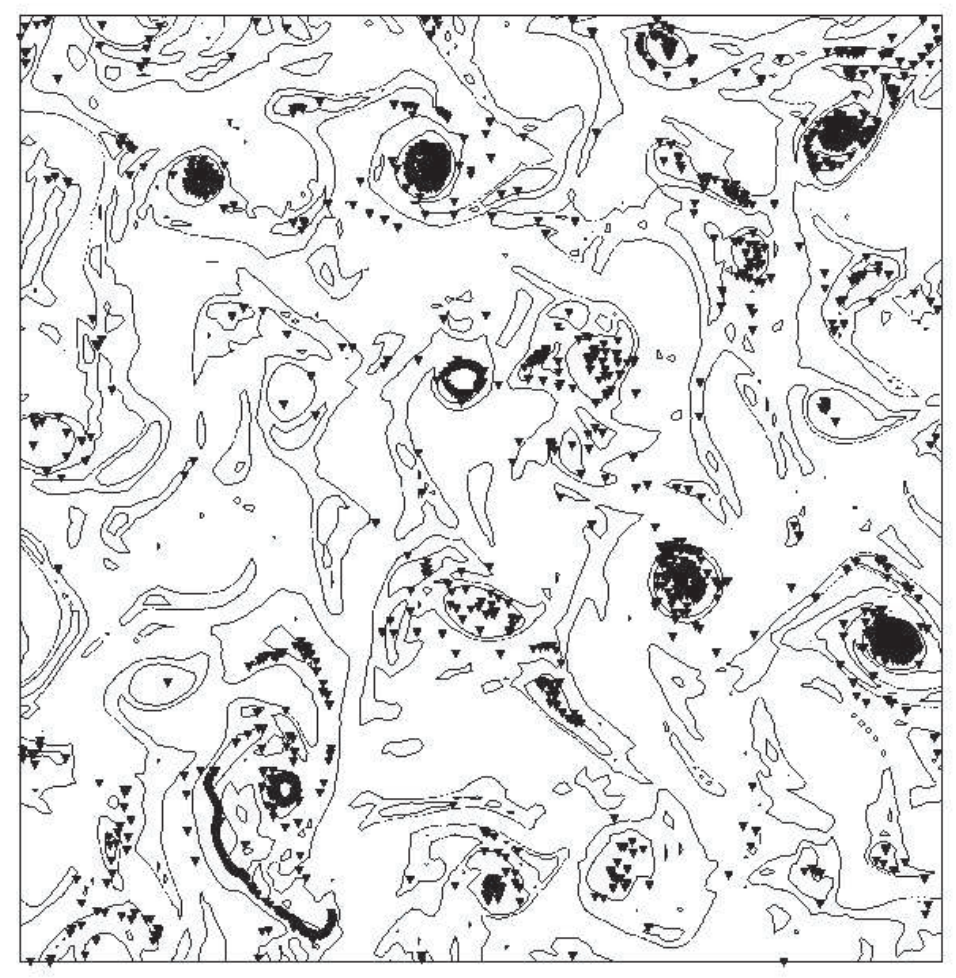

Figure 12: Distribution at $t=6$ non-dimensional time units of 8000 heavy impurities advected by forced, statistically stationary two-dimensional turbulence in a cyclonically rotating reference frame. Small solid triangles indicate the positions of the advected particles and thin solid curves indicate vorticity isolines. The impurities concentrate in the cores of anticyclonic vortices. Parameter values are $\delta=0.8, S t=0.01$, and $R o=1 / 320$.

(a)

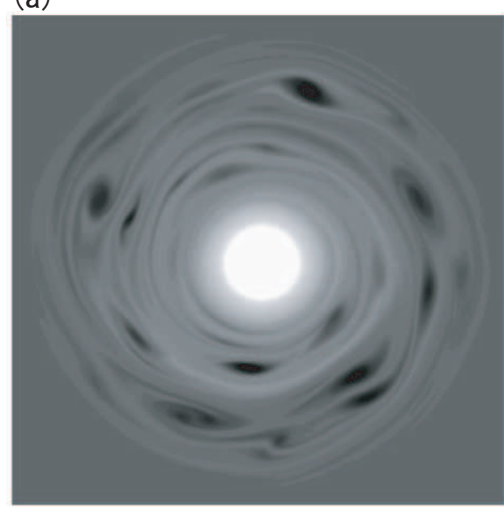

(b)

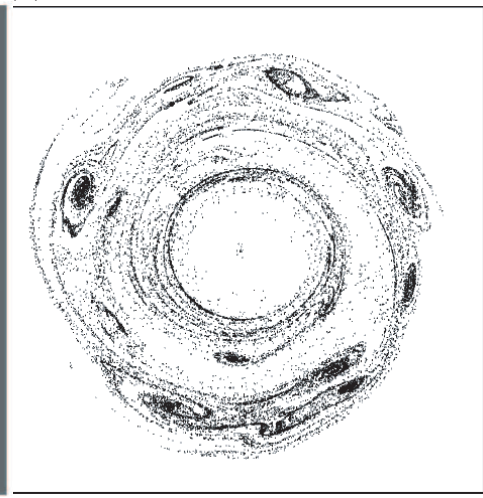

Figure 13: Panel (a) shows the vorticity field at $t=15$ non-dimensional time units for our idealization of the flow in a protosolar nebula. Panel (b) shows the distribution of heavy impurities at the same time. 


\title{
Plankton sinking and turbulence
}

\author{
Lecture given on 1 July 2010 by A. Provenzale
}

\section{Introduction}

In still fluid, heavy particles tend to fall and reach, after some time, a terminal velocity determined by the balance between the pull of gravity and the viscosity of the fluid. Often, plankton cells are slightly heavier than sea water, and as a consequence they tend to sink in the water column. This sinking could then take them below the euphotic layer, arresting photosynthesis.

To overcome sinking, plankton have devised various types of strategies. One is swimming, that is, an active, energy-consuming self-propulsion than can oppose gravitational sinking. Other phytoplankters can modify their buoyancy, becoming, at least for some time, positively buoyant. Others can exploit the turbulence in the mixed layer, using it to stay suspended for longer times. This latter option was discussed at length by Margalef [5], who associated the level of turbulence in the surface layer with the type of organisms that are environmentally favoured. The terminal velocity of spherical impurities with the same density grows with the squared radius of the falling impurity. Thus, larger plankton cells tend to sink faster than smaller ones, provided they can be approximated by a somewhat spherical shape. When the turbulence in the euphotic layer is low, Margalef argued, smaller, possibly swimming phytoplankters such as flagellates are favoured as the larger species sink more rapidly out of the euphotic zone. On the other hand, when the level of turbulence is high, larger plankton can benefit from turbulent suspension and become favoured with respect to the small ones (which feel diffusion limitation of nutrients more severely).

One potential problem with this view is that it is not clear whether turbulence really favours a prolonged suspension of heavy impurities. For example, the results of an experiment by Ruiz et al [8] apparently indicate that turbulence make heavy particles sink faster than in still fluid. However, other experiments considering different flow configurations gave the opposite result, see for example [1]. A careful study of this issue is thus required.

\section{Suspension of heavy impurities}

The non-dimensional equation of motion for a heavy impurity under the influence of gravity is written as

$$
\frac{d \mathbf{V}}{d t}=\delta \frac{D \mathbf{u}}{D t}-\frac{1}{\mathrm{St}}(\mathbf{V}-\mathbf{u}+\hat{\mathbf{z}} W)
$$


where $\mathbf{V}$ is the velocity of the impurity, $\mathbf{u}$ is the fluid velocity, $W$ is the terminal velocity of the impurity in still fluid, $z$ points vertically upwards and St is the Stokes number defined as

$$
\mathrm{St}=\frac{2}{9 \delta}\left(\frac{a}{L}\right)^{2} \operatorname{Re}
$$

where $\delta=\rho_{f} / \rho_{p}$ is the ratio between the density of the fluid and the density of the impurity, $L$ is a typical lengthscale of the flow, $U$ is a characteristic flow velocity and Re the Reynolds number. The terminal velocity $W$ is found by equating the gravitational force to the Stokes drag, and its non-dimensional version is given by

$$
W=\frac{2}{9} \frac{g L}{U^{2}}\left(\frac{a}{L}\right)^{2} \frac{1-\delta}{\delta} \operatorname{Re} .
$$

\subsection{Permanent suspension in a cellular flow field}

Stommel [10] studied the vertical settling of small heavy impurities in a simple flow configuration composed by a network of vortices with horizontal axis; all the dynamics takes plaxe in the vertical plane $(x, z)$ and the vortices are infinitely long in the $y$ direction. Stommel neglected the pressure term $\delta D \mathbf{u} / D t$ (which we shall also do in the rest of this section) and neglected also the inertia of the impurities, imposing $d \mathbf{V} / d t=0$ in equation (1), which gives

$$
\vec{V}=\vec{u}-\hat{z} W .
$$

Defining the two-dimensional stationary streamfunction

$$
\Psi=2(\cos x+\cos z) .
$$

The flow field is given by

$$
\vec{u}=(u, w)=\left(-\frac{\partial \Psi}{\partial z}, \frac{\partial \Psi}{\partial x}\right)
$$

and the velocity of the impurity is

$$
\vec{V}=(u, w-W)=\left(-\frac{\partial \Psi}{\partial z}, \frac{\partial \Psi}{\partial x}-W\right)=\left(-\frac{\partial \tilde{\Psi}}{\partial z}, \frac{\partial \tilde{\Psi}}{\partial x}\right)
$$

where $\tilde{\Psi}=\Psi-W x$. The solution is sketched in Figure 1. The flow is characterized by regions with closed streamlines of $\tilde{\Psi}$, surrounded by jet-like channels with essentially downward velocities, owing to the effects of gravity. The impurities which end up in the downward jets fall faster than in still fluid, but some impurities get trapped in the cells with closed streamlines and remain suspended forever. Stommel [10] thus argued that permanent suspension is possible also for heavy particles and that turbulence (or cellular flow fields) can keep heavy impurities afloat. An extension of this approach to the case of a time-varying stream function was discussed in [9]. 


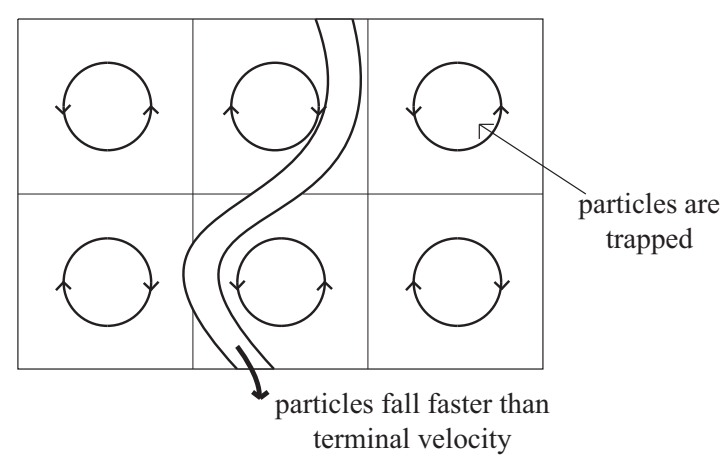

Figure 1: The motion of passively advected heavy impurities in the cellular flow field considered by Stommel [10].

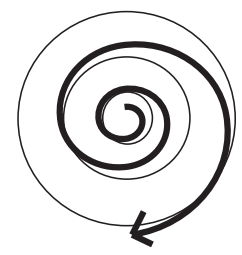

(a) Circular cell

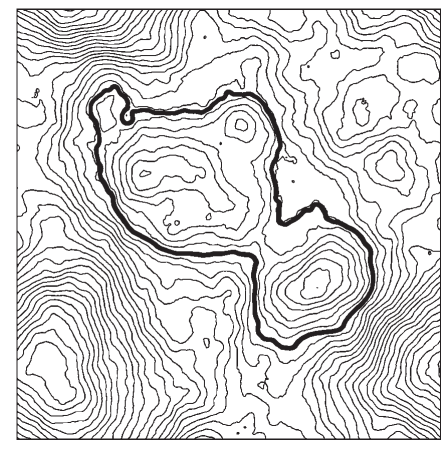

(b) More complex flow field

Figure 2: (a) Heavy impurities in an array of circular flow cells drift away from the center owing to inertial effects. (b) Impurities in a (frozen) flow field with more complex streamlines can stay suspended forever (from [7]).

\subsection{The role of inertia}

According to Maxey and Corrsin [6] permanent suspension is actually not possible when particle inertia is not neglected. Owing to the centrifugal force acting on a particle, in their motion in the circulation cells the impurities will spiral out, away from the cell center, as illustrated in Figure 2a. This has later become known as the sling effect: heavy particles tend to be ejected from high vorticity regions and concentrate in high strain regions between the flow cells; closed orbits no longer exist and heavy particles eventually end up in the downdrafts between the cells, causing an increase of the mean settling rate compared to what happens in still fluid, in keeping with the results of the experiment mentioned above [8].

However, consideration of flows with a more complex streamline pattern, such as that depicted in figure $2 \mathrm{~b}$, can change the picture. The work of [7] considered a spatially random, two-dimensional, incompressible stationary flow with energy spectrum $E(k) \propto k^{-5 / 3}$. In this flow, impurities are now pushed by their own inertia outwards as well as inwards, depending on the streamline curvature. In turn, this can lead to the emergence of closed 
trajectories, which corresponds to a limit cycle in the four-dimensional impurity phase space $\left(X, Y, V_{x}, V_{y}\right)$.

Thus, the study of the motion of small, heavy, spherical particles in a random, stationary two-dimensional flow shows that permanent particle suspension is possible when the randomness suitably alters the curvature of the streamlines around an eddy. A stationary random two-dimensional flow will have two distinct groups of impurities, as shown in figure 3a. The particles in one group will stay very close to where they started (that is, they stay suspended), moving on limit cycles with closed trajectories. The impurities in the other group move away from where they started, reach the downdrafts and sink with an average settling speed which is larger than their terminal velocity in still fluid.

Owing to inertial effects, identical particles can thus have significantly different behavior when moving in a random steady flow. Some particles are swept into the downdrafts between the eddies and settle at rates that are larger than in still fluid. Other particles remain suspended, moving on closed trajectories, so that their vertical motions are oscillatory. These two different types of behavior result in a bimodal distribution of vertical displacements. As a result, the mean settling velocity can be either larger or smaller than the terminal velocity in still fluid depending on the fraction of suspended particles.

For a time-varying random two-dimensional flow, the distinction between the population of suspended impurities and that of rapidly falling ones does not remain so strict as particles can move from one group to the other. In this case, permanent suspension is no longer possible, but suspension can still last for a long time. Overall, these results confirm the view of Stommel and Margalef, who claimed that turbulence could indeed help heavy impurities to stay suspended for longer times than in still fluid.

\section{Phytoplankton competition in turbulent waters}

As turbulence affects the sinking rate of phytoplankton, one may go back to the old Margalef question and ask how populations of phytoplankton species with different size respond to a turbulent environment. Larger plankton species seem to be favoured in conditions of strong turbulent suspension while smaller phytoplankton can thrive in quieter conditions.

To explore this issue, we follow [3] and consider two phytoplankton species with radius $a_{1}$ and $a_{2}$ that compete for the same nutrient, $N$. The population dynamics is described by the following (non-dimensional) NP system:

$$
\begin{aligned}
\frac{D N}{D t} & =I-\beta\left(a_{1}\right) \frac{N P_{1}}{k+N} Q\left(a_{1}\right)-\beta\left(a_{2}\right) \frac{N P_{2}}{k+N} Q\left(a_{2}\right)+\mu_{N} m\left[P_{1} Q\left(a_{1}\right)+P_{2} Q\left(a_{2}\right)\right] \\
\frac{D P_{1}}{D t} & =\beta\left(a_{1}\right) \frac{N P_{1}}{k+N}-\left[m+s\left(a_{1}\right)\right] P_{1} \\
\frac{D P_{2}}{D t} & =\beta\left(a_{2}\right) \frac{N P_{2}}{k+N}-\left[m+s\left(a_{2}\right)\right] P_{2} .
\end{aligned}
$$




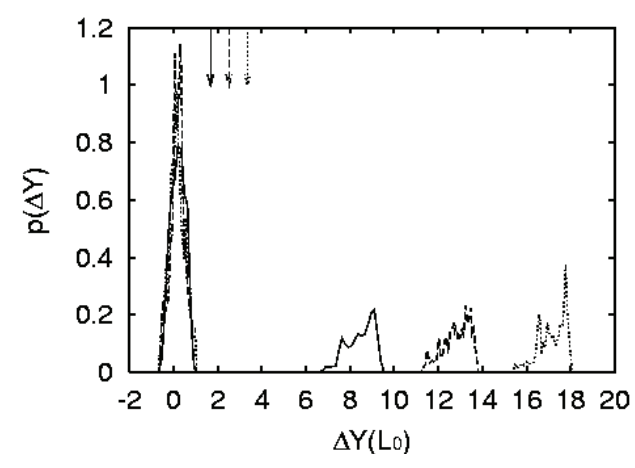

(a) Frozen flow field

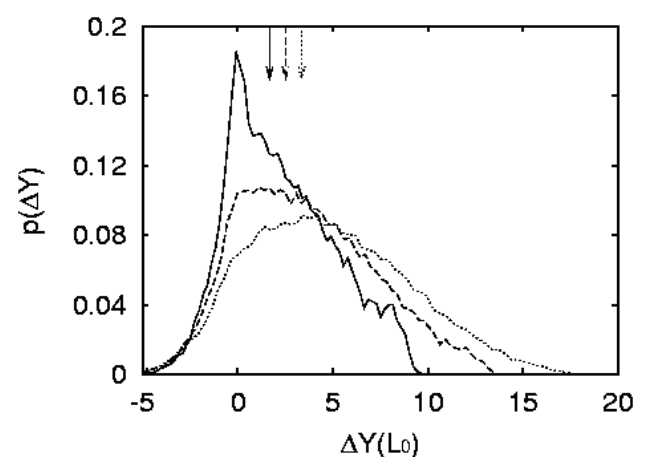

(b) Time-evolving flow field

Figure 3: (a) In a frozen turbulent flow some impurities can stay suspended forever, while others move away from their initial position and fall faster than in still fluid. As a result, the distribution of particle displacements is bimodal. The three curves show the probability distributions of the vertical displacement of falling impurities at different times and the arrows indicate the displacement that the impurities would have had if they moved at the Stokes settling velocity $W$. On the left of the plot, there are particles which stay suspended forever and their displacement from the initial position does not grow with time. (b) In a time-evolving random field there is no strict separation between suspended and falling particles and permanent suspension is not possible, but a large fraction of the impurities can fall more slowly than in still fluid. From [7].

where $P_{1}$ and $P_{2}$ are the concentrations of the two species of phytoplankton, $\beta$ is a growth rate, $k$ is a limiting term for phytoplankton growth, $m$ is a size-independent natural phytoplankton mortality rate, $s$ is the size-dependent sinking rate, and $Q$ is the nutrient quota of phytoplankton, used to transform biomass into nutrient amount.

The terms in the nutrient equation of system (5) describe two sources of nutrients: a constant external nutrient source, $I$, and the nutrients regenerated from dead phytoplankton, as described by the recycling term $\mu_{N} m\left[P_{1} Q\left(a_{1}\right)+P_{2} Q\left(a_{2}\right)\right]$ with $\mu_{N}<1$. Nutrients are consumed by phytoplankton at a rate $\beta$, describing the phytoplankton efficiency to uptake nutrient which is assumed to be a function of phytoplankton size.

In their study, Jiang et al [3] recalled that there is a complex relationship between the efficiency/growth rate $\beta$ and the size of phytoplankton species. Observations show that the growth rate of relatively large phytoplankton species decreases with increasing size while the opposite tends to be true for small phytoplankton species. This suggests that there should be an optimal phytoplankton size at which the growth rate is maximized. A simple analytic form of the phytoplankton growth rate that has these characteristics is

$$
\beta(a)=\frac{a}{\alpha_{1} a^{2}+\alpha_{2} a+\alpha_{3}}
$$

where $\alpha_{1}, \alpha_{2}$, and $\alpha_{3}$ are positive constants. The form of the growth rate described in (6) is only meant to capture the qualitative structure of observed phytoplankton growth rates 


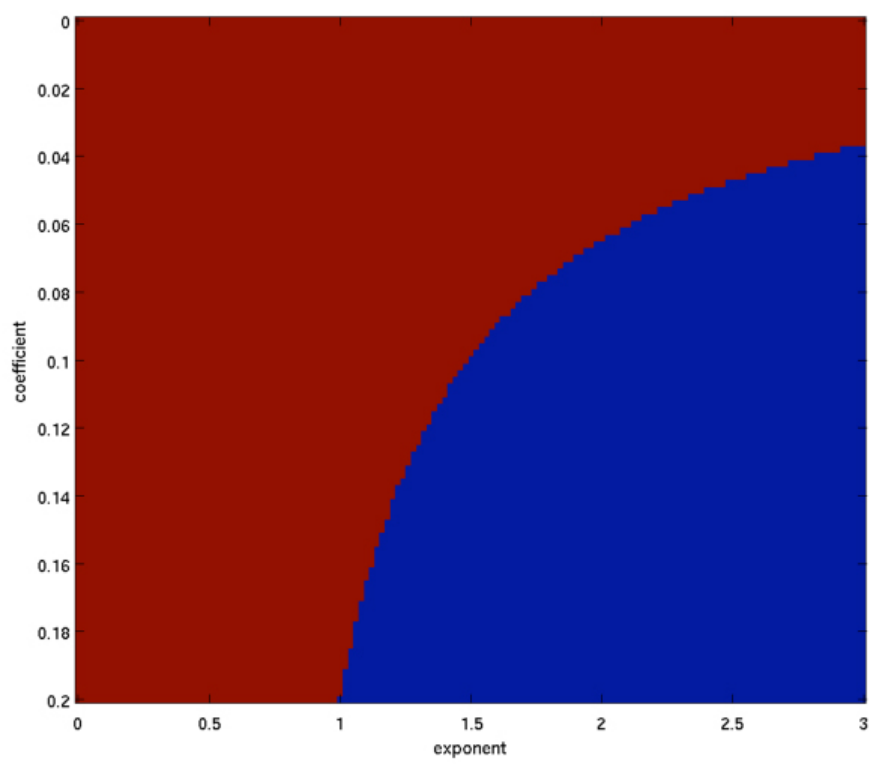

Figure 4: Outcome of species competition in a turbulent environment, parameterized by the values of the exponent $\sigma$ and the coefficient $s_{0}$. Red (in the upper left area) indicates dominance of the larger species with $a=1.4$, blue indicates dominance of the smaller species with $a=0.7$. Cell radii are in non-dimensional units.

and is not necessarily universal.

The growth of phytoplankton populations, thanks to nutrient consumption, is balanced by plankton mortality, $m$, and plankton sinking, $s(a)$. In the phytoplankton competition model (5), the mortality rate $m$ is assumed to be independent of phytoplankton size, unlike the sinking term. In the absence of turbulence and assuming spherical particles, the settling velocity of an individual phytoplankter can be derived from Stokes law obtaining $s(a)=s_{0} a^{2}$; this form was assumed in [3] with $s_{0}=2$. With this choice, it was found that the most favoured species is always the smaller one, and that there should be a natural tendency towards dominance of smaller phytoplankton species. In this view, the higher efficiency in nutrient uptake of the larger species is not enough to balance their faster sinking rate, leading to a dominance of the smaller organisms.

In reality, the sinking rate depends on the level of turbulence, which can alter the above result. In a subsequent study, Demonte et al [2] kept all parameter values as in [3] but assumed the sinking rate to be proportional to a power of the phytoplankton cell radius, $a$, through a simple relationship

$$
s(a)=s_{0} a^{\sigma}
$$

where $s_{0}$ is the coefficient and $\sigma$ is the exponent. The motivation of this choice is that turbulence can alter the simple dependence of sinking velocity on radius observed in still fluid. What is found in this case is that the outcome of species competition depends on the properties of turbulence, that is, on the assumed values of $s_{0}$ and $\sigma$. Figure 4 
shows the outcome of competition for two species with (non-dimensional) radius 0.7 and 1.4, respectively. For the parameter values chosen in [3], the smaller species is favoured. However, by varying the values of $\sigma$ and/or $s_{0}$, one can get the opposite result. Even by keeping $\sigma=2$ but varying the coefficient $s_{0}$, one can obtain dominance of the larger species. This is consistent with the view that the outcome of competition of phytoplankton species with different size depends on the properties of the turbulent environment, and it seems to further confirm Margalef's view.

\section{Vertical velocities in mesoscale flows}

In the previous sections we have seen that turbulent suspension can be an important mechanism in plankton dynamics. We can ask whether these effects are visible also on scales larger than those of homogeneous and isotropic turbulence: for example, whether they can play a role at the ocean mesoscales where fronts, vortices and waves dominate the flow, as discussed in the first lecture.

Although a final answer is still missing, an important point concerns the structure and intensity of the vertical velocity field at the mesoscales. If there are strong and organized vertical velocities, then these can also induce suspension (in upwelling regions) and rapid sinking (in downwelling areas), thus affecting plankton dynamics, besides their role in determining the nutrient input to the euphotic layer.

In proximity of fronts, vertical velocities can be very intense, creating a complex pattern of upwelling and downwelling. However, other mesoscale structures can also play a role: coherent vortices, in particular, have been shown to be associated with a complicated pattern of strong vertical velocities [4]. In their study, Koszalka et al. have considered a primitive equation model, using the ROMS ocean model, with doubly periodic boundary conditions and idealized surface forcing. The simulations show the emergence of coherent vortices, similarly to what happens in two-dimensional or quasi-geostrophic (QG) turbulence. The horizontal dynamics and transport properties of this flow are not too dissimilar from what happens in QG turbulence, suggesting that approaches based on simple QG models capture a relevant portion of the horizontal dynamics. However, the vertical velocities associated with the vortices in the primitive equation setting can be very strong, at variance with the situation found for QG vortices.

Figure 5a shows a slice of the vorticity field in one of the primitive equation simulations discussed in [4], and figure 5b shows the vertical velocity field associated with one of the vortices. The vertical valocities in proximity of the vortex are very intense, and can lead to various consequences for the marine ecosystem, ranging from enahnced nutrient input to the suspension of phytoplankters in the upwelling areas and their rapid sinking in the downwelling regions. 


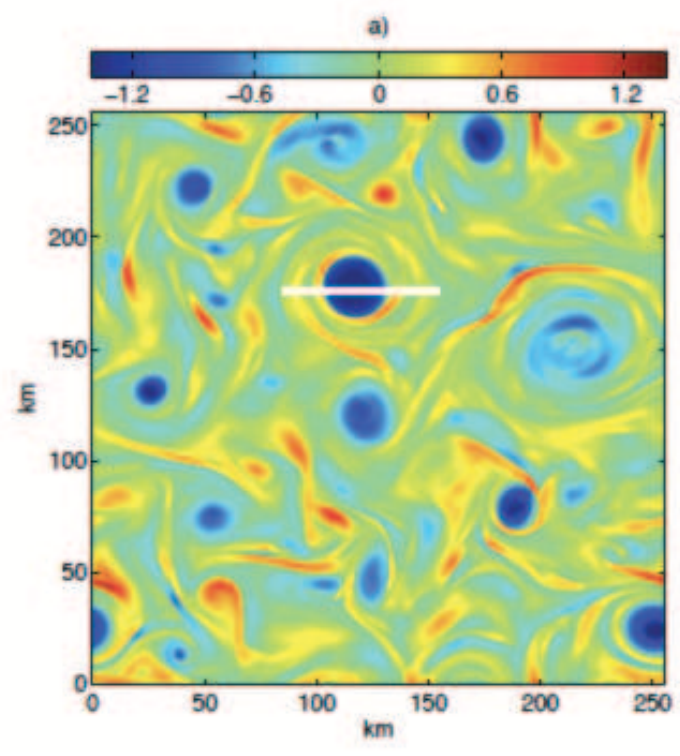

(a) $\zeta / f$

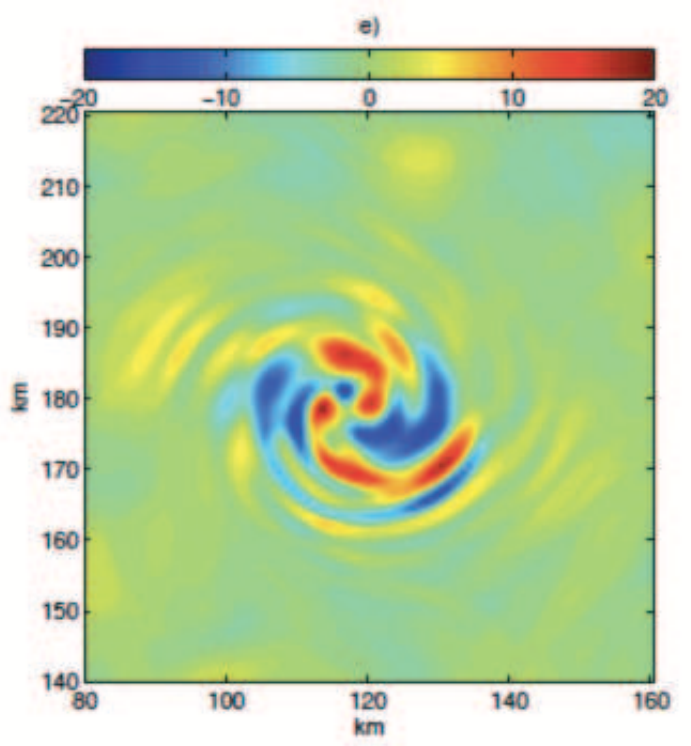

(b) $w \mathrm{~m} /$ day

Figure 5: Panel (a): The surface vorticity field produced by a numerical simulation of forceddissipated primitive equations with doubly periodic boundary conditions in a domain with lateral size $256 \mathrm{~km}$ and depth 1000 meters. Forcing is provided by idealized surface (wind) forcing and relaxation to a given density profile. The relative vorticity, $\zeta$, is normalized by the Coriolis parameter, $f$. Panel (b): The vertical velocity field, in $\mathrm{m} /$ day, at a depth of 78 meters, for the vortex indicated by the white segment in panel (a). From [4].

\section{Conclusion}

In these lectures I tried to illustrate, by various examples, how turbulence affects aquatic organisms. The overall picture shows that turbulence is an important component of these ecosystems and it must be properly considered to obtain a quantitative description of ecosystem functioning. From the small scales of homogeneous and isotropic turbulence, where individual plankton cells swim, sink and are transported by the fluid flow, to the ocean mesoscales where vortices, fronts and waves, with their associated upwelling and downwelling regions determine the plankton distribution, to the large scales where the basin circulation controls the overall structure of the marine ecosystem, fluid dynamics is at the core of the biological processes occurring in aquatic environments. There are still many open questions, some of which can possibly be addressed by the methods of GFD and of dynamical system analysis as illustrated here.

\section{References}

[1] J. DÁvila And J. Hunt, Settling of small particles near vortices and in turbulence, J. Fluid Mechanics, 440 (2001), p. 117. 
[2] S. De Monte, R. Sciascia, And A. Provenzale, Phytoplankton competition and cell size effects in turbulent water, in preparation, (2011).

[3] L. Jiang, O. M. E. Schofield, And P. G. Falkowski, Adaptive evolution of phytoplankton cell size, Am. Nat., 166 (2005), pp. 496-505.

[4] I. Koszalka, A. Bracco, J. McWilliams, and A. Provenzale, Dynamics of wind-forced coherent anticyclones in the open ocean, J. Geophys. Res. - Oceans, 114 (2009), p. C08011.

[5] R. Margalef, Life-forms of phytoplankton as survival alternative in an unstable environment, Oceanologica Acta, 1 (1978), pp. 493-509.

[6] M. Maxey and S. Corrsin, Gravitational settling of aerosol particles in randomly oriented cellular flow fields, Journal of atmospheric science, 43 (1986), pp. 1112-1134.

[7] C. Pasquero, A. Provenzale, and E. Spiegel, Suspension and Fall of Heavy Particles in Random Two-Dimensional Flow, Physical Review Letters, 91 (2003).

[8] J. Ruiz, D. Macias, And F. Peters, Turbulence increases the average settling velocity of phytoplankton cells, PNAS, 101 (2004), pp. 17720-17724.

[9] L. Smith And E. A. Spiegel, in "Macroscopic Modelling of Turbulent Flows", edited by U. Frisch, J. B. Keller, G. Papanicolaou, O. Pironneau, Springer-Verlag, Berlin, 1985.

[10] H. J. Stommel, Trajectories of small bodies sinking slowly through convection cells, J. Mar. Res., 8 (1949), pp. 24-29. 


\title{
Evolutionary Models: Movement and Mixing in Trait and Physical Space
}

\author{
Lecturer: Glenn Flierl
}

May 8, 2011

Over generations, a species will evolve to increase its fitness to its environment. Natural selection is the primary mechanism by which this occurs, wherein environmental pressures cause individuals with more fit phenotypes to reproduce at a higher rate. For the purpose of modeling, the space of possible heritable traits may be viewed as a continuum, and a particular individual as inhabiting a point in trait space. Making the biomass continuous as well, a population is viewed as a distribution in trait space. In this view, natural selection is the process by which the distribution of biomass propagates through trait space, generally increasing its average fitness.

\section{Building blocks for models}

The first step in modeling a system in which the dominant traits can change is defining a suitable measure of how much of a species there is. While the actual number of members of the species is an option, a more useful measure is the biomass, $b$, which readily translates across species. The biomass density can be treated as a function over space, time, and this "trait-space"

$$
b\left(\mathbf{x}, t \mid s_{1}, s_{2}, \ldots\right)
$$

where the variables $\mathbf{s}=\left\{s_{1}, s_{2}, \ldots\right\}$ specify the phenotype, or traits, of the organism. In addition to the biomass, the rates of survival and reproduction depend on the environmental conditions and vary with phenotype. These rates are defined as

$$
\mathcal{R}=\mathcal{R}(\mathbf{s} \mid E) \quad, \quad \mathcal{R}\left(\mathbf{s}_{1} \mid E\right) \neq \mathcal{R}\left(\mathbf{s}_{2} \mid E\right)
$$

where $E$ represents the environmental conditions. The biomass evolution is related to the growth rate (including reproduction) by

$$
\frac{\partial}{\partial t} b(\mathbf{s})=\mathcal{R}(\mathbf{s} \mid E) b(\mathbf{s}) \ldots
$$

indicating that new organisms (represented as biomass) have the same values of $\mathbf{s}$ as their parents. However, in nature genetic mutation can occur causing the offspring of parents to have a slightly different trait values. (And, since these represent phenotypes, there is some degree of natural variation as well.) This can be modeled by changing Equation 1 to

$$
\frac{\partial}{\partial t} b(s)=\mathcal{R}(\mathbf{s} \mid E) b(\mathbf{s})+\nabla_{s}^{2} m b
$$


where $m$ is a type of trait diffusion (which can be proportional to the reproduction rate). The rate appears within the derivatives for the same reasons it does in the Fokker-Planck equation (or the representation of kinesis in a previous lecture) - the length of the random jump in trait space is set at the beginning of the excursion (and there is no reason to expect non-divergence). In any case, we shall just use the over-simplification of treating $m$ as constant.

The simplified model created in Equation 2 obviously has a number of flaws. If a trait is not represented in the initial modeling it will never arise long term meaning that a full trait space needs to be known a priori. Additionally, the time scales are not well-defined. While there is some idea of the rate of genetic changes, not much about how this translates into alterations of the phenotype is understood. Finally, since natural selection acts on an individual basis; a model based on biomass may not accurately model the dynamics for low values where extinction is probable.

If we extend the above model to start accounting for advection and diffusion in space as well as diffusion in trait space, we can use the following equation to model a wide variety of systems

$$
\frac{D}{D t} b-\nabla \kappa \nabla b=b \mathcal{R}(\mathbf{s} \mid E)+\nabla_{s}^{2} m b .
$$

With this governing equation the following topics will be examined

- Evolutionarily stable strategies: what $s$ values are selected for?

- Relationship to stability theory

- Adaptive dynamics in the presence of mutation

- Effects of mixing, diffusion, and advection

\section{A Simple Model}

To make a model which can be studied easily, it is necessary to create a function for the growth rate. For a first model, we make a number of simplifying assumptions: the organisms will have a $1 \mathrm{D}$ trait space, $\mathbf{s}=s$, and use a common resource, $N$, so that the amount available per individual is

$$
N_{I}=N \frac{b_{0}}{\int d s b(s)}
$$

where $b_{0}$ corresponds to the biomass of a single individual and the notation $\langle b\rangle=\int d s b$ has been used. Growth will occur when $N_{I}>N_{\text {crit }}$ but saturates to a maximum value $g_{m}$ when the resources are plentiful. For example,

$$
g=g_{m} \frac{N_{I}-N_{c r i t}}{N_{I}} \text { for } N_{I}>N_{c r i t},
$$

which results in a logistic-like system:

$$
\frac{\partial}{\partial t} b=\left[g_{m}\left(1-\frac{\langle b\rangle}{b_{c}}\right)-d\right] b+m \frac{\partial^{2}}{\partial s^{2}} b
$$


The coefficients $g_{m}$ (maximum growth rate), $d$ (death rate), and $b_{c}=N b_{0} / N_{\text {crit }}$ (carrying capacity) are functions of $s$. For this form,

$$
\mathcal{R}(s \mid E)=\mathcal{R}(s \mid\langle b\rangle)=g_{m}\left(1-\frac{\langle b\rangle}{b_{c}}\right)-d .
$$

The environment here is the density of competitors for the resource as well as the value of $N$ implicit in the carrying capacity $b_{c}$.

\section{Evolutionarily Stable Strategies (ESS)}

If the system modeled by Equation 3 is analyzed without considering mutation $(m=0)$, there are many possible singular solutions of the form

$$
b(s, t)=\bar{b}(t) \delta(s-\bar{s})
$$

with $\bar{b}$ satisfying

$$
\frac{\partial}{\partial t} \bar{b}=\mathcal{R}(\bar{s} \mid \bar{b}) \bar{b}
$$

since $E=\langle b\rangle=\bar{b}$. This results in just the logistic equation and steady solutions where $\mathcal{R}(\bar{s} \mid \bar{b})=0$ indicating

$$
\bar{b} \rightarrow b_{c}(\bar{s})\left(1-\frac{d(\bar{s})}{g_{m}(\bar{s})}\right)
$$

Let us introduce a different organism with phenotype $s^{\prime}: b=\bar{b}(t) \delta(s-\bar{s})+b^{\prime}(t) \delta\left(s-s^{\prime}\right)$; if its biomass is very low when compared to $\bar{b}$, Equation 3 becomes

$$
\frac{1}{b^{\prime}} \frac{\partial}{\partial t} b^{\prime}=\mathcal{R}\left(s^{\prime} \mid \bar{b}\right)=\left[g_{m}\left(s^{\prime}\right)\left(1-\frac{\bar{b}}{b_{c}\left(s^{\prime}\right)}\right)-d\left(s^{\prime}\right)\right],
$$

and when the coefficients are time-independent, we can write this in the simpler form

$$
\frac{1}{b^{\prime}} \frac{\partial}{\partial t} b^{\prime}=g_{m}\left(s^{\prime}\right) \frac{\bar{b}\left(s^{\prime}\right)-\bar{b}(\bar{s})}{b_{c}\left(s^{\prime}\right)}
$$

where $\bar{b}\left(s^{\prime}\right)$ is the equilibrium population for $s=s^{\prime}$. For this model, the population with the highest equilibrium value will exclude all others: $b^{\prime}$ will decay if $\bar{b}\left(s^{\prime}\right)<\bar{b}(\bar{s})$. Alternatively, the ESS maximizes $b_{c}(s)\left[g_{m}(s)-d(s)\right] / g_{m}(s)$, and it's also the one which can survive on the minimum resource $N_{I}$.

In other situations, there may be no single species which can out-compete all others. For example, consider a case in which different species use somewhat different resources so that $N_{I}$ for a species $s$ depends on the nearby biomass but not species far away in trait space. E.g, is $s$ represents the size of a herbivore, it will generally feed on a limited range of plant sizes. This kind of local competition could look like

$$
\mathcal{R}=g_{m}\left[1-\int d s^{\prime} w\left(s \mid s^{\prime}\right) b\left(s^{\prime}\right)\right]-d
$$

with $w$ a peaked function. Since we will take $b_{c}$ to be constant, we can just choose it to be one hereafter; $w$ will be normalized to have unit integral. Figure 1 shows an example with speciation events and a final state with 6 "species." 


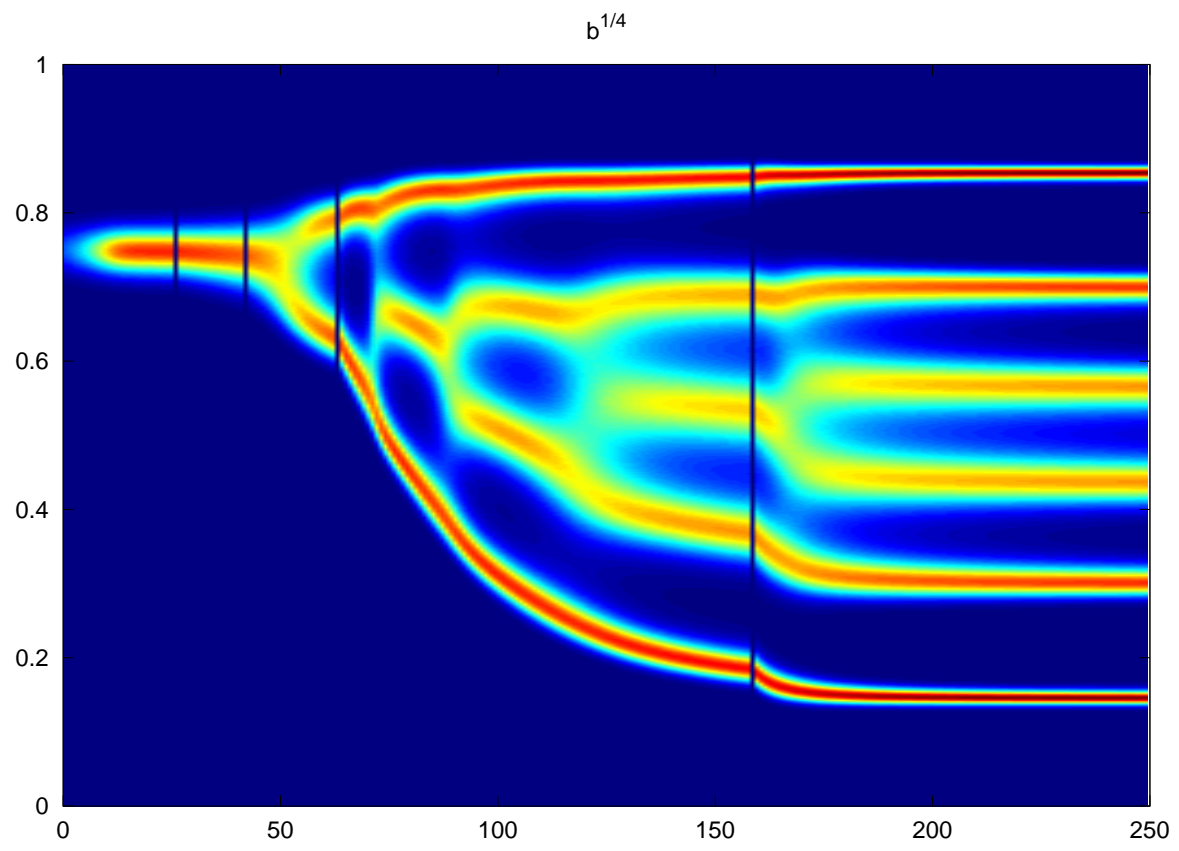

Figure 1: Biomass density vs. time for the case of local competition. The y axis is $s$; the $\mathrm{x}$ axis is time, but on an uneven scale. The black lines show where the time scale changes from 1 day per unit to $5,10,50$, and 500 days.

\section{Adaptive dynamics}

Dieckmann points out that the dynamics may be approximated by equations for the net population and the rate of movement in trait space.

We can look at the (local) mean biomass and the mean value of $s$

$$
\begin{aligned}
& \langle b\rangle=\int_{s_{0}}^{s_{1}} d s b(s) \\
& \langle s\rangle=\langle s b\rangle /\langle b\rangle
\end{aligned}
$$

Applying the local average to a system defined in Equation 2 and ignoring fluxes out of the range by mutation, we get

$$
\frac{\partial}{\partial t}\langle b\rangle=\langle\mathcal{R}(s, b) b\rangle
$$

which is

$$
\simeq \mathcal{R}(\langle s\rangle, \tilde{b})\langle b\rangle+\frac{1}{2} \mathcal{R}^{\prime \prime} \sigma^{2}\langle b\rangle
$$

from Taylor expansion. For evaluating the competition/ environmental effects, we can treat the biomass distribution as a set of singular values

$$
\tilde{b}=\langle b\rangle_{i} \delta\left(s-\langle s\rangle_{i}\right)
$$


Taking the local $s$ moment and expanding gives

$$
\begin{aligned}
\frac{\partial}{\partial t}\langle s\rangle\langle b\rangle & =\langle s \mathcal{R} b\rangle \\
\langle b\rangle \frac{\partial}{\partial t}\langle s\rangle & =\langle(s-\langle s\rangle) \mathcal{R} b\rangle \\
& \simeq \mathcal{R}^{\prime} \sigma^{2}\langle b\rangle \\
\frac{\partial}{\partial t}\langle s\rangle & \simeq \mathcal{R}^{\prime} \sigma^{2}
\end{aligned}
$$

The speed at which the peaks migrate towards the optimal value depends on the gradient and on the width of the distribution $\sigma$. Spread arises from mutation or from migration mixing. We could derive an equation for $\sigma$; it tends to increase because of $m$ and decrease because of negative curvature in $\mathcal{R}$ which acts to sharpen peaks.

As a simple, and analytically tractable example for a single peak, consider a system with

$$
\mathcal{R}=\left[R_{0}-\frac{1}{2} R_{2}\left(s-s_{0}\right)^{2}\right]\left[1-\frac{1}{\sqrt{2 \pi}}\langle b\rangle\right]
$$

where $R_{0}, R_{2}$, and $s_{0}$ could be functions of time as the population or other processes alter the resource. We can find a time-dependent solution (without needing to approximate)

$$
b=b_{0}(t) \exp \left[-\frac{1}{2} \frac{[s-x(t)]^{2}}{\sigma^{2}(t)}\right]
$$

when

$$
\begin{aligned}
r c l \frac{\partial}{\partial t} \sigma & =-\frac{1}{2} R_{2}^{*} \sigma^{3}+\frac{m}{\sigma} \\
\frac{\partial}{\partial t} x & =-R_{2}^{*} \sigma^{2}\left(x-s_{0}\right) \\
\frac{1}{b_{0}} \frac{\partial}{\partial t} b_{0} & =R_{0}^{*}-\frac{1}{2} R_{2}^{*}\left(x-s_{0}\right)^{2}-\frac{m}{\sigma^{2}}
\end{aligned}
$$

with $R_{j}^{*}=R_{j}\left(1-\sigma b_{0}\right)$.

The center of the distribution moves towards the peak in the growth rate curve at a speed which depends of the slope at the current center location $\left[-R_{2}^{*}\left(x-s_{0}\right)\right]$ and the variance of the distribution. The width asymptotes to the value $\left(2 m / R_{2}^{*}\right)^{1 / 4}$ (if $\mathcal{R}^{\prime \prime}$ is negative) which narrows as the growth rate curve becomes more sharply peaked. The population will increase or decrease depending on the sign of $R_{0}^{*}-m / \sigma^{2}$; the dynamics of the resource will adjust $R_{0}^{*}$ until it reaches the equilibrium value.

The environment will be time-dependent because of many external factors, so that $\mathcal{R}$ itself has a whole spectrum of variability. The velocity for movement in trait space will therefore be fluctuating, and we cannot expect the system to be in equilibrium. If the 
external changes are very slow compared to mutation times, the system will be very close to the ESS for current conditions:

$$
\left.\frac{\partial \mathcal{R}(\mathbf{s}, t)}{\partial s_{i}}\right|_{\mathbf{s}=\overline{\mathbf{s}}(t)} \simeq 0
$$

In contrast, if the environmental fluctuations are fast, the center of the distribution will not be able to keep up, and the mean trait value will settle at the point where the time-averaged velocity is zero

$$
\left\langle\left.\frac{\partial \mathcal{R}(\mathbf{s}, t)}{\partial s_{i}}\right|_{\mathbf{s}=\overline{\mathbf{s}}}\right\rangle \simeq 0
$$

For intermediate time-scales, comparable to mutation times, the center of the distribution will partially, but not completely, track the variations. The population dynamics is implicit here, basically maintaining $\mathcal{R}(\bar{s}, t)$; as we shall see, it may play a much more active role with multiple trophic levels.

\section{Time-dependent}

We can also consider a system distributed in trait- and physical space in which a changing environment produces time-dependent forcing. A simple way to do this is to change the reproduction rate in such a way that favors different phenotypes at different times. This could model such changing environmental parameters as mixed layer depth, or sunlight, for example. During the portion of the forcing cycle when a certain phenotype is not fit, mutation can replenish such populations which would otherwise slowly die out. We can then see a seasonal cycle in the community structure as well as the biomass. Alternatively, selective grazing in which the zooplankton feed more heavily on the more abundant species can also prevent the non-optimal species from disappearing.

If the growth rate is time-dependent, $\frac{\partial}{\partial t} \mathcal{R} \neq 0$, a time-average of the biomass can be taken to find

$$
\frac{1}{T} \ln \frac{b^{\prime}(T)}{b^{\prime}(0)}=\left\langle\mathcal { R } \left( s^{\prime}, t|\bar{b}(\bar{s}, t)\rangle \equiv R\left(s^{\prime} \mid \bar{s}\right)\right.\right.
$$

Then, based on this result, $\bar{s}$ will represent an evolutionarily stable strategy if

$$
R(\bar{s} \mid \bar{s})=0 \quad \text { [definition] and } \quad R\left(s^{\prime} \mid \bar{s}\right)<0 \quad \text { for } \quad s^{\prime} \neq \bar{s}
$$

which indicates that organisms with a non-optimal phenotype will die out representing natural selection. Locally, these conditions become

$$
\left.\frac{\partial}{\partial s^{\prime}} R\left(s^{\prime} \mid \bar{s}\right)\right|_{s^{\prime}=\bar{s}}=0 \quad,\left.\quad \frac{\partial^{2}}{\partial s^{\prime 2}} R\left(s^{\prime} \mid \bar{s}\right)\right|_{s^{\prime}=\bar{s}}<0
$$

but since the competition is temporally local, it is possible that several species could coexist with one or another dominant at different times. 


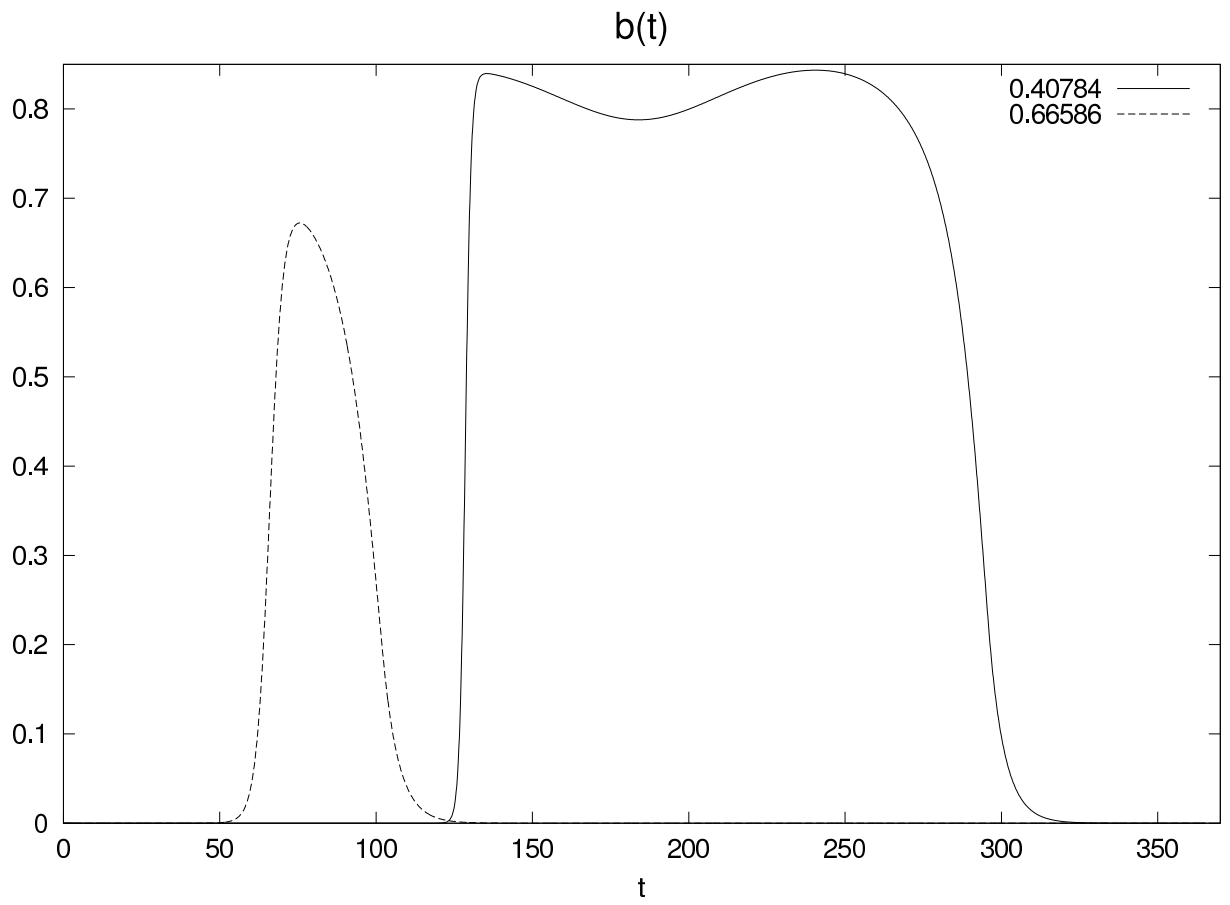

Figure 2: Biomass density vs. time for $s=0.41$ (late) and for the case of local competition. The $\mathrm{y}$ axis is $s$; the $\mathrm{x}$ axis is time, but on an uneven scale. The black lines show where the time scale changes from 1 day per unit to 5, 10, 50, and 500 days.

\section{Relationship to stability theory}

To understand the relationship to stability theory, look again at the linearized model

$$
\frac{\partial}{\partial t} b^{\prime}=\mathcal{R}(s \mid \bar{b}) b^{\prime}+\bar{b} \frac{\partial \mathcal{R}}{\partial E}\left\langle b^{\prime}\right\rangle
$$

with the basic state having

$$
\bar{b}(s) \mathcal{R}(s \mid \bar{b}(s))=0
$$

- Standard problems: $\bar{b}(s) \neq 0 \Rightarrow \mathcal{R}(s, \bar{b}(s))=0$, the first term vanishes, and we deal with the second term, often in the form

$$
\frac{\partial}{\partial t} b_{i}^{\prime}=\left.\bar{b}_{i} \frac{\partial \mathcal{R}_{i}}{\partial b_{j}}\right|_{b=\bar{b}} b_{j}^{\prime}
$$

- Evolutionarily Stable Strategies: $\bar{b}(s)=0$ for $s \neq \bar{s}$ so that $\mathcal{R}(s \mid \bar{b}) \neq 0$ and the first term is the important one.

In the case with mutation

$$
\begin{gathered}
\bar{b} \mathcal{R}(s \mid \bar{b})+\nabla_{s}^{2} m \bar{b}=0 \\
\frac{\partial}{\partial t} b^{\prime}=\mathcal{R}(s \mid \bar{b}) b^{\prime}+\bar{b} \frac{\partial \mathcal{R}}{\partial E}\left\langle b^{\prime}\right\rangle+\nabla_{s}^{2} m b^{\prime}
\end{gathered}
$$


both terms enter. Near the "hot-spots" where $\bar{b}$ is large, $\mathcal{R}$ is relatively small, the second term dominates and the problem looks like a standard stability system modified by diffusion. Far away, however, $\bar{b}$ is small, and the basic state has a decaying form with $\mathcal{R}$ nonzero. If $\mathcal{R}$ changes and becomes positive in that region, the population can "tunnel" into the local maximum and grow. The amount of time this will take depends on distance and the mutation rate.

\section{$7 \quad$ Physical mixing}

We now consider a model with a single spacial dimension, $y$, in addition to the single phenotype dimension, $s$. Our growth rate varies in physical space, and the biomass also diffuses in physical space, but we neglect the diffusion in trait space that represents mutation.

$$
\frac{\partial}{\partial t} b=b \mathcal{R}(s, y \mid\langle b\rangle)+\kappa \frac{\partial^{2}}{\partial y^{2}} b
$$

with

$$
\mathcal{R}=\left[R_{0}-\frac{1}{2} R_{2}\left(s-s_{0}(y)\right)^{2}\right][1-\langle b\rangle]-d .
$$

Figure 3 shows the biomass concentrates into a limited number of species occupying overlapping latitude ranges; intermediate species are excluded.

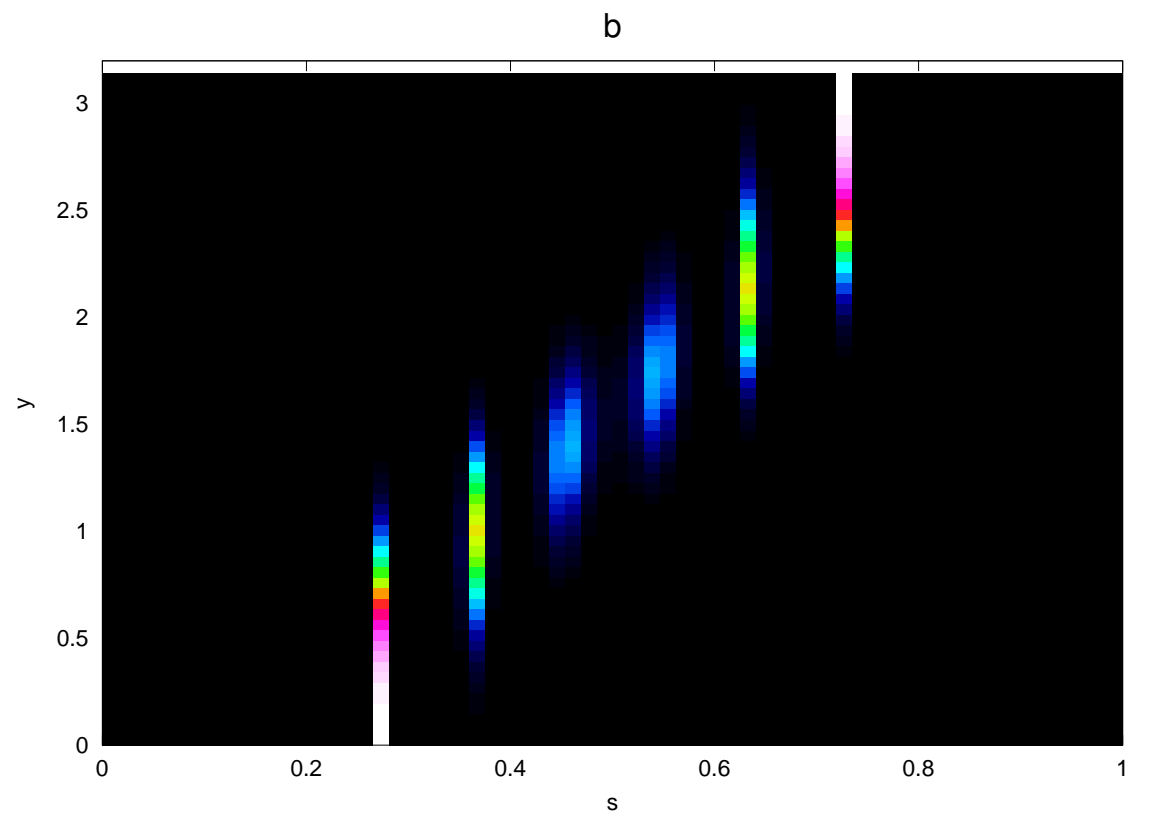

Figure 3: Biomass density $b(s)$ vs. $y$ (non-dimensional) with physical mixing.

Because there is no mutation, we can analyze this behavior by examining singular solutions with the single-phenotype ansatz,

$$
b(s, y, t)=\bar{b}(y, t) \delta(s-\bar{s}) .
$$


Applying this ansatz yields the PDE in $y$ and $t$ that governs $\bar{b}$,

$$
\frac{\partial}{\partial t} \bar{b}=\left(\left[R_{0}-\frac{1}{2} R_{2}\left(\bar{s}-s_{0}(y)\right)^{2}\right][1-\bar{b}]-d\right) \bar{b}+\kappa \frac{\partial^{2}}{\partial y^{2}} \bar{b} .
$$

Note that the linear part of this operator is a Schrödinger equation,

$$
\frac{\partial}{\partial t} \bar{b}=r(y) \bar{b}+\kappa \frac{\partial^{2}}{\partial y^{2}} \bar{b}
$$

which has a trapped mode if the region where $\mathcal{R}(\bar{s}, y \mid 0)>0$ is big enough and strong enough to overcome diffusive losses into the region where the death rate dominates (Kierstead and Slobodkin).

To determine whether a steady single-phenotype solution is linearly stable to nearby species (i.e. is a local ESS), consider small perturbations, $b(s, y, t)=\bar{b}(y)+b^{\prime}\left(\bar{s}+s^{\prime}, y, t\right)$. To linear order in $s^{\prime}$ and $b^{\prime}$, the perturbations are governed by

$$
\frac{\partial}{\partial t} b^{\prime}=b^{\prime} \mathcal{R}(\bar{s}, y, \bar{b})+s^{\prime} \bar{b} \frac{\partial \mathcal{R}}{\partial s}(\bar{s}, y, \bar{b})+\kappa \frac{\partial^{2}}{\partial y^{2}} b^{\prime} .
$$

If $\bar{s}$ is a local ESS, the growth rate for $b^{\prime}$ will be zero, and we would need one higher order is $s^{\prime}$ to decide if the state is a local minimum or maximum. Thus, we want to find the $\bar{s}$ such that

$$
0=b^{\prime} \mathcal{R}(\bar{s}, y, \bar{b})+s^{\prime} \bar{b} \frac{\partial \mathcal{R}}{\partial s}(\bar{s}, y, \bar{b})+\kappa \frac{\partial^{2}}{\partial y^{2}} b^{\prime} .
$$

in the limit as $s^{\prime} \rightarrow 0$. Multiplying this equation by $\bar{b}$ and the equation for the latter by $b^{\prime}$ and integrating over space implies

$$
\int d \mathbf{x} \bar{b} b^{\prime} \frac{\partial \mathcal{R}}{\partial s}(\bar{s}, y, \bar{b})=0
$$

In the limit, $b^{\prime} \rightarrow \bar{b}$, so we can pick an $\bar{s}$, find $\bar{b}$, evaluate this integral, and search for the $\bar{s}$ where it vanishes. Species near this value will be excluded; however, the growth rate for ones far away, found from

$$
\sigma b^{\prime}=\mathcal{R}(s, y, \bar{b}) b^{\prime}+\kappa \frac{\partial^{2}}{\partial y^{2}} b^{\prime},
$$

may be positive. In that case, another band will appear. This can happen because $\bar{b}$ varies spatially, so it is not effective at excluding others in the regions where $\bar{b}$ is small and is dying out but sustained by diffusion from the positive growth regions.

Simulations of this spatial-diffusion model give the following qualitative results:

- The single species solution has reduced amplitude and will die out if $\kappa$ is big and the volume average of $\mathcal{R}(s \mid 0)$ is negative.

- If $b(\bar{s}, y)$ is large enough, single solutions may damp the rest of the field, even when it would otherwise grow.

- Discrete species with different phenotypes emerge in spatially-separated bands. 


\section{Predator-prey}

We now present a model with a predator species (zooplankton, $Z$ ) and a prey species (phytoplankton, $P$ ) evolving in a one-dimensional trait space. The prey has a trait-dependent birth rate, the predator has a constant death rate, and the predator feeds on the prey according to their respective traits, as governed by the interaction kernel, $G$.

$$
\begin{aligned}
& \frac{D}{D t} P(s)=\left[\mu(s) N-\int d s^{\prime} G\left(s-s^{\prime}\right) Z\left(s^{\prime}\right)\right] P \\
& \frac{D}{D t} Z\left(s^{\prime}\right)=\left[a \int d s G\left(s-s^{\prime}\right) P(s)-d_{z}\right] Z,
\end{aligned}
$$

with $\mu$ peaked at $s=0$ and $G\left(s-s^{\prime}\right)$ peaked at zero. We also require that the total biomass be preserved:

$$
N+\int d s P(s)+\int d s^{\prime} Z\left(s^{\prime}\right)
$$

This system has a singular solution,

$$
(P, Z)=\left(\bar{P} \delta(s), \bar{Z} \delta\left(s^{\prime}\right)\right)
$$

which is an ESS, but it may be unstable if

$$
\left|G^{\prime \prime}(0)\right| \bar{Z}>\left|\mu^{\prime \prime}(0)\right| \bar{N} .
$$

or if

$$
\frac{\left|G^{\prime \prime}(0)\right|}{G(0)}>\frac{\left|\mu^{\prime \prime}(0)\right|}{\mu(0)}
$$

so that the predation function is sharply curved and inhibits growth only in a small neighborhood of the single-phenotype solutions. The figures show two examples of $P(s, t)$ and $Z\left(s^{\prime} t\right)$ for weakly and more strongly unstable situations.

\section{Advection}

Another way to make the environment change periodically is to make the time derivative into an advective derivative, and to impose a flow that models ocean circulation. In the model that was simulated, the "ocean" is wind-driven, two layers with warm water above the thermocline and cold water below), and quasi-geostrophic. Figure 6 shows a snapshot of the mean $s$ value from such a model with a single nutrient and a single predator. The diversity is highest in the region where the western boundary currents meet and proceed offshore as an eddying jet. 
$P$
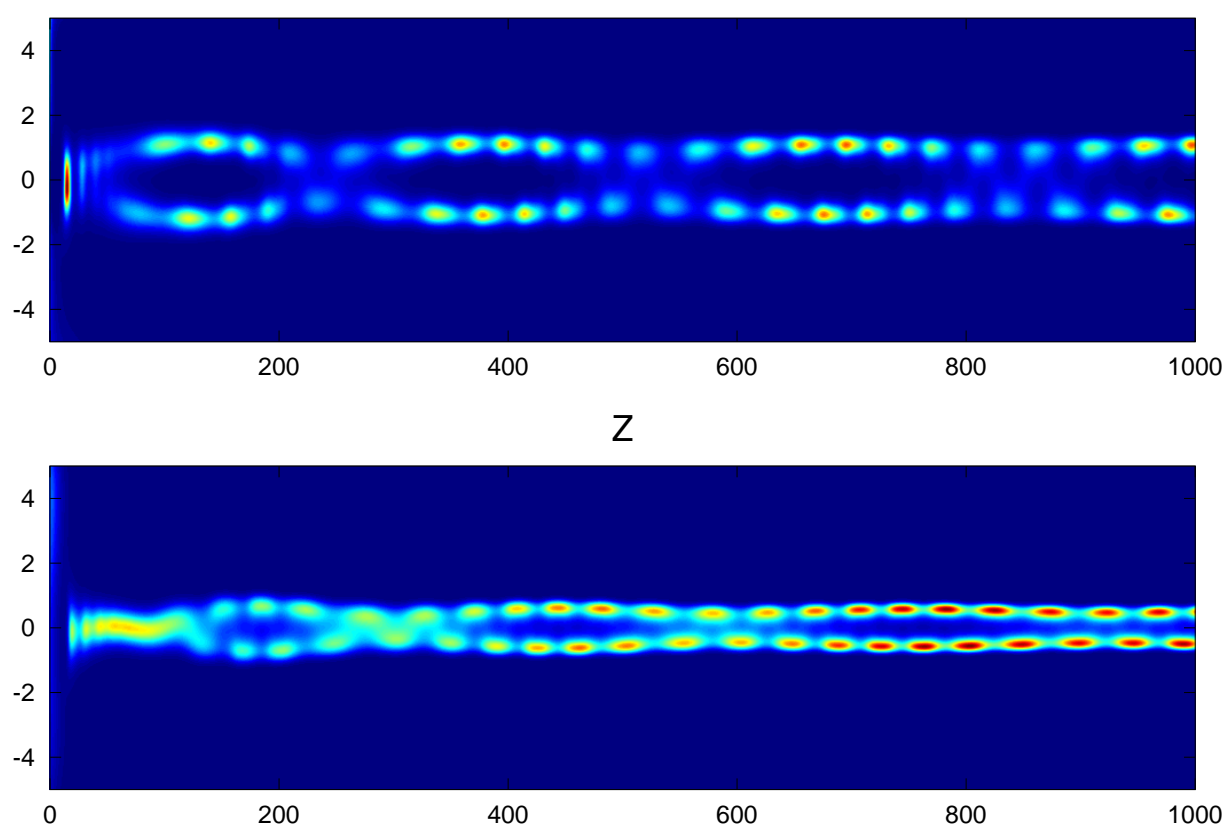

Figure 4: Predator-prey biomass densities for $G^{\prime \prime} / G=1.6 \mu^{\prime \prime} / \mu$.
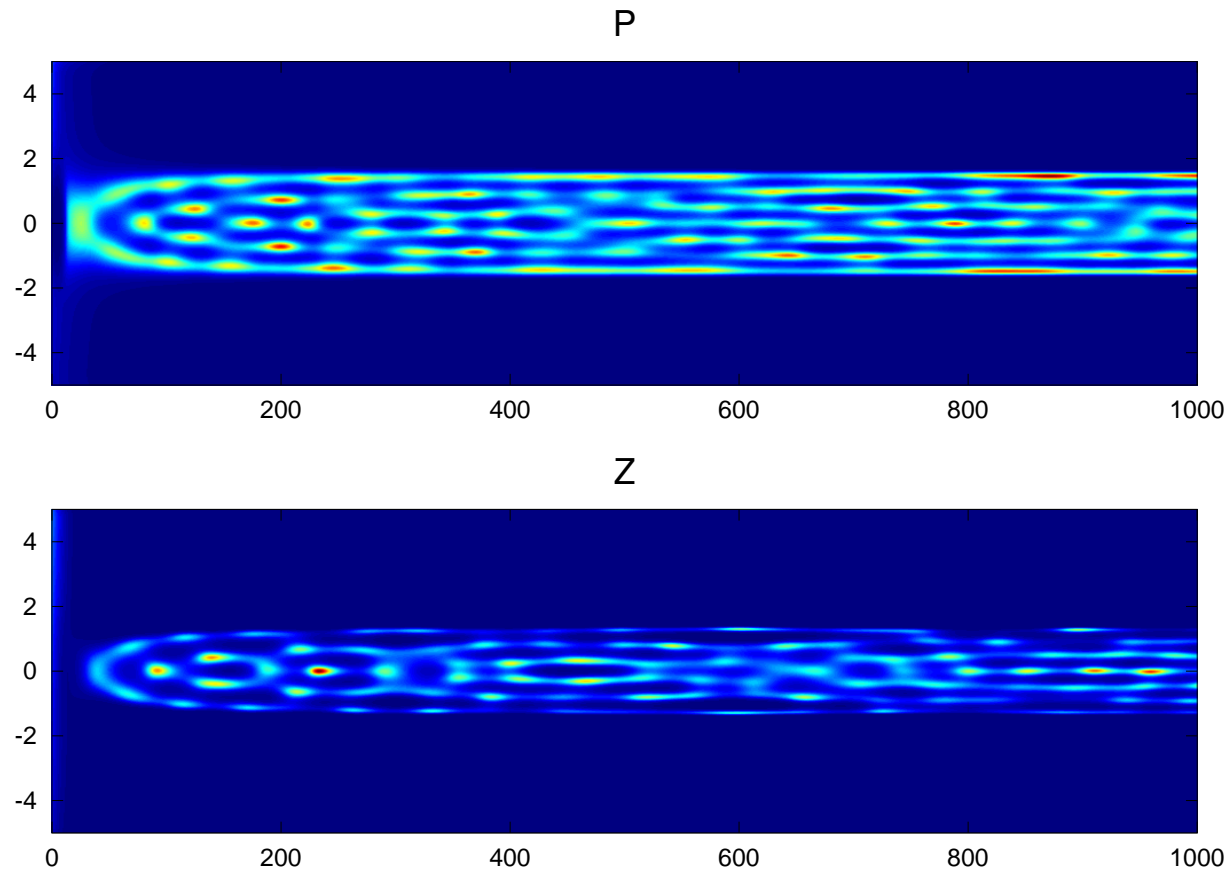

Figure 5: Predator-prey biomass densities for $G^{\prime \prime} / G=25 \mu^{\prime \prime} / \mu$. 


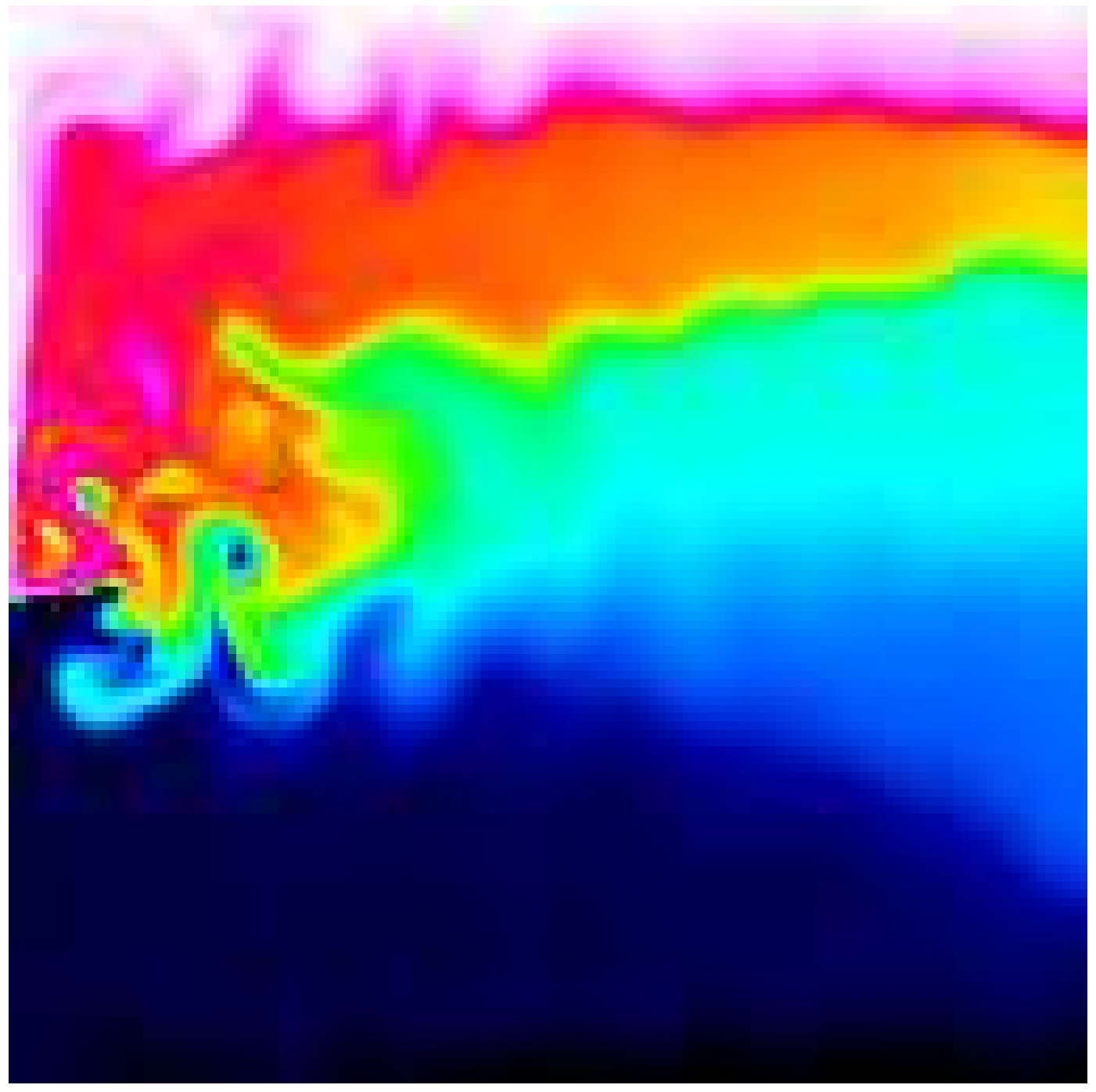

Figure 6: Mean value of $s$ in a two-gyre, eddying model. 


\title{
Finding Lagrangian structures via the application of braid theory
}

\author{
Michael R. Allshouse
}

October 16, 2010

\section{Introduction}

The ability to accurately identify regions of mixing in two dimensional systems has applications in ocean [1] and geophysical systems [5], as well as granular flows [3]. Over the last decade, much work has been put into finding coherent structures in the Lagrangian frame ([7], [8], and [9]). Most of these methods require field data (e.g. velocity or vorticity fields) which in situations like the ocean are not readily available. However, trajectories of tracers for these systems can accurately be measured and are available for analysis. This indicates that a method which estimates the location of Lagrangian structures based on trajectory data would be highly valuable.

The Lagrangian structures we are looking for are defined as regions of mixing surrounded by a transport boundary. These moving regions contain fluid which mixes with other fluid within the boundary but not with fluid outside the region. This self mixing region can move through the full system and can change shape. The transport boundaries in the fluid effectively divide up the fluid into regions of qualitatively different dynamics. What separates these transport boundaries from other material lines in the fluid is that the length of these boundaries stays the same order of magnitude throughout the time evolution. If the material line perimeter stays of the same order of length then there is negligible mixing occurring between fluid inside and outside of the transport boundary.

Throughout this report, a sample system will be used to demonstrate many of the features and concepts presented. The application of this system to two regions of fluid is presented in Section 3.3. Figure 1a depicts thirty trajectories as well as two regions of interest (blue and green) in an initial state. At a later time the position of the trajectories and the regions of interest are presented in Figure 1b.

While the boundaries of the two regions distorts the trajectories that start within a region remain in that region for all time. An important observation is that while the blue 


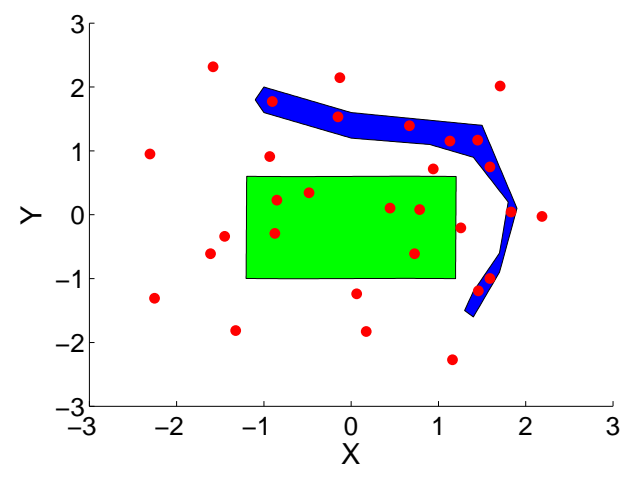

(a)

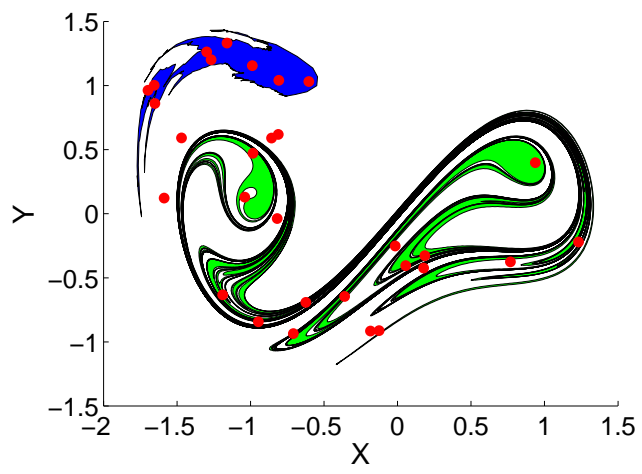

(b)

Figure 1: (a) Initial state (b) State after application of system described in Section 3.3. The blue region is surrounded by a mixing transport boundary. The green region is not. Float positions are shown as red dots.

region has changed shape, the perimeter has remained approximately the same length. This indicates that the boundary to the blue region would be classified as a mixing transport boundary. The green region shows substantial folding and stretching of the boundary as trajectories from outside the region try to mix with points inside the region. Thus it can be concluded that, this boundary would not be classified as a mixing transport boundary.

The goal of this project is to develop a method for finding Lagrangian structures, like the boundary of the blue region in Figure 1, from trajectory data. We will use the property of a negligibly growing perimeter as the differentiating characteristic between transport boundaries and other material lines in the fluid. In order to do this, we have developed an algorithm that analyzes the trajectory data and makes predictions of where these structures are located. The novelty of this algorithm is its reliance on the tools of braid theory.

The concept of using braid theory for the analysis of trajectories was presented by Thieffeault [11] in the context of quantifying Lyapunov exponents in mixing two dimensional fluids. He applies tools from braid theory to calculate the topographical entropy of a system which is a measure of the mixing occurring in the system. The present research takes this work as a basis but develops some of the concepts in a direction which finds the mixing transport boundaries in the system.

This report is structured such that a number of definitions and an introduction of the necessary background of braid theory is first presented. This will be followed by a demonstration that the tools from braid theory can find mixing structures. Next, a revised algorithm will be presented which will make a braid theory based method a reasonable 
option for analyzing large numbers of trajectories in search of transport boundaries. Finally, an application of this method to a mixing system is presented with concluding remarks and future works.

\section{Braid theory}

In order to fully understand the algorithms presented in this report, a number of definitions and tools from braid theory will be provided here. These definitions will be given in the context of applying this theory to a set of trajectories evolving in time on a two dimensional plane. An example set of trajectories for three floats are shown in Figure 2a to provide the reader with a visual example to follow.

The trajectories are constrained to two dimensions in the physical space, and the position of a float at any given time will be referred to as a puncture. For example, there are three punctures in Figure 2a and are represented by colored dots. The initial positions of the punctures are also presented in the figure as crosses.

The trajectories that the punctures traverse can be projected into a three dimensional space where the horizontal plane is the physical domain and the vertical axis is time. These three dimensional trajectories are called strands. Due to the monotonic nature of time, a strand can only travel upwards because it can not travel backwards in time. If these three dimensional strands are projected onto the plane containing the x-axis and time then Figure 2a becomes Figure 2b.

The collection of strands make up what is called the braid. The braid is uniquely defined by how the strands intertwine, or cross in the projected view. The strands itself can be perturbed and the braid will be considered unchanged as long as the perturbations do not change the direction, number or order of the crossings. The braids shown in Figure $2 \mathrm{~b}$ and $2 \mathrm{c}$ are the same braid even though the strands have been transformed to a different location and over a different time. They reason the two images depict the same braid is because the green strand passes in front of the black strand first then the black in front of the red strand in both cases.

\section{$2.1 \quad$ Generators}

Given the importance of the crossing of strands in the braid, it is necessary to define what in braid theory are called generators, $\sigma$. Generators act on a braid by taking a pair of strands and crossing them. For example the braid shown in Figure 2 has two generators acting in a sequence. The first generator causes the green strand to pass in front of the black strand, or equivalently causes the strand in the first index location to pass in front of the strand in

the second index location. The second generator causes the black or second index strand to 


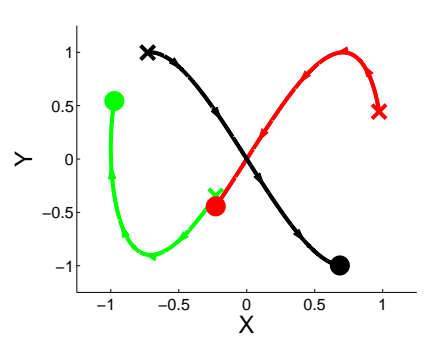

(a)

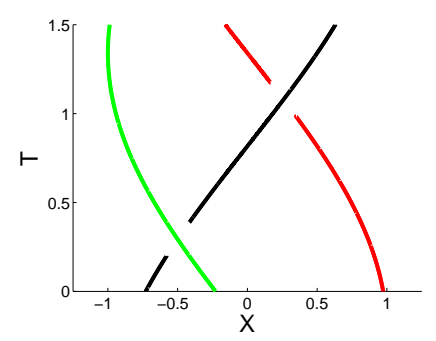

(b)

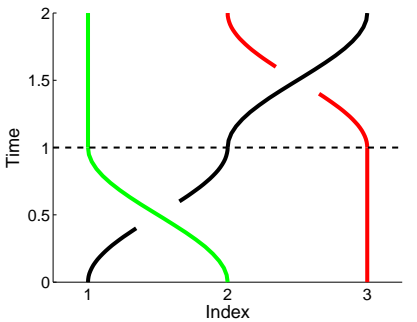

(c)

Figure 2: (a) Three sample trajectories shown in the physical plane. An " $\mathrm{x}$ " represents the initial condition of the trajectory and a dot represents the current position. (b) The projection of the trajectories onto the $\mathrm{x}$-axis as a function of time. When a trajectory passes behind another trajectory a gap is placed in the trajectory. (c) The transformation of the braid in (b) to a plot of their indexes as a function of time.

pass in front of the red or third index strand. Note that the black strand has moved from the first to the second to the third index during the application of these generators.

Based on the description of what the generator does it is clear that there are two defining characteristics: first, what strands are involved in the crossing and second, which strand passes in front. These two components can be used to give a numerical value for a specific generator. The strands involved in the crossing are indicated by the magnitude of the generator, and the direction of crossing is indicated by the generator being positive or negative. Since the two strands which cross must be right next to each other, the magnitude of the generator is equal to the lower index of the strands involved in the crossing.

For example, the braid shown in Figure 2c has two generators acting on three strands. The first generator crosses the lower index strand, black, behind the higher index strand. This direction of crossing is defined as positive. The two strands involved in the first crossing are in positions one and two. This indicates that the first generator is $\sigma_{1}=1$. The same procedure can be repeated for the second crossing which takes the strand in index two and crosses it in front of the strand in index three. Thus, the second generator is $\sigma_{2}=-2$. In this case, the generator sequence $\sigma=\left[\begin{array}{ll}1 & -2\end{array}\right]$ fully defines the three strand braid. It should be stressed that the actual strand involved in a crossing does not mater just the indexes where crossing is taking place.

\subsection{Loops}

The next tool which will be used throughout this report is the concept of a loop. A sample

loop is drawn in Figure 3a. A loop is a boundary of a region that can pass around or encapsulate punctures and provides a way for us to divide up the fluid system into regions. 


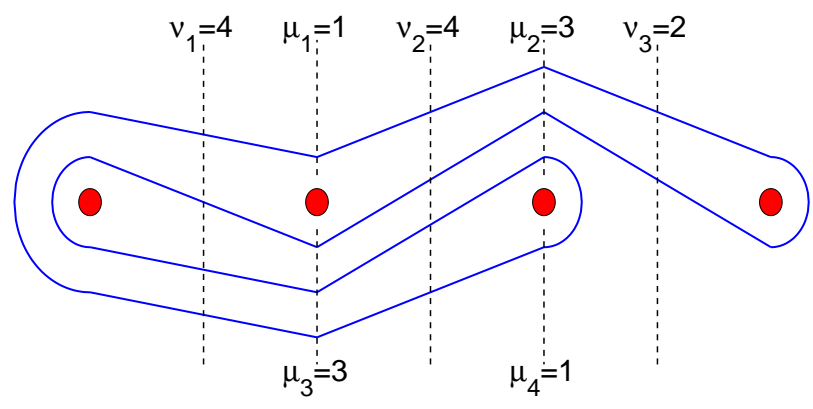

(a)

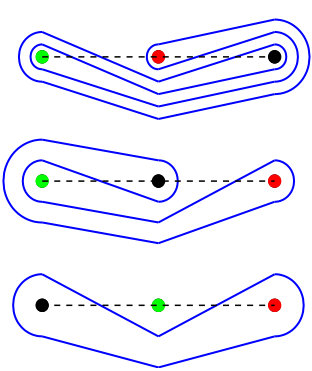

(b)

Figure 3: (a.) Sample loop drawn for a four puncture system. The $\mu$ 's and $\nu$ 's are presented in the plot and the corresponding Dynnikov coordinates are $a=\left[\begin{array}{ll}1 & -1\end{array}\right]$ and $b=\left[\begin{array}{ll}0 & 1\end{array}\right]$. (b.) How an initial loop (shown at the bottom) gets modified by the generators for the system shown in Figure 2. The number of intersection (from bottom to top) are 2,3, and 5.

If a mixing transport boundary exists and contains punctures, it should be possible to find a loop that represents this boundary. A loop representing a mixing transport boundary must have a length that does not grow with time. Clearly if a loop represents the transport boundary it is important to understand some characteristics of loops.

The first important concept is that a loop can be uniquely defined by a coordinate system. The coordinate system uses information on the number of times the loop passes above, below, or between punctures. For example, the number of times the loop passes directly above the second puncture is represented as $\mu_{1}$ and below the second puncture is $\mu_{2}$. Similarly the number of times the loop passes above and below the third puncture are $\mu_{3}$ and $\mu_{4}$ respectively. Additionally the number of times the loop passes the midpoint between punctures is also required for the coordinate system representation. To visualize this, a vertical line at the midpoint between punctures is drawn in Figure 3a and the intersection with the loop can be counted. The three midpoint crossings from left to right are $\nu_{1}, \nu_{2}$, and $\nu_{3}$.

The information about number of passings can be condensed into a minimal coordinate system called the Dynnikov coordinates [4]. Taking the information about number of passes, the Dynnikov coordinates transform the $\mu$ 's and $\nu$ 's by the following relationship

$$
a_{i}=\frac{\mu_{2 i}-\mu_{2 i-1}}{2} \quad b_{i}=\frac{\nu_{i}-\nu_{i+1}}{2}
$$

These coordinates are essentially a comparison of the number of times a loop passes above or below the $i+1$ puncture, $a_{i}$, and the difference in the number of times the loop passes to left and to the right of the $i+1$ puncture, $b_{i}$. The Dynnikov coordinate representation 
for a given loop is $\mathbf{u}=\left(a_{1}, \ldots, a_{n-2}, b_{1}, \ldots, b_{n-1}\right)$. This coordinate system uniquely defines any loop by a sequence of integers, and any even number of integers can be represented as a loop.

With the coordinate system in place it is possible to concisely represent any loop and the next step is to understand how the loop evolves as the generators cause the punctures to change positions. As the generators are applied to the strands, the loop surrounding the strands is also moved. An example application of generators to a loop is shown in Figure $3 \mathrm{~b}$. The initial loop shown at the bottom of the figure is first modified by the generator $\sigma_{1}=1$ then by $\sigma_{2}=-2$ in the same way the strands from Figure $2 \mathrm{c}$ are intertwined.

One of the strengths of braid theory is that there is a set of rules for updating the coordinates presented in [10]. These rules allow for the rapid calculates of how the loops changed position due to a generator and prevent the need for a time intensive advection calculation which would require a velocity field. The simple set of update rules also allows for the analysis of a large number of loops simultaneously which aids in the search for the mixing transport boundaries.

Since we have defined mixing transport boundaries as boundaries with approximately time independent length, we need to establish a way to measure loop lengths. Moussafir demonstrated that the length of a loop is proportional to the number of times the loop crosses a line connecting all the punctures. Figure $3 \mathrm{~b}$ depicts the line connecting the punctures, and an intersection occurs every time the loop crosses this line.

Fortunately, an intersection calculation exists which produces a value proportional to the loop length. The intersection for a given loop, $L(\mathbf{u})[10]$, is calculated by

$$
L(\mathbf{u})=\left|a_{1}\right|+\left|a_{n-2}\right|+\sum_{i=1}^{n-3}\left|a_{i+1}-a_{i}\right|+\sum_{i=0}^{n-1}\left|b_{i}\right|
$$

where

$$
b_{0}=-\max _{i \leq i \leq n-2}\left(\left|a_{i}\right|+\max \left(b_{i}, 0\right)+\sum_{j=1}^{i-1} b_{j}\right) \quad \text { and } \quad b_{n-1}=-b_{0}-\sum_{i=1}^{n-2} b_{i}
$$

This calculation will be used to determine if a loop is growing with time, and thus not a mixing transport boundary, or if the length stays of the same approximately the same length throughout.

Finally, the set of punctures inside by a non-growing loop will be referred to as a structure or puncture structure set. A puncture is considered to be inside a loop if there is no set of generators that will result in the loop not being both above or below the puncture. The punctures (trajectories) inside the loop (transport boundary) will intermix but not mix with 
the punctures outside of the loop.

\subsection{Summary of the application of braid theory}

With the tools and definitions developed in the previous sections, it is now possible to give an overview of the application of braid theory to the problem of finding two dimensional mixing transport boundaries. The trajectories are converted to strands and a generator sequence is calculated from the trajectory crossings. A mixing transport boundary is the equivalent to a loop, which does not show long term growth, surrounding a subset of punctures. The goal of this report is to demonstrate that these loops do exist and can be found.

The search for structures and non-growing loops will be done in two ways. The first method is a methodical search for the non-growing loops by testing Dynnikov coordinates in a sequential manner which is presented in Section 3. This method will be improved upon by a refined searching technique described in Section 4 . For both search methods, a system, see Section 3.3, is analyzed containing a mixing transport boundary.

One assumption made throughout this paper, is that the mixing transport boundaries simply surround a region of the fluid. It seems unlikely that a boundary which weaves in and out of other punctures would not grow with time. Based on this, it is assumed that the Dynnikov coordinates defining the non-growing loops will have small magnitudes for its integer coordinates, because as the coordinate magnitudes grow so does the complexity of the loop.

\section{Proof of concept}

The basis of the proof of concept method is that if a simple non-growing loop, or mixing transport boundary, exists it will be found by analyzing the set of all simple loops. Here we present what is classified as a simple loop, an outline of the code which calculates and analyzes the loops, and a sample system and results of the proof of concept code.

\subsection{Simple loops}

The Dynnikov coordinates provide a compact way of representing loops, and any list of $2 n-4$ integers can create a loop for an $n$ puncture system. Additionally, the loop grows more and more complex as the coordinate values grow larger. Based on the assumption that the non-growing loops are simple loops, the proof of concept code will only search through loops where each of the initial coordinates can have a value between -1 and 1 . Four example loops are presented in Figure 4 demonstrating the range in complexity which is encompassed by this range of Dynnikov coordinates. 

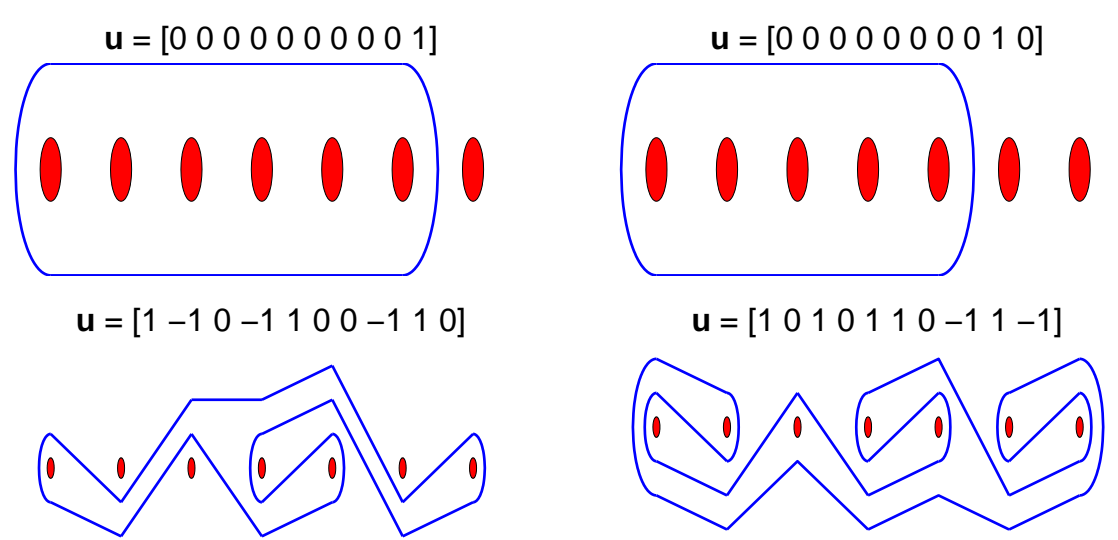

Figure 4: Four loops which fall into the category of simple.

\subsection{Algorithm outline}

In order to find the non-growing loops, an algorithm is developed using Matlab. The objective of this algorithm is to take in a set of trajectories and output the non-growing loop entangling the punctures. The first step is to calculate the generator sequence which defines the braid of the trajectories. Next, for each Dynnikov coordinates which has coordinate values between -1 and 1 inclusively, the entire generator sequence is applied and the resulting loops have their intersections calculated. The loops with the smallest final number of intersections are designated as non-growing loops.

\subsubsection{Calculation of Generators}

The generator sequence for a given set of trajectories is calculated by a procedure called the color braiding method. Pairs of strands are analyzed to determine when the strands cross. This method is called "colored" because each strand is distinguishable throughout searching through the pair and it matters which strand starts in the lower index position. This differs from the final generator sequence where only the indexes of the crossing are considered and not which strands are involved in the crossing.

The color braiding search starts first by ordering the braids based on the initial value of their $x$-coordinate. Then the code loops through each pair of strands where there are $\left(n^{2}-n\right) / 2$ pairs for systems with $n$ strands. For the pair of strands, three pieces of information are saved for each crossing: the time of crossing, the direction (either clockwise or counter-clockwise based on the $y$ value at the time of crossing), and the order of the strands before crossing. After all pairs of strands have been analyzed, the crossings are collected and ordered by time of crossing.

The final step is to convert the list of crossings to a generator sequence. In the cur- 


\begin{tabular}{|c|c|c|c|c|c|c|c|c|}
\hline$t_{\text {crossing }}$ & Direction & Left Strand & Right Strand & $I$ & $=$ & {$\left[\begin{array}{lll}1 & 2 & 3\end{array}\right]$,} & & \\
\hline 1.245 & 1 & 2 & 3 & $I$ & $=$ & {$\left[\begin{array}{lll}1 & 3 & 2\end{array}\right]$,} & $\sigma_{1}$ & $=$ \\
\hline 2.142 & 1 & 1 & 3 & $I$ & $=$ & {$\left[\begin{array}{lll}3 & 1 & 2\end{array}\right]$,} & $\sigma_{2}$ & $=$ \\
\hline 3.145 & $\overline{-1}$ & 1 & 2 & $I$ & $=$ & {$\left[\begin{array}{lll}3 & 2 & 1\end{array}\right]$,} & $\sigma_{3}$ & $=$ \\
\hline
\end{tabular}

Figure 5: A sample system featuring three strands and three crossings. The left table demonstrates the information from the comparison between pairs of strands. The right side shows the index vector as its being changed by the crossings and the resulting generator sequence.

rent state, information on which strands cross is available but the generator sequence is dependent on the index positions involved in crossings. This conversion is accomplished by creating a vector naming each strand after the strands initial index and applying each crossing chronologically. For each crossing, the two strand names are found and their positions in the vector are switched. The smaller index of the strand locations becomes the generator magnitude and the direction determined by color braiding is applied to calculate the generator. The calculated generator sequence is then saved with the corresponding times. A sample system is shown in Figure 5 and outlines the conversion from an ordered list of color-braiding information to a generator sequence..

\subsubsection{Calculating loops, applying generators, and intersection calculation}

The next step in the algorithm is to cycle through all possible simple loops and apply the generator sequence. As mentioned in Section 2.2, an $n$ puncture system is represented by $2 n-4$ coordinates and only values of $-1,0$, and 1 will be considered for each coordinate. This results in $3^{\wedge}(2 n-4)-1$ possible loops which must be checked (Note: the loop with all coordinates equal to zero is not considered a loop).

A simple methodical method for representing all these loops is to create a list from 1 to $3 \sim(2 n-4)$ and convert the list to their respective values in base 3 . The resulting numbers are then converted to a string of length $2 n-4$ where the necessary number of zeros have been appended to the left side of the string. Finally, each value in the string is reduced by one producing the Dynnikov coordinates. With each loop the generator sequence is applied by utilizing the transformation rules and a final loop is ready to be measured.

The last step of the algorithm is to take each final loop and calculate the number of intersections. This is a simple calculation based on Equation 1. Given that the number of intersections is proportional to its length, a criterion is set by the user which will define a loop as growing or not growing. If the number of intersections falls below this threshold it represents an approximation for the location of a mixing transport boundary in the fluid. 


\subsection{Modified Duffing Oscillator system}

In order to test this algorithm, it is necessary to develop a system where there is a mixing transport boundary. This system's velocity field will be used to calculate trajectories for analysis. While this would not be the target application of the algorithm, having a test case where it is possible to determine a priori which punctures make up a structure.

The system selected for this test is a modification of the Duffing Oscillator. One change is made in order to create two mixing regions and the other change adds solid body rotation. The base system without rotation, $\mathbf{x}=f(\mathbf{x})$ is defined as

$$
\begin{aligned}
& \dot{x}=y+\alpha \cos (\omega t) \\
& \dot{y}=x\left(1-x^{2}\right)+\gamma \cos (\omega t)-\delta y .
\end{aligned}
$$

where $\alpha=.1, \gamma=.14, \delta=.08$, and $\omega=1$. To add rotation to the system, the following transformation is made

$$
\begin{aligned}
\tilde{\mathbf{x}} & =[R(t)] \mathbf{x} \\
\dot{\tilde{\mathbf{x}}} & =\left[\frac{d}{d t} R(t)\right] \mathbf{x}+[R(t)] \dot{\mathbf{x}} \\
\dot{\tilde{\mathbf{x}}} & =\left[\frac{d}{d t} R(t)\right][R(t)]^{-1} \tilde{\mathbf{x}}+[R(t)] f\left([R(t)]^{-1} \tilde{\dot{\mathbf{x}}}\right)
\end{aligned}
$$

where

$$
[R(t)]=\left[\begin{array}{cc}
\cos (\Omega t) & \sin (\Omega t) \\
-\sin (\Omega t) & \cos (\Omega t)
\end{array}\right] .
$$

The key feature of this system is that there are two mixing regions. With the velocity field, it is possible to find these regions at the initial time, and a plot of the two regions is presented in Figure 6. This plot indicates that all points initially in the gray region should make up a structure, and all punctures initially in the white region should make up a second structure. Each of these structures should have a non-growing loop surrounding the punctures in the given structure.

\subsection{Results of basic algorithm}

To test the proof of concept algorithm a variety of sets of initial conditions are tested. A single representative example is presented here. This system consists of seven initial conditions which are shown in Figure 6. Based on that figure, it is expected that four of the punctures should make up one structure and the other three punctures will form a second 


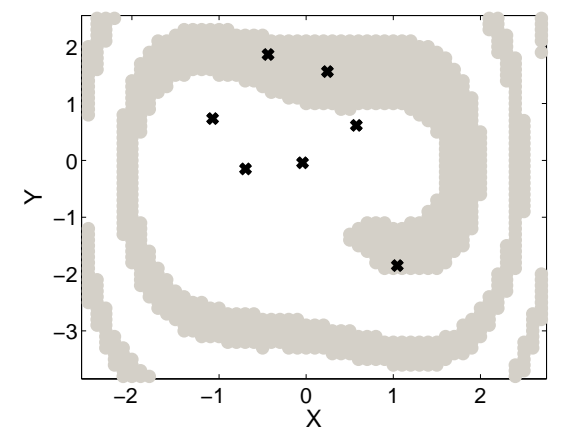

Figure 6: Mixing regions for the modified Duffing oscillator system. The white region collapses to a chaotic mixing region at the center. The gray region approaches a limit cycle around the white region. The black x's are the initial conditions for the trajectories discussed in Section 3.4.

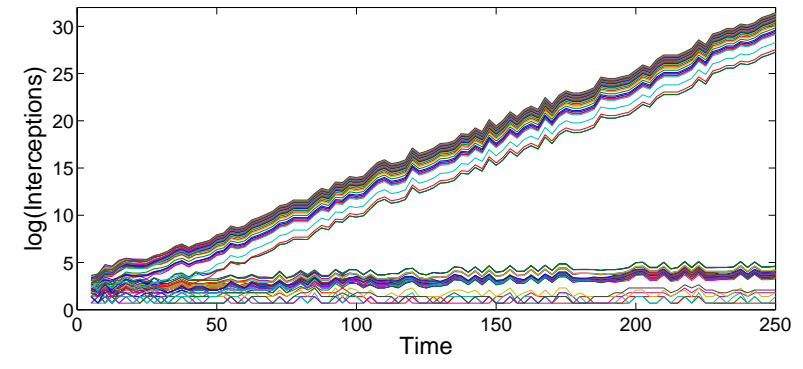

(a)

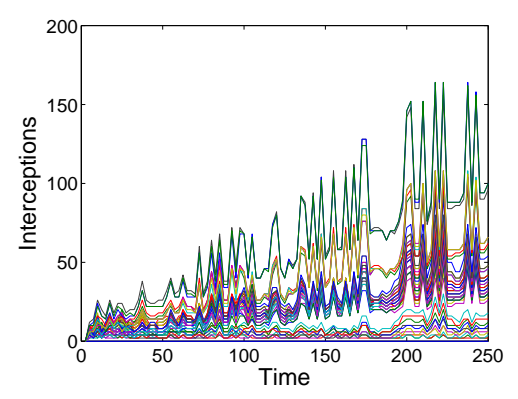

(b)

Figure 7: (a) Semi-log plot of the intersections as a function of time for all possible loops with Dynnikov coordinate values ranging from -1 to 1 . (b) Plot of the intersections as a function of time for loops where growth appears to be non-exponential.

structure. With these trajectories, the corresponding generators are calculated and the $3^{10}$ loops are manipulated via the transformation rules. The lengths of the resulting loops are then calculated.

For a representative set of loops in the defined range, the log of the intersections as a function of time are plotted in Figure 7a. This plot indicates that there are two regimes of growth: loops growing exponentially (linearly increasing lines in the plot) and loops that show non-exponential growth (lines approximately flat in the plot). The loops growing exponentially will not correspond to loops surrounding a structure, so they can be neglected. It should be noted though that they all share the quality that the loops enclose a subset of the four punctures which mix chaotically in the center but never all four.

A closer view of the non-exponentially growing intersections are shown in $7 \mathrm{~b}$. This plot is not on a log scale and shows that some of the loops grow approximately linearly while 
(a)

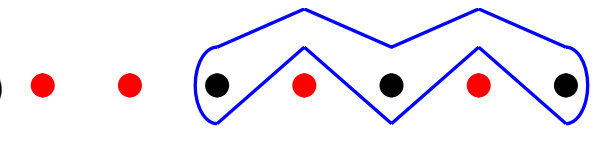

(b)

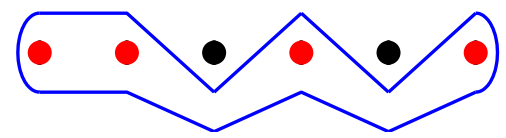

(c)

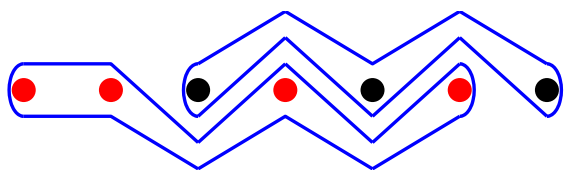

Figure 8: Simplified loop diagrams of the loops which do not show steady state growth. (a) Loop containing the orbiting trajectories (black punctures). (b) Loop containing the inner trajectories (red punctures). (c) Double loop featuring both structure loops.

others don't grow at all. The loops which grow linearly contain all four trajectories in the chaotic region and a subset of the limit cycle region trajectories. The loops which don't show growth, presented in Figure 8, correspond to mixing transport boundaries. Changes in the loop do occur throughout the mixing due to the moving of the particles, but there is no steady state growth of the initial loop.

The expected transport boundaries were found indicating that the proof of concept successfully applied the tools from braid theory to the two dimensional trajectories. One drawback from the proof of concept method is that it is limited in application due to the run times. For systems of eight or fewer punctures, it takes seconds to find the nongrowing loops, but for each trajectory added after eight, the run time increases by almost an order of magnitude. This is due to the bottleneck of the code being the application of generators to loops. This process is repeated for each loop and the number of loops searched is $3^{\sim}(2 n-4) \sim 9^{n}$. This fact renders the method useless for systems with more than eleven punctures.

\section{Refined loop finding algorithm}

While the methodical search through all simple loops produces the non-growing loops, it is not a realistic options for systems where there are thirteen or more punctures (run times would be on the order of four days). The target application for this method is systems with a large number of tracers which mix well so having a capacity for analysis of only thirteen particles is not sufficient. For braid theory to be a reasonable method for finding mixing transport boundaries, it is necessary to develop a code where the time to find non-growing 
loops is not $t \sim O\left(9^{n}\right)$.

One major drawback of the previous method is that no information is gained from a loop which is growing, so every loop has to be tried. The refined algorithm tries a specific set of loops and analyzes their state after the generators have been applied. The loops analyzed connect two punctures. By letting these loops evolve their final state gives an indication of which punctures become entangled by the loop. Now links can be drawn between which punctures entangle which other punctures. This is the basis for the refined algorithm.

The objective of the refined algorithm is still to take in a set of trajectories and output the punctures within a structure and the non-growing loop which surrounds the loop. The outline for this algorithm is still to first calculate the generator sequence which defines the braid. This generator sequence will be applied to a set of Dynnikov coordinates which connect pairs of punctures, described in Section 4.1. The resulting loops are then analyzed to determine which punctures are entangled by the loop, Section 4.2. With the information on entangled sets, a code determines which punctures form a structure, Section 4.3. Finally, the punctures which make up a structure are surrounded by a loop which does not grow, Section 4.4. After the new algorithm has been described, its application to the modified Duffing oscillator will be presented.

\subsection{Pair-loops}

As mentioned in the description of the algorithm, the loops of initial interest are ones which connect a pair of punctures. While there are an infinite number of ways for a loop to connect punctures, the main concern here is how simple loops entangle the other punctures. Given this consideration, punctures that neighbor each other will be connected like loop $(1,2)$ in Figure 9a. For punctures that are not neighbors, two loops are created where one loop passes above intermediate punctures, loops $(1,3)$ and $(1,4)$ in Figure 9a, and the other passes below, loop $(3,1)$ or $(4,1)$ in Figure 9 a.

For a system of $n$ particles there are $n-1$ loops connecting neighbors and $(n-2)(n-1)$

loops connecting above and below resulting in a total of $(n-1)^{2}$ loops to analyze. In the last method, the bottle neck was the application of the generators to the $3 \sim(2 n-4)-1$ loops causing the run time to be $t \sim O\left(9^{n}\right)$. Now if the bottle neck of the algorithm is still the application of the generators, then at least the run time has become $t \sim O\left(n^{2}\right)$ which grows substantially slower particularly for large $n$. Figure 9 a is an example for the initial loops which are considered for any five puncture system. 


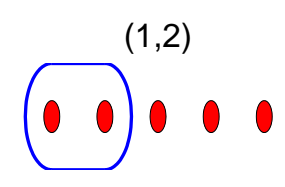

$(3,1)$

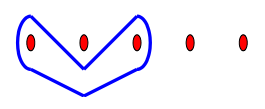

$(4,1)$

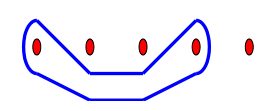

$(5,1)$

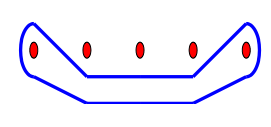

$(1,2)$

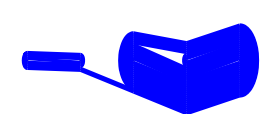

$(3,1)$

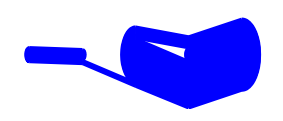

$(4,1)$

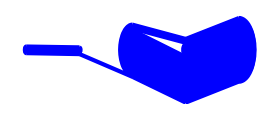

$(5,1)$

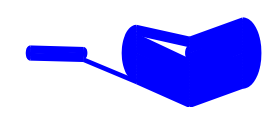

$(1,3)$
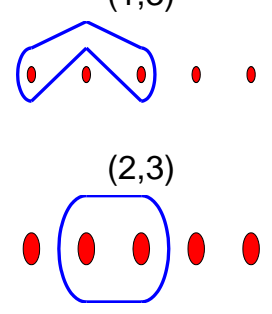

$(4,2)$

-

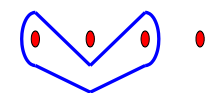

$(5,2)$

-

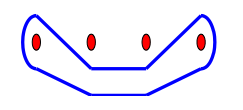

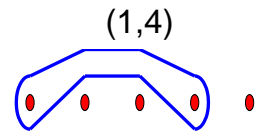
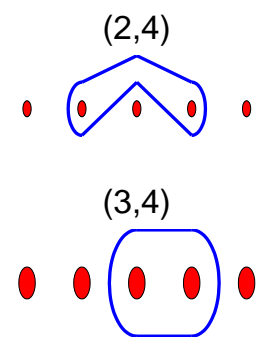

$(5,3)$

0

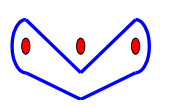

(a)

$(1,3)$

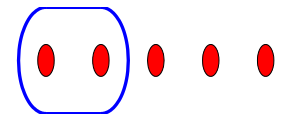

$(2,3)$

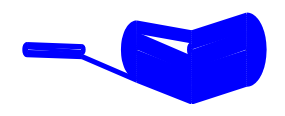

$(4,2)$

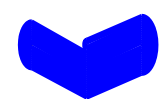

$(5,2)$

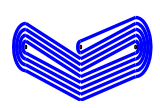

$(1,4)$

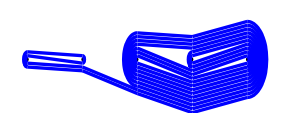

$(2,4)$

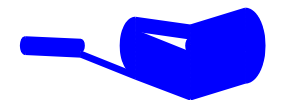

$(3,4)$

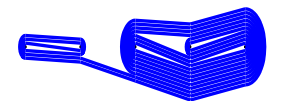

$(5,3)$

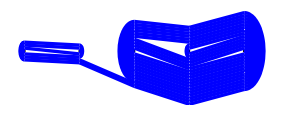

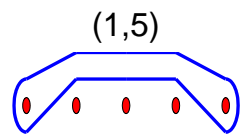

$(2,5)$

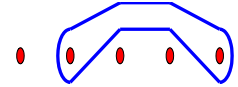

$(3,5)$

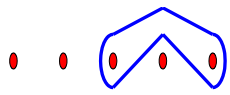

$(4,5)$

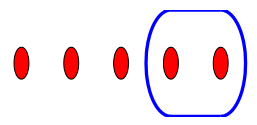

$(1,5)$

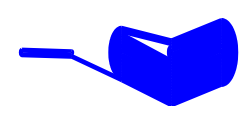

$(2,5)$

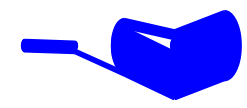

$(3,5)$

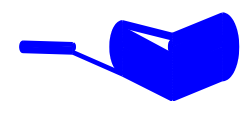

$(4,5)$

(b)

Figure 9: (a.) The initial loops for a five puncture system. The notation for each initial loop is $(a, b)$ where the loop goes from $a$ to $b$ in a clockwise direction. (b.) The loops which result from applying a generator sequence. Only loops $(1,3),(4,2),(5,2)$, and $(4,5)$ do not entangle all punctures after generator application. This group of trajectories form two puncture structure sets $(1,3)$ and $(2,4,5)$ 


\subsection{Calculation of entangled punctures sets}

After the generator sequence has been applied to the pair-loops, the code needs to determine which punctures are entangled by the loops. We will describe a puncture as disentangled with a loop if the loop does not pass above and below the puncture. To determine this the final loop Dynnikov coordinates are converted to the PMN-coordinate system. The PMN-coordinate system calculates the number of times the loop passes above $\left(M_{i}\right)$ and below $\left(N_{i}\right)$ the $i$ th puncture as well as half the number of intersections of the loop with the mid-line between punctures $\left(P_{i}=\nu_{i} / 2\right)$. For example the loop presented in Figure 3a has $M=\left[\begin{array}{llll}2 & 1 & 3 & 1\end{array}\right], N=\left[\begin{array}{llll}2 & 3 & 1 & 1\end{array}\right]$, and $P=\left[\begin{array}{lll}2 & 2 & 1\end{array}\right]$.

With this new coordinate representation, it is straightforward to determine if a puncture is entangled or not. For the $i$ th puncture to be disentangled either $M_{i}$ or $N_{i}$ is zero for the loop after applying the generator sequence. The code searches for all entangled punctures for a given loop and then saves entangled set and the corresponding initial loop. This process is repeated for each pair-loop. After all loops have been processed, the entangled sets are condensed to remove any repeated sets.

For the example presented in Figure 9, the generators have been applied to the loops in Figure 9a and they produce the loops in Figure 9b. Of the sixteen loops only four do not entangle all the punctures. The code only considers entangled sets which are a subset of the full set of punctures. The loop $(1,3)$ entangles only punctures 1 and 3 . The loops $(4,2)$, $(5,2)$, and $(4,5)$ entangle only the punctures 2,4 , and 5 . This means the example results in two entangled sets which also happen to be the structures for this system. It is possible to find a non-growing loop around punctures 1 and 3 and one around 2,4,and 5 .

\subsection{Determining punctures in a structure}

While the example presented in Sections 4.1 and 4.2 produce the structure sets directly from the entangled puncture sets, this is not always the case. For a pair-loop, the two punctures are either within the same structure or not. If a loop connects points within a structure, the loop can either entangle a subset of punctures within the same structure, all punctures within the structure, or all punctures within the structure as well as punctures from another structure. In the simple example, loops connecting points within a structure always only entangled all the points within the same structure. Alternatively, if a loop connects punctures from different structures they will entangle all the punctures from the two structures and possibly other structures as well. The code attempts to take these entangled sets and decipher which punctures form a structure.

To create the structures, the code runs through the entangled sets and allocates a structure index to the set. The entangled sets are read in from largest to smallest, so the 
first and largest set is labeled as structure 1 and then the next entangled set is analyzed. If the second set intersects a structure, then all points in the second set are labeled with the same structure index. However, if the second set is disjoint from the structures, it is given a new structure index. This process is repeated for each of the remaining entangled puncture sets. It is possible for an entangled set to intersect multiple structures in which case the structures as well as the entangled set will all merge into one structure.

At the end of this process, there are two options either a set of disjoint structures have been created or a single structure containing all punctures. If the latter option is produced, the code reruns but instead starts with the next smallest entangled set and repeats the process till it creates multiple structures. Based on the method of finding structures, it is very easy for multiple structures to get combined into one larger structure. To combat this issue the code has the capability to rerun the full algorithm on a structure to determine if it can be broken down into smaller structures. If the code runs through all the possible starting positions, and it can still only find a structure containing all punctures, it concludes that there are no structures present and the absence of non-growing loops.

For clarity, the following is an example where there are three known structures $\{1,2\},\{3,4\}$ and $\{5,6\}$. The entangled sets are

$$
\begin{gathered}
\{1,2,3,4\}, \\
\{3,4,5,6\}, \\
\{1,2\}, \\
\{3,4\}, \\
\{5,6\} .
\end{gathered}
$$

Starting with the first entangled set leads to the formation of one structure after trying four sets. Since only one structures is found, the code restarts from the second entangled set. This results in two structures $\{1,2\}$ and $\{3,4,5,6\}$. The first structure is one of known structure while the second combines two of the known structures. This fact would be resolved after rerunning the $\{3,4,5,6\}$ trajectories through the full algorithm.

\subsection{Calculating Dynnikov coordinates for a structure}

The final task for the algorithm is to find a loop for each structure which surrounds the punctures and does not grow. A loop connecting the structure's puncture set can be viewed as the sum of the loops connecting punctures in a structure. An example is presented in Figure 10 where three loops connect the punctures in a structure then are merged to form one loop. The task of this portion of the algorithm is to determine how to connect the punctures in a structure then merge these loops together.

If a loop surrounding a structure does not grow and it is composed of links between punctures, then the links necessarily should not grow due to being mixed by punctures 


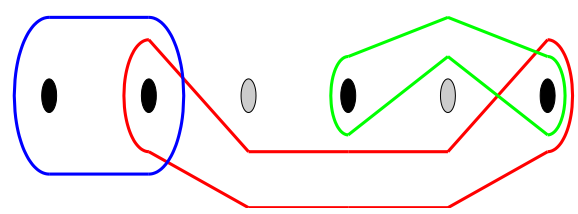

(a)

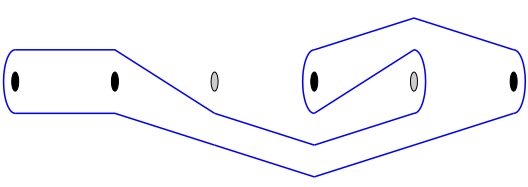

(b)

Figure 10: Punctures 1,2,4, and 6 are in a structure. (a.) The loops shown here connect the punctures. Blue connects 1 to 2 , red connects 2 to 6 , and green connects 6 to 4 . (b.) The connecting loops can be merged to form a single loop around the punctures.

outside the structure. This means that if all punctures from a structure are removed except for two that are linked then the loop that links the remaining structure-punctures should not grow. From the example in Figure 10, if punctures 4 and 6 were removed, the loop connecting punctures 1 and 2 should not grow in a braid of strands 1,2,3, and 5 . The code takes advantage of this property, in order to find the links.

This portion of the code takes in the punctures that are in a structure and outputs the loop surrounding the punctures which does not grow. Using the property previously described as the basis for this method, the code selects a pair of punctures in the structure then calculates the generator sequence for a braid containing only these two punctures and the punctures not in the structure. With the generator sequence simple loops connecting the two punctures of interest are created and the generators are applied. The code calculates the number of intersections and determines if there is a non-growing loop. Once all the punctures are linked together, the loops are merged and the final loop is analyzed to confirm it does not grow. This concludes the algorithms task.

\subsection{Application to modified Duffing oscillator}

The goal of the refined method is to turn the use of the braid theory into a potential method for finding mixing transport boundaries. The run times from the proof of concept code significantly limit that approaches applicability, but by changing the number of loops searched from $O\left(n^{2}\right)$, there is potential in the refined method. For a direct comparison, a set of five $n$ puncture systems are randomly created and run for both algorithms. The results of these times are presented in Table 1.

The run time results demonstrate the drastic advantages that the refined method offers. While systems with fewer than nine punctures actually complete their runs in a faster time using the proof of concept algorithm, the refined algorithm allows the braid theory approach to be applied for systems with much larger numbers of punctures. While the time growth rate for the proof of concept clearly becomes the predicted $t \sim O\left(9^{n}\right)$, the refined algorithm 


\begin{tabular}{|l||c|c|c|c|c|c|c|c|}
\hline \# of punctures & 5 & 6 & 7 & 8 & 9 & 10 & 11 & 20 \\
\hline Proof of Concept & 0.33 & 0.46 & 0.70 & 5.98 & 53.20 & 462.18 & 3445.39 & N/A \\
\hline Refined & 6.71 & 9.46 & 11.61 & 12.34 & 12.87 & 15.71 & 20.48 & $127.86^{*}$ \\
\hline
\end{tabular}

Table 1: Run times for the modified Duffing oscillator using the proof of concept and refined algorithms. * The run with 20 generators had over four times as many generators which contributes to this being higher than the trend would suggest.

does not demonstrate a specific trend in growth time. This is due to the fact that the application of generators is not the bottle neck of the algorithm. The larges amount of time is spent calculating the non-growing loop from the structures.

\section{Finding non-growing loops in a rod mixed system}

The final step in this report is to apply this algorithm to an actual fluid system. The system selected for a first application test is the mixing performed by the translatingrotating mixing system presented by Finn and Cox [6]. Their mixing mechanism features a circular domain of fluid and a circular stirring rod located somewhere in the fluid. The rod has the capability of both translation and rotation about its center and the outer boundary of the domain has the ability to rotate about its axis. The authors are able to develop an analytic solution for the fluid motion when the rod is moved slowly through the fluid.

An interesting result from this type of mixing is the appearance of islands of poor mixing when the circular rod traverses a periodic trajectory through the fluid. These islands have a transport boundary around them which remains the same order of size and mixing inside and outside don't interact. The presence of these islands is undesirable because the purpose of the mixing is to homogenize the entire domain. With the authors analysis it is possible to make accurate estimations on the size and locations of these islands by calculating an iterated or Poincare map. Our objective is to determine if the refined braid theory algorithm can also find these islands.

A sample system is specified by setting the trajectory of the rod, presented in Figure 11a, and a set of initial conditions for the trajectories, presented in Figure 11b. A set of forty initial conditions with half distributed in seven islands and the other half distributed throughout the well mixed chaotic sea. The rods position is also taken as a trajectory bringing the total to forty-one. The initial conditions are tracked for five periods of the mixing, and their trajectories produce a seven thousand generator long sequence.

The application of the refined algorithm takes approximately five and a half minutes and produces the results roughly drawn in Figure 12. The loops plotted are drawn by hand but accurately represent the non-growing loops. An algorithm to represent the non-growing 


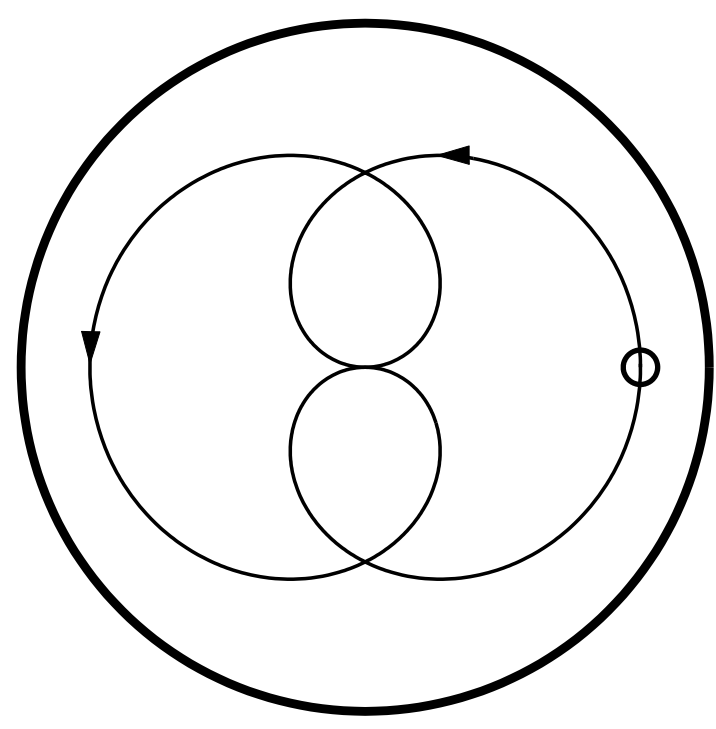

(a)

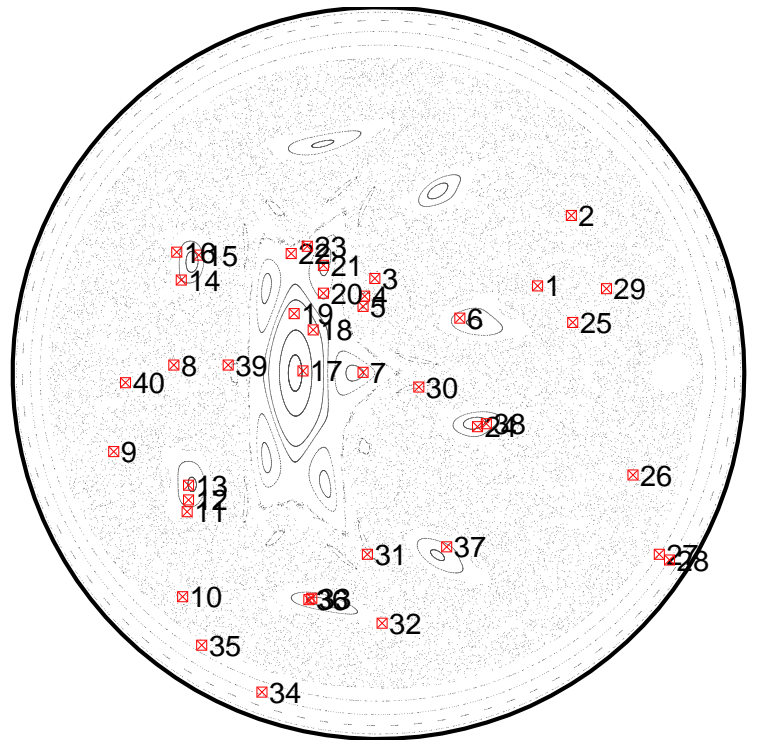

(b)

Figure 11: (a.) Mixing geometry for sample system. (b.) Iterated map demonstrating the islands and the initial positions of the forty trajectories.

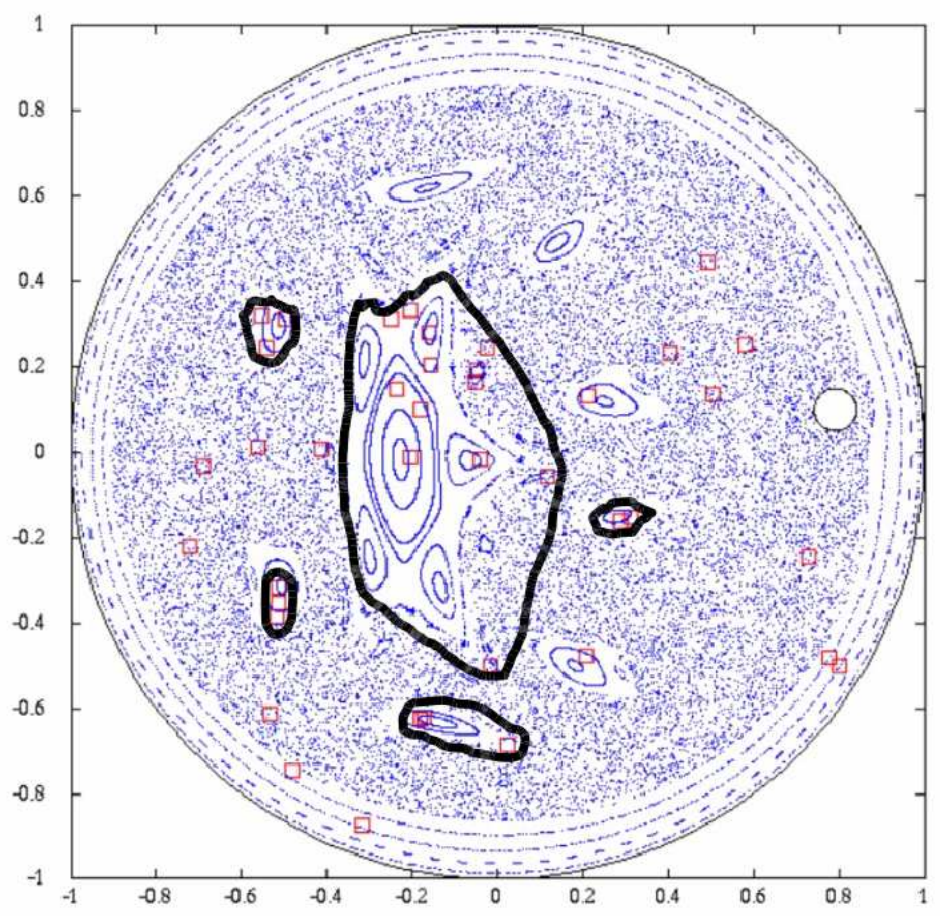

Figure 12: Mixing rod system with the initial conditions and the non-growing loops drawn in. 
loops in development.

The first observation is that not all of the islands have a non-growing loop around them. The reason for this is that there are two islands which don't have any trajectories in them and two with only one trajectory. This algorithm relies on multiple trajectories being inside a mixing transport region in order to find them. If there are no trajectories, then the code is unaware that the region exists, and if there is only one trajectory then the code does not recognize a non-growing loop around a single point.

The other observation is that some of the loops include nearby trajectories in the chaotic sea. The largest loop and the loop at the bottom of Figure 12 both are examples of nongrowing loops beyond the boundaries of the island. The justification for this is that in the period of time analyzed, none of the punctures in the chaotic sea, the other islands, or the rod pass between the islands and its nearby neighbors which end up in the structure. If more time were analyzed or a larger number of punctures, the probability that a puncture would pass between the island and the nearby puncture becomes higher, and if this passing were to occur the code would find a loop around only punctures in the island.

\section{Conclusion}

This report has presented the concept of using braid theory to find mixing transport boundaries in two dimensional systems using float data. Introductory information on braid theory is provided for a foundation to the reader, and then a proof of concept algorithm is developed and demonstrated. With the conclusion that the proof of concept algorithm is not applicable to systems with more than thirteen trajectories, a refined algorithm is presented which uses a pair wise method for searching for non-growing loops. This method successfully reduced the computation time and significantly increased the number of trajectories which can be analyzed. This algorithm is then applied to the real fluid system of a viscous fluid being mixed by a moving rod.

Some observations on the limitations of this algorithm are presented in Section 5, but there are more issues than these system specific considerations. In order for the algorithm to work it is necessary that the significant mixing is occurring in the system. The accuracy of the sets of punctures forming structures is directly proportional to the number of generators in the system.

Another potential issue is that the float trajectories have to be highly accurate. As previously stated, it is necessary to have the correct generator sequence in order to find loops which do not grow. If the position data does not have a high resolution, it is possible a crossing will be missed leading to inaccurate structures and false non-growing loops. While it is straightforward to refine a trajectory from a velocity field, this may not be an option 
for trajectory data taken from a float in the ocean.

Even though these issues do limit the application of the refined algorithm and braid theory somewhat, there is still the potential to find structures in well mixed systems with a large number of particles. One potential area of application is the search for clustering in granular flow data. There is a need to be able to find clusters of particles which move together and mix amongst themselves [2]. The refined algorithm could be used to take trajectories of grains and find these clusters.

In addition to finding applications of this algorithm there are a number of directions for future work. One improvement on the algorithm would be to add the ability to plot the nongrowing loops on the data as the system evolves. Current attempts to plot the non-growing loops has been unsuccessful. It would also be valuable to determine the success rates of the refined code for various crossings per trajectory values. This would give a sharper range of applicability of the refined algorithm.

\section{Acknowledgments}

I would like to thank Jean-Luc Thiffeault for introducing me to the world of braid theory, helping with development of the proof of concept algorithm, and general guidance throughout the project. Matt Finn offered help in testing the refined algorithm on the rod stirred system. Additionally, the author would like to thank the rest of the faculty that aided in the Woods Hole summer program as well as the other fellows for an enjoyable and educational summer. Finally, I would like to thank the NSF for providing funding for this wonderful opportunity.

\section{References}

[1] F. Beron-Vera, M. J. Olascoaga, and G. J. Goni, Oceanic mesoscale eddies as revealed by lagrangian coherent structures, Geophysical Research Letters, 35 (2008).

[2] D. Bonamy, F. Daviaud, L. Laurent, M. Bonetti, and J. P. Bouchaud, Multiscale clustering in granular surface flows, Physical Review Letters, 89 (2002).

[3] T. G. Drake, Structural features in granular flows, Journal of Geophysical Research, 95 (1990), pp. 8681-8696.

[4] I. A. Dynnikov, On a yang-baxter map and the dehornoy ordering, Russian Math Surveys, 57 (2002), pp. 592-594.

[5] C. G. Farnetani And H. Samuel, Lagrangian structures and stirring in the earth's mantle, Earth and Planetary Science Letters, 206 (2003), pp. 335-348. 
[6] M. D. Finn And S. M. Cox, Stokes flow in a mixer with changing geometry, Journal of Engineering Mathematics, 41 (2001), pp. 75-99.

[7] G. HALLER, Lagrangian structures and the rate of strain in a partition of twodimensional turbulence, Physics of Fluids, 13 (2001).

[8] G. Haller AND G. YUAN, Lagrangian coherent structures and mixing in twodimensional turbulence, Physica D, 147 (2000), pp. 352-370.

[9] M. Mathur, G. Haller, T. Peacock, J. Ruppert-Felsot, and H. L. Swinney, Uncovering the lagrantian skeleton of turbulence, Physical Review Letters, 98 (2007).

[10] J.-O. Moussafir, On computing the entropy of braids, Functional Analysis and Other Mathematics, 1 (2006), pp. 37-46.

[11] J.-L. Thiffeault, Braids of entangled particle trajectories, Chaos, 20 (2010). 


\title{
Cave Rings
}

\author{
Emma Boland ${ }^{12}$
}

October 13,2010

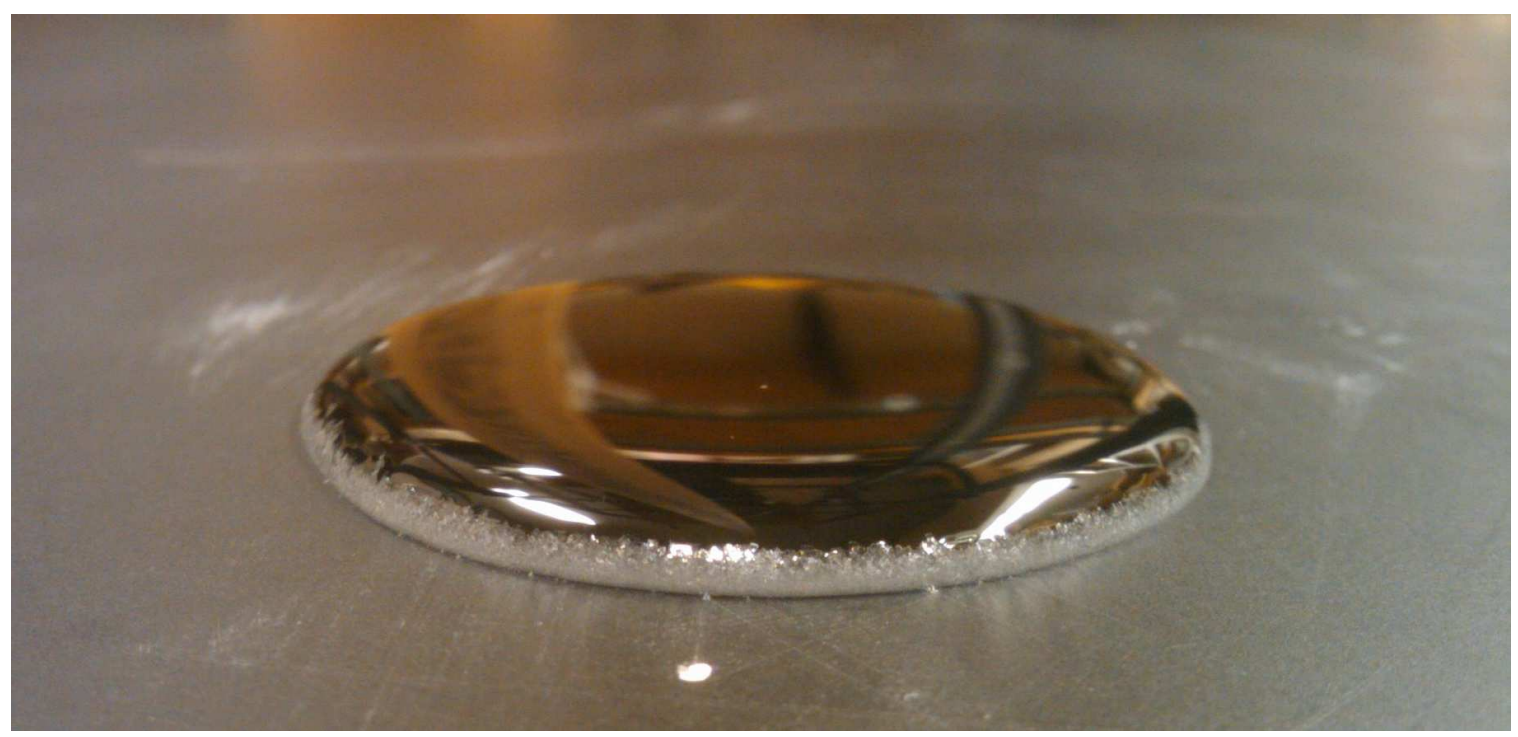

with Neil Balmforth ${ }^{3}$ and Colm-Cille Caulfield ${ }^{1}$

1. The University of Cambridge, Cambridge, United Kingdom. 2. The British Antarctic Survey, Cambridge, United Kingdom. 3. The University of British Columbia, Vancouver, Canada.

\section{Introduction}

Cave Rings are cave formations, or speleothems, consisting of thin circles on cave floors, normally found surrounding a water splash point, beneath stalactites. The rings range in diameter from a few centimetres to roughly two metres, and the width and height of the deposit ranges from a few millimetres to a few centimetres. Examples from caves in Italy can be seen in figure 1. Elliptical formations have also been observed in locations with tilted cave floors. The rings tend to occur in locations where the floor is without strong flows, and with a layer of dust or mud, although it is not clear whether this merely aids their discovery, or is related to their formation. 

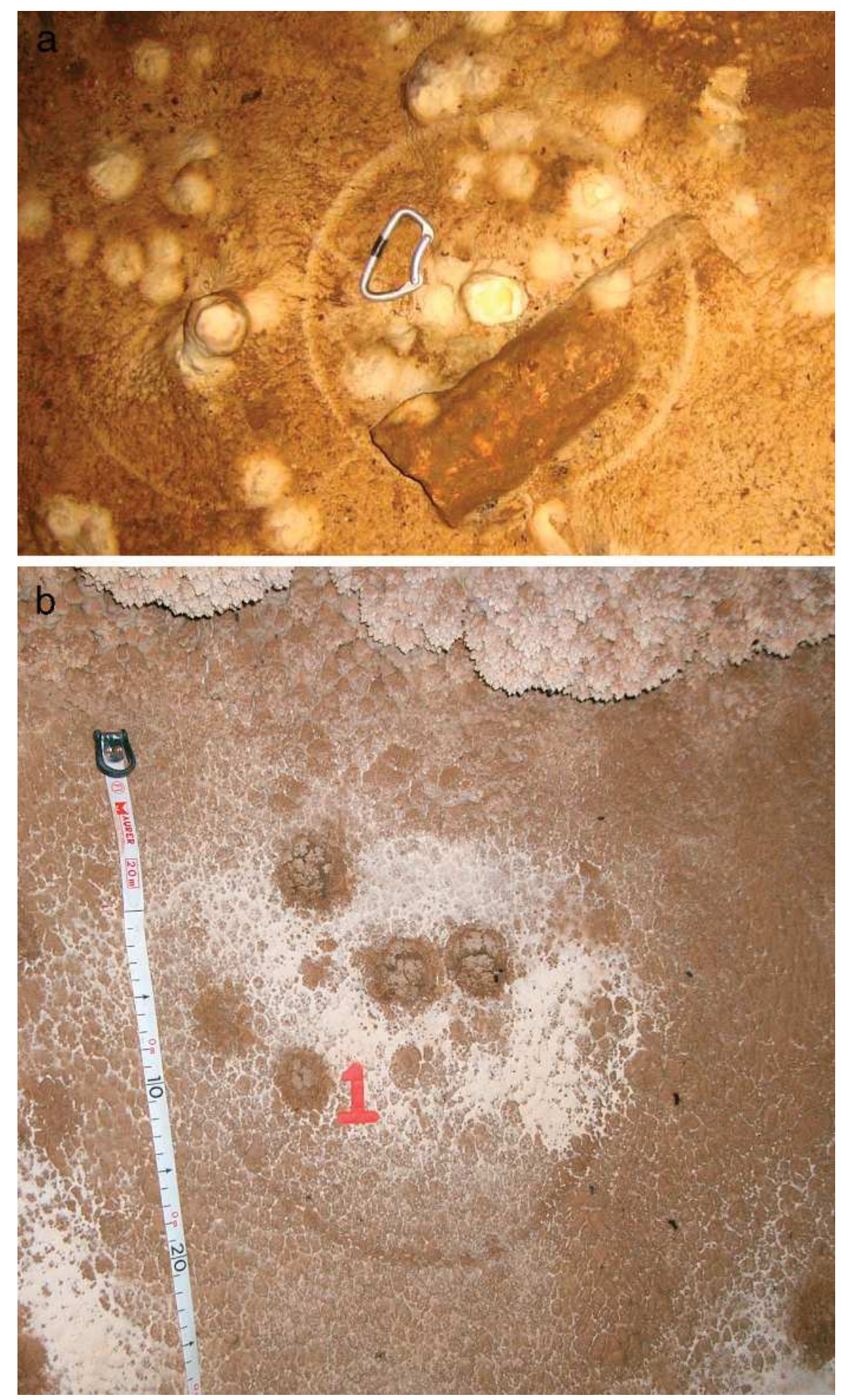

Figure 1: Rings photographed in a) Grotta Imbroglita and b) Grotta del Secchio in Italy, from [9] 
There are currently two theories within the caving community as to the method of ring formation. The first, designated the 'splash ring' mechanism, is thought to be the method responsible for producing the larger rings, and postulates that the rings are formed due to the accumulation of deposits from secondary drops being ejected from the primary drop site $[5,9]$. This requires that the majority of the secondary drops fall to the same radius. This is explained in [5] as being due to the majority of the drops ejecting at $45^{\circ}$ and then travelling a distance determined by the kinetic energy of the primary drop, dependent on the height of the source above the floor.

The second theory, designated the 'fall down ring' mechanism, thought to be responsible for the smaller sized rings, was first proposed in [8] and theorises that the rings are formed due to the accumulation of secondary drops produced from the spontaneous breakup of the primary drop during its fall. In [9], Nozzoli et al. claim to prove through analysis of collected data from various rings that this is in fact the method responsible for the formation of all cave rings. They suggest that all of the primary drops break up at $142.7 \pm 7.2 \mathrm{~cm}$ from the stalactite tip and then fall in a negative arc to the floor, due to 'other forces' than gravity acting on the small secondary drops.

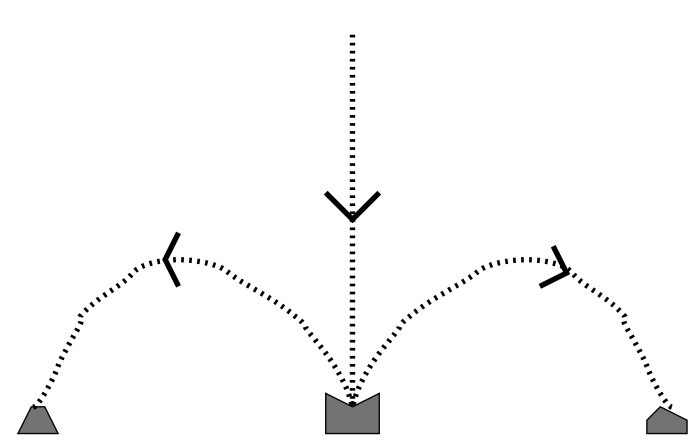

(a)

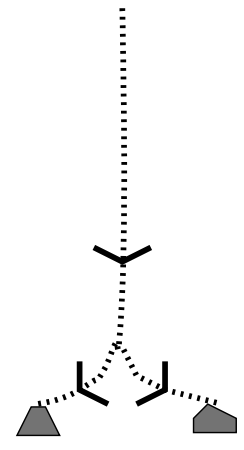

(b)

Figure 2: Depiction of a) the 'splash ring' hypothesis and b) the 'fall down ring' hypothesis.

The aim of this project is to show, through analysis and experiments, that both the 'splash ring' and 'fall down ring' hypotheses are clearly false. We also test a new hypothesis, that cave rings are formed in the same manner as coffee rings[3], that is, due to the enhanced deposition at the edges of sessile drops.

\section{Experimental Setup}

In the following section, the experimental setup used throughout is described. A depiction of the equipment used can be seen in figure 3. Throughout salt water was used as an analogue to the water found in caves. The accuracy of this replacement is discussed in section 6

A black aluminium sheet, $122 \mathrm{~cm}$ by $66 \mathrm{~cm}$, was used as a surface which could be easily heated and also provide contrast to the white salt crystals. This was raised a few $\mathrm{cm}$ off the floor, and levelled using a spirit level, although the sheet was not flat to high precision. Underneath were 6 fluorescent light tubes, centred in the width of the sheet and evenly 


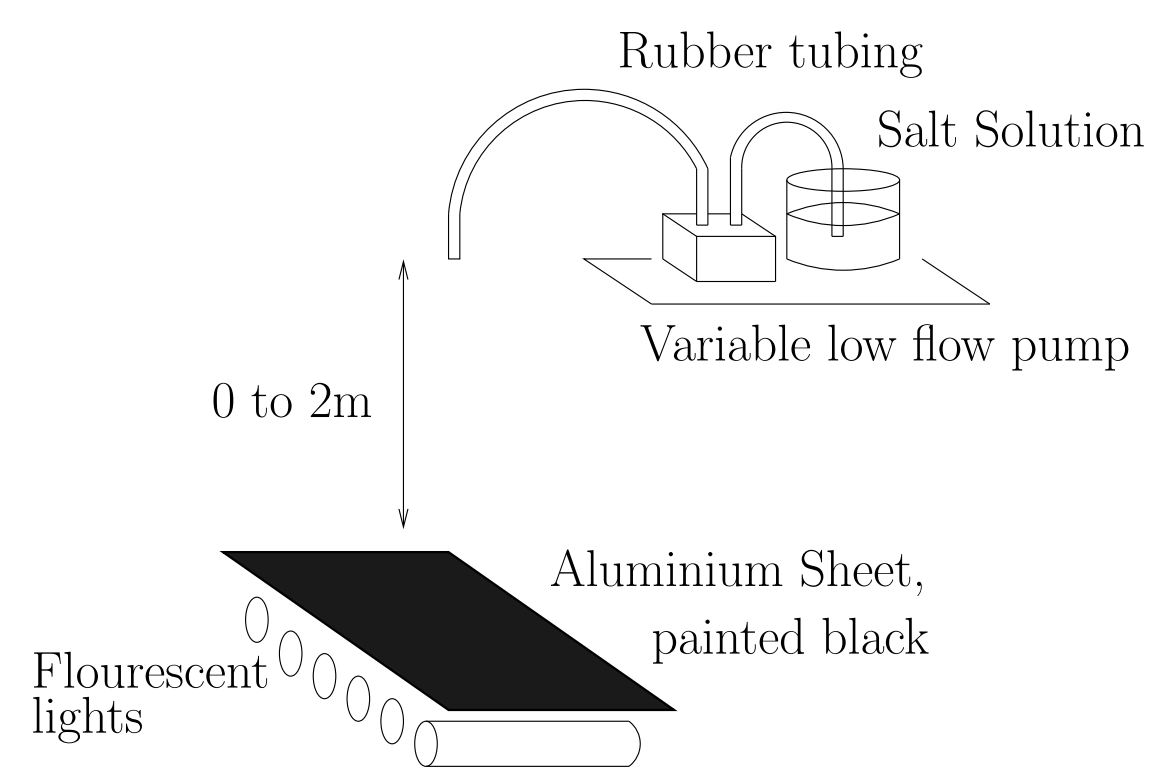

Figure 3: Depiction of the experimental setup used throughout.

distributed along the length of the sheet. These were on for the duration of all experiments, and left on for several hours before any experiment began, to provide even heating to encourage evaporation, reducing the time scales of the experiments. The sheet reached temperatures of $25-27^{\circ} \mathrm{C}$, and was fairly consistent in time, though not evenly distributed across the sheet.

Above this sheet was a system of rubber tubing connecting a variable low flow pump with a source of salt solution, which was varied in concentration for different experiments. The solution was pumped at a constant, controllable rate. The calibration of the pump was carried out by collecting the liquid pumped over a period of time for a specific setting, then using a combination of accurate weight and density measurements, the volume pumped per hour was found. It was noted that the rate was very consistent between different measurements. The end of the tube was suspended over the table using system of pulleys and string, and thus the height above the table could be adjusted between 0 and $2 \mathrm{~m}$.

A Nikon D200 model digital SLR camera was set up on a tripod next to the table to record images from experiments. This was set on a timer to take images at 15 minute intervals throughout all experiments. The images were then processed using the Image Processing Toolbox in the Matlab 2010a software program.

\section{Splash Ring Conjecture}

\subsection{Literature}

The 'splash ring' conjecture is described in [5]. It is claimed that $45^{\circ}$ is the most probable angle for secondary drops to be ejected at, and that the subsequent radius of the ring is determined by the amount of kinetic energy imparted to the primary drop on its fall from the stalactite tip. This has several logical flaws, the most obvious being the assumption that the 
secondary drops will almost always gain the same amount of kinetic energy from the splash, and therefore land at the same radius from the splash site every time. The work of [1] shows that, in the case of drops hitting a very shallow liquid layer, as is likely under stalactites, there is a range of emission angles and a wide range of emission velocities, resulting in a range of splash radii. The literature surrounding the investigation of the transport of soil and plant pathogens via raindrops uses a 'Fundamental Splash Distribution Function' which assumes that the mass splashed falls exponentially with radius, see e.g. [10], and is confirmed experimentally.

\subsection{Experiment}

In order to experimentally disprove the splash ring conjecture, the setup described in section 2 was used, with the source raised $2 \mathrm{~m}$ above the sheet, and a $15 \%$ salt solution was dripped at a rate of $17.7 \pm 0.5 \mathrm{ml}$ per hour. The resulting pattern of salt crystals left on the sheet after 19 hrs 15 min can be seen in figure 4(a), where the image has been manipulated to highlight the location of the salt crystals as follows, using the Image Processing Toolbox in Matlab 2010a:

1. Background gradients due to lighting removed.

2. Image converted to greyscale.

3. Exposure adjusted to rescale pixels with an intensity index of greater than 0.5 to an index of 1 .

4. Image converted to black and white.

5. Image taken before experiment started removed to eliminate background items.

The pattern seen in the bottom right of the photo is due to reflections of drops from a piece of equipment located next to the sheet, and so all numerical calculations from this image use the upper half of the splash pattern only. This experiment was not designed to imitate cave conditions, but to demonstrate clearly the distribution of secondary drops produced by a single drop.

The distribution of salt coverage, a proxy for drop mass, versus radius can be seen in figure 4(b), as calculated from the image in figure 4(a). The salt coverage ratio is calculated from the ratio of white to black pixels within a given radius range. The radius is calculated by finding the length of a pixel in an image using the known dimensions of the sheet. At small radii, there is no salt due to the constant recycling of the water beneath the source preventing crystallisation from occurring. From roughly $4-10 \mathrm{~cm}$, the surface is completely covered in salt crystals, and so the Salt Coverage Ratio is saturated at a value of 1 . The method of using photographs from above the table means that the thickness of the salt at this point cannot be determined. The fact that some radii appear to have coverage ratios greater than one is due to the limitations of the technique employed: because the camera could not be placed directly over the sheet due to the presence of the source equipment, the pictures are taken at a slight angle, and so each pixel is not the same length, as assumed in calculations. There are also inherent errors in taking radial calculations from gridded data. However, this experiment was not intended to provide accurate numerical data, merely 
to provide a qualitative demonstration of the inaccuracy of the 'splash ring' conjecture. The clear exponential tail to the distribution at radii greater than $10 \mathrm{~cm}$ shows qualitative agreement with the literature as discussed in section 3.1.

To determine whether the presence of a central stalagmite could have an effect on the location of splashes, the same experiment was repeated, but with an upturned Pyrex beaker placed under the centre of the source, see figure 5(a). A $20 \%$ salt solution was dripped at a rate of $10.1 \pm 0.6 \mathrm{ml}$ per hour, and the resulting pattern left after 21.5 hours can be seen in figure 5(b). Clearly there is no ring formed, and the pattern formed is similar to that formed with the stalagmite absent, although, as the flow rate was lower, the pattern is not as large.

\section{Fall Down Ring Conjecture}

\subsection{Literature}

The 'fall down' ring conjecture was first proposed in [8] as an explanation for the smaller sized cave rings observed. In [9], Nozzoli et al. claim to prove that all cave rings are produced by this method. They collected data from 67 rings in 5 caves in Central Italy, as well as creating some artificial rings by placing wooden tablets covered in soot underneath several stalactites, over rings. The data they collected can be seen in figure 6 , where an apparent correlation between the height of the source stalactite and the ring radius can be seen. They fit this data to a quadratic function, and then conclude that all the rings are produced by drops that break up at $142.7 \pm 7.2 \mathrm{~cm}$ below the source stalactite, then execute a negative arc to the ring radius. To conclude, because a curve can be fitted to the data, that the theory must therefore be correct, is optimistic at best. There could be many ways to explain a relationship between the two quantities measured, and there is no explanation as to the mechanism behind the proposed breakup, or the physically unlikely negative curvature, which is put down to 'unknown' forces. There is also an apparent contradiction in their explanation - if the drops always break up, then what forms the central stalagmite visible at the centre of all the rings? The artificial rings, which they claim were formed on the wooden tablets over a period of roughly 20 days, would perhaps be more convincing if the paper contained any pictures of these rings or a fuller explanation of what was found. The only thing to be concluded from this work is that any successful production theory must explain this apparent correlation between source height and ring radius.

A review of the literature involving drop breakup revealed the extensive study of Villermaux and Bossa[11] into the dynamics of single drop deformation and breakup, with the aim of explaining the distribution of raindrop sizes found at ground level. The condition for drop break up is $W_{e}>6$, where $W_{e}$ is the Weber number of the drop. For such a drop, first it pancakes and then the radius increases exponentially with time until breakup. If $W_{e}<6$, the drop pancakes but then oscillates around a mean radius. For a drop falling in static air, the Weber number is defined as the ratio between the gravitational force on the droplet and the surface tension on the drop:

$$
W_{e}=\frac{\rho g d^{2}}{\sigma}
$$




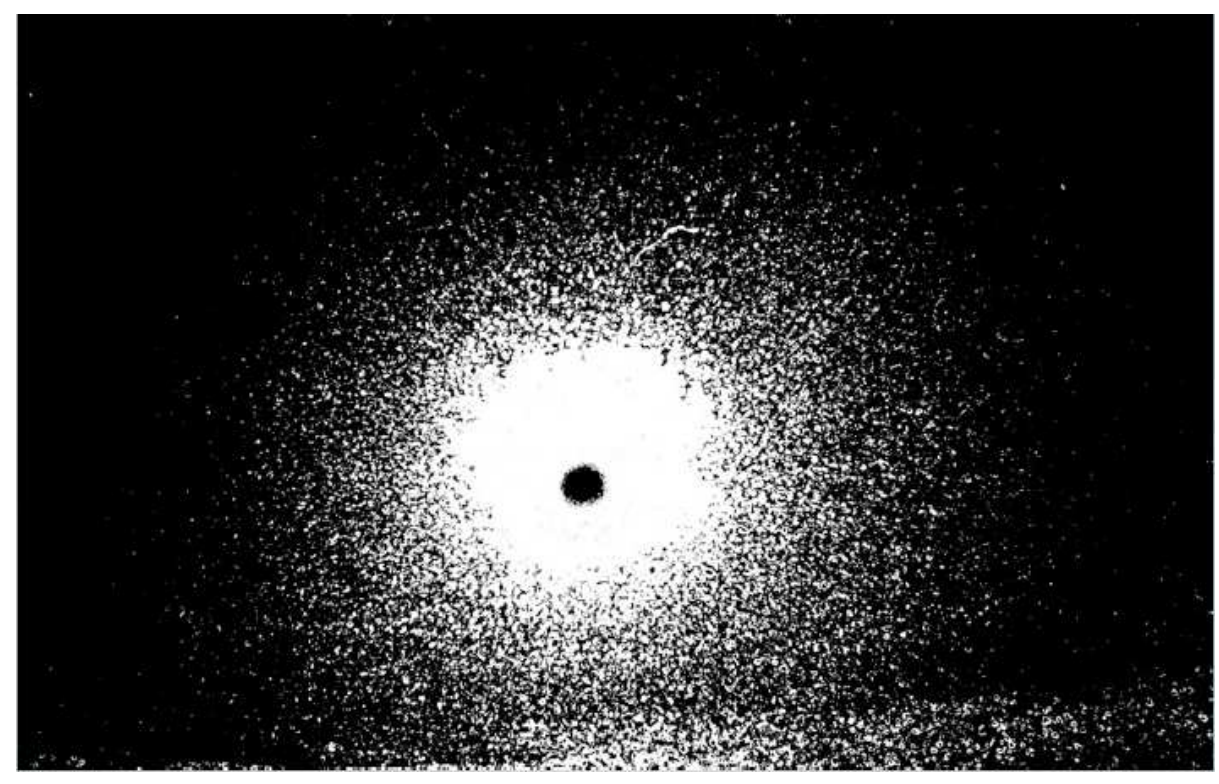

(a)

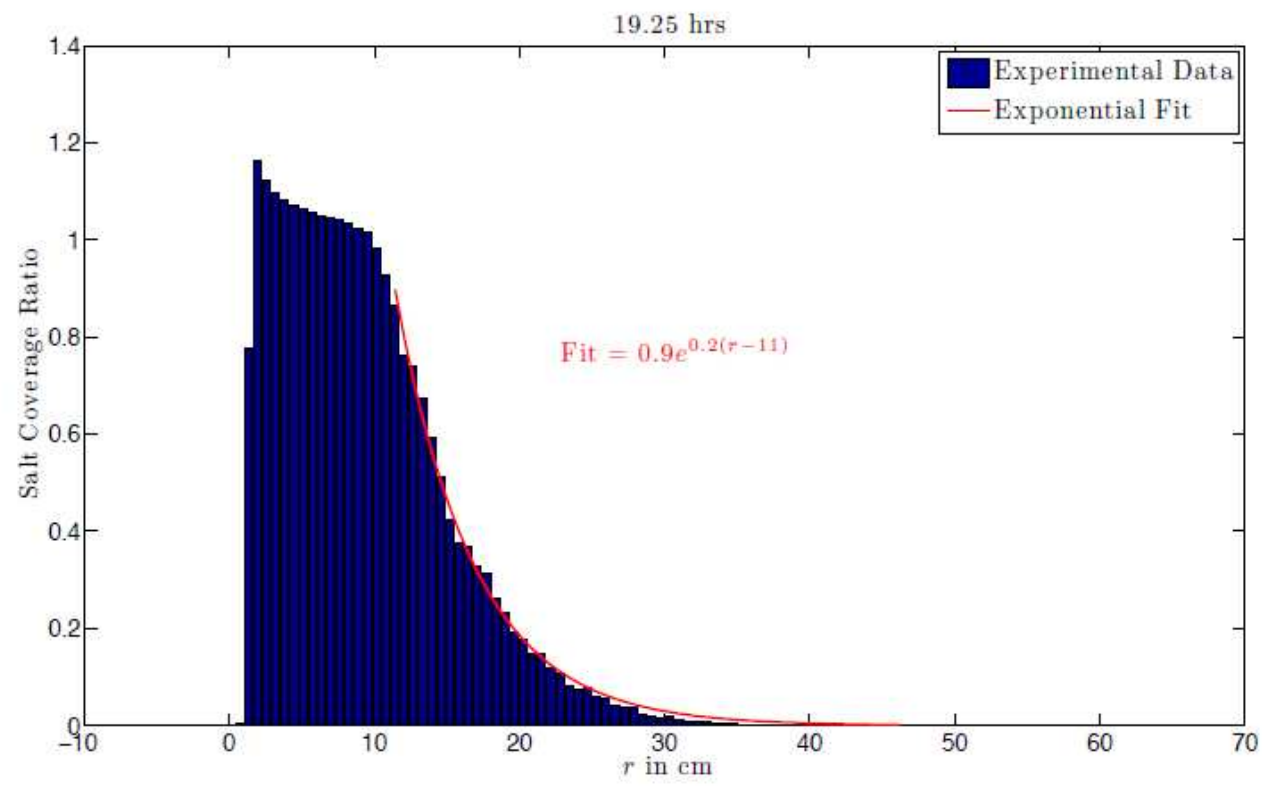

(b)

Figure 4: Experiment described in section 3.2 after 19.25 hours. (a) shows a photo of the sheet, with the exposure manipulated to highlight the salt crystals. (b) shows the salt coverage ratio versus radius, calculated from the image in (a), showing a clear exponential tail to the distribution. 


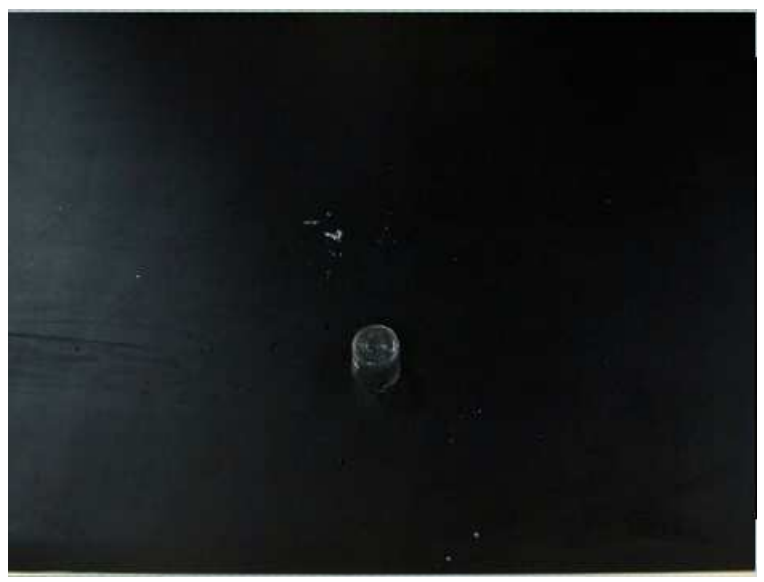

(a)

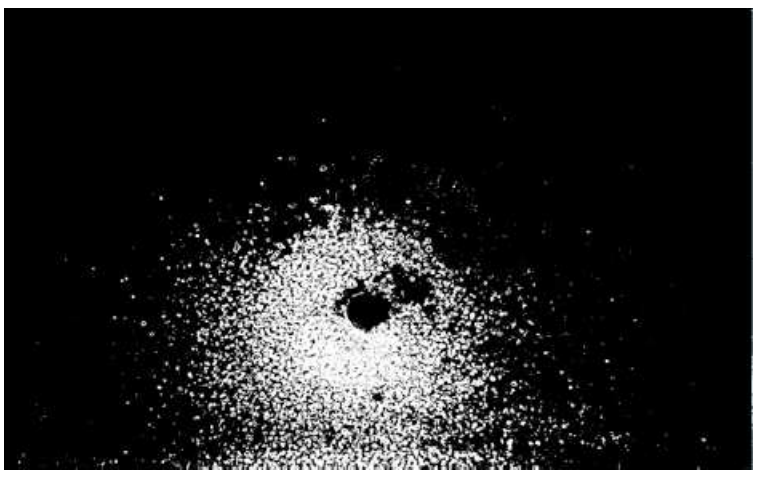

(b)

Figure 5: Experiment as described with Pyrex beaker to represent a stalagmite. (a) shows the beaker in place on the aluminium sheet. (b) shows a manipulated photo to highlight the salt crystals present after 21.5 hours.
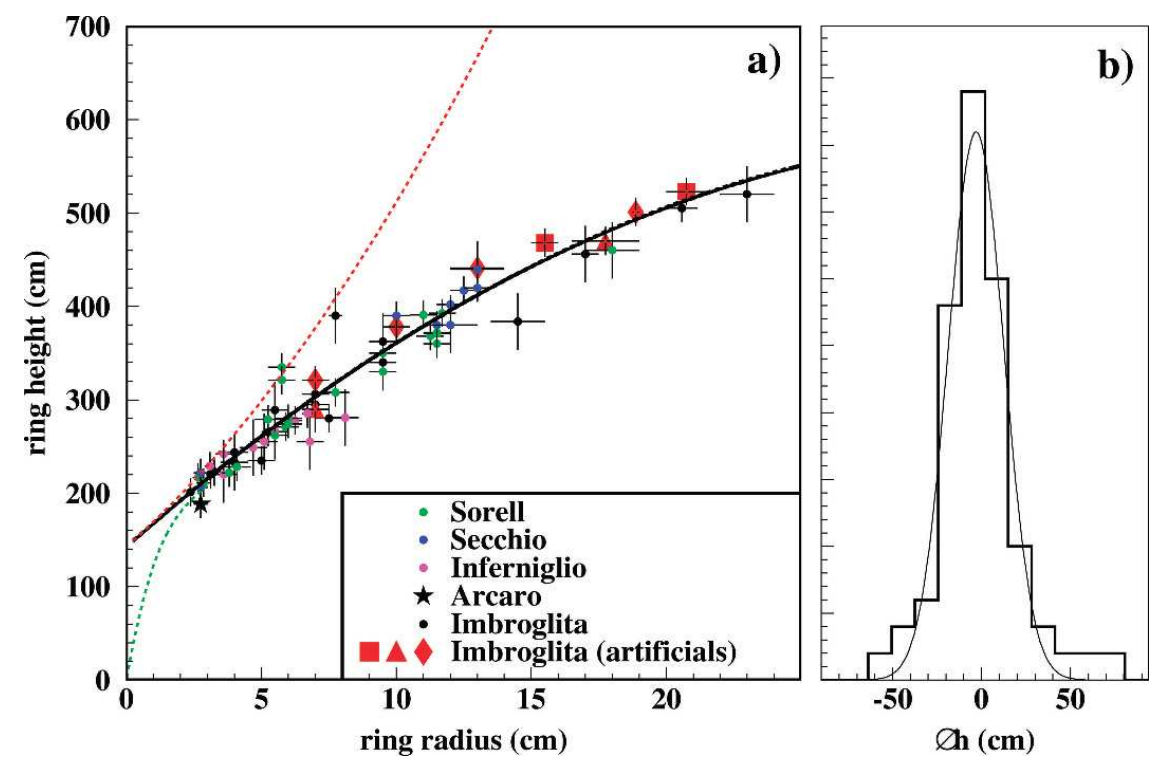

Figure 6: Figures from [9], showing a) the measured height of the source above the rings vs the radius of the ring, for the in situ rings and the artificial rings (large red symbols). The solid black line shows the best fit 2nd degree polynomial. b) shows the distribution of the difference between the best fit height and the actual height. 
where $\rho$ is the density of the water, $g$ is gravitational acceleration, $d$ is the diameter of the drop and $\sigma$ is the surface tension between the water and the air. For a pendant drop, such as one forming on a stalactite, at detachment the gravitational force on the drop just exceeds the surface tension, i.e.

$$
\frac{1}{6} \pi g \rho d^{3} \approx \pi D \sigma
$$

where $D$ is the diameter of the source. Combining (1) and (2) gives $W_{e} \approx 6 D / d$. and thus the condition for the drop to breakup becomes $D>d$, i.e. the drop must detach whilst its diameter is less than that of the source. This is physically unlikely for a detaching pendant drop, see for example [4] and figure 7, and implies the drop would initially have a diameter of over $7 \mathrm{~cm}$.

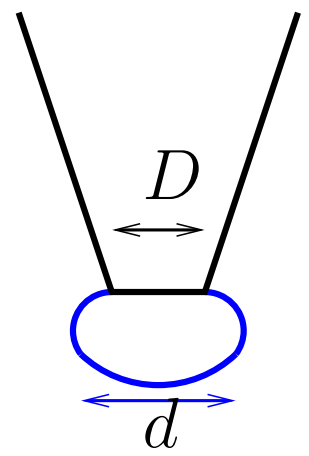

Figure 7: Depiction of a pendant drop from a stalactite. $D$ is the diameter of the source of liquid and $d$ is the diameter of the drop itself.

\subsection{Experiment}

To disprove the 'fall down' conjecture experimentally, the setup described in section 2 was used, with the source raised $2 \mathrm{~m}$ above the sheet, and a $20 \%$ salt solution was pumped at a rate of $17.7 \pm 0.5 \mathrm{ml}$ per hour. A plastic beaker was placed on the sheet directly below the source, in order to catch the primary drop. The experiment was run for 22 hours, after which the beaker contained the majority of the water; a small amount had bounced out as the beaker began to fill, see figure 8. No drops were observed breaking up throughout any of the experiments. It must be noted that $2 \mathrm{~m}$ is at the lowest end of the observed rings, see figure 6 , and that a higher fall height would be more rigorous in disproving the 'fall down' conjecture, however $2 \mathrm{~m}$ was the limit of the equipment available.

\section{$5 \quad$ Coffee Ring Theory}

A 'coffee ring' is the deposit formed when a sessile drop of a solution containing dissolved particles, such as coffee or salt, dries. This was investigated by Deegan et. al in [3], and shown to be the result of a combination of contact line pinning and non-uniform evaporation flux. If the contact line of a drop is pinned, by a slightly rough surface for example, then, 


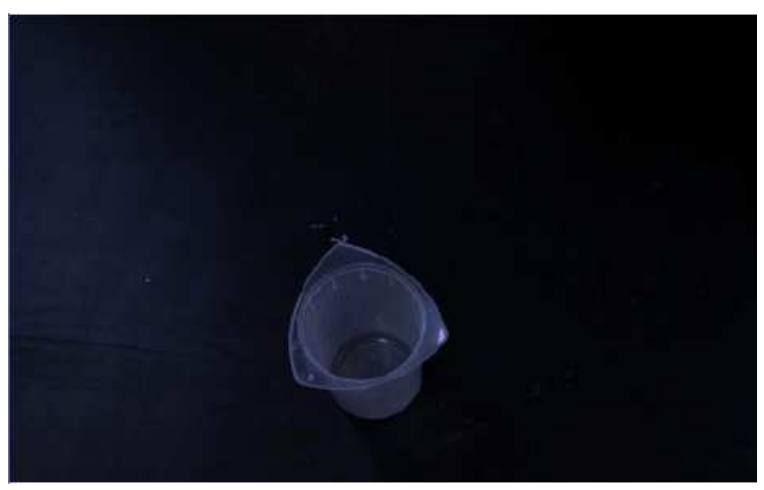

(a)

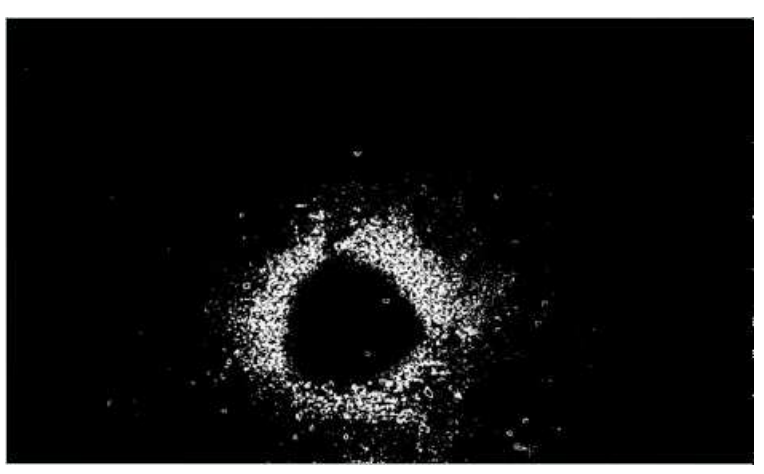

(b)

Figure 8: Experiment as described with plastic beaker to catch primary drops. (a) shows the beaker in place on the aluminium sheet. (b) shows a manipulated photo to highlight the salt crystals present after 22 hours.

when volume is lost, the majority is taken from the centre of the drop, see figure 9 . The rate limiting step in evaporation from a drop is the diffusion of water vapour away from the saturated region around the drop. Due to the geometry of the drop, assumed to be close to a spherical cap, the evaporation is enhanced at the contact line, where it is easier for a water vapour particle to escape, see figure 10(a).

(a)

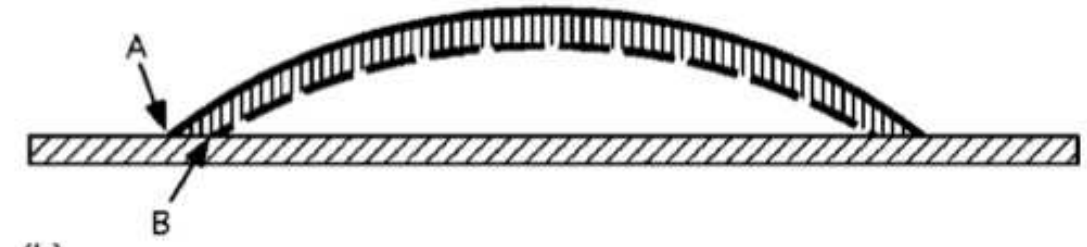

(b)

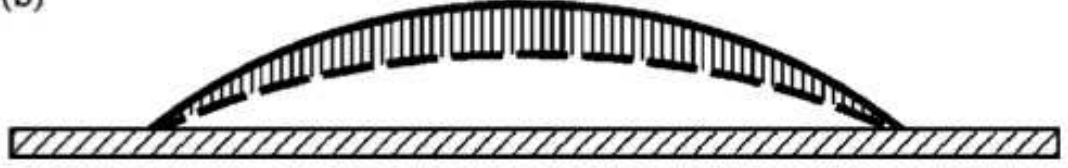

Figure 9: Depiction of volume lost from (a) a drop where the contact line is free to move and (b) a drop where the contact line is pinned. [3]

Because of the mis-match in the location of maximum mass loss and maximum evaporation flux, see figure 10(b), there is a constant flux of fluid towards the edge of the drop to replace the evaporated water. As the majority of the evaporation occurs at the edge of the drop, the majority of the dissolved particles are deposited at the edge of the drop, resulting in the visible ring left behind.

While this is a well formed theory for drops of the size of a few $\mathrm{cm}$ or less, it was proposed that this could also be the mechanism behind the formation of cave rings. If the 


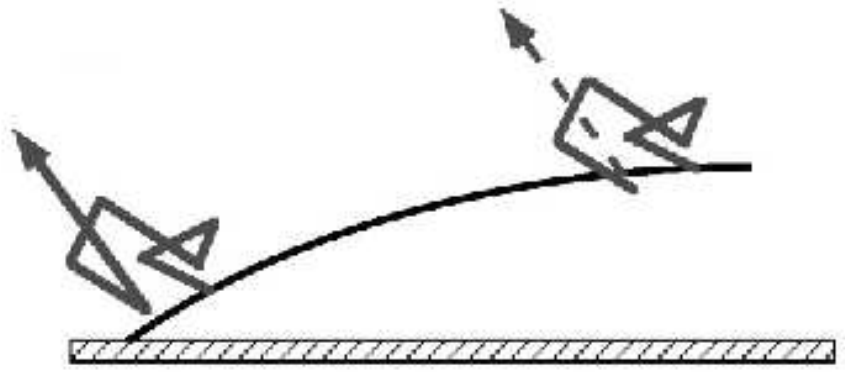

(a)

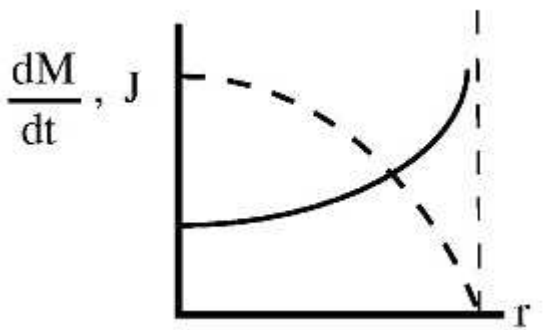

(b)

Figure 10: (a) Depiction of a water vapour particle performing a random walk at the edge of the drop. It is more likely to escape near the contact line. (b) Magnitude of mass loss, $d M / d t$, dashed line, and evaporation flux, $J$, line, versus radius of the drop. The evaporation flux is singular at the contact line. [3]

flow rate in the cave was high enough to produce a large puddle, perhaps perhaps during a rain storm, then the coffee ring mechanism could produce a ring of limestone deposit around the edge of the puddle, forming the cave ring left behind.

\subsection{Large Coffee Ring model}

\subsubsection{Large Puddle}

To test the 'coffee ring' hypothesis, we developed a model for large coffee rings. We begin by modelling a large puddle, fed by a source and losing water to evaporation, with a surface described by $h(r, t)$, using the 2-D quantities depicted in figure 11, under the following assumptions:

- slow, viscous flow,

- shallow puddle, $R>>H$,

- source within $r=a$,

- no slip at $z=0$,

- no stress at $z=h$.

The momentum balance in the radial and vertical directions can then be written as follows:

$$
\begin{aligned}
& \frac{1}{\rho} \frac{\partial p}{\partial r}=\nu \frac{\partial^{2} u}{\partial z^{2}}+\text { smaller } \\
& \frac{1}{\rho} \frac{\partial p}{\partial z}=g+\text { smaller }
\end{aligned}
$$

where $p(r, z, t)$ is the pressure in the puddle, $\rho$ is the density of the fluid (assumed constant), $\nu$ is the molecular diffusivity of the fluid, and $g$ is gravitational acceleration. These 


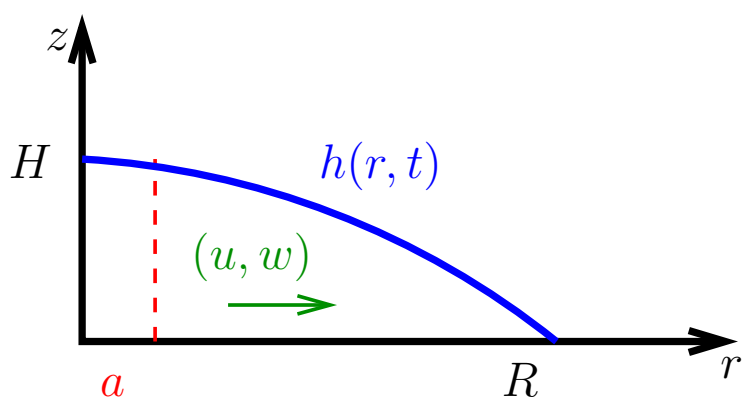

Figure 11: Depiction of the quantities described in the large puddle model. $h(r, t)$ is the surface of the puddle, $(u, w)$ is the velocity field withing the puddle, $H$ and $R$ are the vertical and radial scales respectively, and $a$ is the radius containing the source.

expressions, along with the boundary conditions previously mentioned, lead to the following definitions of the pressure, $p$ and horizontal velocity, $u$ :

$$
p=\rho g(h-z), \quad u=\frac{g}{\nu} \frac{\partial h}{\partial r} z(z-2 h) .
$$

Mass conservation leads to the following relation between the horizontal and vertical velocities, $u$ and $w$ respectively:

$$
\nabla \cdot v=\frac{1}{r} \frac{\partial}{\partial r}(r u)+\frac{\partial w}{\partial z}=0 .
$$

Integrating (6) in the vertical:

$$
\int_{0}^{z} \frac{1}{r} \frac{\partial}{\partial r}(r u) d z+w(z)-w(0)=0
$$

The dynamical boundary conditions for $w$ are as follows:

$$
\begin{aligned}
w(z=h) & =\frac{\partial h}{\partial t}+\left.u \cdot \frac{\partial h}{\partial r}\right|_{z=h}+w_{\text {evap }} \\
w(z=0) & =H(a-r) w_{\text {source }}
\end{aligned}
$$

where $w_{\text {evap }}$ and $w_{\text {source }}$ are the vertical velocities of fluid leaving via evaporation and entering from the source respectively, and $H(a-r)$ is a Heaviside function. We have assumed here that the source is placed at $z=0$, but the results that follow are the same if the source is instead assumed to be at $z=h$. If we evaluate $(7)$ at $z=h$ :

$$
\begin{aligned}
\int_{0}^{h} \frac{1}{r} \frac{\partial}{\partial r}(r u) d z+\left.u \cdot \frac{\partial h}{\partial r}\right|_{z=h}+\frac{\partial h}{\partial t}+w_{\text {evap }}-H(a-r) w_{\text {source }} & =0 \\
\frac{1}{r} \frac{\partial}{\partial r}\left(r \int_{0}^{h} u d z\right)+\frac{\partial h}{\partial t}+w_{\text {evap }}-H(a-r) w_{\text {source }} & =0 .
\end{aligned}
$$

The form of $w_{\text {evap }}$ can be found from Hu and Larson[6], who expanded on Deegan et al.[3] to find an exact form for the evaporation flux over a sessile drop. It turns out that solving 
for the flux is equivalent to the problem of deriving the electrostatic potential formed by the union of two spherical caps, as previously solved by Lebedev[7]. Taking the form for a spherical cap with contact line angle equal to zero, assuming that for a large puddle that this will be approximately correct, then

$$
w_{\text {evap }}=\frac{\kappa}{\sqrt{R^{2}-r^{2}}}, \quad \kappa=\frac{2 D \rho_{v}(1-\theta)}{\pi \rho},
$$

where $R$ is the radius of the puddle, $D$ is the molecular diffusivity of water vapour in air, $\rho_{v}$ is the saturated vapour density of water, $\rho$ is the density of liquid water, and $\theta$ is the ambient relative humidity.

Combining (5), (11) and (12), the evolution of the puddle height can be written as follows:

$$
\frac{\partial h}{\partial t}=H(a-r) \frac{q}{\pi a^{2}}-\frac{\kappa}{\sqrt{R^{2}-r^{2}}}+\frac{2 g}{3 \nu} \frac{1}{r} \frac{\partial}{\partial r}\left(r \frac{\partial h}{\partial r} h^{3}\right),
$$

where $q$ is the source flux in $m^{3} / s$. Assuming a steady state, i.e. $\partial h / \partial t=0$, then we can solve for the height profile of the puddle, $h$ :

$$
\begin{aligned}
& h(r)=2^{1 / 4}\left[g(r)+H(r-a)\left[\frac{1}{2}\left(1-\frac{r^{2}}{a^{2}}\right)+\log \left(\frac{r}{a}\right)\right]\right]^{1 / 4}, \\
& g(r)=\log \left(\frac{r}{1-\sqrt{1-r^{2}}}\right)-\sqrt{1-r^{2}}
\end{aligned}
$$

where the parameters have been non-dimensionalised as follows: $h \rightarrow h / H, r \rightarrow r / R$, $a \rightarrow a / R$. The non-dimensional height and radial scales are defined as follows:

$$
R=\frac{q}{2 \pi \kappa}, \quad H=\left(\frac{3 \nu q}{2 \pi g}\right)^{1 / 4} .
$$

The form of $h(r)$ can be seen in figure 12 for $a=0.1$.

\subsubsection{Solute Dynamics}

We now include the concentration of a passive solute, such as salt or coffee, $c(r, z, t)$, and consider a section of the puddle from $r_{1}$ to $r_{2}$ and $z=0$ to $z=h$, as depicted in figure 13 . If we require conservation of $c$ over the section, then this implies:

$$
\frac{\partial}{\partial t} \int_{r_{1}}^{r_{2}} \int_{0}^{h(r, t)} c(r, z, t) d z r d r=-\left[r \int_{0}^{h(r, t)} J_{r}(r, z, t) d z\right]_{r_{1}}^{r_{2}}+\int_{r_{1}}^{r_{2}} r c(r, 0, t) w(r, 0, t) d r,
$$

where $J_{r}(r, z, t)$ is the radial flux of solute, and the solute arrives through the bottom with flux $J_{z}(r, z=0, t) \equiv w(r, z=0, t) c(r, z=0, t)=w_{\text {source }} c_{\text {source }}$, but the solute cannot leave the puddle through evaporation, and so the flux of concentration at $z=h$ is zero. If we rewrite $(17)$ as a single integral over $\left(r_{1}, r_{2}\right)$, set the integrand to zero since the limits are arbitrary, and use the definition of $\mathbf{J}=c \mathbf{u}-\chi \nabla c$, we get

$$
\frac{\partial}{\partial t} \int_{0}^{h(r, t)} c(r, z, t) d z=-\frac{1}{r} \frac{\partial}{\partial r}\left[r \int_{0}^{h(r, t)}\left(c(r, z, t) u(r, z, t)-\chi r \frac{\partial c}{\partial r}\right) d z\right]+c_{\text {source }} w_{\text {source }}
$$




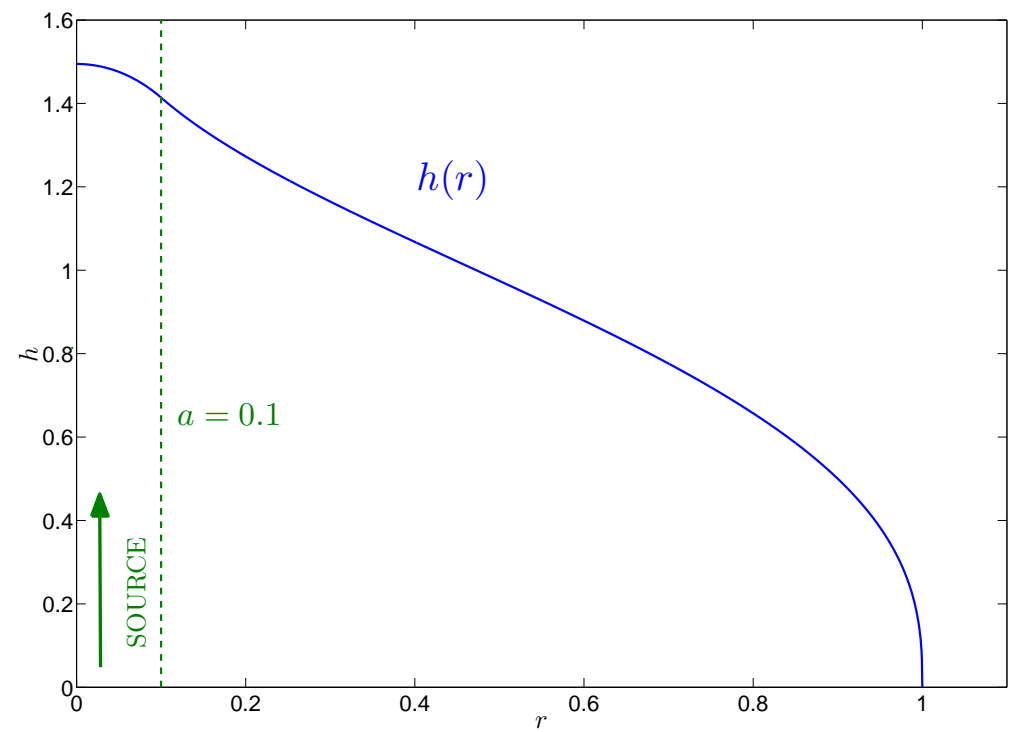

Figure 12: The steady state solution $h(r)$, the height of the puddle, see (14), for $a=0.1$.

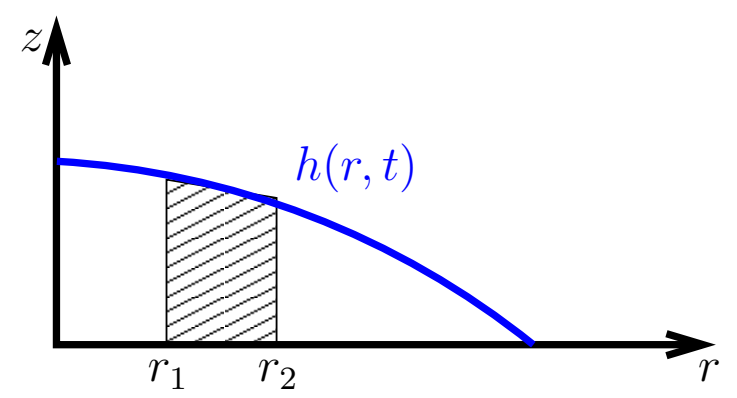

Figure 13: Depiction of section of puddle considered in section 5.1.2. 
where $\chi$ is the molecular diffusivity of the passive solute.

If we now consider the dimensions of each of the terms in the advection-diffusion equation, then we find the following balance:

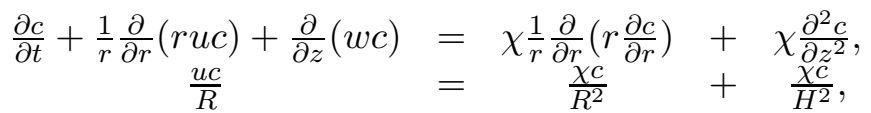

where the bottom row indicates the dimension. The second term on the RHS dominates on that side due to the shallow puddle assumption made previously, $R>>H$. Thus, the ratio between the left and right sides becomes $u H^{2} / R \chi=H / R$.Pe, where Pe is the Peclet number of the fluid. The Peclet number is likely to not be large in a dripping cave fluid, and so the vertical diffusion term clearly dominates. Thus, we can assume that vertical diffusion acts fast enough to homogeonize the solute over the depth of the fluid, i.e. $c(r, z, t) \approx \bar{c}(r, t)$, and we find the following expression for the evolution of the concentration flux from (18):

$$
\frac{\partial}{\partial t}(h \bar{c})=-\frac{1}{r} \frac{\partial}{\partial r}(r \bar{c} \bar{u})+H(a-r) w_{s} c_{s}+\chi \frac{1}{r} \frac{\partial}{\partial r}\left(r \frac{\partial \bar{c}}{\partial r} h\right),
$$

where the subscript $s$ denotes source properties, and $\bar{u}$ is the vertically integrated horizontal velocity $u$. From now on we will drop the bar over $c$.

If we first consider the simpler case of the steady state, $\partial h / \partial t$, then we can rewrite $(20)$ as follows:

$$
h \frac{\partial c}{\partial t}=H(a-r) \frac{c_{s}}{a^{2}}+\frac{1}{r} \frac{\partial}{\partial r}\left[r\left(c \frac{\partial h}{\partial r} h^{3}+K \frac{\partial c}{\partial r} h\right)\right],
$$

where $K=\pi \chi H / q, \tau=H q / 4 \pi \kappa^{2}$, and the parameters have been non-dimensionalised as follows: $c \rightarrow c / c_{0}, c_{s} \rightarrow c_{s} / c_{0}, t \rightarrow t / \tau$, and $h$ and $r$ as previously, where $c_{0}$ is the initial concentration of the solute within the puddle, and $h$ is described in (14).

Solving this numerically as an initial value problem using the pdepe routine in Matlab 2010a, the results up to $t=0.9$ can be seen in figure 14, where $a=0.1, c_{s}=1$ and $K=0.01$. It can be seen that, as expected, the solute is swept to the edge of the puddle over time, where it collects.

(21) can also be solved analytically in certain regimes. It can be seen from figure 14 that, away from the edge of the puddle $(r=1)$, the solution for $c(r)$ approaches a single solution over time. To solve for this solution we thus set $\frac{\partial c}{\partial t}=0$. We also assume that, as the gradients in $c$ are not large away from $r=1$, that diffusion can be neglected ( $K$ being small). We are thus left with the following expression to solve:

$$
\frac{1}{r} \frac{\partial}{\partial r}\left(r c \frac{\partial h}{\partial r} h^{3}\right) \approx-H(a-r) \frac{c_{s}}{a^{2}}
$$

Using (14), we can evaluate:

$$
r \frac{\partial h}{\partial r} h^{3}=-\frac{1}{2}\left[\sqrt{1-r^{2}}+H(a-r)\left(\frac{r^{2}}{a^{2}}-1\right)\right],
$$

and so can solve (22), using the boundary condition of zero flux at $r=0$, and matching fluxes at $r=a$, to find:

$$
c \approx \begin{cases}c_{s}, & r<a, K \approx 0, \\ c_{s} / \sqrt{1-r^{2}}, & r>a, K \approx 0 .\end{cases}
$$




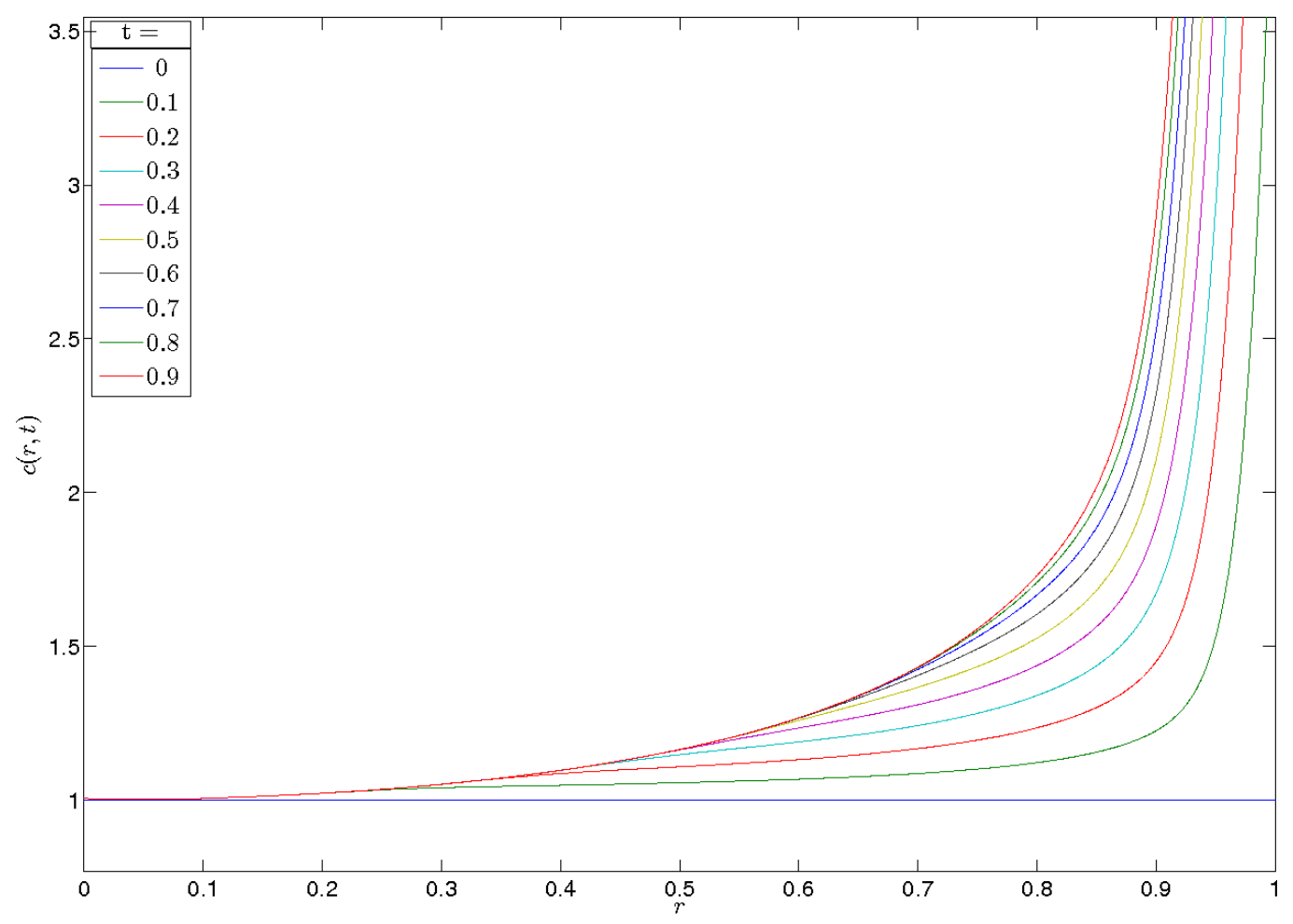

Figure 14: Solutions of (21) for $t=0$ to 0.9 , with $a=0.1, c_{s}=1$ and $K=0.01$.

Thus, the solution matches the source concentration within the source area, and outside follows the evaporation profile (12). Comparing with the numerical solution, see figure 15, it can be seen that the analytical solution is best for low $r$, and becomes worse as the gradient of $c$ increases, and diffusion becomes more important. At values of $r$ close to to 1 and large times, the numerical solution for $c$ becomes linear, see figure 16.

Thus, we can assume that $c(r, t)$ takes the form $A(r) t+B(r)$ near $r=1$. Introducing this into (21), we get two coupled equations describing $A$ and $B$ :

$$
\begin{aligned}
0 & =\frac{1}{r} \frac{\partial}{\partial r}\left[r\left(A \frac{\partial h}{\partial r} h^{3}+K \frac{\partial A}{\partial r} h\right)\right], \\
h A & =\frac{1}{r} \frac{\partial}{\partial r}\left[r\left(B \frac{\partial h}{\partial r} h^{3}+K \frac{\partial B}{\partial r} h\right)\right] .
\end{aligned}
$$

We initially concentrate on (25), and redefine $r$ in terms of the parameter $\zeta$, which is small near the puddle edge: $r=1-K^{\alpha} \zeta$. We can approximate $h$ near $r=1$, and find:

$$
h \approx\left(\frac{2}{3}\right)^{1 / 4} 2^{3 / 8}(1-r)^{3 / 8}=\left(\frac{2}{3}\right)^{1 / 4} 2^{3 / 8} K^{3 \alpha / 8} \zeta^{3 / 8},
$$

and from (23) we can rewrite:

$$
r \frac{\partial h}{\partial r} h^{3} \approx-\frac{\sqrt{2}}{2} \sqrt{1-r}=-\frac{1}{\sqrt{2}} K^{\alpha / 2} \zeta^{1 / 2},
$$




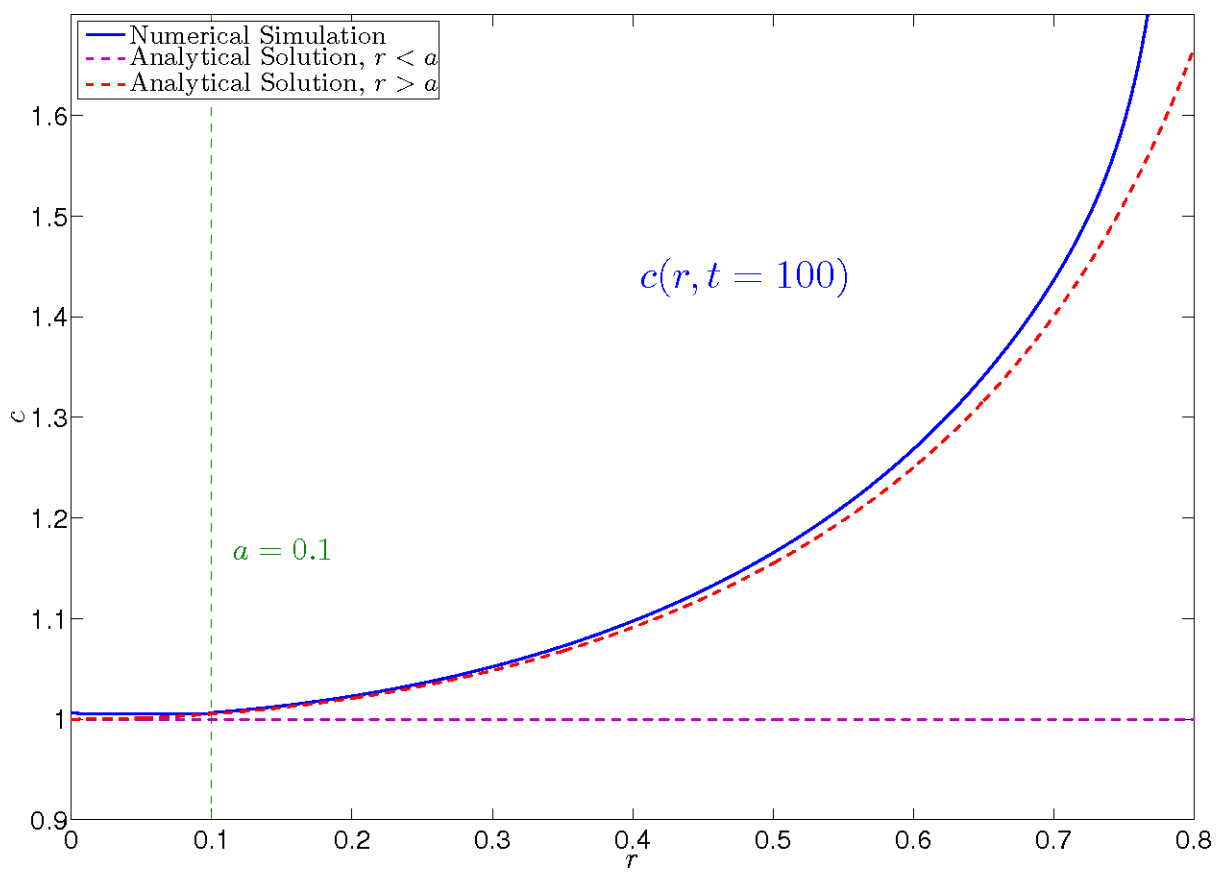

Figure 15: Comparison of the analytical and numerical solutions for $c(r)$ at large times away from $r=1$.

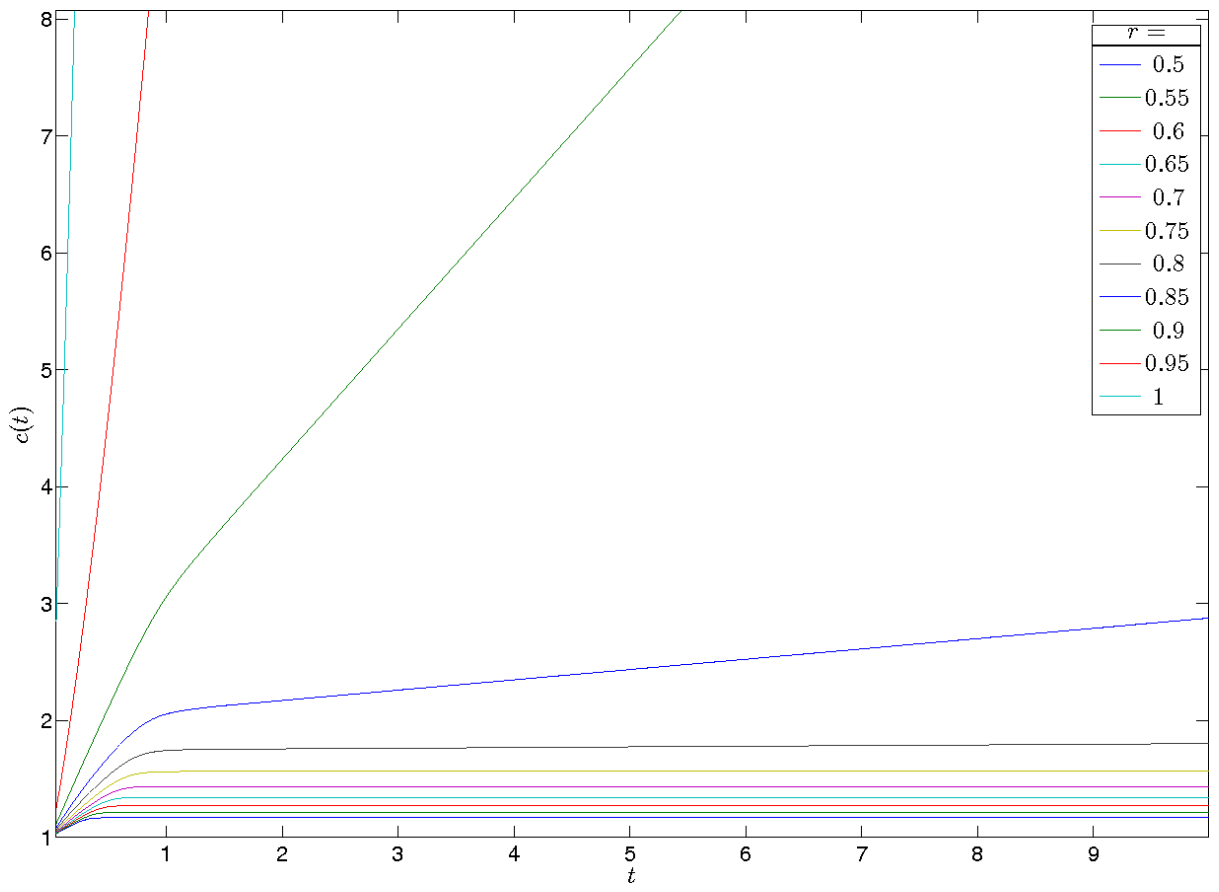

Figure 16: Numerical solutions for $c(t)$ at various radii. Close to $r=1$, the concentration becomes linear with time. 
and so (25) can be rewritten:

$$
r\left(A \frac{\partial h}{\partial r} h^{3}+K \frac{\partial A}{\partial r} h\right) \approx-A \frac{1}{\sqrt{2}} K^{\alpha / 2} \zeta^{1 / 2}+K^{1-\alpha} \frac{\partial A}{\partial \zeta}\left(\frac{2}{3}\right)^{1 / 4} 2^{3 / 8} K^{3 \alpha / 8} \zeta^{3 / 8}=0
$$

where we have integrated both sides. The integration constant is zero, as the expression is equal to zero at $r=0$. Rearranging:

$$
\left(\frac{2}{3}\right)^{1 / 4} 2^{7 / 8} K^{1-\alpha} \frac{\partial A}{\partial \zeta}=K^{\alpha / 8} A \zeta^{1 / 2}
$$

and choosing $\alpha=8 / 9$, we can integrate to find

$$
A(r)=k \exp \left\{-\frac{2^{15 / 8}}{3^{7 / 4} K}(1-r)^{9 / 8}\right\}
$$

where $k$ is a constant to be determined.

Considering the form of the solution, $A(r) t+B(r)$, we know that $A(r)$ vanishes away from the edge of the puddle, as we have already shown that there is no time dependence to the solution within the interior. Thus, $B(r)$ must match the solution at the interior at some point away from the edge. Equivalently, the total flux associated with $B$ must match the total flux of the interior solution. Thus we can find the constant $k$ by evaluating both sides of the following expression, a rewriting of (26):

$$
-\int_{0}^{\infty} r h A K^{\alpha} d \zeta=\int_{0}^{\infty} \frac{\partial}{\partial \zeta}\left[r\left(B \frac{\partial h}{\partial r} h^{3}+K \frac{\partial B}{\partial r} h\right)\right] d \zeta=\text { interior flux }
$$

or

$$
-k\left(\frac{2}{3}\right)^{1 / 4} 2^{3 / 8} K^{3 / 9} \int_{0}^{\infty} \zeta^{3 / 8}\left(1-K^{8 / 9} \zeta\right) \exp \left\{-\frac{2^{15 / 8}}{3^{7 / 4}} \zeta^{9 / 8}\right\} K^{8 / 9} d \zeta=-c_{s} / 2,
$$

which can be re-arranged to find $k\left(c_{s}, K\right)$; the full expression can be found in appendix A. We compare this with the numerical solution, see figure 17, using a logarithmic axis for the concentration. The solution is fairly accurate above $r=0.85$, improving closer to $r=1$ as would be expected.

\subsubsection{Deposition Dynamics}

In order to simulate the production of speleothems, we now include deposition via a sink term in the concentration evolution equation, assuming immediate sedimentation to the bottom. We must also include an equation for the evolution of the depth of the deposit, $d(r, t)$, and therefore allow the height, $h(r, t)$ to evolve also. This results in a system of three coupled equations: 


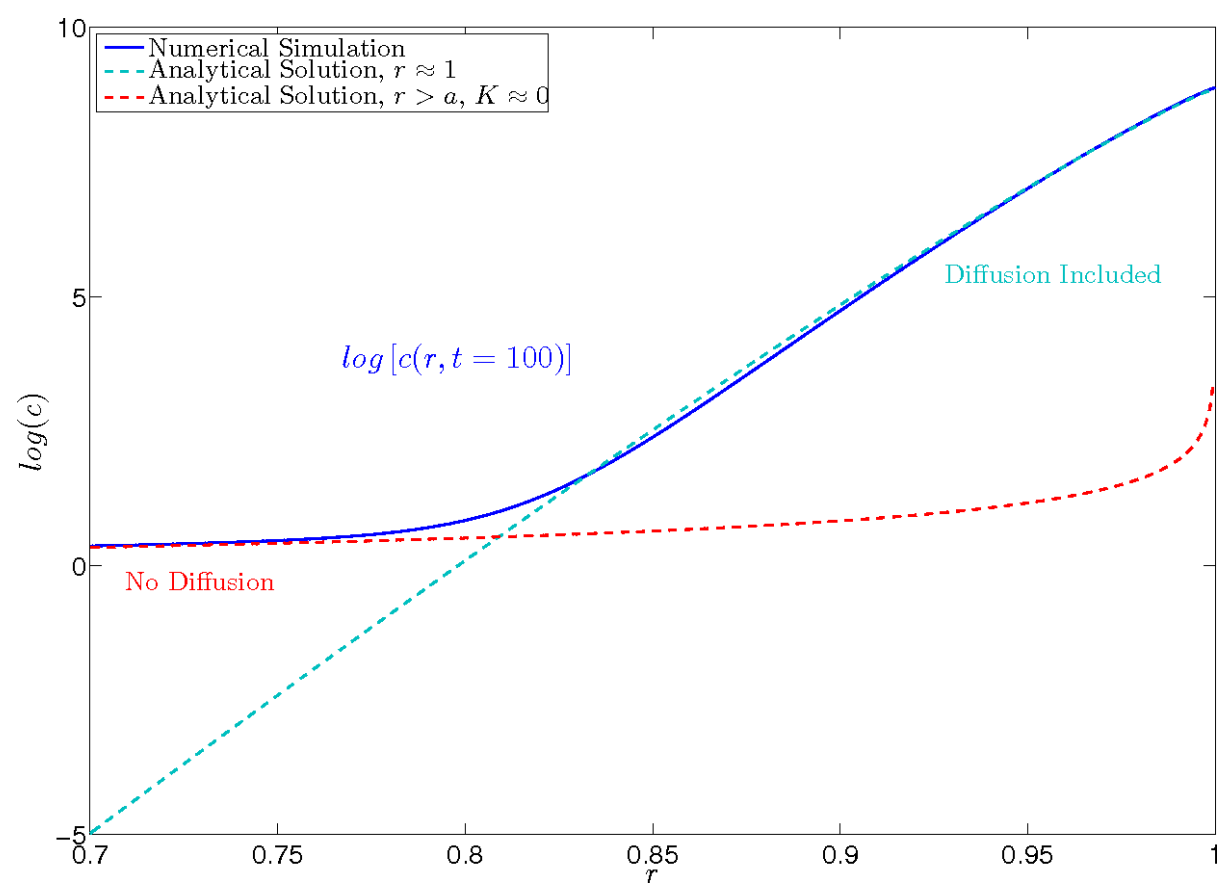

Figure 17: Comparison of the analytical solutions (31) and (24) for the concentration, $c$, at $t=100$, with the numerical solution near $r=1$. As before, $c_{s}=1, a=0.1$ and $K=0.01$. The solution for $r \approx 1$ clearly improves as it approaches 1 .

$$
\begin{aligned}
\frac{\partial(h-d) c}{\partial t}= & H(a-r) \frac{c_{s}}{a^{2}}+\frac{\tau p}{H}\left(c_{s a t}-c\right) \\
& +\frac{1}{r} \frac{\partial}{\partial r}\left[r\left(c \frac{\partial h}{\partial r}(h-d)^{3}+K \frac{\partial c}{\partial r}(h-d)\right)\right], \\
\frac{\partial(h-d)}{\partial t}= & H(a-r) \frac{1}{a^{2}}-\frac{L}{\sqrt{R^{2}-r^{2}}}+\frac{1}{r} \frac{\partial}{\partial r}\left[r \frac{\partial h}{\partial r}(h-d)^{3}\right], \\
\frac{\partial d}{\partial t}= & -\frac{\tau p}{H s}\left(c_{s a t}-c\right)(h-d),
\end{aligned}
$$

where $c_{s a t}$ is the saturation concentration of the solute, $p$ is a precipitation rate, $s$ is a size scale for the concentration of crystals per depth of deposit, $R(t)$ is the boundary of the puddle and $L=\pi \kappa / q$. $h$ and $c$ parameters have been non-dimensionalised as before, however the time and radial parameters have been non-dimensionalized as follows: $r \rightarrow r / \lambda$, $a \rightarrow a / \lambda, R \rightarrow R / \lambda, t \rightarrow t / \sigma$, where $\lambda^{2} / \sigma=q / \pi H$. For limestone (Calcium Carbonate), $p$ is a function of temperature, $\mathrm{CO}_{2}$ pressure, and the depth of the fluid, and we take the value from [2]. The evolution equation for the depth of liquid, $(h-d)$, clearly contains a singularity at $r=R$ in the evaporation term. This unfortunately makes the system impossible to solve, as previously, using the pdpe routine in Matlab. Due to the limited time available on the project, it was not possible to develop an alternative method for finding a numerical solution before completion. It must also be noted that this system 


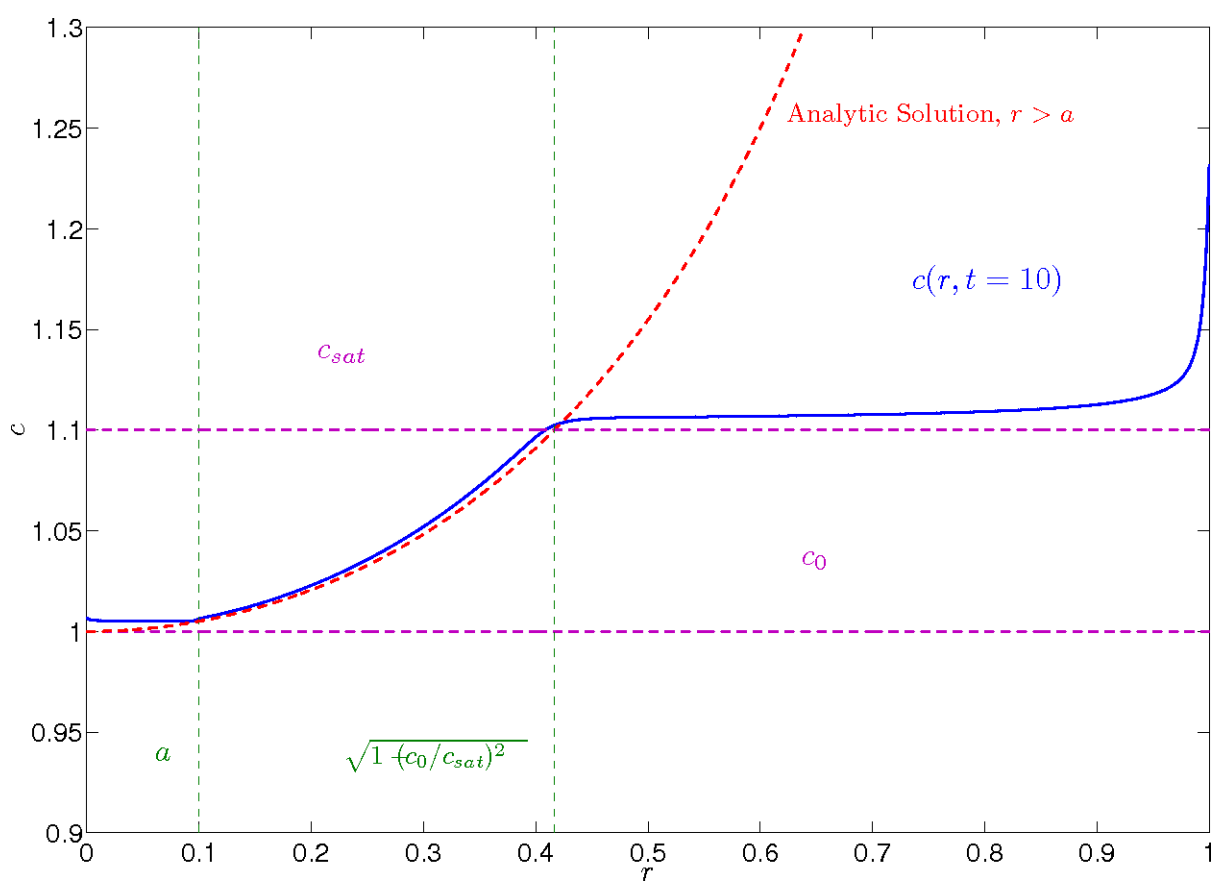

Figure 18: Numerical and analytical solutions to (34), where the solid blue line is the concentration profile $c(r)$ at $t=10$.

now has variable boundaries, and $r=1$ does not necessarily correspond to the edge of the puddle, and so any alternate method for solving the system would need to consider the variation in the outer boundary, $R(t)$.

We can, for small values of $d$, make the approximation $h>>d$, i.e. $h-d \approx h$, and so again use use the steady state form of $h$ derived in section 5.1.1, under the assumption $\partial h / \partial t=0$. We then calculate the depth profile, $d(r, t)$, independently from the concentration profile, $c(r, t)$. The resulting concentration profile at $t=10$ can be seen in figure 18 , where $c_{s}=1, c_{s a t}=1.1, a=0.1$, and $K=0.01$. For $c<c_{s a t}$, the same analytical solutions apply as previously, (24). For $c \geq c_{\text {sat }}$, the steady state solution is $c=c_{\text {sat }}$. It is possible to find the transition point at which $c=c_{\text {sat }}$ from (24):

$$
r\left(c_{\text {sat }}\right)=\sqrt{1-\left(\frac{c_{0}}{c_{\text {sat }}}\right)^{2}},
$$

and the analytical solutions can be seen plotted in figure 18. It can be seen that the analytical solution doesn't hold near $r=1$, where the advection and diffusion terms become important. The resulting depth profile, $d(r)$ can be seen in figure 19, along with the height profile $h(r)$, where the deposit of solute has clearly created a ring shape.

\subsection{Experiments}

To test the 'coffee ring' theory we first lowered the source to within a few $\mathrm{mm}$ of the aluminium sheet in order to first 'grow' a puddle to test the concept. Initially, the results 


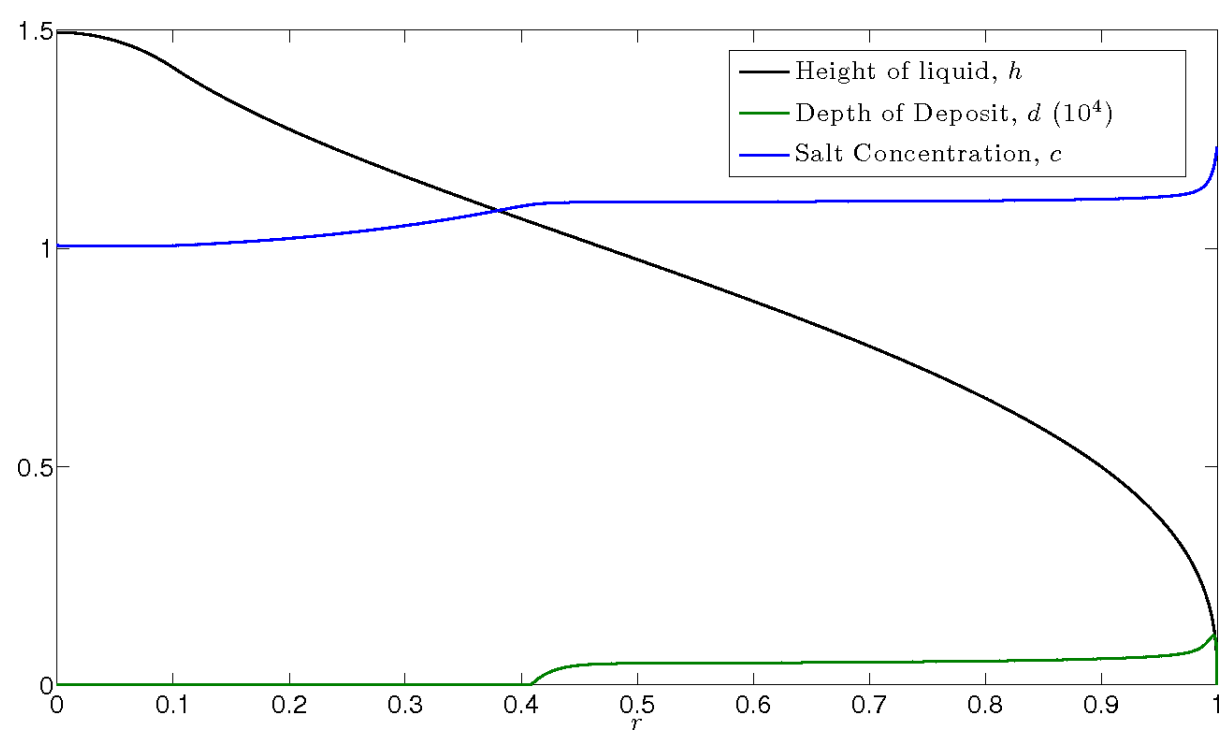

Figure 19: Solute concentration, blue, deposit depth, green, and liquid height, black, profiles at $t=10$. Note that the deposit depth profile has been multiplied by $10^{4}$ for clarity.

looked promising. Figure 20 shows the ring of salt produced by a puddle a few $\mathrm{cm}$ in diameter. However, increasing the rate of flow to grow puddles of the size required to explain the cave rings did not produce the results hoped for. Past a few $\mathrm{cm}$, growth is dominated by surface tension effects at the contact line of the puddle, see figure 21 . Whilst coating the aluminium sheet in vegetable oil to reduce this effect did result in a smoother boundary to the puddle, it still was not possible to produce a large, round puddle. Given that the aluminium sheet was kept as flat as was possible, and that cave floors are hardly uniform surfaces (see figure 1), this would seem to disprove the 'coffee ring' hypothesis. It was possible to find a flow rate at which evaporation matched the source flux, see figure 22, but this was definitely not a circular puddle.

The second set of experiments involved raising the source by $2 \mathrm{~m}$ in order to see if the dynamics of the source dropping from above would affect the puddle formation. The source now arrived in drops of just under $1 \mathrm{ml}$ in volume, which is similar to the volumes measured in situ by Nozzoli et al.[9]. It was found that, because the fraction of mass ejected as secondary drops was quite high, a higher flow rate was required to produce a puddle of a substantial size underneath the source. The correlation between puddle size and flow rate was expected from our model, however, while a substantial amount of crystals formed from the secondary splashes, none formed on the puddle itself, see figure 23. It is speculated that, from observations of the experiment in process, the large amount of kinetic energy imparted into the puddle by each drop effectively mixed the puddles so that the concentration profile predicted did not evolve, the solution within the puddles never exceeding saturation concentration once equilibrium was reached. As with the previous splashing experiments, see section 3.2, the salt splash formed a distribution with an exponential tail, see figure 24 .

We speculated that perhaps the porosity of the cave floor might dictate the formation of the cave rings, and so repeated the experiment with piece of black cloth on top of the 


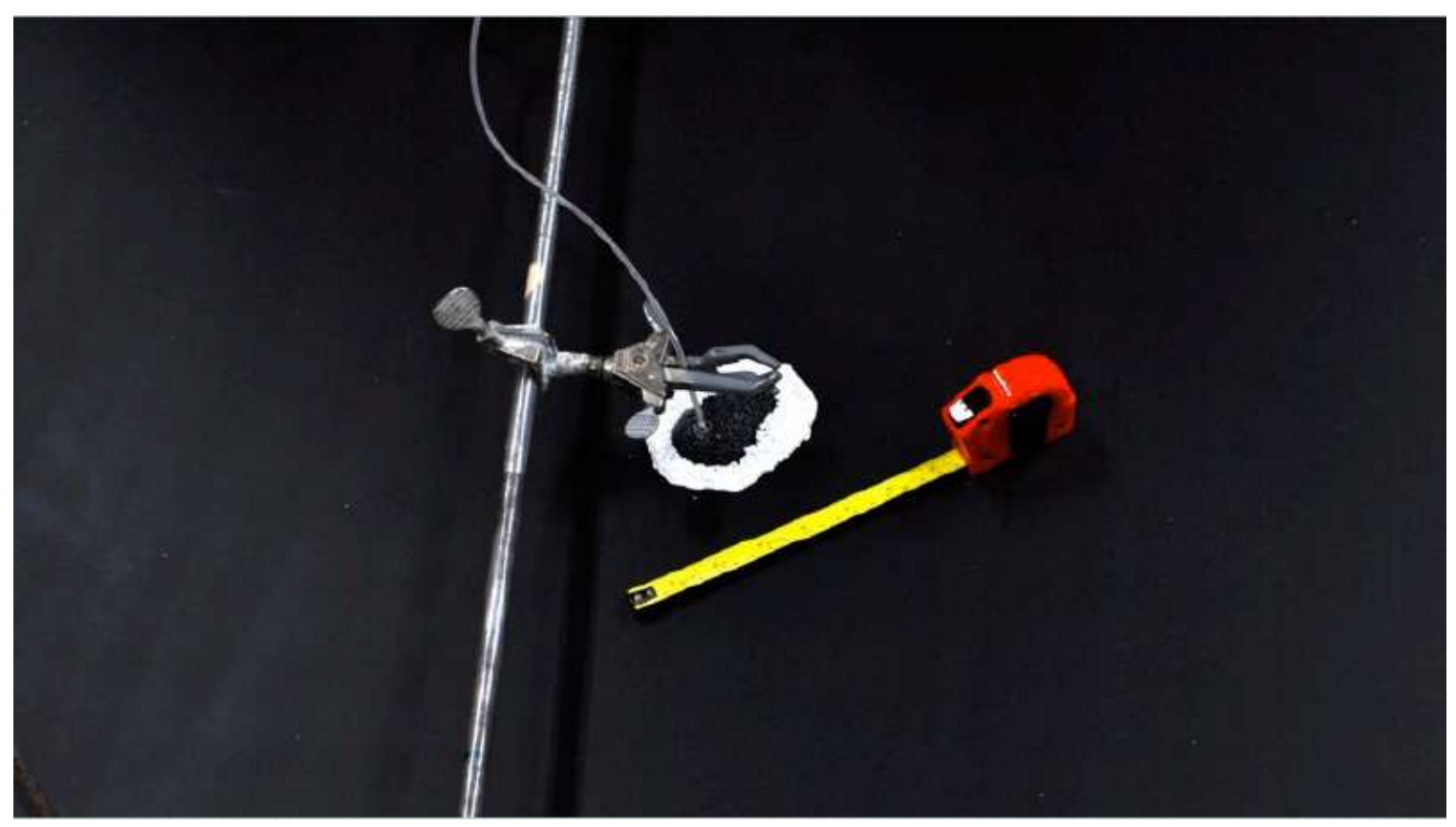

Figure 20: Initial experiment with the source close to the sheet, producing a small ring of salt. $10 \%$ salt solution was pumped at a rate of $1.4 \pm 0.2 \mathrm{ml}$ per hour for 46.5 hours

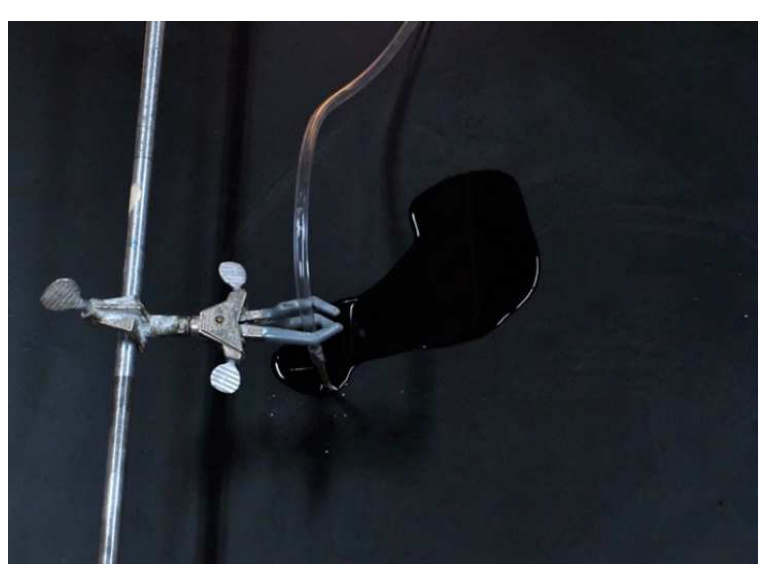

(a)

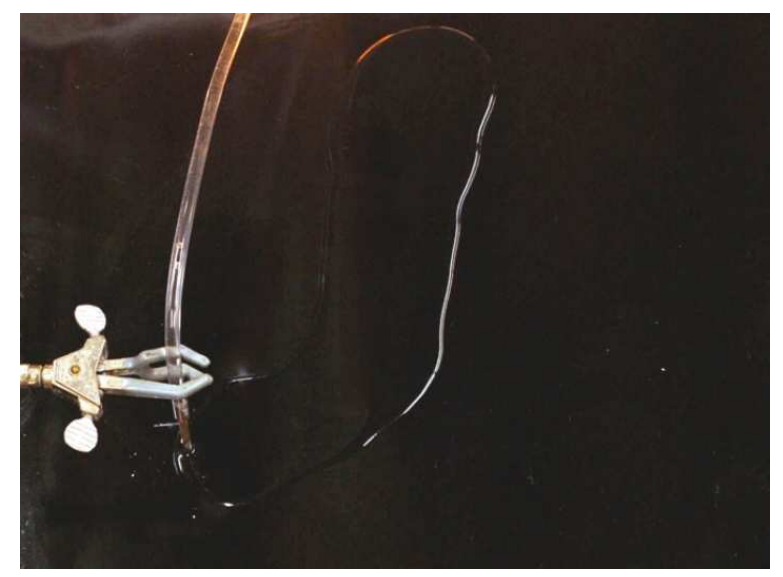

(b)

Figure 21: Two experiments that show the difficulties in creating a perfectly circular puddle, both with a $10 \%$ salt solution pumped at a rate of $14.7 \pm 0.7 \mathrm{ml}$ per hour. (a) shows a puddle after $2.25 \mathrm{hrs}$ with the original setup. (b) shows a puddle after $9 \mathrm{hrs}$, where the sheet has had a thin layer of vegetable oil applied to reduce surface tension effects. 


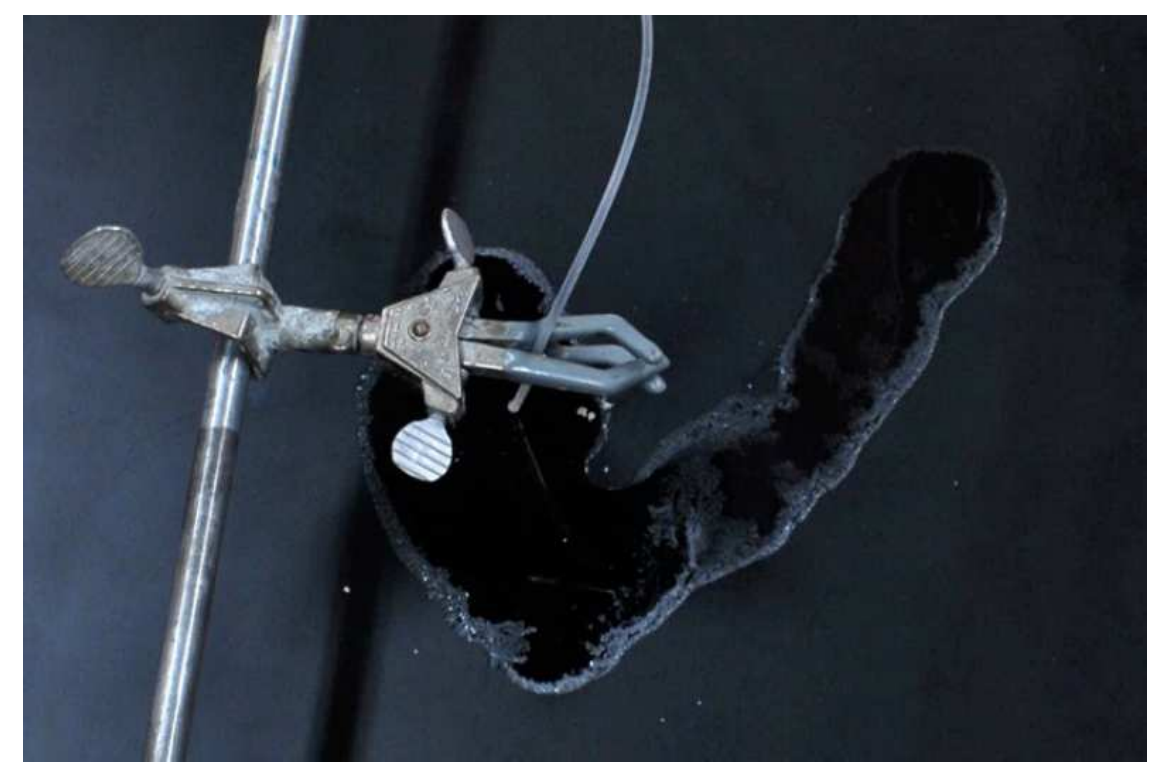

Figure 22: Experiment with flow rate of $6.4 \pm 0.4 \mathrm{ml}$ per hour after $5.25 \mathrm{hrs}$. Equilibrium has been reached and salt crystals are being deposited at the edge of the puddle, but the puddle is not circular.

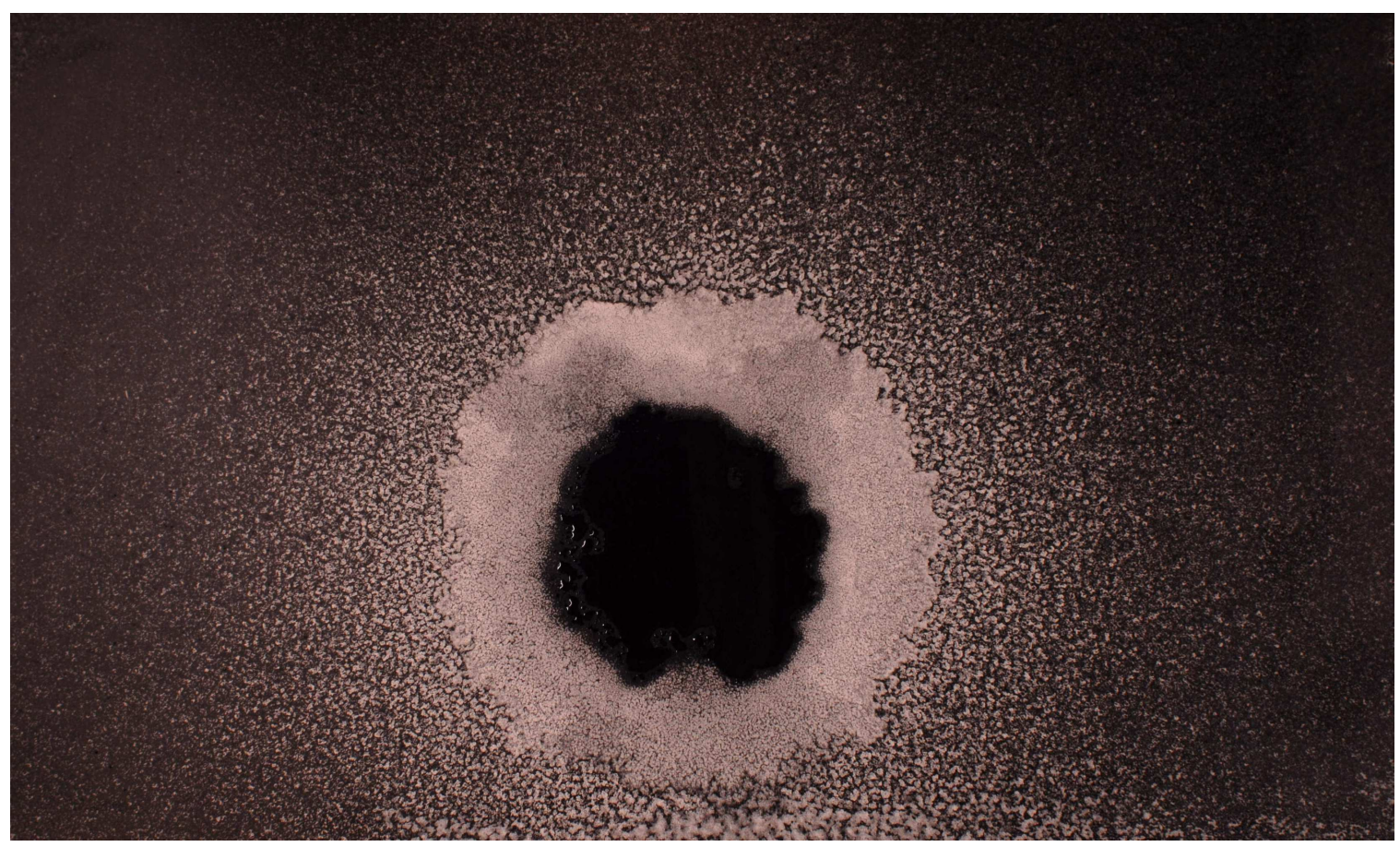

Figure 23: Experiment with source $\sim 2 \mathrm{~m}$ above the sheet, with $10 \%$ solution being pumped at a rate of $17.17 \pm 0.5 \mathrm{ml}$ per hour, after $26.75 \mathrm{hrs}$. The salt deposits are produced by secondary droplets ejected from the puddle, which can be seen in the centre of the picture. 


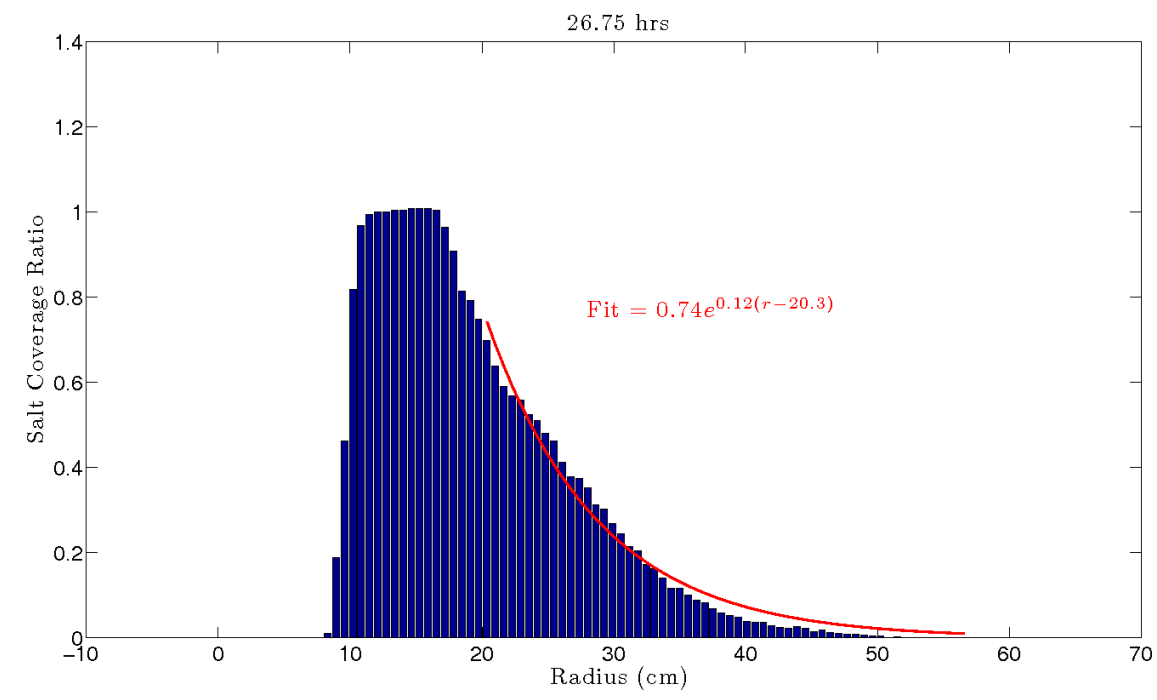

Figure 24: Salt coverage vs radius for figure 23 with an exponential curve fitted. There is $\mathrm{a} \sim 10 \mathrm{~cm}$ ring where the area is saturated.

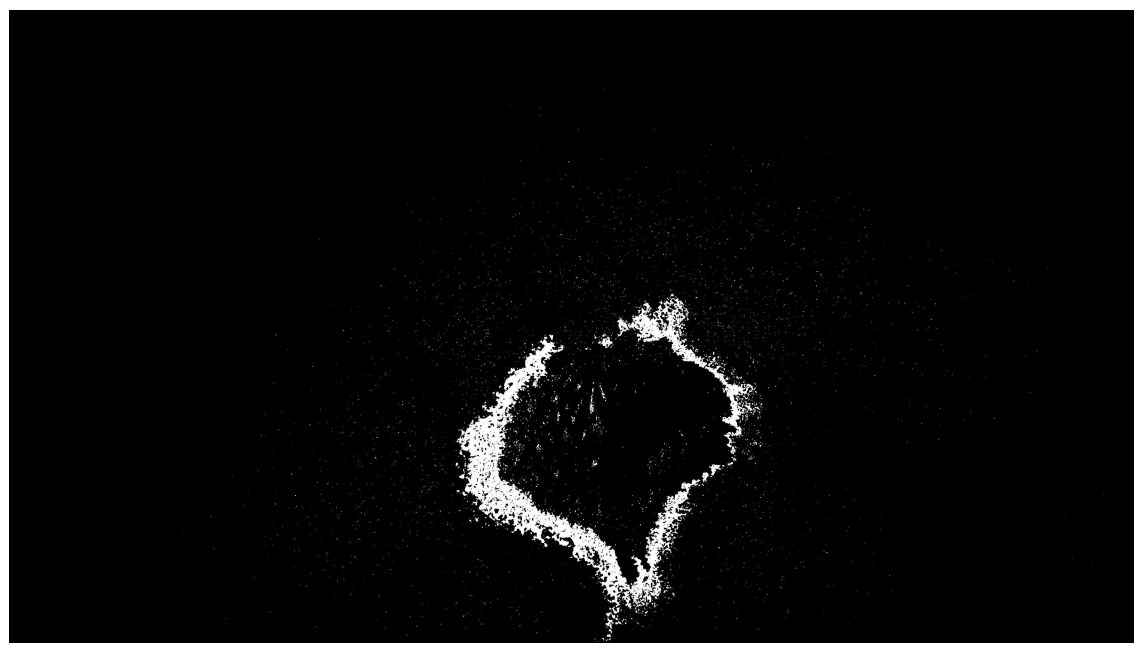

Figure 25: Experiment as previously, with black cloth covering the sheet, after 21.5 hrs. Salt has been deposited at the boundary of the wetted region, but the shape is non-uniform. 
sheet, in order to test whether the spread of the puddle through a porous medium might be more uniform. The resultant salt formation can be seen in figure 25. A ring has formed, as the previous mixing of the solution within the puddle is not possible, however the shape is still non-uniform.

\subsection{Speleothem Science}

As it appears that the coffee ring theory is not able to describe the large cave rings observed, we turned to the science of cave formations, or speleothems. There is a huge variety of formations, but the basic method of formation is the same for most, depicted in figure 26 :

- Rain water seeps down through the top layer of soil, dissolving Carbon Dioxide to form Carbonic acid.

- On contact, the acid dissolves the bedrock, normally Calcium Carbonate.

- The solution comes into contact with air on meeting a cave, and the dissolved stone is then deposited out.

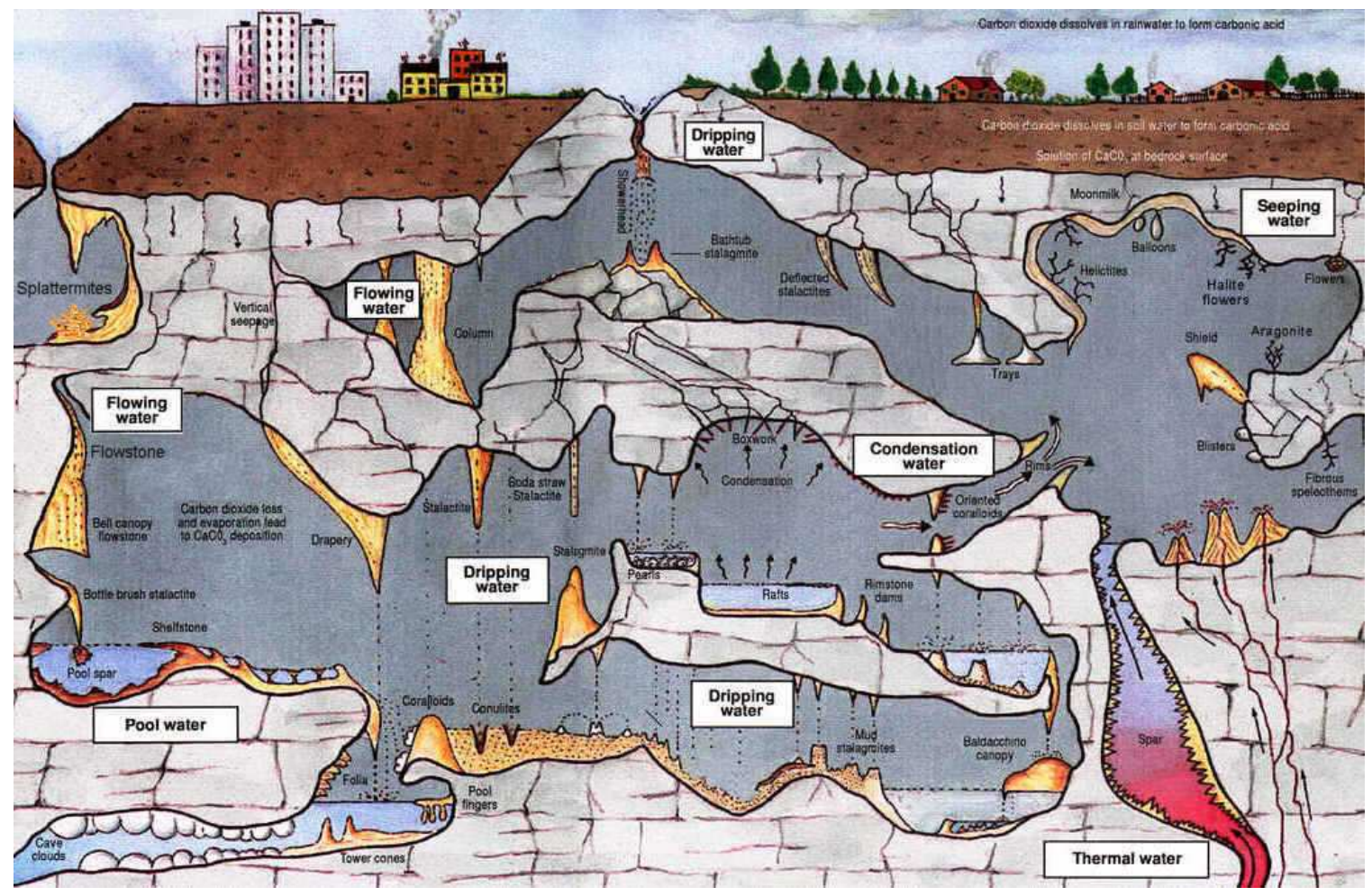

Figure 26: Depiction of the formation of speleothems, through the formation of Carbonic acid and the dissolving of Calcium Carbonate.

The overall chemical reaction involved is as follows:

$$
\mathrm{CaCO}_{3}+\mathrm{CO}_{2}+\mathrm{H}_{2} \mathrm{O} \leftrightarrow \mathrm{Ca}^{2+}+2 \mathrm{HCO}_{3}^{-} .
$$


The equilibrium for the reaction is towards the right, the ionic solution, when the solution is within the rock, not in contact with air. However, when the solution is in contact with the air, the chemical equilibrium moves to the left, and so $\mathrm{CO}_{2}$ is out-gassed quickly and $\mathrm{CaCO}_{3}$ deposited, as the solution is over-saturated. This is the case for the majority of formations, although in some situations, such as in highly humid regions or when the path the liquid takes is not sealed from air, the solution is under-saturated. The modelling for this project assumed that the solution was under-saturated, as is the case for the salt solution used in the experiments. As cave rings are relatively rare, it could be that they are in one of the unusual situations mentioned, however there is currently no data on the concentrations of Calcium Carbonate in the solutions forming cave rings.

It is possible to solve the model described in section 5.1.3 under the condition that the source concentration is greater than the saturation concentration, i.e. $c_{s}>c_{\text {sat }}$. The resulting solution at $t=10$ for $c_{s a t}=0.5, c_{s}=1$, is shown in figure 27 . It can be seen that, although some of the solute is still swept to the edge of the puddle, most is deposited in the centre, where the source is. The resultant deposit is shaped like a classic stalagmite.

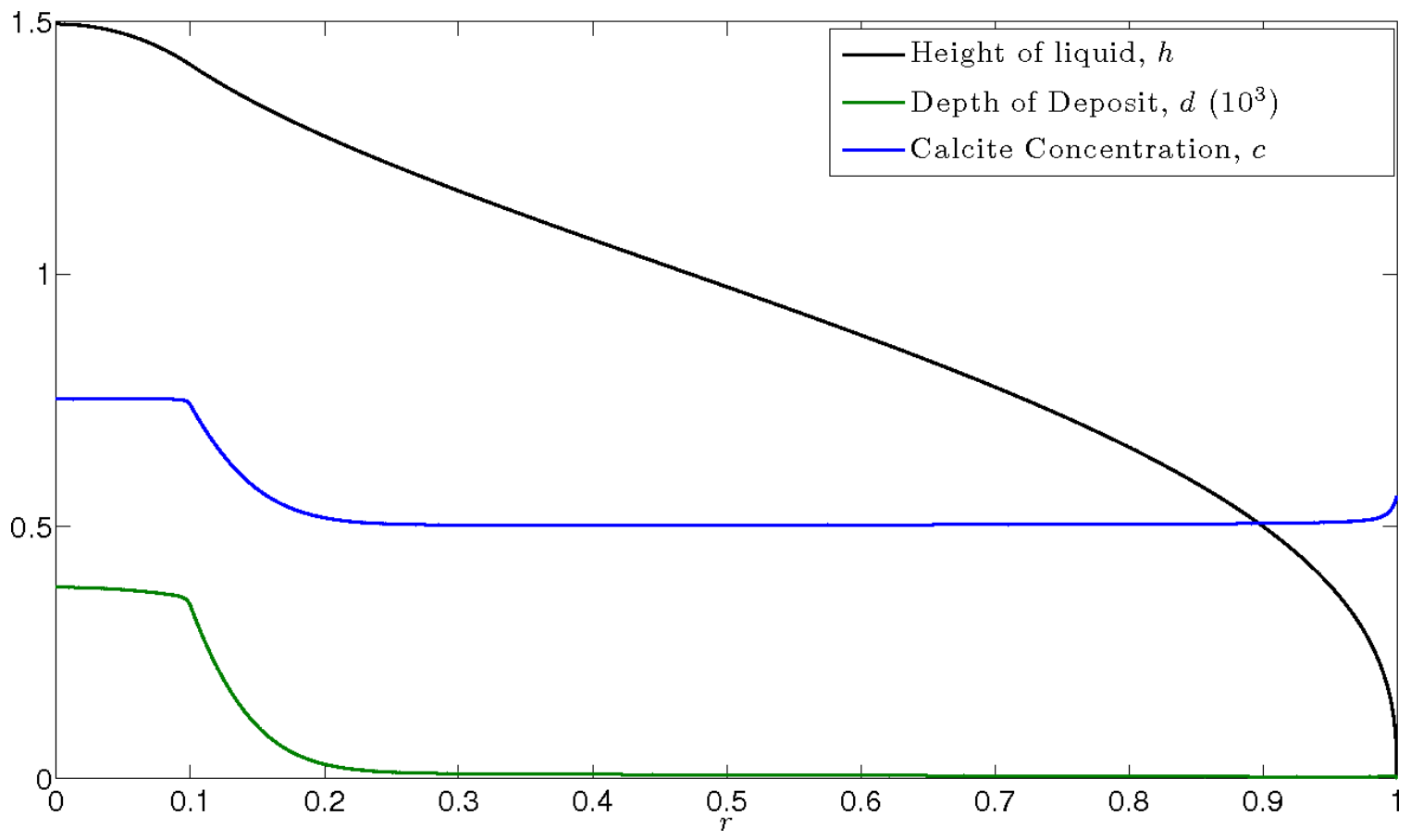

Figure 27: Numerical solution with $c_{s a t}=0.5, c_{s}=1, K=0.01$, and $a=0.1$, at $t=10$. The deposit depth, green line, has been scaled by $10^{3}$ to allow for comparison.

\section{Conclusion}

This project set out to test the hypotheses set out in Nozzoli et al.[9] for the formation of cave rings, namely the 'splash ring' and 'fall down ring' hypotheses, and to test our 'coffee ring' theory. It was shown clearly that both the 'splash' and 'fall down' hypotheses were 
unverifiable experimentally.

A mathematical model for large coffee rings was developed, and numerical solutions that matched our expectations for ring formation were produced. Unfortunately, experimentation showed that the flaw in our theory was that the formation of large, circular puddles seems to be impossible on a realistic surface, as surface tension at the contact line seems to dominate while the puddle grows, producing non-uniform shapes. Experiments also showed that the kinematics of drops arriving from $2 \mathrm{~m}$ produces significantly different sized puddles than those where the source was close to the sheet. The majority of cave formations result from solutions that are over-saturated in Calcium Carbonate when in contact with air, whereas our experiments used under-saturated salt solution.

It seems clear that coffee rings are unlikely to be the formation method for cave rings, although there is still a little work to be done before it can be ruled out completely. Due to the limitations of the equipment and time available, it wasn't possible to create puddles larger than c. $10 \mathrm{~cm}$ when the source was raised $2 \mathrm{~m}$ above the sheet. Using a higher source flux, it should be possible to see if the kinematics of the arriving drops affect the shape of larger puddles. Also, to rule out the 'fall down ring' hypothesis more comprehensively, an experiment could be designed where the source passes through a hole in a sheet, and the water collected below, in order to show that no drops split and land on the sheet.

In general, there appears to be a dearth of mathematical work in the area of speleothem formation, with an astounding range of speleothems, both beautiful and bizarre, still not understood. The development of a more accurate mathematical model for such formations could only be of benefit to the geological community.

\section{Acknowledgements}

Many thanks to both of my supervisors for the summer, Neil and Colm, who both, in their different ways, helped me stay inspired and focused throughout the project. I also wish to thank all the GFD summer fellows, who made the summer an incredibly enjoyable experience. Finally I want to thank my husband, Robert, for his continued support.

\section{References}

[1] R. F. Allen, The mechanics of splashing, Journal of Colloid and Interface Science, 124 (1988), pp. 309-316.

[2] D. Buhmann and W. Dreybrodt, The kinetics of calcite dissolution and precipitation in geologically relevant situations of karst areas. 1. open system, Chemical Geology, 48 (1985), pp. 189-211.

[3] R. D. Deegan, O. Bakajin, T. F. Dupont, G. Huber, S. R. Nagel, and T. A. Witten, Contact line deposits in an evaporating drop, Physical Review E, 62 (2000), pp. $756-765$.

[4] G. German and V. Bertola, Formation of viscoplastic drops by capillary breakup, Physics of Fluids, 22 (2010). 
[5] C. Hill And P. Forti, Cave minerals of the world, National Speleological Society, 2nd ed., 1998, pp. 94-95.

[6] H. Hu And R. G. Larson, Evaporation of a sessile droplet on a substrate, The Journal of Physical Chemistry B, 106 (2002), pp. 1334-1344.

[7] N. N. Lebedev, Special functions and their application, Prentice-Hall, 1965.

[8] L. Montanaro, Osservazioni sui "cherchi" della Grotta del Sorell, Bollettino del Gruppo Speleologico Sassarese, 13 (1991-1992), pp. 21-22.

[9] F. Nozzoli, S. Bevilacqua, and L. Cavallari, The Genesis of Cave Rings explained using Empirical and Experimental Data, Journal of Cave Karst Studies, 71 (2009), pp. 130-135.

[10] A. I. J. M. Van Dijk, A. G. C. A. Meesters, and L. A. Bruijnzeel, Exponential distribution theory and the interpretation of splash detachment and transport experiments, Soil Science Society of America Journal, 66 (2002), pp. 1466-1474.

[11] E. Villermaux and B. Bossa, Single-drop fragmentation determines size distribution of raindrops, Nature Physics, 5 (2009), pp. 697-702.

\section{A Full Concentration Solution}

In section 5.1.2, the time dependent part of the solution to (21) close to the edge of the puddle at large $t, A$, is set out in (31), where the constant, $k$, is given by (33). Rearranging, we find

$$
\begin{aligned}
k\left(c_{\text {sat }}, K\right)= & \frac{3^{1 / 4}}{2^{13 / 8}} c_{\text {sat }}\left[K^{11 / 9} \int_{0}^{\infty} \zeta^{3 / 8} \exp \left\{-\frac{2^{15 / 8}}{3^{7 / 4}} \zeta^{9 / 8}\right\} d \zeta\right. \\
& \left.-K^{19 / 9} \int_{0}^{\infty} \zeta^{11 / 8} \exp \left\{-\frac{2^{15 / 8}}{3^{7 / 4}} \zeta^{9 / 8}\right\} d \zeta\right]^{-1}, \\
\approx & \frac{3^{1 / 4}}{2^{13 / 8}} c_{\text {sat }}\left[1.74 K^{11 / 9}-3.48 K^{19 / 9}\right]^{-1},
\end{aligned}
$$




\title{
Dynamics of Air-Blown Dimples
}

\author{
Renske Gelderloos \\ Advisors: C.P. Caulfield and A. Belmonte
}

\section{Introduction}

A classical problem in fluid mechanics is that of a jet impinging on a solid surface. During the 1960's, partly driven by applications in the steel industry, the exploration of this field of study shifted towards the interaction between a gas jet and a deformable liquid surface (Figure 1), where often the shape of the deformation and its relation to the balance of forces were studied. This is also a very simplified representation of the interaction between the wind and the ocean.

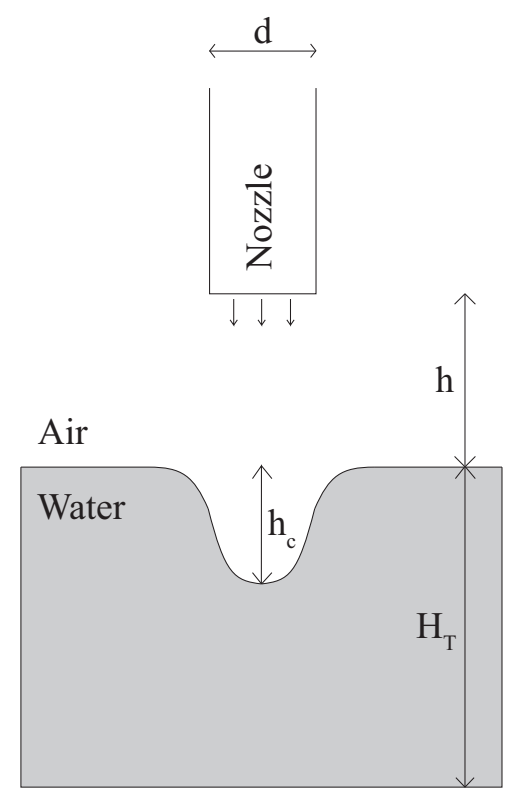

Figure 1: Schematic drawing of the experimental set up. Air is blown from a nozzle at right angles with a still water surface in a tank.

The first report of work on this subject is by Banks and Chandrasekhara (1), who performed experiments with an air jet impinging on a water surface. They identified three regimes: a steady cavity, an oscillating cavity, and splashing. Only the first of these three regimes was studied in more detail and scaling approaches were suggested to establish a relation between the impact of the jet and the depth of the cavity. A few years later Turkdogan (9) performed similar experiments, but included liquids of different densities 
in the analysis and especially focused on the relation between the nozzle height and the depth and width of the cavity. Cheslak et al. (3) also studied the geometric properties of a steady air-blown dimple and found simple relations predicting the depth and diameter of the cavities.

A different approach was taken by Olmstead and Raynor (6), who solved the whole shape of steady shallow dimples using conformal mapping. Almost two decades later, new numerical procedures enabled Vanden-Broeck (10) to study slightly deeper cavities with this method, but a conformal mapping approach to this problem without limitations to the depth of the cavity was only provided recently by He and Belmonte (5).

Although instability of the cavity at higher jet-impact velocity was already reported by Banks and Chandrasekhara (1), surprisingly little attention has been given to this phenomenon. The available literature is restricted to predicting the onset of instability, using energy balances to determine the decisive factors. A pioneering study by Rosler and Steward (8) identified two unstable regimes in their experiments: an unstable oscillating regime and a splashing regime. In the former regime three modes of oscillation were identified which were described qualitatively (Figure 2). The onset of the unstable and splashing regimes were shown to depend on a critical jet velocity, with a strong dependence on the interfacial surface tension. Berghmans (2) showed in a theoretical study that increasing liquid density or surface tension is stabilizing, while increasing the gas density is destabilizing. The jet radius was also found to be important.

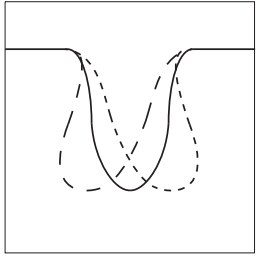

E-mode

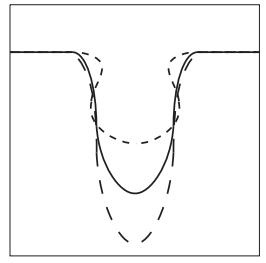

V-mode

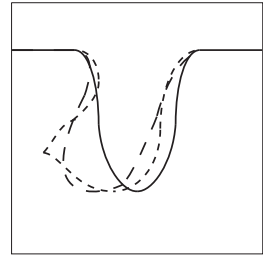

B-mode

Figure 2: The three modes of instability. In the E-mode the tip of the dimple traces out an ellipse. In the $\mathrm{V}$-mode, the tip oscillates in the vertical direction only. In the B-mode, the tip rolls up the side of the dimple.

The reason behind the sparse coverage of this very interesting problem is likely in the limitations imposed by the available observational equipment. While with the cameras used four decades ago this regime was very difficult to study experimentally, today's high-speed cameras offer the opportunity to capture the movements of the cavity and study them in detail. To our knowledge there is one study (4) in which an attempt was made to study the oscillations of the cavity using a video camera, but no conclusions could be drawn from these results. Here, we use a high speed camera to obtain movies of unstable dimples in a variety of regimes for an air-water system. We explore the dependence of the regimes on key nondimensional parameters and study the spectral properties of the dimple oscillations.

The experimental set up and image processing are discussed in Section 2. The results are first discussed from a qualitative point of view in Section 3. Then a quantitative analysis 
is given in Section 4, on the geometric properties of the dimple as well as a spectral analysis of the dimple oscillations. Section 5 discusses the results and gives an overview of the conclusions from this study.

\section{Method}

\subsection{Experimental set up}

The jet in the experiment was produced by attaching the wall air supply to a nozzle, which was fixed to an adjustable frame above a layer of water in a tank. The circular nozzle had an inner diameter of $1.35 \mathrm{~mm}$. The square Plexiglas tank was $11.2 \mathrm{~cm}$ in height and $20 \mathrm{~cm}$ wide. The water depth in the tank was $7 \mathrm{~cm}$ unless stated otherwise. To regulate the air flow rate in the jet, a pressure meter (Digital air regulator, Central Pneumatic Professional, model no. 98426) was used. The pressure was calibrated with a volume rate meter (Omega Engineering Gas Mass Flow Meter FMA 1818) to obtain the flow rate $Q$. The frame to which the nozzle was attached could be moved up and down to adjust its height above the water surface; the position could be read from a calibrated scale. The fluid properties of interest to this experiment are the kinematic viscosity of the gas $\left(\nu_{g}=15.68 \cdot 10^{-6} \mathrm{~m}^{2} \mathrm{~s}^{-1}\right)$, the densities of the gas and the liquid $\left(\rho_{g}=1 \mathrm{~kg} \mathrm{~m}^{-3} ; \rho_{l}=1000 \mathrm{~kg} \mathrm{~m}^{-3}\right)$, and the interfacial surface tension $\left(\sigma=71.97 \mathrm{dyn} \mathrm{cm}^{-1}=71.97 \cdot 10^{-3} \mathrm{~N} \mathrm{~m}^{-1}\right)$. The data acquisition is done with a digital high-speed camera (Phantom v5.0) at a 3000-fps frame rate. Only for the surface views (Section 3.2) the frame rate was adjusted to $300 \mathrm{fps}$.

\subsection{Parameter regime}

The two key nondimensional parameters that are varied in this study are the nondimensional nozzle height $(\zeta=h / d)$, which determines the distance over which the spreading jet can evolve, and the Reynolds number, which (for fixed $\zeta$ ) determines the impact pressure of the jet. The Reynolds number is here defined as

$$
\operatorname{Re}=\frac{4 Q}{\pi d \nu_{g}}
$$

and varies between $8.5 \cdot 10^{2}$ and $3.4 \cdot 10^{3}$. $\zeta$ varies between 7.4 and 37.0, equivalent to 1 and $5 \mathrm{~cm}$ above the water surface, respectively.

Four other nondimensional parameters, which are mentioned in literature to be important for this problem, are not consciously changed but vary along with $\zeta$ and Re. The first is the nondimensional depth of the dimple $\eta=h_{c} / d$ (variation between 0.8 and 10.0). The second and third are a measure for the importance of surface tension. The Weber number $(2 ; 5)$, defined as the ratio between inertial forces and surface tension, is given by

$$
\mathrm{We}=\frac{\rho_{g}\left(\frac{Q}{1 / 4 \pi d^{2}}\right)^{2} r_{\text {jet }}}{\sigma},
$$

and varies in our experiments between 1.4 and 105 The Bond number $(2 ; 5)$, which is the ratio between gravitational forces and surface tension, is given by

$$
\mathrm{Bo}=\frac{\left(\rho_{l}-\rho_{g}\right) g r_{\text {jet }}^{2}}{\sigma},
$$


and varies between 0.1 and 3 . The last nondimensional number of importance to this study is the Froude number (1), which is defined as the ratio between a characteristic velocity and a gravitational wave velocity:

$$
\mathrm{Fr}=\frac{\left(\frac{Q}{1 / 4 \pi d^{2}}\right)}{\sqrt{g h_{c}}},
$$

and varies between 107 and 127 in our experiments. The dynamics of the surface waves were however not studied here quantitatively.

\section{$2.3 \quad$ Image processing}

A five-step process was followed in obtaining the contours of the cavity from the movies (Figure 3). First the black and white avi movies, captured with the high-speed camera, were loaded into Matlab and separated into individual frames (Figure 3a). Second, to maximize the amount of information that could be extracted from the movie frame, the contrast in the images was enhanced using CLAHE (Contrast-Limited Adaptive Histogram Equalization, Figure $3 \mathrm{~b}$ ). This technique determines the histogram of the grayscale values of the pixels for small regions in the image and then enhances the contrast in such a way that the histogram of the output approximately matches a uniform distribution. Neighbouring tiles are combined using bilinear interpolation to eliminate artificially induced boundaries. An edge detection scheme is applied on the filtered image to define the shape of the cavity (Figure 3c). Note that the contrast enhancement step improves the result of the edge detection considerably, especially tracing the shallow part of the cavity (Figure 4). The Sobel method was found to give the best representation of the deep as well as the shallow part of the cavity shape. This method calculates the first derivative of the grayscale intensity in both $\mathrm{x}$ and $\mathrm{y}$ directions, and locates the edge at minimum and maximum values of this gradient as long as this value exceeds a certain threshold value. Last, the outer contour of the cavity was traced by moving upward along the vertical direction in the frame and tracing the first 'edge-point' for every column (Figure 3d). The shapes thus obtained were lowpass filtered to reduce artificial noise introduced in the detection process, before statistics such as the cavity depth, the angle with the vertical, and frequencies were calculated. These are discussed in Section 4. For the spectral analysis the time series were first detrended, after which a Hann filter was applied. The frequency spectrum was calculated using a FFT algorithm.

\section{$3 \quad$ Experimental phenomenology}

\subsection{Cavity}

A variety of shapes and movements of the dimple are observed in the experiments. In Figure 5 the sequence of modes is shown when the Reynolds number of the jet is increased while the nozzle height is fixed at the lowest position considered in this study $(\zeta=7.4)$. For low jet Reynolds numbers the cavity is stable and slowly deepens when the jet flow is increased (Figure 5a). We will refer to this state as the S-mode. At a critical value of $R e=1075$ instability sets in and the tip of the dimple traces out an ellipse (E-mode, Figure 5b). This mode only exists for a very narrow range of the jet Reynolds number, as it quickly changes 


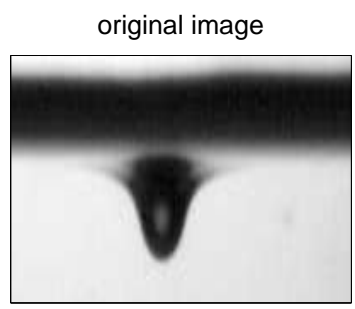

(a) enhanced contrast

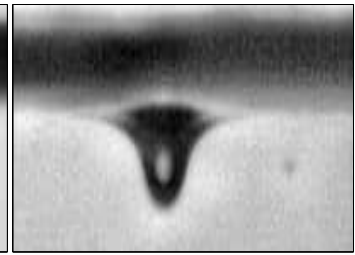

(b)

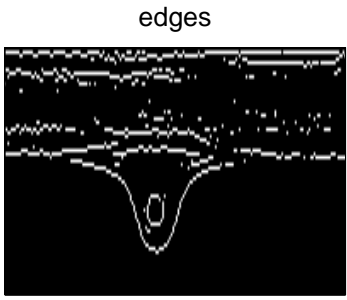

(c)

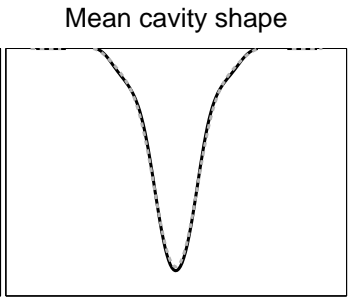

(d)

Figure 3: From the original image frame to a cavity outline. First the contrast is enhanced using CLAHE (b), then the edge is detected using the Sobel method (c). The contour of the cavity is found from the detected edges (black solid line in (d)), and lowpass filtered for further analysis (gray dotted line in $(\mathrm{d})) .(\zeta=7.4, \operatorname{Re}=1050)$

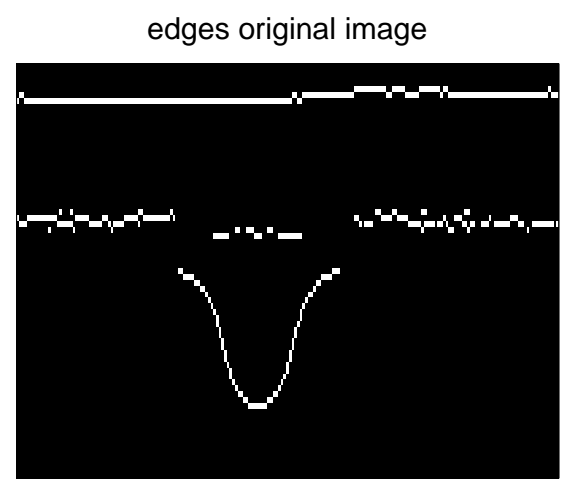

(a) edges filtered image

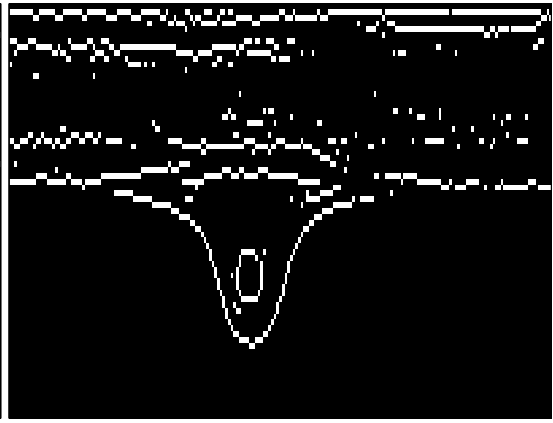

(b)

Figure 4: Improvement in the edge detection results due to contrast enhancement.

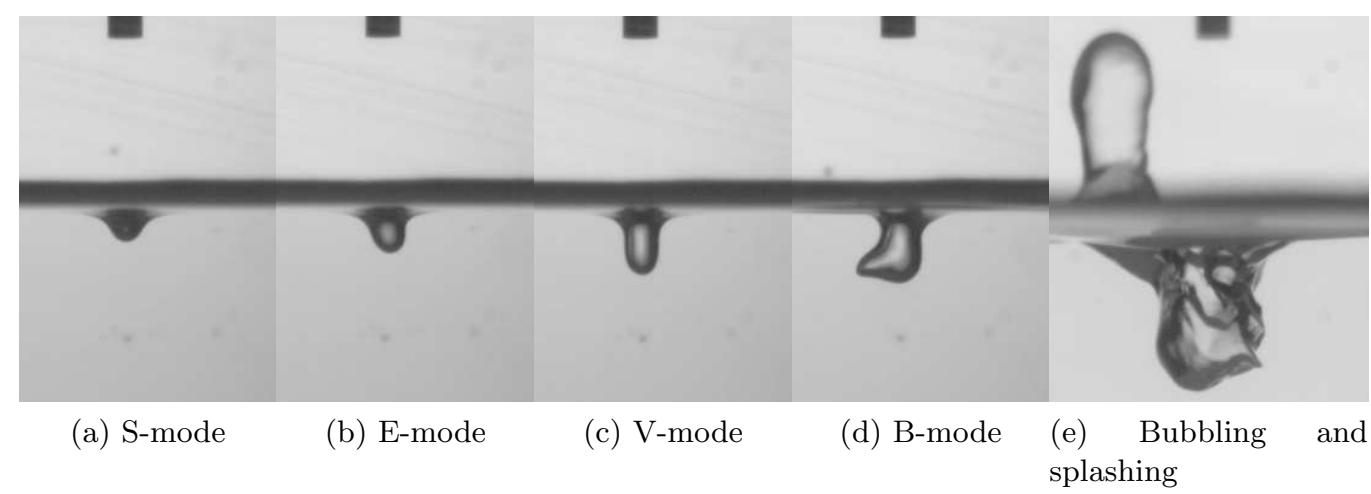

Figure 5: Subsequent stages of the dimple with increasing jet Reynolds number for a fixed nozzle height $(\zeta=7.4)$. (a) A stable dimple $(\operatorname{Re}=1030)$. (b) The tip traces out an ellipse $(\operatorname{Re}=1075)$. (c) The tip moves up and down vertically $(\mathrm{Re}=1095)$. (d) The tip bends and rolls up the side of the dimple $(\operatorname{Re}=1230)$. (d) Bubbling and splashing $(\operatorname{Re}=3360)$. 
into a purely vertical oscillation of the tip, here referred to as the V-mode (Figure 5c). If the flow rate in the jet is increased even further, the tip starts to bend and roll up the side of the dimple, which we call the B-mode (Figure 5d). At very high Reynolds numbers (Figure 5e) bubbling and splashing is observed.

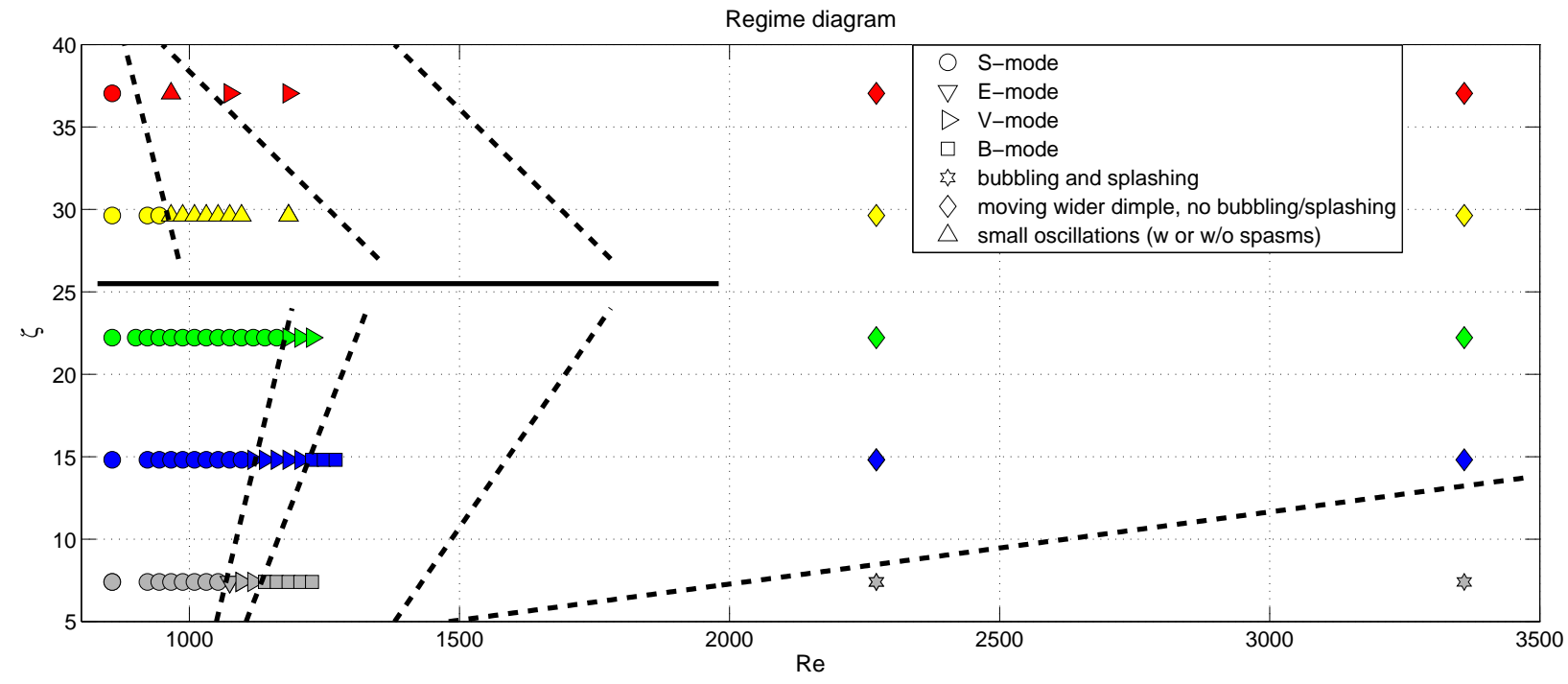

Figure 6: Overview of the observed regimes as a function of the jet Reynolds number Re and the nondimensional nozzle height $\zeta$.

The onset of the next mode shifts to different Reynolds numbers at the other nozzle heights considered in this study. The regime diagram in Figure 6 shows the observed modes for several combinations of $\zeta$ and Re. A first interesting feature from this graph is the increase in Reynolds number required for instability with $\zeta$ for the lowest three values of $\zeta$ considered (beneath the solid black line in Figure 6). For the larger nozzle heights, the required Reynolds number decreases with $\zeta$. Although the measurements are sparser in the remainder of the regime diagram, this seems to be a robust feature as indicated by the speculative dashed lines. Also, in the two largest $\zeta$ cases the dimple starts 'shivering' when it is still stable, i.e. there are very small, noise-like oscillations. It is well known that it takes a distance of about 30 nozzle diameters for a turbulent jet to become fully developed (7). We therefore believe that the horizontal solid line indicates the nozzle height above which the jet shows fully developed turbulence. The noise-like behaviour above this height is then due to instability of the jet rather than an instability of the dimple.

A second result from this figure is that for all the nozzle heights except for the smallest one, the dimple widens at high Reynolds number (Figure $7 \mathrm{~b}$, a stable small dimple is given in Figure $7 \mathrm{a}$ for comparison). We speculate that the width of the dimple is in general restricted by the interfacial surface tension, but that the high impact pressure of the higher Reynolds number jet is able to overcome the surface tension and creates a wider dimple. At very small $\zeta$ the dimple displays bubbling and splashing behaviour. It is expected however that the dimple will enter the splashing state at the larger nozzle heights as well at even higher jet Reynolds numbers, which were not currently studied. It should be noted that the 
modes are not entirely identical for the different nozzle heights. For example, at $\zeta=24.4$ the $\mathrm{V}$-mode is a very elegant one and resembles a Mexican hat (Figure 7c). However, the overall behaviour is the same.

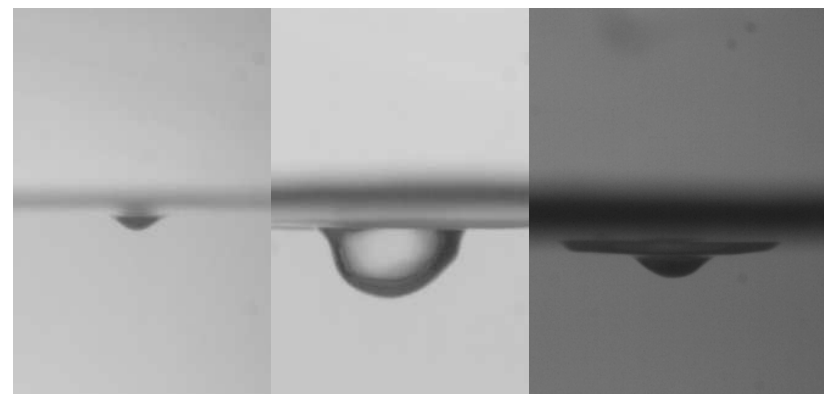

(a)

(b)

(c)

Figure 7: Snapshots (a) Stable dimple: $\zeta=22.2$, Re $=990$ (b) Wide oscillating dimple: $\zeta=14.8, \operatorname{Re}=2270$ (c) The "mexican hat", a very elegant V-mode: $\zeta=24.4, \operatorname{Re}=1400$

In this study we focused on an air-water system. The influence of interfacial surface tension $\sigma$ and liquid viscosity $\nu_{l}$ were studied briefly as well from a qualitative point of view only. The surface tension was altered by adding 2-3 drops of dish-washing liquid (Ajax Lemon) to the water in the tank, which decreased the stability of the dimple. To test the influence of viscosity, the tap water was replaced by glycerol and water-glycerol mixtures. The experiments clearly showed increased stability of the dimple, but were not conclusive quantitatively.

\subsection{Surface}

While motions of the liquid phase are consistently neglected in analyses presented in literature, the observed motion shows an interesting pattern. When the cavity is in the S-mode and thus not moving, the flow at the surface displays a saddle-point like pattern at the location of the dimple, with water flowing in from two sides and flowing out in the perpendicular directions. It starts up as soon as the jet is switched on, and ceases when the air source is switched off. When the air rate is turned up, waves are formed at the surface which spiral out from the cavity (Figure 8a) accompanying the E-mode. At higher Reynolds number the surface waves were more axisymmetric (Figure 8b) and increased in amplitude with increasing jet Reynolds number. The wave formation was clearly triggered by the dimple oscillations, but a quantitative analysis of the surface phenomena are beyond the scope of this study.

\section{Data analysis}

\subsection{Geometric properties of the dimple}

The cavity depth $\left(h_{c}\right.$ in Figure 1$)$ increases with increasing jet strength and decreases with increasing nozzle height. This is shown in Figure 9a, where the symbols correspond to the 


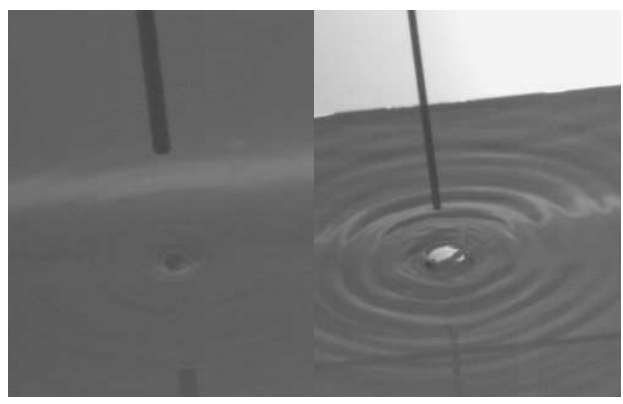

(a)

(b)

Figure 8: Snapshots of the surface wave field (a) E-mode: $\zeta=7.4, \operatorname{Re}=1075$ (b) Bubbling and splashing: $\zeta=14.8, \operatorname{Re}=2270$

cavity depth, averaged over all frames of a single movie with the corresponding jet strength $\operatorname{Re}$ (x-axis) and nozzle height $\zeta$. The colour coding for $\zeta$ and symbols for mode of instability are the same as used in Figure 6. The low-Reynolds number regime is enlarged in Figure $9 \mathrm{~b}$. The maximum cavity depth, which is a more objective measure for oscillating dimples, is plotted in Figures 10a and 10b. (Note that cavity depths on the red line $(\zeta=37.0)$ exceed the values on the yellow line $(\zeta=29.6)$ in the low-Reynolds number regime. This is not a physical feature however, but a result of increasing difficulty in obtaining sharp images as the bottom of the cavity approached the meniscus.) The area of the two-dimensional image of the cavity shows a similar behaviour (not shown).

The depth of the stable cavities slowly increases when the jet strength is raised (generally $\operatorname{Re}<1050$ ). Then a clear difference is observed between the three smallest nozzle heights and the two larger ones. In the former case, the cavity depth raises quickly between a Reynolds number of approximately 1050 and 1200, around the point of instability. In the latter case the cavity depth only increases slightly over this range of Reynolds numbers. This is in line with the preliminary conclusion from Figure 6 in Section 3.1 that the behaviour of the dimple is different for the larger two nozzle heights: the turbulence in the jet is already fully developed and therefore the peak velocity is lower at impact. The jet is therefore unable to exert a large enough pressure on the water surface to deepen the dimple further.

A second interesting property is the evolution of the curvature of the cavity as the jet strength is increased. In Figure 11 the maximum curvature is given for the lowest three nozzle heights (the jet does not form a stable cavity over a range of Re at larger nozzle heights and the curvature can therefore not be determined well). As the point of instability is approached, the maximum curvature quickly increases. The lines suggest that instability sets in when a critical value of the curvature is exceeded, around $11 \cdot 10^{-3}$. Figure 10a suggests that a similar critical value of the cavity depth (or jet impact velocity) may exist for the onset of the bubbling and splashing phase. In the current set of experiments this critical depth, which is at 11 to $12 \mathrm{~mm}$, is only reached in the smallest $\zeta$ case. 


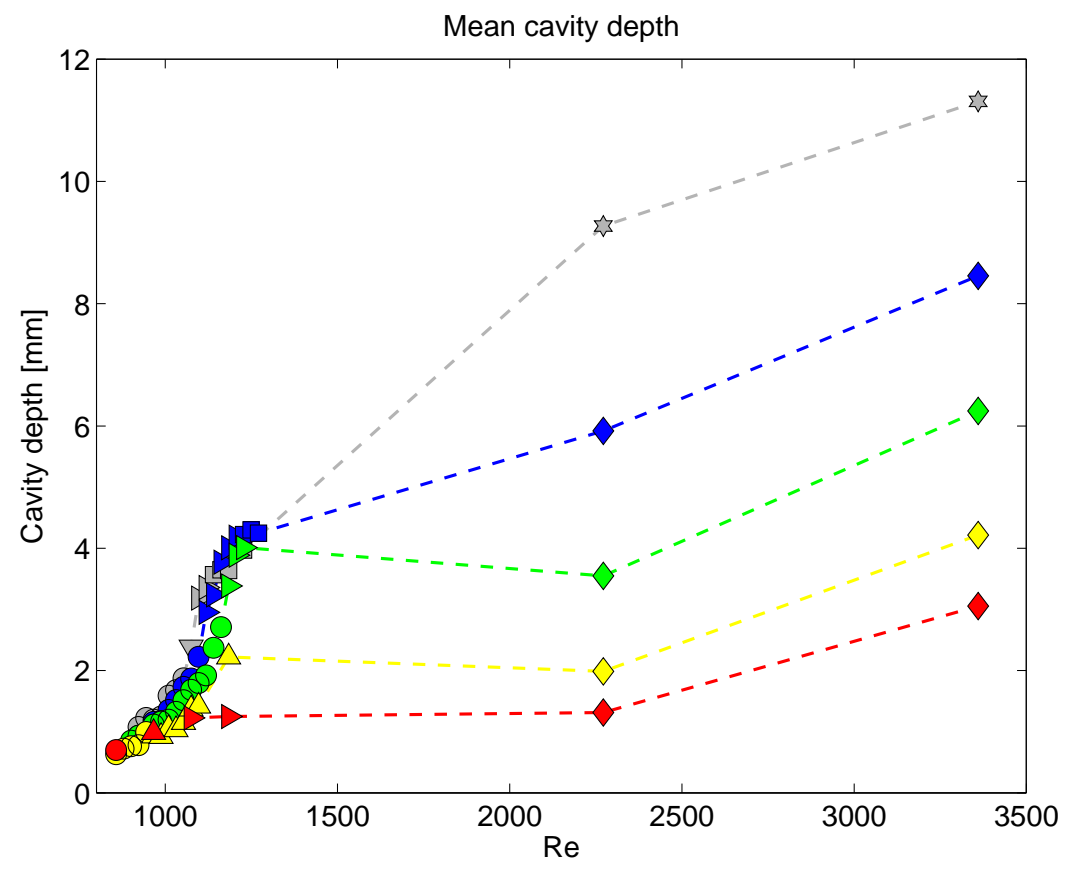

(a)

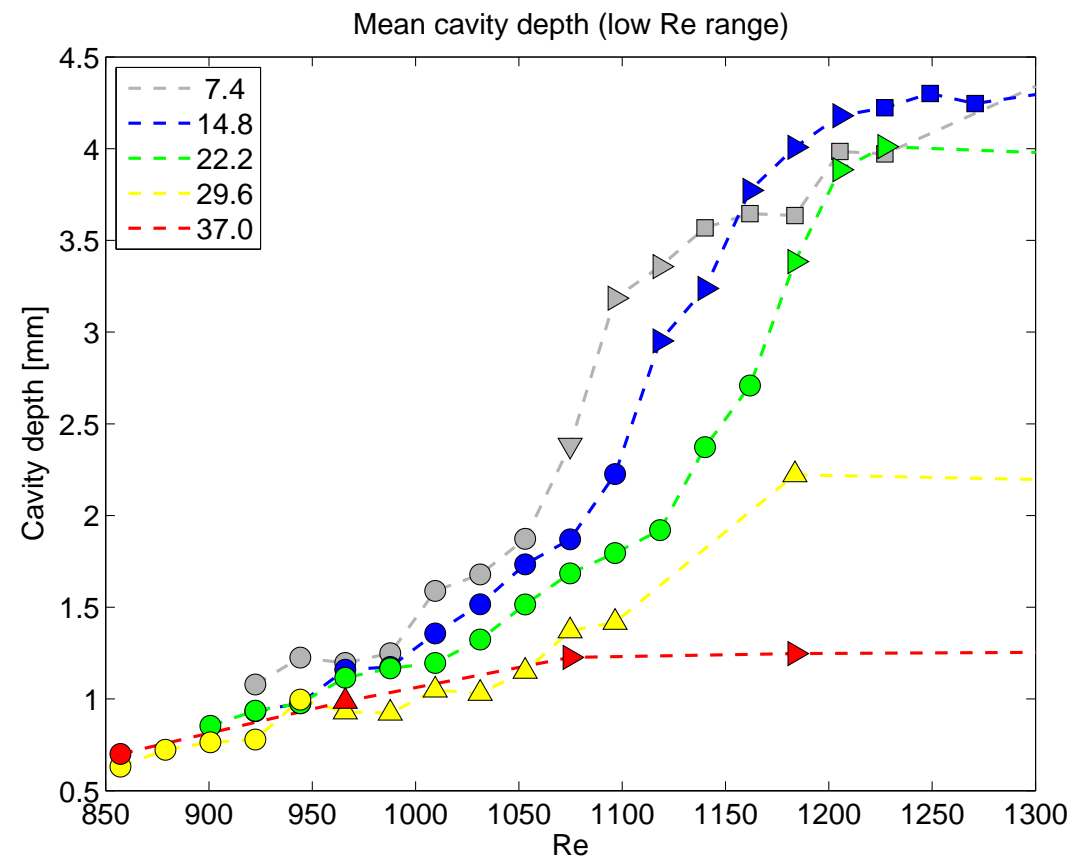

(b)

Figure 9: Mean cavity depth $h_{c}$ as a function of the jet Reynolds number. The colours and symbols are as in Figure 6. 


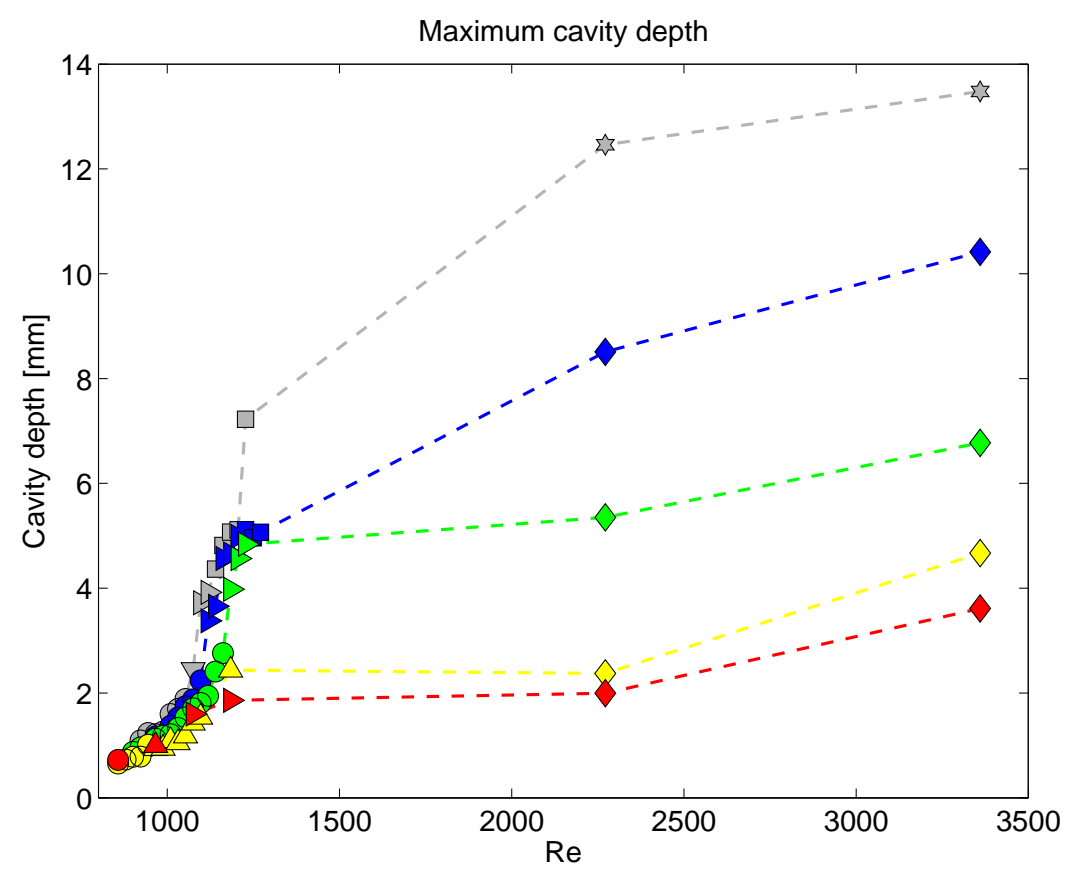

(a)

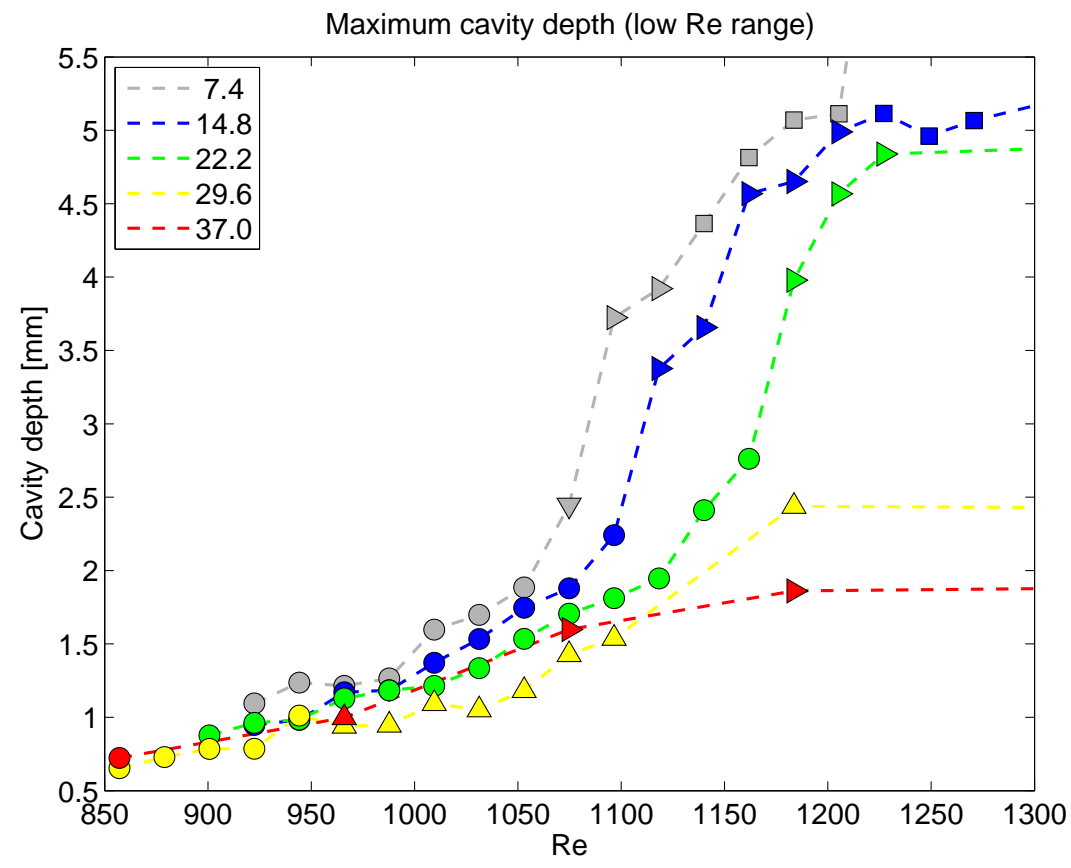

(b)

Figure 10: Maximum cavity depth $h_{c}$ as a function of the jet Reynolds number. The colours and symbols are as in Figure 6. 


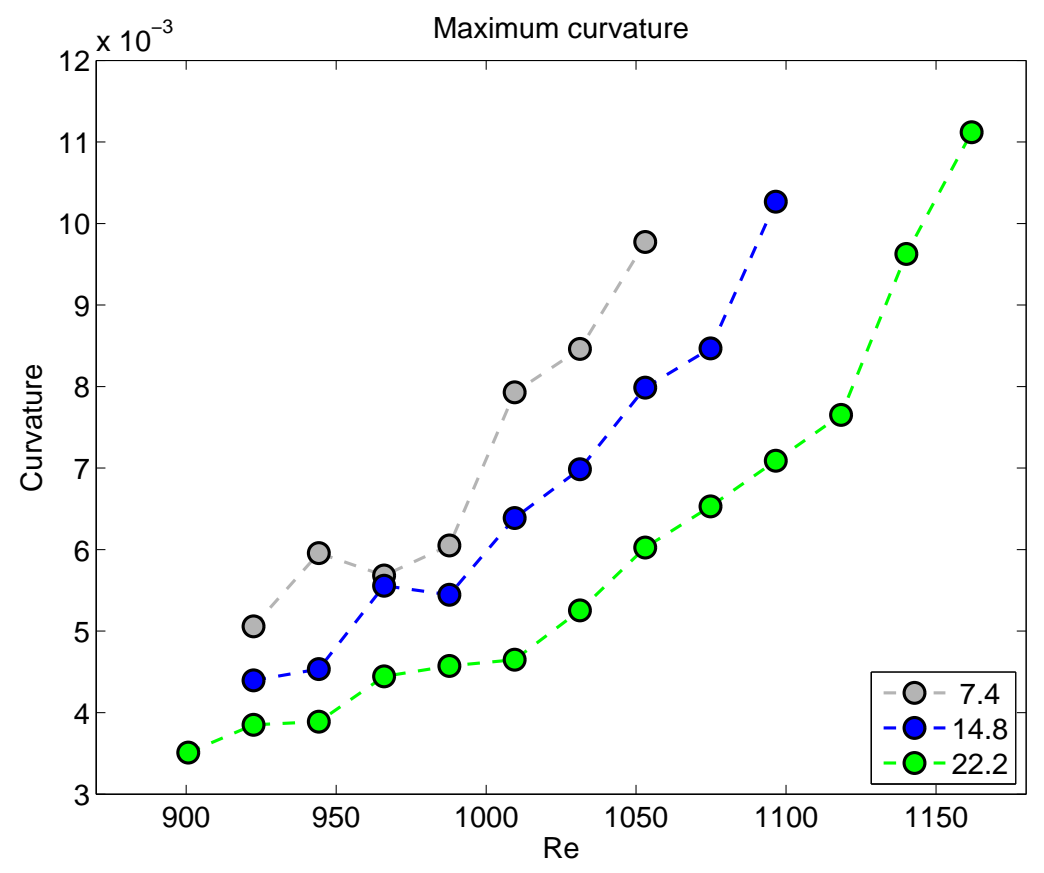

Figure 11: Maximum curvature of the cavity outline as a function of the jet Reynolds number for the stable cavities. The colours and symbols are as in Figure 6 .

\subsection{Dimple dynamics}

The geometric properties of the dimple were described above. In this section the behaviour of the dimple in time is analyzed. A first impression of the differences between different modes of oscillation is presented in Figure 12. The cavity depth and angle with respect to the vertical ${ }^{1}$ are drawn for the E-mode (left panels), the V-mode (middle panels), and the B-mode (right panels) at nozzle height $\zeta=7.4$. The E-mode only shows very slight oscillations in depth, but very regular oscillations in the angle time series. The angle in the $\mathrm{V}$-mode on the other hand is, as expected, almost zero, with clear oscillations in the depth series. The B-mode shows oscillations in both depth and angle. As the behaviour of the dimple in the B-mode seemed fairly chaotic at first sight, the oscillations are remarkably regular. In contrast to the E-mode, with one clear frequency, and the V-mode, which has a more intermittent behaviour, the signal shows multiple frequencies with a clear periodicity.

The frequency spectra of the three modes of instability (Figure 13) show spectral peaks at slightly different frequencies. The E-mode has a peak around $55 \mathrm{~Hz}$ in the power spectra of cavity depth and angle with the vertical. A second peak is found in the depth spectrum at twice the $55 \mathrm{~Hz}$ frequency, around $108 \mathrm{~Hz}$, that is larger than the $55 \mathrm{~Hz}$ peak. This higher frequency mirrors the double crossing of the vertical in the apparent pendulumlike movement of the dimple in the 2-dimensional image of the 3 -dimensional elliptical

\footnotetext{
${ }^{1}$ This angle is defined as the angle between the axis of symmetry (the continuation of the centerline of the jet) and the line drawn from the crossing of the centerline of the jet and the water surface to the tip of the dimple.
} 

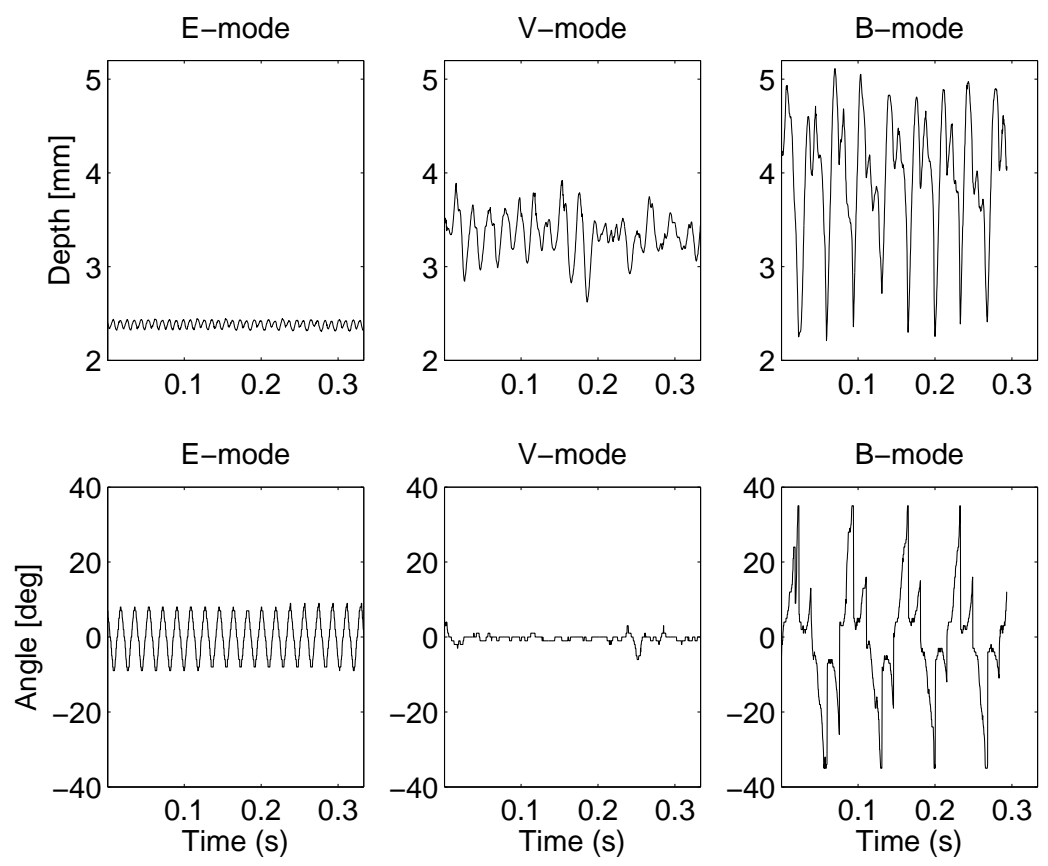

Figure 12: Cavity depth (upper panels) and angle of the cavity with the vertical (lower panels) from time series in the E-mode (left panels), the V-mode (middle panels), and the B-mode (right panels) at nozzle height $\zeta=7.4$.

movement. The $\mathrm{V}$-mode spectrum is more noisy, but shows the highest peak around 52 $\mathrm{Hz}$ in the depth series, comparable to the E-mode. As expected, no distinct peaks are observed in the angle power spectrum. Last, the spectra of the B-mode time series show a high peak at low frequency $(14.5 \mathrm{~Hz}$ in the angle spectrum and twice this value, $29 \mathrm{~Hz}$, in the depth spectrum) with lower peaks at higher harmonics. One of the peaks is at $57 \mathrm{~Hz}$, again comparable to the $55-\mathrm{Hz}$ peak in the E-mode spectra. Although the exact values of the frequency peaks slightly differ, we not believe them to be actually different and the fundamental oscillation frequency does therefore not seem to change as the dimple oscillation enters another mode.

Figure 14 shows the frequency spectra for the highest Reynolds number considered at the different nozzle heights. Unlike the spectra at one nozzle height, the spectral peak shows up at increasingly lower frequency for increasing nozzle height. (An exception is in the $\zeta=29.6$ line, which does not show one clear peak at all because the jet is going turbulent around this nozzle height.) The same behaviour, but less distinct, is observed at the other high-Reynolds number measurements. For the lower Reynolds number regime, no such pattern is found.

In the regime diagram (Figure 6) a separate mode was assigned to the seemingly stable cavities at higher nozzle heights which showed very small and rapid oscillations. This shivering-like behaviour does not seem to be an oscillation of the dimple itself. An example of a time series in this mode is give in Figure 15. The larger troughs were probably dust particles or other disturbances swept through the cavity by the surface flow. The smaller 


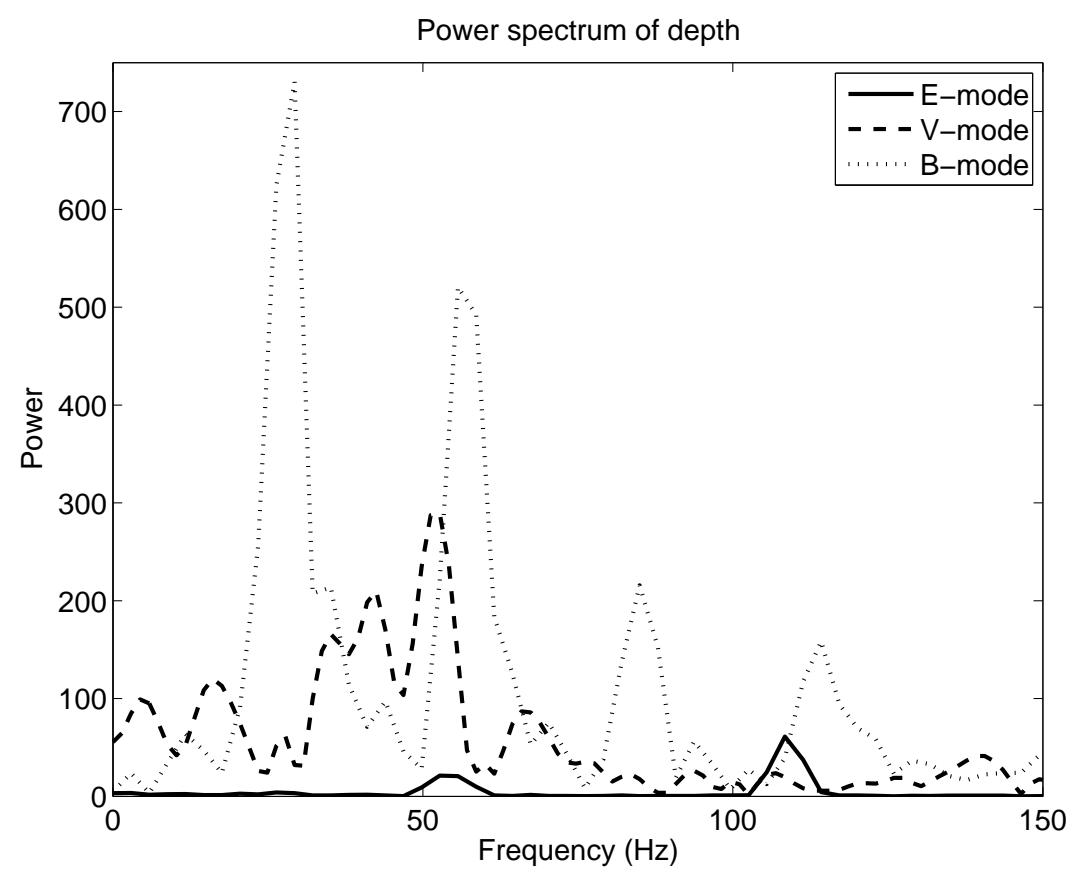

(a)

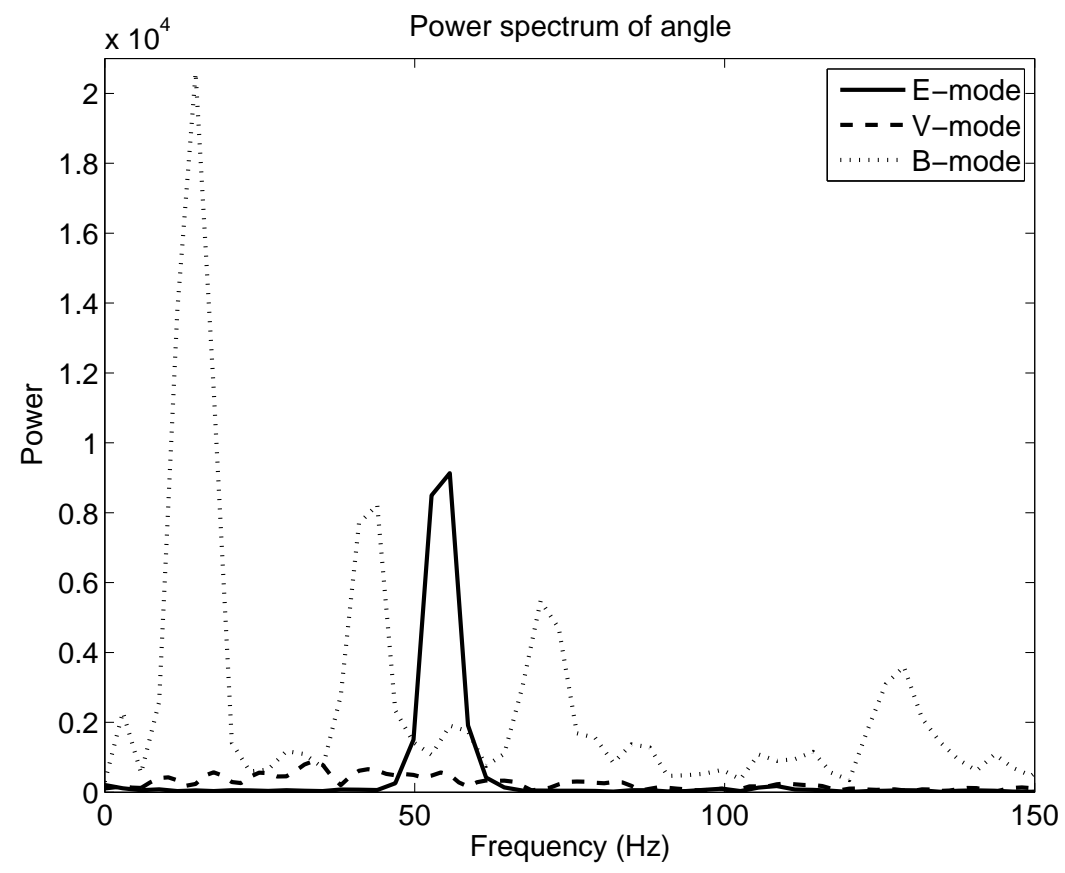

(b)

Figure 13: Power spectra of the time series from Figure 12. 


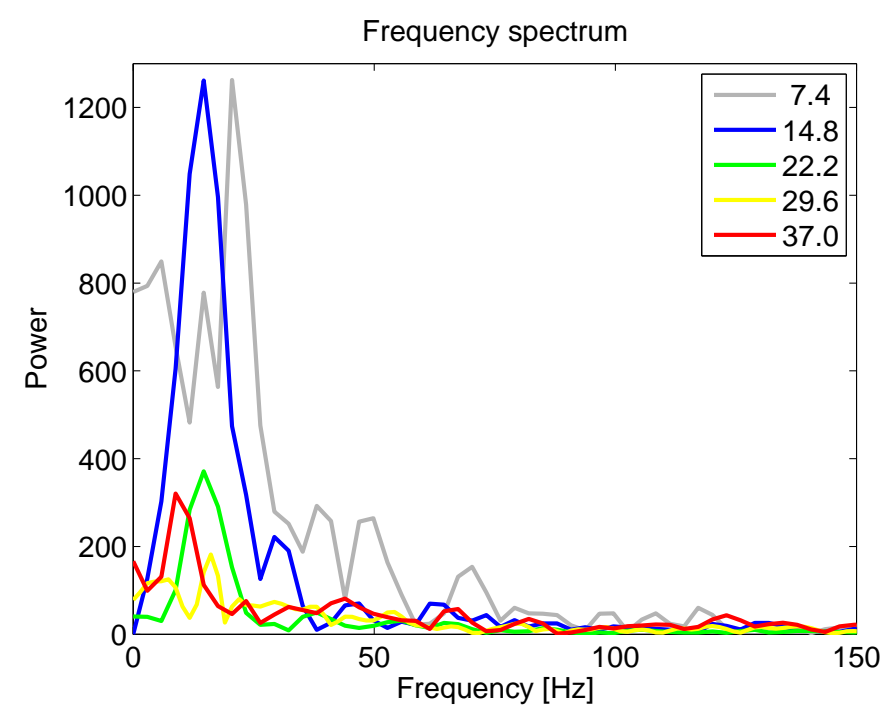

Figure 14: Frequency spectra of the time series at $R e=3360$. The line colours for nozzle heights are as in Figure 6.

noisy oscillations are the observed small and rapid oscillations. The frequency spectrum of the middle part of the time series confirms that there is no clear spectral peak at high frequency. These oscillations are thus indeed noise. The noise is likely caused by the increased turbulence in the jet, which has more time to develop at the larger nozzle height.
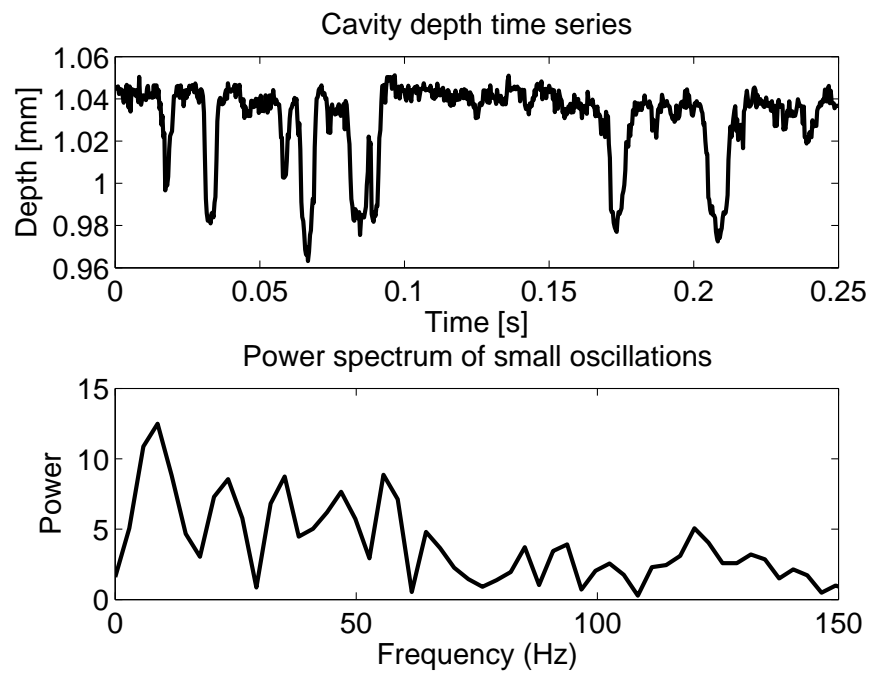

Figure 15: Time series of the cavity depth and its frequency spectrum at $\zeta=29.6$ and $\operatorname{Re}=1030$. 


\section{$5 \quad$ Discussion and Conclusions}

The behaviour of a dimple that is formed by an air jet impinging at right angles on a water surface was studied. In particular the dependence of the geometric properties, onset of oscillation and modes of oscillation were studied as a function of the air flow rate at the nozzle, defined as the jet Reynolds number, and the source height, given by the nondimensional nozzle height.

At low jet Reynolds number a stable cavity is formed. When the air flow rate is turned up, a sequence of oscillation modes is observed. The range over which the modes of oscillations are found depends on the nozzle height. Also not all unstable modes are observed at every nozzle height considered. The unstable modes identified were:

- E-mode: The tip of dimple traces out an ellipse. This mode was only found at the smallest nozzle height and shows very regular oscillations.

- V-mode: The dimple oscillates vertically. This mode was found at all nozzle heights except for the fourth one. The regime diagram however indicates that the Re-range where this mode is expected at the fourth nozzle height is not sampled in this study. The oscillations in this mode are sometimes rather regular, but other times they showed more intermittent behaviour (see e.g. the upper middle panel in Figure 12).

- B-mode: The oscillation overshoots, and the tip of the dimple bends and rolls up the side of the dimple. This mode was observed at the lowest two nozzle heights. Again, the Re-range at which this mode is expected at the higher nozzle heights was not sampled. The oscillations in this mode are again very regular.

- At the largest nozzle heights, very small oscillations of the dimple are observed at low jet Reynolds number, which shows up as noise in the time series. We speculate this is due to an instability of the jet.

- In the high Reynolds number range two other modes are found:

- Bubbling and splashing. This mode is only observed at the lowest nozzle height, but will probably eventually set in at higher nozzle heights as well if the flow rate is increased further. It seems that a critical dimple depth exists above which this mode sets in.

- A widening of the dimple. We believe this shape to arise when the jet overcomes the surface tension. This mode is not observed at the lowest nozzle height.

Two distinctively different regimes are found. For the smallest three nozzle heights, the Reynolds number at which a next mode of oscillation is entered increases with increasing nozzle height. For the largest two nozzle heights on the other hand the required Reynolds number for a certain mode of oscillation decreases with increasing nozzle height. Also, the cavity depth increases rapidly around the point of instability for the smallest three nozzle heights, while it only increases slightly in the larger two cases. Overall, the depth of the cavity increased with increasing jet strength and decreases with increasing nozzle height. The difference in cavity depth with nozzle height is small at low jet Reynolds number and larger at high jet Reynolds number. 
Clear evidence is found for two competing effects in the system as the air flow rate in the jet is increased. If both the nozzle height and the flow rate are small, the dimple depth increases with increasing flow rate until the dimple becomes unstable. If the nozzle is sufficiently far away from the water surface however, turbulence develops in the jet when the flow rate is high enough. In a turbulent jet the velocity decreases with distance from the nozzle and the rate of increase of impact of the jet therefore levels off.

The time dependent behaviour of the dimple oscillations was studied using their frequency spectra. The spectra suggest that the frequency does not change much when the nozzle height is fixed (spectral peaks at different higher harmonics do show up with increasing Reynolds number). For the smallest nozzle height a fundamental frequency around $55 \mathrm{~Hz}$ is found. This frequency can be linked to the waves produced on the surface. The dispersion relation for deep water gravity waves is $\omega=\sqrt{g k}$. With $\omega=c k, c=\lambda / T$, and $k=2 \pi / \lambda$, the frequency of the wave is found from

$$
\frac{1}{T}=\sqrt{\frac{g}{2 \pi \lambda}}
$$

where $\omega$ is the angular frequency, $c$ the phase velocity, $k$ the wave number, $T$ the wave period, and $\lambda$ the wave length. If the dimple represents half a wave, the wave length is twice the dimple width. The small dimples are about $1 \mathrm{~mm}$ wide, giving a frequency of 28 $\mathrm{Hz}$. The fundamental frequency of $55 \mathrm{~Hz}$ is twice this frequency. At the higher Reynolds numbers the largest peak in the spectrum shifts to a lower frequency with increasing nozzle height. It should be noted that not all spectra show clear narrow peaks. As the filter removes part of the time series, perhaps longer time series would give a better result. Also ensemble measurements with more than one time series per $\operatorname{Re}-\zeta$ pair could increase the signal-to-noise ratio, especially in the modes where the dimple oscillations are irregular.

A few open question remain after this study. In particular, it would be very helpful to know more about the air jet itself. Visualization of the jet would provide information on the formation of turbulence, the radius at impact and its relation to the dimple radius. Without specific information on the jet, it is very difficult to form an idea of the physics involved, and results remain speculative.

Secondly, surface tension seem to be an important factor in the dimple stability. This was already confirmed by earlier studies (in particular Rosler and Steward (8)). If the radius of the jet could however be measured accurately in this study, the Bond and Weber number can be calculated and related to the jet Reynolds number and the nozzle height (the jet spreads and thus reaches a larger radius at impact at larger nozzle height). This is likely to shed a more physical light on the now speculative lines in the regime diagram.

Thirdly, measurements should be added in the regime diagram in the 700 to 1500 Reynolds number range, in particular where the speculative lines suggest the presence of a certain mode at a certain nozzle height which is not yet confirmed due lack of data. 


\section{References}

[1] R. Banks And D. Chandrasekhara, Experimental investigation of the penetration of a high-velocity gas jet through a liquid surface, Journal of Fluid Mechanics, 15 (1963), pp. 13-34.

[2] J. Berghmans, Theoretical investigation of the interfacial stability of inviscid fluids in motion, considering surface tension, Journal of Fluid Mechanics, 54 (1972), pp. 129141.

[3] F. Cheslak, J. Nicholls, And M. Sichel, Cavities formed on liquid surfaces by impinging gaseous jets, Journal of Fluid Mechanics, 36 (1969), pp. 55-63.

[4] M. Evestedt And A. Medvedev, Gas jet impinging on liquid surface: cavity shape modelling and video-based estimation, (2005).

[5] A. He And A. Belmonte, Deformation of a liquid surface fue to an impinging gas jet: A conformal mapping approach, Physics of Fluids, 22 (2010), pp. 042103-1 - 042103-7.

[6] W. Olmstead and S. Raynor, Depression of an infinite liquid surface by an inconpressible gas jet, Journal of Fluid Mechanics, 19 (1964), pp. 561-576.

[7] S. Pope, Turbulent flows, Cambridge University Press, Cambridge, 2000.

[8] R. Rosler and G. Stewart, Impingement of gas jets on liquid surfaces, Journal of Fluid Mechanics, 15 (1968), pp. 13-34.

[9] E. Turkdogan, Fluid dynamics of gas jets impinging on surface of liquids, Chemical Engineering Science, 21 (1966), pp. 1133-1144.

[10] J. Vanden-Broeck, Deformation of a liquid surface by an impinging gas jet, SIAM Journal of Applied Mathematics, 41 (1981), pp. 306-309. 


\title{
Who ate whom: \\ Population dynamics with age-structured predation
}

\author{
David Goluskin
}

15 October 2010

\begin{abstract}
We study a population model in which there are two species, one of which has a juvenile and adult life stage. The adults of the first species prey on the second species, which in turn preys on the juveniles of the first. One version of the model represents systems where neither species can survive on its own, although we find that both can survive through mutual predation. To avoid extinction, the two types of predation must be of sufficient strength and in appropriate proportion to one another. Another version of the model represents systems where each species can survive without the other, and there we find that mutual predation is capable of increasing both of their equilibrium populations or creating stable limit cycles.
\end{abstract}

\section{Introduction}

An organism's trophic level is the position that it occupies in the food web, defined by the organisms that it eats and vice versa. However, few organisms occupy the same trophic levels throughout their lives. Typically an organism's predators and prey change over its lifetime, as do the organisms of similar trophic level with which it competes for food. Werner and Gilliam [2] review many examples of ecosystems in which competitive and predatory relationships are age- and size-dependent. As an instance of the latter, adult salamanders and newts prey on one another's juveniles. Similarly, frogs eat insects, while insect larva eat tadpoles. Although competition is important, we do not consider it in this report, where our object is to explore solely the dynamics that arise in ecological models with age-structured predation.

\section{Development of the models}

Any model for age-structured predation must have at least two species, and at least one of the species must have multiple life stages. Some ecological models have used continuous age or size variables to describe life stage, which requires partial differential equations to describe the change of populations in time, but the simplest possibility is to have two discrete life stages. Thus, the simplest possible model has three populations in total: one species with one life stage and one species with both juvenile and adult life stages. For concreteness we shall call our populations tadpoles $(T)$, frogs $(F)$, and insects $(I)$, where the frogs eat the insects, the insects eat the tadpoles, and the tadpoles and frogs beget one another through recruitment and reproduction. For simplicity we shall assume that recruitment occurs at a rate proportional to tadpole biomass, that 
reproduction occurs at a rate proportional to frog biomass, and that a constant fraction of the biomass being transferred through predation is lost to metabolic inefficiencies. The model as described thus far is diagramed in Figure 1. Through the "feeding/death" fluxes in Figure 1, each population may gain biomass by feeding on the external environment (which excludes the other two populations explicitly modeled), or lose biomass through death. All that remains to fully define our population model is to specify the functional forms of the predation and feeding/death terms.

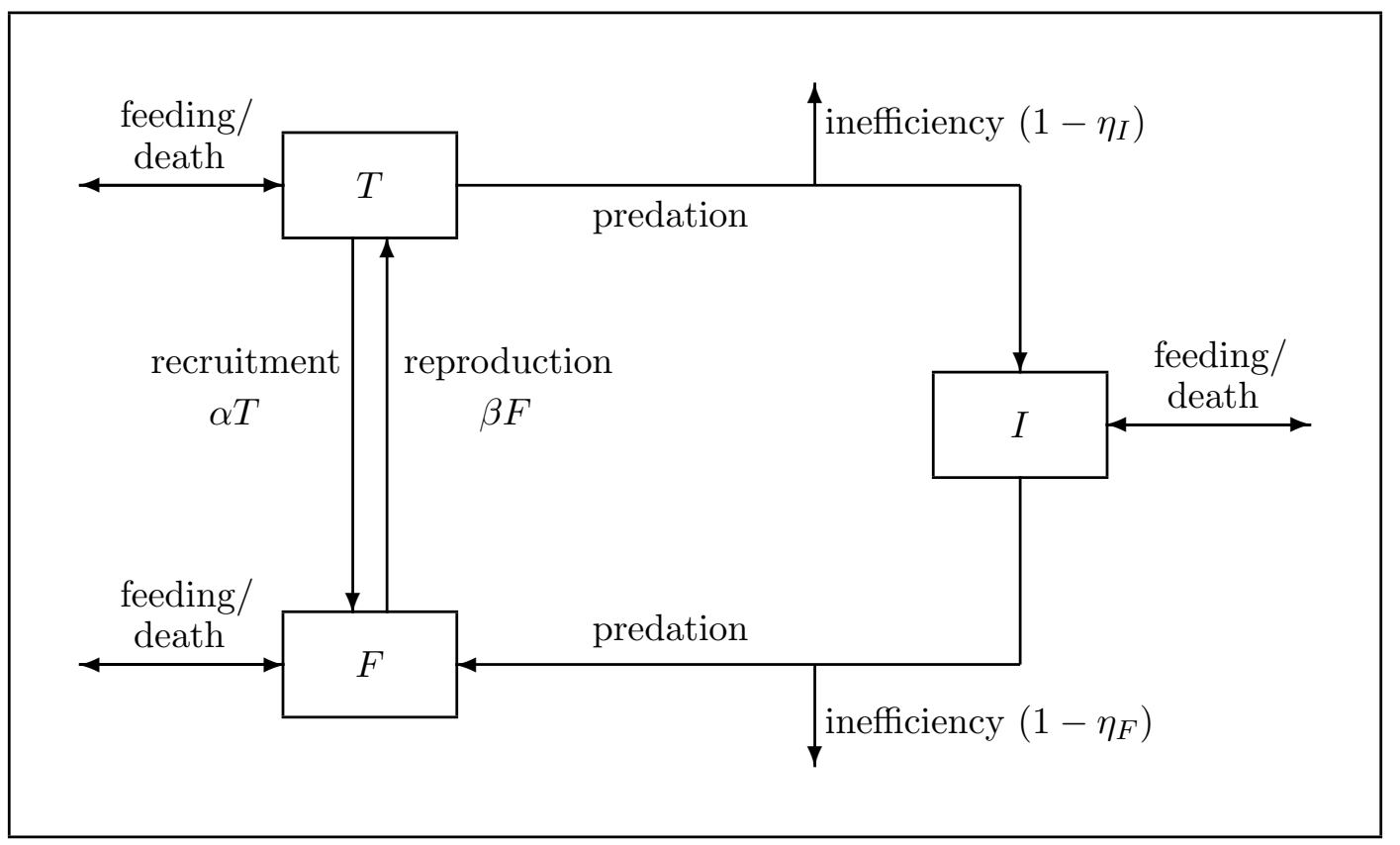

Figure 1: Biomass flux in the tadpole-frog-insect system.

\section{$2.1 \quad$ Feeding or death terms}

Assuming the tadpoles, frogs, and insects do not compete with one another, each population's feeding/death term should be independent of the other two populations. The simplest choice is a proportional law, which in the absence of predation creates exponential growth or decay, depending on the parameter values. Unbounded exponential growth is unrealistic, but exponential decay is feasible: it represents a case where the two species cannot survive without predation, and we analyze such a model in Section 3. We also wish to consider a scenario in which both species would survive without the predation. This requires that the system have carrying capacities, which cause initially exponential growth to saturate at finite values. We must put a carrying capacity on the insects, but we have a choice between putting one on the frogs, the tadpoles, or both. We shall somewhat arbitrarily place a carrying capacity on frogs and not on tadpoles. In the biological reality of frogs and tadpoles this is usually accurate; frogs will typically run out of environmental resources before tadpoles do. But for other species, resources may certainly be scarcer for juveniles than for adults. We analyze the carrying capacity model in Section 4. 


\section{$2.2 \quad$ Predation terms}

The simplest possible predation law is a quadratic one in which the rate of predation is proportional to both predator and prey populations, the so-called Holling type I functional form [1]. Some models of age-structured predation employing these forms were studied by Whitehead and Doering [3], but such a form fails to reflect the fact that increasing the amount of prey beyond a certain point stops benefiting the predators because there is a limit to how fast they can eat and metabolize the prey. The Holling type II functional form avoids this problem by saturating as the amount of prey becomes large:

$$
\text { rate of predation }=C_{1} \frac{\text { (prey population })(\text { predator population })}{\left.1+C_{2} \text { (prey population }\right)},
$$

where $C_{1}$ and $C_{2}$ are constant parameter. We have examined some models employing the type I form, but it leads to unbounded growth in certain cases, so we shall henceforth always use the type II form. It is not hard to justify needing a predation law with saturation, but it is certainly not clear a priori that the type II form is the best choice. We justify this choice in the appendix, where we derive the type II form as an asymptotic limit of a higher-dimensional dynamical system and numerically compare the reduced system with the higher-dimensional system.

\section{Model without carrying capacities}

Let the feeding/death term for tadpoles add biomass at the rate $\epsilon_{T} T$, where $\epsilon_{T}$ can take either sign, and likewise for frogs and insects. Then, the dimensional ODE governing the biomass of each population is

$$
\begin{aligned}
& \dot{T}=-\gamma T+\beta F-\kappa \frac{I T}{1+\mu T} \\
& \dot{F}=\alpha T-\zeta F+\eta_{F} \lambda \frac{F I}{1+\nu I} \\
& \dot{I}=\epsilon_{I} I+\eta_{I} \kappa \frac{I T}{1+\mu T}-\lambda \frac{F I}{1+\nu I},
\end{aligned}
$$

where

$$
\begin{aligned}
& \gamma=\alpha-\epsilon_{T} \\
& \zeta=\beta-\epsilon_{F} .
\end{aligned}
$$

Without frogs or insects, tadpoles should die out, and likewise for frogs, so $\gamma$ and $\zeta$ must be positive. Only the $\epsilon_{I}$ parameter may be negative. If $\alpha<\gamma$, tadpole feeding is adding biomass to the system, and the same is true for frogs if $\beta<\zeta$. The parameters $\eta_{I}$ and $\eta_{F}$ are metabolic efficiencies, so they must fall between 0 and 1 . We now nondimensionalize the system by

$$
t \mapsto \frac{1}{\gamma} t \quad T \mapsto \frac{1}{\mu} T \quad F \mapsto \frac{\gamma}{\beta \mu} F \quad I \mapsto \frac{1}{\nu} I .
$$

This particular nondimensionalization is attractive because it retains unique coefficients on all the nonlinear predation terms and minimizes the number of free parameters (seven). Its drawback is that the different populations are no longer in equivalent units of biomass, so they cannot be meaningfully compared. This is tolerable because we are more interested in qualitative system behavior than relative population biomasses. The 
nondimensional ODE is

$$
\begin{aligned}
& \dot{T}=-T+F-d \frac{I T}{1+T} \\
& \dot{F}=a T-b F+g \frac{F I}{1+I} \\
& \dot{I}=c I+e \frac{I T}{1+T}-f \frac{F I}{1+I},
\end{aligned}
$$

where

$$
\begin{aligned}
a & =\frac{\alpha \beta}{\gamma^{2}} \\
b & =\frac{\zeta}{\gamma} \\
c & =\frac{\epsilon_{I}}{\gamma} \\
d & =\frac{\kappa}{\gamma \nu} \\
e & =\frac{\eta_{I} \kappa}{\gamma \mu} \\
f & =\frac{\lambda}{\beta \mu} \\
g & =\frac{\eta_{F} \lambda}{\gamma \nu} .
\end{aligned}
$$

\subsection{Model without predation}

Without predation, the model is linear, and the $T-F$ system decouples from the insects:

$$
\frac{d}{d t}\left(\begin{array}{c}
\Delta T \\
\Delta F \\
\Delta I
\end{array}\right)=\left(\begin{array}{ccc}
-1 & 1 & 0 \\
a & -b & 0 \\
0 & 0 & c
\end{array}\right)\left(\begin{array}{c}
\Delta T \\
\Delta F \\
\Delta I
\end{array}\right)
$$

The tadpoles and frogs decay exponentially if $a<b$ and grow exponentially if $a>b$, while the insects decay exponentially if $c<0$ and grow exponentially if $c>0$. The behaviors of the uncoupled systems split the full system rather naturally into four separate cases. (Structurally unstable parameter values such as $a=b$ or $c=0$ will not be considered in anything that follows.) The only equilibrium of this system is the origin, and the decoupled systems are rather boring without predation. Predation can create stable fixed points and limit cycles for all four combinations of signs of $(a-b)$ and $c$, but only the case in which the $T-F$ and $I$ systems both decay without predation is biologically reasonable, so we shall restrict ourselves to this case. Henceforth in this section, $a<b$ and $c<0$.

When $I$ and the $T-F$ system both decay without predation, the mechanism by which predation can stabilize the system is the following. Suppose that biomass enters the system though frog feeding $(\zeta<\beta)$ and leaves the system through tadpole death $(\gamma>\alpha)$, with tadpole death dominating when the $T-F$ system is isolated. In other words, the rate of reproduction is higher than ideal. Introducing insects creates a flux of biomass from tadpoles to frogs, where it is used to create more biomass from external feeding, rather than lost to tadpole death. This effect can prevent the entire system from decaying to zero, even with the additional sources of biomass loss by insect death and metabolic inefficiencies.

\subsection{Lyapunov bound}

Not only is the origin linearly stable when $a<b$ and $c<0$, the Lyapunov functional $L \equiv \frac{e}{d} T+\frac{f}{g} F+I$ suffices to show that all solutions decay to the origin. This is a rather 
narrow range of validity, but it suggests the possible importance of the ratio $\frac{e g}{d f}$. In terms of dimensional variables,

$$
\frac{e g}{d f}=\eta_{I} \eta_{F} \frac{\beta}{\gamma}
$$

Biologically, $\eta_{I} \eta_{F}$ is the squared geometric mean of the metabolic efficiencies, so we can think of this term roughly as the metabolic efficiency of the entire system.

\subsection{Nontrivial equilibria}

At nontrivial fixed points,

$$
\begin{aligned}
F & =T\left(1+\frac{d I}{1+T}\right) \\
a T & =F\left(b-\frac{g I}{1+I}\right) \\
0 & =c+\frac{e T}{1+T}-\frac{f F}{1+I} .
\end{aligned}
$$

From Equation (8), $I$ and $T$ uniquely define $F$ at a fixed point. Using this equation to eliminate $F$ from the latter two yields two polynomials equations in $I$ and $T$ :

$$
\begin{gathered}
a(1+I)(1+T)=(1+T+d I)(b+b I-g I) \\
c(1+I)(1+T)+e T(1+I)=f T(1+T+d I) .
\end{gathered}
$$

Equation (11) is linear in $T$, and Equation (12) is linear in $I$, so they may be used to find explicit expressions for $T(I)$ and $I(T)$, respectively:

$$
\begin{aligned}
T & =\frac{d I(b+(b-g) I)}{(a+g-b) I+a-b}-1 \\
I & =\frac{f T^{2}-(c+e-f) T-c}{(c+e-d f) T+c} .
\end{aligned}
$$

Evidently, $I$ and $T$ define one another uniquely. Applying $T(I)$ to Equation 12 gives a cubic polynomial for $I$ at the equilibria, so there are at most three positive real population equilibira.

$$
i_{3} I^{3}+i_{2} I^{2}+i_{1} I+i_{0}=0, \text { where }
$$

$$
\begin{aligned}
& i_{0}=-e(a-b)^{2}<0 \\
& i_{1}=(a-b)[2 e(b-g)+b d(c+e)+a(d f-2 e)] \\
& i_{2}=d(c+e)[(a-b)(b-g)+b(a+g-b)]-e(a+g-b)^{2}+a d f(a+g-b-b d) \\
& i_{3}=d(b-g)[(c+e)(a+g-b)-a d f]
\end{aligned}
$$

The cubic equation for $I$ of course has explicit algebraic solutions, but they are too messy to be of use, so although we have explicit expressions for $F$ and $T$ in terms of $I$, we cannot generally predict whether $F$ and $T$ will be positive when $I$ is positive. The best we can do analytically is infer some partial information about the signs of polynomial roots. For this, we also need the first and last coefficients of the cubic polynomial governing $T$, which we derive by applying $I(T)$ to Equation 11:

$$
\begin{aligned}
& t_{3} T^{3}+t_{2} T^{2}+t_{1} T+T=0, \text { where } \\
& T=-c^{2} g<0 \\
& t_{3}=f[(c+e)(a+g-b)-a d f] .
\end{aligned}
$$


Theorem Let $a<b$ and $c<0$. If $g<b$ and $a d f<(c+e)(a+g-b)$, there are either 1 or 3 positive equilibria. Otherwise, there are either 0 or 2 positive equilibria.

Proof The $I$ polynomial is cubic, and $I$ uniquely defines $F$ and $T$, so there are at most three real, positive equilibria. It is clear from Equation 8 that $F$ is positive when $I$ and $T$ are, so it suffices to know when the $I$ and $T$ polynomials have corresponding positive roots. There are three ways in which the number of positive roots of the $I$ or $T$ polynomials may change. Firstly, a root may remain real but leave the positive octant if $i_{0}$ or $t_{0}$ becomes negative, but we have eliminated this possibility by assumption. Secondly, the number of positive equilibria may change by two when a pair of equilibria become complex simultaneously, a saddle-node bifurcation. Thirdly, an equilibrium may move off to infinity when $i_{3}$ or $t_{3}$ pass through zero. By dividing parameter space into four regions in which $i_{3}$ and $t_{3}$ do not change sign, we are assured that the number of equilibria within each region may change only by saddle-node bifurcations. These regions are

$$
\begin{aligned}
I & \equiv\left\{i_{3}>0, t_{3}>0\right\}=\{g<b, \text { adf }<(c+e)(a+g-b)\} \\
I I & \equiv\left\{i_{3}<0, t_{3}>0\right\}=\{g>b, \text { adf }<(c+e)(a+g-b)\} \\
I I I & \equiv\left\{i_{3}>0, t_{3}<0\right\}=\{g<b, \text { adf }>(c+e)(a+g-b)\} \\
I V & \equiv\left\{i_{3}<0, t_{3}<0\right\}=\{g>b, \text { adf }>(c+e)(a+g-b)\} .
\end{aligned}
$$

If we know the number of positive equilibria at one point in each parameter region, we know the number of positive equilibria at all points in that parameter region, modulo 2. So, we chose one such test point in each parameter region and computed the equilibria numerically, inferring from this the possible number of positive equilibria in each parameter region. The results are tabulated in Table 1. Evidently, there may be 1 or 3 positive equilibria in parameter region I, and 0 or 2 otherwise. Parameter region I is defined precisely by the condition that $g<b$ and $a d f<(c+e)(a+g-b)$, so the theorem is proved.

Table 1: Number of nontrivial equilibria in each of the four parameter regions, as inferred from a test points, $(a, b, c, d, e, f, g)$, in parameter space.

\begin{tabular}{|c|c|c|c|}
\hline Parameter region & Test point & $\begin{array}{c}\text { Number of positive } \\
\text { equilibria at test point }\end{array}$ & $\begin{array}{c}\text { Number of positive } \\
\text { equilibria in region }\end{array}$ \\
\hline$I$ & $(0.9,1,-0.5,1,1.5,0.1,0.5)$ & 1 & 1 or 3 \\
\hline$I I$ & $(0.9,1,-0.5,1,1.5,0.1,2)$ & 2 & 0 or 2 \\
\hline$I I I$ & $(0.9,1,-0.5,100,1.5,0.1,0.5)$ & 0 & 0 or 2 \\
\hline$I V$ & $(0.9,1,-0.5,100,1.5,0.1,2)$ & 0 & 0 or 2 \\
\hline
\end{tabular}

By the preceding theorem, we have partial information about the number of equilibria inside and outside region I. Because a saddle-node bifurcation creates two equilibria of opposite stability, we also know that there can be at most two stable equilibria inside parameter region I, and at most one stable equilibrium outside it. We would of course like precise conditions on when the saddle-node bifurcations occur, thereby further dividing our parameter regions into ones in which the numbers of positive equilibria are exactly known. This requires knowing when $I$ or $T$ become complex. Since $I$ and $T$ are 
governed by cubic equations, this can in principle be determined from the discriminants of those equations. The $I$ equation has one real and two complex roots precisely when

$$
\Delta_{I} \equiv 18 i_{3} i_{2} i_{1} i_{0}-4 i_{2}^{3} i_{0}+i_{2}^{2} i_{1}^{2}-4 i_{3} i_{1}^{3}-27 i_{3}^{2} i_{0}^{2}<0,
$$

and likewise for the coefficients of the $T$ equation, so the parameter regimes we seek are divided by the surfaces on which the discriminants vanish. Unfortunately, these inequalities are prohibitively messy when expressed in terms of the problem parameters. In biological modeling, one is more interested in qualitative behaviors than the precise values at which bifurcations occur, so we will be content to observe the saddle-node bifurcations numerically.

\subsection{Numerical exploration of parameter space}

Asymptotic analysis shows that the predation parameters cannot be small compared to the population decay rates if predation is to stabilize the decay. In fact, numerical experiments reveal that the predation parameters must typically be about an order of magnitude larger than the decay rates. In light of this, we shall fix $(a, b, c)=(0.9,1,-0.1)$ and expect interesting behavior when predation parameters are $O(1)$.

\subsubsection{Looking for bifurcations}

When $(d, e, f, g)=(0.2,1,0.2,0.5)$, there are three positive equilibria, the maximum number possible, so we choose this as a starting point from which to explore the fourdimensional space of predation parameters. Linearizing the system about these equilibria and computing matrix eigenvalues numerically, we find that none of the fixed points are stable. Biologically, we are interested in regimes where there are stable fixed points, so we use the bifurcation continuation package MATCONT to continue these equilibria in parameter space. Arbitrarily choosing the parameter $e$ in which to continue the equilibria, we obtain the bifurcation diagram of Figure 2. We have chosen the coordinate $I$ for the ordinate of our bifurcation diagrams because the value of $I$ sometimes becomes unrealistically small, and we wish to see when this is so.

Continuing in $e$, we find a saddle-node bifurcation $(L P)$, a subcritical Hopf bifurcation $\left(H^{+}\right)$, and a neutral saddle $(N S)$. These are all bifurcations of codimension 1 , as we would expect to find when varying only one parameter. To access the higher-level structure of parameter space, we would like to find higher-codimension bifurcations. The MATCONT package can in general only find bifurcations up to codimension-2, so we shall settle for this, though ideally we would like to find bifurcations of codimension up to the dimension of our parameter space. Codimension-2 bifurcations are typically found by continuing a codimension-1 bifurcation in two parameters, so we shall continue all of the bifurcations of Figure 2. Continuing in $e$ and $g$ yields the bifurcation diagram of Figure 3, in which we see two types of codimension-2 bifurcations: Bogdanov-Takens bifurcations $(B T)$, and generalized Hopf bifurcations $(G H)$, also known as Bautin bifurcations. Continuing the codimension- 1 bifurcations of Figure 2 in any other combination of two parameters does not yield any other types of codimension-2 bifurcations.

In the neighborhood of a Bogdanov-Takens bifurcation, there are guaranteed to be a saddle-node bifurcation and a Hopf bifurcation (both of which we have seen already), and also a saddle homoclinic bifurcation. In the neighborhood of a generalized Hopf bifurcation, there are guaranteed to be both supercritical- and subcritical Hopf bifurcations, and a fold bifurcation of limit cycles. Although we are only assured of these system behaviors in local neighborhoods of the codimension-2 bifurcations, we can reasonably expect to see them all around parameter space. 


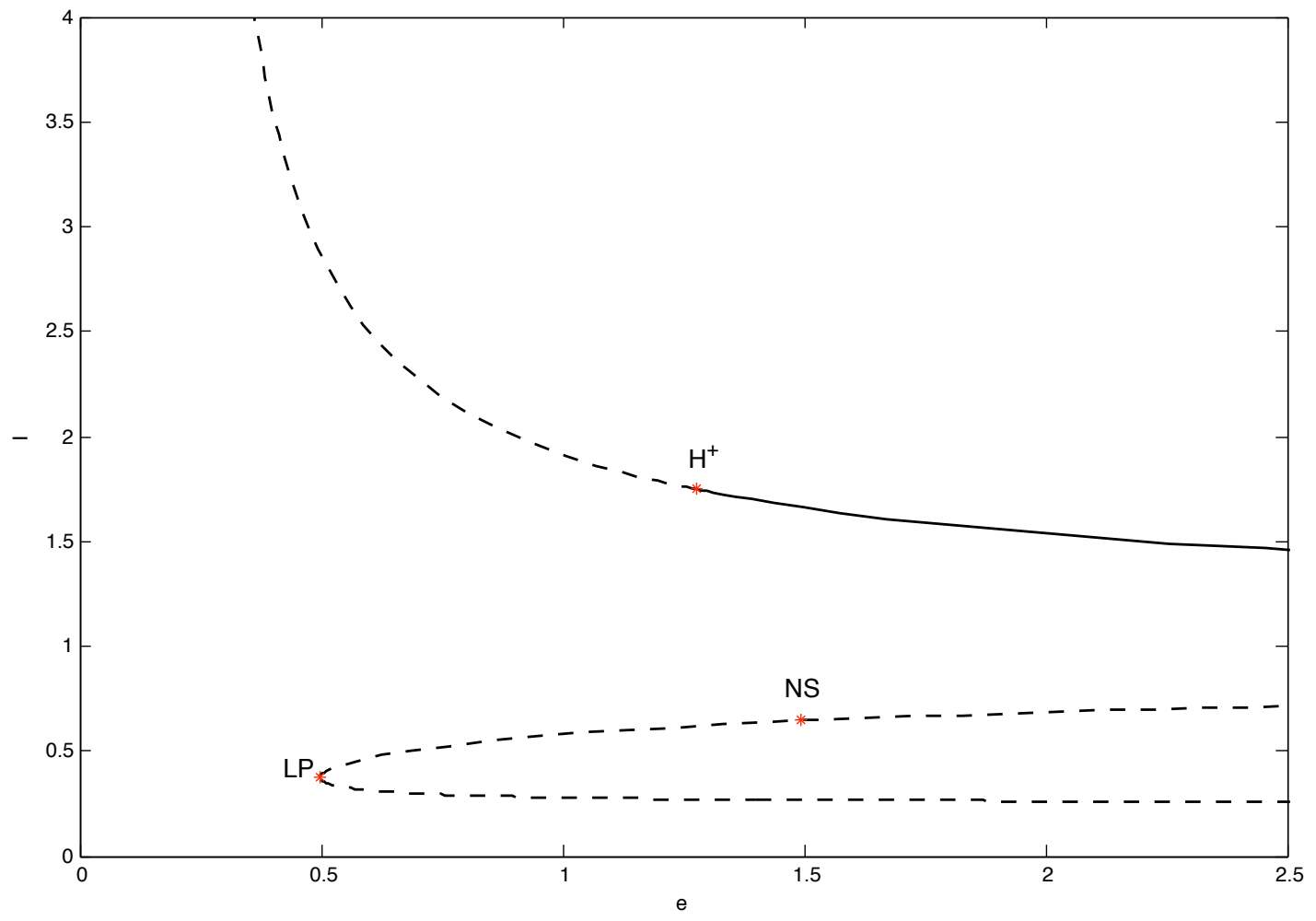

Figure 2: Continuation in $e$ of the three starting equilibria.

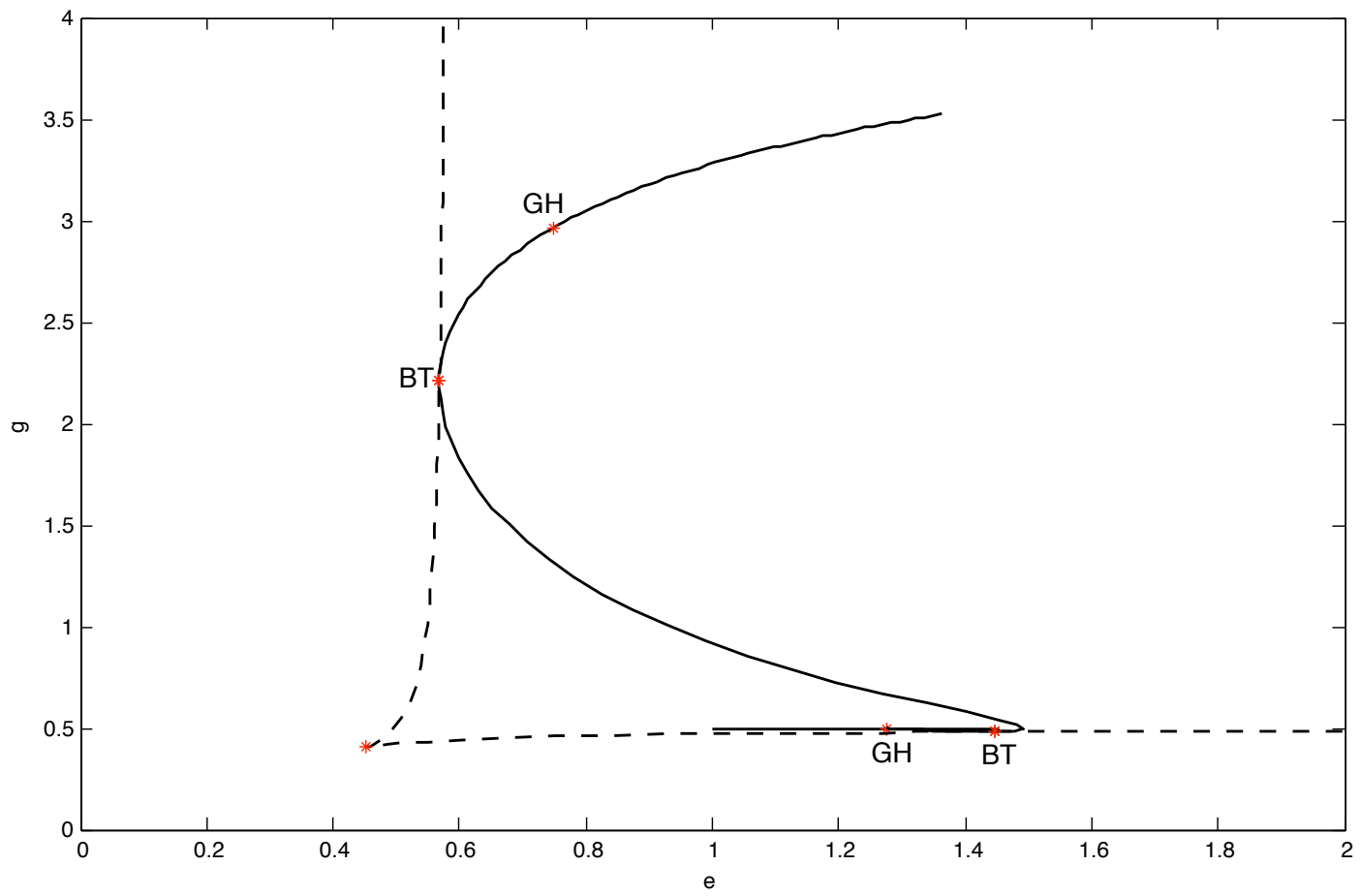

Figure 3: Continuation in $e$ and $g$ of the Hopf bifurcation of Figure 2. 
Before exploring the effects of each parameter, we would like to find a baseline point in parameter space at which all parameters are of similar magnitude, and at which a stable fixed point exists. There is always a stable fixed point near a Hopf bifurcation, so we continue the Hopf bifurcation of Figure 2 in various pairings of the predation parameters, arriving at a point where $(d, e, f, g)$ is near $(2,4,2,2)$. We shall use this and other nearby values as our baseline parameter points.

\subsubsection{Overall predation strength}

To study the effect of the overall predation strength in the system, we fix the ratios between all four predation parameters and vary them proportionally. The resulting diagrams are shown in Figure 4, where we have used two sightly different parameter ratios to exhibit one case each where the Hopf bifurcation is supercritical or subcritical. In any of the bifurcation diagrams that follow, the predation parameter ratios could be chosen to realize either type of Hopf bifurcation, but the distinction is not very important because limit cycles in this system are not robust biologically, for the following reason. In the chosen regime of $a, b$ and $c$, the coordinates of the stable fixed point appear to always be such that $I \ll T, F$. As the limit cycle emerging from a Hopf bifurcation grows, the oscillations become strongly nonlinear, consisting of a fast part and slow part. During the slow part of the cycle, the value of $I$ is very small. Without traveling very far in parameter space, either the limit cycle is destroyed in a homoclinic bifurcation, or $I$ becomes so small during the slow part of the cycle that in the noncontinuum of reality, the insect population would go extinct, bringing the same fate to the frogs and tadpoles. If we altered the model to include stochasticity or discrete populations, we could reasonably expect it to display this biologically-realistic extinction during such troughs of $I$. The type of Hopf bifurcation controls whether or not a stable limit cycle coexists with a stable fixed point, but since the limit cycle is a much less robust structure than the fixed point, the distinction is not very important.

It is evident from Figure 4 that the system has no stable fixed points or limit cycles when the predation is too weak. This is expected, since the predation must stabilize the exponential decays of the uncoupled systems, and it makes clear that predation cannot do this as a mere perturbation on the uncoupled systems. As predation increases, the fixed point not only remains stable, but its basin of attraction enlarges. Simultaneously, however, the equilibrium insect population decreases toward zero, and the frog and tadpole populations decrease asymptotically toward identical finite values. When the basin of attraction is too small, a perturbation could send the system across the separatrix and onto a trajectory heading toward the origin (extinction). When the equilibrium insect population is too small, it is vulnerable to eradication by some catastrophic event, in which fate the frogs and tadpoles would follow. The fitness of the insect population would be maximized at some intermediate predation strength that balances these two factors.

\subsubsection{Relative strengths of the two types of predation}

The parameters $d$ and $e$ convey the strength of the insect-tadpole predation, and the parameters $f$ and $g$ do likewise for the frog-insect predation. To study the effect of the relative strengths of the two types of predation, we vary the insect-tadpole predation parameters in fixed proportion while holding the other two constant, and likewise for the frog-insect parameters. The resulting bifurcation diagrams are presented in Figure 5, and they both suggest the same conclusions. The process in which insects move biomass from tadpoles to frogs is essential to stabilizing the system, and when $I-T$ predation 

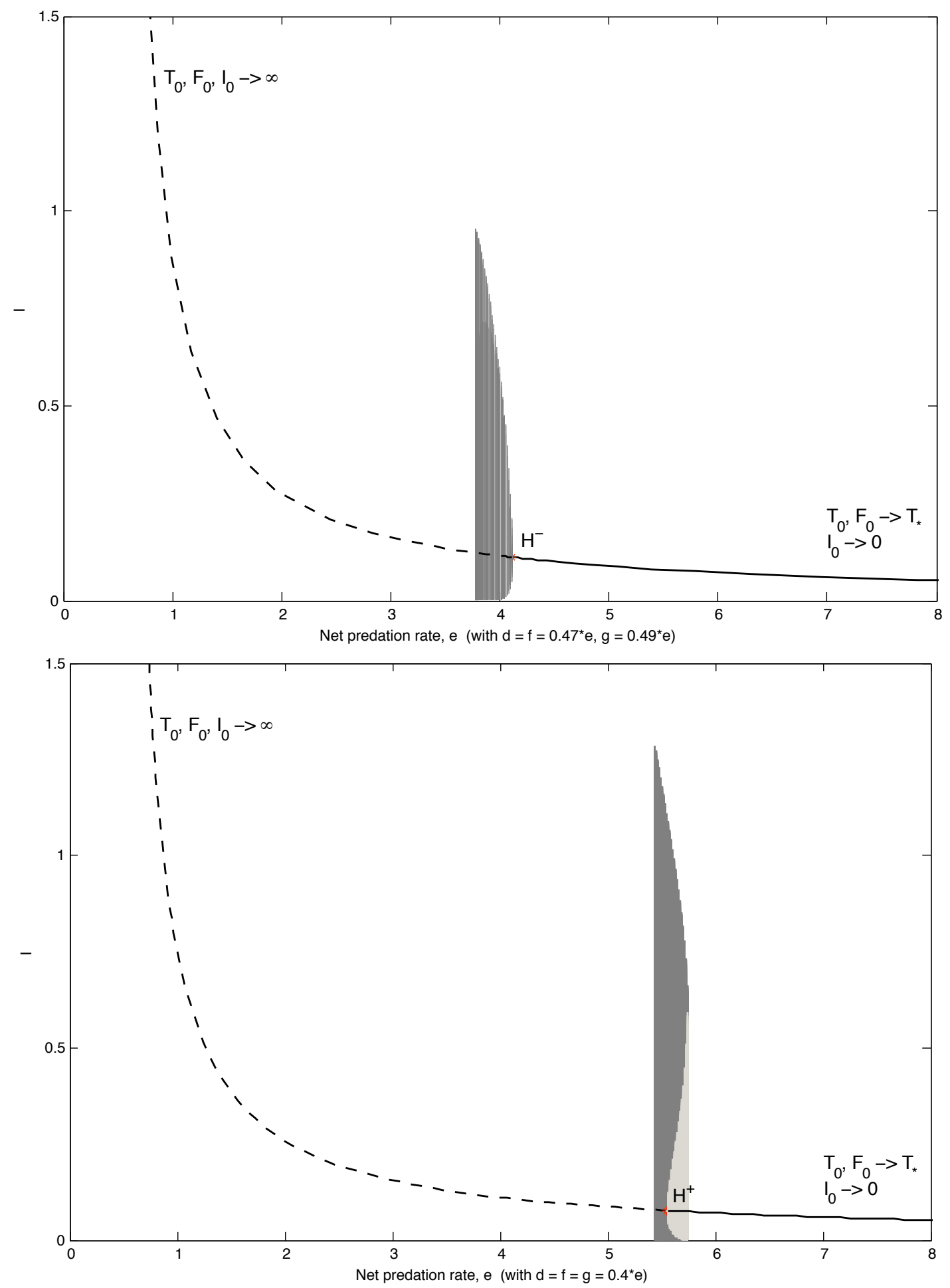

Figure 4: Bifurcation diagrams in which all four predation parameters are varied proportionally to study the overall effect or predation strength. Different proportions between the parameters can produce either supercritical (top) or subcritical (bottom) Hopf bifurcations. Light gray depicts unstable limit cycles, while dark gray depicts stable limit cycles. 
is too weak relative to $F-I$ predation, the insect population is kept low and cannot transfer enough biomass, so the stable fixed point disappears through a saddle-node bifurcation. Conversely, when the $I-T$ predation is relatively strong, the fixed point will lose stability to stable oscillations. In such oscillations, insects eat tadpoles quickly and deplete the tadpole population, after which frogs eat the plentiful insects, depleting that population, and then frogs give birth to many tadpoles, losing biomass themselves until the insects rebound. When these oscillations become too dramatic, extinction is the likely result.

\section{Model with carrying capacities}

With carrying capacities on frogs and insects, the dimensional governing equations become

$$
\begin{aligned}
& \dot{T}=-\gamma T+\beta F-\kappa \frac{I T}{1+\mu T} \\
& \dot{F}=\alpha T-\zeta F\left(1+\frac{1}{N} F\right)+\eta_{F} \lambda \frac{F I}{1+\nu I} \\
& \dot{I}=\epsilon_{I} I\left(1-\frac{1}{M} I\right)+\eta_{I} \kappa \frac{I T}{1+\mu T}-\lambda \frac{F I}{1+\nu I} .
\end{aligned}
$$

\subsection{Nontrivial equilibria}

Without predation, a nontrivial equilibrium exists only if $\frac{\alpha \beta}{\gamma \zeta}>1$, which is also the condition under which the origin is unstable. We wish to study a parameter regime in which the system has nontrivial behavior before predation is added, so we shall assume this inequality always holds. With predation, a nontrivial fixed point must satisfy

$$
\begin{aligned}
& T=\frac{1}{\alpha} F\left[\zeta\left(1+\frac{1}{N} F\right)-\eta_{F} \lambda \frac{I}{1+\nu I}\right] \\
& F=\frac{1}{\beta} T\left(\gamma+\kappa \frac{I}{1+\mu T}\right) \\
& I=M\left[1+\frac{1}{\epsilon_{I}}\left(\eta_{I} \kappa \frac{T}{1+\mu T}-\lambda \frac{F}{1+\nu I}\right)\right] .
\end{aligned}
$$

These algebraic equations cannot be solved explicitly for the equilibrium populations, so we must resort to perturbation expansions and numerical solutions.

\subsubsection{Perturbation of the nontrivial equilibrium by weak predation}

Let the predation strength be small:

$$
\kappa=\epsilon \kappa_{1} \quad \lambda=\epsilon \lambda_{1},
$$

where $\epsilon \ll 1$. We can expand the equilibrium populations in $\epsilon$ (e.g. $T \sim T_{0}+\epsilon T_{1}+O\left(\epsilon^{2}\right)$ ), going to first order to obtain the leading order impact of predation. At zeroth order, i.e. without predation,

$$
\begin{aligned}
T_{0} & =\frac{\beta}{\gamma}\left(\frac{\alpha \beta}{\gamma \zeta}-1\right) N \\
F_{0} & =\left(\frac{\alpha \beta}{\gamma \zeta}-1\right) N \\
I_{0} & =M .
\end{aligned}
$$



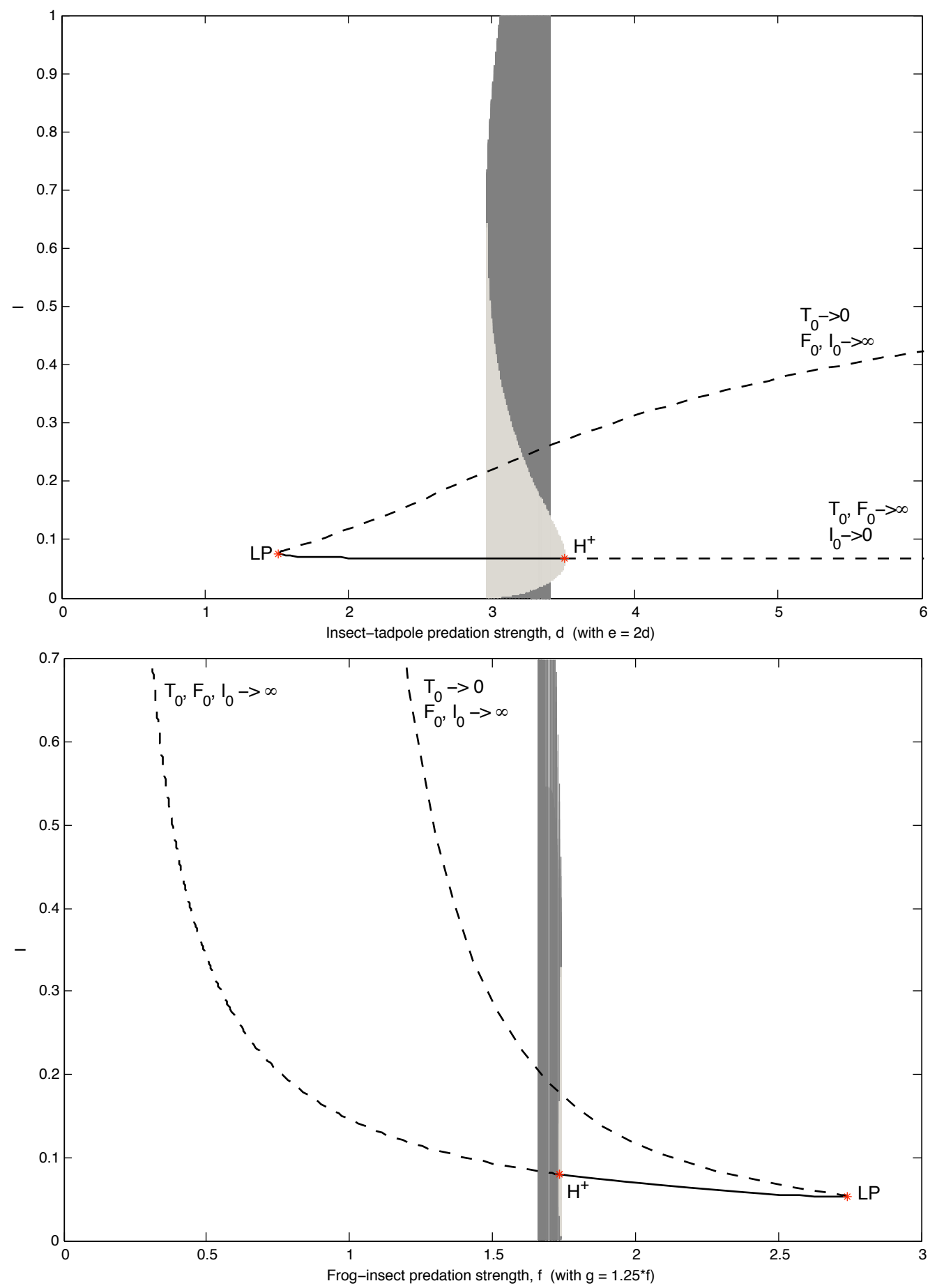

Figure 5: Bifurcation diagrams in which the relative strengths of the two types of predation are varied. To study the variation of insect-tadpole predation strength (top), $d$ and $e$ are varied proportionally $(e=2 d)$, while frog-insect predation is kept constant $(f=2, g=2.5)$. To study the variation of frog-insect predation (bottom), $f$ and $g$ are varied proportionally $\left(f=\frac{5}{4} g\right)$, while insect-tadpole predation is kept constant $(d=2, e=4)$. Light gray depicts unstable limit cycles, while dark gray depicts stable limit cycles. 
The first order coefficients in the expansions are

$$
\begin{aligned}
T_{1} & =\frac{\beta}{\gamma}\left[\eta_{F} \frac{\lambda_{1}}{\zeta} \frac{1}{1+\nu M}+\frac{\kappa_{1}}{\gamma} \frac{\left(1-2 \frac{\alpha \beta}{\gamma \zeta}\right)}{1+\mu \frac{\beta}{\gamma}\left(\frac{\alpha \beta}{\gamma \zeta}-1\right) N}\right] M N \\
F_{1} & =\left[\eta_{F} \frac{\lambda_{1}}{\zeta} \frac{1}{1+\nu M}-\frac{\kappa_{1}}{\gamma} \frac{\frac{\alpha \beta}{\gamma \zeta}}{1+\mu \frac{\beta}{\gamma}\left(\frac{\alpha \beta}{\gamma \zeta}-1\right) N}\right] M N \\
I_{1} & =\left[\eta_{I} \frac{\kappa_{1}}{\epsilon_{I}} \frac{\frac{\beta}{\gamma}}{1+\mu \frac{\beta}{\gamma}\left(\frac{\alpha \beta}{\gamma \zeta}-1\right) N}-\frac{\lambda_{1}}{\epsilon_{I}} \frac{1}{1+\nu M}\right]\left(\frac{\alpha \beta}{\gamma \zeta}-1\right) M N .
\end{aligned}
$$

Unlike the system without carrying capacities, this system has stable fixed points without predation, so we may compare populations with and without predation. There are parameter regimes in which only amphibians, or only insects, benefit from the predation. There is also a regime in which predation hurts both species, for instance when both species are bad at metabolizing each other. This situation is essentially a prisoner's dilemma; both species would benefit from a truce, but once a system of reciprocal predation has evolved, it is hard to see how it could stop. Finally, there is a parameter regime where the predation benefits both species by improving the efficiency with which the entire tadpole-frog-insect system uses environmental resources. We will call this a regime of population increase by mutual predation, or PIMP, and we now look at this regime in further detail.

\subsubsection{Population increase by mutual predation}

Suppose the two types of predation occur with relative strengths given by $K=\frac{\lambda}{\kappa}$. We consider turning on predation with fixed $K$, so the signs of the total derivatives of $T$, $F$, and $I$ with respect to $\kappa$ (or $\lambda$ ) dictate whether predation increases or decreases the population biomasses at equilibrium. When $\epsilon$ is small,

$$
\frac{d T}{d \kappa}=\frac{\partial T_{1}}{\partial \kappa_{1}}+K \frac{\partial T_{1}}{\partial \lambda_{1}}+O\left(\epsilon^{2}\right),
$$

and likewise for the other populations. Evaluated at $\kappa=0$, just as predation is turned on,

$$
\begin{aligned}
& \left.\frac{d T}{d \kappa}\right|_{0} \sim \frac{1}{\gamma}\left[K \eta_{F} \frac{\beta}{\zeta} \frac{1}{1+\nu M}-\frac{2 \frac{\alpha \beta}{\gamma \zeta}-1}{\frac{\gamma}{\beta}+\mu\left(\frac{\alpha \beta}{\gamma \zeta}-1\right) N}\right] M N \\
& \left.\frac{d F}{d \kappa}\right|_{0} \sim \frac{1}{\gamma}\left[K \eta_{F} \frac{\gamma}{\zeta} \frac{1}{1+\nu M}-\frac{\frac{\alpha}{\zeta}}{\frac{\gamma}{\beta}+\mu\left(\frac{\alpha \beta}{\gamma \zeta}-1\right) N}\right] M N \\
& \left.\frac{d I}{d \kappa}\right|_{0} \sim \frac{1}{\epsilon_{I}}\left(\frac{\alpha \beta}{\gamma \zeta}-1\right)\left[\frac{\eta_{I}}{\frac{\gamma}{\beta}+\mu\left(\frac{\alpha \beta}{\gamma \zeta}-1\right) N}-K \frac{1}{1+\nu M}\right] M N .
\end{aligned}
$$

The signs of the above derivatives determine whether predation hurts or helps a species, insofar as its equilibrium biomass decreases or increases. The regime of PIMP could be defined by the condition that both species' biomasses increase with predation, or by the stronger condition that all three populations' biomasses increase, and it is not obvious which definition is more useful. Biologically, the advantage of a larger population is that it is more fit because it is more genetically diverse and robust to catastrophe. In that sense, it does not matter what life stage the amphibians are in because one life 
stage begets another, and our two-stage model is a simplification anyway. On the other hand, it could still be catastrophic for an entire generation of tadpoles to be wiped out. We will thus derive the conditions for PIMP using both the strong and weak definitions.

\subsubsection{Conditions for strong population increase by mutual predation}

All three derivatives with respect to $\kappa$ are positive, meaning the equilibrium biomasses of insects and both amphibian life stages increase, if and only if

$$
\frac{\zeta}{\beta}\left(2 \frac{\alpha \beta}{\gamma \zeta}-1\right) \text { and } \frac{\alpha}{\gamma}<K \eta_{F}\left[\frac{\frac{\gamma}{\beta}+\mu\left(\frac{\alpha \beta}{\gamma \zeta}-1\right) N}{1+\nu M}\right]<\eta_{I} \eta_{F} .
$$

By the assumption that $\frac{\alpha \beta}{\gamma \zeta}>1, \frac{\alpha}{\gamma}<\frac{\zeta}{\beta}\left(2 \frac{\alpha \beta}{\gamma \zeta}-1\right)$, so strong PIMP occurs if and only if

$$
\frac{\zeta}{\beta}\left(2 \frac{\alpha \beta}{\gamma \zeta}-1\right)<K \eta_{F}\left[\frac{\frac{\gamma}{\beta}+\mu\left(\frac{\alpha \beta}{\gamma \zeta}-1\right) N}{1+\nu M}\right]<\eta_{I} \eta_{F}
$$

The ratio of predation rates, $K$, and the rate of reproduction, $\beta$, can be changed by behavior, so they can vary on much shorter time scales than the other parameters, which are controlled by physiology. Thus, we may regard $K$ and $\beta$ as control parameters. Recalling that $\zeta=\beta-\epsilon_{T}$, it is clear from Equations (13) and (14) that without predation, changing the birth rate cannot increase frog and tadpole populations simultaneously; only predation can do that. There exists an interval of $K$ in which strong PIMP occurs precisely when

$$
\frac{\zeta}{\beta}\left(2 \frac{\alpha \beta}{\gamma \zeta}-1\right)<\eta_{I} \eta_{F}
$$

For this inequality to hold, it is necessary that metabolic losses not be too large, and also that $\alpha<\gamma$ and $\beta>\zeta$. That is, predation can only increase all three equilibrium biomasses when frogs gain biomass through their interaction with the external environment, while tadpoles lose it. The biological interpretation of this parameter regime is that frogs are more fit for their environment than tadpoles. Indeed, one could expect this to be true; there are morphogenetic tradeoffs between juvenile and adult fitness, and since frogs spend the majority of their lives in the adult stage, it is likely that they would evolve to be maximally fit as adults. It is less clear that tadpoles would be so unfit as to lose biomass without a constant input from reproduction, but this is certainly feasible in harsh environments that create high juvenile mortality. So, in such a regime, predation can increase all three biomasses by increasing the ratio of frog biomass to tadpole biomass, with insects profiting as middlemen.

\subsubsection{Conditions for weak population increase by mutual predation}

Let $A \equiv T+F$, the total amphibian biomass. Without predation, the equilibrium amphibian biomass is

$$
A_{0}=\left(\frac{\beta+\gamma}{\gamma}\right)\left(\frac{\alpha \beta}{\gamma\left(\beta-\epsilon_{F}\right)}-1\right) N
$$

There is an optimal birth rate, $\beta$, that maximizes $A_{0}$. In the strong definition of PIMP there is no such way to define an optimal $\beta$ since there is always a tradeoff between $T$ and $F$. When $\beta$ is above its optimal value, too much of the frogs' biomass is going into tadpoles, who are less fit than the frogs. When $\beta$ is below its optimal value, the frog 
population becomes so large than environmental pressures make frogs less successful than tadpoles.

The dependence of equilibrium amphibian biomass on weak predation is

$$
\left.\frac{d A}{d \kappa}\right|_{0} \sim \frac{1}{\gamma}\left[K \eta_{F} \frac{\beta+\gamma}{\zeta} \frac{1}{1+\nu M}-\frac{2 \frac{\alpha \beta}{\gamma \zeta}-1+\frac{\alpha}{\zeta}}{\frac{\gamma}{\beta}+\mu\left(\frac{\alpha \beta}{\gamma \zeta}-1\right) N}\right] M N .
$$

Weak predation increases the equilibrium values of both $A$ and $I$, thus satisfying the definition of weak PIMP, if and only if

$$
2 \frac{\alpha \beta}{\gamma \zeta}-1+\frac{\alpha}{\zeta}<K \eta_{F}\left(\frac{\beta+\gamma}{\zeta}\right)\left[\frac{\frac{\gamma}{\beta}+\mu\left(\frac{\alpha \beta}{\gamma \zeta}-1\right) N}{1+\nu M}\right]<\eta_{I} \eta_{F}\left(\frac{\beta+\gamma}{\zeta}\right),
$$

and clearly such a $K$ exists if and only if

$$
2 \frac{\alpha \beta}{\gamma \zeta}-1+\frac{\alpha}{\zeta}<\eta_{I} \eta_{F}\left(\frac{\beta+\gamma}{\zeta}\right) .
$$

This condition is harder to interpret biologically than the stronger condition of Equation (eq: strong PIMP necessary), but it has roughly the same necessary conditions; in the parameter regime for which predation improves efficiency, the metabolisms cannot be too inefficient, frogs cannot be too unfit, and tadpoles cannot be too fit.

Adding predation is like decreasing the rate of reproduction in that it transfers biomass from tadpoles to frogs, though it is certainly not identical. We have not fully explored the relationship between optimal $\beta$ and optimal $K$, but this would be a good topic for future work. For instance, we would like to know whether Equation (18) can hold when the reproduction rate is at its no-predation optimal value, and how the optimal reproduction rate changes in the presence of predation.

\subsubsection{Beyond weak predation}

We have seen analytically that there is a parameter regime in which PIMP occurs when predation is weak, but we would like to know whether increasing the strength of the predation will increase the equilibrium populations indefinitely. We have not tackled this question analytically, but in all numerical experiments the populations reach a maximum value before decreasing as predation is strengthened further. To use MATCONT, we prefer to work with dimensionless parameters, so we apply the nondimensionalization of Equation (4), the same one used for the system without carrying capacities.

$$
\begin{aligned}
\dot{T} & =-T+F-d \frac{I T}{1+T} \\
\dot{F} & =a T-b F\left(1+\frac{1}{N} F\right)+g \frac{F I}{1+I} \\
\dot{I} & =c I\left(1-\frac{1}{M} I\right)+e \frac{I T}{1+T}-f \frac{F I}{1+I},
\end{aligned}
$$

where $M$ and $N$ have been nondimensionalized in the same way as $I$ and $F$, respectively.

In terms of dimensionless quantities, the regime of strong PIMP given by Equation (16) becomes

$$
(2 a-b) \frac{d}{g}<\frac{1+\left(\frac{a}{b}-1\right) N}{1+M}<\frac{e}{f} .
$$

An example case that falls in this regime has $(a, b, c)=(2,1,1),(M, N)=(1,1)$, and the predation parameters in fixed proportion such that $e=2 d, f=d$, and $g=4 d$. Figure 6 
displays the variation of equilibrium populations with predation strength. Starting with no predation and increasing the predation parameters in fixed proportion, we initially see increases in all three equilibrium populations, as predicted by the asymptotic analysis, followed by decreases in all three populations. Insect population appears to go to zero as predation goes to infinity, while frog and tadpole populations appear to decrease asymptotically to the same finite value. Although it might be possible for the amphibians to predate the insects to extinction, it is not in their interest to do so; this strategy does not maximize their equilibrium population.

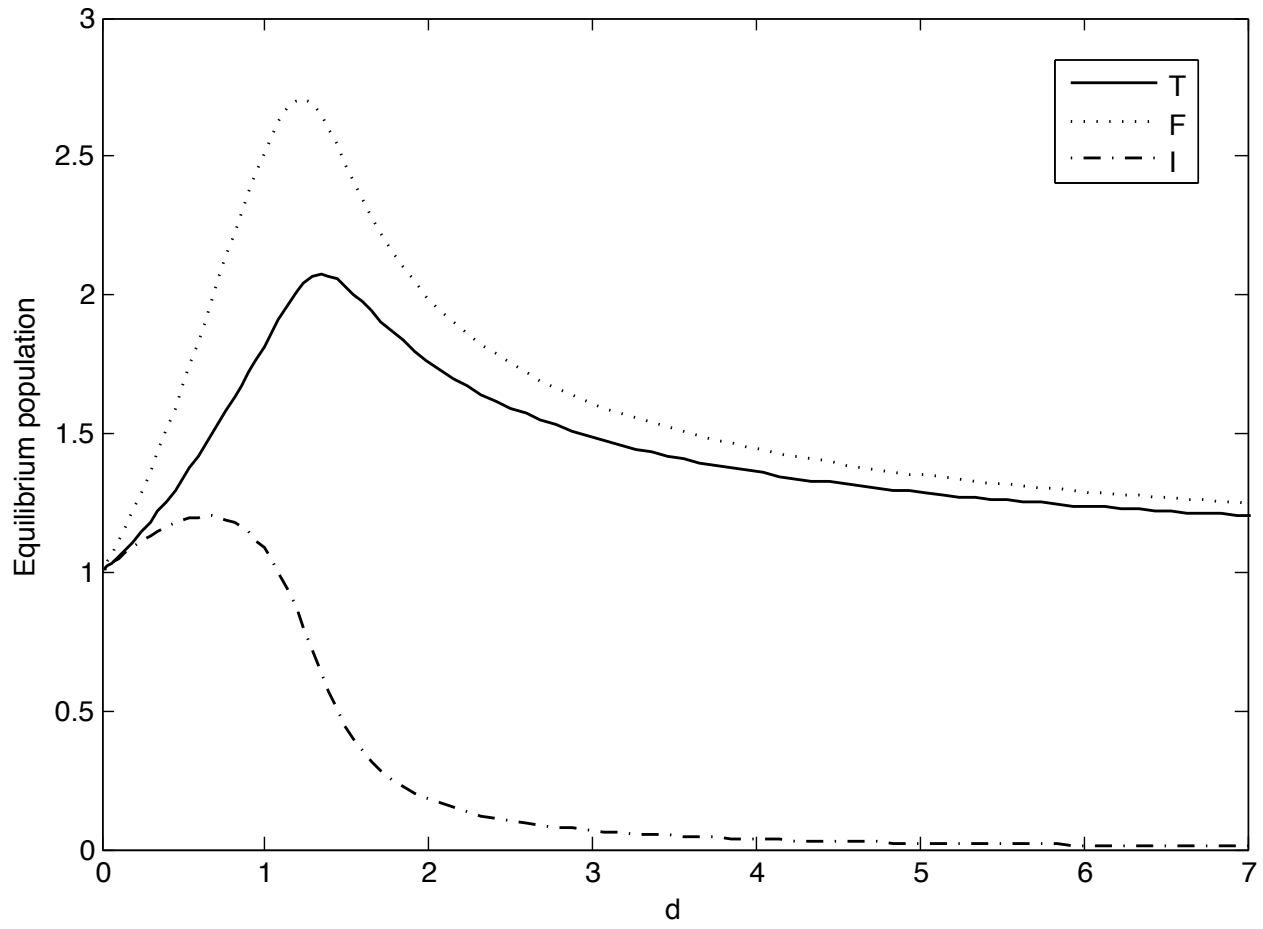

Figure 6: Equilibrium populations as predation strength is varied in the strong PIMP regime. Fixed parameters parameters are $(a, b, c)=(2,1,1)$ and $(M, N)=(1,1)$. Predation parameters are varied in constant proportion with $e=2 d, f=d$, and $g=4 d$.

\subsection{Numerical exploration of parameter space}

We have searched for bifurcations in parameter space using MATCONT, but we have not found any in regimes where PIMP occurs; we simply observe the equilibrium changing its coordinates while remaining stable. In other parameter regimes we find supercritical Hopf bifurcations but no saddle-node bifurcations. The Hopf bifurcations observed in this system were of a different character than those in the system without carrying capacities. In that system, continuing in parameter space past a Hopf bifurcation typically led to growing oscillations and, ultimately, extinction. In the system with carrying capacities, a limit cycle may appear and grow as we move through a Hopf bifurcation, but as we continue through parameter space, it ultimately shrinks and disappears back into a stable equilibrium. So, it seems limit cycles occur only in isolated regions of parameter space where certain resonances are strong. This significant qualitative dif- 
ference from the system without carrying capacities is due to the damping effect of the carrying capacities.

\section{Conclusions}

In the model without carrying capacities, we examined the regime in which the insect and frog-tadpole systems decay without predation. Predation can create stable fixed points and limit cycles, though the limit cycles are not robust and rather unrealistic biologically, so in this model the two species are completely codependent. For this stabilization to occur, predation must be above a certain minimum strength, and the relative strengths of insect-tadpole and frog-insect predation must be within a certain interval. There are biological parameters for which a fixed point and a limit cycle are simultaneously stable, but we have not found parameters for which nontrivial stable fixed points coexist.

In the model with carrying capacities, both species exist stably without predation. Depending on the biological parameters, predation may increase or decrease the equilibrium populations in any combination. Fixing all biological parameters other than predation rates, we have seen that mutual predation can increase all of the equilibrium populations simultaneously. However, there always exists an optimal rate of reproduction that maximizes the equilibrium biomass of amphibians, and we have not determined whether predation can only increase all populations simultaneously by effectively decreasing the rate of reproduction toward its optimal value. If this is the case, we must ask whether it is biologically realistic for the amphibians to habitually reproduce at a non-optimal rate, and if not, we would conclude that population increase by mutual predation is not biologically relevant in the long term. This is both a mathematical and biological question for future study.

\section{Acknowledgments}

I would like to thank all the staff members of the 2010 GFD program who had helpful discussions with me over the summer, particularly Charles Doering, Antonello Provenzale, and Glenn Flierl, who between them gave me constant guidance on this project.

\section{Appendix: The Holling type II functional form}

The simplest system in which the Holling type II functional form could appear is a 2D predator-prey model. However, the justification for using this specific form in such a model is not clear a priori. A quadratic predation term that is proportional to both predator and prey populations would not require much justification since it represents leading order behavior at the very least, but such a term produces unrealistic exponential growth in the system. However, building on the work of Whitehead and Doering [4], we can create a dichotomy between hungry predators sated predators and use only linear and quadratic terms in the resulting 3D system, and then find that in the appropriate limit it reduces to a $2 \mathrm{D}$ predator-prey system with Holling type II predation laws. The functional forms of the $3 \mathrm{D}$ model do not require justification since they are the simplest laws that could capture the necessary behavior. To evaluate the accuracy of the reduced system with Holling type II laws, we shall compare its features to those of the full 3D system. 


\subsection{The 3D system}

The 3D system consists of hungry predator $(H)$, sated predators $(S)$, and prey $(P)$ :

$$
\begin{aligned}
\dot{H} & =-\phi P H+\mu S-\delta H+\beta S \\
\dot{S} & =\phi P H-\mu S-\delta S \\
\dot{P} & =-\phi P H+\gamma P\left(1-\frac{P}{n}\right) .
\end{aligned}
$$

Hungry predators eat prey at rate $\phi$ and become sated predators, while sated predators metabolize and become hungry at rate $\mu$. Both hungry and sated predators die at rate $\delta$, and sated predators give birth to hungry ones at rate $\beta>\delta$. The prey is being born according to a logistic law. In a more accurate model, the $\phi$ and $\delta$ constants in the different equations could take different values, but this would only add parameters without changing any qualitative results.

To reduce the order of the system, we will consider the limit of large metabolism rate, i.e. $\mu \gg \beta, \delta, \gamma$. To see how each variable should scale with $\mu$, we consider the three-species equilibrium,

$$
\left(H_{0}, S_{0}, P_{0}\right)=\left(\frac{\gamma}{\phi}, \frac{\gamma}{\phi} \frac{\delta}{\beta-\delta}, \frac{\mu+\delta}{\phi} \frac{\delta}{\beta-\delta}\right) .
$$

At equilibrium, there is $O(\mu)$ more prey than predators. This motivates us to scale the prey variable in $\mu$, so we nondimensionalize the system by

$$
H \mapsto \frac{\delta}{\phi} h \quad S \mapsto \frac{\delta}{\phi} s \quad P \mapsto \frac{\delta}{\phi} X \quad t \mapsto \frac{1}{\delta} \tau
$$

The nondimensional 3D system is

$$
\begin{aligned}
\dot{h} & =-\frac{1}{\epsilon} X h+\frac{1}{\epsilon} s-h+b s \\
\dot{s} & =\frac{1}{\epsilon} X h-\frac{1}{\epsilon} s-s \\
\dot{X} & =-X h+g X\left(1-\frac{X}{N}\right),
\end{aligned}
$$

where

$$
\begin{aligned}
b & \equiv \frac{\beta}{\delta}>1 \\
g & \equiv \frac{\gamma}{\delta} \\
\epsilon & \equiv \frac{\delta}{\mu} .
\end{aligned}
$$

We shall consider the singular limit where a predator eats many times in its life, i.e. $\epsilon \ll 1$. The parameter $b$ is the ratio of predator birthrate to death rate, $g$ is the ratio of prey birthrate to predator death rate, and $N$ is the dimensionless carrying capacity of the environment for prey. Note that predator and prey populations are in different units, so their numerical values can not be meaningfully compared.

\subsection{Reduced systems}

The 3D system's behavior can only be well approximated by a two dimensional system if its behavior is roughly two dimensional, for instance this implies that it must not be chaotic. There must be a two-dimensional slow manifold in phase space on which all solutions approximately lie, possibly after some transient behavior as the component of the solution on the fast manifold rapidly decays. Reduction of order is achieved by 
projecting the full system onto the slow manifold. To find the slow manifold, we observe that the system is linear in $h$ and $s$ if $X$ is regarded as a known function of time:

$$
\left(\begin{array}{c}
\dot{h} \\
\dot{s}
\end{array}\right)=\left(\begin{array}{cc}
-\left(\frac{1}{\epsilon} X+1\right) & \frac{1}{\epsilon}+b \\
\frac{1}{\epsilon} X & -\left(\frac{1}{\epsilon}+1\right)
\end{array}\right)\left(\begin{array}{l}
h \\
s
\end{array}\right) .
$$

The eigenvalues of this system are

$$
\lambda_{ \pm}=-\frac{1}{\epsilon} \frac{X+1}{2}-1 \pm \frac{1}{\epsilon} \frac{X+1}{2} \sqrt{1+\epsilon \frac{4 b X}{(X+1)^{2}}} .
$$

To first order in $\epsilon$,

$$
\begin{aligned}
& \lambda_{+}=-1+\frac{b X}{X+1}+O(\epsilon) \\
& \lambda_{-}=-\frac{1}{\epsilon}(X+1)-1+\frac{b X}{X+1}+O(\epsilon) .
\end{aligned}
$$

The $\lambda_{+}$eigenvalue is $O(1)$, while the $\lambda_{-}$eigenvalue is negative and $O\left(\frac{1}{\epsilon}\right)$, so solutions decay quickly along the direction of the $\lambda_{-}$eigenvector and move more slowly in the $\lambda_{+}$direction. Thus at a given $X$, the $\lambda_{-}$vector is tangent to the fast manifold, and the $\lambda_{+}$vector is tangent to the slow manifold.

\subsubsection{Full 2D system}

The $\lambda_{+}$eigenvector yields a proportionality between $s$ and $h$ on the slow manifold as a function of $X$,

$$
s=\frac{1}{2(1+\epsilon b)}\left(X-1+\sqrt{(X+1)^{2}+4 \epsilon b X}\right) h .
$$

This relation may be used to reduce the dynamical system by eliminating either $h$ or $s$, but since we are ultimately concerned with the total number of predators, we define $Y \equiv h+s$ and work in this variable. Applying the above $s(h)$ relation to the 3D system, we obtain our $2 \mathrm{D}$ reduction,

$$
\begin{aligned}
& \dot{X}=g X\left(1-\frac{X}{N}\right)-\frac{2(1+\epsilon b) X Y}{X+1+\sqrt{(X+1)^{2}+4 \epsilon b X}+2 \epsilon b} \\
& \dot{Y}=-Y+\frac{\left(X-1+\sqrt{(X+1)^{2}+4 \epsilon b X}\right) b Y}{X+1+\sqrt{(X+1)^{2}+4 \epsilon b X}+2 \epsilon b} .
\end{aligned}
$$

\subsection{2 $\mathrm{O}(\epsilon)$ 2D system}

If we approximate the $s(h)$ relation to $O(\epsilon)$, we obtain a simpler relation that still captures some affects of finite $\epsilon$,

$$
s=\left(X-\epsilon \frac{b X^{2}}{1+X}\right) h+O\left(\epsilon^{2}\right) .
$$

The $O(\epsilon)$ truncated reduced system is

$$
\begin{aligned}
& \dot{X}=g X\left(1-\frac{X}{N}\right)-\frac{X Y}{1+X}-\epsilon \frac{b X^{3} Y}{(X+1)^{3}} \\
& \dot{Y}=-Y+\frac{b X Y}{1+X}-\epsilon \frac{b^{2} X^{2} Y}{(X+1)^{3}} .
\end{aligned}
$$




\subsubsection{Holling type II 2D system}

If we truncate further and retain only the $O(1)$ term in $\epsilon$,

$$
s=h X+O(\epsilon)
$$

Using this simple proportionality we recover the Holling type II functional form.

$$
\begin{aligned}
& \dot{X}=g X\left(1-\frac{X}{N}\right)-\frac{X Y}{1+X} \\
& \dot{Y}=-Y+\frac{b X Y}{1+X} .
\end{aligned}
$$

The form $\frac{X Y}{1+X}$ goes by various other names in other fields, such as the Jacob-Monod form in microbiology or the Michaelis-Menten form in enzyme kinetics.

\subsection{Comparison of system behaviors}

To understand what has been lost by projecting onto the slow manifold, as well as by truncating in $\epsilon$, one must compare the behavior of the full $3 \mathrm{D}$ system with the behaviors of the reduced $2 \mathrm{D}$ systems. There is no unique measure of the quality of the approximation; different properties are approximated better than others, so the value of the approximation depends ultimately on what properties are of interest.

For the 3D system, we ignore the separate dynamics of the $h$ and $s$ variables and consider only their sum, $Y$, because this is the quantity of interest and the one that compares directly to the $2 \mathrm{D}$ models. As we demonstrate below, all four models have the same qualitative behavior, some representative phase portraits of which are given in Figure 7 . There is a trivial equilibrium at the origin representing mass extinction, but it is always unstable. There is a prey-only equilibrium, stable only when $b$ and $N$ are small. That is, when predators are not born too fast, and saturation population of prey is not too large, both factors that would inhibit predator success. When $b$ and $N$ are a bit larger so conditions are a bit better for predators, the prey-only equilibrium becomes unstable as a stable two-species equilibirum becomes physical and splits off from it in a transcritical bifurcation. The two-species equilibrium is a plain sink initially and becomes a spiral sink at larger $b$ and $N$. When $b$ and $N$ are increased further still, the two-species equilibrium undergoes a Hopf bifurcation, losing its stability to a limit cycle. All orbits are bounded for all parameter values. Since all four systems share this qualitative picture, the effects of approximation appear only in the quantitative differences between, say, equilibria and limit cycle locations, or bifurcation values.

\subsubsection{Equilibria}

At all parameter values, all four systems have equilibria at the origin and at $(X, Y)=$ $(N, 0)$. Solving for the nontrivial two-species equilibria, we obtain $Y_{0}=\frac{b g}{b-1}\left(1-\frac{X_{0}}{N}\right)$ for all four systems, though the $X_{0}$ value may vary between systems. Clearly the nontrivial equilibrium is only physical $\left(Y_{0}>0\right)$ when $X_{0}$ is less than $N$, the prey-only saturation population. That is, such equilibria never represent mutualistic solutions. The $X_{0}$ values for the two-species equilibria in each system are tabulated in Table 2 . The $X_{0}$ value for the $O(\epsilon)$ system is more cleanly expressed implicitly by the cubic equation of which it is the only positive real root. The full $2 \mathrm{D}$ system has exactly the same equilibrium as the $3 \mathrm{D}$ system, while the truncated systems have different two-species equilibria, which converge to the $3 \mathrm{D}$ value as $\epsilon \rightarrow 0$. 

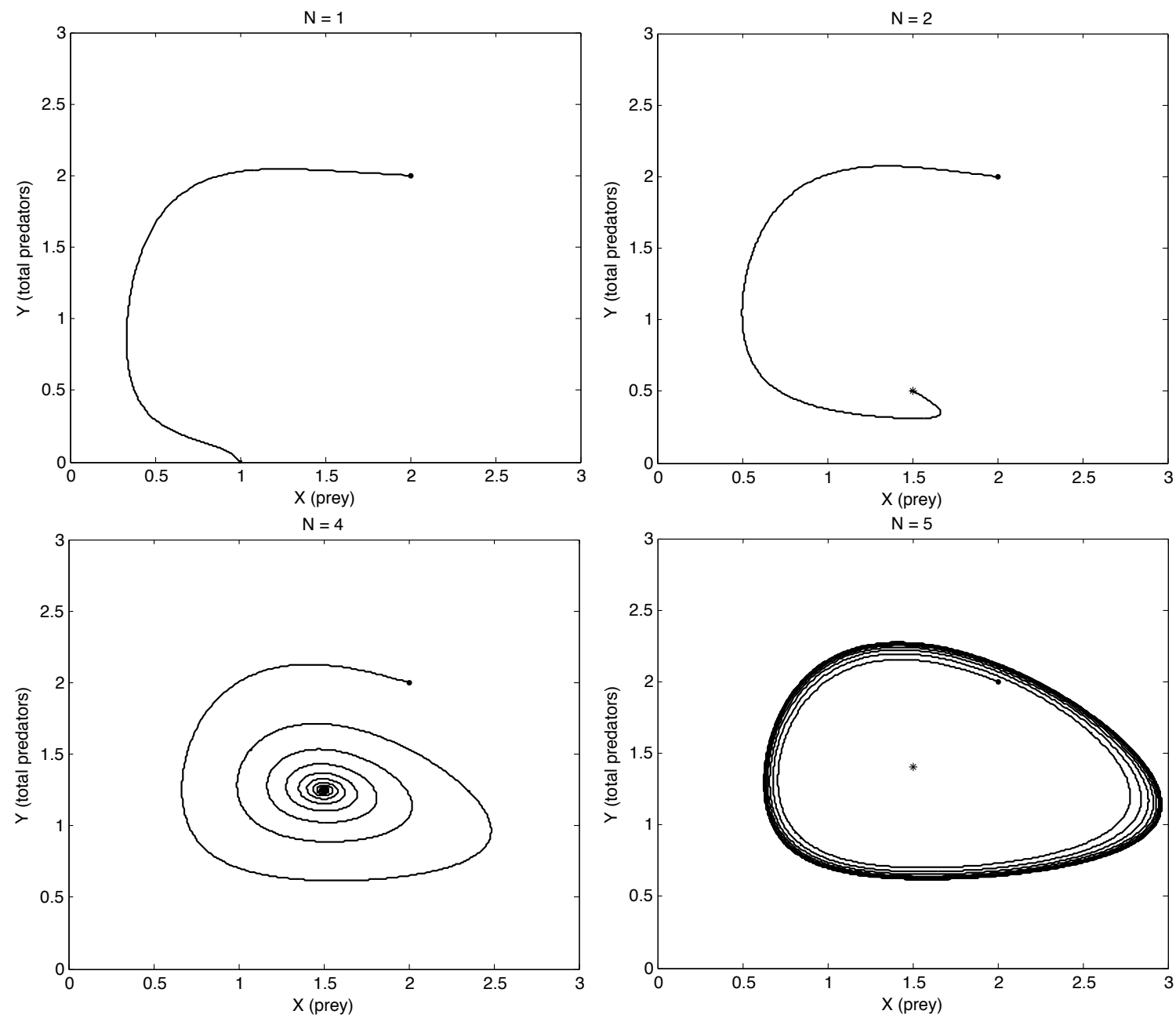

Figure 7: Sample phase portraits representing all four possible qualitative behaviors, considering $N$ as the control parameter: stable prey-only equilibrium (upper left), stable twospecies equilibrium with plain sink (upper right), stable two-species equilibrium with spiral sink (lower left), and a limit cycle around the two-species equilibrium (lower right). These portraits were generated by integrating the $3 \mathrm{D}$ system with $b=2, g=1$, and $\epsilon=0.5$. 
Table 2: Locations of the two-species equilibria in the four models.

\begin{tabular}{|c|c|c|}
\hline Model & $X_{0}$ & $Y_{0}$ \\
\hline 3D & $\frac{1+\epsilon}{b-1}$ & $\frac{b g}{b-1}\left(1-\frac{1+\epsilon}{N(b-1)}\right)$ \\
\hline Full 2D & $\frac{1+\epsilon}{b-1}$ & $\frac{b g}{b-1}\left(1-\frac{1+\epsilon}{N(b-1)}\right)$ \\
\hline$O(\epsilon)$ 2D & $(b-1) X_{0}^{3}+\left(2 b-\epsilon b^{2}-3\right) X_{0}^{2}+(b-3) X_{0}-1=0$ & $\frac{b g}{b-1}\left(1-\frac{X_{0}}{N}\right)$ \\
\hline Holling type II 2D & $\frac{1}{b-1}$ & $\frac{b g}{b-1}\left(1-\frac{1}{N(b-1)}\right)$ \\
\hline
\end{tabular}

\subsubsection{Linear stability}

To analyze the linear stability of the three equilibria in all four systems, we linearize each system about an arbitrary point, $\left(h_{0}, s_{0}, X_{0}\right)$, or $\left(X_{0}, Y_{0}\right)$.

\section{D system}

$$
\frac{d}{d t}\left(\begin{array}{c}
\Delta h \\
\Delta s \\
\Delta X
\end{array}\right)=\left(\begin{array}{ccc}
-\left(\frac{1}{\epsilon} X_{0}+1\right) & \frac{1}{\epsilon}+b & -\frac{1}{\epsilon} h_{0} \\
\frac{1}{\epsilon} X_{0} & -\left(\frac{1}{\epsilon}+1\right) & \frac{1}{\epsilon} h_{0} \\
-X_{0} & 0 & -h_{0}+g\left(1-\frac{2 X_{0}}{N}\right)
\end{array}\right)\left(\begin{array}{c}
\Delta h \\
\Delta s \\
\Delta X
\end{array}\right)
$$

\section{Full 2D system}

$$
\begin{gathered}
\frac{d}{d t}\left(\begin{array}{c}
\Delta X \\
\Delta Y
\end{array}\right)=\left(\begin{array}{ll}
m_{X X} & m_{X Y} \\
m_{Y X} & m_{Y Y}
\end{array}\right)\left(\begin{array}{c}
\Delta X \\
\Delta Y
\end{array}\right) \text {, where } \\
m_{X X}=g\left(1-\frac{2 X_{0}}{N}\right)-\left(1-\frac{X_{0}}{\sqrt{\left(X_{0}+1\right)^{2}+4 \epsilon b X_{0}}}\right) \frac{2(1+\epsilon b) Y_{0}}{1+X_{0}+\sqrt{\left(X_{0}+1\right)^{2}+4 \epsilon b X_{0}}+2 \epsilon b} \\
m_{X Y}=\frac{2(1+\epsilon b) X_{0}}{1+X_{0}+\sqrt{\left(X_{0}+1\right)^{2}+4 \epsilon b X_{0}}+2 \epsilon b} \\
m_{Y X}=\left(\frac{b}{\sqrt{\left(X_{0}+1\right)^{2}+4 \epsilon b X_{0}}}\right) \frac{2(1+\epsilon b) Y_{0}}{1+X_{0}+\sqrt{\left(X_{0}+1\right)^{2}+4 \epsilon b X_{0}}+2 \epsilon b} \\
m_{Y Y}=-1-\frac{1}{2 \epsilon}\left(X_{0}+1+\sqrt{\left(X_{0}+1\right)^{2}+4 \epsilon b X_{0}}\right)
\end{gathered}
$$

\section{$\mathrm{O}(\epsilon)$ 2D system}

$$
\frac{d}{d t}\left(\begin{array}{c}
\Delta X \\
\Delta Y
\end{array}\right)=\left(\begin{array}{cc}
g\left(1-\frac{2 X_{0}}{N}\right)-\frac{Y_{0}}{\left(1+X_{0}\right)^{2}}-\epsilon \frac{3 b X_{0}^{2} Y_{0}}{\left(1+X_{0}\right)^{4}} & -\frac{X_{0}}{1+X_{0}}-\epsilon \frac{b X_{0}^{3}}{\left(1+X_{0}\right)^{3}} \\
\frac{b Y_{0}}{\left(1+X_{0}\right)^{2}}+\epsilon \frac{b^{2}\left(X_{0}^{2}-2 X_{0}\right) Y_{0}}{\left(1+X_{0}\right)^{4}} & \frac{b X_{0}}{1+X_{0}}-1-\epsilon \frac{b^{2} X_{0}^{2}}{\left(1+X_{0}\right)^{3}}
\end{array}\right)\left(\begin{array}{c}
\Delta X \\
\Delta Y
\end{array}\right)
$$

\section{Holling type II 2D system}

$$
\frac{d}{d t}\left(\begin{array}{c}
\Delta X \\
\Delta Y
\end{array}\right)=\left(\begin{array}{cc}
g\left(1-\frac{2 X_{0}}{N}\right)-\frac{Y_{0}}{\left(1+X_{0}\right)^{2}} & -\frac{X_{0}}{1+X_{0}} \\
\frac{b Y_{0}}{\left(1+X_{0}\right)^{2}} & \frac{b X_{0}}{1+X_{0}}-1
\end{array}\right)\left(\begin{array}{c}
\Delta X \\
\Delta Y
\end{array}\right)
$$

About the origin, every linearized system has an eigenvalue of $g$, which is positive, so the extinction equilibrium is always unstable. Linearizing about the prey-only equilibrium, one finds that each system goes unstable according to the same parameter 
inequalities that determine when the two-species equilibrium exists. This is as expected from a transcritical bifurcation; the stability of the prey-only equilibrium changes precisely when it collides with the two-species equilibrium, which is also the moment when that equilibrium becomes physical. As for the two-species equilibrium, we can study its stability analytically in the Holling type II system, but the other linearized systems are messy, so we solve their stability eigenproblems numerically.

\subsubsection{Stability of the two-species equilibrium in the Holling type II 2D system}

The Holling type II system linearized about its two-species equilibrium is

$$
\frac{d}{d t}\left(\begin{array}{c}
\Delta X \\
\Delta Y
\end{array}\right)=\left(\begin{array}{cc}
\frac{g}{b}\left(1-\frac{b+1}{N(b-1)}\right) & -\frac{1}{b} \\
g\left(b-1-\frac{1}{N}\right) & 0
\end{array}\right)\left(\begin{array}{c}
\Delta X \\
\Delta Y
\end{array}\right) .
$$

The stability of the two-species equilibria is not hard to compute analytically for the Holling type II system. The characteristic equation of the linearized system is

$$
\lambda^{2}-\frac{g}{b}\left(1-\frac{b+1}{N(b-1)}\right) \lambda+\frac{g}{b}\left(b-1-\frac{1}{N}\right)=0 .
$$

For the two-species equilibrium to exist, the $O(1)$ coefficient of the characterisitc equation must be positive. Thus, solving the quadratic equation for $\lambda$, the discriminant will either be imaginary or of smaller magnitude than the $O(\lambda)$ coefficient. Either way, both eigenvalues will be negative (the equilibrium will be stable) if and only if the $O(\lambda)$ co-

efficient is positive, i.e. when $N<\frac{b+1}{b-1}$. When $N$ exceeds this value, all three equilibria are unstable. We later prove that all orbits are bounded, so the Poincaré-Bendixon theorem will guarantee that the system converges to a limit cycle.

\subsubsection{Bifurcations}

The transcritical bifurcation occurs when $Y_{0}$ exceeds zero, which occurs when the $X_{0}$ expressions reported in Table 2 are less than $N$. For each system, this is possible only when $b>1$. The exact relation between $b$ and $N$ at the bifurcations are given in Table 3. Note that from a point in parameter space where the prey-only equilibrium is stable, the bifurcation may be produced by increasing either $b$ or $N$. The point in parameter space where the Hopf bifurcation occurs has a simple analytic expression for the Holling type II model, so this is also given in Table 3 . For the other models, the $N$ at which the Hopf bifurcation occurs was computed numerically for given values of $b, g$ and $\epsilon$, and some representative results are plotted in Figure 8. The 3D transcritical bifurcation depends only on $b, N$ and $\epsilon$, while the Hopf bifurcation depends also on $g$, but quite weakly so. It is clear from Figure 8 that the full $2 \mathrm{D}$ model captures the transcritical bifurcation perfectly, while the truncated models are inaccurate when $\epsilon$ becomes large. At the Hopf bifurcation, the full 2D model captures the 3D behavior imperfectly, but again much better than the truncated models.

\subsubsection{Lyapunov stability}

Each system undergoes only the two bifurcations we have studied and has no other fixed points. All that remains is to verify that in each system the orbits are bounded for all parameter values. We shall do this by the Lyapunov method for the 3D system and the Holling type II 2D system. We shall not prove boundedness for the other two systems, whose algebraic nonlinearities would make it a cumbersome task, but we can feel confident in its veracity. 
Table 3: Bifurcation points of the four models. For the $O(\epsilon)$ model, $X_{0}$ is defined implicitly by the formula given in Table 2 .

\begin{tabular}{|c|c|c|}
\hline Model & Transcritical bifurcation & Hopf bifurcation \\
\hline 3D & $0<\frac{1+\epsilon}{b-1} \leq N$ & \multirow{3}{*}{ Found numerically } \\
\hline Full 2D & $0<\frac{1+\epsilon}{b-1} \leq N$ & \\
\hline$O(\epsilon)$ 2D & $0<X_{0} \leq N$ & $\frac{b+1}{b-1} \leq N$ \\
\hline Holling type II 2D & $0<\frac{1}{b-1} \leq N$ & \\
\hline
\end{tabular}

Holling type II 2D system Examining the $\dot{X}$ equation, we see that $\dot{X}<0$ whenever $X>N$. Thus, if $X<N$ at the initial condition, it remains true for all time. To put an upper bound on $Y$ that is valid for all parameters, we must consider $X$ and $Y$ together in a Lyapunov functional.

Let $L \equiv X+\frac{1}{b} Y$. The proportionality between $X$ and $Y$ is chosen such that the nonlinear Holling type II terms in $\dot{L}$ cancel:

$$
\dot{L}=g X\left(1-\frac{X}{N}\right)-\frac{1}{b} Y .
$$

Our goal is to bound $\dot{L}$ by an affine function of $L$, i.e. $\dot{L} \leq \alpha-\beta L$, where $\beta>0$. This will imply that $L<\frac{\alpha}{\beta}$ for all time if it is true initially. To bound $\dot{L}$ by such a term we must bound the quadratic $X$ term by an affine function of $X$ with a negative coefficient on $X$. Any line tangent to the parabola at $X_{*}>\frac{N}{2}$ will suffice, but we seek the line that minimizes $\frac{\alpha}{\beta}$, thereby providing the optimal bound on $L$. An arbitrary tangent line gives the bound

$$
X\left(1-\frac{X}{N}\right) \leq \frac{X_{*}^{2}}{N}-\left(\frac{2 X_{*}}{N}-1\right) X,
$$

which induces a bound on $\dot{L}$,

$$
\dot{L} \leq \frac{X_{*}^{2}}{N}-\min \left\{\frac{2 X_{*}}{N}-1, \frac{1}{b}\right\} L .
$$

Thus,

$$
L<\inf _{X_{*}>N / 2} \frac{X_{*}^{2}}{N \min \left\{\frac{2 X_{*}}{N}-1, \frac{1}{b}\right\}} .
$$

Assuming the above infimum occurs at an $X_{*}$ such that $\frac{2 X_{*}}{N}-1<\frac{1}{b}$, the optimal choice of $X_{*}$ in fact contradicts the assumption when $b>1$. So, the minimum must be $\frac{1}{b}$, meaning that $X_{*} \geq \frac{N}{2}\left(1+\frac{1}{b}\right) \geq N$. The bound on $L$ then becomes

$$
L<\inf _{X_{*} \geq \frac{N}{2}\left(1+\frac{1}{b}\right)} \frac{b X_{*}^{2}}{N}=\frac{N}{4 b}(b+1)^{2} .
$$

Putting this in terms of $X$ and $Y$, and adding the known bound on $X$ alone,

$$
X<\min \left\{\frac{N}{4 b^{2}}(1+b)^{2}-\frac{1}{b} Y, N\right\} .
$$

3D system Let $L \equiv h+\alpha s+\beta X$. To make the $h X$ terms to vanish from $\dot{L}$, we let $\beta=\frac{\alpha-1}{\epsilon}$, which clearly requires $\alpha>1$ for $\beta$ to be positive. Thus,

$$
\begin{aligned}
\dot{L} & =-\left[\frac{1}{\epsilon}+1-\left(\frac{1}{\epsilon}+b\right) / \alpha\right] \alpha s-h+\frac{1}{\epsilon}(\alpha-1) g X\left(1-\frac{X}{N}\right) \\
& \leq-m(\alpha) L+\frac{1}{\epsilon}(\alpha-1) X\left[(g+m(\alpha))-\frac{g X}{N}\right],
\end{aligned}
$$




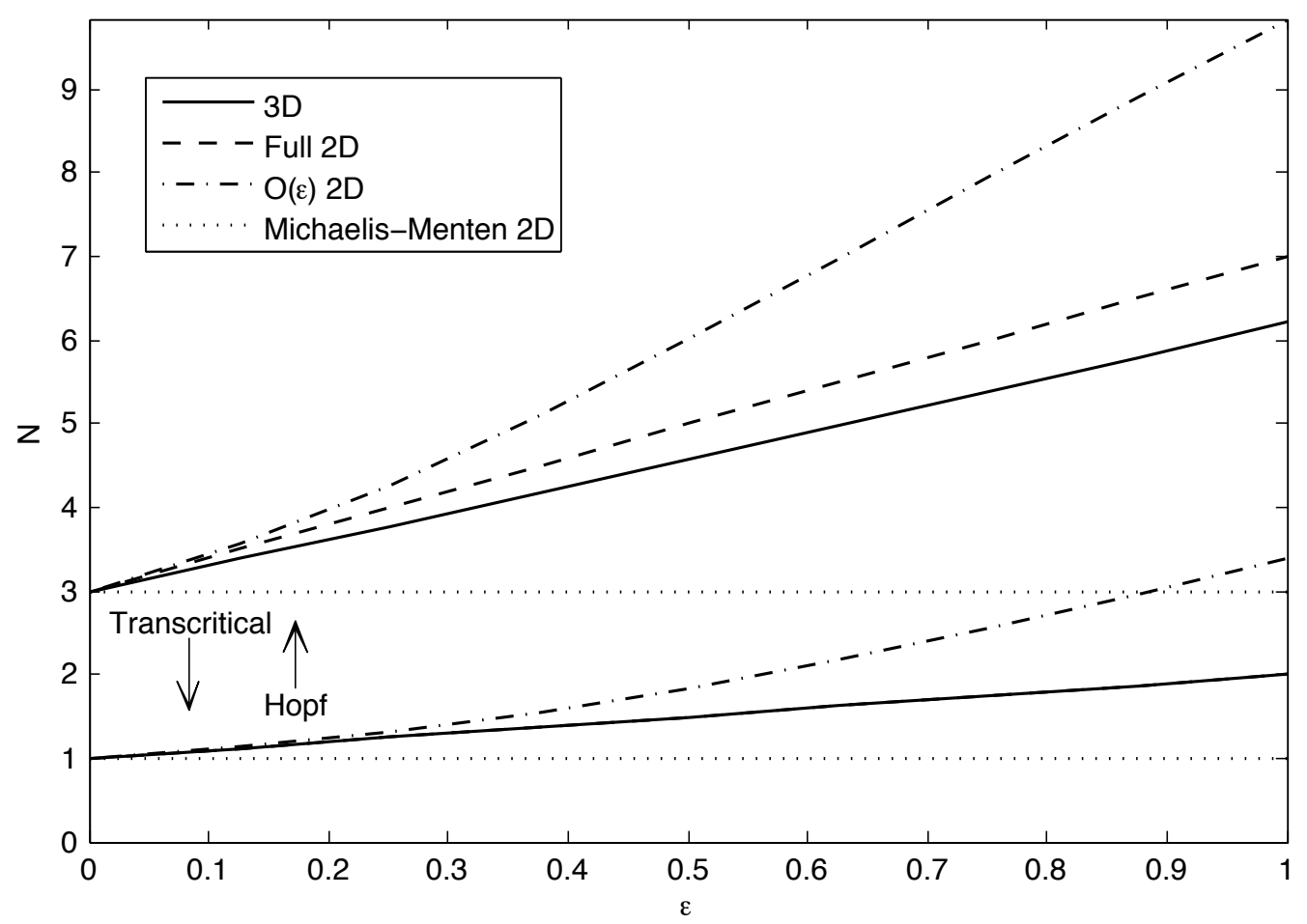

Figure 8: Values of $N$ as a function of $\epsilon$ at the transcritical and Hopf bifurcations for all four systems with $b=2$ and $g=1$. For the transcritical bifurcation, the full $2 \mathrm{D}$ systems coincides with the $3 \mathrm{D}$ system.

where $m(\alpha) \equiv \min \left\{1, \frac{1}{\epsilon}+1-\left(\frac{1}{\epsilon}+b\right) / \alpha\right\}$. We now bound the quadratic $X$ term by its maximum value without bothering to find the optimal bound linear in $X$.

$$
\dot{L} \leq-m(\alpha) L+\frac{N}{4 \epsilon g}(\alpha-1)(g+m(\alpha))^{2} .
$$

This yields a bound on $L$ that can be optimized over all allowable $\alpha$ :

$$
L<\inf _{\alpha>1} \frac{N}{4 \epsilon g} \frac{\alpha-1}{m(\alpha)}(g+m(\alpha))^{2} .
$$

Assuming the infimum occurs when $m(\alpha)=1$ implies that the optimal bound is obtained by choosing $\alpha=1^{+}$, which contradicts $m(\alpha)=1$. Let $\alpha \geq \frac{1+\epsilon b}{1+\epsilon}$, giving our best result for a bound on the Lyapunov functional:

$$
L<\inf _{\alpha \geq \frac{1+\epsilon b}{1+\epsilon}} \frac{N}{4 \epsilon^{2} g} \frac{\alpha-1}{1+\epsilon-\frac{1+\epsilon b}{\alpha}}\left(g+1+\epsilon-\frac{1+\epsilon b}{\alpha}\right)^{2} .
$$

The optimal bound can be calculated given the other parameters, but the more important conclusion is that some such finite bound always exists for $L$.

\subsubsection{Summary of results}

Our analysis strongly suggests that $X$ and $Y$ have the same qualitative behavior in all four systems, though to make this result rigorous, one needs Lyapunov bounds on the 
full 2D and $O(\epsilon) 2 \mathrm{D}$ systems, and one needs to show analytically that a Hopf bifurcation occurs in all systems as it does in the Holling type II system. Trusting that the systems indeed all have the same behavior, they differ only quantitatively. We have seen that the full 2D system has the same two-species equilibrium as the $3 \mathrm{D}$ system, while the truncated systems do not, and quantitative differences in bifurcation values were shown already in Figure 8.

Phase portraits produced by the different models appear in Figure 9. Although the $2 \mathrm{D}$ systems began with the same initial conditions, we must compare them each to their own corresponding 3D solution because they each correspond to slightly different decompositions of $Y_{0}$ into $h_{0}$ and $s_{0}$. However, the three $3 \mathrm{D}$ solutions tend to be quite similar. The top row of Figure 9 shows solutions at small $N$, when the prey-only equilibrium is stable. The prey-only equilibrium is identical in all four systems, so the

phase portraits agree well even for $\epsilon=0.5$. The middle row of Figure 9 shows solutions for larger $N$, when the two-species equilibrium is stable. The different systems agree well when $\epsilon$ is 0.05 , but at 0.5 the locations of the equilibria differ significantly, so the respective phase trajectories spiraling towards them are quantitatively quite different. The bottom row of Figure 9 shows long-time solutions at still larger $N$. When $\epsilon$ is 0.05 , the limit cycles of the full $2 \mathrm{D}$ and $O(\epsilon) 2 \mathrm{D}$ systems approximate the $3 \mathrm{D}$ limit cycle quite well, while the Holling type II system does a bit worse. When $\epsilon$ is 0.5 , the Holling type II system's limit cycle is much too large, the full $2 \mathrm{D}$ system's is too small but a bit better, and the $O(\epsilon) 2 \mathrm{D}$ system has not yet gone through the Hopf bifurcation.

The full 2D system approximates the $3 \mathrm{D}$ system well for $\epsilon \lesssim 0.5$, while the Holling type II 2D system is quantitatively accurate only when $\epsilon$ is an order of magnitude smaller. We wish to extrapolate these truths to other models where the Holling type II or full-order-in- $\epsilon$ functional forms might be used as predation laws without repeating their rigorous derivation from a higher-order dynamical system. If $\epsilon$ is very small, or if one is only concerned with qualitative features, as is often the case in biological modeling, the Holling type II functional form is certainly satisfactory. If $\epsilon$ is closer to unity, and the quantitative properties of the system matter, as is often the case in enzyme kinetics, the full-order-in- $\epsilon$ functional form would be a better choice. The $O(\epsilon)$ functional form probably offers neither enough simplicity nor accuracy to be chosen over the other two.

\section{References}

[1] C. S. Holling, Some characteristics of simple types of predation and parasitism, Canadian Entomologist, (1959).

[2] E. E. WERner AND J. F. Gilliam, The ontogenetic niche and species interactions in size-structured populations, Ann. Rev Ecol. Syst., (1984).

[3] J. P. Whitehead And C. R. Doening, Notes on some age structure trophic dynamics, (2007, unpublished).

[4] — Simple hungry and satiated predator, (2007, unpublished). 

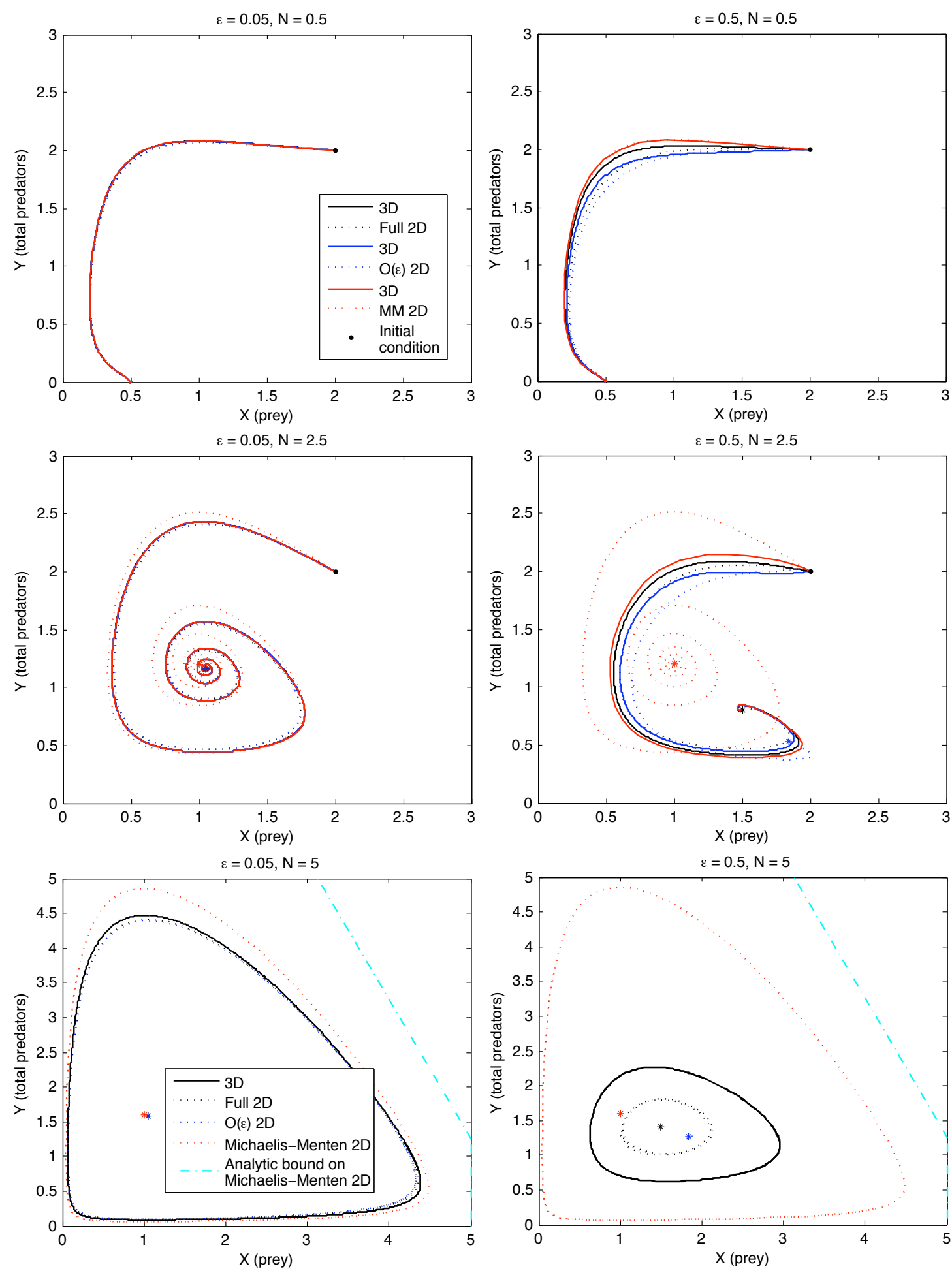

Figure 9: Phase portraits of all four systems for values of $N$ with $\epsilon=0.05$ (left) and $\epsilon=0.5$ (right), and $b=2, g=1$. All solutions began at $(2,2)$, but only the late-time behavior is shown in the bottom two plots to make the limit cycles clear. The asterisks are the equilibria of the different systems, which always coincide for the $3 \mathrm{D}$ system and the full $2 \mathrm{D}$ system. 


\title{
Dynamics of the Mixed Layers in Stratified Sheared Flows
}

\author{
Georgy Manucharyan
}

October 18, 2010

\section{Introduction}

Vast verity of geophysical flows occur in density stratified fluids in a presence of sheared mean velocity profiles. Under such conditions it's been observed that vigorous turbulence leads to generation of mixed layers within the fluid, e.g. the regions of homogeneous density separated from each other by strong density gradients (interfaces). These layers could be of large vertical scales as well as of fine scales. An example of such flows is the destratification of the upper layer of the ocean under the action of the surface wind stress and the formation of multiple mixed layers which are observed within the seasonal thermocline in the ocean as well as in fresh lakes [10]. Such structures in a turbulent flows are thought to play a crucial role in the determining the turbulent fluxes of momentum, buoyancy and other tracers which in turn affect the further dynamics of the flow. Nonetheless, the problem of turbulent transport remains one the most difficult ones in geophysics.

Previous studies investigated mechanisms acting in stratified turbulent flows - a review of those could be found in [4]. The formation of small scale mixed layers in stratified fluids driven by external mechanical sources of turbulence were investigated by theoretically by Balmforth et al [1], and experimentally by Park et al [6]. In the present study the dynamics of layers is explored within a turbulent fluid in a presence of a sheared mean flow. A set of experiments were performed in which a linearly stratified fluid is driven by the stress applied by a rotating horizontal disk at the surface. This setting leads to the formation of the surface mixed layer extending from the the disk into the interior of the fluid. Its evolution with time and its dependence of the stratification and the rotation of the disk are obtained. Furthermore, small scale mixed layers were observed in below the surface mixed layer. They have a complicated dynamics with multiple regimes, which were attempted to characterize. The description of the experiment and the behavior of the observed mixed layers is presented in section (2). Further, a phenomenological model is developed that explains the mechanism under which the secondary mixed layers are formed. It parametrized the action of the two mechanisms: shear generation of the turbulence due to Kelvin-Helmholtz type of instabilities, and the mean flow generated turbulence though the vortex scraping mechanism. The resulting gradient-type mixing model predicts the formation of the instabilities in a particular parameter regime that lead to mixed layers formation. The instabilities are related to those described by Phillips [7] and Posmentier [8]. The model description, its analysis and numerical simulations are presented in section (3). The summary of the work is in section 4 . 


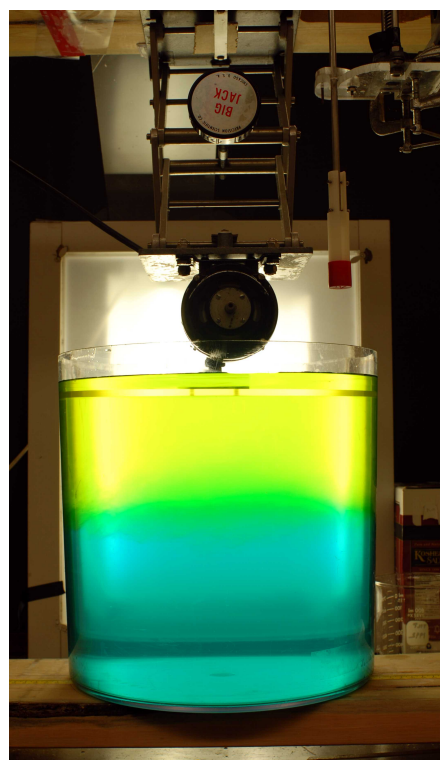

Figure 1: Experimental set-up: cylindrical tank filled with stratified fluid, rotating disk at the surface of the fluid, conductivity probe

\section{Experiments}

A series of experiments were made where a stratified fluid was forced by a rotating horizontal disk at the surface with the objective to investigate the dynamics of the formed mixed layers under various cases of stratification and the disk rotating speeds. The experimental setting is similar to that used by Davies et al [3], who investigated the flow generated during the spin up process and further established secondary circulation.

\subsection{Experiment arrangement}

Experimental set-up is shown on Figure 1. A cylindric tank of $30 \mathrm{~cm}$ height and $30 \mathrm{~cm}$ diameter was filled with a salt stratified water. Only linear stratifications were considered, which were obtained with a standard double bucket technique [5]. The range of density variations within the fluid was from $2 \%$ to $20 \%$. A horizontal disk with the diameter of $24 \mathrm{~cm}$ is located at the surface of the fluid, with its axis of rotation aligned with the axis of the cylinder. The rotation speed of the disk is controlled by the motor (attached above the disk) that gives a range of $0.5-10 \mathrm{rad} / \mathrm{s}$. Measurements of vertical density profiles were obtained using a conductivity probe which was taking 100 measurements per $1 \mathrm{~cm}$ of fluid, moving vertically with a speed of $3 \mathrm{~mm} / \mathrm{s}$. Measurements were taken only when the probe was moving down to avoid fluxes of water along the probe that would contaminate the data. The probe Reynolds number is of the order of 100 ( this is an upper bound, which is reached at the edge of a disk); thus, measurements are not considerably affected by the presence of the probe on a scales considered in the current study - $O(\mathrm{~cm})$. The profiled depth of the fluid was $20 \mathrm{~cm}$, whereas the height of it is $27 \mathrm{~cm}$. Density was calculated based on conductivity with a calibration using a 3rd order polynomial as a mapping function. To 

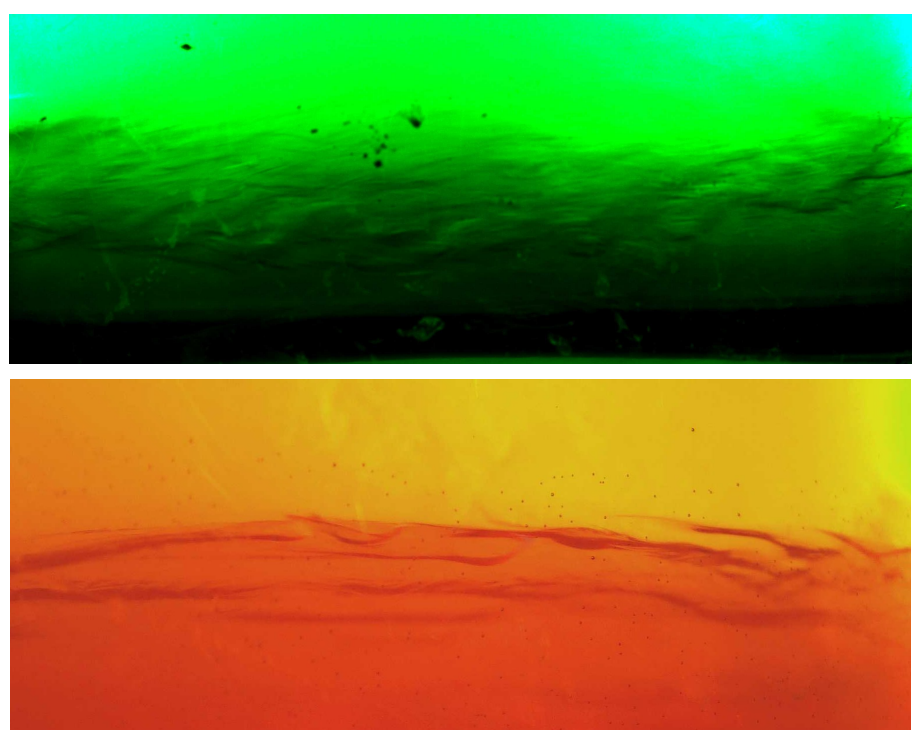

Figure 2: Images of the typical interfaces between the well mixed layer and a linearly stratified layer below it.

reduce temperature effects on conductivity the fluid allowed to reach the room temperature $\left(20^{\circ} \mathrm{C}\right)$ before the start of the experiment. Thus, the control parameters that define the outcome of the experiment were the stratification of the fluid (expressed as a Brunt-Väisälä frequency $N$ ) and the rotating speed of the disk $\Omega$.

There are multiple non-dimensional parameters involved in current experiments. The most relevant one is the Richardson number, which will be defined here as $R i=\frac{N^{2}}{\Omega^{2}}$. This definition should not be confused with the local Richardson number $\frac{N^{2}}{U_{z}^{2}}$ which varies with time and the location in the fluid. The explored range of $R i \in(0.15-2.3)$. Another parameter would be the disk edge Reynolds number: $R e=\frac{U L}{\nu} \sim 10^{5}$, where $U$ is the azimuthal velocity at the edge of the disk, $L$ is the radius of the disk, and $\mu$ is the kinematic viscosity of water. A flow with such high values of Reynolds number could be considered turbulent and independent from the exact value of $R e$. The Schmidt number for the salt stratified water is $\sigma=\frac{\nu}{\kappa} \sim 700$, where $\kappa$ is the salt diffusivity. The tank aspect ratio as well as the ration of the disk radius to the height of the water column is $\sim 1$. All geometrical parameters as well as the Schmidt number were fixed throughout all experiments. Thus, the only non-dimensional parameter that describes the different outcomes of the experiments is the Richardson number.

\subsection{Observations of density profiles}

After the tank is filled with the stratified fluid the disk is set in motion. As was mentioned previously, the disk Reynolds number is big enough that the flow adjacent to it becomes turbulent. Such flows are very effective in diffusing buoyancy and momentum. Thus, a mixed layer forms below the disk, with almost homogeneous density and velocity distributions. The growth of such a layer however is suppressed by the stable stratification of the 


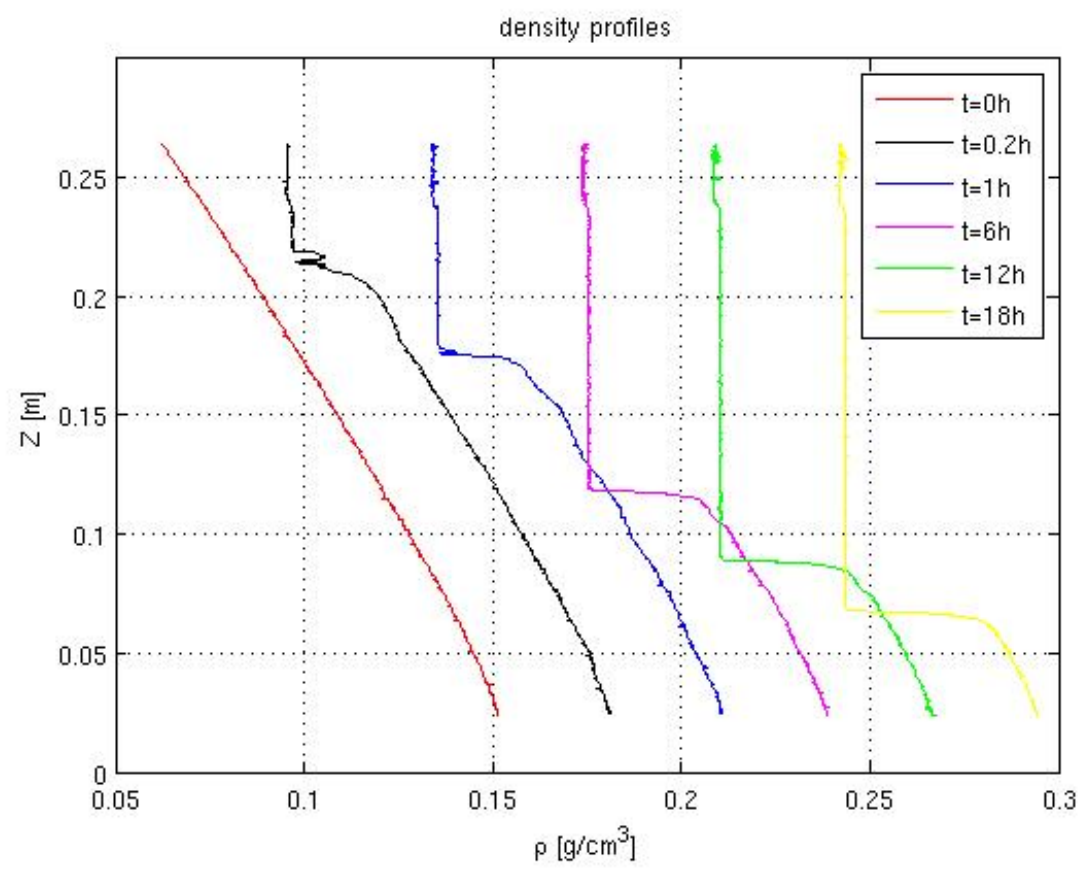

Figure 3: Density profiles plotted for different times after the start of the experiment. Profiles are shifted along the X-coordinate by $0.03 \mathrm{~g} / \mathrm{cm}^{3}$ from each other.

fluid. As the mixed layer deepens the gravitational potential energy of the fluid increases because mixing process effectively lifts the center of the mass of a water column by mixing dense waters upwards against the gravitational field. Furthermore, mixing in a stratified fluid leads to formation of a density jump at the base of the mixed layer. The density jump suppresses turbulence and effectively separates the turbulent flow in the upper mixed layer from almost quiescent layer underneath it. Within the mixed layer there is a mean azimuthal flow due to the coherent rotation of the disk. This flow is also suppresses when it reaches strong stratification at the base of the layer creating a strong shear and providing other mechanisms of the generation of turbulence which would be discussed further.

Figure 2 shows images of two interfaces. The top one appears at early stages in the development of the mixed layer and is characterized by weak density jump across it, energetic eddies and large thickness (few $\mathrm{cm}$ ). The one shown at the bottom, forms during later stages and has low energy eddies of smaller sizes (less then a $\mathrm{cm}$ ) and much stronger density jump across it. The signature of these interfaces is clearly present in the vertical density profiles, the time evolution of which is shown on Figure 3. Initially, linearly stratified fluid get mixed from the top and forms a mixed layer as well as the density jump at the base of it, both of which are growing in magnitude. The interface separating the mixed layer from almost undisturbed bottom layer changes its characteristics as well. The profile taken at $t=0.2 h$ (hours) shows the presence of unstable stratification, which resulted from the overturning of energetic eddies at the base of the mixed layer. The density interface for this profile corresponds to a type shown on Figure 2 (top). At the profile taken at $t=6 h$ it is hard to see the density structures resulted from overturning of eddies because turbulence is 


\begin{tabular}{llllll}
\hline Exp\# & $\mathrm{N}$ & $\Omega$ & $R i$ & $\alpha$ & $A$ \\
\hline 9 & 0.5 & 1.13 & 0.2 & 0.2 & 0.42 \\
13 & 0.5 & 1.34 & 0.14 & 0.18 & 0.5 \\
14 & 0.52 & 0.72 & 0.52 & 0.2 & 0.32 \\
15 & 1.37 & 1.7 & 0.65 & 0.23 & 0.27 \\
17 & 1.3 & 0.95 & 1.87 & 0.246 & 0.19 \\
18 & 1.1 & 1.5 & 0.54 & 0.22 & 0.29 \\
19 & 1.12 & 1.7 & 0.43 & 0.2 & 0.36 \\
20 & 1.18 & 1.13 & 1.09 & 0.25 & 0.21 \\
21 & 1.3 & 1.34 & 0.95 & 0.24 & 0.23 \\
22 & 0.98 & 0.95 & 1.05 & 0.24 & 0.24 \\
23 & 0.84 & 2.08 & 0.16 & 0.21 & 0.46 \\
24 & 0.78 & 0.72 & 1.18 & 0.24 & 0.23 \\
25 & 1.46 & 1.13 & 1.67 & 0.25 & 0.18 \\
26 & 1.7 & 1.7 & 1 & 0.225 & 0.22 \\
27 & 1.7 & 1.13 & 2.25 & 0.225 & 0.24 \\
28 & 1.72 & 3.14 & 0.3 & 0.18 & 0.41 \\
29 & 1.6 & 1.94 & 0.67 & 0.22 & 0.29 \\
30 & 1.42 & 1.13 & 1.57 & 0.23 & 0.22 \\
31 & 1.5 & 1.18 & 1.6 & 0.24 & 0.22 \\
32 & 0.94 & 1.5 & 0.4 & 0.23 & 0.29 \\
\hline
\end{tabular}

Table 1: The table shows the experiment settings: initial stratification of the fluid $(\mathrm{N})$, the disk angular velocity $\Omega$. The last two columns show the coefficients of the power law fit for evolution of the mixed layer depth $\hat{h}=A \hat{t}^{\alpha}$. The values are organized and sorted by the experiment number.

highly suppressed there and eddies are of smaller scale; this interface corresponds the one on Figure 2(bottom).

The presence of the density jump and a velocity shear across it points to a possibility of generation of the Kelvin-Helmholtz type of instabilities at the interface that leads to its further erosion. However, these type of instabilities would be present if the local Richardson number $\left(N^{2} / U_{z}{ }^{2}\right)$ is sufficiently small. Eventually the flow would reach conditions stable to $\mathrm{K}-\mathrm{H}$ instabilities as the velocity of the mixed layer would gradually slow down, whilst the density jump across the interface would increase. At this point, the erosion of the interface would continue due to the mean flow acting frictionally on turbulent eddies at the interface (vortex scraping). Thus, whether it's the mean flow or its shear, there is a mechanism which would generate mixing at the stable interface and lead to its further deepening.

\subsection{Development of the primary mixed layer}

As was described earlier, a mixed layer forms at the disk and expands into the fluid reaching the thickness of the order of the tank size. It will be referred to as the primary mixed layer. In certain cases, there are other layers that form below it (secondary mixed layers) which 

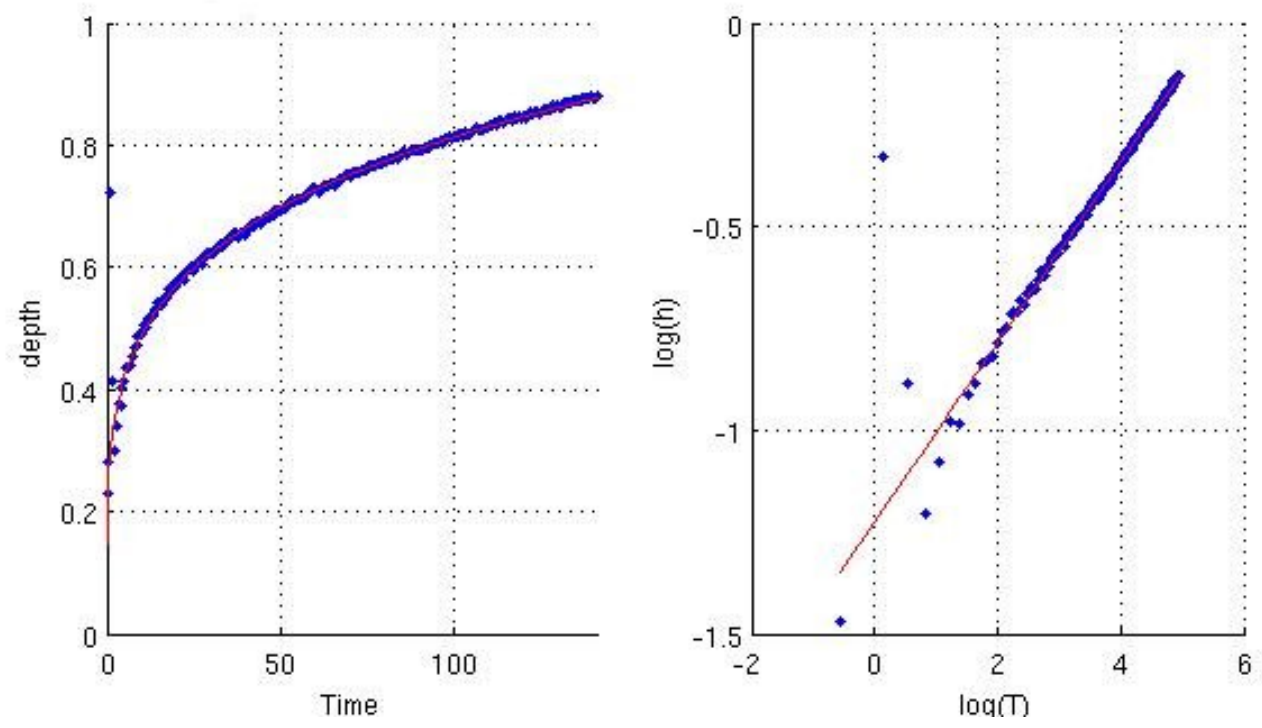

Figure 4: A non-dimensional depth of the primary mixed layer is plotted as a function of non-dimensional time (left) and on a log-log plot (right).

are of smaller scales and have much weaker interfaces. These will be discussed in the next section.

For many geophysical applications it is important to know the growth of the primary mixed layer with time as it controls the distribution of tracers as well as the dynamics of the flow . To present the observations, the mixed layer depth $h$ is non-dimensionalized by the depth of the fluid $\left(\hat{h}=h / H_{w}\right)$, and time $t$ by the period of rotation of the disk $(\hat{t}=\Omega t)$. Figure 4(left) shows the time evolution of the primary mixed layer for a typical experiment: the layer grows rapidly at first, then the growth slows down due to the increasing density jump at the base of the layer. Figure 4(right) shows the thickness of the layer as a function of time on a log-log plot with a corresponding linear fit $\left(R^{2}=0.89\right)$. This fit suggests a power law $\hat{h}=A \hat{t}^{\alpha}$, where $A$ and $\alpha$ presumably are functions of the Richardson number the control parameter of the experiment. Indeed, Figure 5 shows that there is a particular dependence of the fit coefficients on the Richardson number. The power $\alpha$ stays relatively constant at a value of 0.22 with some trend towards smaller values at lower Richardson numbers. The coefficient $A$ of the fit seems to scale with the Richardson number as $A \sim$ $R i^{-0.33}$ as shown in Figure 5(right). Thus, the experimental data shows that the nondimensional mixed layer depth scales as $\hat{h} \sim R i^{-0.33} \hat{t}^{0.22}$.

To understand the obtained dependence of the mixed layer depth evolution, consider the energy balance of the system. The source for the generation of the mean flow as well as the mixing of the stratified fluid is a power produced by frictional stress due to motion of the disk. It scales as $P \sim \tau U \sim U^{3}$, where $\tau \sim U^{2}$ is the frictional stress and $U$ is the characteristic velocity of the mixed layer near the disk. Further, a linearly stratified fluid that was mixed to a depth $h$ would have its gravitational potential energy increased by $\triangle G P E=\int_{-h}^{0}\left(\rho-\rho_{0}\right) g z d z \sim N^{2} h^{3}$, where $\rho_{0}$ is the initial linear density profile and $\rho$ is the one obtained by adiabatic mixing the initial profile within the layer of thickness $h$. At last, 

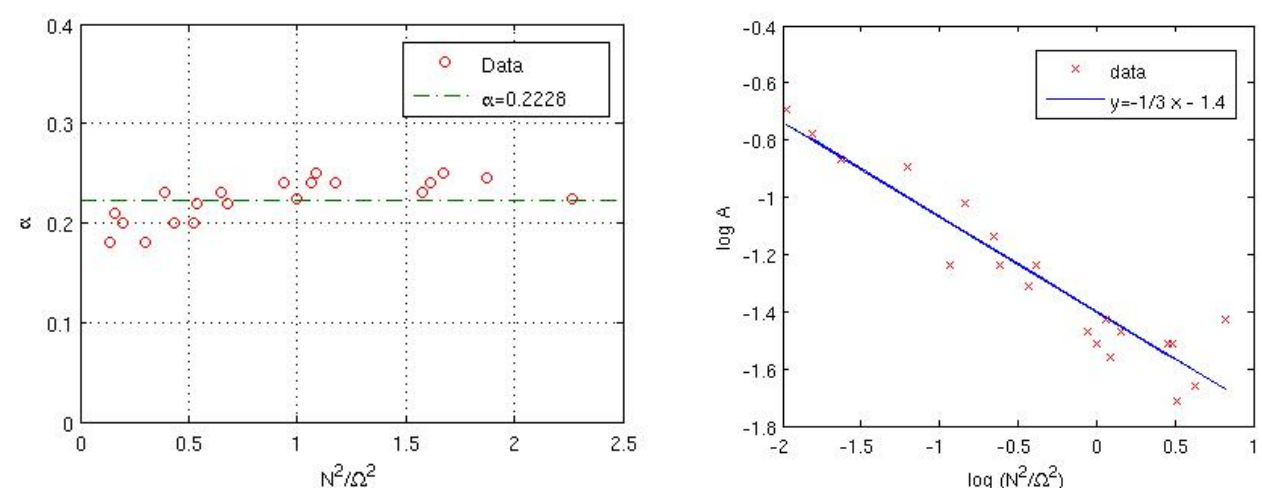

Figure 5: Coefficients of the power fit for the depth of the mixed layer are plotted as functions of $R i$. On the left is the coefficient $\alpha=\alpha(R i)$; coefficient $A=A(R i)$ plotted on a log-log scale (right).

the kinetic energy of the mixed layer would scale as $K E \sim U^{2} h$ and the dissipation $D$ is assumed to be proportional to the energy production $(D=\gamma P)$. Thus, the energy balance is described by the following equation:

$$
\frac{d}{d t}(K E+P E)=P-D \Rightarrow \frac{d}{d t}\left(U^{2} h+N^{2} h^{3}\right) \sim U^{3}
$$

At this point, an assumption is made that the flow reaches a state where the kinetic energy of the mixed layer approaches a constant value and the energy input redistributes between potential energy and dissipation. If $K E=$ cont then $U \sim h^{-1 / 2}$ and the energy balance model becomes $h^{2} h_{t} \sim h^{-3 / 2} \Rightarrow h \sim t^{2 / 9}$. The obtained power law for the growth of the mixed layer is consistent with observations since they indicate a power of 0.22 (Figure 5). The dependence of the fitted coefficient $A \sim R i^{-1 / 3}$ suggests that the assumed constant value of the kinetic energy is a function of $R i$ as well. This relation, could not be determined based on the simple energy balance model. The assumptions made in constructing an energy balance could be verified with measurements of the velocity of the fluid using, for example, PIV techniques. In current experiment these measurements were not produced, but for further progress there are of vital importance.

\subsection{Dynamics of a secondary mixed layer}

Layers of weak stratification that form below the primary layer will be referred to as the secondary mixed layers. Figure 6 shows an example of a developed secondary mixed layer - it has a distinct signature in density profile as well as in Brunt-Väisälä frequency, where bases of layers (interfaces) have strong stratification and the layer itself is weekly stratified. Thus, on Figure 6(right) the strong peak ${ }^{1}$ corresponds to the base of a primary mixed layer, and the peak with smaller magnitude corresponds to the base of a secondary mixed layer.

\footnotetext{
${ }^{1}$ Buoyancy frequency was obtained from smoothed density profile to remove noise - instrumental as well as due to small scale overturning. This leads to broader peaks with smaller magnitudes; nonetheless, a clear signature of mixed layers is preserved.
} 

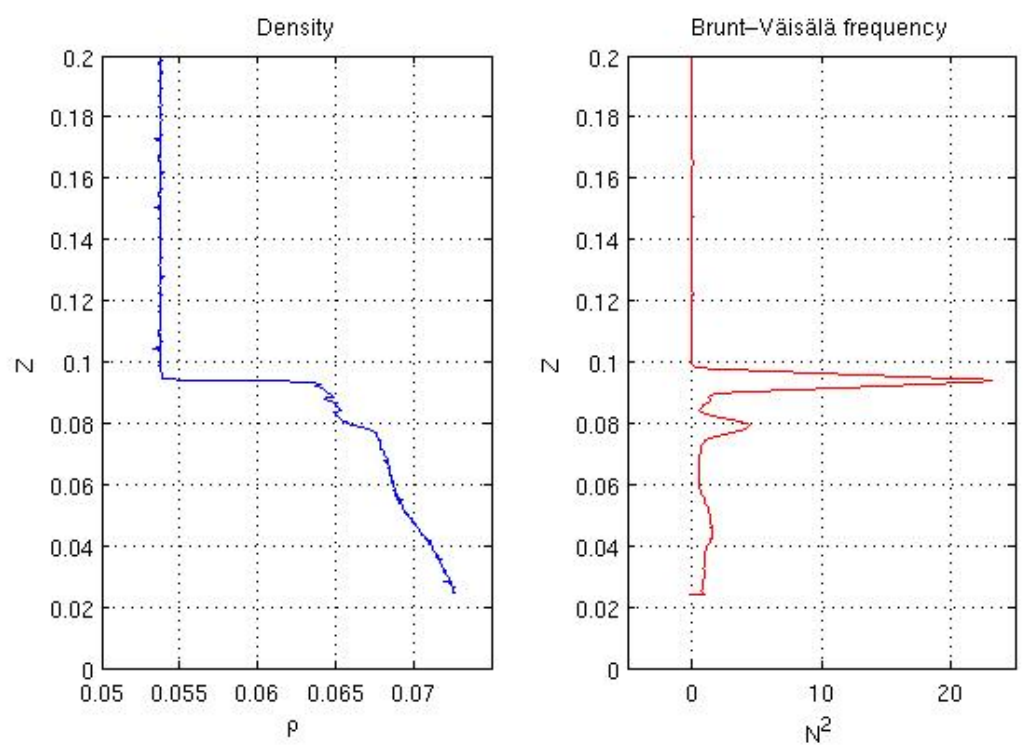

Figure 6: A typical density profile containing a primary mixed layer as well as a secondary one (left).Brunt-Väisälä frequency showing peaks at the bases of mixed layers.

Note that, secondary mixed layer is only partially mixed as apposed to the primary one that is completely destratified. Thus, secondary layers are characterized by the magnitude of a peak in buoyancy frequency at the base of the layer, and by a layer thickness, which is the distance between the two peaks.

As opposed to primary mixed layer, that monotonically grows with time, the secondary layer has a very rich dynamics. Figure 7 shows how the buoyancy frequency evolves with time. Here, there is a clear indication of both mixed layers - the primary one continuously grows and the secondary one forms and decays multiple times throughout the experiment. Characteristic thickness of the secondary layer is of few centimeters. The evolution process could be decomposed in several stages. In the beginning, there is a strong turbulent flow that drives the growth of the primary layer and does not allow the formation and persistence of the fine scale secondary layer. Then follows a period of time when the secondary layer forms and decays with a characteristic time of few tens of minutes. The layer forms spontaneously having a finite thickness which then decreases until this layer merges with the primary one (collision instability) and the process repeats. The next stage is described by a change in the way the layer forms. They appear at the base of a primary mixed layer having small thickness and start to grow building up the density jump at the interface. However, the magnitude of a jump vanishes with time (erosion instability), the layer disappears and the next one forms. Finally, comes the stage when the layers either no longer form or stay in a locked position relative to the primary interface.

The evolution of the secondary mixed layer is highly dependent on the Richardson number for the experiment and in a current time. In some cases, certain stages of the evolution could be skipped which makes it hard to classify all the regimes. Nonetheless, an attempt to plot a regime diagram was made. Figure 8 shows the stages of the evolution of the mixed layer for different experiments as a function of non dimensional time and 

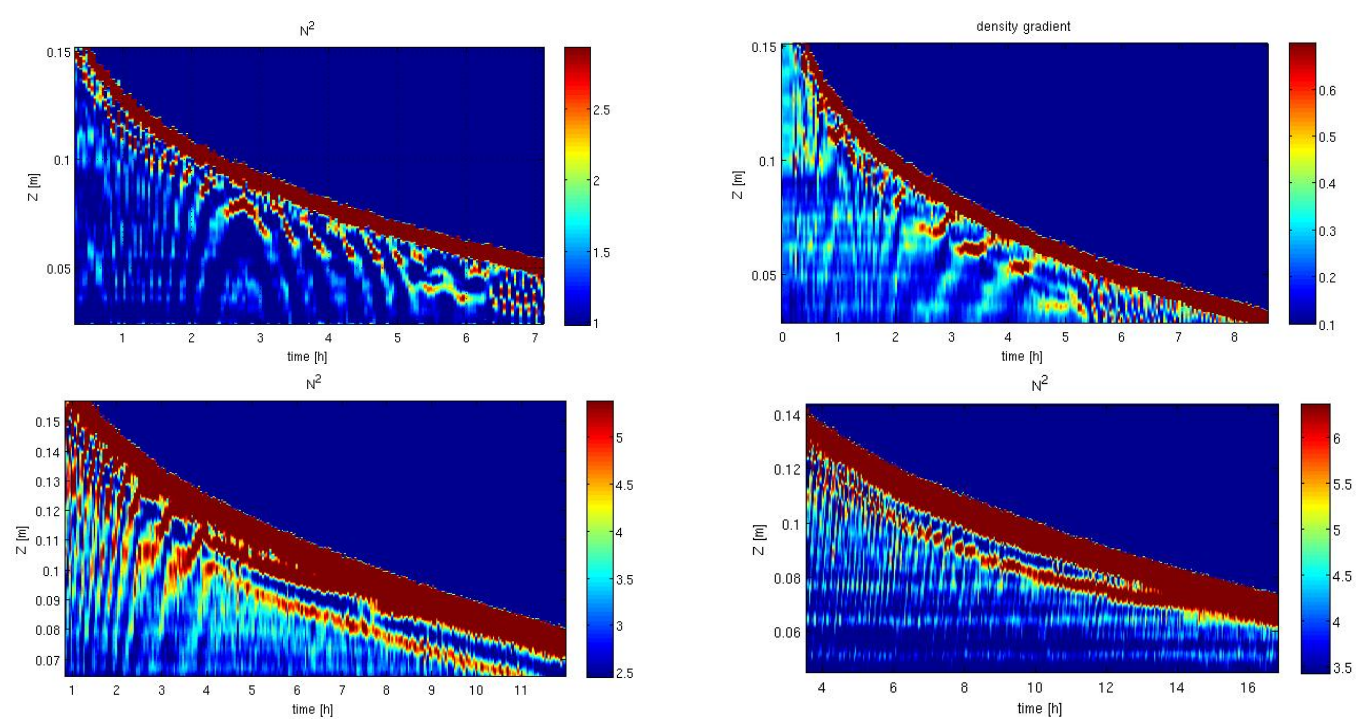

Figure 7: Examples of the time evolution of the square of the Brunt-Väisälä frequency for different values of the Richardson number $(R i=0.54,1,2.25,1.67)$.

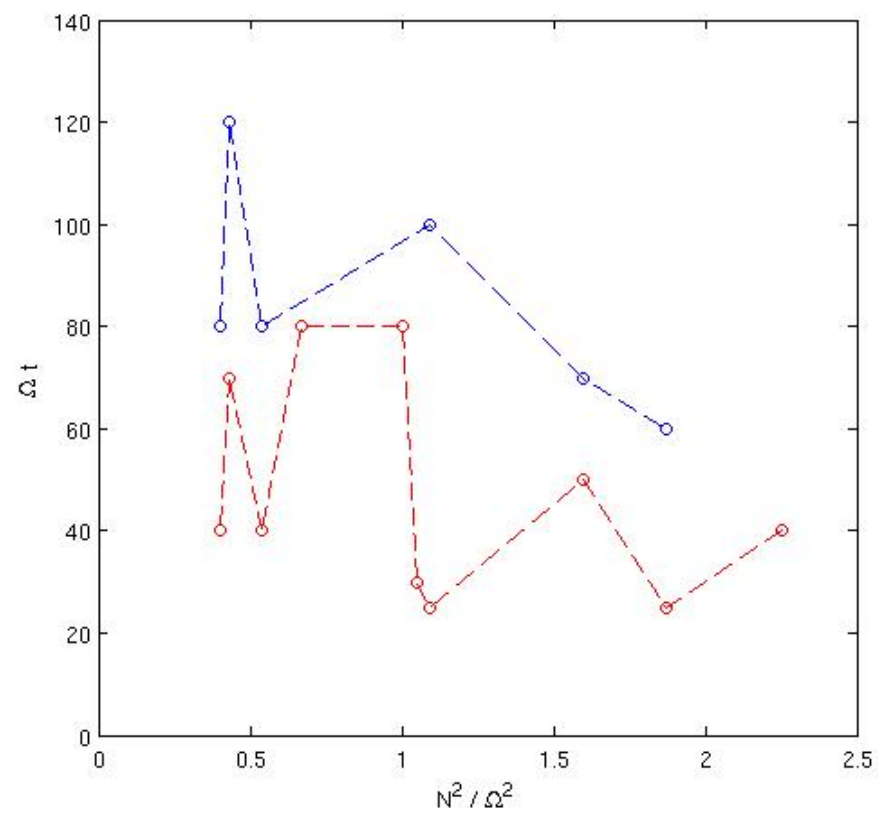

Figure 8: Regime diagram showing different stages of evolution of the secondary mixed layer 
Richardson number. Below the red curve steps generate below the mixed layer and decay by merging, whereas in between the red and blue curves mixed layers generate from the primary mixed layer and decay by erosion. Above the blue line the staircases are either in locked state or do not exist. The diagram does not show any specific dependence, however this does not imply that there is no dependence whatsoever. There is an obvious lack of data: a total of 20 experiments were made and for less then half of them the secondary mixed layers could be clearly classified. Furthermore, since there are no theoretical predictions it is not clear how to present such a diagram: the most obvious choice of presenting in terms of Richardson number and time might not be the clear one to look at.

The dynamics of the secondary mixed layers is extremely complicated. Understanding it to a level of making predictions requires a precise theoretical model bucked up by extensive observations of not only density but also velocity fields, turbulent buoyancy and momentum fluxes. However, due to time constrains these elaborate measurements could not be performed. Thus, the theoretical part of this research would be focused on explaining the formation of secondary layers in an idealized phenomenological framework.

\section{Phenomenological model}

Secondary mixed layers arise in a stratified fluid that is subjected to the action of a turbulent flow with a mean shear. The turbulent nature of the flow would be the key to understanding the phenomenon. The proposed mechanism is as follows. The sheared mean flow driven by the rotating disk is a source of turbulent motion which inhomogeneously distributes within a stratified fluid depending on its stratification. The buoyancy itself is affected by turbulent fluxes. Thus, the formation on the layers could be related to the instability of the turbulent flux-buoyancy relation as was first proposed by Phillips [7] and Posmentier [8]. Suppose, the evolution of buoyancy $b$ is controlled by the divergence of the downward turbulent buoyancy flux $F$ :

$$
\frac{\partial b}{\partial t}=\frac{\partial F}{\partial z}
$$

and this flux is a function of the buoyancy gradient $F=F\left(b_{z}\right)$. The equation has obvious steady state solutions with constant flux, however such solutions would be unstable if the flux would be a decreasing function of buoyancy gradient e.i. $\frac{\partial F}{\partial b_{z}}<0$. Figure 9 shows the mechanisms of the instability. A linear steady state buoyancy profile (dashed line) has a value of buoyancy gradient that corresponds to the negative derivative of the buoyancy flux. After introducing a small perturbation (black line) there will appear anomalous buoyancy fluxes (blue arrows). In regions where the perturbations have higher buoyancy gradient the anomalous flux is negative and in it is positive in regions of lower gradients. As could be seen from the evolution equation the current distribution of the buoyancy fluxes would lead to a growth of the initial perturbation, meaning that the base state is unstable. This instability would lead to buoyancy profiles consisting of a series of mixed layers separated by strong interfaces (staircases). In the case of $\frac{\partial F}{\partial b_{z}}>0$ the created fluxes would lead to the decay of the buoyancy anomaly - this state is stable.

This model considers the buoyancy equation only and assumes a particular shape of the flux-gradient curve. However, the exact form of this curve would depend on particular mixing mechanisms that create the turbulent buoyancy fluxes. In the following section 


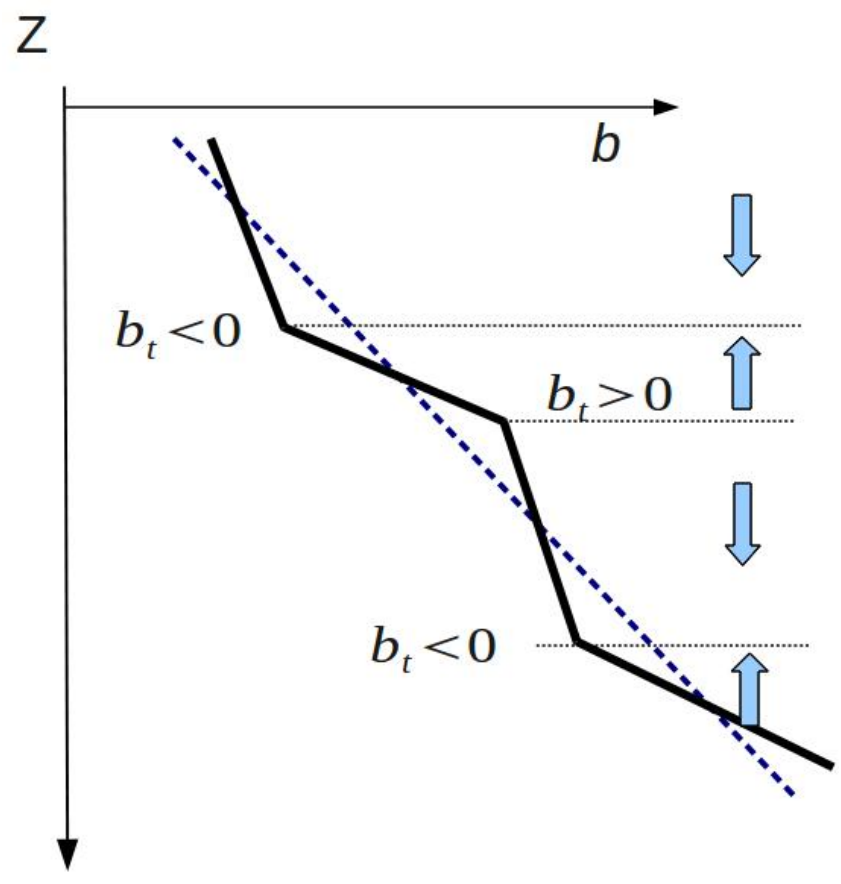

Figure 9: Schematic representation of the instability mechanism that leads to layer formation. Dashed line represents the initial buoyancy profile, black line is the slightly perturbed profile, anomalous buoyancy fluxes denoted by blue arrows.

a gradient-type mixing model would be devised that would describe the development of buoyancy profiles under the action of the mean flow generated turbulence. The formation of the layers in the model would be related to the mechanism described above by calculating the exact form of the flux-gradient relation. The model would consist of evolution equations written for buoyancy as well as for turbulent kinetic energy that is drained from the mean flow. It is aimed to describe the secondary mixed layers only, so it is assumed that the primary layer has been formulated and the mean velocity profile is established.

\subsection{Model formulation}

The approach taken in constructing a mixing model is similar to that implemented by Balmforth [1] and Barenblatt [2]. The model parametrizes turbulent vertical transports as gradient transports using eddy diffusivity and viscosity. Thus, the conservation laws for horizontally averaged buoyancy $b$ and turbulent kinetic energy $e$ are as follows

$$
\begin{aligned}
& \frac{\partial b}{\partial t}=\frac{\partial}{\partial z}\left(\kappa \frac{\partial b}{\partial z}\right) \\
& \frac{\partial e}{\partial t}=\frac{\partial}{\partial z}\left(\kappa \frac{\partial e}{\partial z}\right)+S
\end{aligned}
$$

where $S$ is a source term for the energy and $\kappa$ is the turbulent diffusivity. The source term represents production and destruction of the turbulent energy and in this formulation 
would consist of four terms:

$$
S=-\kappa b_{z}-\alpha \frac{e^{2}}{\kappa}+\frac{1}{1+R i} \kappa U_{z}^{2}+\frac{R i}{1+R i} U^{2} e^{1 / 2} l^{-1}
$$

As it was mentioned previously, the mixing of buoyancy leads to an increase in a gravitational potential energy of the system. Thus, the role of the first term $\left(-\kappa b_{z}\right)$ is to convert the eddy kinetic energy into potential energy preventing the artificial accumulation of the total energy $E=\int_{0}^{H}(e-b z) d z$. The second term $-\alpha \frac{e^{2}}{\kappa}$ is the dissipation, which is on dimensional grounds proportional to the energy $e$ and to the inverse of the eddy turnover time $\tau \sim \kappa / e$; $\alpha$ is a small parameter which controls the intensity of the dissipation and effectively defines the number of turnover periods over which an eddy would dissipate. The last two terms are the production terms due to the action of a mean flow. From visual observations of the experiments there seem to be two mechanism in action. At low local Richardson numbers $\left(R i=N^{2} / U_{z}^{2}\right)$, where the destabilizing effect of the shear is dominating the stabilizing effect of the stratification, the turbulence is generated due to Kelvin-Helmholtz type instabilities. These instabilities, however, are suppressed at high $R i$. Hence the representation of the energy production is of the form $\frac{1}{1+R i} \kappa U_{z}{ }^{2}$. At high $R i$ the turbulence is generated by the mean flow acting on turbulent eddies (vortex scraping) by inducing a frictional stress. The amount of power introduced by such a mechanism is proportional to $U^{2} e^{1 / 2} l^{-1}$, where $U^{2}$ is scaling for the stress exerted by the mean flow, $e^{1 / 2}$ is a characteristic velocity of an eddy and $l$ is its length scale. Factors $\frac{R i}{1+R i}$ and $\frac{1}{1+R i}$ are chosen to ensure that the two mechanisms would act in their corresponding regime of Richardson numbers.

Eddy diffusivity, as well as viscosity, is ${ }^{2}$ proportional to $l e^{1 / 2}$. To close the system the characteristic eddy size $l$ needs to be defined. Since the model aims to describe mixing in the stratified fluid there are several length scales involved. In the limit of strong stratification the size of eddies would be proportional to the Ozmidov's length scale $\left(e / b_{z}\right)^{1 / 2}$. This restriction comes from the fact that eddies overturn density layers leading to increase in potential energy. Thus, for a given energy of an eddy and the ambient stratification there would be a restriction on its size. In the limit of weak stratification, the characteristic length scale for an eddy $l=d$ should be related to the geometrical parameters e.i. the size of the tank $\mathrm{k}^{3}$. Thus, to interpolate the two length scales in the limits of strong and weak stratifications expression for the eddy length scale $l$ is chosen to be

$$
\frac{1}{l^{2}}=\frac{1}{d^{2}}+\gamma \frac{1}{e / b_{z}}
$$

where $\gamma$ is a parameter that determines the length scale in the limit of strong stratification. It should be pointed out that the model is written in terms of locally defined dynamical variables, meaning that all the scales are controlled by the local dynamics and do not depend on global profiles of the variables. However, this doesn't always take place in reality. For example, the model used for the eddy length scale states that in the case of locally weak stratification the length should be equal to $d$ - some geometrical restriction. However, vertical profiles of stratification containing mixed layers bounded by strong stratification

\footnotetext{
${ }^{2}$ For simplicity eddy viscosity and diffusivity are taken to be the same, however this assumption could be relaxed

${ }^{3} \mathrm{~A}$ restriction of the length scale in weakly stratified case avoids singularities in the model.
} 
would impose an equivalent 'geometrical' constrain on an eddy size in the location within the mixed layer - the eddy size could not be greater than the thickness of a mixed layer with strong interfaces. Thus, the parameter $d$ should be considered as effective one that takes into account geometrical constrains as well as the global buoyancy profile. In the current model, for simplicity it is assumed to be constant.

A resulting model is effectively a set of coupled nonlinear diffusion equations, which require boundary and initial conditions to be completed. As boundary conditions, the fluxes of energy and buoyancy are set to be zero at the top and the bottom boundaries of the domain e.i. $e_{z}=0, b_{z}=0$ at $z=0, H$. The initial conditions would be discussed in a section 3.3. Thus, the complete equation set of the mixing model is the following:

$$
\begin{aligned}
b_{t} & =\left(\kappa b_{z}\right)_{z} \\
e_{t} & =\left(\kappa e_{z}\right)_{z}-\kappa b_{z}-\alpha \frac{e^{2}}{\kappa}+\frac{1}{1+R i} \kappa U_{z}^{2}+\frac{R i}{1+R i} U^{2} e^{1 / 2} l^{-1} \\
\kappa & =l e^{1 / 2} \\
\frac{1}{l^{2}} & =\frac{1}{d^{2}}+\gamma \frac{1}{e / b_{z}} \\
R i & =\frac{b_{z}}{U_{z}^{2}}
\end{aligned}
$$

where $\alpha, \gamma, d$ are fixed parameters; characteristic velocity $U$ and velocity shear $U_{z}$ represent the forcing for this system and assumed to be constant. In principle, equations 6 to 9 could be combined to give an evolution equation for the turbulent diffusivity $\kappa$ - the variable that determines the evolution of the buoyancy profile.

Since the purpose of the model is to describe the formation of mixed layers it is convenient to use the buoyancy gradient $\left(g=b_{z}\right)$ instead of buoyancy as a dynamical variable. The mixed layers would have a distinct signature in buoyancy gradient profile: low values within the mixed layer and sharp peaks at the interfaces. Further, it is useful to use non-dimensional equation set with the following definition of dimensionless quantities:

$$
\hat{t}=\frac{U t}{\gamma d}, \quad \hat{z}=\frac{z}{\gamma^{1 / 2} d}, \quad \hat{e}=\frac{e}{U^{2}} \quad \hat{g}=g \frac{\gamma d^{2}}{U^{2}}, \quad \hat{l}=\frac{l}{d}, \quad Q=\left(\frac{U}{U_{z} d}\right)^{2}
$$

In dimensionless form the equations are

$$
\begin{aligned}
g_{t} & =(\kappa g)_{z z} \\
e_{t} & =\left(\kappa e_{z}\right)_{z}-\kappa g-\alpha \gamma \frac{e^{2}}{\kappa}+\frac{\gamma \kappa+Q g^{3 / 2}}{1+g / \gamma} \\
\kappa & =\frac{e}{(e+g)^{1 / 2}} \\
\text { b.c. } & : \quad e_{z}=0, g=0 \text { at } z=0, H
\end{aligned}
$$

where a parameter $Q$ describes the relative importance of the mean flow and the mean shear in the energy production term; the local Richardson number is proportional to the buoyancy gradient only $(R i=g / \gamma)$. 


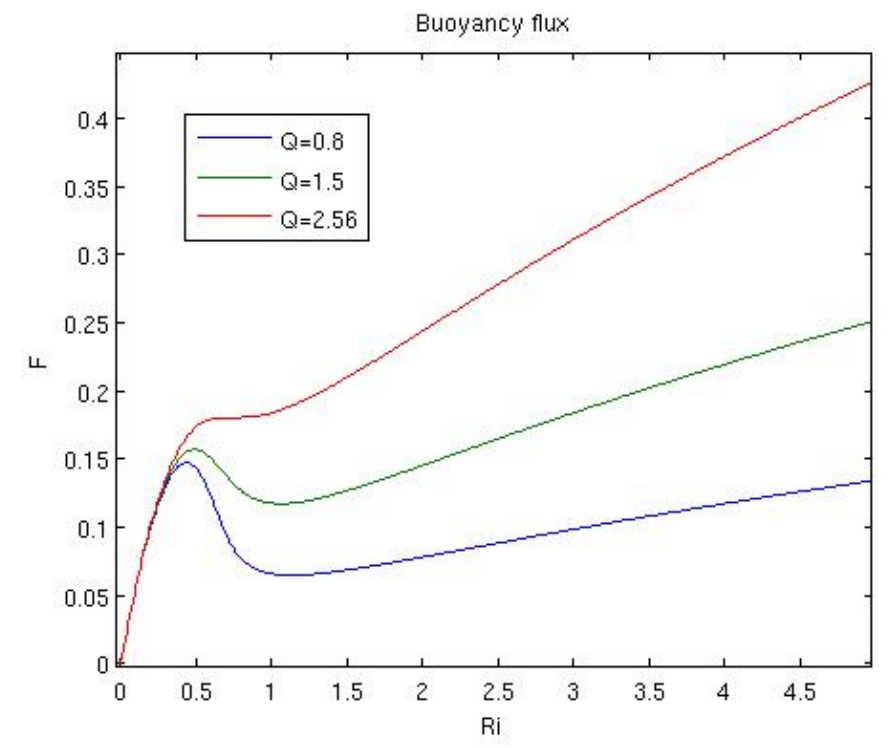

Figure 10: Steady state buoyancy flux plotted as a function of the buoyancy gradient for different values of the forcing parameter $Q$.

\subsection{Steady states and their stability}

Consider a steady state of the model, which has a constant energy and buoyancy gradient $\left(e_{0}, g_{0}\right)=$ const. It violates the boundary conditions, nonetheless away from the influence of the boundaries it is useful to consider its stability. Such steady states should have no production of turbulent kinetic energy $(S=0)$ in order for dynamic variables not to change with time:

$$
\begin{array}{r}
-\kappa_{0} g_{0}-\alpha \gamma \frac{e_{0}^{2}}{\kappa_{0}}+\frac{\gamma \kappa_{0}+Q g_{0}^{3 / 2}}{1+g_{0} / \gamma}=0 \\
\kappa_{0}=\frac{e_{0}}{\left(e_{0}+g_{0}\right)^{1 / 2}}
\end{array}
$$

This sets a constrain, which effectively defines energy and buoyancy flux as a function of buoyancy gradient (or Richardson number) for the considered steady state solutions:

$$
\begin{aligned}
e_{0} & =E\left(g_{0}\right) \\
\kappa_{0} g_{0} & =F\left(g_{0}\right)
\end{aligned}
$$

As was discussed earlier, the instabilities would develop if the initial profile has the Richardson number which is within the range of the negative slope of the buoyancy flux. Figure 10 shows the steady state buoyancy flux as a function of Richardson number $(R i=$ $g / \gamma)$. The numerical values of parameters are chosen as $\alpha=0.1, \gamma=0.2$. The representative mean flow parameter $Q=0.8,1.5,2.56$. Curves with values of $Q<2.56$ show a nonmonotonic behavior, having a region where the derivative of the flux is negative. At low 
values of $\mathrm{Ri}$, the instabilities are not possible due to extremely high turbulent energy created by shear instabilities that are not suppressed by the stratification. At high Richardson numbers the buoyancy flux is controlled by the turbulence produced by the mean flow which is proportional to $g^{1 / 2}$, hence the increase of the buoyancy flux with the increase in stratification. However, the shear production term is $\sim e / g^{3 / 2}$, which means that there is strong penalty in energy production for an increase in stratification - hence the turbulent flux due to shear production should decrease with the stratification at high Richardson numbers. Thus, the effect of the mean shear tends to destabilize the system and create mixed layers, whereas the mean flow turbulence has a stabilizing effect. Hence, in the intermediate range of Richardson the slope has a negative slope due to shear production term, however this region is of finite size due to the action of the mean flow turbulence at higher values of $\mathrm{Ri}$. This means that the instability could not grow unbounded in time: at some point the effect of the mean flow turbulence would suppress the growth. Thus, the existence and development of instabilities depend crucially on the relative importance of the mean flow to shear production terms which is determined by the value of the parameter $Q$. If the forcing $Q$ is greater than 2.56 the flux-gradient curve monotonically increases with the value of Richardson number - stabilizing effect of the mean flow is dominant. For such forcing all the buoyancy profiles would be stable.

The described instabilities could now be identified from the formulated model equations and their growth rates could be quantified. Linearizing the equations around the base state $\left(e_{0}, g_{0}\right)$ and assuming exponentially growing solutions for perturbations $(\hat{e}, \hat{g}) \sim \exp (\lambda t-$ $i k z)$ leads to a linear stability problem:

$$
\lambda\left(\begin{array}{c}
\hat{g} \\
\hat{e}
\end{array}\right)=\left(\begin{array}{cc}
-k^{2} f_{g} & -k^{2} f_{e} \\
S_{g} & -k^{2} \kappa+S_{e}
\end{array}\right)\left(\begin{array}{l}
\hat{g} \\
\hat{e}
\end{array}\right)
$$

where $f=\kappa g$ is the buoyancy flux, $S$ is the source term in the energy equation and all the partial derivatives are calculated at the point $\left(e_{0}, g_{0}\right)$. Thus, for a given value of parameter $Q$ and initial value of the Richardson number a growth rate $\lambda$ could be calculated as a function of the wavenumber $k$. Figure 11 shows growth rates calculated for $Q=1.5$ and for values of base state $R i=0.3,0.7,1.3$. The growth rate is positive for $R i=0.7$ which is located at the negatively sloped part of the flux curve (Figure 10). The growth rate is negative for the values of Richardson numbers of 0.3 and 1.3 that belong to the monotonically increasing part of the slope - these are stable solutions. An important property of the written model is that in the case of the unstable steady states the growth rate of the perturbations has has a high wavenumber cutoff ${ }^{4}$ which means that small scale perturbations do not grow with time - instead, they decay. This property allows to perform direct numerical simulations of the development of the instabilities and their evolution leading to the formation of the mixed layers.

\subsection{Numerical simulations}

Numerical simulations of the mixing model were performed using first order in time and second order in space numerical scheme with an adaptive time stepping. Computational domain is $z=[0,1200]$ having 600 uniformly distributed grid points. Initial conditions for

\footnotetext{
${ }^{4}$ The high wavenumber cutoff is due to the diffusion of the turbulent kinetic energy
} 


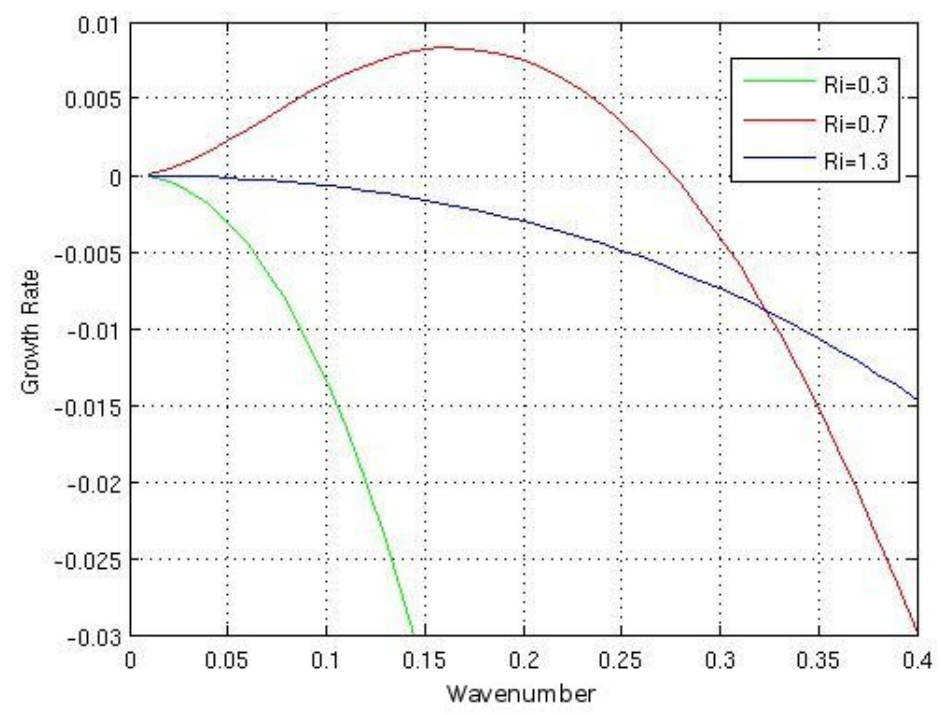

Figure 11: Growth rate plotted as a function of the wavenumber for different values of the base state Richardson numbers and for a fixed parameter $Q=1.5$ (as predicted by the linear stability analysis).

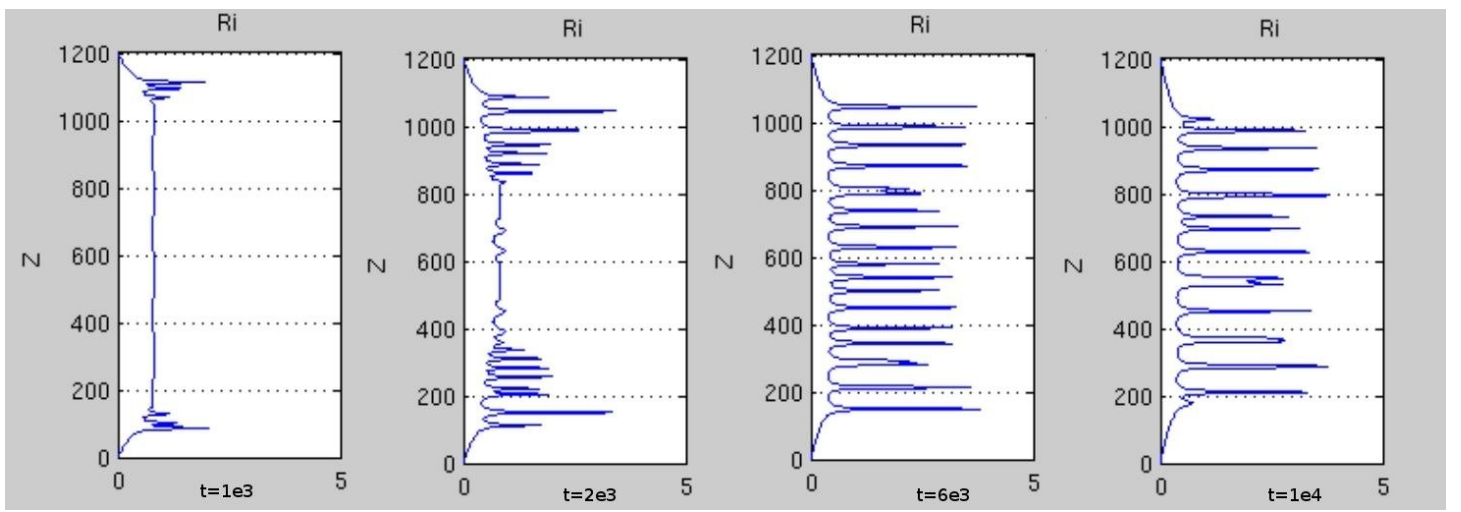

Figure 12: Numerically simulated evolution of the instabilities manifested in the vertical profiles of the Richardson number. 


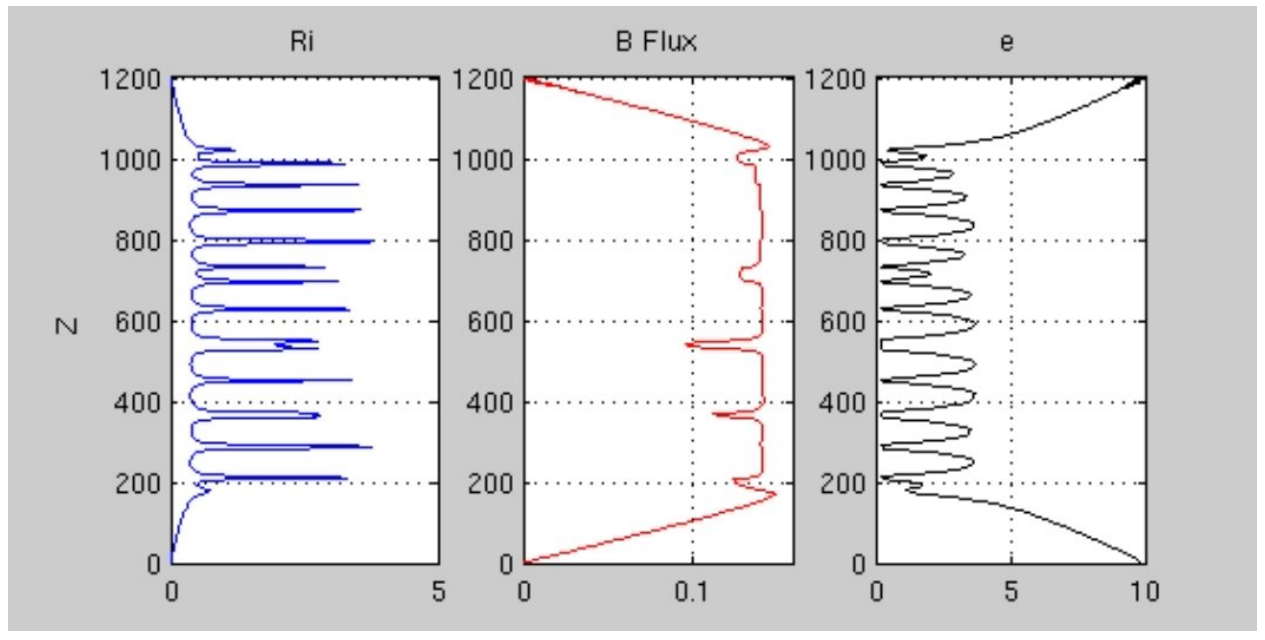

Figure 13: A snapshot of model variables at $t=1 e 4$ : Richardson number (left), buoyancy flux (middle) and turbulent kinetic energy (right).

the numerical simulation are chosen to be $R i=0.8$ for the buoyancy profile ${ }^{5}, e=0.01$ for the turbulent kinetic energy and the forcing parameter $Q=1$. Based on a linear stability analysis such a configuration should be unstable. Indeed, the numerical simulations confirm it. Figure 12 shows snapshots of the modeled buoyancy gradient at different time - it is clearly seen that the instabilities are developing in the form of sharp peaks in buoyancy gradient profiles. First, the instabilities develop close to boundaries because there are larger perturbation from the steady state their. Then, they appear in the interior and eventually fill the whole domain except for small regions adjacent to boundaries which are affected by zero flux boundary conditions.

Figure 13 shows a snapshot of the buoyancy gradient $g$, turbulent kinetic energy $e$ and a corresponding buoyancy flux $f$. The buoyancy flux seems to be relatively constant in the interior region, however having isolated regions of low flux. The energy distribution is higher in the regions of weak stratification and has minimums at within highly stratified interfaces. The instabilities develop having a wavelength corresponding to the one that has a maximum growth rate predicted by a linear stability analysis. However, it has been observed that after the initial development stage the structure tends to coarsen with time by merging or decaying mechanisms. The individual peaks are quasi-steady solutions of the equations and evolve by weakly interacting with each other. The result of the interaction could be considered as a secondary instability that leads to the coarsening through erosion or collision of the adjacent interfaces [9]. The time scales for the two instabilities would depend on the base.

Figure 14 shows the time evolution of the buoyancy gradient and the buoyancy flux throughout the whole simulation. The initial development stage lasts till the time $t \approx 4000$ and is characterized by the formation of the peaks in buoyancy gradient. The overall structure, however, tends to coarsen. The peaks either merge with each other creating a

\footnotetext{
${ }^{5}$ To satisfy initial conditions at the boundaries the distribution of $\mathrm{Ri}$ was chosen such that it is constant within the interior of the domain and continuously approaches zero at the boundaries.
} 

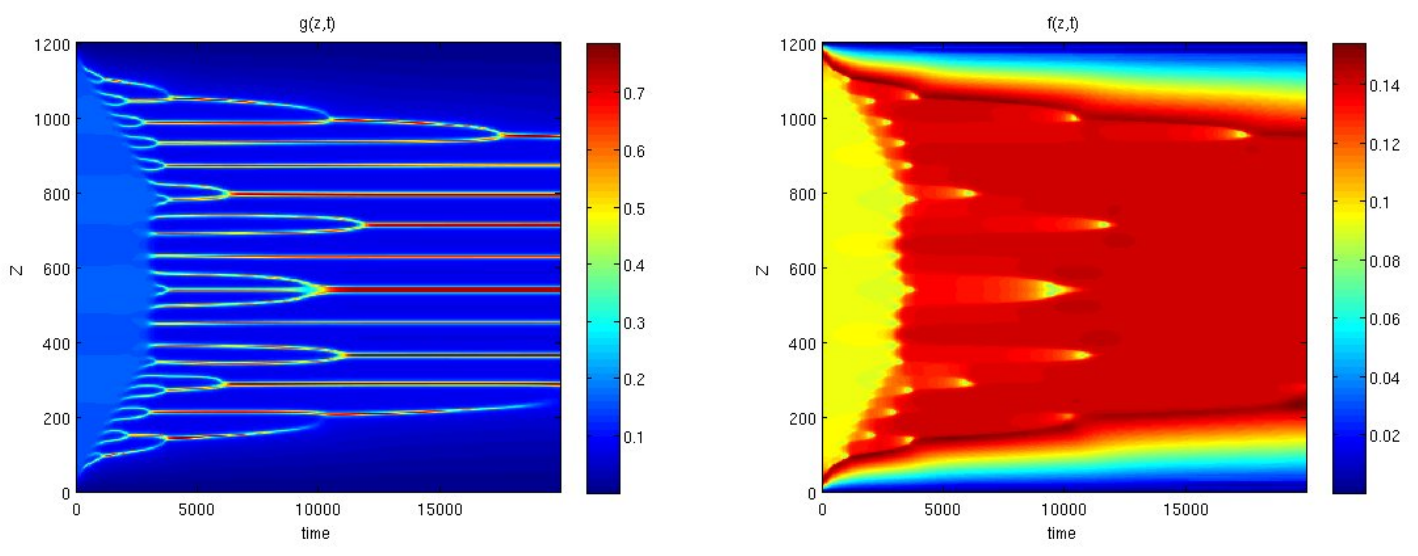

Figure 14: Time evolution of the modeled vertical profiles of the Richardson number (left) and the buoyancy flux (right).

higher and broader peaks, or they decay in amplitude and disappear amplifying the adjacent peaks( Figure 14(top) ). The flux on the other hand, started from an initial condition which has a weak constant value within the interior and after the instabilities have developed its value also remained constant, however at a higher value (Figure 14(bottom)). Note that the merging and decaying events are accompanied by a transient decrease in buoyancy flux. This is clearly seen at Figure13, where, for example, the two peaks at $z \approx 550$ or $z \approx 750$ are about to merge and the corresponding values of the buoyancy flux are decreased. The edge regions which are characterized by weak stratification and buoyancy flux are continuously growing free of instabilities. The end result of such a simulation would be the full homogenization of the buoyancy, since system is forced by the mean flow which is a continuous source of turbulent mixing.

The numerical simulation, showed that a series of mixed layers could form in a region subjected to the action of the mean flow turbulence. However, the simulation was performed in an idealized setting so that it could confirm the results of the linear analysis of the stability of the model. The model only conceptually shows the mechanism of layer formation. In reality, there are many more factors that would contribute to the evolution of the layers. First of all, the mixing model uses constant characteristic shear and the mean flow as a mechanisms for the generation of turbulence. However, the mean flow is affected by changes in stratification and should be accounted by the model if the instabilities develop at a similar time scale as the variations in mean flow. Furthermore, in the experiments there is a nonuniform buoyancy gradient profile, as it has a dominant peak at the base of the primary mixed layer. The existence of this layer affects the evolution of the adjacent secondary mixed layers. Nonetheless, the model shows the important features of the evolution of the secondary mixed layers: spontaneous generation of the layers, the merging of the layers and their decay - all of which have been observed in the experiments. Even though the proposed model simulates similar behavior to the observations, the assumption of the model should be verified experimentally - this would require elaborate measurements of the mean flow as well as the of the turbulent buoyancy and momentum fluxes. 


\section{Conclusions}

A series of experiments were performed on a linearly stratified fluid subjected to the surface frictional stress induced by a rotating disk. The control parameters for the experiments were the magnitude of the stratification $\left(N^{2}\right)$ and the rotating speed of the disk $(\Omega)$ resulting in a single non-dimensional parameter $R i=N^{2} / \Omega^{2}$. Two types of mixed layers have been observed - the primary layer extending from the surface into the interior of the fluid, and the secondary mixed layer of much smaller scale that forms underneath it. The growth of the primary layer with time seems to obey a power law $\hat{h} \sim R i^{-1 / 3} \hat{t}^{2 / 9}$. This result is consistent with the energy balance model that assumes a constant kinetic energy withing the layer.

The secondary mixed layers show a complicated behavior, having several regimes depending on time and the Richardson number of the experiment. A physical mechanism for the formation of the secondary mixed layers has not been clearly identified from the observations due to the lack of observational data, namely the turbulent fluxes and the mean flow. Nonetheless, the hypothesis has been put forward that relates the formation of these layers to an instability of the flux-gradient type that could arise in stratified turbulent flows. A one dimensional mixing model has been devised and the instabilities were investigated theoretically as well as through numerical simulations. The resulting mixed layers form only in a particular regime that puts constraints on the magnitude of the mean flow with respect the its shear and the Richardson number for the profile. Furthermore, the dynamics predicts the destruction of the mixed layers through merging and decay - the precesses observed in experiments.

Based on the mixing model, the results of the experiments could be interpreted as follows. Supported by the disk generated turbulence, the primary mixed layer grows creating a large density gradient at the base of it. At the same time, the mean flow is established within the primary layer. The flow is suppressed as it reaches high stratification and thus the shear is created at the base of the mixed layer. This sets a particular value of the model parameter $Q$, which reflects the relative importance of the mean flow to the mean shear. The magnitude of the flow as well as of its shear changes with time as the mixed layer grows, meaning that $Q$ is a function of time as well as space. The formation of the secondary mixed layers below the primary is observed when the necessary conditions for the instabilities are met: the parameter $\mathrm{Q}$ is smaller then the critical value, and the local Richardson numbers belong to the unstable part of the flux-gradient curve. Such a constrain identifies a confined region in a fluid where the instabilities could occur. The instabilities within this region lead to the development of the layers, however the magnitude of the instabilities is suppressed by the stabilizing effect of the mean flow turbulence. That explains why secondary mixed layers are not completely destratified. After the spontaneous development of the layers, comes the stage of slow evolution when the layers either merge with the primary interface of decay by erosion - consistent with the behavior of the observed layers. The change in the regime is the shift from the dominating collision instability to the erosion instability which is associated with the change in the base state. 


\section{Acknowledgements}

The present study was conducted under the Geophysical Fluid Dynamics Program at Woods Hole Oceanographic Institution. I would like thank Colm Caulfield for advising me on this project and Niel Balmforth for many productive discussions. All the lectures for giving excellent presentations on modern topics of fluid dynamics. And a special thanks to the fellows for the friendly atmosphere and overnight discussions that made this program a unique and unforgettable experience.

\section{References}

[1] N. Balmforth, S. Smith, And W. Young, Dynamics of interfaces and layers in a stratified turbulent fluid, Journal of Fluid Mechanics, 355 (1998), pp. 329-358.

[2] G. Barenblatt, M. Bertsch, R. Passo, V. Prostokishin, and M. Ughi, A mathematical model of turbulent heat and mass transfer in stably stratified shear flow, Journal of Fluid Mechanics, 253 (1993), pp. 341-358.

[3] P. Davies, Y. Guo, D. Boyer, And A. Folkard, The flow generated by the rotation of a horizontal disk in a stratified fluid, Fluid Dynamics Research, 17 (1995), pp. 27-47.

[4] H. Fernando, Turbulent mixing in stratified fluids, Annual review of fluid mechanics, 23 (1991), pp. 455-493.

[5] G. Oster and M. Yamamoto, Density Gradient Techniques., Chemical Reviews, 63 (1963), pp. 257-268.

[6] Y. Park, J. Whitehead, And A. Gnanadeskian, Turbulent mixing in stratified fluids: layer formation and energetics, Journal of Fluid Mechanics, 279 (1994), pp. 279311.

[7] O. PhILlips, Turbulence in a strongly stratified fluid -is it unstable?, in Deep Sea Research and Oceanographic Abstracts, vol. 19, Elsevier, 1972, pp. 79-81.

[8] E. Posmentier, The generation of salinity finestructure by vertical diffusion, Journal of Physical Oceanography, 7 (1977), pp. 298-300.

[9] T. RADKO, Mechanics of merging events for a series of layers in a stratified turbulent fluid, Journal of Fluid Mechanics, 577 (2007), pp. 251-273.

[10] J. Simpson And J. Woods, Temperature microstructure in a fresh water thermocline., Nature, 226 (1970), p. 832. 


\title{
On-off Intermittency in locally coupled maps
}

\author{
Woosok Moon
}

November 2, 2010

\begin{abstract}
On-off intermittency refers to the special pattern of time series which experience long time periods of calm behavior followed by short periods at bursting. In particular, the Ricker Map, which is a model of the discrete evolution of the expected population of one species at a given generation, is implemented to generate on-off intermittency. The on-off intermittency generated by the Ricker Map also exhibits the main characteristics found in other dynamical systems. On-off intermittency in a locally connected Ricker Map is the main focus here. The signal transfer between adjacent maps changes the stability condition compared with that of a single map, which leads to different conditions for on-off intermittency in locally coupled Ricker maps. The evolution and signal transfer in locally connected maps is carefully studied using a simple continuous model which contains similar structure and generates on-off intermittency. Through this analysis, it is found that a specific map in a locally connected structure recieves positively correlated signals from the adjacent maps, which generates more on-stages compared with a single map which has the same parameters.
\end{abstract}

\section{Introduction}

A signal representing on-off intermittency exhibits two different states. One is an 'off' states, where the variable remain almost constant. The other is an 'on' state which is a short time period of sudden bursting in the time series. This on-off intermittency has attracted wide interest due to the potential that many phenomena are related to on-off intermittency. Examples include the solar cycle showing long time periods of calm interrupted by short bursting(Platt et al. 1993a), and earthquake occurrence(Bottiglieri \& Godano 2007).

Suppose that there is a $k$-dimensional invariant manifold in an $n$-dimensional dynamical system, where $n>k$. The invariant manifold has a chaotic attractor whose stability can be controlled by a parameter, $p$. If $p$ varies across a threshold, the distance between the trajectory and the invariant manifold remains very close to 0 for a long time and shows a sudden increase for a short duration. In this context, on-off intermittency can be defined(Platt et al. 1993b). This argument is discussed by considering the skew product structure in the dynamical system. Though the skew structure is not necessary for on-off intermittency, it facilitates the analysis of on-off intermittency. Let us consider the following 
equations :

$$
\begin{aligned}
\dot{X} & =F(X, \mu(t)), \\
\dot{Y} & =G\left(Y, \nu_{0}\right), \text { and } \\
\mu(t) & =M\left(\mu_{0}, Y(t)\right),
\end{aligned}
$$

wherein the parameter $\mu$ for the evolution of $X$ is controlled by $Y$. In this system, if $Y$ is responsible for the parameter $\mu$ traveling above and below a threshold with time, one could observe on-off intermittency in the time series for $X$. Consider an even simpler case of the logistic map for time evolution of $X$ represented as $X_{t+1}=r_{t} X_{t}\left(1-X_{t}\right)$. Here, $r_{t}$ is the parameter determining the stability of the invariant manifold $X_{t}=0$ and hence $r_{t}=A_{0}+\sigma \xi_{t}$, where $A_{0}$ is the average of $r_{t}$ and $\xi_{t}$ is uniformly distributed noise between 0 and 1 . Depending on the choice of $A_{0}$ and $\sigma$, we can generate an on-off intermittency signal in $X$ (Toniolo et al. 2002).

This simple setting for on-off intermittency reveals several important characteristics of an on-off intermittency signal. Heagy et al. (1994) used uniformly distributed noise for driving a parameter to investigate the statistics of the duration of off-stages in on-off intermittency. They found that the probability density function $\phi$ of the duration of off-stages is proportional to $\phi^{-\frac{3}{2}}$, which is considered as the distinguishing characteristic of on-off intermittency relative to other non-intermittent signals or other intermittency signals. They also found numerically that the probability density function does not depend on the choice of the driving parameters, which hints of the possibility that the $-3 / 2$-law could be general for on-off intermittency generated by various dynamical structures. Toniolo et al. (2002) sought out various characteristics of on-off intermittency using power spectrum analysis and found a clear negative slope in a log-log scale plot. These results can be used to identify the on-off intermittency in the time series obtained from observation or experiments. For example, the time series of the earth quake occurence shows the $-3 / 2$-law in the probability density function of the duration of off-stages (Bottiglieri \& Godano 2007). Even if we do not have an exact model for earthquake justifiably, we can speculate that the occurrence of earthquakes shows on-off intermittency.

This previous work opens the possibility that we can determine whether or not a signal observed exhibits on-off intermittency. However, the previous work used a single map to investigate the characteristics of on-off intermittency. It is not realistic that a single dynamical system is totally isolated from other nearby systems. For example, convection cells located in deep clouds can interact with neighboring cells, or the population of insects or fish at one location can be related to those at another location through migration or sharing of resources given by same environment. Considering the possible coupling with neighbors in realistic situations, it is worthwhile to investigate a simple form of interaction between dynamical systems under conditions for on-off intermittency.

In this report, we will use the Ricker Map, which is a generalized version of the logistic map for population dynamics. First, we will check whether the Ricker Map also contains on-off intermittency in trajectories that travel near an invariant manifold. We will also examine whether there is consistency in the characteristics of on-off intermittency that have been identified using different maps. Second, simple local coupling will be defined for the investigation of the role of the interaction. Based on the definition of local coupling, we will try to 
find out how the signals in the local coupling structure evolve and transfer to adjacent maps. Numerical simulation and a possible theoretical approach will be provided.

\section{Single Ricker Map}

The Ricker Map (Ricker 1954) was first suggested by Bill Ricker to study the expected population of one specific species at a given generation. The Ricker Map is represented by a single discrete dynamical model in which the population at the generation $t+1$ is determined by the previous generation $t$ with a prescribed growth rate and restriction given by environmental conditions like food, habitat and water. The Ricker Map is represented as

$$
N_{t+1}=N_{t} e^{r\left(1-\frac{N_{t}}{K}\right)},
$$

where $N_{t}$ is the population at $t$ and $r$ is the growth rate. Th carrying capacity $K$ represents the population size of the species that the environment can hold depending on resources like food or water. For simplicity in this study, $K$ is assumed to be equal to be 1.0 and hence (4) becomes

$$
N_{t+1}=N_{t} e^{r\left(1-N_{t}\right)}
$$

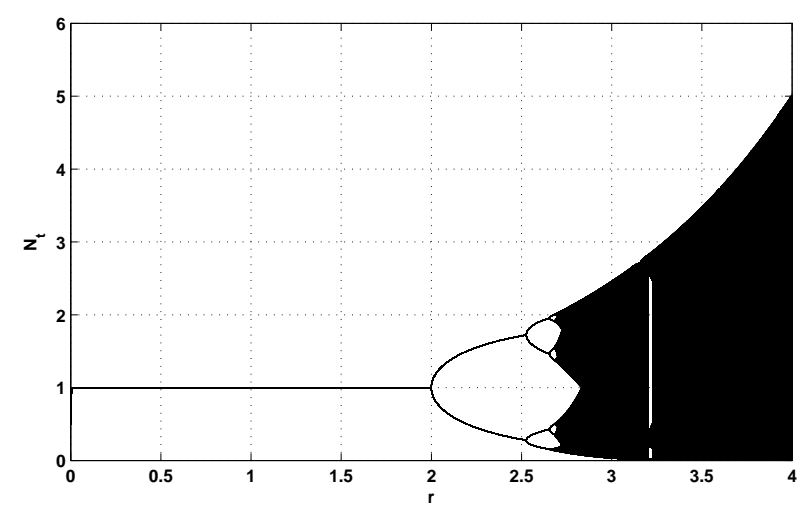

Figure 1: Bifurcation diagram for Ricker Map. If $r<2.0, N_{t}$ converges to 1.0. But if $r>2.0, N_{t}$ oscillates among several values, which demonstrates perioddoubling bifurcation. At $r=2.0$, one solution divides into two branches. Near $r=2.5$, each solution bifurcates again and hence $N_{t}$ travels around four values. As $r$ increases, the periodic-doubling bifurcations continue and the number of solutions grows dramatically.

This discrete map shows different sequences of $N_{t}$ depending on the value of $r$. If $r$ is less than 2.0, $N_{t}$ converges to 1.0. But if $r$ is greater than 2.0, $N_{t}$ starts to oscillate among several values. This situation is well displayed by a bifurcation diagram. Figure 1 shows the steady state solutions as a function of the value of $r$. When $r$ is equal to 2.0, one solution divides into two branches which is called a period-doubling bifurcation. Then near $r=2.5$, each solution 
bifurcates again giving four solutions. As $r$ increases further then, the perioddoubling bifurcations continue and the number of solutions grows dramatically, which is shown in black in figure 1 . Based on the bifurcation diagram, we can see that $N_{t}=1.0$ is the invariant manifold whose stability is determined by $r$. If $r$ is larger than 2.0, $N_{t}=1.0$ becomes unstable. On-off intermittency can be generated when $r$ moves across 2.0 in time. Under the assumption that $r$ is independent of $N_{t}$ (the skew product structure), we have to choose a way of determining $r$ at each time step. Heagy et al. (1994) and Toniolo et al. (2002)'s works indicate that the method of determining $r$ does not influence on-off intermittency. However, without specific physical constraints, we choose Gaussian white noise as our driving method with appeal to the central limit theorem. In realistic cases, $r$ can be affected by various physical variables. The dimension of the dynamical system for $r$ could be large. Therefore, many physical or environmental processes that evolve over a range of frequencies influence the time dependence of $r$. We can, therefore, think of the change of $r$ in the context of Brownian motion as $r=r_{0}+\sigma \xi$, where $r_{0}$ is the mean of $r, \xi$ the Gaussian white noise whose mean and standard deviation are 0 and 1.0, respectively, and $\sigma$ the intensity of noise. Hence the parameter $r$ is controlled by two variables, $r_{0}$ and $\sigma$. We can ask if on-off intermittency is always seen in $\left|N_{t}-1.0\right|$ in the parameter space, $\left(r_{0}, \sigma\right)$, when $r$ travels across 2.0 .

Figure 2 shows time series with different $r_{0}$ and $\sigma$. For (a) and (c), we choose $r_{0}=2.1$, so that $r_{0}$ is in the parameter region where $N_{t}=1.0$ is unstable. Then $r$ is driven by Gaussian white noise whose amplitude is $\sigma$. In (a), $\sigma$ is chosen to be 0.2 , which does not produce an intermittent signal even though $r$ moves through 2.0. The signal shown in figure 2(a) is similar to a noisy chaotic signal. When $\sigma$ is increased to 0.4 in (c), the signal shows clear bursting after long calm period, which can be recognized as on-off intermittency. On-off intermittency is generated through a particular perturbation of $r$. Figure 2(b) shows an intermittent signal where $r=7.0$ is chosen to be constant, but it is not on-off intermittency. On-off intermittency is generated when the perturbation of $r$ changes the stability of the fixed point at each time step. The distinction between the intermittency shown in (b) and the on-off intermittency shown in (c) cannot be understood without information about $r$. We need further analyses to make a clear distinction.

According to figure 2, on-off intermittency emerges only for certain choices of $r_{0}$ and $\sigma$. Metta et al. (2010) suggested a method to find on-off intermittency by first computing the stability curve in a parameter space which consists of the mean value of the parameter and the intensity of the noise. They then used kurtosis to locate on-off intermittency in the parameter space. Larger values of kurtosis represent a signal that can be considered as on-off intermittency. We can follow the procedure here. First, we can think of the stability of $N_{t}$ as a function of $r_{0}$ and $\sigma$. A necessary condition for on-off intermittency is that $r_{0}$ and $\sigma$ must be chosen to lie inside the unstable region in the parameter space, $\left(r_{0}, \sigma\right)$. Unless it is unstable, the time series converges to a fixed point so that the emergence of bursting is unlikely.

Consider the linearized equation near the fixed point, $N_{t}=1.0$, for which we can let $N_{t}=\eta_{t}+1$. After ignoring higher order terms, this gives

$$
\left|\eta_{t+1}\right|=\left|1-r_{0}-\sigma \xi_{t}\right|\left|\eta_{t}\right| .
$$



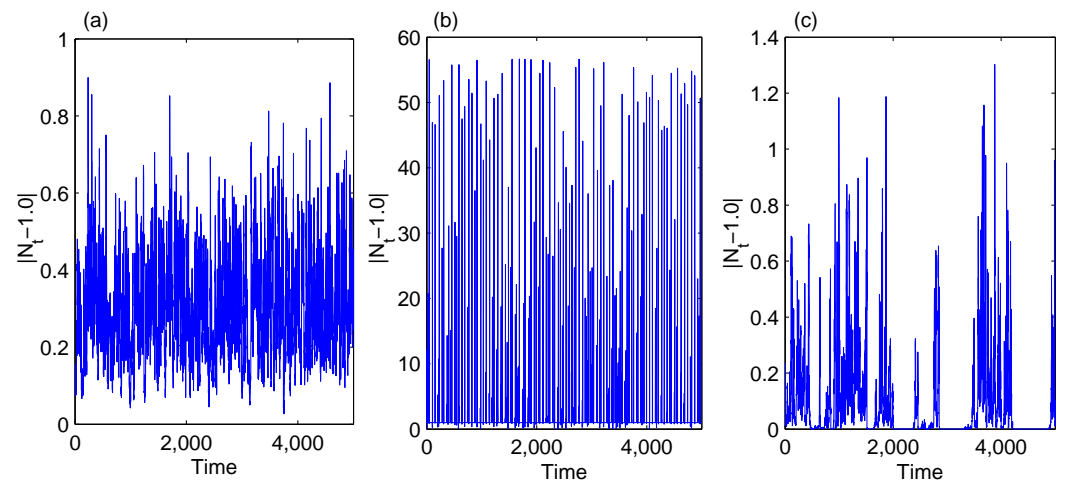

Figure 2: Sample time series of the driven variable $N_{t}$ in the different $r_{0}$ and $\sigma$. (a) $r_{0}=2.1, \sigma=0.2$; (b) $r_{0}=7.0, \sigma=0.0$; (c) $r_{0}=2.1, \sigma=0.4$. (a) shows noisy chaotic signal, which is non-intermittent signal. (b) is a kind of intermittent signal but not on-off intermittency. (c) shows an on-off intermittency.

From this relationship between $N_{t+1}$ and $N_{t}$, we can determine $\left|\eta_{t}\right|$ as

$$
\left|\eta_{t}\right|=\prod_{k=0}^{t-1}\left|1-r_{0}-\sigma \xi_{k}\right|\left|\eta_{0}\right| .
$$

Taking the logarithm of both sides, the equation becomes

$$
\begin{aligned}
\log \left|\eta_{t}\right| & =\sum_{k=1}^{t-1} \log \left|1-r_{0}-\sigma \xi_{k}\right|+\log \left|\eta_{0}\right| \\
& =t<\log \left|1-r_{0}-\sigma \xi_{k}\right|>+\log \left|\eta_{0}\right|
\end{aligned}
$$

Here $<\cdot>$ represents the ensemble average and $<\log \left|1-r_{0}-\sigma \xi_{k}\right|>$ can be considered as the exponent of $\left|\eta_{t}\right|$. Therefore, the stability curve satisfies

$$
<\log \left|1-r_{0}-\sigma \xi_{k}\right|>=0 .
$$

The calculation of the ensemble average can be accomplished using the known probability density function for $\xi_{k}$.

$$
<\log \left|1-r_{0}-\sigma \xi_{k}\right|>=\int_{-\infty}^{\infty} \rho(z) \log \left|1-r_{0}-\sigma z\right| d z
$$

where $\rho(z)$ is the normal distribution whose mean and standard deviation are 0.0 and 1.0 , respectively.

Figure 3(a) shows the stability curve in $\left(r_{0}, \sigma\right)$ parameter space, calculated using the equation (10). In the region bounded by the $r_{0}$ axis and the $\sigma$ axis and the stability curve, all time series converge to the fixed point 1.0. Inside this region, it is impossible to find on-off intermittency. On-off intermittency 

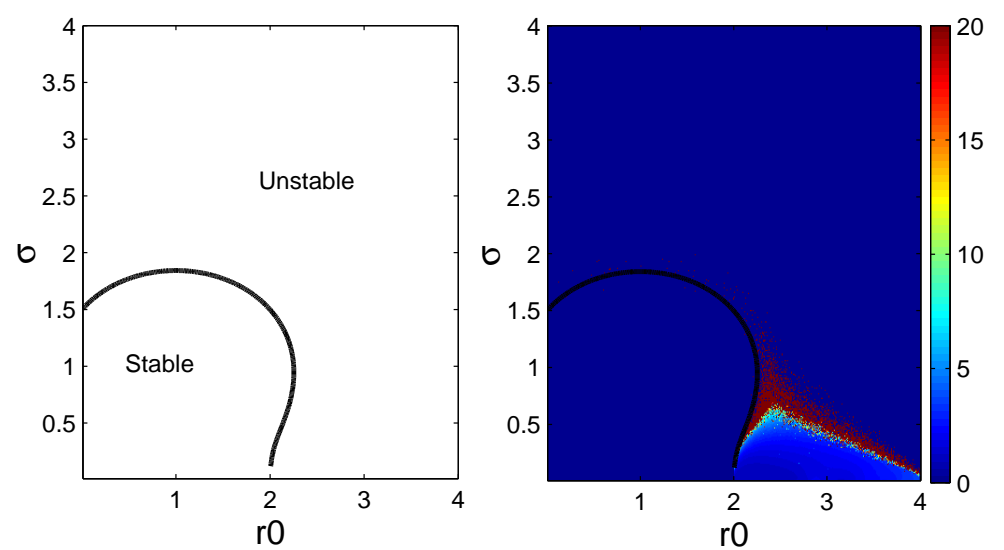

Figure 3: Stability curve and kurtosis at each $r_{0}$ and $\sigma$. (a) The stability curve is calculated using $<\log \left|1-r_{0}-\sigma \xi_{k}\right|>=0.0$. (b) At each $r_{0}$ and $\sigma$, kurtosis is calculated after generating the time series.

only exists outside of this region. To find the on-off intermittency outside of this stable region, kurtosis is a useful measure. Kurtosis is the statistical quantity used to detect infrequent extreme deviations. Higher kurtosis in this parameter space representative of on-off intermittency. In figure 3(b), we can see higher values of kurtosis in the area very close to stability curve. As suggested by previous research (Metta et al. 2010), there is on-off intermittency in the Ricker Map of the unstable region in parameter space and very near the stability curve.

In the Ricker Map, we have information about the parameters, $r_{0}$ and $\sigma$, which can lead to an on-off intermittency signal. Without information concerning $r_{0}$ and $\sigma$, however, we could be led by only looking at the signal itself. We need to seek other characteristics of on-off intermittency. First, we can examine the power spectrum of the time series generated by the Ricker Map. In figure 2 , we used three time series including one on-off intermittency case. We can compare the power spectrum of these three cases. Figure 4 shows the power spectrum of the three cases shown in figure 2. Case (a) shows the power spectrum of the non-intermittency case which looks like a noisy chaotic time series. For lower frequencies, the slope is almost flat, which is analogous to white noise. We can see slope at higher frequencies. Case (b) has intermittency signals but not on-off intermittency. Instead of a slope, the power is intensified in higher frequencies. Case(c) shows on-off intermittency. Unlike the previous cases, a clear negative slope is shown from low frequencies to high frequencies. The slope shown in (c) can be considered as one of main features contained of on-off intermittency.

Another characteristic we have to consider is the duration of off-stages in 

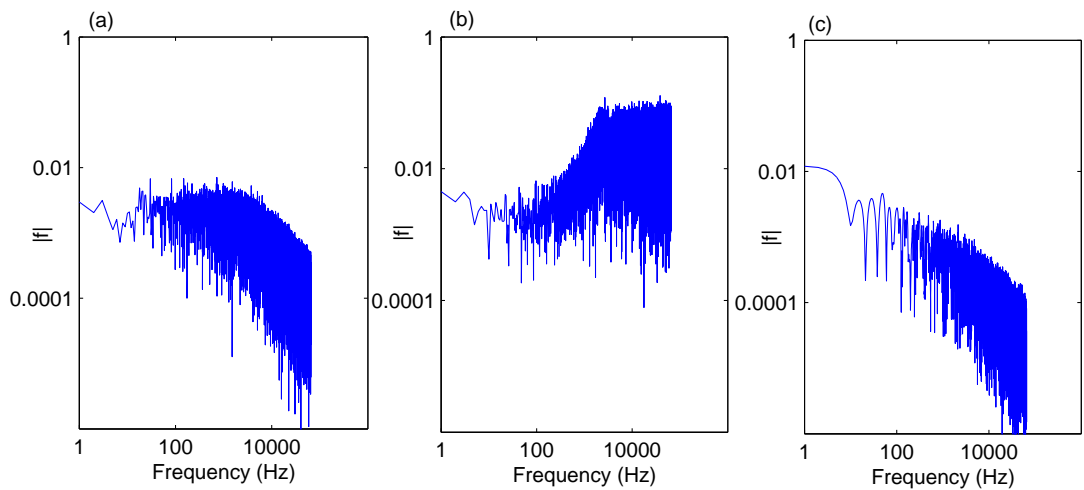

Figure 4: Power spectrum of three different cases.

$r_{0}=7.0, \sigma=0.0 ;$ (c) $r_{0}=2.1, \sigma=0.4$

(a) $r_{0}=2.1, \sigma=0.2$

the time series. The duration of an off stage is the time step between two consecutive on stages. The off stage can be defined in terms of the signal being below a certain value. Here, we will use 0.001 for the criterion for the off stage. Using the logistic map and a random process uniformly distributed between 0 and 1 for driving parameter, Heagy et al. (1994) proved that the probability density function (PDF) of $T_{\phi}$ (the duration of off-stages) is proportional to $T_{\phi}^{-\frac{3}{2}}$. They also showed numerically that the $-3 / 2$ law is maintained regardless of the details of the driving method. These could be another random process or any chaotic map. We can expect the same characteristics of on-off intermittency to occur in the Ricker Map. Figure 5 shows the probability density function of the duration of off-stages for three cases. The red line is the PDF for on-off intermittency, which is very close to a $-3 / 2$ slope line in log-log scale plot. The green line represents the PDF for the noisy chaotic case when $r_{0}=2.1$ and $\sigma=0.2$. Here, the slope of the probability density function is quite different from $-3 / 2$. The last line is for another type of intermittency when $r_{0}=7.0$ and $\sigma=0.0$, which is also distinct from on-off intermittency.

The $-3 / 2$ law can be used as a characteristic to determine whether a time series exhibits on-off intermittency. But it must be understood that this is not proven mathematically in general. To determine whether a given time series shows on-off intermittency, we have to use multiple analyses including power spectra and the PDF of the duration of off stages. Finally, if possible, we will try to construct a dynamical system for the time series and find the parameters necessary for the generation of on-off intermittency.

A single Ricker Map with Gaussian white noise generates on-off intermittency near the fixed point $N_{t}=1.0$. The power spectrum and probability density function of the duration of off-stages of the Ricker Map are consistent with the results at several previous studies. The consistencies shown in on-off intermittency of the Ricker Map make it possible for us to use the Ricker Map for further analysis of on-off intermittency. As noted in the introduction, one 


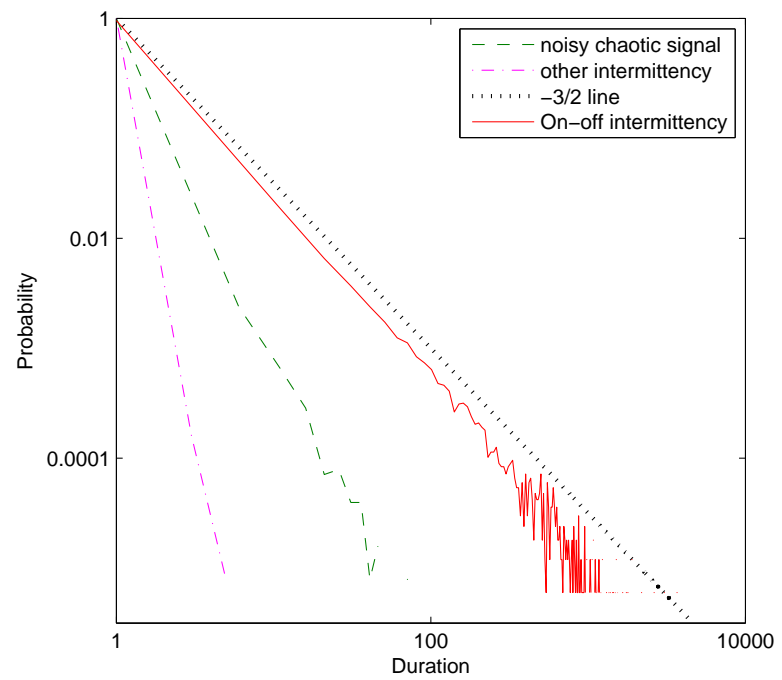

Figure 5: Probability density function of the duration of off-stages in log-log scales. On-off intermittency case is compared with two other cases. One of them is noisy chaotic time weries with $r_{0}=2.1$ and $\sigma=0.2$. The other is other intermittency case when $r_{0}=7.0$ and $\sigma=0.0$.

particular system is not totally isolated from other similar systems. More specifically, a group of species whose population is governed by the Ricker Map can interact with other nearby groups of same species. The interaction can occur in various forms. One example is the migration from one group to nearby groups. Another example is sharing of environmental resources. Therefore, our next step is to examine how on-off intermittency changes through in a single map under the possible interactions with adjacent maps.

\section{Locally Connected Ricker Maps}

Now we examine the dynamics of connected maps. For example, one group of salmon can interact with other groups via migration and sharing food or resources. Depending on the system, the form of the interaction may be different. As a starting point, the interaction can be assumed to be linear and local, which implies that maps are diffusively coupled. In a Ricker Map, local coupling can be understood as the dynamics of a metapopulation composed of many different populations that share individuals between different sites.

Lets consider the case of $K$ locally connected maps all of which have the same parameter values,

$$
N_{t+1}^{k}=(1-\epsilon) f\left(N_{t}^{k} ; r_{t}^{k}\right)+\frac{\epsilon}{2}\left[f\left(N_{t}^{k-1} ; r_{t}^{k-1}\right)+f\left(N_{t}^{k+1} ; r_{t}^{k+1}\right)\right],
$$

where $k=1,2, \ldots, K$, and

$$
f\left(N_{t}^{k} ; r_{t}^{k}\right)=N_{t}^{k} \exp \left[r_{t}^{k}\left(1-N_{t}^{k}\right)\right]
$$


where $r_{t}^{k}=r_{0}+\sigma W_{t}^{k}$, and $W_{t}^{k}$ is Gaussian white noise whose mean and standard deviation are 0 and 1 , respectively. Also $W_{t}^{k}$ is $\delta$-correlated in space and time so that $\left\langle W_{t}^{k} W_{s}^{m}\right\rangle=\delta_{k m} \delta_{t s}$. The coupling coefficient $\epsilon$ determines the intensity of the local coupling. We also assume periodic boundary conditions which means that the $K^{t h}$ map is coupled to the $(K-1)^{t h}$ map and $1^{\text {st }}$ map. The periodic boundary conditions mimic an infinite array of population sites.

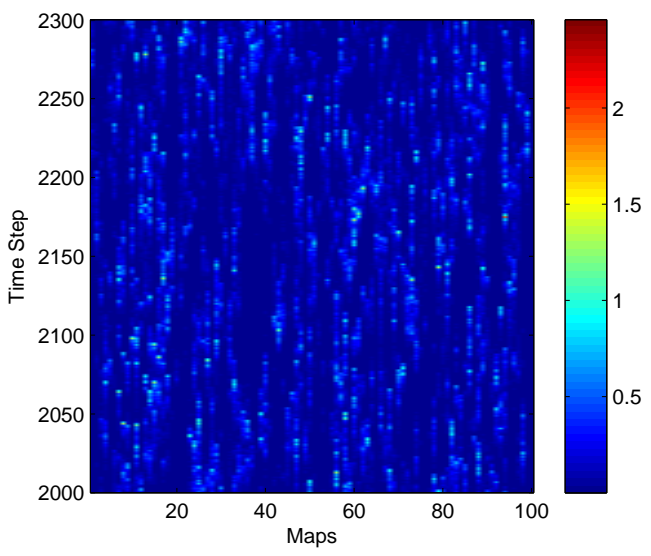

Figure 6: Signal pattern in a locally coupled map. $r_{0}=2 ; 1, \sigma=0.4$, and $\epsilon=0.01$ are used for the map.

Figure 6 shows an example of the local coupling of many maps, $r_{0}=2.1$, $\sigma=0.4$, and $\epsilon=0.01$. "On stages", seen as bright color dots, appear from time to time after long period of "off stages". The on-stages in one specific map seem to be related to that of the adjacent maps. Sometimes, the on-stage in one map seems to trigger in adjacent maps. We see a cluster of on-stages in the same space and time domain. This might be due to transfer between adjacent maps. Signal transfer can shape the on-off intermittency in the case of a local coupling.

A simple question is whether or not the local coupling is helpful in generating more on-stages. Figure 7 shows three examples of time series of one specific map among locally coupled maps. The coupling coefficient $\epsilon$ is different for the three examples. The first case has no coupling, the second has $\epsilon=0.01$ and the third one has $\epsilon=0.1$. According to the figure, the number of on stages generated during a fixed time increases as $\epsilon$ increases. Even though $\epsilon$ is quite small, the effect from this weak coupling is substantial. Figure 7 demonstrates that even a weak coupling can change the statistics of on-off stages dramatically.

In a single map, the most important issue is where on-off intermittency exists in the parameter space; it exists in the unstable region, but very close to the stability curve. Finding where on-off intermittency occurs in a parameter space must begin with the determination of the stability curve. For simplicity, we start with a 3-map local coupling. Even though only three maps are locally connected, we expect to find general characteristics of infinite coupled maps due to locality and periodic boundary conditions. Generally, finding the stability condition for a point in a parameter space is equivalent to finding the Lyapunov exponent. If the exponent is positive, it means the system is unstable. The stability curve 

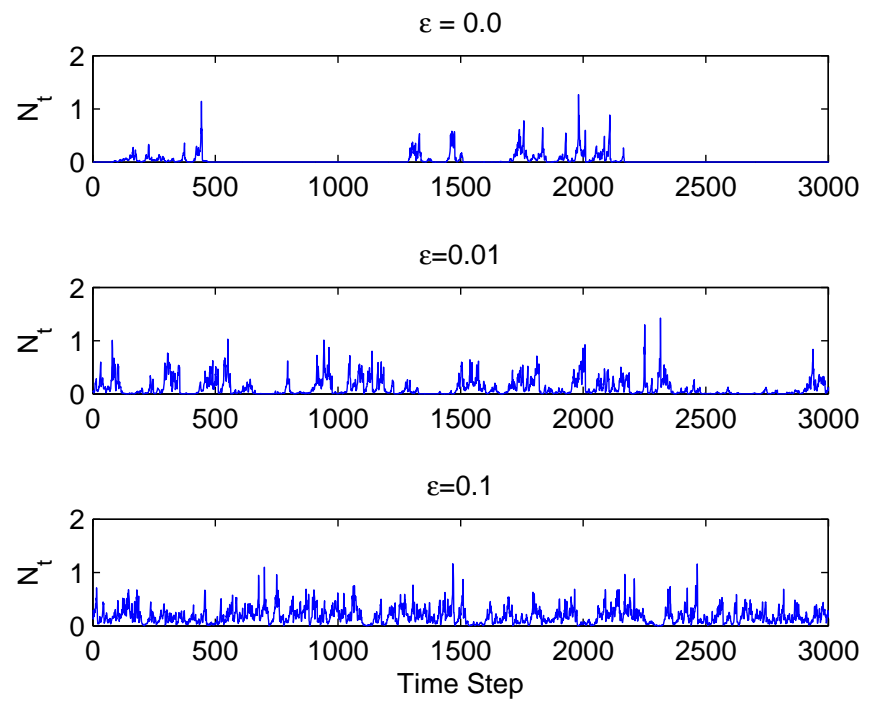

Figure 7: Time series of one specific map in locally coupled maps. Here, $r_{0}=2.1$ and $\sigma=0.4$. The only difference among three examples is the magnitude of $\epsilon$, the coupling coefficient.

is given by the set of the points where Lyapunov exponent is zero. To find the Lyapunov exponent, we have to linearize equation (8) near the fixed point $N_{t}=1.0$. If $K=3$, our linearized equation is

$$
Z_{t+1}=Y_{t} Z_{t}
$$

where $Z_{t}=N_{t}-1.0$ and

$$
Y_{t}=\left[\begin{array}{ccc}
(1-\epsilon)\left(1-r_{0}-\sigma \xi_{1}\right) & \frac{\epsilon}{2}\left(1-r_{0}-\sigma \xi_{2}\right) & \frac{\epsilon}{2}\left(1-r_{0}-\sigma \xi_{3}\right) \\
\frac{\epsilon}{2}\left(1-r_{0}-\sigma \xi_{1}\right) & (1-\epsilon)\left(1-r_{0}-\sigma \xi_{2}\right) & \frac{\epsilon}{2}\left(1-r_{0}-\sigma \xi_{3}\right) \\
\frac{\epsilon}{2}\left(1-r_{0}-\sigma \xi_{1}\right) & \frac{\epsilon}{2}\left(1-r_{0}-\sigma \xi_{2}\right) & (1-\epsilon)\left(1-r_{0}-\sigma \xi_{3}\right)
\end{array}\right] .
$$

Therefore, $Z_{t+1}$ is

$$
Z_{t+1}=\left(\prod_{s=0}^{t} Y_{s}\right) Z_{0}
$$

Here, the Lyapunov exponent $\lambda$ can be found using

$$
\lambda=\lim _{t \rightarrow \infty} \frac{1}{t} \ln \left\|\prod_{s=0}^{t} Y_{s}\right\|
$$

It is known that a limit for the calculation of $\lambda$ exists even though the matrix is randomly determined at each time step. To determine the change of the Lyapunov exponent with local coupling, we fix $r_{0}=2.1$ and then vary $\sigma$ and $\epsilon$. Figure 8 shows the Lyapunov exponent in the parameter space $(\sigma, \epsilon)$ with fixed $r_{0}=2.1$. For a single map, with $\sigma$ larger than 0.42 , the Ricker Map 


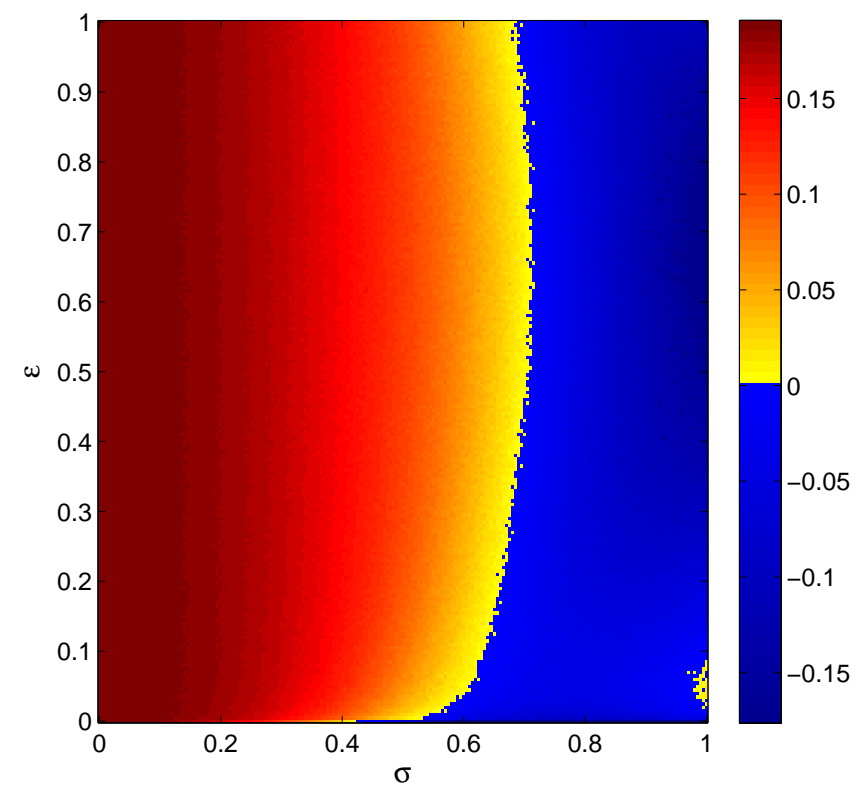

Figure 8: Lyapunov exponent in the parameter space $(\sigma, \epsilon)$ with fixed $r_{0}=2.1$.

becomes stable. In figure 8 we find $\sigma=0.42$ when $\epsilon=0.0$. Interestingly, a sudden change of stability with local coupling is formed for $0.42<\sigma<0.53$ where the stability condition suddenly changes with coupling. Even a weak coupling makes the system unstable. If $\sigma$ is large enough, local coupling cannot change the stability. Lets consider a scenario relevant for this analysis. We think of the population of salmon governed by the Ricker Map. The growth rate $r$ of one generation of salmon can be assumed to fluctuate due to a change in environmental resources at every time step. If the standard deviation of the fluctuation exceeds a certain threshold, for example $\sigma=0.42$, the population of the salmon always maintains the fixed value even after many generations. Under the same environmental conditions, which implies that the mean and standard deviation of $r$ are same, we can think of a case where salmon in a group start to migrate to nearby population groups. In this case, we expect a sudden sharp increase of the salmon population at some time step.

Figure 8 shows the increase of Lyapunov exponent with $\epsilon$ within some range of $\sigma$. If we remember that on-off intermittency is seen near the stability curve in the parameter space, we surmise that on-off intermittency formed in a single map could disappear with strong local coupling. First, we can check the power spectrum of the time series at each coupling coefficient. We know that a clear negative slope is found for on-off intermittency in the single map analysis. Figure 9 shows the power spectra for several values of $\epsilon$. These power spectra are made by averaging the signals in $2^{13}$ maps after $2^{14}$ time steps. For the weak couplings, when $\epsilon$ is 0.1 or 0.2 , the clear negative slope is shown in power spectrum. However, when $\epsilon=0.5$ or $\epsilon=0.8$, flatness over low frequencies appear, which also appeared in the noisy chaotic signal in the single map when 
$r_{0}=2.1$ and $\sigma=0.2$.

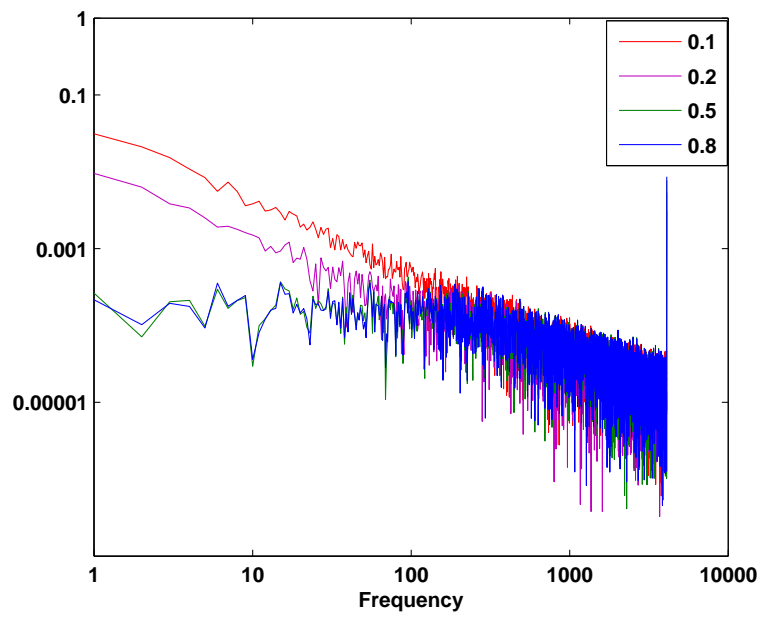

Figure 9: Power Spectra for several local couplings.

We have seen the change of stability and the statistics of on and off stages due to local coupling. This change could be made more evident if we trace how a certain on-stage is generated and how it interacts with other maps. For this job, we create $2^{13}$ maps locally coupled and run the system for $2^{14}$ time steps. A substantial number of on-stages can be sampled in the time and space domain. In this sampling, we also collect adjacent points centered on the onstage in both the spatial and time domains. Figure 10 shows the average map over samples collected as described above. For this figure, we chose 20 adjacent maps and 40 time steps for sampling. The time step is from lag -20 to lag 20 centered on the on-stage. From this figure, we see how the signal in locally coupled maps evolves. Before the on-signal is generated, we see an increase of the signal of the map together with the adjacent maps. The on signal is not generated alone randomly. It can be correlated with adjacent maps so that the signals all increases together for the on-signals. This argument is refined further in the next section. After an on-signal is generated, the signal decays and, at the same time, diffuses away to adjacent maps. It is seen as a sharp V-shape, which may be related to the $\mathrm{V}$-shape propagation of on-signals in the spatial and time domains from time to time.

The characteristics of on-off intermittency shown in this local coupling seems to be determined by how the signal is transfered and evolves with adjacent maps. We can conclude from numerical analysis that more on-signals are generated through the coupling. In a certain range of $\sigma$, the coupling even changes the stability of the map. This motivates a theoretical approach to explain how the signal of a specific map evolves with that of adjacent maps.

\section{Signal Transferring in locally connected maps}

Compared with a single Ricker Map, without rigorous quantification, we surmise that signals are coming to each map from adjacent maps, which may lead 


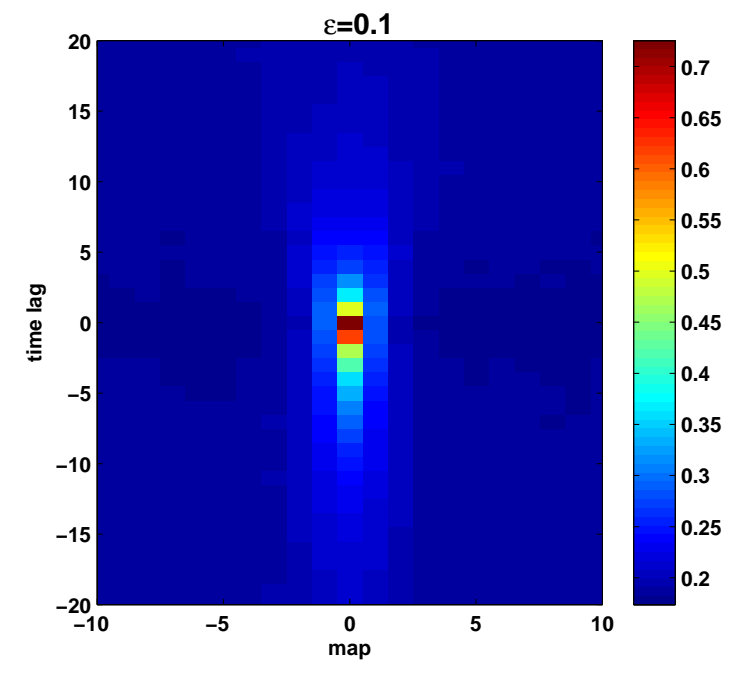

Figure 10: Average map over the area centered on on-stages in the spatial and time domains.

to more on stages in time series of locally coupled maps. However, without aid from numerical results, we can think of two different effects due to local coupling. As suggested above, more signals will come into each map from adjacent maps. Alternatively, the growing signal in each map could be diffused and transfered to adjacent maps, which could lengthen the duration of the off stage. Finding which one is more dominant or how to determine which case is preferred requires more precise analysis. However, these two possible effects only treat signal transferring. As we know, each map has its own system for increasing or decreasing signals, which might be a broader sort of reaction. Therefore, the combination of diffusion and reaction, which are randomly controlled, influences the signal pattern in the locally coupled maps.

On-off intermittency is characterized by long periods of calm stages and short periods of bursting. During a long period time of the off-stage, the signal, which is the distance from the fixed point, is negligibly small. Even though we can easily ignore the tiny signals during off-stages, the occurrence of the on-signal is completely governed by how the signal evolves during the off-stage, which implies that the generation of the on-stage is completely explained by the linearized equation near the fixed point or more generally the invariant manifold. The role of nonlinearity is to prevent further increase of the signal after the emergence of the on-signal and to make the signal return to the off stage. Heagy et al. (1994) proved the $-3 / 2$ law for the PDF of the duration of off stages using a linearized equation near the fixed point, which was possible due to the fact that the generation of the on-signal is governed by the signal growth during the off stage. Metta et al. (2010) explained the signal synchronization shown in two-map coupling based on the signal matching during the off stage. So, how the signal grows during the off period is the key process for explaining the statistics of the on-stages. Returning to the locally coupled maps, the main question is how signals grow and exchange information during off stages in 
locally coupled maps. From this perspective, linearized equations near the fixed point during a particular off stage will be considered.

Despite the use of linearized equations, discrete maps are difficult to analyze analytically. A continuous model is more useful and can be constructed using stochastic calculus. Therefore, we use a time-continuous model qualitatively similar to the Ricker map to try to generate on-off intermittency. The model is a canonical cubic ODE,

$$
\frac{d x}{d t}=\mu x-x^{3},
$$

whose stability near $x=0$ is determined by the sign of $\mu$; if $\mu$ is positive (negative), equation (16) is unstable (stable). The similarity to the Ricker map is seen as

$$
\frac{d x}{d t}=-\frac{d}{d x} V=-\frac{d}{d x}\left(-\frac{1}{2} \mu x^{2}+\frac{1}{4} x^{4}\right),
$$

where the shape of $V$ can tell us the stability of $x=0$ and where stable solutions exist. Figure 11 shows the shape of $V$ depending on the sign of $\mu$ near $x=$ 0 . When $\mu$ changes from negative to positive, the stable solution near $x=$ 0 becomes unstable and two stable solutions near $x= \pm \sqrt{\mu}$ appear. This situation is analogous to a period-doubling bifurcation when $r$ becomes slightly larger than 2 in the Ricker map. Therefore, if $\mu$ is perturbed near 0 , on-off intermittency may be generated. Due to this qualitative similarity, we can use this continuous model to investigate on-off intermittency in locally connected maps.
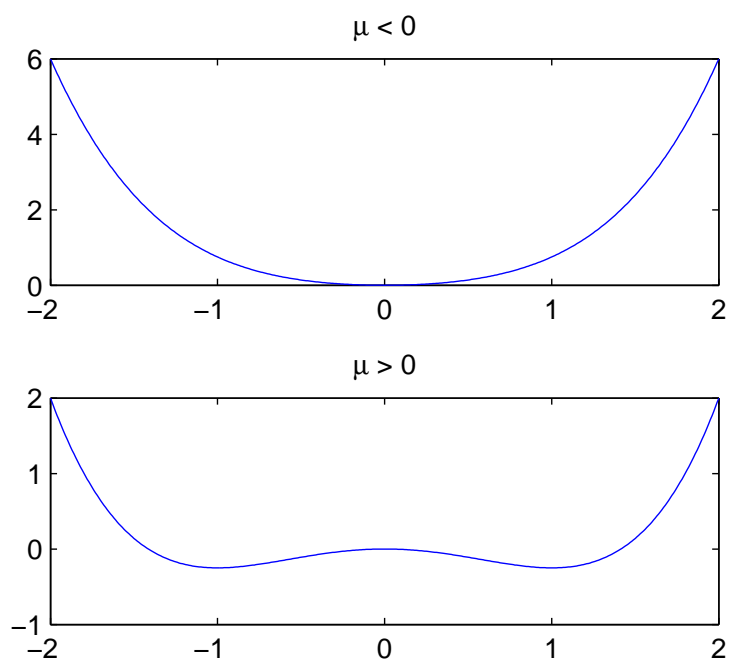

Figure 11: Shapes of $V$ depending on the sign of $\mu$

First, it is necessary to analyze on-off intermittency of the single map in this continuous model. Near $x=0$, the linearized equation is

$$
\frac{d x}{d t}=\mu x,
$$


where $\mu$ is perturbed by some external process. Here, we simply choose Gaussian white noise to perturb $\mu$ as $\mu=\mu_{0}+\sigma \xi$, where $\xi$ and $\sigma$ represent Gaussian white noise and intensity of the noise respectively. Moreover Gaussian white noise $\xi$ can be written as time derivative of a Wiener process $d W / d t$. Equation (18) becomes and

$$
d x=\mu_{0} x d t+\sigma x d W .
$$

Among other systems, this equation describes the evolution of option prices, where it is called the Black-Scholes model. Ito-calculus provides us with an exact solution, which is

$$
x=\exp \left[\left(\mu_{0}-\frac{1}{2} \sigma^{2}\right) t+\sigma W\right] .
$$

We can determine the stability condition from the solution as

$$
<\left(\mu_{0}-\frac{1}{2} \sigma^{2}\right) t+\sigma W>=\left(\mu_{0}-\frac{1}{2} \sigma^{2}\right) t>0 .
$$

Therefore, the stability curve in the $\left(\mu_{0}, \sigma\right)$ domain is $\mu=\frac{1}{2} \sigma^{2}$. We expect on-off intermittency. Figure 12 shows a example of on-off intermittency near

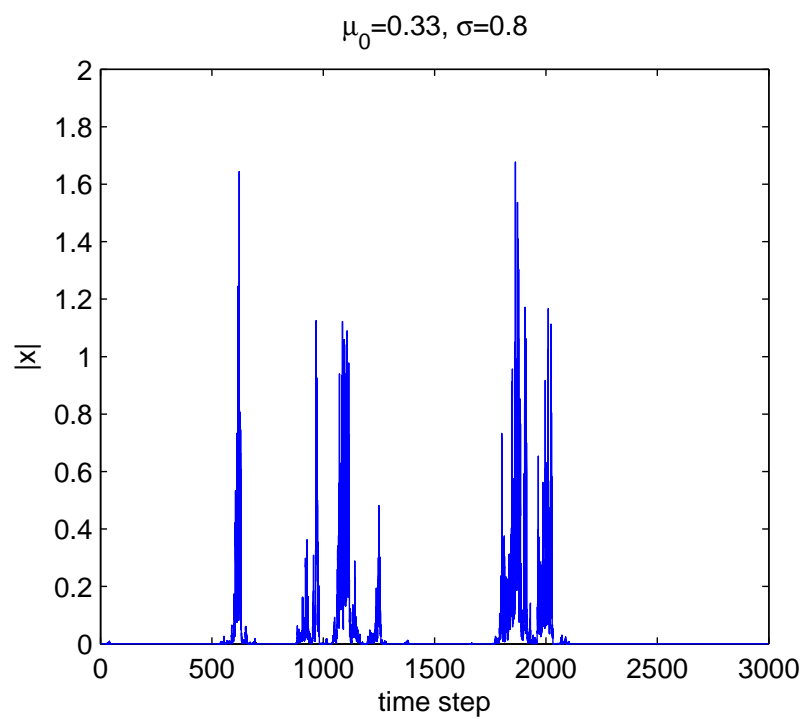

Figure 12: On-off intermittency when $\mu_{0}=0.33$ and $\sigma=0.8$

stability curve in this continuous model.

We now construct the locally coupled maps. We focus on signal evolution and transfer during a certain off-stage so that the linearized equation near $x=0$ will be considered in local coupling. Our equation is

$$
\begin{aligned}
\frac{d x_{k}}{d t} & =\mu_{0} x_{k}+\sigma \xi_{k} x_{K}+\epsilon \mu_{0}\left(\frac{x_{k+1}+x_{k-1}}{2}-x_{k}\right) \\
& +\epsilon \sigma\left(\frac{\xi_{k+1} x_{k+1}+\xi_{k-1} x_{k-1}}{2}-\xi_{k} x_{k}\right),
\end{aligned}
$$


where $k$ and $\epsilon$ are the index of each map and the coupling coefficient, respectively. We can let $x_{k}=y_{k} e^{\mu_{0} t}$ to remove the first term in the equation (21), thereby giving

$\frac{d y_{k}}{d t}=\epsilon \mu_{0}\left(\frac{y_{k+1}+y_{k-1}}{2}-y_{k}\right)+\sigma \xi_{k} x_{k}+\epsilon \sigma\left(\frac{\xi_{k+1} y_{k+1}+\xi_{k-1} y_{k-1}}{2}-\xi_{k} y_{k}\right)$,

which can be written in matrix form as

$$
\frac{d Y}{d t}=\epsilon \mu_{0} A Y+\sigma(I+\epsilon A) Y \xi
$$

where

$$
A=\left(\begin{array}{cccccc}
-1 & \frac{1}{2} & 0 & 0 & \cdots & \frac{1}{2} \\
\frac{1}{2} & -1 & \frac{1}{2} & 0 & \cdots & 0 \\
0 & \frac{1}{2} & -1 & \frac{1}{2} & \cdots & 0 \\
\vdots & & & & \ddots & \vdots \\
\frac{1}{2} & & \cdots & & \frac{1}{2} & -1
\end{array}\right)
$$

and

$$
\begin{aligned}
Y & =\left(y_{1}, y_{2}, \ldots \ldots, y_{n}\right)^{T}, \\
Y \xi & =\left(y_{1} \xi_{1}, y_{2} \xi_{2}, \ldots \ldots ., y_{n} \xi_{n}\right)^{T} .
\end{aligned}
$$

Here, $A$ describes the structure of the periodic connection and is a symmetric matrix. We can easily construct the integral form of $Y(t)$, which is written as

$$
Y(t)=\exp \left(\epsilon \mu_{0} A t\right) Y(0)+\sigma(I+\epsilon A) \int_{0}^{t} \exp \left(\epsilon \mu_{0} A\left(t-t^{\prime}\right)\right) Y d W
$$

where

$$
Y d W=\left(y_{1} W_{1}, y_{2} W_{2}, \ldots \ldots . ., y_{n} W_{n}\right),
$$

and again $W$ represents Wiener process. Because $A$ is symmetric, it can be diagonalized and represented as $A=Q D Q^{T}$ where $D$ is the diagonal matrix whose diagonal terms are the eigenvalues of $A$. The above integral equation becomes

$$
Y(t)=Q \exp \left(\epsilon \mu_{0} D t\right) Q^{T} Y(0)+\sigma Q(I+\epsilon D) \int_{0}^{t} \exp \left[\epsilon \mu_{0} D\left(t-t^{\prime}\right)\right] Y d W
$$

This integral form does not immediately give us any information. Even though we cannot expect a Gaussian distribution for the PDF of $Y(t)$, which means we have to know higher order moments, the correlation could give us some important information regarding the signal transfer between adjacent maps. From the above integral equation, we can construct the covariance matrix. Multiplying the transpose of $Y(t)$ and taking ensemble average, the covariance matrix can be represented as

$$
\begin{aligned}
& <Y(t) Y(t)^{T}>=\exp \left(\epsilon \mu_{0} D t\right) Q^{T}<Y(0) Y^{T}(0)>Q \exp \left(\epsilon \mu_{0} D t\right) Q^{T} \\
& +\sigma^{2} Q(I+\epsilon D) \int_{0}^{t} \int_{0}^{t} \exp \left(\epsilon \mu_{0} D\left(t-t^{\prime}\right) Q^{T}<(Y d W)(Y d W)^{T}>Q \exp \left[\epsilon \mu_{0} D\left(t-t^{\prime}\right)\right](I+\epsilon D) Q^{T} .\right.
\end{aligned}
$$


To simplify equation (26), we need information about the initial covariance structure. We seek the average behavior of signal transfer and hence it is reasonable to initially assume that each map is uncorrelated with any other map and the second moment of each map is identical. Mathematically, the assumption is

$$
\begin{aligned}
& <y^{2}(0)>=<y_{1}^{2}(0)>=<y_{2}^{2}(0)>=\ldots \ldots=<y_{n}^{2}(0)> \\
& <y_{i}(0) y_{j}(0)>=0, i \neq j
\end{aligned}
$$

and $d W$ represents a Wiener process which is independent at each time step and the noise input at each map is independent from other maps. Based on these facts and $d W \sim N(0, \sqrt{d t})$, we can deduce $<d W_{i} d W_{j}>=\delta_{i j} d t$. A more rigorous explanation is given by Ito-calculus as

$$
\int_{0}^{t} \int_{0}^{t} f<(Y d W)(Y d W)^{T}>g=\int_{0}^{t} f g S,
$$

where $f$ and $g$ are integrable functions and

$$
S=\left(\begin{array}{cccc}
<y_{1}^{2}>d t & & & \\
& <y_{2}^{2}>d t & & \\
& & \ddots & \\
& & & <y_{n}^{2}>d t
\end{array}\right) .
$$

There is no distinction between any of $n$ maps because each map starts from the same condition. The statistical structure of all maps must be the same in time, which leads to

$$
<y_{1}^{2}>=<y_{2}^{2}>=\ldots \ldots=<y_{n}^{2}>.
$$

Therefore, the integral equation for covariance can be simplified as

$$
\begin{aligned}
<Y(t) Y^{T}(t)> & =\operatorname{Qexp}\left(2 \epsilon \mu_{0} D t\right) Q^{T} \\
& +\sigma^{2} Q(I+\epsilon D) \int_{0}^{t} \exp \left(2 \epsilon \mu_{0} D t\right)(I+\epsilon D) Q^{T}<y^{2}>d t^{\prime} .
\end{aligned}
$$

A technical difficulty exists for handling the $n \times n$ matrix in this equation. The simplest case is of 3 identical maps that are connected locally. Even in the 3 -map case, we expect that the most important aspect for signal transference remains. For 3-map case we have

$$
\begin{gathered}
A=\left(\begin{array}{ccc}
-1 & \frac{1}{2} & \frac{1}{2} \\
\frac{1}{2} & -1 & \frac{1}{2} \\
\frac{1}{2} & \frac{1}{2} & -1
\end{array}\right), \\
Q=\left(\begin{array}{ccc}
-\frac{1}{\sqrt{6}} & -\frac{1}{\sqrt{2}} & \frac{1}{\sqrt{3}} \\
\frac{2}{\sqrt{6}} & 0 & \frac{1}{\sqrt{3}} \\
-\frac{1}{\sqrt{6}} & \frac{1}{\sqrt{2}} & \frac{1}{\sqrt{3}}
\end{array}\right) \text {, and } D=\left(\begin{array}{ccc}
-\frac{3}{2} & 0 & 0 \\
0 & -\frac{3}{2} & 0 \\
0 & 0 & 0
\end{array}\right),
\end{gathered}
$$


If we insert $Q$ and $D$ into the integral equation, we find the integral equation for $<y^{2}>$ as

$$
\begin{aligned}
<y^{2}> & =\left[\frac{1}{3}+\frac{2}{3} \exp \left(-3 \epsilon \mu_{0} t\right)\right]<Y^{2}(0)> \\
& +\sigma^{2} \int_{0}^{t}\left[\frac{1}{3}+\frac{2}{3}\left(1-\frac{3}{2} \epsilon\right)^{2} \exp \left(-3 \epsilon \mu_{0}\left[t-t^{\prime}\right]\right)\right]<y^{2}>d t^{\prime} .
\end{aligned}
$$

Differentiating with respect to $t$, we get

$$
\begin{aligned}
& \frac{d}{d t}<y^{2}>=-2 \epsilon \mu_{0} \exp \left(-3 \epsilon \mu_{0} t\right)<y^{2}(0)>+\sigma^{2}\left(\frac{1}{3}+\frac{2}{3}\left(1-\frac{3}{2} \epsilon\right)^{2}\right)<y^{2}> \\
& -2 \epsilon \mu_{0} \sigma^{2}\left(1-\frac{3}{2} \epsilon\right)^{2} \int_{0}^{t} \exp \left(-3 \epsilon \mu_{0}\left(t-t^{\prime}\right)\right)<y^{2}\left(t^{\prime}\right)>d t^{\prime},
\end{aligned}
$$

and multiplying both sides by $\exp \left(3 \epsilon \mu_{0} t\right)$ and letting $q=<y^{2}>\exp \left(3 \epsilon \mu_{0} t\right)$, we find

$$
\begin{aligned}
\frac{d q}{d t}-3 \epsilon \mu_{0} q & =-2 \epsilon \mu_{0}<y^{2}(0)>+\sigma^{2}\left[\frac{1}{3}+\frac{2}{3}\left(1-\frac{3}{2} \epsilon\right)^{2}\right] q \\
& -2 \epsilon \mu_{0} \sigma^{2}\left(1-\frac{3}{2} \epsilon\right)^{2} \int_{0}^{t} q d t^{\prime}
\end{aligned}
$$

Differentiating again with respect to $t$ on both sides, we get

$$
\frac{d^{2} q}{d t^{2}}-\left(3 \epsilon \mu_{0}+\frac{1}{3} \sigma^{2}+\frac{2}{3} \sigma^{2}\left(1-\frac{3}{2}\right)^{2}\right) \frac{d q}{d t}+2 \epsilon \sigma^{2} \mu_{0}\left(1-\frac{3}{2} \epsilon\right)^{2} q=0 .
$$

The initial conditions are determined in the previous steps as

$$
q(0)=<y^{2}(0)>, q^{\prime}(0)=\left(\epsilon \mu_{0}+\sigma^{2}+\frac{2}{3} \sigma^{2}\left(1-\frac{3}{2} \epsilon\right)^{2}\right)<y^{2}(0)>.
$$

A solution can be easily constructed for the second order differential equation with constant coefficients.

$$
<y^{2}>=C_{1} e^{\left(\lambda_{1}-3 \epsilon \mu_{0}\right) t}+C_{2} e^{\left(\lambda_{2}-3 \epsilon \mu_{0}\right) t},
$$

where

$$
\begin{aligned}
\lambda_{1,2} & =\frac{1}{2}\left(3 \epsilon \mu_{0}+\frac{1}{3} \sigma^{2}+\frac{2}{3} \sigma^{2}\left(1-\frac{3}{2} \epsilon\right)^{2}\right) \\
& \pm \sqrt{\frac{1}{4}\left(3 \epsilon \mu_{0}+\frac{1}{3} \sigma^{2}+\frac{2}{3}\left(1-\frac{3}{2} \epsilon\right)^{2}\right)^{2}-2 \epsilon \sigma^{2} \mu_{0}\left(1-\frac{3}{2} \epsilon\right)^{2}}
\end{aligned}
$$

and

$$
\begin{aligned}
& C_{1}=\frac{<y^{2}(0)>}{\lambda_{1}-\lambda_{2}}\left(\left(\epsilon \mu_{0}+\frac{1}{3} \sigma^{2}+\frac{2}{3} \sigma^{2}\left(1-\frac{3}{2} \epsilon\right)^{2}\right)-\lambda_{2}\right), \\
& C_{2}=\frac{<y^{2}(0)>}{\lambda_{1}-\lambda_{2}}\left(\lambda_{1}-\left(\epsilon \mu_{0}+\frac{1}{3} \sigma^{2}+\frac{2}{3} \sigma^{2}\left(1-\frac{3}{2} \epsilon\right)^{2}\right)\right) .
\end{aligned}
$$

The coefficients, $C_{1}$ and $C_{2}$, are calculated from the two initial conditions. For $t \gg 0,<y^{2}>$ is governed by $C_{1} e^{\left(\lambda_{1}-3 \epsilon \mu_{0}\right) t}$ and hence we can write

$$
<y^{2}>\sim C_{1} e^{\left(\lambda_{1}-3 \epsilon \mu_{0}\right) t},
$$


and we can compare this result with the single map. For the single map, the stochastic solution is $x(0) e^{\left(\mu_{0}-\frac{1}{2} \sigma^{2}\right) t+\sigma W}$. Because $W$ follows $N(0, t)$, it is possible to find the $\left\langle x^{2}\right\rangle=x^{2}(0) e^{\left(\mu_{0}+\sigma^{2}\right) t}$. Figure 13 shows the comparison of $<y^{2}>$ between the single map and the locally connected map. The $x$-axis represents the interaction coefficient $\epsilon$ and the $y$-axis is the difference of exponential exponent between the two cases. In this figure, $\lambda_{1}-3 \epsilon \mu_{0}-\sigma^{2}$ is negative for all interaction coefficients. The decrease of growth rate compared with that of the single map is caused by the local connection which suppresses the growth of the second moment of each map in the locally connected maps.

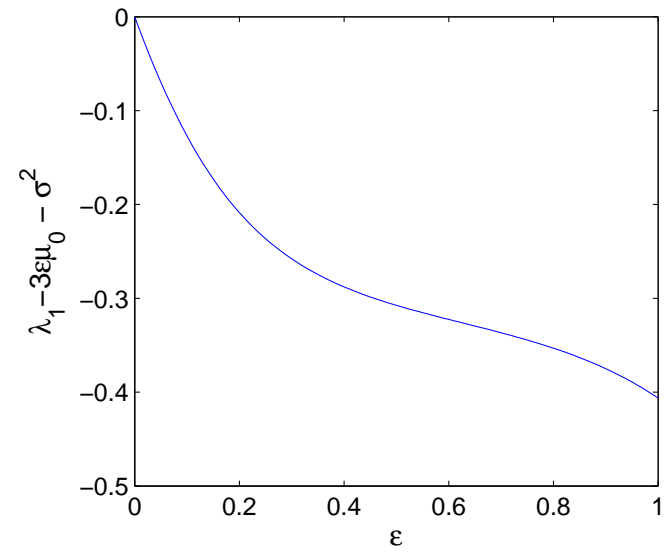

Figure 13: comparison of $<y^{2}>$ between the single map and the locally connected maps

This result seems not to support the generation of more on stages in locally connected maps. A more revealing aspect may be the correlation between two adjacent maps. From the equation (12), we can find an equation for $\left\langle y_{1} y_{2}\right\rangle$.

$$
\begin{aligned}
<y_{1} y_{2}> & =\left(\frac{1}{3}-\frac{1}{3} e^{-3 \epsilon \mu_{0} t}\right)<y^{2}(0)> \\
& +\sigma^{2} \int_{0}^{t}\left(\frac{1}{3}-\frac{1}{3}\left(1-\frac{3}{2} \epsilon\right)^{2} e^{-3 \epsilon \mu_{0}\left(t-t^{\prime}\right)}\right)<y^{2}>d t^{\prime}
\end{aligned}
$$

In this equation $<y_{1} y_{2}>$ is determined by $\left\langle y^{2}>\right.$ which we calculated previously. Before finding the value of the integral, we discuss its structure. According to our previous calculation $\left\langle y^{2}\right\rangle$ is an exponentially increasing function with time. The correlation $\left\langle y_{1} y_{2}\right\rangle$ is the time integral of $\left\langle y^{2}\right\rangle$. Therefore, we can expect that $\left\langle y_{1} y_{2}\right\rangle$ increases exponentially with time. If we only care about the leading order form of the solution, we can approximate $\left\langle y_{1} y_{2}>\right.$ as

$$
<y_{1} y_{2}>\sim \frac{1}{3} C_{1}\left[\frac{1}{\lambda_{1}-3 \epsilon \mu_{0}}-\frac{\left(1-\frac{3}{2} \epsilon\right)^{2}}{\lambda_{1}}\right] e^{\left(\lambda_{1}-3 \epsilon \mu_{0}\right) t} .
$$

Obviously, the coefficients multiplying the exponential are positive. So, the correlation between two adjacent maps increases exponentially.

Returning to our linearized coupled equations, we can apply the above results to interpret each term in the equation (22). First, our underlying assumption 
is that the system is unstable such that the signal, $y_{k}$, increases on average. Temporarily, $y_{k}$ can experience a sudden or steady decrease but $y_{k}$ eventually increases if we consider long time periods. We found from the above analysis that the $y_{k}$ are positively correlated with the $y_{k+1}$ and the $y_{k-1}$. In equation (22), the term $-\epsilon \mu_{0} y_{k}$ decreases the growth rate of $\left\langle y_{k}^{2}\right\rangle$ compared with that of a single model. The term $\epsilon \mu_{0}\left(y_{k+1}+y_{k-1}\right) / 2$ has the most important role in this locally connected system due to the fact that $y_{k+1}$ and $y_{k-1}$ are positively correlated with $y_{k}$. If $y_{k}$ starts to move away from the fixed point $\left(y_{k}=0\right)$, it provides positively correlated signals to $y_{k}$. More specifically, if $y_{k}$ is negative, this term provides negative values for $y_{k}$ at the next time step. If $y_{k}$ is positive, the opposite sequence occurs. This term drives $y_{k}$ away from the fixed point. When we consider other terms $(1-\epsilon) \sigma \xi_{k} y_{k}+\epsilon \sigma\left(\xi_{k+1} y_{k+1}+\xi_{k-1} y_{k-1}\right) / 2$, we must recall that $y_{k}, y_{k+1}$ and $y_{k-1}$ are positively correlated, and $\xi_{k+1}, \xi_{k}$ and $\xi_{k-1}$ are independent Gaussian white noise terms. The sum of these noisy terms is negligible compared with the other terms. As a result, local connection sets up the condition for the positive correlation between adjacent systems, which provides the positively correlated signals to one specific map.

Our calculation in the context of a linearized local connection gives a picture of how the signals among adjacent maps evolve and transfer during a long time period of the off stage. Under unstable conditions, adjacent maps together have the tendency to escape from the fixed point. This process is rapidly accelerated due to the transfer of positively correlated signals from one specific map to adjacent maps. This mechanism can explain the generation of more on stages when we increase the coupling coefficient $\epsilon$.

\section{Conclusion}

The Ricker Map which is represented as $N_{t+1}=N_{t} e^{r_{t}\left(1-N_{t}\right)}$ has a fixed point at $N_{t}=1.0$. The fixed point is stable or unstable depending on the value of $r_{t}$ relative to 2.0. If $r_{t}$ is driven by Gaussian white noise near $r_{t}=2.0$, on-off intermittency is generated. The on-off intermittency in the Ricker Map has characteristics consistent with those discussed in previous work. The power spectrum for on-off intermittency shows a clear slope in log-log scale plots. Most importantly, the probability density function $\phi$ for the duration of the off stages in the Ricker Map is also proportional to $\phi^{-3 / 2}$. This consistency leads us to a more realistic consideration of the Ricker Map.

The Ricker Map is used for the evolution of the population of one species at one generation. A realistic environment in this species is not isolated from other similar systems. Therefore, on-off intermittency in the Ricker Map is investigated under a locally connected situation. That mimics the possible interaction among neighboring species under the same environmental conditions. Even though the exact form of interaction is not shown, as a first step, we use a linear coupling with adjacent maps based on the coupling coefficient $\epsilon$. The magnitude of $\epsilon$ represents the intensity of the interaction. According to numerical simulations, the statistics of on-off intermittency are changed relative to a single map that has the same parameters. Moreover, the stability condition changes due to local coupling. Within some range of $\sigma$, the locally connected Ricker maps become unstable even though the single map is stable under the same parameters. The mechanism for explaining the change caused by the lo- 
cal coupling must be strongly related to the evolution and transfer of signals between adjacent maps.

Signal evolution and transfer of information in a locally coupled system was considered theoretically using a time-continuous model similar to the Ricker Map. This continuous model enables us to use stochastic calculus to make progress. We found that the signal in a specific map belonging to the locally coupled system is positively correlated with the signal in adjacent maps. The positively correlated signals that come from adjacent maps are added to the signal in the map for the next time step. Under unstable conditions, this feedback due to correlations with adjacent maps causes the signal to accelerate away from the fixed point, which explains the change of statistics of on-off intermittency under the local coupling.

\section{Acknowledgments}

I am grateful to Antonello Provenzale for initiating this project and his helpful advice. I am also thankful to Neil Balmforth. After discussion with him, I could realize that I was making mistakes in my calculation. Finally, I thank to all the GFD fellows for sharing great experience with me and especially to those who have spent long night with me in Walsh cottage during this summer.

\section{References}

Bottiglieri, M. \& Godano, C. (2007), 'On-off intermittency in earthquake occurrence', Phys. Rev. E 75(2), 026101.

Heagy, J. F., Platt, N. \& Hammel, S. M. (1994), 'Characterization of on-off intermittency', Physical Review E 49(2), 1140-1150.

Metta, S., Provenzale, A. \& Spiegel, E. A. (2010), 'On-off intermittency and coherent bursting in stochastically-driven coupled maps', Chaos, Solitons 83 Fractals .

Platt, N., Spiegel, E. A. \& Tresser, C. (1993a), 'The intermittent solar cycle', Geophys. Astrophys. Fluid Dynamics 73, 147-161.

Platt, N., Spiegel, E. A. \& Tresser, C. (1993b), 'On-off intermittency : A mechanism for bursting', Physical Review Letters 70(3), 279-282.

Ricker, W. E. (1954), 'Stock and recruitment', J. Fisheries Res. Board Can. 11, 559-623.

Toniolo, C., Provenzale, A. \& Spiegel, E. A. (2002), 'Signature of on-off intermittency in measured signals', Phys. Rev. E 66(6), 066209. 


\title{
Trajectories of a low Reynolds number treadmilling organism near a half-infinite no-slip wall
}

\author{
Kiori Obuse, Supervised by Jean-Luc Thiffeault
}

June-August 2010

\begin{abstract}
This project investigates the behaviour of a treadmilling microorganism in a twodimensional unbounded domain with a half-infinite non-slip wall. The governing evolution equations for such a treadmilling organism are derived in an analytical form and trajectories of a microorganism for several different initial positions and heading directions are numerically calculated. Then we define and numerically calculate the escaping probability $P_{E}$, the probability that the treadmilling organism can escape from its image on the wall. This $P_{E}$ does not take 0 or 1 value for any physically reasonable initial point. This suggests that the treadmilling organism feels the presence of its image on the wall even when it is placed at a position far from the wall at the initial time, and that, if the initial heading direction is appropriately chosen, it is possible for the microorganism to escape from the wall.
\end{abstract}

\section{Introduction}

The locomotion of microorganisms has received much attraction in fluid dynamics and Biology recently $[1,2]$. As their motion possesses very small length scales and speeds, the dynamics is governed by low Reynolds number hydrodynamics, ${ }^{1}$ where inertial forces are negligible in comparison to the viscous effects of the fluid.

Many studies have been undertaken on such dynamics in unbounded low Reynolds number flows $[3,4,5]$. In reality, however, most organisms are in the vicinity of other bodies or boundaries, where their hydrodynamic interactions with these have a significant effect on their motion. The importance of the existence of boundaries has also been suggested by many experimental observations. For example, some experimental observations $[6,7,8]$ found a qualitative feature of microorganism near a solid wall to be attracted to no-slip boundaries. Berke etal. [9] measured the steady-state distribution of smooth-swimming Escherichia Coli (E. Coli) between two glass plates and found a strong increase of the cell concentration at the boundaries. They also theoretically demonstrated that hydrodynamic interactions of swimming cells with solid surfaces lead to their reorientation in the direction parallel to the surfaces, as well as their attraction to the closest wall. Lauga etal. [10] showed that circular trajectories are natural consequences of their force-free and torque-free swimming and hydrodynamic interactions with the boundary. This leads to a hydrodynamic

\footnotetext{
${ }^{1}$ For example, the Reynolds number associated with the motion of Escherichia coli (E. Coli) is $\mathrm{O}\left(10^{-4}\right)$
} 
trapping of the cells close to the surface. Drescher etal. [11] found that when two nearby Volvox colonies swim close to a solid surface, they attract one another and can form stable bound states in which they waltz or minuet around each other. These observations suggest that, in order to obtain a comprehensive understanding of low Reynolds number locomotions, it is necessary to study hydrodynamic interactions between microorganism and the boundaries.

Some of the phenomena stated above have already been verified by numerical simulations $[12,13]$. However, not many physical explanations have been given to the locomotion of microorganism near boundaries. Berke etal. [9] studied the swimming microorganisms' attraction to boundaries by modeling the swimmer as a force dipole singularity. This simple model captures the attraction of the microorganisms to boundaries. However, contrary to the experimental findings, the microorganism in this model crashes into the boundary in finite time. Moreover, their analysis did not investigate the dynamics of microorganism near the boundary. Or and Murray [14] studied the dynamics of low Reynolds number swimming organism near a plane wall. They analysed the motion of a swimmer consisting of two rotating spheres connected by a thin rod, as a simple theoretical model of swimming organisms. They found that when the spheres are rotated in unequal velocities, the swimmer has a solution of steady translation parallel to the wall, and that under small perturbation, the swimmer exhibits wave-like motions along the wall. These results have recently been verified experimentally on a macroscale robotic prototype swimming in a highly viscous fluid [15]. Furthermore, Crowdy and Or [16] have proposed a singularity model for swimming microorganisms placed near an infinite no-slip boundary. Their model was based on a circular treadmilling organism which has no means of self-propulsion. They proposed the appropriate Stokes singularities that represented the flow field created by this treadmilling organism in the global fluid. By studying the interaction between these singularities and the no-slip wall, they formulated the nonlinear dynamics of the treadmilling organism explicitly, and fully characterised its motion near the wall. The trajectories parallel to the wall and periodic bouncing motion along the wall found in Crowdy and Or [16] had remarkable similarities to the trajectories shown in Or and Murray [14]. Crowdy and Samson [17], utilising the singularity model, investigated the dynamics of treadmilling organism near an infinite no-slip boundary with a gap of a fixed size. They employed a conformal mapping technique to avoid the difficulty in treating the image of the treadmilling organism on the wall. In their model, the treadmilling organism was found to make several qualitatively different trajectories; jumping over the gap, rebounding from the gap, trapping near the gap, and escaping the gap region even when the organism has the initial point in the gap. They also performed a bifurcation analysis as the size of the organism varies, and demonstrated a presence of stable equilibrium points in the gap region as well as Hopf bifurcations to periodic bound states. This reduced model system also exhibited a global gluing bifurcation in which two symmetric periodic orbits merge at a saddle point into symmetric bound states having more complex spatio-temporal structure.

The present project examines the dynamics of a treadmilling organism near a halfinfinite no-slip wall (a corner whose angle is $\pi$ ). This is an important preliminary step to clarifying the dynamics of a treadmilling organism near a no-slip corner with an arbitrary angle, which will shed light on the cases with more complicated realistic geometries. 


\section{Two-dimensional Stokes flow problem and Goursat func- tions}

\subsection{General Solution for Stokes equation}

In many of existing three-dimensional theoretical models of low Reynolds number locomotions, swimming microorganisms possess symmetry about their sagittal plane $[14,18]$ or even about their longitudinal axis $[4,5,19]$. This suggests that even in a fully three-dimensional flow, the motions of microorganisms are confined to a two-dimensional plane. Consequently, here we consider the two-dimensional model for a microorganism in a $(x, y)$-plane, in which we shall be free to treat the flow in a complex plane (z-space), where $z \equiv x+i y$. While the complex function method is one of the classical methods applied to ideal flows, a similar analytical method may also be applied to two-dimensional Stokes flows [20, 21]. Here, the

complex method for Stokes flows is introduced to show that the two-dimensional Stokes flow is expressed through a couple of complex functions. This helps us to understand the flow clearly.

Stokes equations which describe the motion of an incompressible viscous fluid are

$$
\begin{aligned}
& \nabla p=\eta \Delta \mathbf{u}, \\
& \nabla \cdot \mathbf{u}=0,
\end{aligned}
$$

where $\Delta$ is a Laplace operator, $\mathbf{u}=\left(u_{x}, u_{y}\right)$ is the fluid velocity, $p$ and $\eta$ are the pressure distribution and dynamic viscosity, respectively. As we are considering a two-dimensional flow, we can introduce the stream function $\psi$, such that the velocity is given by

$$
u_{x}=\partial \psi / \partial y, \quad u_{y}=-\partial \psi / \partial x
$$

then the Stokes equations (1) reduce to biharmonic equation

$$
\Delta^{2} \psi=0
$$

Now we define complex velocity $W$ as

$$
\begin{aligned}
u_{x}+i u_{y} & =\frac{\partial \psi}{\partial y}-i \frac{\partial \psi}{\partial y} \\
& =-2 i \frac{\partial \psi}{\partial \bar{z}} .
\end{aligned}
$$

The vorticity $\omega=-\nabla^{2} \psi$ is then given by

$$
\omega=-\nabla^{2} \psi=2 i \frac{\partial W}{\partial \bar{z}} .
$$

By substituting (2) and (4) into the Stokes equations (1), we obtain

$$
\frac{\partial p}{\partial x}=-\eta \frac{\partial \omega}{\partial y}, \quad \frac{\partial p}{\partial y}=\eta \frac{\partial \omega}{\partial x} .
$$

The equations in (5) are the Cauchy-Riemann equations on the pair $p$ and $-\eta \omega$. It follows that $p-i \eta \omega$ is an analytic function of $z$, and so that it can be written as

$$
p-i \eta \omega=4 \eta f^{\prime}(z)
$$


(a)
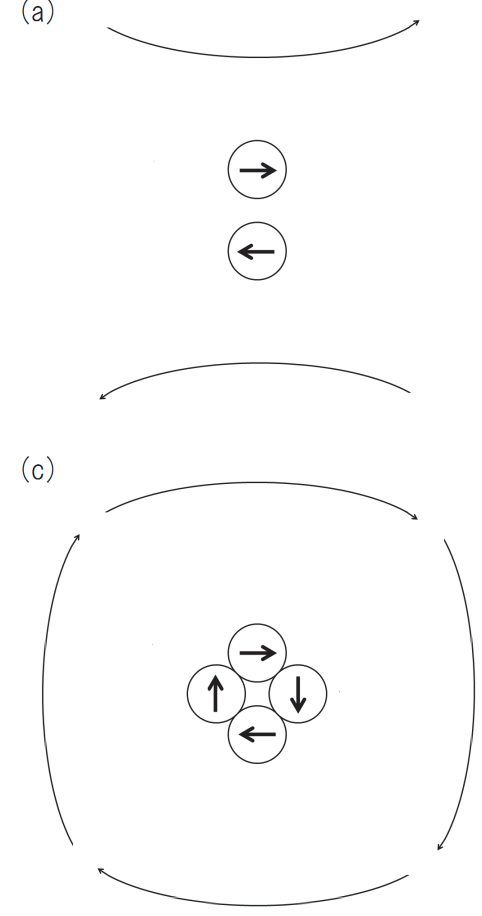

(b)

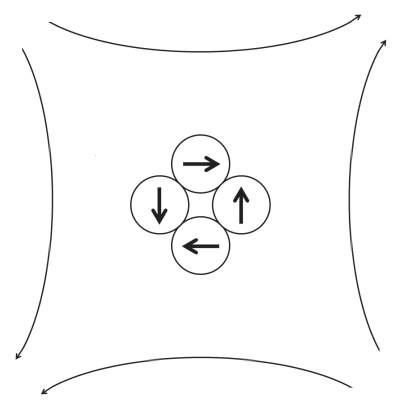

Figure 1: Schematic description of a (a): Stokes dipole (b): stresslet (b): rotlet.

where $f^{\prime}(z)$ is an arbitrary analytic function of $z$. Taking the imaginary part of (6), it is obvious that, by means of the function $f(z), \omega$ can be written as

$$
\omega=-4 \operatorname{Im}\left[f^{\prime}(z)\right] .
$$

Substituting (7) into (4) and integrating it with respect to $z$, we obtain

$$
W=u_{x}+i u_{y}=f(z)+z \overline{f^{\prime}(z)}+\overline{g^{\prime}(z)},
$$

where $g^{\prime}(z)$ is an arbitrary analytic function of $z$. Again, substituting (8) into (4) and integrating it with respect to $z$, we finally obtain the general solution for $\psi$ in (3) as

$$
\operatorname{Im}[\bar{z} f(z)+g(z)]
$$

The fictions $f(z)$ and $g(z)$, which are called Goursat functions, are analytic everywhere in the considered domain, except when isolated singularities are introduced in order to model various flows of interest. The functions $\bar{z} f(z)$ and $g(z)$ in $(9)$ correspond to the viscous and potential part of the flow, respectively. Note that $g(z)$ does not appear (7), since potential flow has zero vorticity.

\subsection{Singular solutions of Stokes flow}

The Goursat functions $f(z)$ and $g(z)$ which determine the stream function of the flow are analytic almost everywhere in a considered domain except at isolated singular points. It is 
necessary to choose appropriate singularities for the Goursat functions in order to describe the flow of interest. What kind of singularities should we use in order to model the flow generated by a treadmilling organism near a no-slip half-infinite wall? This is one of the main problem we should cope with for the formulation of the governing evolution functions of the treadmilling organism, and this will be discussed later in $§ 3.1$. Here in this section, the basic singularities of the Stokes flow are discussed. The flow generated by a treadmilling organism may be modeled with some of these singularities.

First of all, let us consider $f(z)$ of the form

$$
f(z)=\mu \log \left(z-z_{d}\right),
$$

where $\mu \in \mathbf{C}$ is the strength of the singularity. Then we insist that the complex velocity, given by (8),

$$
\begin{aligned}
u_{x}+i u_{y} & =-\mu \log \left(z-z_{d}\right)+\frac{\bar{\mu} z}{\bar{z}-\overline{z_{d}}}+\bar{g}^{\prime}(\bar{z}) \\
& =-\mu \log \left(z-z_{d}\right)+\frac{\bar{z}\left(z-z_{d}\right)}{\bar{z}-\overline{z_{d}}}+\frac{\bar{\mu} z_{d}}{\bar{z}-\overline{z_{d}}}+\bar{g}^{\prime}(\bar{z})
\end{aligned}
$$

should be both single-valued and, at least, logarithmically singular at $z_{d}$. From these constraints, $g(z)$ should be chosen to have the form which satisfies

$$
g^{\prime}(z, t)=-\frac{\mu \overline{z_{d}}}{\bar{z}-\overline{z_{d}}}-\bar{\mu} \log \left(z-z_{d}\right) .
$$

Hence, if the Goursat functions $f(z)$ and $g(z)$ are

$$
\begin{aligned}
f(z) & =\mu \log \left(z-z_{d}\right)+\text { analytic function, } \\
g^{\prime}(z) & =-\frac{\mu \overline{z_{d}}}{\bar{z}-\overline{z_{d}}}-\bar{\mu} \log \left(z-z_{d}\right)+\text { analytic function, }
\end{aligned}
$$

the flow described by these Goursat functions (13) is the one generated by Stokeslet at $z_{d}$. The complex velocity (11) takes the form

$$
u_{x}+i u_{y} \sim-\mu \log \left|z-z_{d}\right|
$$

and grows logarithmically as $z \rightarrow \infty$. This is the heart of the Stokes Paradox. In order to avoid the Stokes paradox, we must restrict our attention to Stokes flow problems which do not contain any Stokeslet. Note that imposing certain singularities to $f(z)$ forces $g$ some concomitant singularities. Stokes dipole is obtained by superposing two Stokeslet, drawing infinitesimally close together, and with equal and opposite strengths tending to infinity at a rate inversely proportional to their separation. This is schematically shown in Fig.1 (a).

Next, if we allow $f(z)$ to have a simple pole at $z_{d}$,

$$
f(z)=\frac{\mu}{z-z_{d}}
$$

where $\mu \in \mathbf{C}$, and insist that the complex velocity, given by (8), should be singular like $1 /\left|z-z_{d}\right|$, not line $1 /\left|z-z_{d}\right|^{2}$. Then, again, the singularity in $f(z)$ forces $g(z)$ to have singularities, and we find that $g(z)$ should be chosen to have the form which satisfies

$$
g^{\prime}(z)=\frac{\mu \overline{z_{d}}}{\left(\bar{z}-\overline{z_{d}}\right)^{2}} \text {. }
$$


This $g(z)$ corresponds to an irrotational dipole contribution of strength $-\mu \overline{z_{d}}$. If

$$
\begin{aligned}
& f(z) \frac{\mu}{z-z_{d}}+\text { analytic function, } \\
& g^{\prime}(z)=\frac{\mu \overline{z_{d}}}{\left(\bar{z}-\overline{z_{d}}\right)^{2}}+\text { analytic function, }
\end{aligned}
$$

we say that there is a stresslet of strength $\mu$ at $z_{d}$. This is given by the addition of two Stokes dipoles oriented at $90^{\circ}$ with respect to each other as schematically shown in Fig.1 (b).

When $f(z)$ has singularities, as already stated above, $g(z)$ should have concomitant singularities to those in $f(z)$, but not conversely; $g(z)$ can have singularities which is independent of those of $f(z)$. These are kinematically identical to the singularities of the complex potential flow in two-dimensional incompressible and irrotational flow. Let us allow $g(z)$ to admit, for instance, a logarithmic singularity, which means simple pole in $g^{\prime}(z)$

$$
g(z)=c \log \left(z-z_{d}\right)
$$

where $c \in \mathbf{R}$ or $\in \mathbf{C}$. If $c \in \mathbf{R} z_{d}$ is a source/sink, whilst if $c \in \mathbf{C} z_{d}$ is a rotlet(or couplet). A rotlet is given by the difference between two Stokes dipoles oriented at $90^{\circ}$ with respect to each other as schematically shown in Fig.1 (c), and this is kinematically identical to a point vortex [22]. Similarly, a double pole of $g^{\prime}(z)$ corresponds to an irrotational dipole singularity, a triple pole corresponds to an irrotational quadrupole and so on.

With regard to modelling a swimming micro-organism, we are free to choose any combination of such Stokes flow singularities which seems to be appropriate for the problem at hand. However, each of these singularities will be a function of the swimmers size, shape, its swimming protocol and its local effect on the fluid around it. The attention is therefore focused on what specific combination of singularities can be used to accurately model a physical microorganism.

\section{The model for a treadmilling tiny swimming organism}

\subsection{Basic settings and boundary conditions}

Here we describe our simple model for a treadmilling organism. Any swimming organism in a Stokes flow locally generates a flow that can be modelled by some combination of Stokes flow singularities described in $\S 2.2$ placed on its position or its boundary. This singularity distribution is generally a complicated function of the swimming organism's basic properties such as size, shape, and configuration of cilia. The treadmilling organism does not have any self-propulsion in itself, but moves around in the considered domain when there is a boundary near it $[6,7,10,9,11,12,13,9,14,15,16,17]^{2}$. It suggests that if a treadmilling organism is influenced by its surroundings such as other swimming organisms or a solid boundary, and behaves in a different way from when it is in isolation.

\footnotetext{
${ }^{2}$ Strictly, "treadmilling" and "swimming" describe different behaviours of the organism, and here in this project, we are only considering treadmilling organisms. However, as a treadmilling organism can move in the flow when there is a no-slip wall around it, we occasionally use the description "swimming organism" when we focus upon the organism's moving behaviour in the flow.
} 


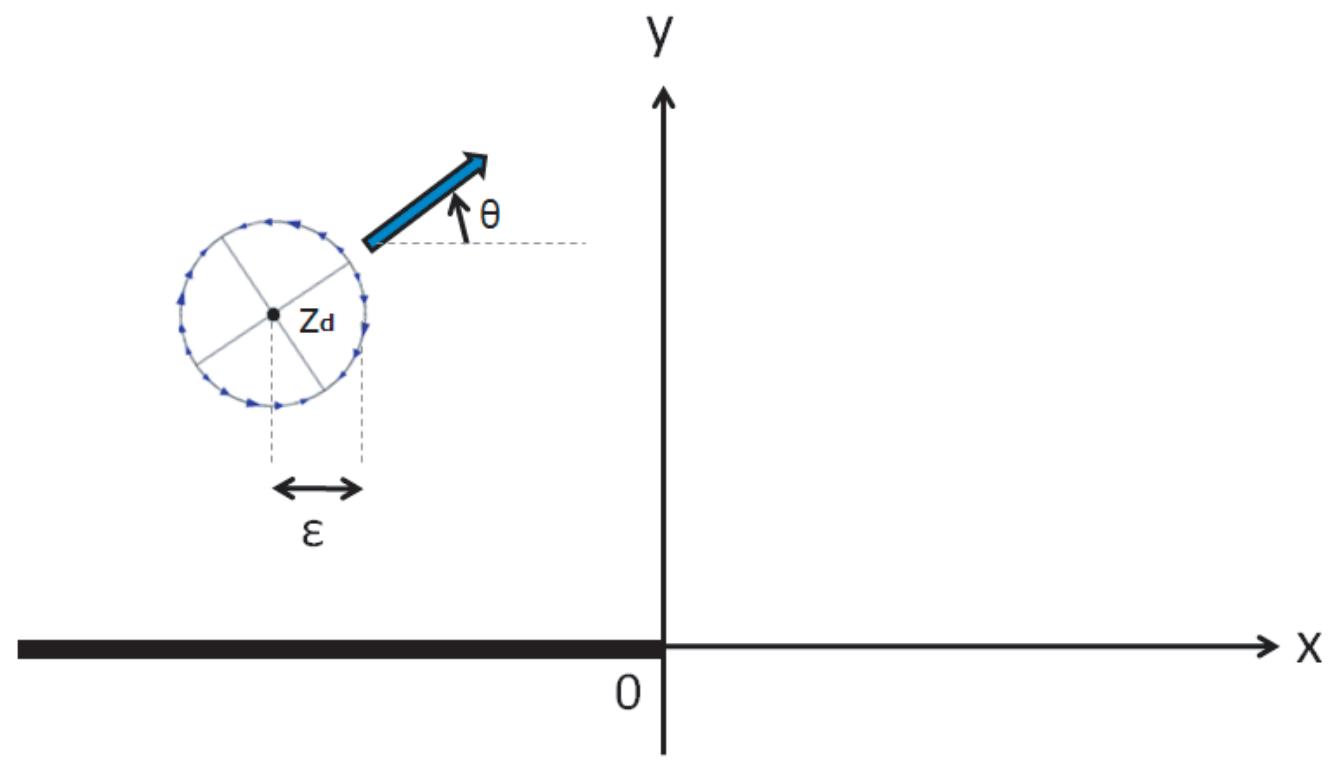

Figure 2: Simple model for a treadmilling organism. $z_{d}, \epsilon$, and $\theta$ are position of the organism, radius of the circular body of the organism, and the angle of the head of the organism from the real axis, respectively.

This means that, strictly speaking, the singularities in the Goursat functions referred above should be replaced by effective singularities, which will be complicated functions, according to the treadmilling organism's surroundings. However, as a first approximation model of a treadmilling organism's hydrodynamic interaction effects with a wall, it seems to be reasonable to consider the treadmilling organism as passive. It follows that the treadmilling organism always keeps its characteristics irrelevant to its surroundings.

In a simple theoretical model, we assume that the treadmilling organism has a circle body of radius $\epsilon$, with a moving centre $z_{d}(t)=x_{d}(t)+i y_{d}(t)$ in the fluid. We also assume that, with respect to the angle of the head of the treadmilling organism from the real axis, $\theta(t)$, surface actuators of the treadmilling organism induce a tangential velocity profile given by

$$
\begin{aligned}
U(\phi, t) & =2 V \sin (2(\phi-\theta)) \\
& =c(t) \exp (2 i \phi)+\overline{c(t)} \exp (-2 i \phi), \quad c(t) \equiv-i V \exp (-2 i \theta(t)) .
\end{aligned}
$$

Here $V$ is a constant whose magnitude sets the time scale for the treadmilling action, $\phi$ is the angle measured from the positive $x$ direction and $\phi=\theta$ is the direction of the head of the treadmilling organism (Fig.2). Note that when the treadmilling organism produces a time-dependent tangential velocity profile, the Stokes equations imply that the fluid reacts to it instantaneously. Also note that in unbounded fluid, this velocity profile does not result in any self-propulsion of the organism due to its symmetry about real and imaginary axes.

The two Goursat functions $f(z, t)$ and $g(z, t)$, which give the stream function of the flow, should satisfy two boundary conditions: boundary conditions on the no-slip wall and 
on the surface of the treadmilling organism. The boundary condition on the no-slip wall is, from (8),

$$
u+i v=-f(z, t)+z \overline{f^{\prime}(z, t)}+\overline{g^{\prime}(z, t)}=0 \text { on the wall. }
$$

Next, suppose that the centre of the treadmilling organism $z_{d}$ moves in a translational velocity $\dot{x_{d}}(t)+i \dot{y_{d}}(t)$, and the organism rotates with an angular velocity $\omega(t)$. Then the boundary condition of the velocity field around the treadmilling organism can be written as

$u_{x}+i u_{y}=-f(z, t)+z \overline{f^{\prime}(z, t)}+\overline{g^{\prime}(z, t)}=\dot{x_{d}}(t)+i \dot{y}_{d}(t)+[\epsilon \Omega+U(\phi, \theta(t))] \frac{d z}{d s}, \quad$ at $\left\{z|| z-z_{d} \mid=\epsilon\right\}$,

where $d z / d s$ is the complex unit tangent to the boundary. Here, the relations

$$
z-z_{d}=\frac{\epsilon^{2}}{\bar{z}-\overline{z_{d}}}, \quad \frac{d z}{d s}=i \frac{\left(z-z_{d}\right)}{\epsilon}
$$

have been used.

\subsection{Singularities of $f(z, t), g(z, t)$ and governing evolution equations of a treadmilling organism near a half-infinite wall}

As it was mentioned in $\S 2$, the flow generated by a treadmilling organism in a Stokes flow can be locally modelled by some combination of Stokes flow singularities. However, it is not obvious what singularities should be chosen for each considered situation. A low Reynolds number treadmilling organisms exert no net force or torque upon the flow around it. Consequently, here we introduce a mathematical representation of the treadmilling organism as a two-dimensional stresslet, which is accompanied by additional singularities to those for the stresslet in its Goursat functions. Note that neither a Stokeslet nor a rotlet should be used for modelling the treadmilling organism, since the treadmilling organism should be force-free and torque-free. The type of the singularities in Goursat functions should be chosen such that the Goursat functions satisfy two boundary conditions (19) and (20). For this, we employ "image system method" $[23,16]$. It is done by introducing additional singularities at $z=\overline{z_{d}}$, physically understood as the image of the treadmilling organism, to the Goursat functions.

The determination of the Goursat functions is basically performed by trial and error. Here we seek solutions for these Goursat functions having the form

$$
\begin{aligned}
& f(z, t)=\frac{\mu}{z-z_{d}(t)}+f_{0}+f_{1}\left(z-z_{d}(t)\right)+\cdots, \\
& g^{\prime}(z, t)=\frac{b}{\left(z-z_{d}(t)\right)^{3}}+\frac{a}{\left(z-z_{d}(t)\right)^{2}}+\cdots .
\end{aligned}
$$

$f(z, t)$ having no Stokeslet and $g(z, t)$ having no rotlet imply that the treadmilling organism is force-free and torque-free. Now we use the boundary condition (20) to find relations between the coefficients in (21) and the velocity of the treadmilling organism. On the surface of the treadmilling organism, where $\left|z-z_{d}\right|=\epsilon$,

$$
z-z_{d}=\frac{\epsilon^{2}}{\bar{z}-\overline{z_{d}}}, \quad \frac{d z}{d s}=i \frac{z-z_{d}}{\epsilon},
$$


hold. Substituting (18) and (22) into (20) then equating coefficients of different powers of $z-z_{d}$, we obtain

$$
\begin{aligned}
\dot{x_{d}}+i \dot{y}_{d} & =-f_{0}+\overline{f_{1}} z_{d}+\overline{g_{0}}, \\
i \Omega & =-\left(f_{1}-\overline{f_{1}}\right), \\
\mu & =-i \epsilon \bar{c}, \\
a & =\mu \overline{z_{d}}, \\
b & =\mu \epsilon-i \bar{c} \epsilon^{3}=2 \mu \epsilon^{2} .
\end{aligned}
$$

Thus the Goursat functions $f(z, t)$ and $g^{\prime}(z, t)$ have the form

$$
\begin{aligned}
& f(z, t)=\frac{\mu(t)}{z-z_{d}(t)}+f_{0}(t)+f_{1}(t)\left(z-z_{d}(t)\right)+\mathcal{O}\left(\left(z-z_{d}(t)\right)^{2}\right), \\
& g^{\prime}(z, t)=\frac{2 \mu(t) \epsilon^{2}}{\left.\left(z-z_{d}(t)\right)^{3}\right)}+\frac{\mu(t) \overline{z_{d}(t)}}{\left.\left(z-z_{d}(t)\right)^{2}\right)}+g_{0}(t)+\mathcal{O}\left(\left(z-z_{d}(t)\right)\right),
\end{aligned}
$$

around $z_{d}$. This means that the treadmilling organism has an equivalent point singularity description with a stresslet of strength $\mu$, corresponds to the terms $\mu /\left(z-z_{d}\right)$ in $f(z)$ and $\left(\mu z_{d}\right) /\left(z-z_{d}\right)^{2}$ in $g^{\prime}(z)$, and the quadrupole of strength $2 \epsilon^{2} \mu$ at $z_{d}$, corresponds to the term $\left(2 \epsilon^{2} \mu\right) /\left(z-z_{d}\right)^{3}$ in $g^{\prime}(z)$. Note that the existence of the quadrupole enables this model to describe a treadmilling organism with a finite size; for the pure point treadmilling organism, $\epsilon=0$, the quadrupole vanishes. We also set the time scale of the motion by letting $V=\epsilon^{-1}$ so that

$$
\mu(t)=\exp (2 i \theta(t))
$$

Since the treadmilling organism is considered as a passive scalar here, the temporal change of its position follows the velocity of the fluid around it. Here, however, we should be aware that the velocity components which originates from the position of the treadmilling organism cannot affect the treadmilling organism itself by definition of "treadmilling". With these consideration, $\dot{z}=\dot{x_{d}}+i \dot{y}_{d}$ is given by the analytic part of the flow velocity (8) at $z=z_{d}$, and can be exactly written as

$$
\frac{d z_{d}}{d t}=-f_{0}+z_{d} \overline{f_{1}}+\overline{g_{0}}
$$

Similarly, the temporal change of the heading angle of the treadmilling organism is given from the half of the analytic part of the vorticity of the flow $(7)$ at $z=z_{d}$ :

$$
\frac{d \theta}{d t}=-2 \operatorname{Im}\left[f_{1}\right]
$$

Hence, from (26) and (27), if we know $f_{0}, f_{1}$, and $g_{0}$ of $f(z)$ and $g(z)$ that satisfy the conditions (19) and (20), we obtain the governing dynamical system of the treadmilling organism in an explicit form and know its trajectory. 

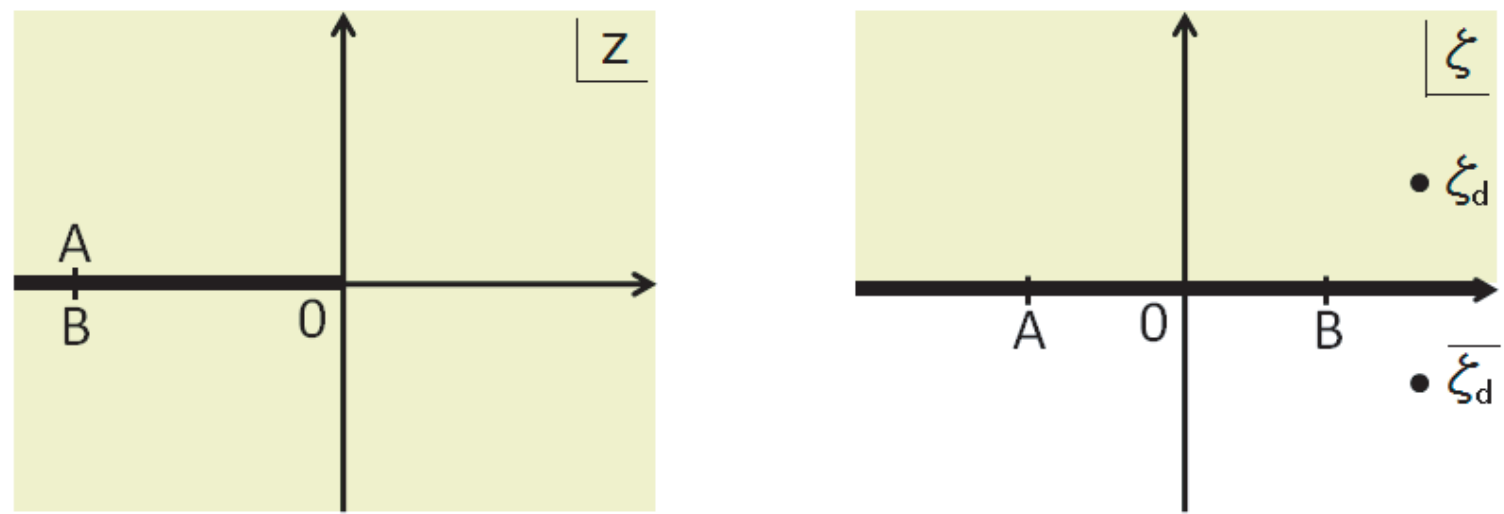

Figure 3: Considered domain in $z$-space and $\zeta$-space. The points $A$ and $B$ in both spaces are mapped to each other by the conformal mapping $\zeta=i z^{1 / 2}$ and its inverse mapping $z=z(\zeta)=-\zeta^{2}$, then the infinite wall in $\zeta$-space corresponds to the half-infinite wall in $z$-space.

\subsection{Conformal mapping}

The Goursat functions in (24) are the solution for the stream function for the flow generated in the vicinity of the treadmilling organism without accounting for the effect of the wall. To know the Goursat functions that have the form (24) near $z_{d}$ and that also satisfy a boundary condition $u_{x}+i u_{y}=0$ on the wall, we have to find the coefficients $f_{0}, f_{1}$ and $g_{0}$ such that the velocity satisfies the boundary condition on the wall. It, however, is not straightforward to determine such coefficients in a region near a half-infinite wall of an infinitesimal width in $z$-space, since where to place the image of the swimmer is not clear as we are now considering a half-infinite wall of infinitesimal width. To treat this complicated situation, here we employ conformal mapping techniques. Although the boundary value problem we have here is not conformally invariant, these techniques still work, since we always come and go to the two spaces.

let us consider a mapping

$$
\zeta=i z^{1 / 2}
$$

This maps the domain $\{z \in \mathbf{C}\}$ to $\{\zeta \mid \operatorname{Im}(\zeta) \geq 0\}$, especially, the domain $\{z \mid \operatorname{Im}(z)=$ $0, \operatorname{Re}(z) \leq 0\}$. The half-infinite wall we are considering in $z$-space are mapped to the domain $\{\zeta \mid \operatorname{Im}(\zeta)=0\}$, the infinite wall in $\zeta$-space(Fig.3). The inverse of this conformal mapping (28) can be explicitly written as

$$
z=z(\zeta)=-\zeta^{2}
$$

When written as a function of $z$, the function $\zeta(z)$ has the same type of point singularity with the one required for $f(z)$ and $g(z)$. It follows that $\zeta$ can serve as a uniformisation variable for the problem, and that if we define the composed functions

$$
\begin{aligned}
F(\zeta) & \equiv f(z(\zeta)), \\
G(\zeta) & \equiv g^{\prime}(z(\zeta)),
\end{aligned}
$$

$F(\zeta)$ and $G(\zeta)$ can be written as single-valued functions of the variable $\zeta$ and are analytic except at the position of the isolated singularities we impose to these functions to describe 
the treadmilling organism and its image. In other words, by utilising this uniformising $\zeta$ $(\zeta$-space), we can write two analytic functions which determine the flow as single-valued functions with no branch point singularities.

From Crowdy and Or [16] and Crowdy and Samson [17], we propose the function $F(\zeta)$ to have the form

$$
F(\zeta)=\frac{A}{\zeta-\zeta_{d}}+\frac{B}{\left(\zeta-\overline{\zeta_{d}}\right)^{3}}+\frac{C}{\left(\zeta-\overline{\zeta_{d}}\right)^{2}}+\frac{D}{\left(\zeta-\overline{\zeta_{d}}\right)}+E,
$$

where we can set $E$ to be zero without loss of generality because of an additive degree of freedom in the definition of $f(z) . \overline{\zeta_{d}}$ corresponds to the image of the swimmer on the wall, and in the $\zeta$-space, this is simply a complex conjugate of $\zeta_{d}$. Utilising this and the inverse mapping (29), we define the image of the swimmer in $z$-space as

$$
\overline{z_{d}} \equiv \overline{z\left(\zeta_{d}\right)} \equiv z\left(\overline{\zeta_{d}}\right)
$$

Recalling the non-slip boundary condition on the wall in $z$-space $(19), G(\zeta)$ should satisfy

$$
\begin{aligned}
G(\zeta) & =\overline{f(z)}-\bar{z} f^{\prime}(z) \\
& =\overline{F(\zeta)}-\bar{z} \frac{d \zeta}{d z} F^{\prime}(\zeta) \\
& =\overline{F(\zeta)}-\overline{(-i \zeta)^{2}} \frac{1}{2} \zeta(-i \zeta)^{-2} F^{\prime}(\zeta) \\
& =\bar{F}(\zeta)-\frac{1}{2} \zeta \overline{(-i \zeta)^{2}}(-i \zeta)^{-2} F^{\prime}(\zeta)
\end{aligned}
$$

where, we have utilised the fact that $\bar{\zeta}=\zeta$ on the wall in $\zeta$-space. Now, as $\zeta=|\zeta| \exp (i \phi), \phi=$ 0 or $\pi$ on the wall,

$$
\begin{aligned}
\overline{(-i \zeta)^{2}}(-i \zeta)^{-2} & =\overline{[|\zeta| \exp (i(-\pi / 2+\phi))]^{2}}[|\zeta| \exp (i(-\pi / 2+\phi))]^{-2} \\
& =[\exp (i(\pi-2 \phi))]^{2}=1
\end{aligned}
$$

holds, so that

$$
G(\zeta)=\bar{F}(\zeta)-\frac{1}{2} \zeta F^{\prime}(\zeta)
$$

By substituting (31) into (32), we obtain

$$
\begin{aligned}
G(\zeta)= & \frac{\bar{A}}{\zeta-\overline{\zeta_{d}}}+\frac{\bar{B}}{\left(\zeta-\zeta_{d}\right)^{3}}+\frac{\bar{C}}{\left(\zeta-\zeta_{d}\right)^{2}}+\frac{\bar{D}}{\left(\zeta-\zeta_{d}\right)} \\
& -\frac{1}{2} \zeta\left[\frac{-A}{\left(\zeta-\zeta_{d}\right)^{2}}+\frac{-3 B}{\left(\zeta-\overline{\zeta_{d}}\right)^{4}}+\frac{-2 C}{\left(\zeta-\overline{\zeta_{d}}\right)^{3}}+\frac{-D}{\left(\zeta-\overline{\zeta_{d}}\right)^{2}}\right] .
\end{aligned}
$$

As $\overline{\zeta_{d}}$ is outside the considered domain in $\zeta$-space, i.e. in the wall, $G(\zeta)$ only has singularity at $\zeta=\zeta_{d}$. Then it follows that $g(z)$ has singularities at $z=z_{d}$ and Moffatt type singularity[24] at $z=0$. 
Now we have to determine the unknown coefficients $A-D \in \mathbf{C}$. For A, we use the fact that $f(z(\zeta))$ has to have the singularity near $z_{d}$ as shown in (24). Performing the Laurent expansion of $1 /\left(\zeta-\zeta_{d}\right)$ in terms of $z$ around $z_{d}$ and comparing (24) and (31), we obtain

$$
A=i \frac{\mu}{2 z_{d}^{1 / 2}} \text {. }
$$

To determine the rest of the unknown coefficients, $B-D$, we insist that $g^{\prime}$ has the required singularity structure in (24) at $z=z_{d}$. If we rewrite $G(\zeta)$ as a function of $z$ and perform Laurent expansion in terms of $z$ around $z_{d}$ and compare it with $g^{\prime}(z)$ in (24), we obtain

$$
\begin{aligned}
& B=i \frac{\epsilon^{2} \bar{\mu}}{4{\overline{z_{d}}}^{3 / 2}}, \\
& C=\frac{\bar{\mu}\left(3 \epsilon^{2}-4 z_{d} \overline{z_{d}}+4{\overline{z_{d}}}^{2}\right)}{8{\overline{z_{d}}}^{2}}, \\
& D=i-\frac{\bar{\mu}\left(3 \epsilon^{2}-4 z_{d} \overline{z_{d}}-4{\overline{z_{d}}}^{2}\right)}{8{\overline{z_{d}}}^{5 / 2}},
\end{aligned}
$$

and $g_{0}$ in $(24)$,

$$
\begin{aligned}
& g_{0}=-\frac{3 \mu \overline{z_{d}}}{16 z_{d}^{2}}+\frac{10 \epsilon^{2} \mu}{32 z_{d}^{3}}+\frac{3 \epsilon^{2} \bar{\mu}}{8\left(z_{d}^{1 / 2}+\bar{z}_{d}^{1 / 2}\right)^{4} \overline{z_{d}}} \\
& -\frac{z_{d}-\overline{z_{d}}}{4\left(z_{d}^{1 / 2}+{\overline{z_{d}}}^{1 / 2}\right)^{3} z_{d}^{1 / 2}}-\frac{\left(-2 z_{d}{\overline{z_{d}}}^{1}+6{\overline{z_{d}}}^{2}+3 \bar{\epsilon}^{2}\right) \bar{\mu}}{16\left(z_{d}^{1 / 2}+{\overline{z_{d}}}^{1 / 2}\right)^{2}{\overline{z_{d}}}^{2}} \\
& -\frac{\left(-2 z_{d} \overline{z_{d}}+6{\overline{z_{d}}}^{2}+3 \bar{\epsilon}^{2}\right) \bar{\mu}}{16\left(z_{d}^{1 / 2}+{\overline{z_{d}}}^{1 / 2}\right){\overline{z_{d}}}^{5 / 2}}
\end{aligned}
$$

Similarly, rewriting $F(\zeta)$ as a function of $z$ and perform Laurent expansion in terms of $z$ around $z_{d}$ and compare with $f(z)$ in (24), and utilising (34) and (35)-(37), we obtain $f_{0}$ and $f_{1}$,

$$
\begin{aligned}
& f_{0}=\frac{\mu}{4 z_{d}}-\frac{\bar{\epsilon}^{2} \bar{\mu}}{4\left(z_{d}^{1 / 2}+{\overline{z_{d}}}^{1 / 2}\right)^{3}{\overline{z_{d}}}^{3 / 2}}+\frac{\left(2 z_{d}{\overline{z_{d}}}^{1 / 2}{\overline{z_{d}}}^{2}-3 \bar{\epsilon}^{2}\right) \bar{\mu}}{8\left(z_{d}^{1 / 2}+{\overline{z_{d}}}^{1 / 2}\right)^{2}{\overline{z_{d}}}^{2}} \\
& -\frac{\left(-2 z_{d} \overline{z_{d}}-2{\overline{z_{d}}}^{2}+3 \bar{\epsilon}^{2}\right) \bar{\mu}}{8\left(z_{d}^{1 / 2}+{\overline{z_{d}}}^{1 / 2}\right){\overline{z_{d}}}^{5 / 2}} \\
& f_{1}=\frac{1}{12 z_{d}^{2}}\left(-\frac{3 \mu}{4}+\frac{9 z_{d}^{3 / 2} \bar{\epsilon}^{2} \bar{\mu}}{2\left(z_{d}^{1 / 2}+\bar{z}_{d}^{1 / 2}\right)^{4}{\overline{z_{d}}}^{3 / 2}}-\frac{3 z_{d}^{3 / 2}\left(2 z_{d}{\overline{z_{d}}}^{2}-2{\overline{z_{d}}}^{2}-3 \bar{\epsilon}^{2}\right) \bar{\mu}}{2\left(z_{d}^{1 / 2}+{\overline{z_{d}}}^{1 / 2}\right)^{3}{\overline{z_{d}}}^{2}}\right. \\
& \left.+\frac{3 z_{d}^{3 / 2}\left(-2 z_{d}{\overline{z_{d}}}^{3}-2{\overline{z_{d}}}^{2}+3 \bar{\epsilon}^{2}\right) \bar{\mu}}{4\left(z_{d}^{1 / 2}+{\overline{z_{d}}}^{1 / 2}\right)^{2}{\overline{z_{d}}}^{5 / 2}}\right) .
\end{aligned}
$$



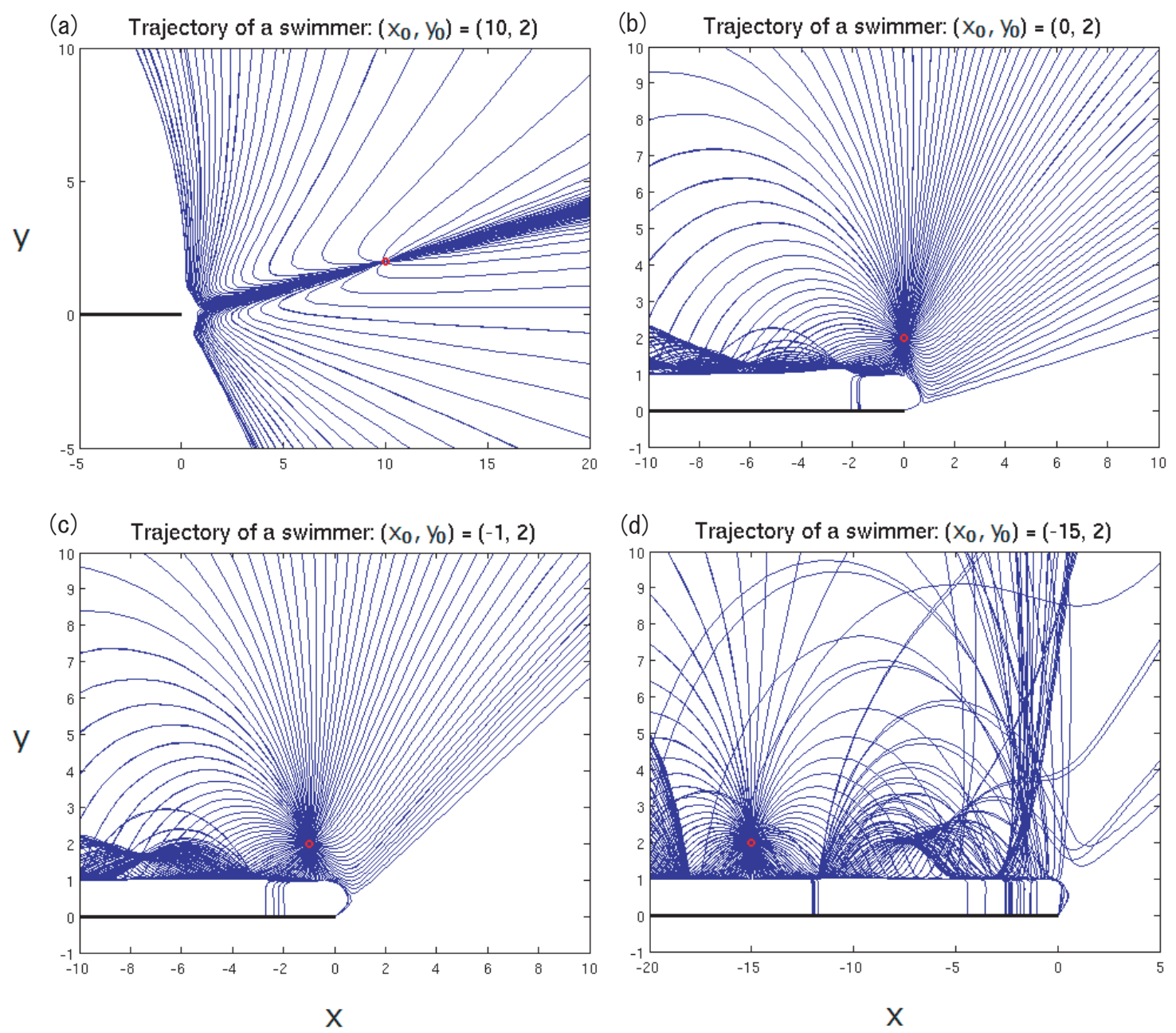

Figure 4: Examples of trajectories from the initial points $\left(x_{d 0}, y_{d 0}\right)$ marked with red circles, each with 200 different initial heading directions $\theta_{0}$. ( $\left.x_{d 0}, y_{d 0}\right)$ is $(\mathrm{a}):(10,2),(\mathrm{b}):(0,2)$, $(\mathrm{c}):(-1,2)$, and $(\mathrm{d}):(-15,2)$.

\section{Results of numerical simulations}

Results of numerical simulations using the governing evolution equations for a treadmilling organism (26) and (27) are shown in this section. The time integration for all the simulations here were performed by Matlab ode45 solver with the absolute error tolerance $10^{-6}$ and relative error tolerance $10^{-3}$. The radius of the circular body of the treadmilling organism $\epsilon$ is set to be unity, giving the reference length scale to the system. For initial conditions $\left(x_{d 0}, y_{d 0}, \theta_{0}\right)$, we took 3237 different $\left(x_{d 0}, y_{d 0}\right) \in[-20,1] \times[1,10] \oplus(1,20] \times[0,20]$ every 0.5 in both $x$ and $y$ direction, and 200 different $\theta_{0} \in[0,2 \pi)$ every $\pi / 100$. 
(a)

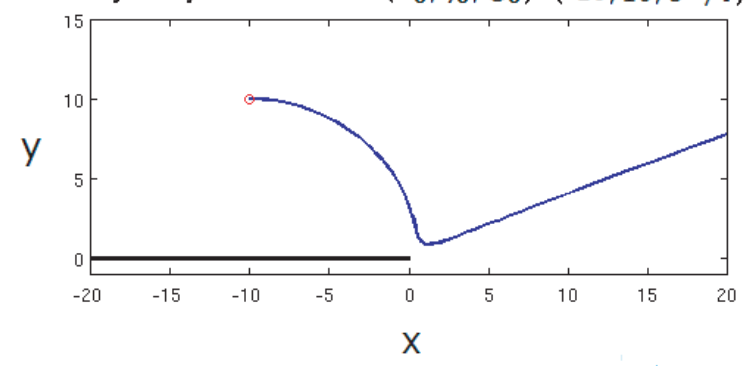

(c) Trajectory of a swimmer: $\left(x_{0}, y_{0}, \Theta_{0}\right)=(5,0,51 \pi / 50)$

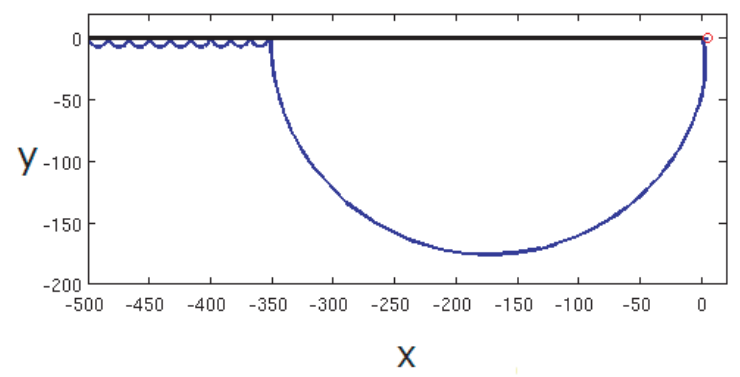

(e) Trajectory of a swimmer: $\left(x_{0}, y_{0}, \Theta_{0}\right)=(10,10,6 \pi / 5)$

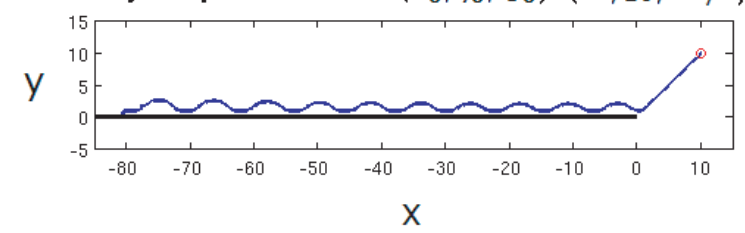

(b) Trajectory of a swimmer: $\left(\mathrm{x}_{0}, \mathrm{y}_{0}, \Theta_{0}\right)=(-10,10,18 \pi / 25)$

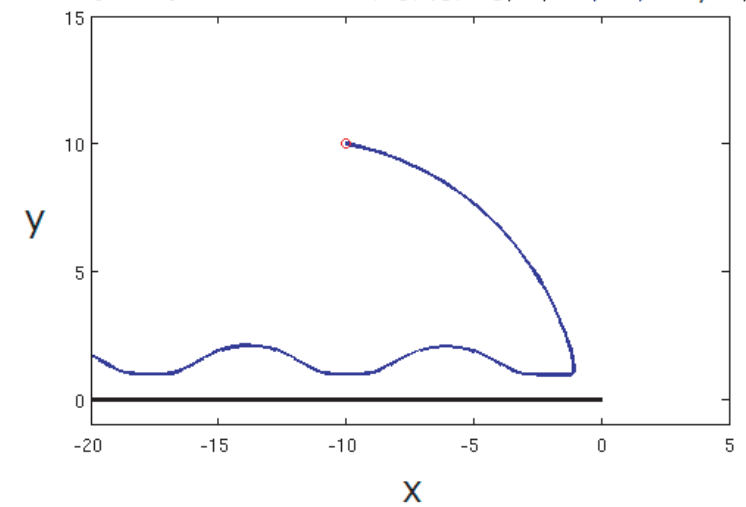

(d) Trajectory of a swimmer: $\left(x_{0}, y_{0}, \Theta_{0}\right)=(5,5,3 \pi / 10)$

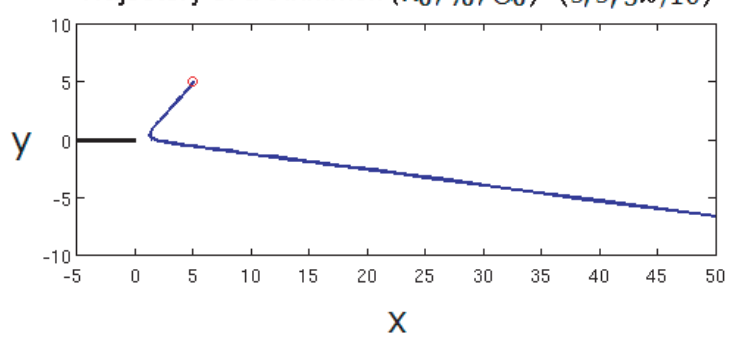

Figure 5: Examples of trajectories from the initial points $\left(x_{d 0}, y_{d 0}\right)$ marked with red circles each, with a certain initial heading direction $\theta_{0}$. Treadmilling organism can escape from the wall, (a) and (d), be above the wall (b), be underneath the wall (d), or crush into the wall (e). 


\subsection{Various trajectories}

By solving the governing equations of the swimming organism (26) and (27) utilising (38)(40), we obtain many different trajectories depending upon different initial conditions. Figure 5 shows examples of such trajectories. At sufficiently large time, the treadmilling organism is possible to in any quadrant if we choose appropriate initial conditions, as we can see in Fig.5 (a)-(d). There also is a case that the treadmilling organism crushes into the wall in finite time as reported in [9]. An example of this unphysical trajectory is shown in Fig.5 (e), and will be discussed in $§ 4.4$. Both experimental observations and preceding theoretical studies suggest that, when there is a no-slip wall near a treadmilling organism, the organism tends to be attracted to its own image on the wall and move towards the wall $[6,7,10,9,12,13,9,16,17]$. This behaviour is clearly seen in all the trajectories shown in Fig.5 at an early stage of time integration. Nevertheless, in the cases shown in Fig.5 (a) and (d), the treadmilling organism moves away from the wall after it has come close to the edge of the wall once. Apparently, there are several cases that the treadmilling organism hangs around/above/underneath the wall as shown in Fig.5 (b) and (c). This is consistent with the results in several preceding study with a no-slip wall $[6,7,10,9,12,13,9,16,17]$. In these case, the treadmilling organism shows the trajectories bouncing along the wall as those in the case with an infinite no-slip wall[16], but the bouncing pattern is, unlike the case with the infinite no-slip wall, irregular and sometimes not periodic even after sufficiently large time has passed. This is also qualitatively consistent with the results of the study with a no-slip infinite wall with a gap [17]. Note that the treadmilling organism make changes its heading direction and the quality of its trajectory significantly when it arrived in the vicinity of the edge of the wall, where the image of the organism cannot move to the positive $\mathrm{x}$-direction any more. Note also that, at a single glance, the initial heading direction $\theta_{0}$ cannot be judged from the trajectory even approximately. This is a both natural and striking difference from the case with an infinite wall [16]. The strong asymmetry due to the absence of the wall in the region $x>0$ gives a great influence to the trajectories, and even the trajectory for $\theta_{0}$ doesn't make a straight vertical line. This feature can be clearly seen in Fig.4 in which 200 different trajectories for 200 different $\theta_{0}$ for several combination of $\left(x_{d 0}, y_{d 0}\right)$ are shown. In the cases with the initial point of the organism is close to or above the wall (Fig.4 (b)-(d)), it is somewhat possible to predict the direction that the treadmilling organism heads for at an early stage, but when the initial point is far from the wall (Fig.4 (a)), the heading direction is unpredictable from the knowledge of the trajectories for the cases with an infinite wall in [16]. However, we see a remarkable line on which many of the trajectories with different $\theta_{0}$ seem to converge in all the cases in Fig.4, especially clearly in Fig.4 (a). This line may give us some kind of criterion in speculating the trajectory. As such lines seem to fall upon the lines which connect the edge of the wall $x=y=0$ and the initial points of the treadmilling organism $\left(x_{d 0}, y_{d 0}\right)$ which is marked by red circles in Fig.4, here, we introduce a critical linear line $\ell_{c}$,

$$
\ell_{c}(x) \equiv \frac{y_{d 0}}{x_{d 0}} x .
$$

Whether or not $\ell_{c}$ acts as a critical line in considering the direction of trajectory in the long run will be checked in $§ 4.2$. Another noticeable feature we can see from Fig.fig:AllThetas is the complexity of the trajectories for initial point with a large negative $\mathrm{x}$-coordinate. 


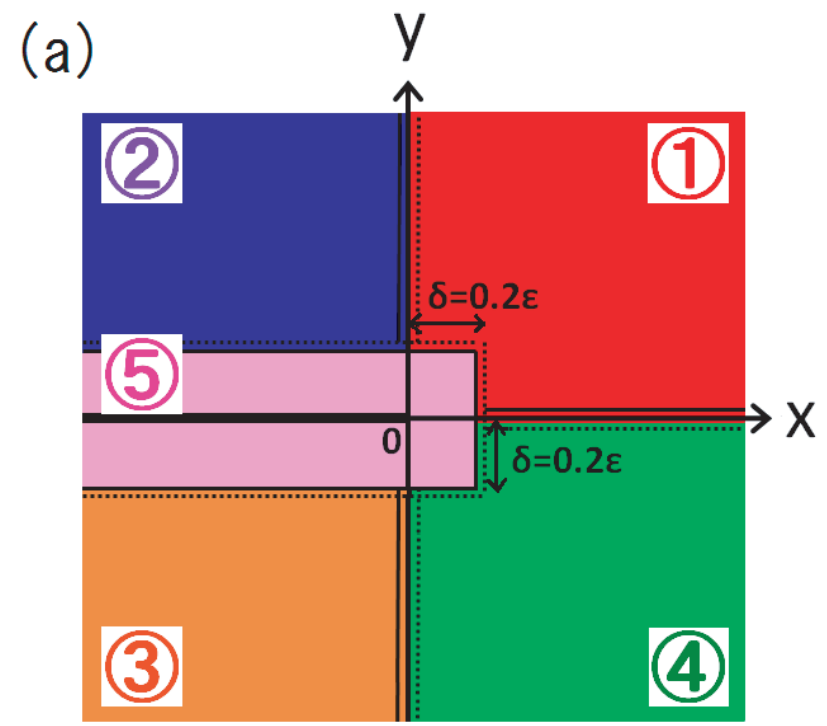

(b)

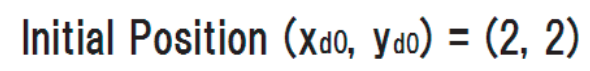

Figure 6: (a): Five regions. The thick black line corresponds to the wall. (b): An example of a pie chart. The different colours indicate in what region shown in the panel (a) the treadmilling organism from a certain initial point is at sufficiently large time. Dotted line corresponds to the slope of the critical line $\ell_{c}$ for the considered initial point.

As already seen in 5 and mentioned above, the treadmilling organism changes its heading direction and the quality of its trajectory greatly when it comes to the vicinity of the edge of the wall, $x=y=0$. The behaviour of the organism after reaching such area significantly depends upon $\left(x_{d}, y_{d}, \theta\right)$ of the trajectory at the time. This makes the trajectories from large negative $x_{d 0}$ unpredictable and complicated. For such cases, even a very small difference in initial condition can result in a huge difference to the trajectory of the treadmilling organism, and this tendency is expected to become more distinct when the initial x-coordinate $x_{d 0}$ has larger negative value. We will come back this point in $\S 4.2$ again.

\subsection{Escaping probability}

Now, we classify a wide variety of trajectories in terms of the regions in which the treadmilling organism is at sufficiently large time. For this purpose, we first introduce five regions shown in Fig.6 (a). Region 1 is $\{(x, y) \in(0.2 \epsilon, \infty) \times[0,0.2 \epsilon] \oplus(0, \infty) \times(0.2 \epsilon, \infty)\}$, and is coloured red in Fig.6 (a). This is the region in which the treadmilling organism that escapes from the wall to $y \geq 0$ direction is. Region 2 is $\{(x, y) \in(-\infty, 0] \times(0.2 \epsilon, \infty)\}$, and is coloured blue in Fig.6 (a). This is the region in which the treadmilling organism that goes back to above the wall is. Region 3 is $\{(x, y) \in(-\infty, 0] \times(-\infty,-0.2 \epsilon)\}$, and is coloured orange in Fig.6 (a). This is the region in which the treadmilling organism that escapes from the wall to $y<0$ direction is. Region 4 is $\{(x, y) \in(0.2 \epsilon, \infty) \times[-0.2 \epsilon, 0) \oplus(0, \infty) \times(-\infty, 0.2 \epsilon)\}$, and is coloured green in Fig.6 (a). This is the region in which the treadmilling organism that goes back to underneath the wall is. Then Region 5 is $\{(x, y) \in(-\infty, 0.2 \epsilon] \times[-0.2 \epsilon, 0.2 \epsilon]\}$, and is coloured pink in Fig.6 (a). This is to describe the case that the treadmilling organism 


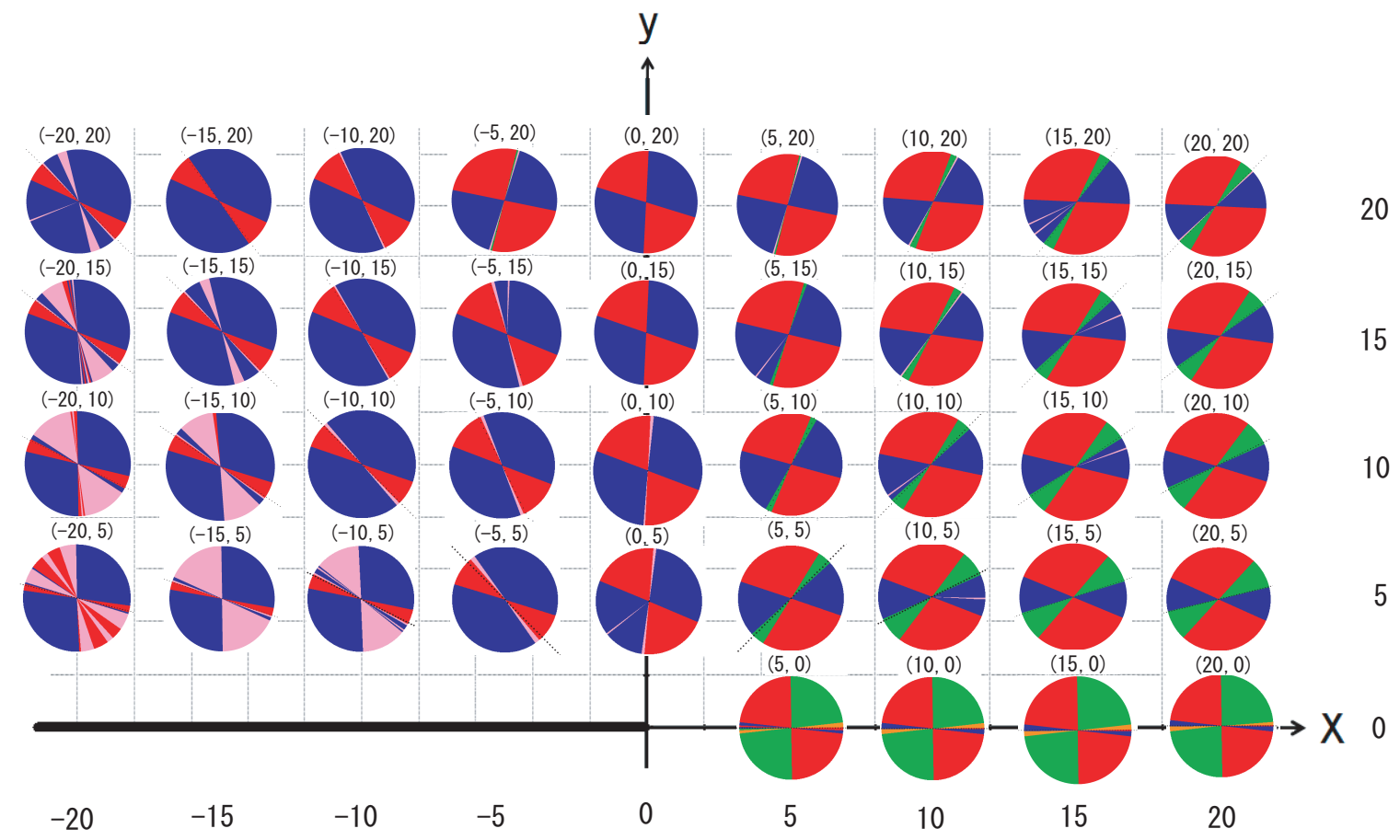

Figure 7: Pie charts at $t=1500$ for several initial points. $x$ and $y$ coordinates of the point on which each pie chart is placed and correspond to the initial point for the pie chart. Thick black line represents the wall.

crushes into the wall.

Utilising these five regions, we make a pie chart for each considering initial point. The pie chart shows in what region the treadmilling organism will be at a large time, here $t=1500$, if it is given a certain initial heading direction $\theta_{0}$. An example of such pie chart is given in Fig.6 (b). Each colour appear in this pie chart corresponds to the colour given for each region in Fig.6 (a). The circular sector where the angle measured from the solid black arrow is in $\left[\theta_{0}-\pi / 200, \theta_{0}+\pi / 200\right]$ corresponds to the initial heading direction $\theta_{0}$ of the treadmilling organism, and the colour there indicate the region in which the treadmilling organism is at sufficiently large time. For instance, the pie chart in Fig.6 (b) shows that the treadmilling organism initially placed at the point $\left(x_{d 0}, y_{d 0}\right)=(2,2)$ and given the heading direction $\theta_{0}=3 / 4 \pi$ will be in the Region 1 at time $t=1500$. The dotted black line in Fig.6 (b) corresponds to the slope of the critical line $\ell_{c}$ (41) for the considered initial point $\left(x_{d 0}, y_{d 0}\right)=(2,2)$. This is to see whether or not, or to what degree, $\ell_{c}$ can act as a critical line in predicting the asymptotic position of the treadmilling organism. For the initial position $\left(x_{d 0}, y_{d 0}\right)=(2,2)$, we can see that, from Fig.6 (b), the dotted line fall near on the boundary between the blue(Region 2) and the green(Region 4) regions.

Figure 7 shows pie charts for several initial points $\left(x_{d 0}, y_{d 0}\right)$. The most impressive feature is the complex city of the pie charts for the initial points with large negative x-coordinates. This reflects the fact, which has been discussed in $\S 4.1$, that the treadmilling organism changes its heading direction and the quality of its trajectory significantly when it has 
come to the vicinity of the edge of the wall, $x=y=0$ depending upon its $\left(x_{d}, y_{d}, \theta\right)$ at the time, and so even a very small difference in initial condition can result in a huge difference to its trajectory when the initial x-coordinate $x_{d 0}$ has large negative value. Note that, however, Pie charts tend to become rather simpler for larger $y_{d 0}$ for any fixed $x_{d 0}$. One more thing we can easily notice is that there is some limitation for the region that the treadmilling organism is in at $t=1500$ depending upon its initial position. Broadly, the treadmilling organism is in regions 1,2 , or 5 when its $\left(x_{d 0}, y_{d 0}\right) \in[-20,0] \times[1,10]$; regions $1,2,4$, or 5 when its $\left(x_{d 0}, y_{d 0}\right) \in(0,20] \times[1,10]$; regions 1,2 , and 4 when $x_{d 0} \in(0,20], y_{d 0}=0$. The possibility of going the region 3 where is underneath the wall that the cases the initial $y$ position $y_{d 0}$ is zero is natural because of the symmetry about $y=0$ the they have. When we compare the pie charts at a certain x-coordinate, it is apparent that the pie charts become simpler for larger $y_{d 0}$. This is because of the effect of asymmetry that the wall gives to the system. Also, although all the pie charts almost have 2-fold rotational symmetry, desymmetrised part becomes obvious when the initial point is near the wall, where the asymmetric effect of the system becomes strong. From the pie charts in Fig.7, it is apparent that $\ell_{c}$ does not act as a critical line as we expected in $\S 4.1$ in any way in considering the position of the treadmilling organism at sufficiently large time, at least at $t=1500$. However, ell still falls near one of the boundaries of two different regions in the cases with positive initial x-coordinate $x_{d 0}>0$ and in the cases whose initial positions are far from the wall.

Now let us consider the escaping probability $P_{E}$, the probability that the treadmilling organism can escape from the wall, or its own image on the wall. Although, ideally, this is to be defined by means of the positions of the treadmilling organism at $t \rightarrow \infty$, here we define this $P_{E}$ as the probability of the treadmilling organism's being in the region 1 or 4 at sufficiently large time $t=t_{l}$, i.e.,

$$
P_{E} \equiv \frac{\left\{\# \theta_{0} \mid z_{d} \in \text { regions } 1 \text { or } 4 \text { at } t=t_{l}\right\}}{\# \theta_{0}} .
$$

The escaping probability $P_{E}$ for $t_{l}=1500$ for different initial conditions are shown in Fig.8, where each $(x, y)$ coordinate corresponds to each considered initial position of the treadmilling organism. $P_{E}$ takes the value $0<P_{E}<1$ for almost all the initial positions in Fig8; $P_{E}$ is zero for only several points with $\left(x_{d 0}<0, y_{d 0}=0\right)$, which is the case that the treadmilling organism touches the wall at the initial time and rather meaningless in this context, and there is no point where $P_{E}$ is unity. This suggests that treadmilling organism has the possibility of both escaping from the wall and being above/underneath the wall for any initial position. Even when the treadmilling organism is initially placed at a position far from the wall, it feels the presence of its own image on the wall and move towards the wall if its initial heading direction $\theta_{0}$ is properly chosen. Similarly, even when the treadmilling organism is initially placed at a point with large negative $\mathrm{x}$-coordinate, above the wall and far from the edge of the wall, for some $\theta_{0}$, it finally moves away from its own image on the wall. The distribution and the monotonic change of the value of $P_{E}$ in the region where $x>0$ seems to be reasonable. However, in the region where $x<0$, there are couple of lines which has larger $P_{E}$ value than naive expectation. This might be the result of the complex pie charts in these region, but the reason why we see such fairly larger $P_{E}$ value on the points on certain lines is not clear and needs further investigations. 


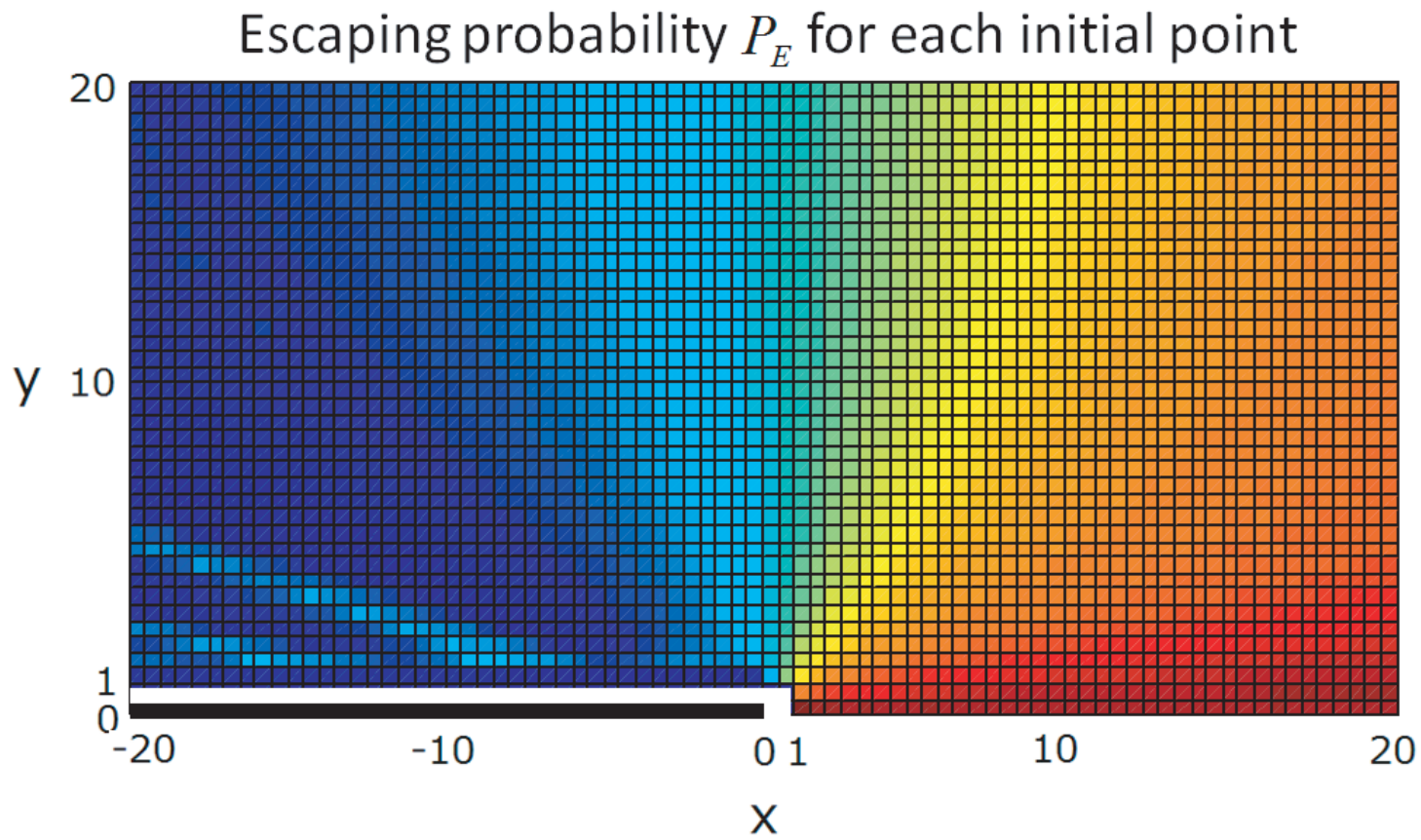

$0.2 \quad 0.4 \quad 0.6 \quad 0.8$

Figure 8: Escaping probability at $t=1500$ for different initial points. Each $(x, y)$ coordinate and the thick black line corresponds to the considered initial position of the treadmilling organism and the wall, respectively. 
(a)

Trajectory of a swimmer

$:\left(\mathrm{X}_{\mathrm{d} 0}, \mathrm{Y}_{\mathrm{d} 0}, \Theta_{0}\right)=(-10,10,3 \pi / 4)$

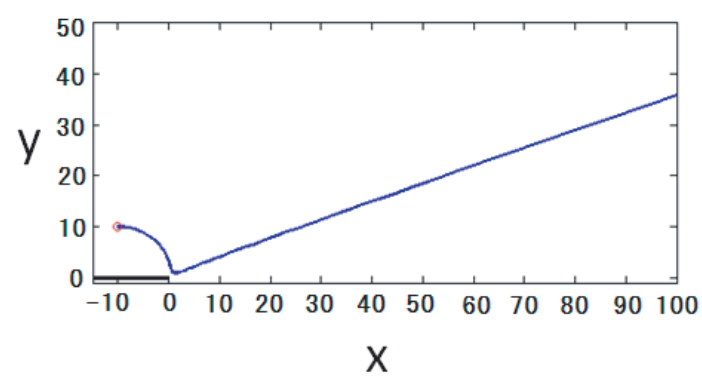

(b)

Speed and y-position of a swimmer

$:\left(X_{\mathrm{d} 0}, Y_{\mathrm{do}}, \Theta_{0}\right)=(-10,10,3 \pi / 4)$

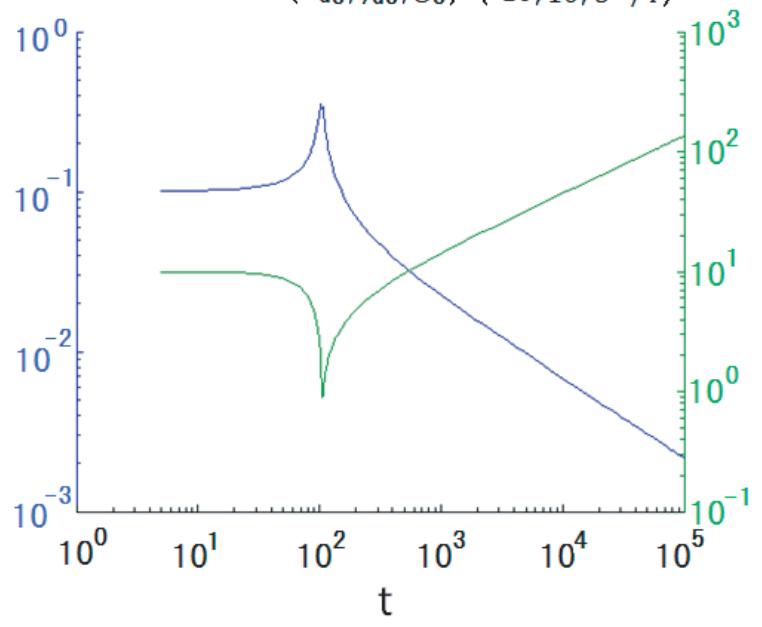

Figure 9: Trajectory of the temporal change of the speed of the treadmilling organism for the case initial condition $\left(x_{d 0}, y_{d 0}, \theta_{0}\right)=(-10,10,3 \pi / 4)$. (a): the trajectory (b): the temporal variation of the $y$-coordinate of the organism $z_{d}$ (blue solid line) and the temporal variation of the speed of the organism (green solid line).

\subsection{Temporal change of the speed of the treadmilling organism in region 1 and 4}

For the definition of the escaping probability $P_{E}$ in $\S 4.2$, we have considered the probability of the treadmilling organism's being in the region 1 or 4 at large time $t=t_{l}$. Then the following questions may always exit: has the treadmilling organism surely escaped from the wall when it is in these region at large time?, does it stop if it reaches a point sufficiently far from the wall? An example of the temporal variation of the speed of the treadmilling organism $\sqrt{{\dot{x_{d}}}^{2}+\dot{y d}^{2}}$ is shown in Fig.9. When the treadmilling organism comes in the vicinity of the edge of the wall and changes its heading direction, the speed of the organism shows a steep increase. After that the treadmilling organism moves away from the wall. Then, as the distance from the wall increase, the moving speed of the organism decreases. The speed is $\sim 10^{-2}$ at $t \sim 10^{3}$ and keep decreasing. This is because that the farther away from the wall the treadmilling organism goes, the effect of its image on the wall becomes weaker since the image cannot follow the organism any further than the edge of the wall. Nevertheless, we cannot be perfectly sure whether or not the speed of the treadmilling organism approaches zero as $t \rightarrow \infty$, and whether or not the treadmilling organism has really escaped from the wall in this case, only from Fig.9. To confirm these points, we need a longer time integration with much higher accuracy; this should be a further work.

\subsection{Velocity field}

Fig.10 shows the velocity field calculated from the two Goursat functions when the treadmilling organism is placed at the point $(x, y)=(2,2)$. It can be confirmed from Fig.10(a) 
(a) Velocity field, $u_{x}(x, y)$

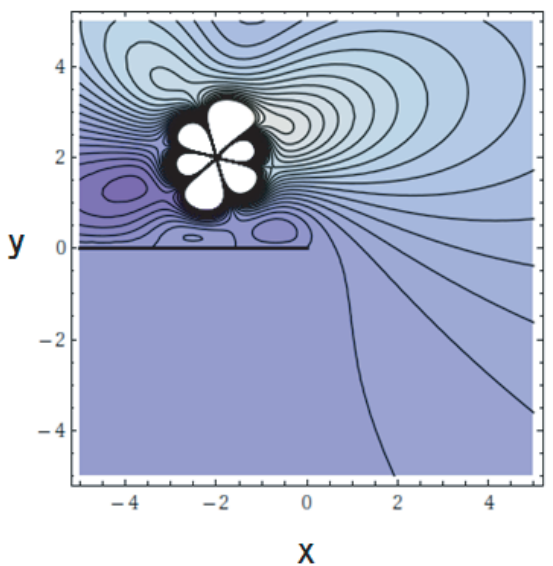

(c) Velocity field, $u_{x}(x, y=2)$

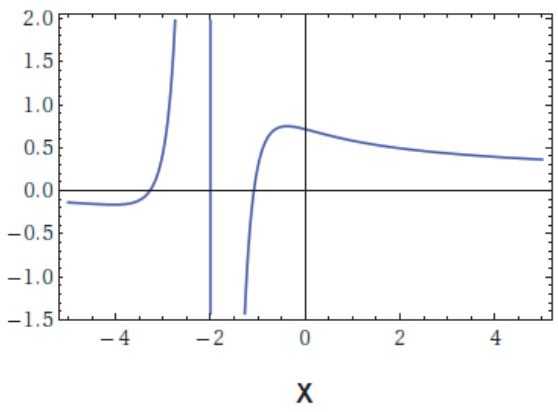

(b) Velocity field, $u_{y}(x, y)$

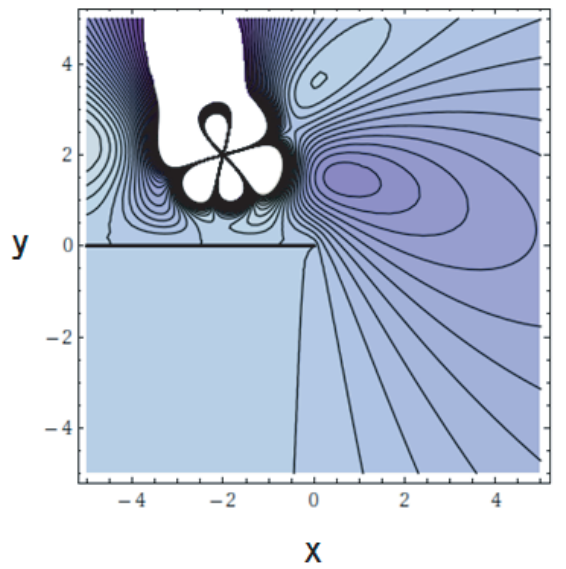

(d) Velocity field, $u_{y}(x, y=2)$

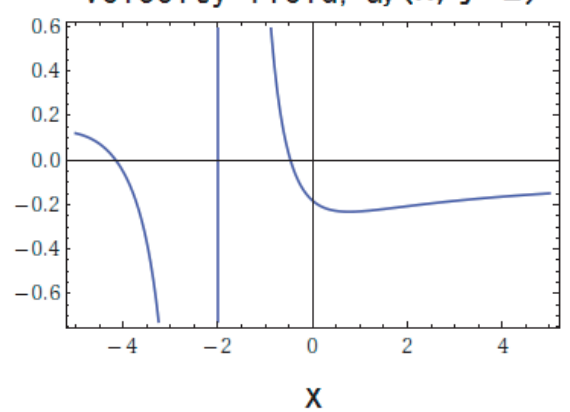

Figure 10: Velocity field when the treadmilling organism is at the point $(x, y)=(2,2)$. (a) and (b)(a): x-velocity $u_{x}(x, y)$ (b) : y-velocity $u_{y}(x, y) \quad$ (c): x-velocity at $\mathrm{y}=2$ $u_{x}(x, y=2)(\mathrm{d}): \mathrm{y}$-velocity at $\mathrm{x}=2 u_{y}(x=2, y)$. The darkness of colour represents the value of the velocity, the thin and the thick solid line are contour of the velocity and the no-slip wall, respectively. 
and (b) that the boundary condition on the no-slip wall is satisfied, and the velocity diverges at the point $(x, y)=(2,2)$ where the singularity, which represents the treadmilling organism, is placed. The boundary condition on the surface of the treadmilling organism, on the contrary, is not satisfied; the contours of the both $\mathrm{x}$ - and $\mathrm{y}$ - velocity are not circles around the treadmilling organism. This is because the model used in this project is very simple and has failed to capture all the features of the treadmilling organism we need to consider. This may affect the behaviour of the treadmilling organism, especially when it is near the wall. This may be one of the reasons why we had unphysical trajectories such as the treadmilling organism crushes into the wall. Nevertheless, at least for the case that the treadmilling organism bounces back on the wall, we have confirmed that the qualitative behaviour of the organism does not change even when the model which shows better boundary condition. This suggests that it may probably be possible to consider that the trajectories, apart from those that the treadmilling organism crushes into the wall, we have found in this report are reasonable.

\section{Discussion and conclusions}

The governing evolution equations for a treadmilling organism when there is a half-infinite non-slip wall in the considered unbounded domain have been derived, by means of an image system method, in $\S 3$. The treadmilling organism was assumed to have a circle body of radius $\epsilon$ and a moving centre $z_{d}(t)=x_{d}(t)+i y_{d}(t)$ in such a domain. It has been modelled as an equivalent point singularity description with stresslet of strength $\mu$ and the quadrupole of strength $2 \epsilon^{2} \mu$ at $z_{d}$ in Stokes flow. The existence of the wall of an infinitesimal thickness causes a critical problem when an image system method is employed; it is not straightforward to determine where to place the image of the treadmilling organism on the wall. To avoid this problem, a conformal mapping $\zeta-i z^{1 / 2}$ was employed, which maps $\{z \in \mathbf{C}\}$ to $\{\zeta \mid \operatorname{Im}(\zeta) \geq 0\}$, especially, the half-infinite wall in $z$-space to the infinite wall in $\zeta$-space. Then $z_{d}$ and its image were defined through $\zeta_{d}$, the image of $\zeta_{d}$ on the infinite wall in $\zeta$-space overline $\zeta_{d}$, and the conformal mapping. By imposing two boundary conditions, one on the body of the organism and the other on the no-slip wall, the coefficients of two Goursat functions $f(z, t)$ and $g(z, t)$, which gives the stream function of the flow, were determined. The governing evolution equations of the position and the heading direction of the treadmilling organism, $z_{d}$ and $\theta_{d}$, were obtained by taking the analytic part of the flow velocity and the half of the analytic part of the vorticity of the flow, respectively.

The trajectories of the treadmilling organism were numerically calculated in $\S 4$. The different trajectories of the treadmilling organism depending upon different initial conditions were discussed in $\S 4.1$. The treadmilling organism was attracted to its image on the wall, as suggested in previous experimental and theoretical studies $[6,7,10,9,12,13,9,16,17]$, at an early stage of the time integration. Nevertheless, in some cases, the treadmilling organism moved away from the wall after it had come to the vicinity of the edge of the wall once. To investigate this behaviour further, in the first part of $\S 4.2$, we have divided $\mathbf{R}^{2}$ domain into five regions and made pie charts which show in what region the treadmilling organism is at sufficiently large time. The pie charts for different initial positions provided in $\S 4.2$, demonstrated that those for the initial points with larger negative $\mathrm{x}$-coordinates

have more complex structures compared to those with smaller negative x-coordinates. This 
reflects the fact that the treadmilling organism changes in its heading direction and the quality of its trajectory significantly when it comes to the vicinity of the edge of the wall, $x=y=0$ depending upon its $\left(x_{d}, y_{d}, \theta\right)$ at the time, and that even a very small difference in initial condition can make a huge difference to its trajectory when the initial x-coordinate $x_{d 0}$ has larger negative value as seen in $\S 4.1$. We further defined and numerically calculated the escaping probability $P_{E}$, the probability that the treadmilling organism can escape from the wall, or its image on the wall in the latter part of $\S 4.1$. This $P_{E}$ did not take 0 or 1 value for any physically reasonable initial point. This suggests that treadmilling organism has the possibility of both escaping from the wall and being above/underneath the wall for any initial position. Even when the treadmilling organism is initially placed at a position far from the wall, it feels the presence of its image on the wall and move towards the wall if its own initial heading direction $\theta_{0}$ is appropriately chosen. Similarly, even when the treadmilling organism is initially placed at a point with large negative $\mathrm{x}$-coordinate, above the wall and far from the edge of the wall, for some $\theta_{0}$, it can finally move away from its own image on the wall. When the treadmilling organism moves away from the wall, its speed decreases as the distance from the wall increases as discussed in $\S 4.3$. This is because that the treadmilling organism feels less effect of its own image on the wall as it moves farther from the wall, since the image cannot follow the organism any further than the edge of the wall.

The results in this project seem reasonable in some respects. However, the obtained $P_{E}$ does not take 0 or 1 for any physically reasonable initial point, even with large positive/negative $\mathrm{x}$-coordinate. This may suggest that the combination of the singularities used required some modifications when the treadmilling organism was far from the edge of the wall. The situation with a half-infinite no-slip wall in an unbounded domain corresponds to the case in which the no-slip wall has a gap as studied in [17] but of an infinite width. Checking whether or not the results gained in this report and those for the case with noslip wall with a gap in the limit of width of the gap $\rightarrow \infty$ may help us gain some further understanding of this problem. The behaviour of a treadmilling organism near a $90^{\circ}$ no-slip corner or even a no-slip corner with arbitrary angle in an unbounded domain will be our next problem to investigate. These will be an important step to clarifying how a treadmilling organism behaves and the effect of its mixing when it is in a bounded domain with no-slip boundaries of complicated forms.

\section{Acknowledgements}

I would like to thank the Woods Hole Oceanographic Institution for the opportunity to participate in Geophysical Fluid Dynamics Program 2010. I would also like to express my sincere gratitude to Jean-Luc Thiffeault for his great patience, and for the fruitful discussions. My thanks also goes to Matthew Finn for his helpful advice and suggestions. Last but not least, I would like to say a big thank you to the staff members and the fellows, without whom the programme would have been much less fruitful.

Some of the numerical calculations for this project were performed on the computer systems of the Institute for Information Management and Communication (IIMC) of Kyoto University. 


\section{References}

[1] Lauga, E. and T.R. Powers, "The hydrodynamics of swimming micro-organisms", Rep. Prog. Phys., 72, 096601 (2009)

[2] Pedley, T. J. and J. O. Kessler, "Hydrodynamic phenomena in suspensions of swimming microorganisms", Annu. Rev. Fluid Mech., 24313 (1992)

[3] Shapere, A. and F. Wilczek, "Geometry of self-propulsion at low Reynolds numbers", J. Fluid Mech., 189, 557 (1989)

[4] Najafi, A. and R. Golestanian, "Simple swimmer at low Reynolds number: Three linked spheres", Phys. Rev. E, 69062901 (2004)

[5] Avron, J. E., O. Kenneth, and D. H. Oakmin, "Pushmepullyou: an efficient microswimmer", New J. Phys., 7, 234 (2005)

[6] Rothchild, A. J., "Non-random distribution of bull spermatozoa in a drop of sperm suspension", Nature(London), 198, 1221 (1963)

[7] Winet, H., G.S. Bernstein, and J. Head, "Observations on the response of human spermatozoa to gravity, boundaries and fluid shear", Reprod. Fert., 70, 511523 (1984)

[8] Cosson, J., P. Huitorel, and C. Gagnon, "How spermatozoa come to be confined to surfaces", Cell Motil. Cytoskel., 54, 5663 (2003)

[9] Berke, A. P., L. Turner, H. C. Berg, and E. Lauga "Hydrodynamic attraction of swimming microorganisms by surfaces", Phys. Rev. E, 101, 038102 (2008)

[10] Lauga, E., W. R. DiLuzio, G. M. Whitesides, and H. A. Stone, "Swimming in circles: motion of bacteria near solid boundaries", Biophys. J., 90, 400 (2006)

[11] Drescher, K., K. C. Leptos, I. Tuval, T. Ishikawa, T. J. Pedley, and R. E. Goldstein, "Dancing Volvox: Hydrodynamic Bound States of Swimming Algae", Phys. Rev. Lett., 102, $168101(2009)$

[12] Ramia, M., D.L. Tullock, and N. Phan-Thien, "The role of hydrodynamics interaction in the locomotion of microorganism", Biophys. J., 65, 755 (1993)

[13] Hernandez-Ortiz, J. P., C.G. Stoltz, and M.D. Graham, "Transport and collective dynamics in suspensions of confined swimming particles", Phys. Rev. Lett., 95, 204501 (2005)

[14] Or, Y. and R. M. Murray, "Dynamics and stability of a class of low Reynolds number swimmers near a wall", Phys. Rev. E, 79045302 (2009)

[15] Zhang, S., Y. Or, and R.M. Murray, "Experimental demonstration of the dynamics and stability of a low Reynolds number swimmer near a plane wall", Proc. Amer. Cont. Conf. (accepted) 
[16] Crowdy, Darren. and Yazhar Or, "Two-dimensional point mdoel of a low-Reynoldsnumber swimmer near a wall", Phys. Rev. E, 81030313 (2010)

[17] Crowdy, Darren. and Ophir Samson, "Hydrodynamic bound states of a low-Reynoldsnumber swimmer near a gap in a wall", J. Fluid.Mech. (accepted)

[18] Becker, L. E., S. A. Koehler, and H. A. Stone, "Hydrodynamic attraction of swimming microorganisms by surfaces", J. Fluid Mech., 490, 15 (2003)

[19] Leshansky, A. M. and O. Kenneth, "Surface tank treading: Propulsion of Purcell's toroidal swimmer", Phys. Fluids, 20063104 (2008)

[20] Imai, I., "Ryuutai Rikigaku (Fluid Dynamics)", Shokabo, Tokyo (1973)

[21] Milne-Thomson, L. M., "Theoretical Hydrodynamics, Fifth Edition", The Macmillan And Company, New York, pp.683-684, (1996)

[22] Batchelor, G. K., "An introduciton to fluid dynamics", Cambridge University Press, Cambridge (1967)

[23] Blake, J. R. and A. T. Chwang, "Fundamental singularities of viscous flow. part 1: the image systems in the vicinity of a stationary no-slip boundary", J. Eng. Math., 8, 23 (1974)

[24] Moffatt, H. K., "Viscous and resistive eddies near a sharp corner", J. Fluid Mech., 18, $1(1964)$ 


\title{
Optimal Mixing on the Sphere
}

\author{
Amanda K. O’Rourke
}

November 10, 2010

\section{Introduction}

The mixing of tracers on the surface of a sphere has many geophysical applications from the advection of volcanic ash to the global redistribution of temperature and angular momentum by atmospheric general circulation. Due to the difficulties of spherical geometry, such as the poles and the isotropy between the meridional and zonal directions, previous work on optimal mixing focuses instead on the $d$-dimensional torus $[5,7,8]$. Here we are motivated by the geophysical problem of temperature redistribution over the surface of the sphere given a heat source at the equator and cooling at the poles. What flow most efficiently mixes the temperature field and how do the characteristics of these optimal flows compare to similar flows on the torus?

Before describing the properties of optimal flows, we must first define a measure of mixing efficiency that will then be used to define a flow that most optimally mixes a tracer field. One measure of mixing is the variance of the tracer concentration. If we consider the time evolution of a tracer field with zero mean subject to advective stirring and diffusivity, the tracer concentration $\theta$ obeys the advection-diffusion equation

$$
\frac{\partial \theta}{\partial t}+\boldsymbol{u} \cdot \nabla \theta-\kappa \Delta \theta=0
$$

where $\boldsymbol{u}$ is the advective velocity (independent of the tracer concentration $\theta$ ) and where $\kappa$ is the molecular diffusivity coefficient. Multiplying (1) by $\theta$ and averaging over the domain yields the time rate of change of the variance of $\theta$ given by

$$
\frac{\partial\left\langle|\theta|^{2}\right\rangle}{\partial t}=-2 \kappa\left\langle|\nabla \theta|^{2}\right\rangle
$$

where the integral over the domain $V$ is denoted by

$$
\langle\theta\rangle=\frac{1}{V} \int_{V} \theta d V .
$$

From (3) we see that the variance of the tracer concentration $\left\langle|\theta|^{2}\right\rangle$ decreases monotonically as a function of the molecular diffusivity $\kappa$ and the magnitude of the gradients of the tracer field. As both $\kappa$ and $\left\langle|\nabla \theta|^{2}\right\rangle$ are positive definite, the variance of the tracer tends to a constant as the gradients of the tracer field tend to zero in the absence of sources or sinks of the tracer. In other words, diffusion acts to destroy gradients in the tracer field and homogenize $\theta$. 
Diffusivity can reduce the variance of a tracer field in the absence of stirring, however this process of diffusive mixing is quite inefficient. We can significantly enhance the process of mixing and homogenization by including a stirring flow $\boldsymbol{u}$. The mechanical act of stirring stretches and folds material lines of the tracer field, enhances the gradients of $\theta$, and ultimately leads to a more rapid homogenization of the tracer field.

If there are no source terms to maintain gradients in $\theta$ diffusivity will lead to a spatially homogeneous tracer field over time. We can obtain a nonzero $\left\langle|\theta|^{2}\right\rangle$ as $t \rightarrow \infty$ by including a spatially varying tracer source term in (1),

$$
\frac{\partial \theta}{\partial t}+\boldsymbol{u} \cdot \nabla \theta-\kappa \Delta \theta=s(\boldsymbol{x})
$$

The homogenization of the tracer field by mixing can then balance the production of tracer variance by the source in steady state. It is within this steady state that we will evaluate the effect of stirring on the mixing process.

As we expect stirring to enhance mixing, we anticipate that for the same value of $\kappa$ the variance of a stirred tracer field will be less than that of an unstirred tracer in steady state. Thiffeault et al. [7] measure the relative mixing enhancement due to stirring through a measure given by ratio of the unstirred to stirred steady state variances,

$$
\mathcal{E}^{2}=\frac{\left\langle\left|\theta_{0}\right|^{2}\right\rangle}{\left\langle|\theta|^{2}\right\rangle}
$$

where $\theta_{0}$ satisfies the unstirred, steady state diffusion equation

$$
-\kappa \Delta \theta_{0}=s(\boldsymbol{x})
$$

and where $\theta$ satisfies the stirred, steady state advection-diffusion equation

$$
\boldsymbol{u} \cdot \nabla \theta-\kappa \Delta \theta=s(\boldsymbol{x}) .
$$

A mixing efficiency of $\mathcal{E}=1$ would imply that the velocity field does not enhance mixing relative to diffusive processes, as would be the case if $\boldsymbol{u}$ were parallel to lines of constant $\theta$ such that the advective term $\boldsymbol{u} \cdot \nabla \theta$ of (4) vanished. Mixing efficiencies of $\mathcal{E}<1$ will be obtained if $\boldsymbol{u}$ enhances gradients of $\theta$ in steady state. Such small mixing efficiencies are counterintuitive if we assume that stirring tends to improve the homogenization of a tracer with time, as is the case for flows with mixing efficiencies of $\mathcal{E}>1$.

By defining the mixing efficiency factor $\mathcal{E}$ we can then create an optimization problem to determine the most efficient flow fields over the surface of the sphere by maximizing $\mathcal{E}$. Many approaches have been taken to this problem in the past, however all have focused on flows over the torus. There are two primary methods of maximizing $\mathcal{E}$ : finding the flow that most efficiently mixes a tracer field for a given source function or, alternatively, finding the source function that is most efficiently mixed by a fixed velocity field. Thiffeault and Pavliotis [8] consider the problem of optimizing the source function for a fixed velocity field and find that these sources tend to have maxima and minima located near in regions of strongest velocity and are aligned such that the flows transport sources over sinks.

Alternatively, Lin et al. [3] and Thiffeault [6] maximize the mixing efficiency for a fixed source by varying the velocity field. Lin et al. [3] determine the velocity field that most 
quickly reduces a measure of mixing similar to $\mathcal{E}$ in the absence of diffusivity. In contrast to the transient problem considered by Lin et al. [3], Thiffeault [6] introduce a means of solving for the velocity fields that most efficiently mix a tracer concentration maintained by a spatially varying source term and subject to molecular diffusivity.

Both Lin et al. [3] and Thiffeault [6] find optimal velocity fields on the torus. Here we will expand on the approach of Thiffeault [6] to consider optimal flows over the surface of the sphere. We choose to focus on three points of comparisons between optimal flows on the torus and the sphere: the issue of upper and lower bounds on the mixing efficiencies, the structure of the most optimal flow configurations, and the scaling of the efficiencies in the Péclet number.

In Section 2 we describe the numerical model we will use to find velocity fields that maximize $\mathcal{E}$. We then compare the analytical lower bound of the mixing efficiencies on the torus to that of the sphere in Section 3 and see if the efficiencies scale as a function of the Péclet number on the sphere as they do on the torus in Section 4. We will then discuss the possibility of an efficient 'sweeping' flow for the sphere in Section 5. In Section 6 we consider future directions of this work.

\section{The Model}

\section{$2.1 \quad$ Euler-Lagrange Equations}

We maximize $\mathcal{E}$ in a spherical geometry by varying the advective velocity field while holding constant the structure of the source function and the diffusivity. The mixing efficiency $\mathcal{E}$ as given in (5) has two components: the variance of the unstirred steady state tracer field $\left\langle\left|\theta_{0}\right|^{2}\right\rangle$ and the variance of the stirred steady state tracer field $\left\langle|\theta|^{2}\right\rangle$. These tracer fields can be written as

$$
\theta=\mathcal{L}^{-1} s
$$

where $\mathcal{L}$ and $\mathcal{L}_{0}$ are the advection-diffusion and diffusion operators given by

$$
\mathcal{L}=\boldsymbol{u} \cdot \boldsymbol{\nabla}-\kappa \Delta \quad \mathcal{L}_{0}=-\kappa \Delta .
$$

From (8) and (9), we see that the solution $\theta_{0}$ to the diffusion-only differential equation will be constant under a change in $\boldsymbol{u}$. Changing $\boldsymbol{u}$ will only influence the steady state stirred tracer field $\theta$ such that maximizing $\mathcal{E}$ is equivalent to minimizing $\left\langle|\theta|^{2}\right\rangle$. In order to minimize $\left\langle|\theta|^{2}\right\rangle$ with respect to $\boldsymbol{u}$, we first define the variation of $\left\langle|\theta|^{2}\right\rangle$ following Thiffeault $[6]$

$$
\delta\left\langle|\theta|^{2}\right\rangle=\delta\left\langle\left|\mathcal{L}^{-1} s\right|^{2}\right\rangle
$$

where we have used $\theta=\mathcal{L}^{-1} s$ from (8).

As we are holding the source function constant, only the integral operator $\mathcal{L}^{-1}$ changes under a change in $\boldsymbol{u}$ such that

$$
\delta\left\langle|\theta|^{2}\right\rangle=2\left\langle\mathcal{L}^{-1} s \delta \mathcal{L}^{-1} s\right\rangle
$$


which can be simplified using the identity $\delta \mathcal{L}^{-1}=-\mathcal{L}^{-1} \delta \mathcal{L} \mathcal{L}^{-1}$,

$$
\delta\left\langle|\theta|^{2}\right\rangle=-2\left\langle\mathcal{L}^{-1} s \mathcal{L}^{-1} \delta \mathcal{L} \mathcal{L}^{-1} s\right\rangle
$$

After integrating (12) by parts, $\delta\left\langle|\theta|^{2}\right\rangle$ can be written in terms of the self-adjoint operator

$$
\mathcal{A}=\mathcal{L} \mathcal{L}^{\dagger}
$$

where $\mathcal{L}^{\dagger}=-\boldsymbol{u} \cdot \boldsymbol{\nabla}-\kappa \delta$ is the adjoint of the advection-diffusion operator, such that

$$
\delta\left\langle|\theta|^{2}\right\rangle=-2\left\langle\mathcal{A}^{-1} s \delta \mathcal{L} \mathcal{L}^{-1} s\right\rangle
$$

As the variation in the advection diffusion operator only depends on the variation of the velocity field, $\delta \mathcal{L}=\delta \boldsymbol{u} \cdot \boldsymbol{\nabla}$, we can write the variation of $\left\langle|\theta|^{2}\right\rangle$ in terms of $\delta \boldsymbol{u}$,

$$
\delta\left\langle|\theta|^{2}\right\rangle=-2\left\langle\mathcal{A}^{-1} s \delta \boldsymbol{u} \cdot \nabla \mathcal{L}^{-1} s\right\rangle .
$$

The functional derivative of $\left\langle|\theta|^{2}\right\rangle$ with respect to $\boldsymbol{u}$ then is

$$
\frac{\delta\left\langle|\theta|^{2}\right\rangle}{\delta \boldsymbol{u}}=-2 \mathcal{A}^{-1} s \nabla \mathcal{L}^{-1} s
$$

We could find the extrema of $\left\langle|\theta|^{2}\right\rangle$ by finding the zeroes of (16), however this would tell us little about the structure of the most efficient flows without additional constraints. We place a constraint on the energy and restrict our attention to non-divergent flows by creating an extended functional of $\left\langle|\theta|^{2}\right\rangle$

$$
\mathcal{F}(\boldsymbol{u})=\left\langle|\theta|^{2}\right\rangle+\gamma\left(\left\langle|\boldsymbol{u}|^{2}\right\rangle-U^{2}\right)+\langle\nu \nabla \cdot \boldsymbol{u}\rangle
$$

whose variation with respect to $\boldsymbol{u}$ is

$$
\frac{\delta \mathcal{F}[\boldsymbol{u}]}{\delta \boldsymbol{u}}=-\mathcal{A}^{-1} s \nabla \mathcal{L}^{-1} s+\gamma \boldsymbol{u}-\nabla \nu=0 .
$$

Equation (18) is the Euler-Lagrange equation for the constrained optimization problem where $\gamma$ and $\nu$ are the Lagrange multipliers for the energy and non-divergence constraints, respectively. Flows that satisfy these constraints have a kinetic energy $\left\langle|\boldsymbol{u}|^{2}\right\rangle$ equal to a constant value $U^{2}$ and are non-divergent such that $\boldsymbol{\nabla} \cdot \boldsymbol{u}=0$.

\section{$2.2 \quad$ Numerical Model}

There are numerous methods of finding velocity fields that maximize $\mathcal{E}$ and minimize $\left\langle|\theta|^{2}\right\rangle$. Thiffeault [6], for example, suggests a method to directly solve the Euler-Lagrange equations given in (18) for the velocity field. Here we consider an alternate means of finding the minima of $\left\langle|\theta|^{2}\right\rangle$. We perform a two-part optimization process based on Matlab's built-in nonlinear optimization routine fmincon. This routine, a part of Matlab's Optimization Toolbox, minimizes $\left\langle|\theta|^{2}\right\rangle$ subject to the non-divergence and energy constraints.

The fmincon routine minimizes $\left\langle|\theta|^{2}\right\rangle$ using the iterative interior point algorithm. At each iteration of $\boldsymbol{u}$ and $\mathcal{L}[\boldsymbol{u}]$, we must solve the advection-diffusion equation (8) for $\theta$. For 
the torus problem, we can solve (8) for $\theta$ by generating an invertible, spectral advectiondiffusion operator matrix $\mathcal{L}[\boldsymbol{u}]$. The tracer concentration $\theta$ is then obtained by solving the system of linear equations using this non-singular operator. Due to time limits, we were unable to generate a non-singular, spectral advection-diffusion operator for the sphere. In order to find optimal solutions, we instead solve (8) for $\theta$ using fsolve where fsolve is a built-in Matlab routine that solves a system of nonlinear equations. Resorting to fsolve leads to an increased computational cost in the spherical problem relative to the torus problem.

In addition to solving for $\theta$ using fsolve for varying $\boldsymbol{u}$, we must consider that not all variations in $\boldsymbol{u}$ are permitted due to constraints. The optimization problem is constrained in two ways: the kinetic energy of the flow is held fixed at a constant value and the flow field must be non-divergent. The energy constraint limits the complexity and strength of the flow such that the optimal flow field $\boldsymbol{u}$ in steady state satisfies

$$
\left\langle|\boldsymbol{u}|^{2}\right\rangle=U^{2}
$$

where $U$ is a constant equal to unity for all optimal and sub-optimal flows considered here.

The energy constraint (19), along with the coefficient of diffusivity $\kappa$ and the radius of the sphere $a$, allow us to calculate the Péclet number for a given flow,

$$
P e=\frac{U a}{\kappa}
$$

where $P e$ is a nondimensional parameter relating the relative magnitude of the advective stirring to the molecular diffusivity in the advection-diffusion equation (1) such that mixing processes with large $P e$ are advection-dominated. The radius of the sphere is held fixed at $a=1$ so that the Péclet number of all experiments here is a function of the diffusivity only.

The non-divergence constraint $\boldsymbol{\nabla} \cdot \boldsymbol{u}$ can be satisfied by defining a streamfunction $\psi$ such that the zonal and meridional velocities over the sphere, $u$ and $v$ respectively, satisfy

$$
u=-\frac{1}{a} \frac{\partial \psi}{\partial \phi} \quad v=\frac{1}{a \cos \phi} \frac{\partial \psi}{\partial \lambda}
$$

where $-\pi \leq \phi \leq \pi$ is the latitude and $0 \leq \lambda \leq 2 \pi$ is the longitude. It should be mentioned that we are using a geophysical coordinate system where $\phi=0$ is at the equator rather than standard spherical coordinates where the polar angle is typically zero at the north pole.

As we are interested in flows over the surface of a thin spherical shell, it is useful to recast all operators and variables in terms of spherical coordinates. We use a pseudo-spectral approach to calculating the advection-diffusion operator $\mathcal{L}$. Transformations between grid space and spherical harmonics in addition to the calculation of derivatives in spherical coordinates are performed using Algorithm 888, a package of Matlab routines for spherical harmonic functions [1]. Using Algorithm 888, we cast all scalars in terms of their spherical harmonics,

$$
\theta(\lambda, \phi)=\sum_{n=0}^{\infty} \sum_{m=-n}^{n} \tilde{\theta}_{n}^{m} Y_{n}^{m}(\lambda, \mu)
$$




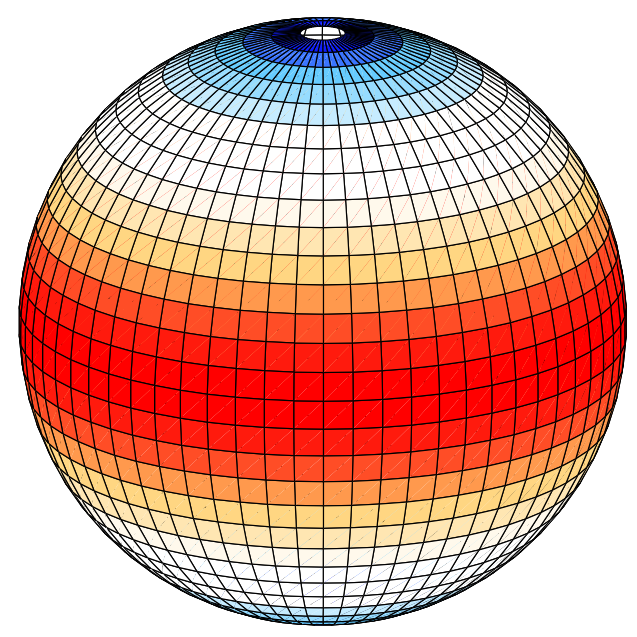

Figure 1: The geophysical source in arbitrary units, with heating (red) at the equator and cooling (blue) at the poles.

where $\tilde{\theta}$ is the spectral coefficient of $\theta, n$ is the total wavenumber, $m$ is the zonal wavenumber, and $Y_{n}^{m}(\lambda, \mu)$ is the spherical harmonic of longitude $\lambda$ and of $\mu=\sin \phi$. The spherical harmonic is given by

$$
Y_{n}^{m}(\lambda, \mu)=e^{i m \lambda} P_{n}^{m}(\mu) .
$$

where $P_{n}^{m}(\mu)$ are the associated Legendre functions defined by

$$
P_{n}^{m}(\mu)=\frac{1}{2^{n} n !} \sqrt{\frac{(2 n+1)(n-m) !}{2(n+m) !}}\left(1-\mu^{2}\right)^{m / 2} \frac{d^{n+m}}{d \mu^{n+m}}\left(\mu^{2}-1\right)^{n} .
$$

The wavenumbers $n$ and $m$ describe the structure of the scalar field in latitude and longitude. In the zonal direction, $m$ is similar to the $x$-wavenumber on the torus and is simply a superposition of Fourier modes. The geometries of the torus and the sphere, however, differ significantly in the meridional direction. Instead of a $y$-wavenumber as on the torus, spherical harmonics are cast in terms of a total wavenumber $n$ where $n-m$ are the number of nodes in the meridional direction.

The geophysical source function we consider here is given by the $n=2, m=0$ spherical harmonic with a spectral amplitude of -1 . The source function can then be written as

$$
s(\lambda, \phi)=(-1) Y_{2}^{0}(\lambda, \mu)=-P_{2}^{0}(\mu) .
$$


As $n-m=2$ and $m=0$ for the source function, $s(\lambda, \phi)$ has two nodes in latitude located in the midlatitudes and has no zonal structure. The amplitude is chosen so that there is a source of 'heat' at the equator and a sink at the poles, as shown in Figure 1.

\subsection{Efficiencies of Sub-Optimal Flows}

The fmincon routine is an iterative process that requires an initial velocity field sufficiently close to the optimal solution in order to converge on a minima in $\left\langle|\theta|^{2}\right\rangle$ with respect to $\boldsymbol{u}$. Fmincon can only identify local minima and, at this time, we are unable to state whether or not these optimal solutions minimize $\left\langle|\theta|^{2}\right\rangle$ globally. The solution space is littered with local minima such that a 'good' choice of an initial velocity field is crucial for both convergence and for the best chance at finding a true optimal velocity field $\boldsymbol{u}$.

In order to determine what constitutes a 'good' initial guess, we begin our investigation of optimal flows by determining the efficiencies of the sub-optimal flows generated by monochromatic streamfunctions. Thiffeault [6] note that the velocity fields that most efficiently mix a tracer in the presence tends to be flows that directly transport the tracer from regions of excess $\theta$ ('hot' regions, if we imagine the tracer is temperature) to sink ('cold') regions.

For the geophysical source in Figure 1 and following the observations of optimized source functions in Thiffeault and Pavliotis [8], the most optimal flow would likely be cellular with the strongest velocities over the maxima or minima of the source and nodes located near the mean value of $s(\boldsymbol{x})$. The geophysical source function achieves its mean value in the midlatitudes such that we would expect

$$
\boldsymbol{u}=\left(-\frac{1}{a} \frac{\partial \psi}{\partial \phi}, \frac{1}{a \cos \phi} \frac{\partial \psi}{\partial \lambda}\right) \approx 0
$$

in these regions. Using (21), the streamfunction $\psi$ of this efficient flow would then likely achieve its extrema in the midlatitudes and have an inflection point near the equator. In terms of spherical harmonics, a streamfunction with wavenumbers satisfying $n-m=1$, such as the monochromatic spherical harmonic functions $Y_{2}^{1}$ and $Y_{3}^{2}$, are good choices for $\psi$.

Indeed, $\psi \propto Y_{3}^{2}$ is the most efficient sub-optimal streamfunction at $P e=10$ over the range of monochormatic streamfunctions shown in Figure 2. Streamfunctions proportional to spherical harmonic modes $Y_{n}^{m}$ with $n-m=1$ tend to have the highest mixing efficiencies, as expected, while those streamfunctions with $n-m=0$ (inter-hemispheric flows) are slightly less efficient. In both the $n-m=1$ and $n-m=0$ flows, stirring enhances mixing such that $\mathcal{E}>1$. Velocity fields described by streamfunctions with less zonal structure, or with smaller $m$, tend to be more efficient than those with larger values of $m$, with an optima in $\mathcal{E}$ around $m=2$ for both $n-m=1$ and $n-m=0$. Good initial conditions for $\psi$ in the optimization algorithm would then be the monochromatic streamfunctions proportional to $Y_{3}^{2}$ and $Y_{2}^{1}$.

One surprising conclusion from Figure 2 is that some flows with $n-m=2$ are in fact inefficient. For these flow fields, stirring actually increases $\left\langle|\theta|^{2}\right\rangle$ relative to the unstirred

solution $\left\langle\left|\theta_{0}\right|^{2}\right\rangle$ resulting in $\mathcal{E}<1$. It is clear that such flow fields would not be good initial guesses for the optimization problem as stirring acts to increase the variance of the steady 


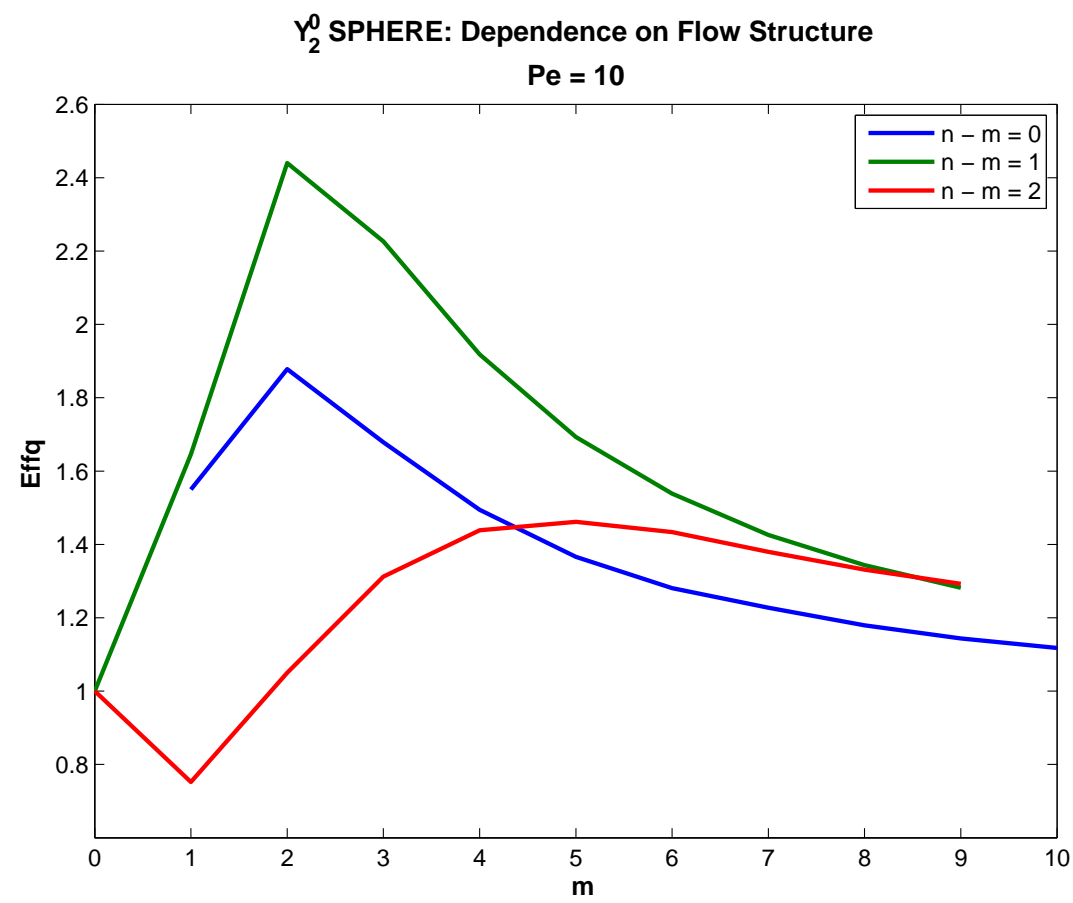

Figure 2: Sub-optimal efficiencies $\mathcal{E}$ for streamfunctions of the form $\psi \propto Y_{n}^{m}(\lambda, \mu)$ stirring the geophysical source field with $n-m=0$ (blue), $n-m=1$ (green), and $n-m=2$ (red) for zonal wavenumbers $m=0$ to 10 at $P e=10$.

state tracer field. These inefficient flows are a unique feature of spherical geometry and of our choice of steady, time-independent velocity fields as will be shown in Section 3.

\section{Lower Bounds and Inefficient Stirring}

In Section 2.3 we determined that not all stirring by a velocity field $\boldsymbol{u}$ enhances diffusive mixing. We obtained a range of sub-optimal efficiencies spanning both both positive and negative values of $\mathcal{E}$. What is the range of possible $\mathcal{E}$ for the sphere and how does this compare to that of the torus? We will answer this in two parts, focusing on the lower bounds and the existence of inefficient flows here and on the scaling of the most efficient flows in Section 4.

Previous work by Shaw et al. [5] obtained analytic upper and lower bounds on $\mathcal{E}$ for the torus by optimizing over the tracer field $\theta$. For tracer fields stirred by a uniform velocity field, where $\nabla \boldsymbol{u}(\boldsymbol{x}, t)=0$ at each instant in time, and supplied by a monochromatic source, Shaw et al. [5] find that the mixing efficiencies are bounded by

$$
1 \leq \mathcal{E}^{2} \leq 1+P e^{2} / d L^{2} k_{s}^{2}
$$

where $d$ is the dimension of the torus, $L$ is the domain size, and $k_{s}$ is the wavenumber of the source. The Péclet number for the torus is the same as that of the sphere given in (20) with the domain size $L$ being the characteristic length in the place of the radius $a$. 


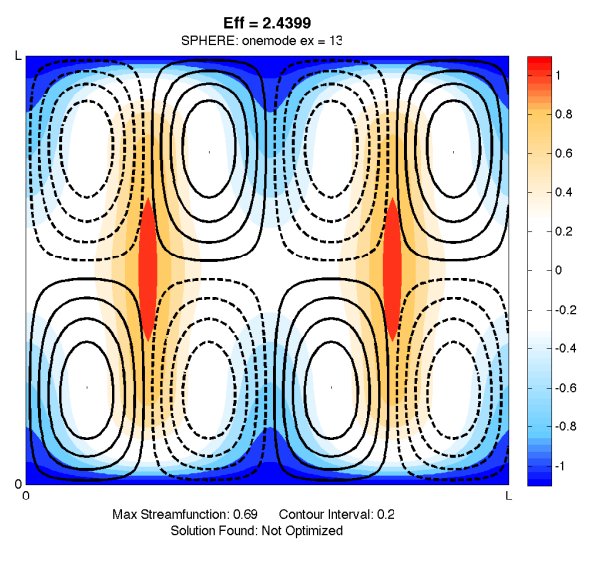

(a) Efficient Flow, $\mathcal{E}=2.44$

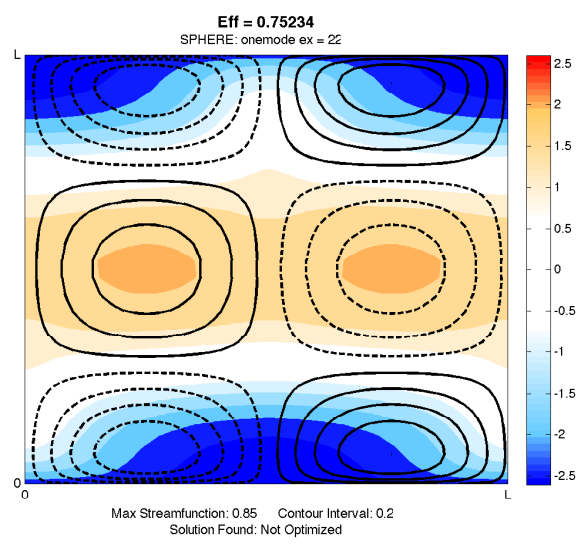

(b) Inefficient Flow, $\mathcal{E}=0.75$

Figure 3: Two of the sub-optimal flows associated with the parameter sweep in Figure 2 where Panel 3(a) is associated with the relatively efficient flow of mode $m=2, n=3$ and where Panel 3(b) is the inefficient flow of mode $m=1, n=3$. The streamfunction of the velocity field are plotted on top of colored contours of the steady-state tracer field $\theta$.

Shaw et al. [5] found that inefficient flows do not exist on the torus for monochromatic sources, such as the geophysical source function $s(\boldsymbol{x}) \propto Y_{2}^{0}(\lambda, \mu)$ that we consider here. For such monochromatic sources, stirring over the torus always acts to decrease the $\left\langle|\theta|^{2}\right\rangle$ in steady state relative to the unstirred solution. As we have already seen from Figure 2, inefficient flow not only exist for the geophysical source on the sphere-they are, in fact, fairly common. What are the properties of these inefficient flows that lead to an increase in the variance of $\theta$ relative to the unstirred solution?

If we compare the most efficient sub-optimal flow of Figure 2 to the most inefficient flow, we find that the concentration of the tracer field in steady state between Panel 3(a) and Panel 3(b) are quite different. The efficient flow has stagnation points centered near the mean value of the source function (located in the midlatitudes for the geophysical source) and strong zonal velocities near the source maxima and minima. In steady state, the warm patches of tracer are advected off equator in thin jets while cold regions are advected equatorwards, leading to efficient mixing of the tracer field relative to the diffusive-only solution.

The inefficient flow in Panel 3(b) has stagnation points centered on the equator at the region of maximum tracer input and strong flows parallel to lines of constant $\theta$. The maximum 'heating' at the equator is trapped at the stagnation points of the flow while the meridional flow everywhere is relatively weak. Without the ability to remove excess tracer at the equator and transport it to the sink at the poles, this flow tends to increase the tracer variance in steady state relative to the diffusive-only solution and thus has a mixing efficiency of $\mathcal{E}<1$.

As for inefficient flows on the torus, Shaw et al. [5] identify that $\mathcal{E}$ satisfies

$$
\mathcal{E}^{2}=\frac{\left\langle\left|\theta_{0}\right|^{2}\right\rangle}{\left\langle|\theta|^{2}\right\rangle} \geq \frac{4 \pi^{2}}{L^{2}} \frac{\left\langle\left|\Delta^{-1} s\right|^{2}\right\rangle}{\left\langle\left|\nabla^{-1} s\right|^{2}\right\rangle}=\frac{\sum_{k}(L k / 2 \pi)^{-4}\left|\tilde{s}_{\boldsymbol{k}}\right|^{2}}{\sum_{k}(L k / 2 \pi)^{-2}\left|\tilde{s}_{\boldsymbol{k}}\right|^{2}}
$$


which they then solve using a variational problem in $\theta$ to maximize the stirred, steady state $\left\langle|\theta|^{2}\right\rangle$ subject to the steady state constraint $\kappa\left\langle|\nabla \theta|^{2}\right\rangle=\langle s \theta\rangle$.

The results of Shaw et al. [5] for a monochromatic source are due in part to the similarity between the the gradient and Laplacian operators in Fourier space, evident in the similarity in the numerator and denominator of the last term in (28). For a monochromatic source of wavenumber $k_{s}$, the Laplacian and gradient operators are simply

$$
\nabla=i k_{s} \quad \Delta=-k_{s}^{2}
$$

so that the Laplacian operator is the square of the gradient operator.

In terms of spherical harmonics, however, the gradient operator is significantly different from the Laplacian. The Laplacian of the spherical harmonic function $Y_{n}^{m}$ is given by

$$
\Delta Y_{n}^{m}(\lambda, \mu)=-\frac{n(n+1)}{a^{2}} Y_{n}^{m}(\lambda, \mu) .
$$

While the gradient operator is similar to that of the torus in the zonal direction,

$$
\frac{\partial}{\partial \lambda} Y_{n}^{m}(\lambda, \mu)=i m Y_{n}^{m}
$$

the meridional derivative of $Y_{n}^{m}(\lambda, \mu)$ is a recursion relation

$$
\left(1-\mu^{2}\right) \frac{\partial}{\partial \mu} Y_{n}^{m}(\lambda, \mu)=-n v_{n+1, m} Y_{n+1}^{m}(\lambda, \mu)+(n+1) v_{n, m} Y_{n-1}^{m}(\lambda, \mu)
$$

where $v_{n, m}=\left(\frac{n^{2}-m^{2}}{4 n^{2}-1}\right)^{1 / 2}$. On the sphere, the Laplacian operator of a monochromatic source function is not equal to the square of the gradient operator. The simplification of the efficiency given in (28) from Shaw et al. [5] will not apply here. It will thus be necessary to find an alternative approach to solving for the analytic lower bound on $\mathcal{E}$ in spherical coordinates.

\section{Upper Bounds and Péclet Number Scaling}

The upper bound given in (27) from Shaw et al. [5] suggests that the efficiencies on the torus scale linearly in the Péclet number for large $P e$. Thiffeault [6] also notes that for optimization problems on the torus, the optimal efficiencies scale quadratically for small $P e$. Do these scalings in Péclet number hold for the sphere?

In order to determine the efficiencies of optimal flows, we must first solve the optimization problem given in (18). We can use the highly efficient, sub-optimal flows from Figure 2, namely the flows with $\psi \propto Y_{3}^{2}(\lambda, \mu)$ and $\psi \propto Y_{2}^{2}(\lambda, \mu)$ as initial guesses at $P e=10$ to obtain the two optimized flows shown in Figure 4.

Both flows in Figure 4 have characteristics similar to the efficient, but sub-optimal flows of Figure 2. The optimal flows tend to have stagnation points near the mean value of the source function and both have inter-hemispheric cells to transport the tracer from high concentrations at the equator to low concentrations near the poles. The efficiency of the optimized flow with $m=2$ (Panel 4(a)) is slightly higher than that of the optimized flow 


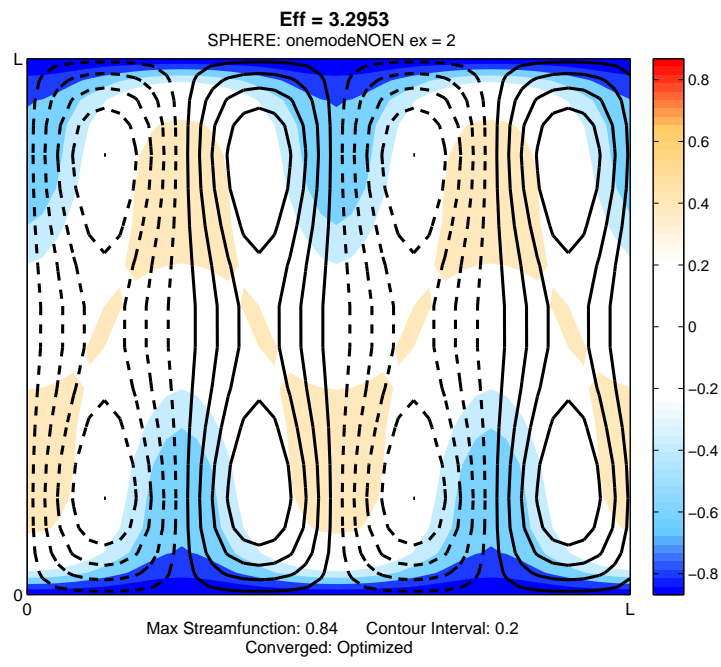

(a) Optimized Flow, $\mathcal{E}=3.30$

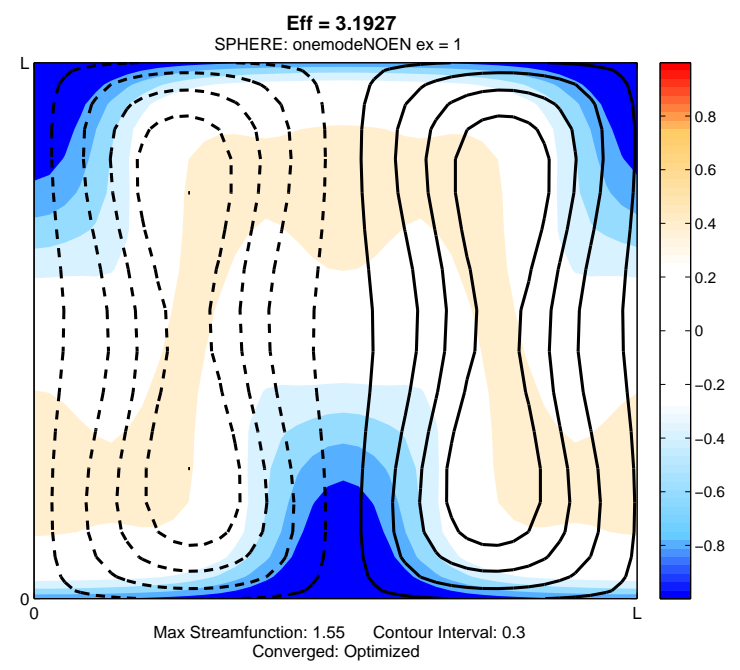

(b) Optimized Flow, $\mathcal{E}=3.19$

Figure 4: Two optimized flows with initial guesses guided by the efficient, sub-optimal flows $Y_{2}^{2}$ and $Y_{3}^{2}$ shown in Figure 2. Contours of the streamfunction are superimposed on filled, colored contours of the steady state tracer concentration.

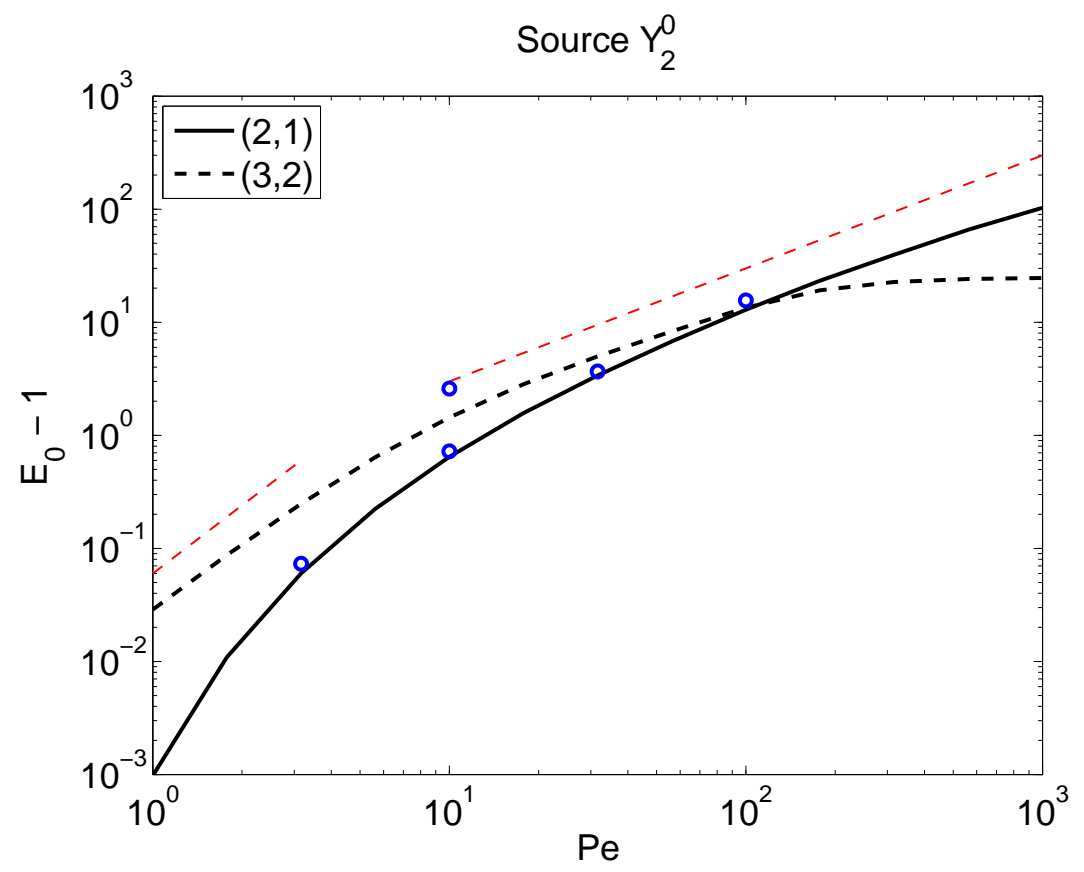

Figure 5: Efficiencies $\mathcal{E}-1$ of optimized flows (blue circles) and sub-optimal flows (black lines) as a function changing Péclet number. The efficiencies of sub-optimal flows are those arising from streamfunctions proportional to $Y_{3}^{2}$ (dashed) and $Y_{2}^{1}$ (solid). Red dashed lines are the predicted scaling for the most efficient flows at low Pe (quadratic scaling) and at high $P e$ (linear scaling). 


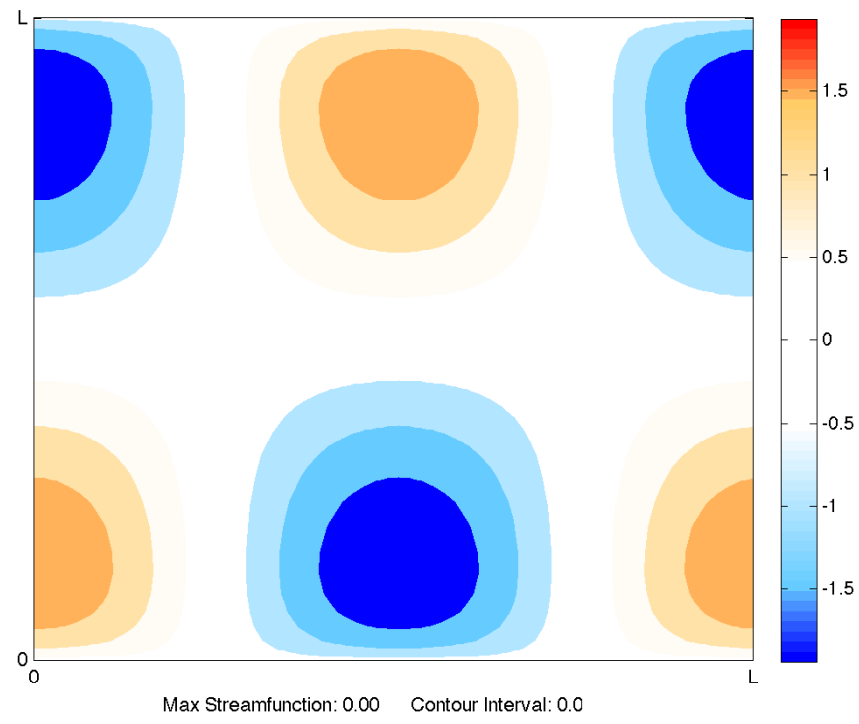

Figure 6: The cellular source on the sphere mapped in latitude-longitude coordinates.

with $m=1$ (Panel $4(\mathrm{~b}))$ at $\mathcal{E}=3.30$ as compared to $\mathcal{E}=3.19$. Expectedly, both flows have efficiencies greater than the most efficient sub-optimal flow in Figure 2.

While we were able to obtain these two optimal flows at $P e=10$, it was not without difficulty. As state in Section 2.3, the optimization algorithm for the sphere is an iterative process that will converge on local minima, if it converges at all. Unfortunately, due to time limitations, very few optimal solutions were found while for varying $P e$. Those that we could identify are plotted in Figure 5 along with the efficiencies of sub-optimal flows. With the exception of one point at $P e=10$, most all optimized solutions lie very near that of the initial guess (the $Y_{2}^{1}$ mode).

In place of looking at the optimized flows, we examine the scaling of the efficiencies of the sub-optimal 'best guess' streamfunctions with monochormatic $Y_{2}^{1}$ or $Y_{3}^{2}$ modal structure instead of the true optimal solutions. From Figure 5, we see that the maximum efficiencies of the $Y_{2}^{1}$ and $Y_{3}^{2}$ modes appear to scale in agreement with the observations of Thiffeault [6] and of Shaw et al. [5] for the torus, namely that the mixing efficiencies scale as $P e$ for small $P e$ and transition to a $P e^{2}$ scaling at high $P e$.

The scaling of $\mathcal{E}$ with $P e$ even holds if we move away from the geophysical source. If we consider a cellular source on the sphere (Figure 6) and its equivalent on the torus, we can compare the scaling of the efficiencies between the two geometries directly. The efficiencies of sub-optimal flows on the sphere (Panel 7(a)) and both optimal and sub-optimal flows on the torus (Panel 7(b)) are plotted against $P e$ in Figure 7.

There are two primary observations to note in Figure 7: the close proximity of optimized flows on the torus to the sub-optimal cellular flows and the agreement with $\mathrm{Pe}$ scaling arguments. For optimized flows on the torus, as with the optimized flows on the sphere in Figure 5, the optimal solutions lie very close to the 'best guess' sub-optimal flows. This suggests that the optimized efficiencies on the sphere for the cellular source should be 


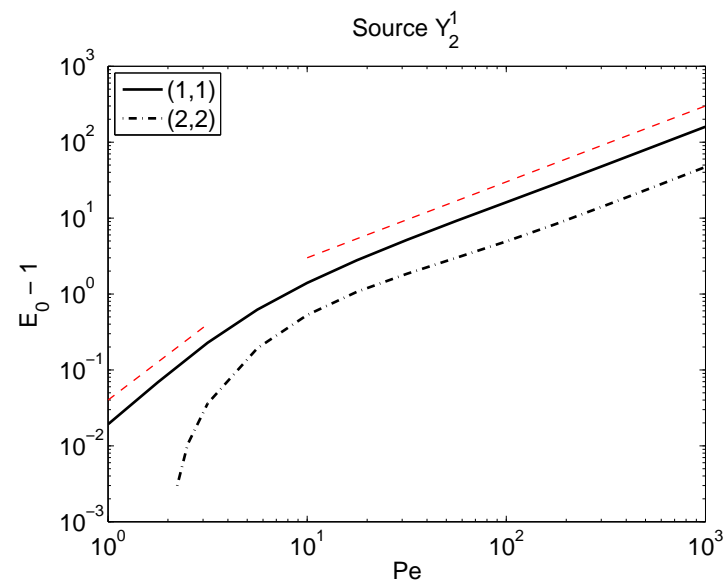

(a) Sphere

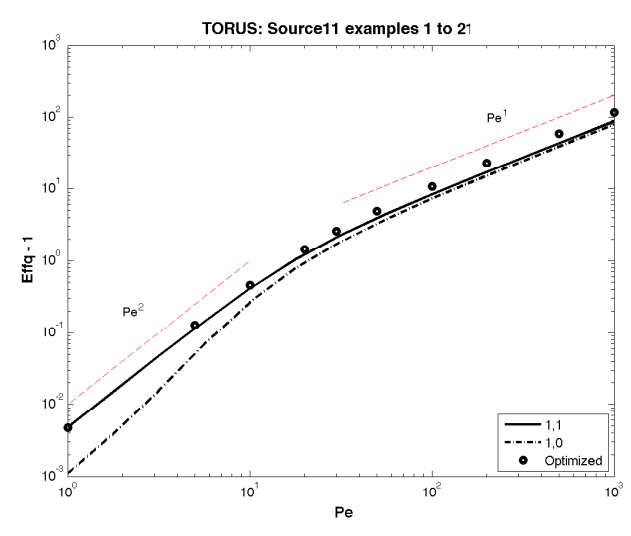

(b) Torus

Figure 7: Scaling of efficiencies $\mathcal{E}-1$ with respect to changing $P e$ for the cellular source function (shown in Figure 6 for the sphere) on the sphere (Panel 7(a)) and for the equivalent flow on the torus (Panel 7(b)). Efficiencies of sub-optimal flows of $Y_{1}^{1}$ (solid) and $Y_{2}^{2}$ (dashed) over the surface of the sphere are shown in Panel 7(a). Similarly, sub-optimal flows on the torus are shown for $k_{x}=1, k_{y}=1$ (solid) and $k_{x}=1, k_{y}=0$ (dashed) in Panel 7(b). Optimized flows for the torus are shown in circles. Quadratic and linear $P e$ slopes are red-dashed.

relatively close to the sub-optimal flows shown in Figure 7, however this still needs to be shown in future work.

The close agreement to the $P e$ scaling between the torus and the sphere suggests that, unlike the lower bound on $\mathcal{E}$, one property of maximally efficient flows from the torus does carry over to the sphere. Why, then, does the efficiency scale linearly in $P e$ at high $P e$ and as $P e^{2}$ at small $P e$ ? Why is this result independent of geometry?

At high $P e$, we can approximate the effect of stirring by and eddy diffusivity, $\kappa_{e f f}$, that is significantly larger than the molecular diffusivity, $\kappa$. For an advection-dominated regime, an effective diffusivity can be generated by $\kappa_{\text {eff }} \sim U L$, where $U$ is the characteristic length of the advective velocity and $L$ is a characteristic mixing length $[4,5]$. For the cellular flows we've considered here, where the most efficient flows tend to be those of the largest scale permissible, the characteristic length scale is that of the domain for the torus or that of radius for the sphere.

The stirred steady state advection-diffusion equation (1) is then parameterized by

$$
\kappa_{e f f} \Delta \theta \approx s
$$

while the unstirred, diffusive steady state problem is unchanged

$$
\kappa \Delta \theta_{0}=s
$$

so that the solutions to the steady state stirred and unstirred systems are

$$
\theta \approx \frac{1}{\kappa_{e f f}} \Delta^{-1} s \quad \theta_{0}=\frac{1}{\kappa} \Delta^{-1} s
$$


The efficiency $\mathcal{E}$ is then approximated by

$$
\mathcal{E}=\sqrt{\frac{\left\langle\left|\theta_{0}\right|^{2}\right\rangle}{\left\langle|\theta|^{2}\right\rangle}} \approx \sqrt{\frac{\kappa^{-2}\left\langle\left|\Delta^{-1} s\right|^{2}\right\rangle}{\kappa_{e f f}^{-2}\left\langle\left|\Delta^{-1} s\right|^{2}\right\rangle}}
$$

which scales as

$$
\mathcal{E} \sim \frac{\kappa_{e f f}}{\kappa} \sim \frac{U L}{\kappa}=P e
$$

where we have used $\kappa_{e f f} \sim U L$. From (37), the mixing efficiency $\mathcal{E}$ should scale linearly with $P e$ so long as $P e$ is sufficiently large such that the mixing is advection-dominated and the advection-diffusion equation can be approximated by an eddy diffusivity.

The diffusion-dominated small $P e$ regime, on the other hand, appears to scale quadratically in $P e$ for the cases we have considered here. Consider the advection-diffusion operator $\mathcal{L}$ given in (9) in the limit of small Pe. By definition the Péclet number is the scale of the advection term relative to the diffusive term in (1) and could be considered a small parameter in the steady-state, nondimensional advection-diffusion equation

$$
(P e \hat{\boldsymbol{u}} \cdot \hat{\nabla}-\hat{\Delta}) \hat{\theta}=\hat{s}
$$

where hatted variables are nondimensional. We have assumed here that, to a first approximation, the size of the source function scales as $\Delta \theta$ in the limit of small $P e$.

The inverse of the nondimensional operator $\hat{\mathcal{L}}^{-1}=(P e \hat{\boldsymbol{u}} \cdot \hat{\nabla}-\hat{\Delta})^{-1}$ can then be approximated by expanding about $P e \ll 1$. Let $A=\hat{\boldsymbol{u}} \cdot \hat{\nabla}$ be the advection operator and let $D=-\hat{\Delta}$ be the diffusion operator. Additionally, let $\epsilon=P e$ be the small parameter. The nondimensional advection-diffusion operator (dropping the hats) is then

$$
\mathcal{L}=D+\epsilon A .
$$

The inverse of (39) can then be expanded out to $O(\epsilon)$

$$
\mathcal{L}^{-1}=D^{-1}-\epsilon D^{-1} A D^{-1}+O\left(\epsilon^{2}\right)
$$

such that $\left\langle|\theta|^{2}\right\rangle=\left\langle\left|\mathcal{L}^{-1} s\right|^{2}\right\rangle$ is

$$
\begin{aligned}
\left\langle|\theta|^{2}\right\rangle & =\frac{1}{V} \int\left(\mathcal{L}^{-1} s\right)^{2} d V \\
& =\frac{1}{V} \int\left(D^{-1} s-\epsilon D^{-1} A D^{-1} s+O\left(\epsilon^{2}\right)\right)^{2} d V
\end{aligned}
$$

Expanding the square of (41) yields

$$
\left\langle|\theta|^{2}\right\rangle=\frac{1}{V} \int\left(D^{-1} s\right)^{2}-2 \epsilon\left(D^{-1} s\right)\left(D^{-1} A D^{-1} s\right) d V+O\left(\epsilon^{2}\right)
$$

The advection operator can be written in terms of, $A \xi=[\psi, \xi]$, where

$$
[\psi, \xi]=\frac{\partial \psi}{\partial x} \frac{\partial \xi}{\partial y}-\frac{\partial \xi}{\partial x} \frac{\partial \psi}{\partial y}=v \frac{\partial \xi}{\partial y}+u \frac{\partial \xi}{\partial x}
$$


on the torus, noting that the non-divergent advective velocities are defined using a streamfunction $\psi$ by $u=-\frac{\partial \psi}{\partial y}$ and $v=\frac{\partial \psi}{\partial x}$. On the sphere,

$$
[\psi, \xi]=\left(\frac{1}{a \cos \phi} \frac{\partial \psi}{\partial \lambda}\right)\left(\frac{1}{a} \frac{\partial \xi}{\partial \phi}\right)-\left(\frac{1}{a \cos \phi} \frac{\partial \xi}{\partial \lambda}\right)\left(\frac{1}{a} \frac{\partial \psi}{\partial \phi}\right)
$$

for the non-divergent velocities given in (21).

Replacing the advection operator in $(42)$ with $[\psi, \xi]$ yields

$$
\left\langle|\theta|^{2}\right\rangle=\frac{1}{V} \int\left(D^{-1} s\right)^{2}-2 \epsilon\left(D^{-1} s\right)\left(D^{-1}\left[\psi, D^{-1} s\right]\right) d V+O\left(\epsilon^{2}\right)
$$

such that the variation in $\left\langle|\theta|^{2}\right\rangle$ given a change in the streamfunction $\delta \psi$ is

$$
\begin{aligned}
\delta\left\langle|\theta|^{2}\right\rangle & =-\frac{2 \epsilon}{V} \int\left(D^{-1} s\right) D^{-1}\left[\delta \psi, D^{-1} s\right] d V+O\left(\epsilon^{2}\right) \\
& =-\frac{2 \epsilon}{V} \int \delta \psi\left[D^{-1} s, D^{-2} s\right] d V+O\left(\epsilon^{2}\right) .
\end{aligned}
$$

To $O\left(\epsilon^{2}\right)$, the variation of $\left\langle|\theta|^{2}\right\rangle$ is then

$$
\frac{\delta\left\langle|\theta|^{2}\right\rangle}{\delta \psi}=-2 \epsilon\left\langle\left[D^{-1} s, D^{-2} s\right]\right\rangle+O\left(\epsilon^{2}\right)
$$

For any source that satisfies $\left[D^{-1} s, D^{-2} s\right]=0$, such as monochromatic sources, the variation of $\left\langle|\theta|^{2}\right\rangle$ with respect to a change in the streamfunction $\delta \psi$ will be zero at $O(\epsilon)$. As both the geophysical and the cellular sources considered here are monochromatic, we can expect $\left\langle|\theta|^{2}\right\rangle$ to vary as $\epsilon^{2}=P e^{2}$, hence the quadratic scaling in the Péclet number at small $P e$. If we were to instead look flows which optimized $\mathcal{E}$ on the sphere with a source function satisfying $\left[D^{-1} s, D^{-2} s\right] \neq 0$, we might expect linear scaling at small $P e$. Experiments so far, however, have been inconclusive. Finding sources where efficiencies scale linearly in $P e$ is left for future work.

\section{Sweeping Flow}

It is interesting to note that the $Y_{3}^{2}$ flow appears to saturate in efficiency around $P e=10^{2}$ in Figure 5 for the geophysical source. This saturation leads to a crossing of the efficiencies of sub-optimal flows. Although streamfunctions with a modal structure of $Y_{3}^{2}$ are more efficient mixers than those of $Y_{2}^{1}$ at small $\mathrm{Pe}, Y_{2}^{1}$ is more efficient than the $Y_{3}^{2}$ mode at high $P e$.

The saturation of $\mathcal{E}$ at high $P e$ for the $Y_{3}^{2}$ streamfunction can be understood by looking at the steady state snapshots of $\theta$ and $\psi$ in Figure 8. At $P e=10^{3}$, the tracer field becomes trapped near the stagnation points of $\boldsymbol{u}$. For the saturated streamfunction in Panel $8(\mathrm{~b})$, the flow velocity is largely parallel to contours of constant $\theta$ in steady state such that the velocity field is no longer stirring the tracer field as, for these flows, $\boldsymbol{u} \cdot \nabla \theta \approx 0$. As the advective term of (7) is small, increasing the Péclet number further by either decreasing the diffusivity or increasing the strength of the velocity field $\boldsymbol{u}$ will do little to change the mixing efficiency, hence $\mathcal{E}$ will not change greatly with increasing $P e$ as observed in Figure 5. 


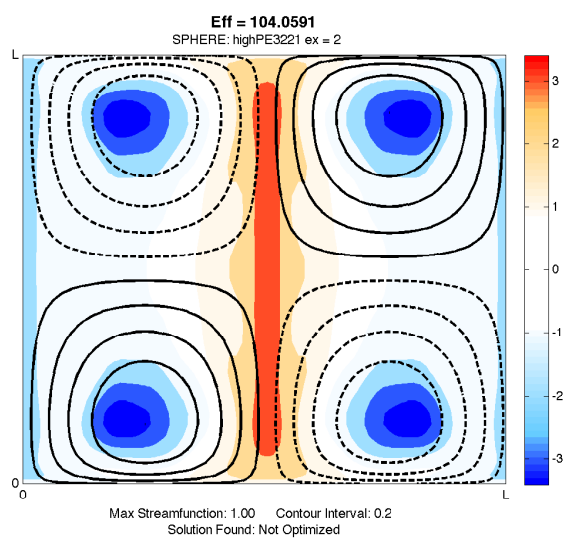

(a) High $P e, \mathcal{E}=104.06, Y_{2}^{1}$

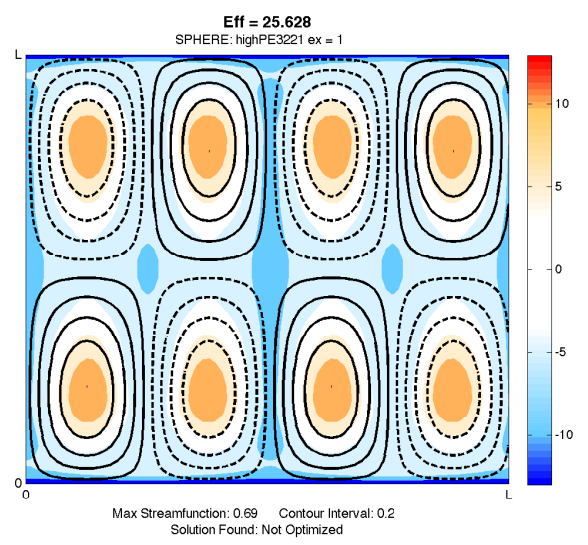

(b) High $P e, \mathcal{E}=25.63, Y_{3}^{2}$

Figure 8: Flows given with a streamfunction structure of $Y_{2}^{1}$ in Panel 8(a) and $Y_{3}^{2}$ in Panel 8(b) at $P e=10^{3}$ in the region of efficiency saturation. Streamlines (black) are superimposed on colored contours of the stirred, steady-state $\theta$.

We are interested in finding the velocity field that most efficiently mixes the a tracer over the surface of the sphere for a given source function, however we have only considered time independent velocity fields that contain stagnation points in $\boldsymbol{u}$. The presence of these stagnation points limits the mixing efficiency of these steady flows at high $P e$. We may be able to achieve higher $\mathcal{E}$ if we allow the flow to vary in time such as to effectively remove these stagnation points on average.

One example of such an efficient, time-varying flow for the torus is found in Shaw et al. [5]. Shaw et al. [5] are able to saturate the upper bound of $\mathcal{E}$ given in (27) using a 'sweeping' flow. This flow is characterized by a constant velocity, $U$, that alternates between a purely zonal flow and a purely meridional flow over the torus over long timescales. Does such a sweeping flow exist for the sphere?

A sweeping flow as described in Shaw et al. [5] for the torus cannot be defined on the sphere in the same manner as it is on the torus due to the presence of the poles. The velocity field $\boldsymbol{u}$ must be zero at the poles because of artificial discontinues in the gradient operator in spherical coordinates. If we were to consider a velocity field that was purely zonal at one instant in time it would be require to have the amplitude of $\boldsymbol{u}=u(\phi) \hat{\lambda}$ to go to zero as $\phi \rightarrow \pm 90^{\circ}$. A rotation of this flow by $90^{\circ}$ on the sphere would not produce the same purely meridional flow as in the sweeping flow on the torus. Instead, it would produce a flow with strong meridional velocities over the poles and with two stagnation points at the equator. With a fixed coordinate system this rotated flow violates the condition that $|\boldsymbol{u}|=0$ at the poles.

A sweeping flow on the sphere would then require more than just a rotation of the velocity field. One method to obtain a sweeping flow on the sphere is described by Finn [2]. Finn [2] considers a sweeping flow produced by a rotation of both the velocity field and the source function or, equivalently, a rotation of the coordinate system. 
Perhaps the simplest sweeping flow over the sphere would have an initial velocity field

$$
\boldsymbol{u}=\sqrt{\frac{3}{2}} U \cos (\phi) \hat{\lambda}
$$

mixing a tracer supplied by the source function given by

$$
s(\lambda, \mu)=S P_{1}(\mu)(\exp (-i \lambda)+\exp (i \lambda))=S\left(Y_{1}^{1}(\lambda, \mu)+Y_{1}^{-1}(\lambda, \mu)\right) .
$$

This source function is not identical to the rotate geophysical source. It instead describes a sphere heated in the western hemisphere and cooled in the eastern hemisphere. We choose this particular source instead of the rotated geophysical source in Finn [2] as simplifies the advective term significantly upon rotation of the velocity field.

We allow the velocity field given in (49) to mix the source from (50) to steady state before rotating the velocity field by $90^{\circ}$. Upon rotation, the velocity field will lie parallel to lines of constant $\phi$ as supplied by the source function $s(\lambda, \mu)$ from (50) such that $\boldsymbol{u} \cdot \nabla \theta=0$. As the velocity field does not advect the tracer away from the source, all stirring enhanced mixing occurs in the first half of the sweeping flow when $\boldsymbol{u}$ is given by (49).

The mixing efficiency of the sweeping flow given by (49) over the sphere can be calculated by first finding the steady state tracer concentration satisfying the steady-state advective diffusive equation of (7). Using the gradient and Laplacian formulations given in (31) and (30), the tracer concentration in steady state satisfies

$$
\begin{aligned}
\sqrt{\frac{3}{2}} \frac{U}{a}\left(\sum_{n=0}^{\infty} \sum_{m=-n}^{n} i m \tilde{\theta}_{n}^{m} Y_{n}^{m}(\lambda, \mu)\right)+\kappa \sum_{n=0}^{\infty} \sum_{m=-n}^{n} \frac{n(n+1)}{a^{2}} \tilde{\theta}_{n}^{m} Y_{n}^{m}(\lambda, \mu) \\
=S\left(Y_{1}^{1}(\lambda, \mu)+Y_{1}^{-1}(\lambda, \mu)\right)
\end{aligned}
$$

such that the we can solve for the $\tilde{\theta}_{n}^{m}$ by matching coefficients,

$$
\begin{aligned}
\tilde{\theta}_{1}^{-1} & =S\left(-i \sqrt{\frac{3}{2}} \frac{U}{a}+\kappa \frac{2}{a^{2}}\right)^{-1} \\
\tilde{\theta}_{1}^{1} & =S\left(+i \sqrt{\frac{3}{2}} \frac{U}{a}+\kappa \frac{2}{a^{2}}\right)^{-1} .
\end{aligned}
$$

By Parsevals' theorem, the variance of $\theta$ is then

$$
\left\langle|\theta|^{2}\right\rangle=2 \pi \sum_{n=0}^{\infty} \sum_{m=-n}^{n}\left|\tilde{\theta}_{n}^{m}\right|^{2}
$$

which, given the coefficients from (52), allows us to find the steady state tracer variance of the stirred problem

$$
\left\langle|\theta|^{2}\right\rangle=4 \pi S^{2}\left(4 \frac{\kappa^{2}}{a^{4}}+\frac{3}{2} \frac{U^{2}}{a^{2}}\right)^{-1} .
$$


By taking $U=0$ in (54), we can similarly find the steady state tracer variance of the unstirred problem

$$
\left\langle\left|\theta_{0}\right|^{2}\right\rangle=4 \pi S^{2}\left(4 \frac{\kappa^{2}}{a^{4}}\right)^{-1}
$$

we can calculate the efficiency of the sweeping flow as

$$
\mathcal{E}^{2}=\frac{\left\langle\left|\theta_{0}\right|^{2}\right\rangle}{\left\langle|\theta|^{2}\right\rangle}=\frac{a^{4}}{4 \kappa^{2}}\left(4 \frac{\kappa^{2}}{a^{4}}+\frac{3}{2} \frac{U^{2}}{a^{2}}\right)=1+\frac{3}{8} P e^{2}
$$

where $P e=\frac{U a}{\kappa}$.

If we were to compare the mixing efficiency of the sweeping flow over the sphere to the upper bound for the mixing efficiency of a monochromatic source similar to that of (50) on the torus in (27), we would expect that the most efficient sweeping flow over the torus would have a mixing efficiency of

$$
\mathcal{E}_{\text {torus }}^{2}=1+\frac{1}{2} P e^{2}
$$

where the source function on the $d=2$ torus is described by

$$
s(x, y)=S_{\text {torus }}\left(e^{i x}+e^{-i x}\right)
$$

in a domain of length $L=1$ with a total wavenumber of $k_{s}^{2}=1$.

In comparing (56) to (57), we note that the mixing efficiency of the sweeping flow on the sphere is very close to that of the torus. The effiency $\mathcal{E}^{2}$ of the sweeping flow on the sphere scales as $\frac{3}{8} P e^{2}$ as compared to a scaling of $\frac{1}{2} P e^{2}$ on the torus. For the same Péclet number, the sweeping flow over the torus will be slightly more efficient than the corresponding sweeping flow on the sphere.

Additionally, the sweeping flow over the sphere has an efficiency $\mathcal{E}=\sqrt{1+\frac{3}{8} P e^{2}}$ which scales as

$$
\mathcal{E} \approx\left\{\begin{array}{cc}
\sqrt{\frac{3}{8}} P e & \text { for } P e \gg 1 \\
1+\frac{3}{16} P e^{2} & \text { for } P e \ll 1
\end{array}\right.
$$

such that $\mathcal{E}$ scales linearly in $P e$ at high $P e$ and quadratically in $P e$ at low $P e$ in agreement with the arguments for highly efficient flows found in Section 4.

While Shaw et al. [5] found that the sweeping flow on the torus is the most efficient flow for a monochromatic source function, we are not guaranteed that the sweeping flow described by (49) is the most efficient flow for the the sphere. One particular issue with any flow on the sphere arises due to the location of stagnation points in the flow.

As we must have $|\boldsymbol{u}|=0$ at the poles for any rotated coordinate system we choose, we will always have stagnation points in the velocity field over the poles. It would then always be possible to achieve the trapping behavior described for inefficient flows in Section 3 if these polar stagnation points are located away from the mean value of the source function. (One could consider regions where the source is equal to its mean as already well-mixed, thus stirring by $\boldsymbol{u}$ could do little to reduce the variance in these regions.) This is one reason 
why we chose the source given in (50) rather than the rotated geophysical source. The rotate geophysical source from Finn [2], given by

$$
s(\lambda, \mu)=-\frac{\sqrt{3}}{2} S P_{2}^{2}(\mu)(\exp (-2 i \lambda)+\exp (2 i \lambda))+\frac{1}{\sqrt{2}} S P_{2}^{0}(\mu),
$$

would have a maxima over the poles. These regions could never be mixed for a fixed spherical coordinate system, leading to a saturation of $\mathcal{E}$ with increasing $P e$ similar to the suboptimal flows stirred by a streamfunction proportional to $Y_{3}^{2}$ in Figure 5 and in Panel 8(b).

Finn [2] suggests a more efficient sweeping flow could be achieved by a careful rotation of the coordinate system at each time interval. If the poles of the spherical coordinate system are always located in regions where the source function achieves its mean value one could bypass the trapping issue on the sphere and possibly find the most efficient, time-dependent flows on the sphere.

\section{Discussion and Future Directions}

In this work we sought answers to the two following questions: 1) what flows most efficiently mix a tracer field on the surface of the sphere that is supplied by a spatially-varying source function? and 2) how do the properties of these flows compare to similar velocity fields on the torus? We were able to find flows that maximized the mixing efficiency given in (5) using a numerical optimization scheme. This optimization scheme, however, only optimizes the velocity field according to one measure of the mixing efficiency. In determining the flows that maximize other possible measures of mixing efficiency, such as the multi-scale mixing efficiencies described in Shaw et al. [5] given by

$$
\mathcal{E}_{p}^{2}=\frac{\left\langle\left|\nabla^{p} \theta_{0}\right|^{2}\right\rangle}{\left\langle\left|\nabla^{p} \theta\right|^{2}\right\rangle}
$$

where $p=-1,0,1$, we could emphasize the homogenization of variance of $\theta$ on smaller $(p=1)$ and larger $(p=-1)$ spatial scales.

In changing geometries we find that many of the properties of optimized flows over the torus do not translate directly to that of the sphere. The hard lower bound of $1 \leq \mathcal{E}$ of Shaw et al. [5], for example, is violated for the geophysical source on the sphere due to the inescapable presence of stagnation points in $\boldsymbol{u}$ and the trapping of the tracer concentration at its source.

The issue of stagnation points in the stirring field on the sphere also has consequences for saturating an upper bound, should such an upper bound be discovered in the future. To remove these stagnation points we must consider time-dependent velocity fields similar to the sweeping flow described by Shaw et al. [5] modified by the solid body rotation method introduced by Finn [2]. It may be possible to determine the most efficient time-dependent flow for a given source function on the sphere by using the method of steepest decent described in Lin et al. [3].

One similarity between efficiencies of optimal (or sub-optimal, in the case of the sphere) flows for the two geometries is that of the scaling of $\mathcal{E}$ with changing $P e$. For both the geophysical and the cellular source functions, the efficiency $\mathcal{E}$ scales linearly with $P e$ at high 
$P e$ and quadratically with $P e$ at small $P e$. For diffusion-dominated flows at small Péclet number we expanded the advection-diffusion integral operator, $\mathcal{L}^{-1}$ around the diffusive term and determined that the $\mathcal{E} \sim P e^{2}$ scaling in this regime was a consequence of our choice of monochromatic sources. It is an open question as to whether we can obtain linear Péclet number scaling for non-monochromatic sources, such as sources with off-equatorial heating.

As we are motivated here by the geophysical question of the redistribution of heat over the surface of the sphere, the optimization of $\mathcal{E}$ subject to more complex source functions are of significant interest. Some source functions in particular are those with off-equatorial heating, as mentioned, to simulate seasonal changes in solar insolation. Additional source functions we could consider include those with longitudinally localized heating to simulate land-sea surface temperature contrasts.

Throughout this work we have considered a velocity field that is independent of temperature, however in a geophysical context these two fields are not wholly independent. It may be of further interest to modify the code to allow for coupling between the velocity field and the tracer field.

The energy constraint here follows from previous work on the torus. However, this is not the only dynamical constraint we may wish to explore on the sphere. If we were to include some form of rotation to our model of optimized flows over the sphere, we could additionally consider an angular momentum constraint in the form of

$$
M=\Omega a^{2} \cos ^{2} \phi+u a \cos \phi
$$

where $M$ is the angular momentum, $\Omega$ is the rotation rate, $a$ is the radius of the sphere, and $u$ is the zonal component of the non-divergent velocity field $\boldsymbol{u}$.

On the Earth, the redistribution of heat on the sphere from large-scale atmospheric stirring is not primarily achieved by the barotropic circulations we have considered here, but instead by the near-zonally symmetric circulations of the Hadley and Ferrel cells in height $z$ and latitude $\phi$ coordinates. A question of significant geophysical interest would then be what zonally symmetric circulation pattern in $z-\phi$ coordinates most efficiently mixes temperature on the sphere? Furthermore, can such a flow be obtained using a numerical optimization scheme such as the one used here?

\section{Acknowledgements}

I would foremost like to thank Tiffany Shaw and Jean-Luc Thiffeault for their guidance and advice throughout the summer, particularly in the final days of the program when everything came together. The GFD program wouldn't be the same without softball; I would like to thank George Veronis and Charlie Doering for their coaching and endless enthusiasm through the summer's practices, games, and injuries. Last, but certainly not least, I'd like to thank my fellow fellows-Anubhab, David, Emma, Renske, Georgy, Kiori, Michael, Sam, and Woosok-for all the late nights in Walsh Cottage, fantastic dinners, trips to the beach, and more. 


\section{References}

[1] J. B. Drake, P. Worley, and E. D'Azevendo, Algorithm 888: Spherical harmonic transform algorithms, ACM Trans. Math. Softw., 35 (2008), pp. 23:1-23.

[2] M. D. Finn, Mixing on the sphere. notes, 2010.

[3] Z. Lin, J.-L. Thiffeault, And C. R. Doering, Optimal stirring strategies for passive scalar mixing. Under consideration for publication in J. Fluid Mech., 2010.

[4] J. M. Ottino, Mixing, chaotic advection, and turbulence., Annu. Rev. Fluid Mech., 22 (1990), pp. 207-253.

[5] T. A. Shaw, J.-L. Thiffeault, And C. R. Doering, Stirring up trouble: Multi-scale mixing measures for steady scalar sources, Physica D, 231 (2007), pp. 143-164.

[6] J.-L. Thiffeault, Quantifying fluid mixing using norms. in preparation, 2010.

[7] J.-L. Thiffeault, C. R. Doering, and J. D. Gibbon, A bound on mixing efficiency for the advection-diffusion equation, J. Fluid Mech., 521 (2004), pp. 105-114.

[8] J.-L. Thiffeault and G. A. Pavliotis, Optimizing the source distribution in fluid mixing, Physica D, 237 (2008), pp. 918-929. 


\title{
Snail locomotion
}

\author{
Sam Pegler, \\ advised by Neil Balmforth
}

\section{Introduction}

A snail propels itself by deforming a layer of mucus beneath it via muscular contractions in its foot muscle. This unique form of locomotion allows snails to traverse almost any obstacle, including the ability to climb steep inclines. Throughout the snail kingdom, there exist a number of variations for the pattern of muscular forcing used by the foot to activate locomotion and in particular, the two main classes of snails, namely land snails and water snails, have adopted two very different patterns. Water snails prefer a method in which its foot applies a predominantly vertical force upon the mucus layer as a wave travelling in the opposite direction to that of locomotion. This retrograde locomotion generates forces in a similar manner to that of a peristaltic pump, whereby the forcing surface undergoes significant perpendicular deformation. Land snails prefer a method that applies a wave of tangential muscular contractions that travel in same direction to that of locomotion. Unlike the retrograde case, this prograde locomotion involves significant tangential contraction of the base of the foot muscle, and little vertical deformation. These processes involve a rich mixture between the fluid mechanics of the mucus layer and its interaction with the muscular and elastic mechanics of the snail's foot.

A further complication is introduced by the non-Newtonian rheology of snail mucus, which is known to have an appreciable yield stress, i.e, a minimum stress necessary to deform it. Evolution has lead to this rheology because it allows the snail to remain adhered to a steep incline without having to apply any force to counteract its weight. By selectively forcing the mucus in a wave-like pattern, the snail is able to climb the incline without slipping. In this report we investigate the two forms of snail locomotion outlined above, and further attempt to quantify the role of yield stress in locomotion.

Studies of locomotion in fluids began with the seminal work of Taylor [4], who considered the low-Reynolds number problem of a two-dimensional surface surrounded by a Newtonian fluid, that swims by exerting a normal force as a wave travelling in the opposite direction to that of locomotion. By performing a biharmonic analysis of the flow in the limit of a low-amplitude forcing, he relates the velocity of locomotion to the parameters of the forcing. This problem was generalized by Katz [3] to include a rigid wall lying parallel to the swimmer, which he investigates using both the biharmonic approach of Taylor, and a lubrication theory for the thin-gap limit. More recently, Balmforth, Coombs and Pachman [1] extend this lubrication theory to cases involving non-Newtonian fluids in the thin-gap geometry. This study, which considers the effect of a yield stress, is of relevance to the process of retrograde snail locomotion. A model of prograde snail locomotion is investigated 


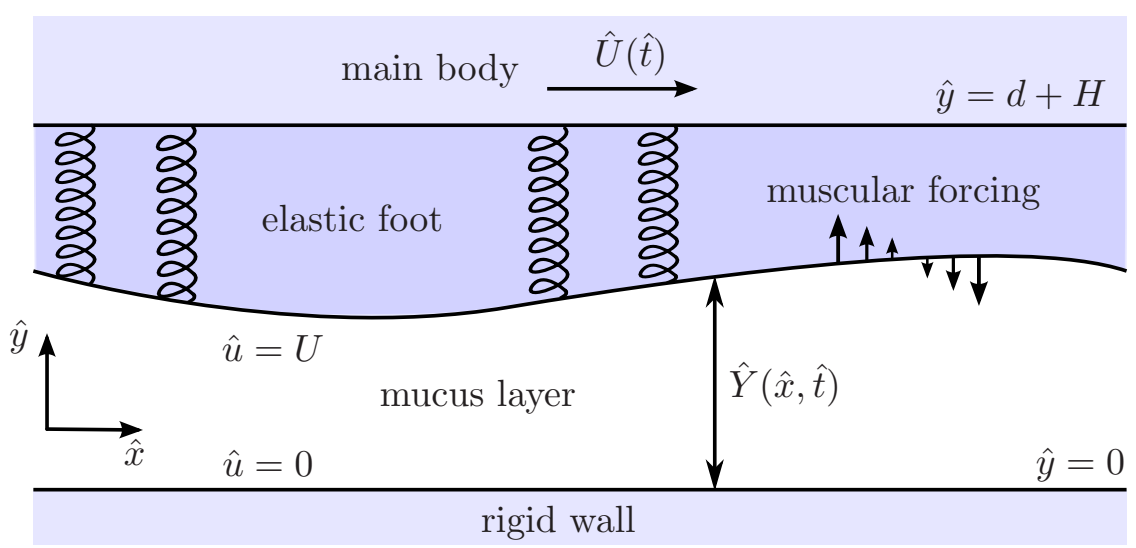

Figure 1: Schematic of the foot and mucus layer in retrograde locomotion.

by Chan, Balmforth and Hosoi [2], in which they model the foot by prescribing its tangential displacement.

In this project, we begin in section 2 with an investigation into the process of retrograde snail locomotion that extends the work of Balmforth et al. [1] by solving the system as an initial-value problem, and further explores the effect of the elastic parameters of the foot on the locomotion velocity. We show that the system converges toward a steady state, which we proceed to study by solving the associated eigenvalue problem. From our solutions, we identify a critical yield stress above which locomotion does not occur, and further relate the locomotion velocity to the parameters of the system. In section 3, we consider the case of prograde snail locomotion in which we extend the work of Chan et al. [2] by developing a fully dynamic model with both a muscular forcing and a consideration of the elastic mechanics of the snail's foot. We proceed to study the resulting system numerically and derive some analytical results.

\section{Retrograde locomotion}

\subsection{The foot}

We assume a configuration in which a layer of mucus of average height $H$, is confined between the foot's surface at $\hat{y}=\hat{Y}(\hat{x}, \hat{t})$, and a stationary rigid wall at $\hat{y}=0$, as depicted schematically in figure 1. The foot is assumed to be composed of a linear elastic material, of average thickness $d$, that lies in contact with the main body of the snail at $\hat{y}=H+d$, which we assume to be a rigid solid that is able to translate horizontally with velocity $\hat{U}(\hat{t})$. Further, we allow the foot to subject itself to forces via internal muscular contractions, which lead to elastic deformation and hence provide a means to activate its motion. Analysis of snail anatomy has shown that these contractions are produced by a series of muscular fibres inclined at varying angles that lie anchored just inside the main body of the snail and the base of the foot. By choosing the contraction pattern of these inclined fibres appropriately, the snail is able to force different points of its foot's surface in a variety of directions and patterns. 
In retrograde locomotion, the contraction pattern is such that the resultant muscular forces act predominantly normal to the interface between the mucus layer and the foot, as shown in figure 1. Anatomical studies of snails have shown that the top surface of the foot does not deform significantly into the main body of the snail. Thus, we assume that the main body of the snail acts as a rigid foundation, such that the top surface of the foot does not penetrate the main body above. Assuming the foot is very slender $d \ll L$, where $L$ is a characteristic length scale for its horizontal variation, the leading-order pressure in the foot is given by

$$
\hat{p}=\hat{A} f(\hat{x}, \hat{t})+\hat{D}_{R}(\hat{Y}-H),
$$

where $\hat{A} f$ represents the muscular forcing, $\hat{A}$ is the amplitude of the forcing, and $\hat{D}_{R}$ is the vertical stiffness coefficient that can be expressed in terms of the elastic parameters of the foot. The elastic force, represented by the second term on the right-hand side of this equation, provides a restoring force that is proportional to the perturbation of the thickness profile from $H$. Thus, we can consider the surface of the foot to be equivalent to a sheet attached to the main body of the snail via Hookean springs of equilibrium height $d$, as illustrated in figure 1 , where $\hat{D}_{R}$ represents the Young's modulus of the springs. Based on observations of wave-like patterns of muscular contraction that propagate along the feet of water snails, we assume a forcing of the form

$$
f=f(k \hat{\xi}),
$$

where $\hat{\xi}=\hat{x}+c \hat{t}$. For positive wave speed $c$, equation (2) corresponds to a wave travelling in the negative $\hat{x}$-direction, chosen in anticipation that this will lead to steady locomotion in the positive $\hat{x}$-direction.

The absence of significant tangential forcing implies that we can assume that the foot undergoes negligible horizontal deformation to leading order. In this case, the horizontal velocity of the entire foot is equal to that of the main body of the snail $\hat{U}$, so that the velocity of the foot's surface is given uniformly by

$$
\hat{u}_{s}=\hat{U}(\hat{t}) .
$$

Having established the fundamentals of the foot mechanics in retrograde locomotion, we proceed to consider the fluid mechanics of the mucus layer, which we subsequently relate to the elastic mechanics of the foot via boundary conditions imposed at the foot-mucus interface.

\subsection{The mucus layer}

We assume that the mucus layer is composed of an incompressible yield-stress fluid that is subject to forcing from the foot above. We proceed to detail a model for the yield-stress rheology of the fluid, and then simplify the theoretical analysis of the resulting equations by applying a lubrication approximation. The general equations of force balance and continuity 


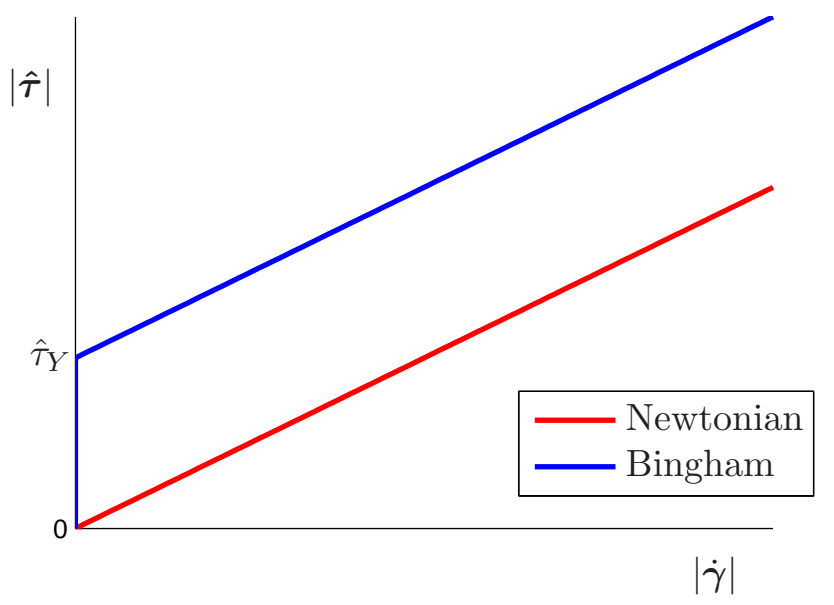

Figure 2: Relationship between the moduli of the deviatoric stress and rate-of-strain tensors for a Newtonian (a) and Bingham (b) fluid.

for an incompressible fluid of velocity $\hat{\boldsymbol{u}}=(\hat{u}, \hat{v})$, are given by

$$
\begin{aligned}
& \nabla \cdot \hat{\boldsymbol{\tau}}=\nabla \hat{p}, \\
& \nabla \cdot \hat{\boldsymbol{u}}=0,
\end{aligned}
$$

where $\hat{\tau}$ is the deviatoric stress tensor, and $\hat{p}$ is the pressure. A constitutive relation that relates the deviatoric stress to the deformation of the fluid is necessary to close the system. The simplest model of a yield-stress fluid is the Bingham model, in which one applies the constitutive relation

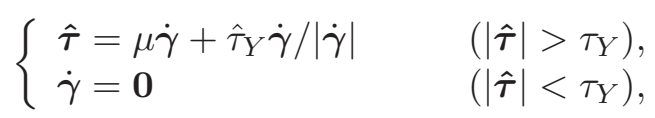

where $\dot{\gamma} \equiv \nabla \hat{\boldsymbol{u}}+(\boldsymbol{\nabla} \hat{\boldsymbol{u}})^{T}$ is the rate-of-strain tensor, $|\dot{\boldsymbol{\gamma}}| \equiv \sqrt{\dot{\gamma}: \dot{\gamma}}$ and $|\hat{\boldsymbol{\tau}}| \equiv \sqrt{\hat{\boldsymbol{\tau}}: \hat{\boldsymbol{\tau}}}$ are the moduli of the rate-of-strain and deviatoric stress tensors respectively, the constant $\tau_{Y}$ is the yield stress, and $\mu$ is the coefficient of viscosity. Relation (6b) ensures that the fluid does not deform if the magnitude of the deviatoric stress at a point does not exceed the yield stress. If the magnitude of the deviatoric stress does exceed the yield stress, then relation (6a) implies that the magnitude of the deviatoric stress in excess of the yield stress is linearly proportional to the deformation of the fluid $\dot{\gamma}$. We sketch this relationship in figure 2, in which we compare it to the Newtonian subcase $\tau_{Y}=0$.

Part of the integration of the boundary-value problem defined by equations (4)-(6) involves identifying the locations of yield surfaces where the magnitude of the deviatoric stress tensor attains the yield stress. These surfaces provide boundaries for rigid regions of unyielded fluid, called rigid plugs, in which the magnitude of the deviatoric stress tensor does not exceed the yield stress. Apart from along the yield surfaces, the stress inside these rigid plugs does not form part of the solution described by the Bingham model. The presence of the non-linear yield-stress term in equation (6a), and the need to identify yield surfaces, have the potential to make problems involving Bingham fluids especially difficult 
to investigate theoretically. However, given that the thickness of the mucus layer is much less than the characteristic length scale $L$ of its horizontal variation, we can simplify the equations of motion substantially by making a lubrication approximation $H \ll L$. Under this assumption, a scaling analysis of the terms in equation (4) implies that the vertical shear stress $\hat{\tau}=\hat{\tau}_{x y}$ is much greater than the other components of the deviatoric stress tensor. Furthermore, the scaling between horizontal and vertical velocity gradients given by the continuity equation (5) implies that the flow is predominantly horizontal. Thus, to leading order in the lubrication approximation, the horizontal and vertical components of equation (4) simplify to

$$
\begin{aligned}
& \hat{\tau}_{\hat{y}}=\hat{p}_{\hat{x}}, \\
& \hat{p}_{\hat{y}}=0,
\end{aligned}
$$

respectively, while the continuity equation (5),

$$
\hat{u}_{\hat{x}}+\hat{v}_{\hat{y}}=0
$$

remains unchanged. Furthermore, the constitutive relation (6) can be simplified to

$$
\begin{cases}\hat{\tau}=\mu \hat{u}_{\hat{y}}+\tau_{Y} \operatorname{sgn}\left(\hat{u}_{\hat{y}}\right) & \left(|\hat{\tau}|>\tau_{Y}\right) \\ \hat{u}_{\hat{y}}=0 & \left(|\hat{\tau}|<\tau_{Y}\right)\end{cases}
$$

where the direction of deformation is given simply by the sign of the vertical shear $\operatorname{sgn}\left(\hat{u}_{\hat{y}}\right)$. The reduced equations (7)-(8) represent a balance between the divergence of vertical shear stress and the horizontal gradient of the pressure, which is independent of the vertical coordinate at each $\hat{x}$-location.

For retrograde snail locomotion, equations (7)-(10) are subject to the following no-slip conditions on the upper and lower surfaces

$$
\begin{array}{ll}
\hat{u}=\hat{U}(\hat{t}) & (\hat{y}=\hat{Y}), \\
\hat{u}=0 & (y=0),
\end{array}
$$

respectively. Furthermore, the equation for the pressure at the foot's surface (1), combined with continuity of normal stress, implies that the fluid pressure is given by

$$
\hat{p}=\hat{A} f+\hat{D}_{R} \hat{Y} \quad(\hat{y}=\hat{Y}),
$$

to leading order in the lubrication approximation, where we have absorbed the constant $\hat{D}_{R} H$ into the pressure. Equation (8) implies that the pressure given by (13) applies throughout the vertical extent of the layer at each $\hat{x}$-location. In the following analysis, we assume a sinusoidal muscular forcing of the form

$$
f=\sin [k(\hat{x}+c \hat{t})]=\sin (k \hat{\xi}) .
$$

Noting that this forcing can be considered steady with respect to the variable $\xi$, we proceed to use $\xi$ in favour of the $\hat{x}$-coordinate. Thus, we recast the system in terms of $(\hat{\xi}, \hat{t})$, and 
transform partial derivatives according to

$$
\begin{aligned}
\frac{\partial}{\partial \hat{x}} & \mapsto \frac{\partial}{\partial \hat{\xi}}, \\
\frac{\partial}{\partial \hat{t}} & \mapsto \frac{\partial}{\partial \hat{t}}+c \frac{\partial}{\partial \hat{\xi}} .
\end{aligned}
$$

We proceed to assume that all variables in the system are $2 \pi$-periodic in $k \hat{\xi}$.

On the assumption that the only surface force applied on the main body of the swimmer is from the mucus layer, we can derive an equation for the acceleration of the main body of the snail in terms of the tangential force applied by the mucus along the underside of the foot,

$$
\hat{M} \frac{d \hat{U}}{d \hat{t}}=\frac{k}{2 \pi} \int_{0}^{2 \pi / k} p \hat{Y}_{\hat{\xi}}+\hat{\tau}_{s} \mathrm{~d} \hat{\xi}=\left\langle\hat{\tau}_{0}\right\rangle
$$

where $\hat{M}$ represents the mass of the main body of the snail per unit wavelength, $\hat{\tau}_{0} \equiv$ $\hat{\tau}(\hat{\xi}, 0, \hat{t}), \hat{\tau}_{s} \equiv \hat{\tau}(\hat{\xi}, \hat{Y}, \hat{t})$, and the angled brackets denote the average $\langle\phi\rangle \equiv \frac{k}{2 \pi} \int_{0}^{2 \pi / k} \phi \mathrm{d} \hat{\xi}$. The second equality in (17) is derived by applying integration by parts to the pressure term, and using equations (7)-(8) to rewrite the resulting integrand in terms of the stress at the lower surface.

We can derive an evolution equation for the thickness profile by depth-integrating the continuity equation (24) over the thickness of the layer, to yield

$$
\frac{\partial \hat{Y}}{\partial \hat{t}}+\frac{\partial \hat{q}}{\partial \hat{x}}=0
$$

or in terms of $(\hat{\xi}, \hat{t})$,

$$
\frac{\partial \hat{Y}}{\partial \hat{t}}+\frac{\partial \hat{Y}}{\partial \hat{\xi}}+\frac{\partial \hat{q}}{\partial \hat{\xi}}=0
$$

where the flux is defined by $\hat{q}(\hat{\xi}, \hat{t})=\int_{0}^{\hat{Y}} \hat{u} \mathrm{~d} \hat{y}$.

We non-dimensionalize the system above according to

$$
\begin{aligned}
& \hat{\xi}=\frac{1}{k} \xi, \quad \hat{y}=H y, \quad \hat{Y}=H Y, \quad \hat{t}=\frac{1}{k c} t, \\
& \hat{u}=c u, \quad \hat{U}=c U, \quad \hat{\tau}=\frac{\mu c}{H} \tau, \quad \hat{p}=\frac{\mu c}{k H^{2}} p .
\end{aligned}
$$

where dimensionless variables are denoted without the hat symbol. In terms of dimensionless variables, equations (7)-(8) become

$$
\begin{aligned}
\tau_{y} & =p_{\xi}, \\
p & =A \sin \xi+D Y,
\end{aligned}
$$


respectively, and the constitutive relation (6) becomes

$$
\begin{cases}\tau=u_{y}+B \operatorname{sgn}\left(u_{y}\right) & (|\tau|>B), \\ u_{y}=0 & (|\tau|<B) .\end{cases}
$$

The equations of average thickness and depth-integrated continuity become

$$
\langle Y\rangle=1, \quad Y_{t}+Y_{\xi}+q_{\xi}=0,
$$

the boundary conditions (11)-(12) become

$$
\begin{array}{ll}
u=U(t) & (y=Y), \\
u=0 & (y=0),
\end{array}
$$

and the equation-of-motion of the body (17) becomes

$$
\dot{U}=\frac{1}{M}\left\langle\tau_{0}\right\rangle,
$$

where

$$
A=\frac{k H^{2} \hat{A}}{\mu c}, \quad D=\frac{k H^{3} \hat{D}_{R}}{\mu c}, \quad B=\frac{H \tau_{Y}}{\mu c}, \quad M=\frac{H c k \hat{M}}{\mu},
$$

are the non-dimensional amplitude, non-dimensional stiffness, Bingham number, and nondimensional mass of the main body per unit wave length, respectively. The Bingham number can be considered a non-dimensional yield stress.

The system above defines an initial-value problem for the evolution of the free boundary $Y(\xi, t)$ and the locomotion velocity $U(t)$. To solve it, we can integrate equations $(21)-(23)$ subject to the boundary conditions (26)-(25) for the velocity profile, given $Y(\xi, t)$ and $U(t)$ at each point in time. With the velocity profiles in hand, we evolve the thickness in time using equation (24), and the locomotion velocity using equation (27), in which the surface stress $\tau_{0}$ is established as part of the integration for the velocity profiles. The main complication in the above procedure is the construction of the velocity field for a given $Y(x, t)$ and $U(t)$, which we proceed to detail in the following section.

\subsection{Construction of the flow profiles}

To begin, we can integrate equation (21) with respect to $y$ to yield

$$
\tau=\tau_{0}+p_{\xi} y,
$$

where $\tau_{0}$ is an as yet undetermined function of $\xi$. This expression for the stress applies in any yielded parts of the flow. Combining equation (29) with the component of the constitutive relation (23a) associated with the yielded regions, provides an equation for the velocity profile in the yielded regions,

$$
u_{y}=\tau_{0}+p_{\xi} y-B \operatorname{sgn}\left(u_{y}\right) .
$$


Integrating this equation provides

$$
u=\frac{1}{2} p_{\xi} y^{2}+\left[\tau_{0}-B \operatorname{sgn}\left(u_{y}\right)\right] y+K,
$$

where $K$ is a constant of integration, which shows that the velocity profiles in the yielded regions of the flow are parabolae.

As part of the solution of the velocity profiles, it is also necessary to determine the locations of any yield surfaces where $|\tau|=B$, which provide the boundaries of any unyielded regions of the flow governed by (23b). We can identify two potential yield surfaces $y=\eta_{ \pm}$ by setting $\tau= \pm B$ in equation (29) to give

$$
\eta_{ \pm}=\frac{\tau_{0}}{p_{\xi}} \pm \frac{B}{\left|p_{\xi}\right|},
$$

where the modulus of the pressure gradient has been used in the second term on the righthand side to ensure that $\eta_{+}>\eta_{-}$independently of the sign of the pressure gradient. We refer to $\eta_{ \pm}$as potential yield surfaces because it is possible for one, or both, of $\eta_{ \pm}$to lie outside the domain of the fluid. Equation (32) implies that there exists a region $\eta_{-}<y<\eta_{+}$ in which the velocity is independent of the vertical coordinate. Within these regions, the flow is dominated by the yield stress to leading-order in lubrication theory and thus exhibits plug-like characteristics in the vertical direction. We remark that these regions are not true rigid plugs unless their velocity is also independent of the $\xi$-coordinate. Thus, we refer to regions bounded by the yield surfaces as pseudo-plugs if they are not strictly rigid.

By adding and subtracting the expressions for the potential yield surfaces (32) we deduce that

$$
\eta_{+}+\eta_{-}=\frac{2 \tau_{0}}{p_{\xi}}, \quad \eta_{+}-\eta_{-}=\frac{2 B}{\left|p_{\xi}\right|},
$$

respectively. The latter of these equations provides the width of the pseudo-plug if it lies fully inside the domain, or a maximum for its width if it intersects one of the side walls. The former can be used to relate the positions of the potential yield surfaces to the unknown $\tau_{0}$.

As mentioned above, it is not necessarily the case that the potential yield surfaces given by equation (32) lie inside the domain of the fluid $0<y<Y$. For example, if $\eta_{+}>0$ and $0<\eta_{-}<Y$, then there exists a pseudo-plug in the region $\eta_{-}<y<Y$ that intersects the upper surface. In this case the velocity of the pseudo-plug is equal to the velocity of the side wall it is in contact with and the construction of the velocity profile is different to, for example, the regime in which the pseudo-plug lies fully inside the domain. Thus, the different arrangements arising from whether or not one or both of the potential yield surfaces lie inside the domain require consideration on a case-by-case basis. We summarize the equations for the five possible arrangements in appendix A. For the purposes of demonstration, we proceed to construct the velocity profile for regime $\mathrm{C}$, in which the potential locations of the yield surfaces lie inside the boundary of the domain $0<\eta_{ \pm}<Y$.

We note, by substituting $\tau= \pm B$ into the constitutive relation (23a), that

$$
u_{y}=0 \quad\left(y=\eta_{ \pm}\right),
$$


on the boundaries of the pseudo-plugs, i.e. the vertical gradient of the velocity is continuous at the potential yield surfaces. Combining this condition with the equation for the parabolic profiles in the yielded regions given by (31), we can write down a profile of the form

$$
u= \begin{cases}u_{p}+\frac{1}{2} p_{\xi}\left(y-\eta_{+}\right)^{2} & \left(\eta_{+}<y<Y\right), \\ u_{p} & \left(\eta_{-}<y<\eta_{+}\right), \\ u_{p}+\frac{1}{2} p_{\xi}\left(y-\eta_{-}\right)^{2} & \left(0<y<\eta_{-}\right),\end{cases}
$$

where the plug velocity $u_{p}=-\frac{1}{2} p_{\xi} \eta_{-}^{2}$ is established on applying condition (26). We determine the remaining unknown in the profile by applying the no-slip condition at the upper interface (25) to give

$$
U=\frac{1}{2} p_{\xi}\left[\left(Y-\eta_{+}\right)^{2}-\eta_{-}^{2}\right] .
$$

Using equation (33b) to eliminate $\eta_{-}$in this equation, and rearranging for the location of the upper yield surface, provides

$$
\eta_{+}=\frac{p_{\xi}^{2} Y^{2}-2 U p_{\xi}-4 B^{2}}{2\left|p_{\xi}\right|\left(Y\left|p_{\xi}\right|-2 B\right)} .
$$

The lower yield surface is then given by

$$
\eta_{-}=\eta_{+}-\frac{2 B}{\left|p_{\xi}\right|}
$$

using equation (33b). Having established the velocity profile (35), we can now derive the flux

$$
q=\frac{1}{6} p_{\xi}\left[\left(Y-\eta_{+}\right)^{3}+\eta_{-}^{3}-3 \eta_{-}^{2} Y\right]
$$

which can be used in equation (18) to find the rate-of-change of the thickness profile at each $\xi$-location. For the evolution of the locomotion velocity, we calculate the integral on the right-hand side of equation (27) using the expression

$$
\tau_{0}=\frac{1}{2} p_{\xi}\left(\eta_{+}+\eta_{-}\right)
$$

which is given by rearranging equation (33a).

As shown in appendix A, different regimes have different expressions for the potential yield surfaces. Thus, in constructing the flow profile we determine the regime at a particular $\xi$-location by calculating the potential yield surfaces in all regimes and then choose the one that is consistent with the regime definition. Note that although the potential yield surfaces for regimes $\mathrm{A}$ and $\mathrm{E}$ are not used in the expressions for the velocity profiles or fluxes, they are still relevant in the equation for $\tau_{0}$ given by (40), which we use to evaluate the right-hand side of equation (27).

\subsection{Initial-value problem}

We solve the initial-value problem described by equations (21)-(27) numerically by applying the Matlab time-integrator ode15s, and using fast Fourier transforms to evaluate spatial derivatives. At each step of the time integration, we evaluate the pressure field using 


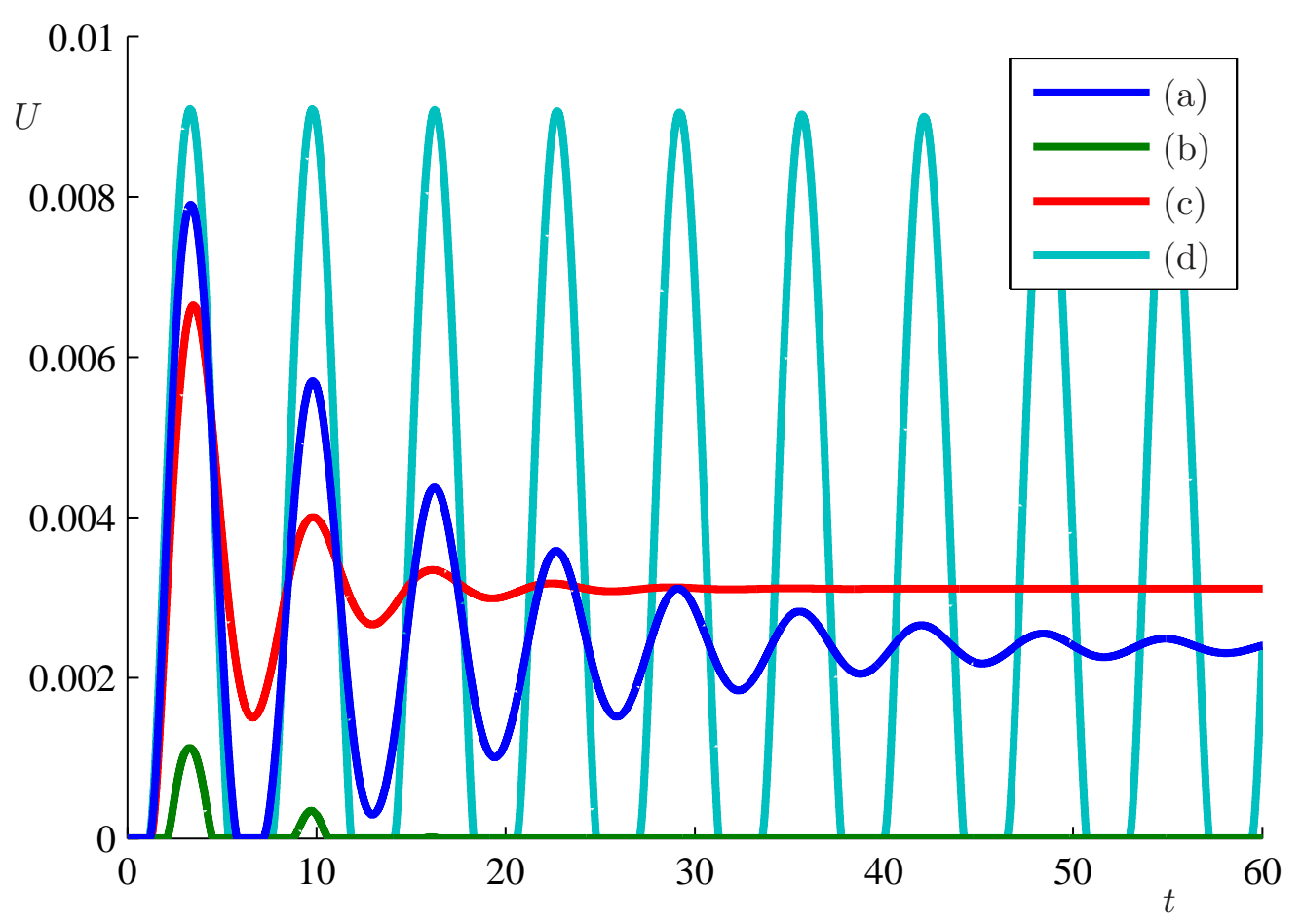

Figure 3: Locomotion velocity as a function of time in the cases (a) $A=1, B=0.1, D=1$, $M=1$, (b) $A=1, B=0.14, D=1, M=1$, (c) $A=1, B=0.1, D=3, M=1$, and (d) $A=1, B=0.1, D=0, M=1$.

equation (22), construct the flow profiles using the formulae in appendix A, and use the resulting flux to determine the rate-of-change of the thickness profile using equation (18). Further, we use the formulae for $\eta_{ \pm}$given in appendix A to evaluate the surface stress $\tau_{0}$ using equation (40), which we then use to determine the rate-of-change of the locomotion velocity using equation (27). Our solutions for the evolution of the locomotion velocity in the four cases (a)-(d) defined by

\begin{tabular}{|c|llll|}
\hline (a) & $A=1$, & $B=0.1$, & $D=1$, & $M=1$ \\
(b) & $A=1$, & $B=0.14$, & $D=1$, & $M=1$ \\
(c) & $A=1$, & $B=0.1$, & $D=3$, & $M=1$ \\
(d) & $A=1$, & $B=0.1$, & $D=0$, & $M=1$ \\
\hline
\end{tabular}

in which we apply the initial conditions

$$
\left.\begin{array}{l}
Y=1 \\
U=0
\end{array}\right\}(t=0)
$$

are shown in figure 3. The corresponding evolutions of the thickness profiles in cases (a) and (b) are shown in the space-time plots of figure 4. 


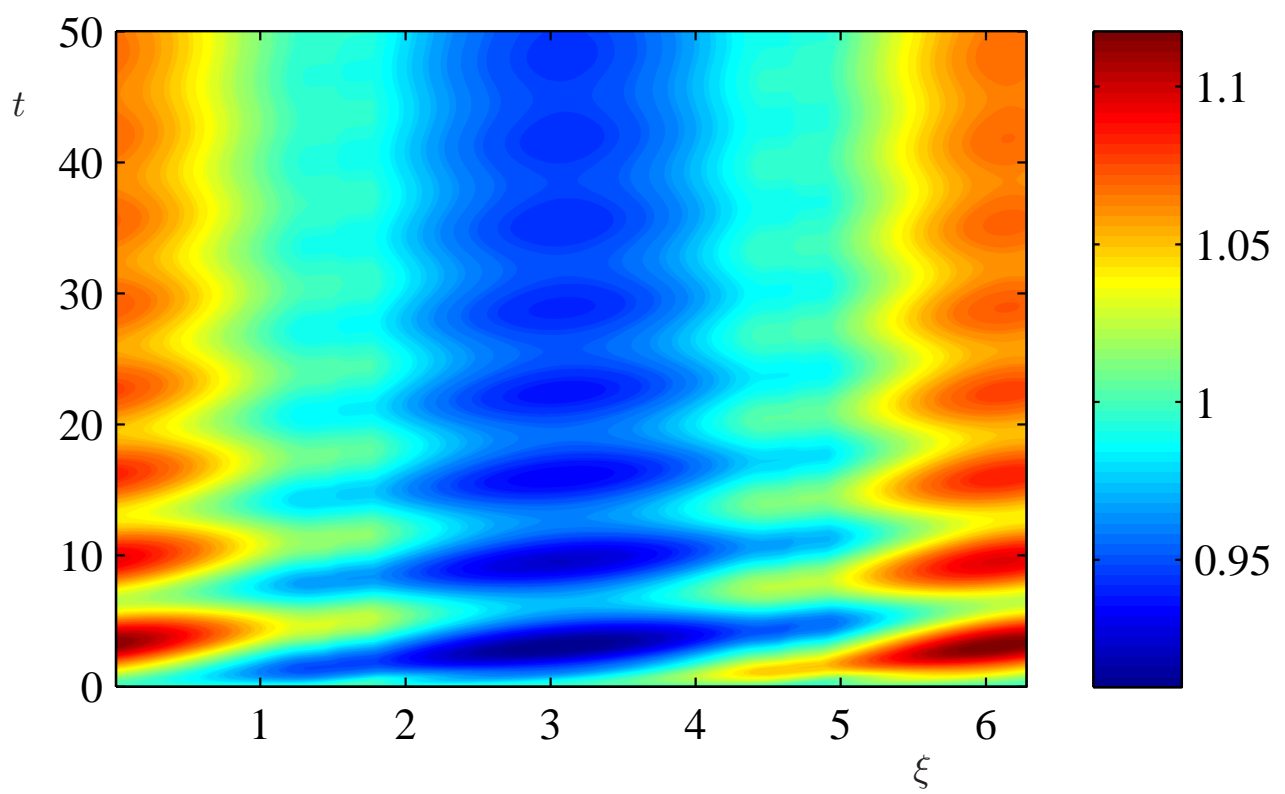

(a)

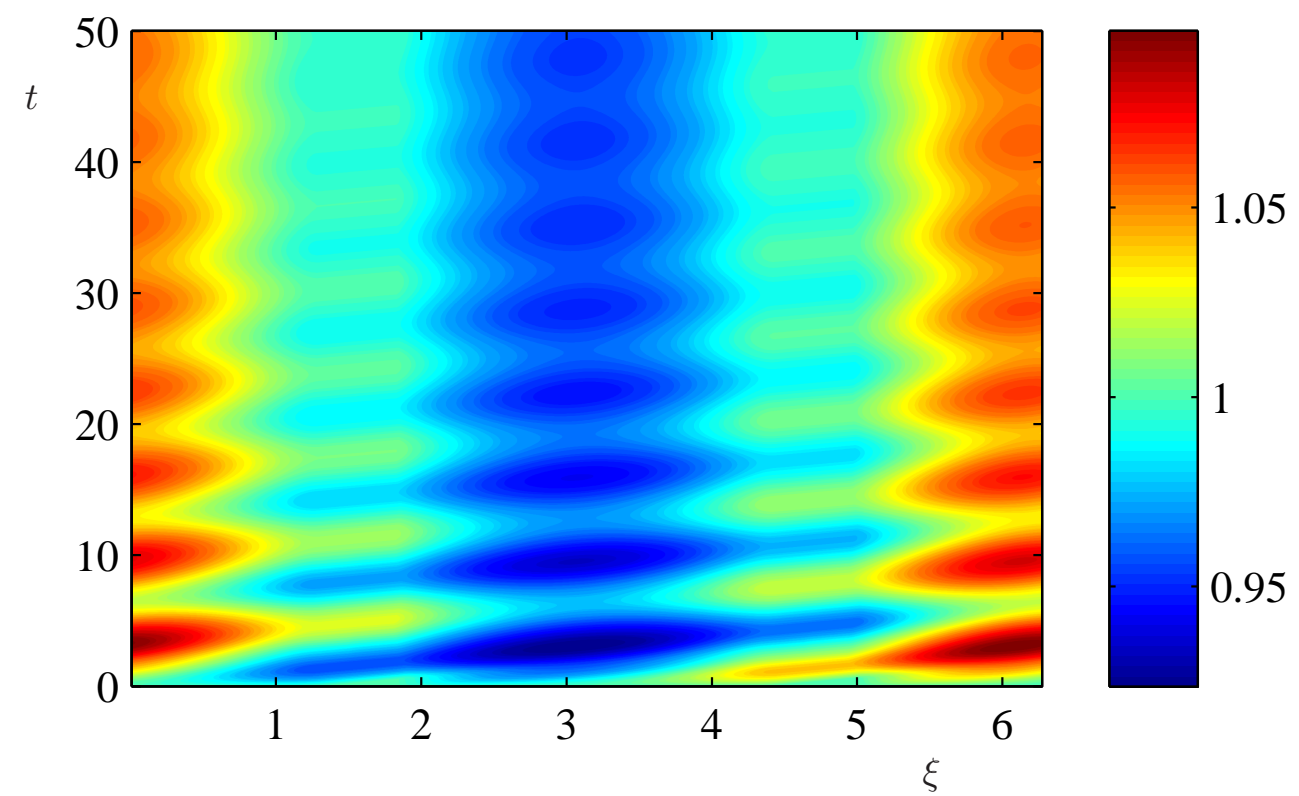

(b)

Figure 4: Space-time plots of the evolution of the thickness profile $Y(\xi, t)$ for cases (a) $A=1, B=0.1, D=1, M=1$, and (b) $A=1, B=0.14, D=1, M=1$. 
For case (a), we observe an initial transient during which the locomotor remains stationary for a short period after its muscles are activated. Although the locomotor is stationary during this initial phase, its thickness profile is strongly time-dependent, as indicated by the space-time plot in figure $4(\mathrm{a})$. At $t \approx 1.2$, the locomotor rapidly accelerates to a maximum velocity in the first half of the forcing period, and subsequently decelerates back to $U=0$. After completing a secondary phase of no locomotion, the locomotor subsequently remains in forward motion with a velocity that converges toward a steady value. The oscillation of the locomotion velocity is due to a periodic change in the direction of the tangential stress on the locomotor due to the muscular force wave as it propagates along the foot. By comparing solutions (a) and (c), we observe that a larger stiffness has the effect of reducing the amplitude of the oscillation. In case (d), in which the foot is perfectly inelastic, the locomotor does converge quickly toward a steady state, and appears to remain in a neutrally stable oscillation for all time.

In the limit of large stiffness and large amplitude, the dominant balance in the pressure equation (22) is between the muscular forcing and the stress due to elastic deformation, which recovers the case of imposing the displacement of the foot surface. In this case it is clear that increasing the stiffness has the same effect as reducing the amplitude. Thus, one could hypothesize that increasing the stiffness always reduces the steady-state locomotion velocity. However, we observe that the steady-state velocity of case (c) is greater than that of case (a). This suggests that it is possible to improve locomotion by using a stiffer material where the resultant force due to the muscular forcing and elastic stress is more efficient at creating the tangential stresses on the lower surface of the foot that leads to locomotion.

The observed regions of no locomotion can be explained on the basis that after its inertia vanishes, the locomotor must wait until the muscular and elastic forces can break the yield stress at each $\xi$-locomotion in order to restart. In case (b), which has a greater Bingham number than case (a), we observe that the locomotor goes through a similar initial transient to that of case (a) but fails to restart on the second oscillation, subsequently converging to a steady state with no locomotion. Thus, we can identify a critical Bingham number $B^{*}(A, D)$ that lies between the Bingham numbers of cases (a) and (b), which defines the value above which no locomotion occurs in the steady state. We observe from the spacetime plot of figure 4(b) that after the locomotor becomes stationary, its thickness profile continues to evolve in time and converges toward a steady-state in a similar manner to that of case (a). Curiously, the locomotor in case (b) has been able to translate forwards despite it coming to a standstill in the steady state, which suggests that it could still be able to locomote by reducing its amplitude over time and then suddenly restarting it at periodic intervals. We proceed to focus our attention on the eventual steady states of the system, in which we identify the value of the critical value $B^{*}$.

\subsection{Steady state}

The steady states of the system can be established directly by considering the equations in the case where the time-derivatives are set to zero. In this case, the thickness evolution equation (19) becomes

$$
\frac{\partial}{\partial \xi}(Y+q)=0,
$$




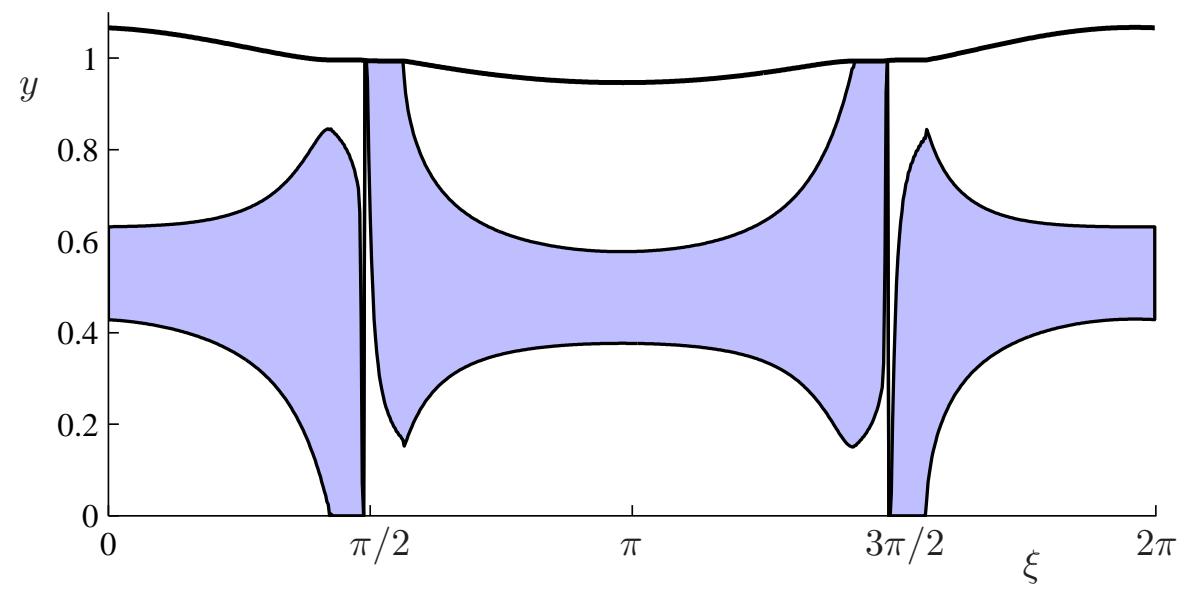

(a)

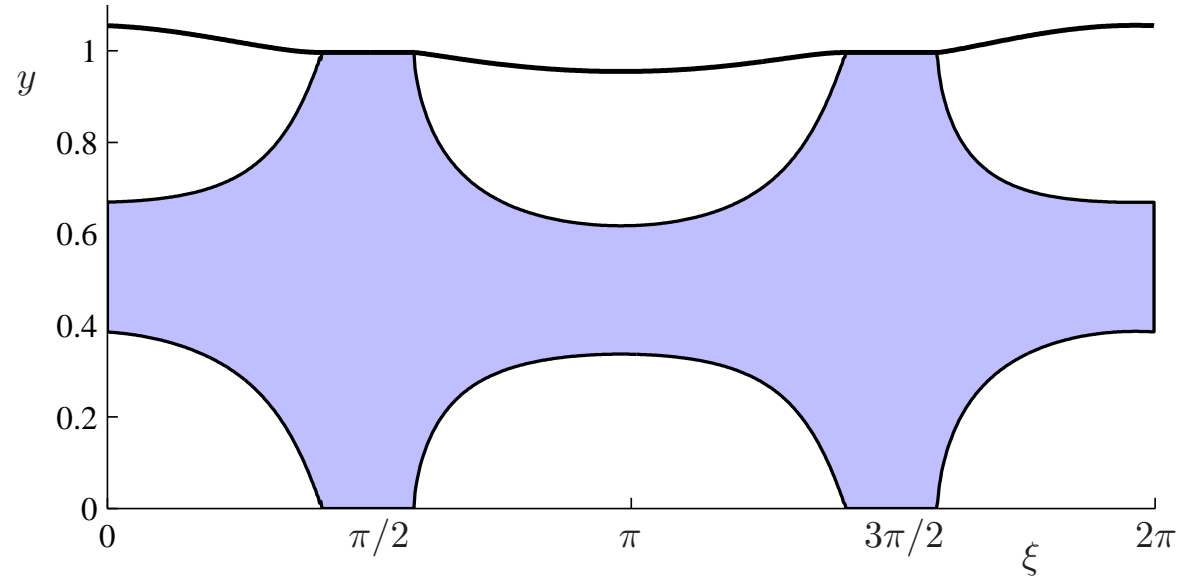

(b)

Figure 5: Steady-state profiles in cases (a) $A=1, B=0.1, D=1$, and (b) $A=1, B=0.14$, $D=1$. Regions of unyielded fluid are shown in blue. 


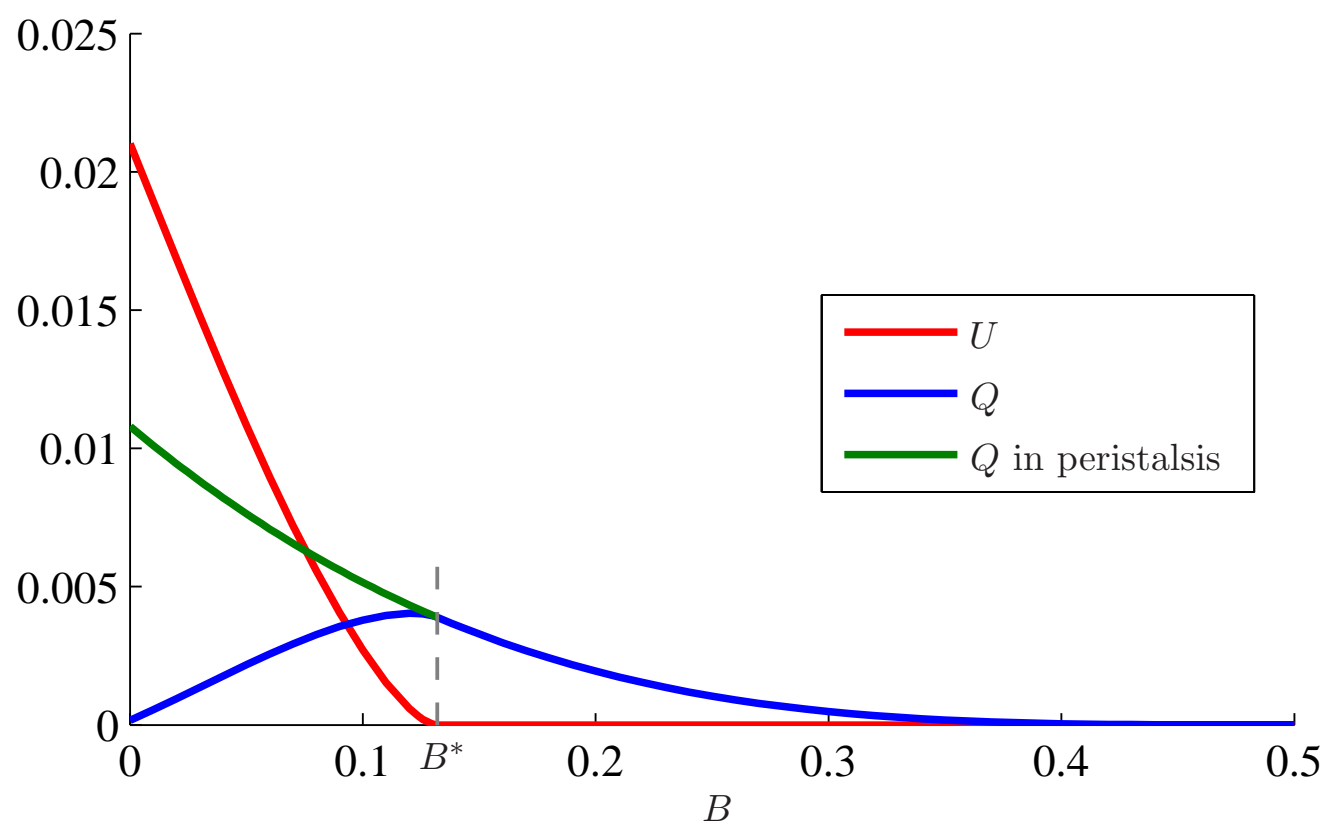

Figure 6: Steady-state locomotion velocity (red), flux (blue), and flux with a fixed locomotor (green), which is relevant to the process of peristalsis, for different values of the Bingham number, in the case $A=1, D=1$.

which integrates to give

$$
Y+q=1-Q,
$$

where $Q$ is the steady-state flux in the negative $\xi$-direction. This equation defines an eigenvalue problem for the unknown steady-state thickness profile $Y$, and the two eigenvalues $Q$ and $U$, for which we apply the two constraints

$$
\begin{aligned}
& \langle Y\rangle=1, \\
& \left\langle\tau_{0}\right\rangle=0,
\end{aligned}
$$

given by the mean thickness condition (24) and the steady form of (27), respectively. We solve this eigenvalue problem numerically using a Newton-Raphson iteration scheme. Noting that trial profiles converge rapidly to the Newtonian case $B=0$, we initialize the scheme with the Newtonian case and progressively increment $B$ in sufficiently small steps to allow convergence at each step.

Our solutions for the steady-states in cases (a) and (b) are shown in figure 5, and the relationships between the locomotion velocity, flux and Bingham Number in the case $A=1$, $D=1$, are shown in figure 6 . In case (b), which lies in $B>B^{*}$, there is no locomotion in the steady state. We see from 5 (b) that the thickness profile becomes raised and depressed near the points $\xi=0$ and $\xi=\pi$, respectively, which correspond to regions where the pressure gradient attains its maximum and minimum values. Furthermore, the yield surfaces touch both the upper and lower boundaries close $\xi=\pi / 2$ and $\xi=3 \pi / 2$, which are regions where 
the pressure gradient becomes close to zero and subsequently changes sign. The plugs in these regions span the vertical extent of the layer, which explains why the locomotor must remain stationary. Although there is no locomotion, there is still fluid motion in the yielded parts of the C-regions, which leads to a net pumping of fluid in the negative $\xi$-direction. Note that although $q=0$ in the rigid plugs, it is the steady flux in the wave frame $q+Y$ that is uniform in the above solutions, so it clear that a net transport of fluid is possible with fluid crossing the yield surfaces. In cases such as this in which the locomotor does not move, we can think of the system as being equivalent to that of a peristaltic pump, in which a condition of no horizontal translation on the upper surface would be imposed at all times.

We observe from figure 6 that the flux eventually vanishes at another critical value of the Bingham number. This can be verified by noting that fluid motion stops when the yield surfaces of the C-regions touch the boundaries of the domain everywhere, which first occurs when $\eta_{+}(\pi)=1$. Thus, by setting $U=0$ in equation (88) of appendix A, we deduce that

$$
\frac{1}{2} Y+\frac{B}{\left|p_{\xi}\right|}=1
$$

at $\xi=\pi$, on simplification. Further, noting that $Y$ is uniformly equal to one in the fully unyielded steady-state system, equation (22) implies that the pressure gradient at this point is given by $p_{\xi}(\pi)=-A$. Substituting this value into equation (46) provides the critical value

$$
B=\frac{1}{2} A \text {. }
$$

The steady state profile for case (a), displayed in figure $5(\mathrm{a})$, shows that the pseudoplugs near $\xi=\pi / 2$ and $\xi=3 \pi / 2$ do not fully span the vertical extent of the layer. Thus, the locomotor is able to break the yield stress at each $\xi$-location, leading to a positive locomotion velocity. Figure 6 shows that the locomotion velocity increases as the Bingham number is reduced from $B^{*}$, with slow growth in the vicinity of $B^{*}$, and approximately linear growth near the Newtonian case $B=0$. In figure 6 , we also compare the corresponding flux in the steady state to that of the peristaltic case, which shows that as the Bingham number is decreased from $B^{*}$, the flux soon starts to decrease, but the flux in the peristaltic case continues to increase.

\section{Prograde locomotion}

\subsection{The foot}

The defining difference between retrograde and prograde locomotion is given by whether the resultant forcing applied by the foot muscle is predominantly tangential or normal to the surface of the foot. For prograde locomotion, we assume that the contraction pattern of the inclined muscular fibres in the snail's foot is such as to provide a predominantly tangential resultant force at the base of the foot. In this case equations (1)-(3), which were relevant in the retrograde case, no longer apply. Assuming that the main body of the snail acts as a lubricated rigid foundation that does not allow vertical penetration but does allow tangential slip with a no-stress condition, it can be shown that the elastic deformation of 


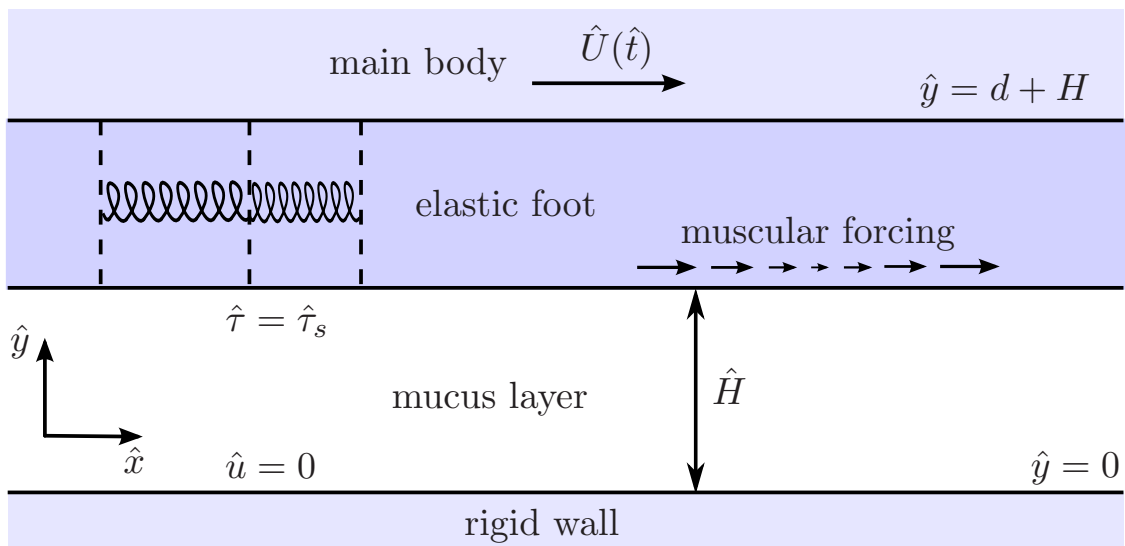

Figure 7: Schematic of the foot and mucus layer in prograde locomotion.

the foot is predominantly horizontal to leading-order in $d \ll L$. Thus, we assume that the base of the foot can undergo significant tangential contraction so that, unlike the retrograde case, we do not impose the condition that the base of the foot moves with the locomotion velocity (3). Instead, we impose a tangential-stress condition,

$$
\hat{\tau}=\hat{A} f(\hat{x}, \hat{t})+\hat{D}_{P} \hat{u}_{s \hat{x}}
$$

where $\hat{A} f$ is the tangential muscular forcing, $\hat{A}$ is the amplitude of the forcing, and the stiffness coefficient $\hat{D}_{P}$ can be related to the elastic properties of the foot. The latter term on the right-hand side of this equation represents the elastic force derived from the relative compression of neighbouring points on the foot's surface. Thus, we can think of the foot as a large collection of vertical sections joined by Hookean springs, as illustrated in figure 7 . Motivated by observations, we assume a travelling-wave forcing of the form

$$
f=f(k \hat{\xi})
$$

where $\hat{\xi} \equiv \hat{x}-c \hat{t}$. For positive wave speed $c$, this corresponds to a wave that propagates in the positive $\hat{x}$-direction, chosen in anticipation that this will lead to locomotion in the positive $\hat{x}$-direction.

The absence of significant vertical deformation implies that we can assume that the surface of the foot remains in its vertical equilibrium position

$$
\hat{Y}=H,
$$

at all times. Assuming that the muscular fibres of the foot are anchored inside the main body of the snail, we can define the locomotion velocity of the main body of the snail as the average of the surface velocity,

$$
\hat{U}=\left\langle\hat{u}_{s}\right\rangle .
$$

The equations above describe the mechanics of a snail's foot undergoing prograde locomotion. We proceed to consider the dynamics of the mucus layer due to the new conditions (48)-(51), and highlight the differences to the retrograde case. 


\subsection{The mucus layer}

In the prograde case, the mechanics of the mucus layer is complicated by the fact that there is no longer a simple expression for the pressure, but is simplified by the fact that it is no longer necessary to evolve the thickness profile in time. The equations of force balance and continuity (7)-(10) are equally relevant in the prograde case, but we no longer assume the surface velocity condition (11) or the pressure condition (13). Instead, equation (48) combined with continuity of tangential stress at the foot's surface, provides a shear-stress condition

$$
\hat{\tau}=\hat{\tau}_{s}=\hat{A} f+\hat{D}_{P} \hat{u}_{s \hat{\xi}} \quad(\hat{y}=H) .
$$

We can non-dimensionalize the relevant equations (7), (8), (10), (12), (19), (50) and (52) according to

$$
\begin{array}{ll}
\hat{\xi}=\frac{1}{k} \xi, & \hat{y}=H y, \quad \hat{Y}=H Y, \quad \hat{t}=\frac{1}{k c} t, \\
\hat{u}=\frac{H \hat{A}}{\mu} u, \quad \hat{\tau}=\hat{A} \tau, \quad \hat{p}=\frac{\hat{A}}{k H} p, &
\end{array}
$$

where dimensionless variables are denoted without the hat symbol. The resulting nondimensional equations of force balance are

$$
\begin{aligned}
\tau_{y} & =p_{\xi}, \\
p_{y} & =0,
\end{aligned}
$$

with constitutive relation,

$$
\begin{cases}\tau=u_{y}+R \operatorname{sgn}\left(u_{y}\right) & (|\tau|>R), \\ u_{y}=0 & (|\tau|<R),\end{cases}
$$

and boundary conditions,

$$
\begin{array}{ll}
\tau=\tau_{s}=f+D u_{s \xi} & (y=1), \\
u=0 & (y=0) .
\end{array}
$$

The thickness and depth-integrated continuity equations are given by

$$
Y=1, \quad q_{\xi}=0,
$$

respectively, where in the equations above,

$$
R=\frac{\tau_{Y}}{\hat{A}}, \quad D=\frac{k H \hat{D}_{P}}{\mu},
$$

are the yield-stress-amplitude ratio, and the non-dimensional stiffness, respectively. The non-dimensionalization above shows that the absence of a scaling between the rate-of-change of the thickness profile and the divergence of flux in the depth-integrated continuity equation (59) implies that unlike the retrograde case, it has been possible to incorporate the yield 
stress and amplitude into a single parameter $R$. We remark that the limit of large stiffness recovers the case of imposing the tangential displacement of the foot's surface.

\subsection{Eigenvalue problem}

The depth-integrated continuity equation (59) can be integrated to give

$$
q=Q,
$$

where $Q$ is the flux of fluid in the positive $\xi$-direction. Thus, the prograde system has been reduced to an eigenvalue problem for the unknown pressure gradient $p_{\xi}$ and eigenvalue $Q$, that we solve subject to the periodicity constraint

$$
\left\langle p_{\xi}\right\rangle=0 .
$$

As in the retrograde case, the main complication is in the construction of the velocity profiles when a yield stress is present. However, before discussing the effects of yield stress, we begin by considering the simpler Newtonian case.

\section{Newtonian solution}

In the Newtonian case $R=0$, we can relate the flux $Q$ and locomotion velocity $U$ to the forcing function analytically. To show this, we begin by integrating equation (70), with the constitutive relation (56) with $R=0$, to give the parabolic velocity profile

$$
u=\frac{1}{2} p_{\xi} y^{2}+\left(\tau_{s}-p_{\xi}\right) y,
$$

in which we have applied the condition of no-slip at the base (58). The associated formula for the flux is then given by

$$
q=\frac{1}{2} \tau_{s}-\frac{1}{3} p_{\xi}=Q,
$$

which is uniformly equal to $Q$ according to (61). Taking the $\xi$-average of this equation yields

$$
Q=\frac{1}{2}\left\langle\tau_{s}\right\rangle,
$$

on applying the continuity constraint (62). Substituting the expression for the surface stress (57) gives

$$
Q=\frac{1}{2}\left\langle f+D u_{s \xi}\right\rangle=\frac{1}{2}\langle f\rangle,
$$

noting that the average of the elastic term vanishes. Thus, we have established the flux in terms of the imposed tangential forcing. To determine the associated locomotion velocity, we evaluate the velocity of the fluid at the surface by setting $y=1$ in equation (63) to give

$$
u_{s}=\tau_{s}-\frac{1}{2} p_{\xi} .
$$

Now, taking the $\xi$-average of this equation yields

$$
U=\left\langle u_{s}\right\rangle=\left\langle\tau_{s}\right\rangle=\left\langle f+D u_{s \xi}\right\rangle=\langle f\rangle,
$$


on noting again that the average of the elastic term is zero.

Expressions (66) and (68) show that both the flux and the locomotion velocity are linearly related to the mean value of the imposed forcing function $f$. Thus, we are motivated in our analysis of the non-Newtonian case to prescribe a forcing with a non-zero mean. In this regard, we proceed to impose

$$
f=\sin ^{2} \xi,
$$

which has mean value $\langle f\rangle=\frac{1}{2}$. We remark that in adopting this forcing, it is not possible to balance the tangential stress exerted on the main body of the snail without the snail placing the front or back of its foot in contact with the ground to provide a frictional force to balance that of the muscular contractions. The resulting process of locomotion is similar to that of an inchworm, which is known to be adopted occasionally by some land snails.

As a side note, we remark that it is possible to locomote in the case $\langle f\rangle=0$ if a yield stress is present. To demonstrate this, consider a forcing pattern with small regions of high magnitude forcing in the positive $\xi$-direction, and with larger regions of low magnitude forcing in the negative $\xi$-direction, constructed with $\langle f\rangle=0$, such that only regions of the forcing directed in the direction of locomotion will break the yield stress. Then it is clear that the velocity of the foot surface will be either stationary or directed only in the positive $\xi$-direction. The mean surface velocity will thus be positive, providing locomotion. Such a pattern may correspond to that adopted commonly by land snails, in cases where the surface of the foot does not make direct contact with the ground.

\section{The effect of yield stress}

The effect of yield stress can be established by considering the eigenvalue problem defined by equations (61)-(62), with the forcing (69), in the general case $R \geq 0$. To simplify the analysis, we assume $D=0$ with the expectation that the resulting system will encapsulate the behaviour of the general case. We solve the eigenvalue problem for the unknown pressure gradient numerically using a Newton-Raphson iteration scheme initialized using the Newtonian solution discussed above. In each iteration, we construct the flow profiles with the pressure gradient form the previous step using an analogous procedure to that outlined in section 2.3. The first step of the integration of the flow profiles can be achieved by integrating (54) subject to (57) to give

$$
\tau=f+p_{\xi}(1-y),
$$

from which we deduce that the potential yield surfaces are given by

$$
\eta_{ \pm}=1-\frac{f}{p_{\xi}} \pm \frac{R}{\left|p_{\xi}\right|}
$$

in all regimes. The other formulae associated with the construction of the velocity profiles are summarized in appendix B. For our solution, we plot the dependence of the locomotion velocity and flux on the yield-stress-amplitude ratio $R$ in figure 8 , and some examples of yield-surface plots and pressure-gradient profiles for specific values of $R$ are shown in figures 9 and 10, respectively.

In all cases of $R$, we can define a region of the fluid $|\zeta|<\zeta_{D}$, where $\zeta \equiv \xi-\pi / 2$, and 


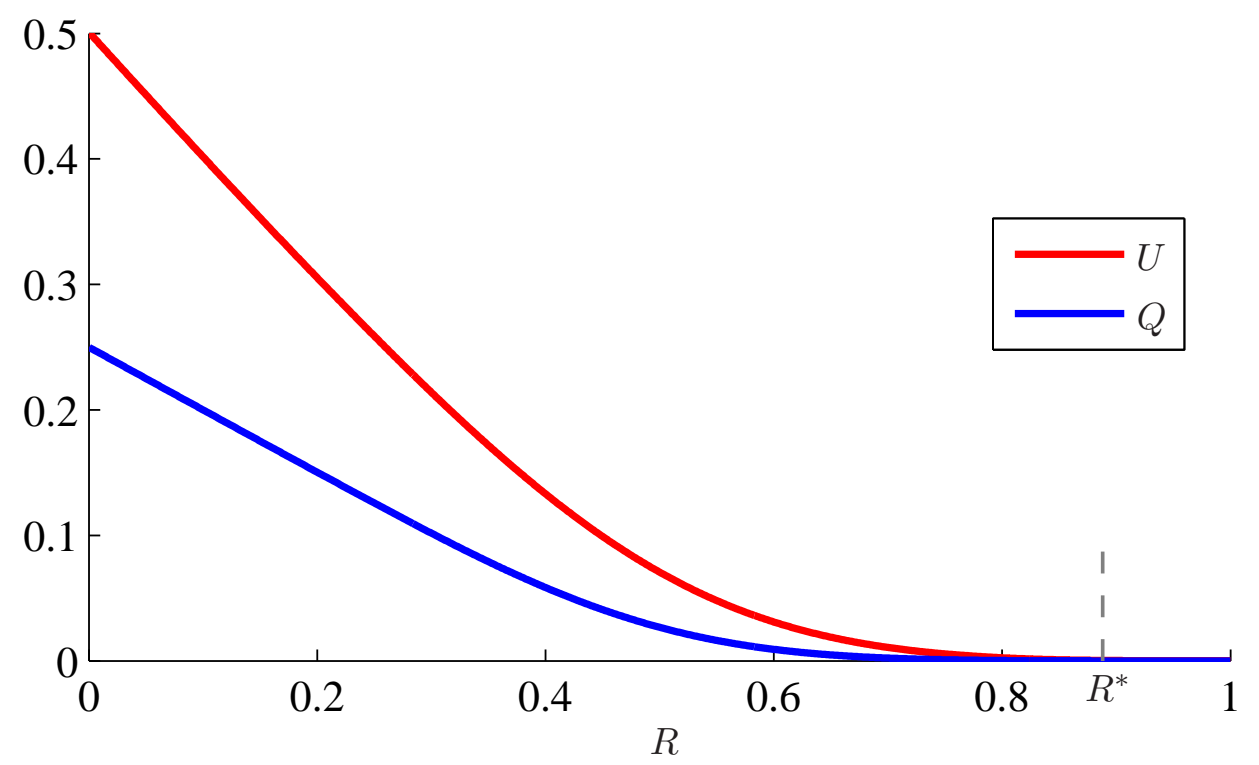

(a)

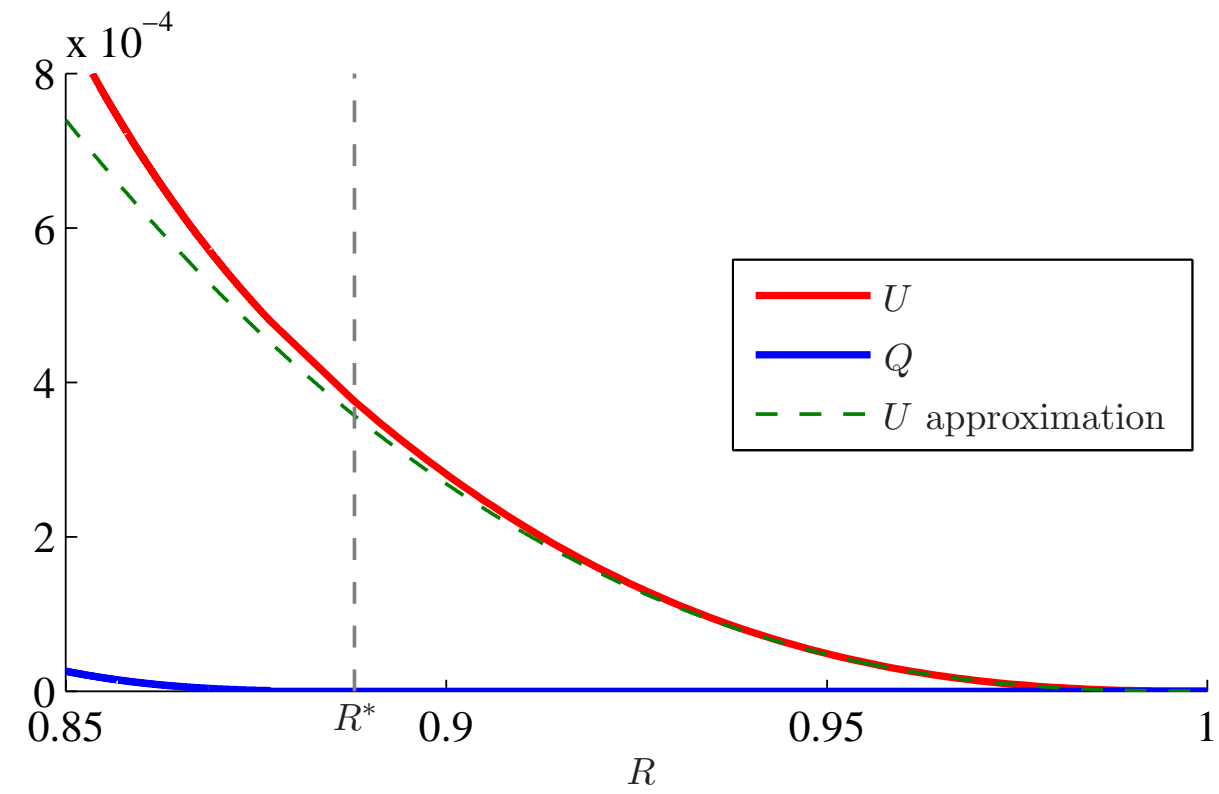

(b)

Figure 8: Locomotion velocity and flux in prograde locomotion (a), and a blow-up (b) of the region $R^{*}<R<1$, in which we plot the asymptotic approximation (80) for $U$. 
$\zeta_{D}$ is defined by

$$
R=\cos ^{2} \zeta_{D},
$$

in which the fluid in contact with the foot must be yielded because the imposed tangential forcing at these points exceeds the yield stress. Conversely, the region of the fluid $|\zeta|>\zeta_{D}$ in contact with the foot $y=1$ must be unyielded. In order to provide a non-zero flux, it is necessary that the structure of the yield surfaces is such that there is a pseudo-plug extending from the top surface and a flow near the lower surface, corresponding to regime $\mathrm{D}$ in appendix B; this is evident in the example profiles of figure 9. Furthermore, we can deduce from equation (70) that the pressure gradient is always negative in the D-region, $|\zeta|>\zeta_{D}$, and positive elsewhere, which is verified by the pressure-gradient plots of figure 10 .

The case $R=0.2$, shown in figure 9 (a), is fully yielded except in the pseudo-plugs of the D-regions discussed above. As $R$ is increased, there is a critical point $R \approx 0.253$, where a pseudo-plug emerges from the base at $\zeta=0$. This B-region, shown in figure $9(\mathrm{~b})$ for the case $R=0.6$, proceeds to grow as $R$ is further increased. We observe from figure 8 that the flux remains approximately half the locomotion velocity in the region $0<R \lesssim 0.4$ and beyond this point, the ratio of the flux to the locomotion velocity begins to decrease at a much faster rate. At $R \approx 0.762$, a C-region appears inside the B-region with a yielded region emerging at the base, and a groove in the upper yield surface, as shown in figure 9 (c) for the case $R=0.8$. At this stage, the regime progression across the period can be summarized

$$
\mathrm{D} \rightarrow \mathrm{E} \rightarrow \mathrm{A} \rightarrow \mathrm{B} \rightarrow \mathrm{C} \rightarrow \mathrm{B} \rightarrow \mathrm{A} \rightarrow \mathrm{E} \rightarrow \mathrm{D},
$$

where the letters correspond to the regime definitions given in appendix B. The fullyyielded A- and E-regions, which are too narrow to be discerned clearly in figure 9(c), lie near locations where the pressure gradient changes sign, as can be verified by plot (c) in figure 10. As $R$ is increased further, there is a critical point $R^{*}$ where the pseudo-plugs in the D-regions touch the base and become rigid plugs that span the thickness of the layer. At this point, the flux vanishes and the velocity of the foot's surface $u_{s}$ becomes zero within the region $|\zeta|>\zeta_{D}$. However, the remaining motion of the foot in the C-region $|\zeta|<\zeta_{D}$ is still able to drive a small locomotion velocity. The behaviour of the flux and locomotion velocity for $R^{*}<R<1$ is shown in figure $8(\mathrm{~b})$.

\section{The onset of locomotion}

It is clear that there is no fluid motion if $R>1$ because in this case the shear stress does not exceed the yield stress anywhere in the fluid. As mentioned above, a C-region emerges about $\zeta=0$ as $R$ is decreased from 1 , such that although the fluid flux remains zero, the foot is still able to drive locomotion. This can be contrasted with the retrograde case in which the flux is always non-zero when locomotion occurs. We proceed to solve the eigenvalue problem for the pressure gradient in the case $R^{*}<R<1$ by applying the constraint $Q=0$ in place of the periodicity constraint (62). The latter is abandoned because a solution for the pressure gradient inside the rigid plugs is not determined as part of the Bingham model. We can assume that the periodicity constraint is accounted for via an elastic force balance within the rigid regions that we need not consider explicitly. 


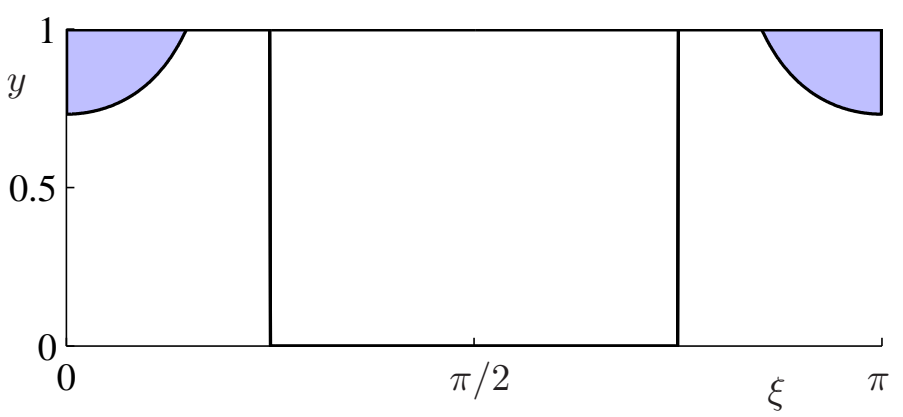

(a)

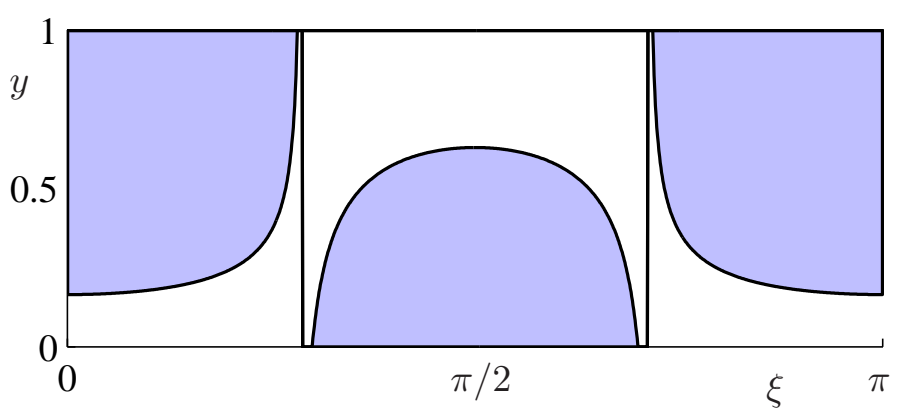

(b)

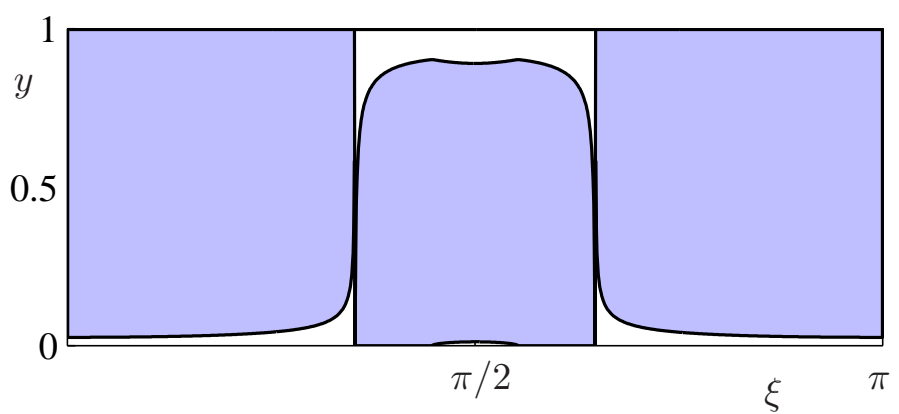

(c)

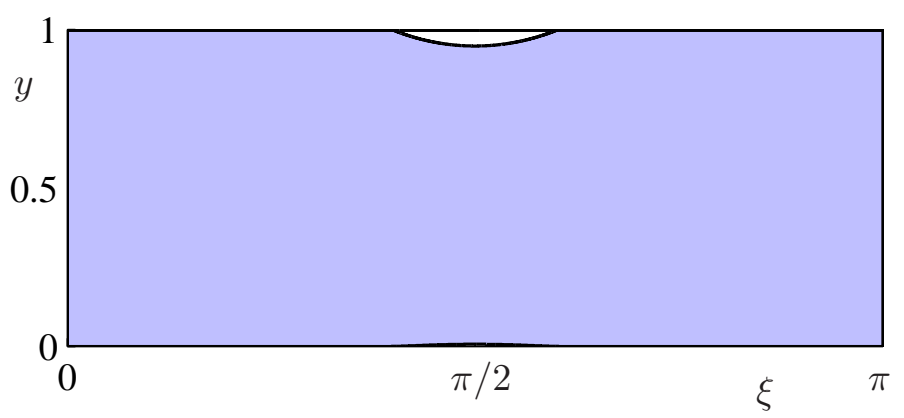

(d)

Figure 9: Yield-surface profiles in the cases (a) $R=0.2$, (b) $R=0.6$, (c) $R=0.8$, and (d) $R=0.9$. Regions of unyielded fluid are shown in blue. 


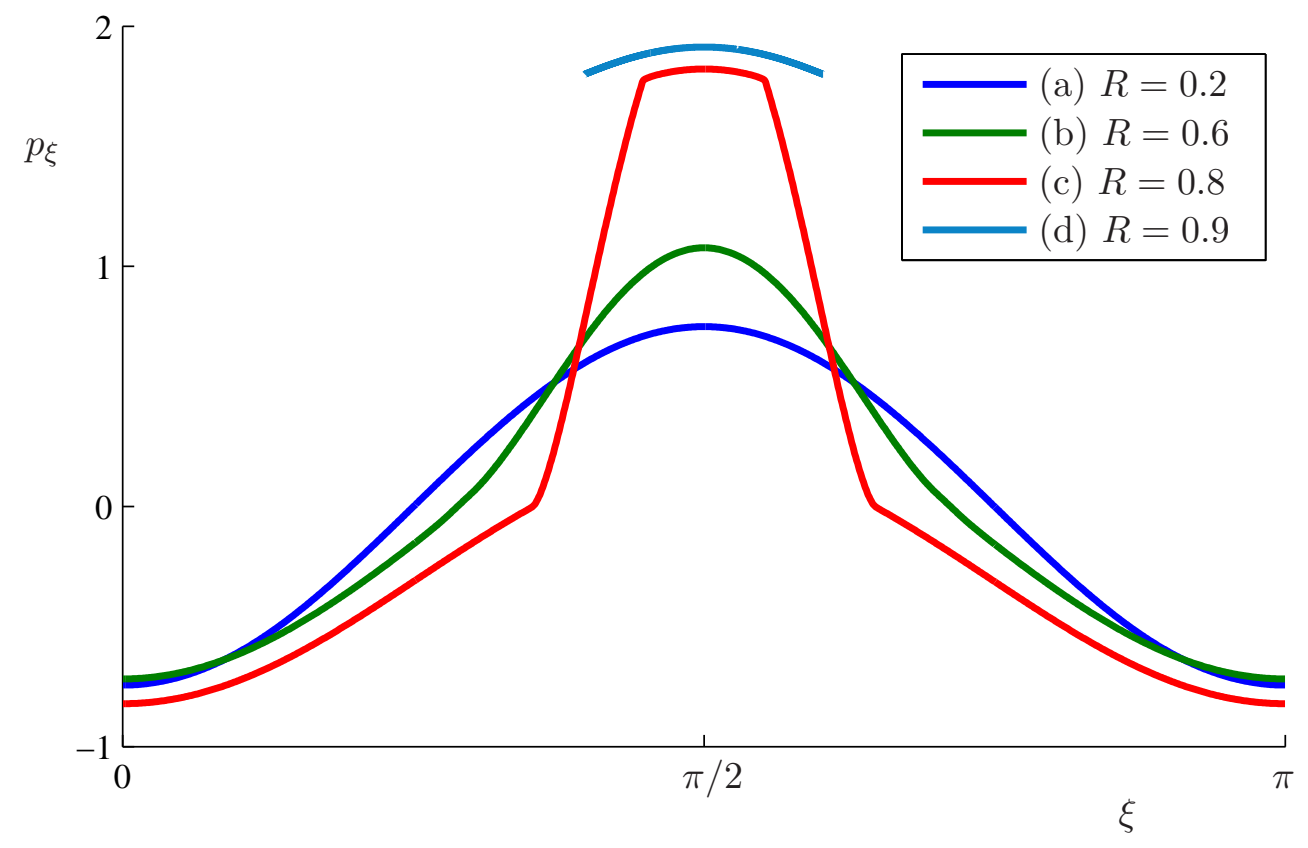

Figure 10: Pressure-gradient profiles in the cases (a) $R=0.2$, (b) $R=0.6$, (c) $R=0.8$, and (d) $R=0.9$. For case (d), the pressure gradient remains undetermined in the region $|\zeta|>\left|\zeta_{D}\right|$ under the Bingham model.

In the C-region, the condition of zero flux implies that

$$
\left(1-\eta_{+}\right)^{3}+\eta_{-}^{2}\left(\eta_{-}-3\right)=0,
$$

using equation (90) from appendix B. Eliminating $\eta_{ \pm}$in favour of the unknown pressure gradient using equations (71), and recalling that $p_{\xi}>0$ for $|\zeta|<\zeta_{D}$, gives

$$
(f-R)^{3}-\left(2 p_{C \xi}+f+R\right)\left(p_{C \xi}-f-R\right)^{2}=0,
$$

which is an algebraic equation for the pressure gradient $p_{C \xi}$ in the C-region. We solve this cubic equation numerically using the Matlab routine fzero. From our solution, we can identify two positive roots if $R<1$, which in the limit of $R \rightarrow 1$, are given by

$$
p_{C \xi} \sim f+R \pm\left[\frac{(f-R)^{3}}{3(f+R)}\right]^{1 / 2},
$$

to leading order. It can be verified numerically that the lesser root always leads to an inconsistent solution with $\eta_{-}<0$. Thus, we use the greater root to calculate the yield surface profiles. Our solution for the yield surface profile in the case $R=0.9$ is shown in figure $9(\mathrm{~d})$, and the corresponding pressure gradient is shown by plot (d) in figure 10. Furthermore, we use the non-dimensional form of equation (51) to evaluate the locomotion 
velocity

$$
U=\frac{2}{\pi} \int_{0}^{\zeta_{D}} u_{s} \mathrm{~d} \zeta
$$

in which the surface velocity is given by

$$
u_{s}=\frac{1}{2} p_{\xi}\left[\left(1-\eta_{+}\right)^{2}-\eta_{-}^{2}\right]=\frac{\left(p_{\xi}-2 R\right)\left(2 f-p_{\xi}\right)}{2 p_{\xi}},
$$

which we have established using equation (89) given in appendix B. We use the resulting solution for $U$ to extend the plot in figure 8(b) to the region $R^{*}<R<1$.

In the limit $R \rightarrow 1$, an approximation for the surface velocity can be derived by substituting the leading-order form of the pressure gradient given by equation (76) into equation (78) to give

$$
u_{s} \sim \frac{1}{4}\left(1-R-\zeta^{2}\right)^{2} .
$$

Using this expression to evaluate the integral in equation (77) provides

$$
U \sim \frac{4}{15 \pi}(1-R)^{5 / 2}
$$

on noting that $\zeta_{D} \sim \sqrt{1-R}$ from equation (72). Figure 8(b) shows that this approximation gives good agreement with the exact solution.

\section{The onset of flux}

The critical value $R^{*}$ defines the point at which the flux first vanishes. Moreover, this point corresponds to the point when both the periodicity constraint (62) and the flux constraint $Q=0$ are satisfied simultaneously. In reference to the profiles shown in figure 8 , we note that as $R$ approaches $R^{*}$ from below, this critical point is reached when the lower yield surface $\eta_{-}$in the D-region first touches the lower surface such that $\eta_{-}=0$. Thus, the pressure gradient in the D-region at the critical point can be found by setting $\eta_{-}=0$ in equation (71) to give

$$
p_{D \xi}=f-R^{*},
$$

recalling that $p_{\xi}<0$ for $|\zeta|>\zeta_{D}$. The pressure gradient in the C-region is given by the numerical solution to (75) described above, which along with (81), enters the continuity constraint (62) according to

$$
\int_{0}^{\zeta_{D}} p_{C \xi} \mathrm{d} \zeta+\int_{\zeta_{D}}^{\pi / 2} p_{D \xi} \mathrm{d} \zeta=0
$$

where we have taken the integral over half the period using the fact that the solution is symmetric about $\zeta=0$. On substituting the pressure gradient in the D-region given by equation (81), and evaluating the second integral, this equation reduces to

$$
\int_{0}^{\zeta_{D}} p_{C \xi} \mathrm{d} \zeta=\left(R^{*}-\frac{1}{2}\right)\left(\frac{\pi}{2}-\zeta_{D}\right)+\frac{1}{4} \sin \left(2 \zeta_{D}\right) .
$$


We solve the integro-algebraic system defined by equation (75) with $R=R^{*}$ and (83) numerically by treating $R^{*}$ as a shooting parameter. For each trial in this method, we solve the algebraic equation (75) for $p_{C \xi}$ using the Matlab routine fzero. Our solution gives the critical value $R^{*} \approx 0.888$.

A near-exact derivation of this result can be derived semi-analytically with less work by making use of the fact that $R^{*} \approx 1$, and recalling that in the limit $R \rightarrow 1$, equation (76) implies that the pressure gradient in the C-region is given approximately by

$$
p_{C \xi} \approx f+R^{*} \text {. }
$$

Substituting this expression into equation (83), and simplifying, provides

$$
\int_{0}^{\pi / 2} f \mathrm{~d} \zeta+R^{*}\left(2 \zeta_{D}-\frac{\pi}{2}\right) \approx 0
$$

and hence

$$
\frac{\pi}{4}+R^{*}\left(2 \zeta_{D}-\frac{\pi}{2}\right) \approx 0 .
$$

Using equation (72) to eliminate $R^{*}$ in this equation leaves the algebraic equation

$$
\frac{\pi}{4}+\left(2 \zeta_{D}-\frac{\pi}{2}\right) \cos ^{2} \zeta_{D}=0,
$$

which can be solved numerically for the solution $\zeta_{D} \approx 0.343$, with corresponding critical value $R^{*} \approx 0.887$ calculated using equation $(72)$. Given that this value is very close to the exact value derived previously, we can conclude that the above approximation is successful at describing the region of the parameter space in which $R$ is close to $R^{*}$.

\section{Conclusion}

We have investigated theoretically and numerically models for retrograde and prograde snail locomotion using a dynamic formulation that accounts for both the elastic and muscular mechanics of the snail's foot, and the yield stress of the mucus layer. Our solution of the initial-value problem in the retrograde case shows an initial transient that involves a highly oscillatory locomotion velocity, with the potential for extended periods of no locomotion. We observe a transition toward a steady state in the wave frame of the forcing, which we investigate by finding solutions of the associated steady-state eigenvalue problem. For given values of the non-dimensional forcing amplitude and stiffness, we identify a critical value of the Bingham number above which there is no locomotion in the steady state. In our analysis of prograde locomotion, we find that the locomotor is able to pump fluid in the direction it is travelling only below a critical value of the yield-stress-amplitude ratio, which we have identified in the case of zero stiffness. Beyond this point, the flux vanishes but the snail is still able to locomote.

A notable difference between the two forms of locomotion we have studied is that the flux in the retrograde case is directed in the opposite direction to that of locomotion, while in the prograde case, the snail is able to pump fluid in the direction it is travelling. Given that mucus production is a substantial chemical cost to the snail, this difference may account 
for why land snails have favoured the prograde form of locomotion. Furthermore, we can hypothesize that a water snail predominantly uses ambient water as a lubricating fluid, and only occasionally produce mucus if, for example, it needs to climb a steep incline.

\section{A Regimes in retrograde locomotion}

Regime A: $\left(p_{\xi} U>0, \eta_{ \pm}<0\right)$

$$
\begin{gathered}
u=\frac{1}{2} p_{\xi} y(y-U)+\frac{U y}{Y}, \quad q=\frac{1}{2} U Y-\frac{1}{12} p_{\xi} Y^{3}, \\
\eta_{+}=\frac{1}{2} Y-\frac{U}{p_{\xi} Y}, \quad \eta_{-}=\eta_{+}-\frac{2 B}{\left|p_{\xi}\right|} .
\end{gathered}
$$

Regime B: $\left(p_{\xi} U>0, \eta_{-}<0<\eta_{+}<Y\right)$

$$
u= \begin{cases}\frac{1}{2} p_{\xi}\left(y-\eta_{+}\right)^{2} & \left(\eta_{+}<y<Y\right), \\ 0 & \left(0<y<\eta_{+}\right),\end{cases}
$$

where the pseudo-plug is stationary.

$$
\begin{gathered}
\eta_{+}=Y-\sqrt{\frac{2 U}{p_{\xi}}}, \quad \eta_{-}=\eta_{+}-\frac{2 B}{\left|p_{\xi}\right|} . \\
q=\frac{1}{6} p_{\xi}\left(Y-\eta_{+}\right)^{3} .
\end{gathered}
$$

Regime C: $\left(0<\eta_{ \pm}<Y\right)$

$$
u= \begin{cases}u_{p}+\frac{1}{2} p_{\xi}\left(y-\eta_{+}\right)^{2} & \left(\eta_{+}<y<Y\right), \\ u_{p} & \left(\eta_{-}<y<\eta_{+}\right), \\ u_{p}+\frac{1}{2} p_{\xi}\left(y-\eta_{-}\right)^{2} & \left(0<y<\eta_{-}\right),\end{cases}
$$

where the plug velocity is given by $u_{p}=-\frac{1}{2} p_{\xi} \eta_{-}^{2}$,

$$
\begin{gathered}
\eta_{+}=\frac{p_{\xi}^{2} Y^{2}-2 U p_{\xi}-4 B^{2}}{2\left|p_{\xi}\right|\left(Y\left|p_{\xi}\right|-2 B\right)}, \quad \eta_{-}=\eta_{+}-\frac{2 B}{\left|p_{\xi}\right|}, \\
q=\frac{1}{6} p_{\xi}\left[\left(Y-\eta_{+}\right)^{3}+\eta_{-}^{3}-3 \eta_{-}^{2} Y\right] .
\end{gathered}
$$

Regime D: $\left(p_{\xi} U<0,0<\eta_{-}<Y<\eta_{+}\right)$

$$
u= \begin{cases}u_{p} & \left(\eta_{-}<y<Y\right), \\ u_{p}+\frac{1}{2} p_{\xi}\left(y-\eta_{-}\right)^{2} & \left(0<y<\eta_{-}\right),\end{cases}
$$


where the plug velocity is given by $u_{p}=-\frac{1}{2} p_{\xi} \eta_{-}^{2}$.

$$
\begin{gathered}
\eta_{-}=\sqrt{-\frac{2 U}{p_{\xi}}}, \quad \eta_{+}=\eta_{-}+\frac{2 B}{\left|p_{\xi}\right|}, \\
q=\frac{1}{6} p_{\xi} \eta_{-}^{2}\left(\eta_{-}-3 Y\right) .
\end{gathered}
$$

Regime E: $\left(p_{\xi} U<0, \eta_{ \pm}>1\right)$

$$
\begin{gathered}
u=\frac{1}{2} p_{\xi} y(y-U)+\frac{U y}{Y}, \quad q=\frac{1}{2} U Y-\frac{1}{12} p_{\xi} Y^{3}, \\
\eta_{-}=\frac{1}{2} Y-\frac{U}{p_{\xi} Y}, \quad \eta_{+}=\eta_{-}+\frac{2 B}{\left|p_{\xi}\right|} .
\end{gathered}
$$

\section{B Regimes in prograde locomotion}

The potential yield surfaces at $y=\eta_{ \pm}$are given by

$$
\eta_{ \pm}=1-\frac{\tau_{s}}{p_{\xi}} \pm \frac{R}{\left|p_{\xi}\right|}
$$

in all regimes.

Regime A: $\left(p_{\xi}>0, \eta_{ \pm}<0\right)$

$$
\begin{aligned}
& u=\frac{1}{2} p_{\xi} y^{2}+\left(\tau_{s}-p_{\xi}-R\right) y \\
& q=-\frac{1}{3} p_{\xi}+\frac{1}{2}\left(\tau_{s}-R\right) .
\end{aligned}
$$

Regime B: $\left(p_{\xi}>0, \eta_{-}<0<\eta_{+}<1\right)$

$$
u= \begin{cases}\frac{1}{2} p_{\xi}\left(y-\eta_{+}\right)^{2} & \left(\eta_{+}<y<1\right), \\ 0 & \left(0<y<\eta_{+}\right),\end{cases}
$$

where the pseudo-plug is stationary.

$$
q=\frac{1}{6} p_{\xi}\left(1-\eta_{+}\right)^{3} .
$$

Regime C: $\left(0<\eta_{ \pm}<1\right)$

$$
u= \begin{cases}u_{p}+\frac{1}{2} p_{\xi}\left(y-\eta_{+}\right)^{2} & \left(\eta_{+}<y<1\right), \\ u_{p} & \left(\eta_{-}<y<\eta_{+}\right), \\ u_{p}+\frac{1}{2} p_{\xi}\left(y-\eta_{-}\right)^{2} & \left(0<y<\eta_{-}\right),\end{cases}
$$

where the plug velocity is given by $u_{p}=-\frac{1}{2} p_{\xi} \eta_{-}^{2}$.

$$
q=\frac{1}{6} p_{\xi}\left[\left(1-\eta_{+}\right)^{3}+\eta_{-}^{3}-3 \eta_{-}^{2}\right] .
$$


Regime D: $\left(p_{\xi}<0,0<\eta_{-}<1<\eta_{+}\right)$

$$
u= \begin{cases}u_{p} & \left(\eta_{-}<y<1\right), \\ u_{p}+\frac{1}{2} p_{\xi}\left(y-\eta_{-}\right)^{2} & \left(0<y<\eta_{-}\right),\end{cases}
$$

where the plug velocity is given by $u_{p}=-\frac{1}{2} p_{\xi} \eta_{-}^{2}$.

$$
q=\frac{1}{6} p_{\xi} \eta_{-}^{2}\left(\eta_{-}-3\right)
$$

Regime E: $\left(p_{\xi}<0, \eta_{ \pm}>1\right)$

$$
\begin{aligned}
u & =\frac{1}{2} p_{\xi} y^{2}+\left(\tau_{s}-p_{\xi}-R\right) y \\
q & =-\frac{1}{3} p_{\xi}+\frac{1}{2}\left(\tau_{s}-R\right) .
\end{aligned}
$$

\section{References}

[1] N. J. Balmforth, D. Coombs, and S. Pachman, Microelastohydrodynamics of swimming organisms near solid boundaries in complex fluids, Q. J. Mechanics Appl. Math, 63 (2009), pp. 267-294.

[2] B. Chan, N. J. Balmforth, and A. E. Hosoi, Building a better snail: lubrication and adhesive locomotion, Phys. Fluids, 17 (2005), p. 113101.

[3] D. F. Katz, Propulsion of microorganisms near solid boundaries, J. Fluid Mech., 64 (1974), pp. 33-49.

[4] G. I. TAYlor, Analysis of the swimming of microscopic organisms, Proc. R. Soc. A, 209 (1951), pp. 447-461. 


\title{
Dynamics of Vorticity Defects in Stratified Shear
}

\author{
Anubhab Roy
}

October 19, 2010

\section{Introduction}

Stability of shear flows at high Reynolds number has been one of the cornerstones of hydrodynamic stability. A canonical problem which contains the basic features is that of a shear layer - two streams of different velocity flowing past each other with different density. Helmholtz[18] and Kelvin[21] were probably the earliest to consider evolution of disturbances in a stratified vortex sheet. While attempting a more general case Rayleigh[27] approximated the shear layer by piecewise-linear velocity profiles.

A kinematic description of the instability for the unstratified vortex sheet was given by Batchelor[1] purely based on vortex dynamics (figure 1(a)). Imagine perturbing the vortex sheet by a sinusoidal disturbance so that the perturbed interface is located at $\eta=\sin k x$. In the neighbourhood of the nodes (A and $\mathrm{B}$ ), the positive vorticity induces a clockwise circulating velocity field. If $\partial \eta / \partial x>0$, the crest and trough move away from each other, leading to vorticity being swept off from nodes like A, whereas if $\partial \eta / \partial x<0$, the crest and trough come closer to each other, leading to vorticity being swept into nodes like B. Thus, accumulation of vorticity at points like B takes place unboundedly in the linear, non-dissipative scenario, giving exponential growth. In absence of stratification the vortex sheet problem as studied by Helmholtz and Kelvin is unstable to infinitely large wavenumbers. This ill-conditioned nature of the system gets removed in Rayleigh's problem with the introduction of a length scale (figure 1(b)). Rayleigh's problem consists of two interfaces of vorticity discontinuity. The Kelvin-Helmholtz (KH) instability of a shear layer was the

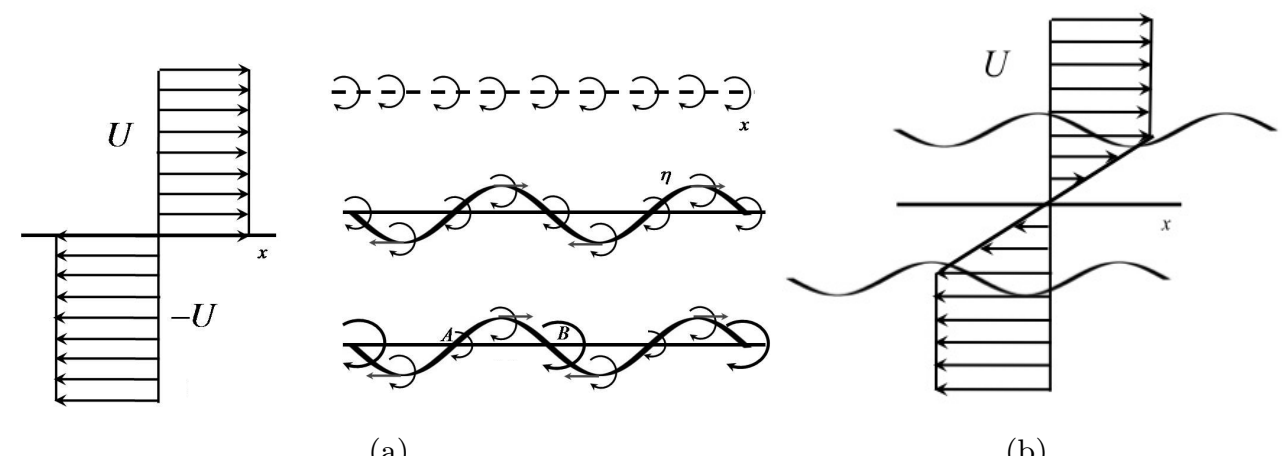

(a)

(b)

Figure 1: (a) Vortex sheet (b) Mixing layer 


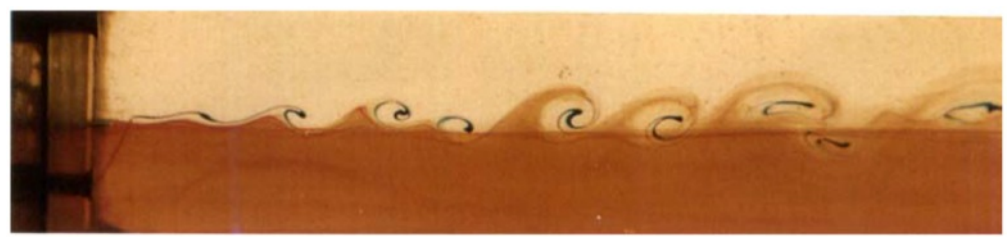

(a) Mixing Layer - Lasheras and Choi (1988)

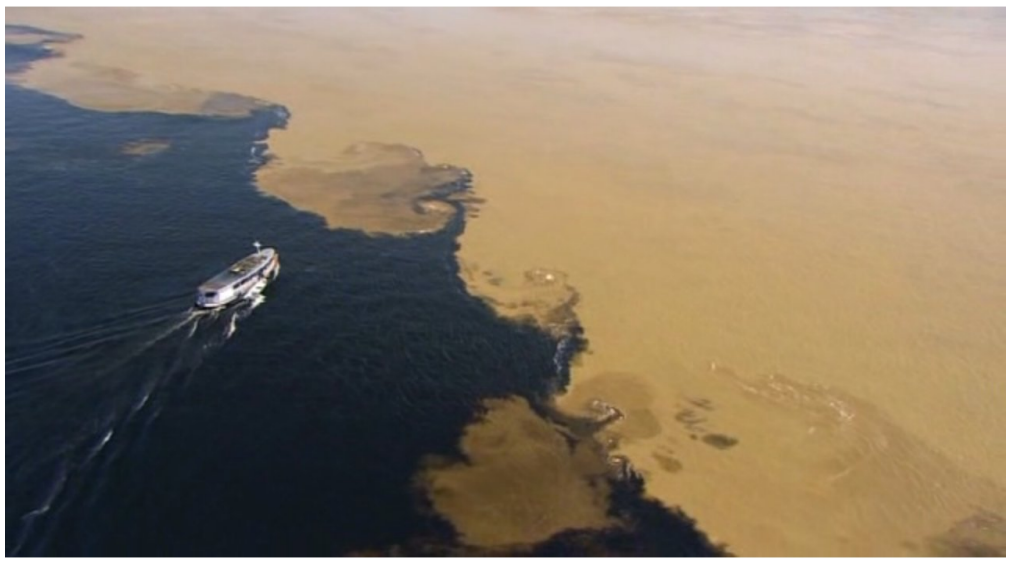

(b) The Meeting of Waters - Confluence of Rio Negro and Rio Solimões near Manaus, Brazil to form the Amazon (Amazon Abyss, BBC)

Figure 2: Kelvin-Helmholtz instabilties

earliest demonstrations of how inviscid instabilities can occur due to presence of vorticity extremum in the flow.

$\mathrm{KH}$ instability is commonly observed in nature. In atmospheric context clouds often help to visualise them. In figure $2 \mathrm{KH}$ instabilities are seen in a laboratory experiment and also in rivers.

Though $\mathrm{KH}$ instability considers the two layers to be of two different density, for stable stratification the role of gravity is purely stabilising. Taylor[32] and Goldstein[17] simultaneously showed how stable stratification can have a destabilising role when it interacts with shear. Considering various multi-layer velocity/density profiles Taylor studied instabilities arising out of interaction of vorticity and/or gravity waves. We will revisit one of Taylor's problems later in the analysis.

Subsequently to model a stratified shear layer relevant for geophysical problems, Holmboe[20] considered Rayleigh's piecwise-linear approximation with an embedded density interface at the shear layer centre (figure 3). For weak stratification a KH like behaviour was observed, instabilities stationary with respect to the flow. On increasing stratification a new class of instabilities emerged - a pair of counter propagating waves known thereafter as Holmboe waves. Unlike $\mathrm{KH}$ instability Holmboe waves are not easily visualised in nature due to the nonstationary behaviour of the waves. They have been experimentally realised and considered to be present in exchange flows. Often a distinction is made between $\mathrm{KH}$ and Holmboe 


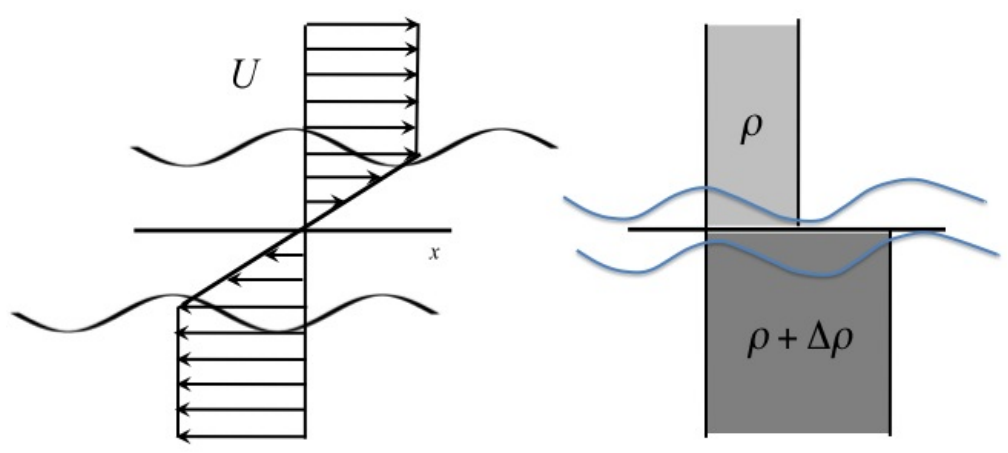

Figure 3: Stratified shear layer

waves based on the stationarity nature as one moves with the speed of the shear layer centre. Such a classification though accurate in the symmetrical case becomes ambiguous in the asymmetrical case. Recently[8] it was shown that a classification based on wave interactions - vorticity-vorticity or vorticity-gravity - provides a consistent explanation.

Approaches towards understanding evolution of disturbances in stratified shear layers can be mostly classified in two categories - Linear stability calculations and direct numerical simulations (DNS). While doing linear stability studies if one restricts to piecewise linear profiles then dispersion relations, eigenfunctions can be analytically computed. To model realistic profiles if one considers the smoother counterparts then solving the formidable Taylor-Goldstein equation (TGE) is the only recourse. For a complete nonlinear description beyond linear instability, DNS holds the key. DNS enables a complete exploration of wide range of length and time scales in flows transitioning towards a turbulent state. Having said that the computational cost and effort involved in a complete DNS severely limits an exhaustive study in nonlinear dynamics of stratified shear layers, many questions go unanswered. How does increasing stratification alter the nonlinear states in stratified shear layers? Does the flow attain a nonlinear stationary state? Gravity waves interact in presence of shear to give rise to Taylor-Caulfield instability, how does the nonlinear state evolve? To answer many such questions full nonlinear simulations are needed. Is there a reduced description of the governing equations which captures the essence of full numerical simulations while giving a stronger analytical handle on the physics? The goal of the present work is to outline a reductive perturbation theory for stratified shear layers. The methodology will rely on a previously used technique, the 'vorticity defect' method.

\section{2 'Defect' theory}

Inviscid shear flow instabilities occur in presence of vorticity maximum, e.g. - KH. For KH like flows one can immediately notice that the base-flow quantities vary across a very small region. This is also true for stratified shear layers where besides vorticity, variations of density occur over a small region. To obtain a reduced description we will try to exploit the smallness of the shear-layer thickness. The perturbative technique to be adopted will in spirit be akin to boundary layer theory. We will have an 'outer' region where the pertur- 

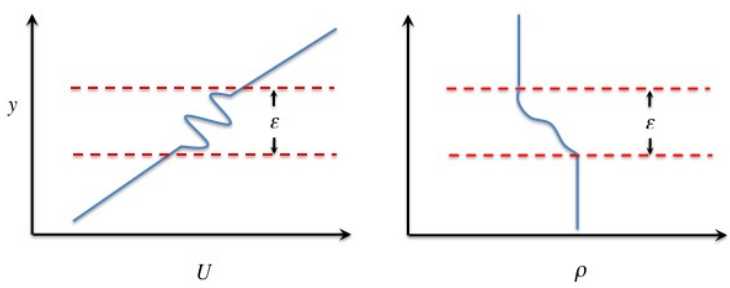

Figure 4: Velocity and density profiles with 'defects'

bations will be assumed to obey linear, steady and inviscid dynamics and an 'inner' region where nonlinearity, dissipation and unsteadiness will be important. A key assumption of the analysis will be considering the base-flow quantities (velocity, density) to be monotonically varying quantity with sharp variations or 'defects' of width $\epsilon$ at the shear layer centre.

While studying wake behind a airfoil, Gill[16] modelled the base-state to be a Couette flow with slight distortions. This severely simplified the linear stability calculations and provided integral dispersion relationships. Next Lerner and Knobloch[24] performed long-wavelength stability studies on a distorted Couette flow. The slight modification to Couette flow was assumed to arise due to finite amplitude perturbations. But it wasn't until the works of Balmforth and co-workers that the 'defect' model - a Couette flow with slight distortions - got a strong theoretical foundations and its immense applications appreciated. Using an analysis similar to critical layer theory del-Castillo Negrete et al.[15] (Balmforth et al. [3]) showed that for inviscid shear flows one can reduce 2D Euler equations to a nonlinear integro-differential equation for the 'defect' vorticity. Exploiting the 'defect' equations analogy with the Valsov-Poisson equation ${ }^{1}$ explicit dispersion relations were obtained for various distortion imposed on the Couette flow. Balmforth and Young[5] went ahead and highlighted evaluation of nonlinear stationary states from the 'defect' equations. The linear viscous problem was addressed by Balmforth [6] and the intriguing connection between viscous and inviscid eigensolutions was discussed in context to various 'defect' profiles. The applicability of the approach for visco-elastic flows has also been studied[4]. In the present work we will try to obtain better understanding of stratified shear layers aided by the 'defect' theory.

Consider a Bousinessq fluid of density,

$$
\rho(x, y, t)=\rho_{m}+\delta \rho(x, y, t)
$$

The evolution equations, vorticity-buoyancy formulation, in the Bousinessq approximation can be written in the following non-dimensional form,

$$
\begin{aligned}
& \frac{\partial \omega}{\partial t}+\frac{\partial(\Psi, \omega)}{\partial(x, y)}=\frac{\partial B}{\partial x}+\frac{1}{R e} \nabla^{2} \omega \\
& \frac{\partial B}{\partial t}+\frac{\partial(\Psi, B)}{\partial(x, y)}=\frac{1}{R e S c} \nabla^{2} B
\end{aligned}
$$

\footnotetext{
${ }^{1}$ Vlasov-Poisson equation describes time evolution of the distribution function of plasma consisting of collision-less charged particles in the zero magnetic field limit.
} 
The horizontal and vertical coordinates have been non-dimensionalised by a characteristic length-scale $L_{0}$ and time by $U_{0} / L_{0}, U_{0}$ being a velocity scale. Stream-function is nondimensionalised by $U_{0} L_{0} . \quad \omega=\nabla^{2} \Psi$ and $B=-g \delta \rho / \rho_{m} L_{0} / U_{0}^{2}$ are the non-dimensional vorticity and buoyancy respectively. Reynolds number, $R e=U_{0} L_{0} / \nu$ ( $\nu$ is the kinematic viscosity), denotes a ratio of inertial to viscous forces and Schmidt number, $S c=\nu / \kappa(\kappa$ is the mass diffusivity), provides a ratio of momentum to mass diffusivity. We will focus on $S c \rightarrow \infty$, the relevant regime for stratified shear layers ${ }^{2}$.

As evident in figure 4, velocity is assumed to be a localised 'defect' imposed on a Couette flow and similarly buoyancy remains largely uniform but for a sharp variation at the 'defect' level. Thus if the variations occur over a length-scale $\epsilon$, we can write,

$$
\begin{aligned}
& \Psi=-\frac{1}{2} y^{2}+\epsilon^{2} \psi(x, y, t) \\
& B=b_{m}+\epsilon^{2} b
\end{aligned}
$$

Hence the vorticity-buoyancy equation can be reduced to,

$$
\begin{aligned}
& \nabla^{2} \psi_{t}+y \nabla^{2} \psi_{x}+\epsilon^{2}\left(\psi_{x} \nabla^{2} \psi_{y}-\psi_{y} \nabla^{2} \psi_{x}\right)=b_{x}+R e^{-1} \nabla^{4} \psi \\
& b_{t}+y b_{x}+\epsilon^{2}\left(\psi_{x} b_{y}-\psi_{y} b_{x}\right)=(\operatorname{Re} S c)^{-1} \nabla^{2} b
\end{aligned}
$$

The above equations can be solved using matched asymptotic theory by splitting the flow into an exterior region where base flow quantities remain uniform or vary monotonically and an interior region corresponding to the 'defect'.

\section{$2.1 \quad$ Outer solution}

The dynamics in the outer region is assumed to be steady, inviscid and linear in the leading order. Another important assumption made regarding the outer solution is that of weak stratification. It is assumed that the outer solution for $\psi$, in the leading order, is uninfluenced by buoyancy. As would become evident that in doing so we deal with a weaker singularity in the outer problem. An assumption of these form becomes unphysical for strongly stratified flows, scenarios beyond the scope of stratified 'defects'.

A regular perturbation expansion of the solution in the outer region provides,

$$
\begin{aligned}
& \psi=\psi_{0}+\epsilon \psi_{1}+\ldots \\
& b=\epsilon b_{1}+\ldots
\end{aligned}
$$

Thus the leading order dynamics is governed by the following pair of equations.

$$
\begin{aligned}
& y \nabla^{2} \psi_{0 x}=0, \quad y b_{1 x}=0 \\
\Rightarrow \quad & \nabla^{2} \psi_{0}=-2 A(x, t) \delta(y)
\end{aligned}
$$

Thus the outer solution behaves like an irrotational flow being forced by a vortex sheet, a $\delta$ function singularity, at the 'defect' level $-y=0$ (buoyancy also gets forced by a density

\footnotetext{
${ }^{2}$ In the context of passive scalar turbulence $S c \rightarrow \infty$ is known as the Batchelor regime. Such a regime allows for rapid variations in passive scalar field due to stirring by a smooth velocity field.
} 
sheet). This necessitates a jump in tangential velocity (while maintaining continuity of normal velocity),

$$
\left[\psi_{0 y}\right]_{y=0^{-}}^{y=0^{+}}=-2 A(x, t), \quad\left[\psi_{0}\right]_{y=0^{-}}^{y=0^{+}}=0
$$

As will be seen this jump in the outer solution will be smoothened out over a region $\epsilon$ by the inner solution. If we had allowed for leading order buoyancy effects in the outer vorticity equation, the flow would have been forced by a vortex dipole instead of a vortex sheet. The consequence of these stronger singularity, a $\delta^{\prime}$ singularity, would have then resulted in a jump in normal velocity. Weak stratification allows us to overlook such a possibility in the present analysis.

Introducing Fourier transform of the streamfunction as,

$$
\tilde{\psi}(k, y, t)=\mathcal{F}[\psi(x, y, t)]=\int_{-\infty}^{\infty} \psi(x, y, t) e^{-i k x} d x
$$

equation 11 can be written as,

$$
\tilde{\psi}_{0 y y}-k^{2} \tilde{\psi}_{0}=-2 \tilde{A}(k, t) \delta(y)
$$

Thus one obtains,

$$
\psi_{0}=\mathcal{F}^{-1}[\mathcal{G}(k) \hat{A}(k, t)]
$$

where, $\mathcal{F}^{-1}$ denotes the inverse Fourier transform and the Green's function, $\mathcal{G}(k)$, can be computed for both unbounded and bounded domains $(|y| \leqslant 1)$ as,

$$
\begin{aligned}
\mathcal{G}(k) & =\frac{1}{|k|} \operatorname{sech}|k| \sinh |k|(1-|y|), & & \text { bounded } \\
& =\frac{1}{|k|} e^{-|k||y|}, & & \text { unbounded }
\end{aligned}
$$

Henceforth all analysis will be presented for the unbounded case but the results have been extended to the bounded case too. The 'defect' analysis is not solely restricted to the outer flow being Couette and can be extended to more general flows by the method of constructing Green's function for flow profiles with curvature[2].

Next we will regularise the discontinuity in outer velocity field by evaluating the inner solution.

\subsection{Inner solution}

Similar to a traditional boundary-layer like analysis, the inner solution must contain at least one of the physics that have been overlooked in the outer region - viscosity, nonlinearity and unsteadiness. Working with our small parameter, the defect thickness $\epsilon$, we define a stretched vertical coordinate, $\eta=y / \epsilon$ and a slow time-scale $\tau=\epsilon t$. It needs to be mentioned that the method of analysis bears similarities with standard critical layer theories ([33], [31]). Beyond the initial transients the $O\left(\epsilon^{2}\right)$ nonlinear term acting on a critical layer of width $O(\epsilon)$ becomes important after time $O(1 / \epsilon)$. As would become clear that the 'defect' theory 
is considerably simpler than critical layer analysis. We pose the following expansion in the inner variables,

$$
\begin{array}{ll} 
& \psi=\psi_{0}(x, 0, t)+\epsilon \phi_{1}(x, \eta, \tau)+O\left(\epsilon^{2}\right), \\
\Rightarrow \quad & \psi_{y}=\phi_{1 \eta}+\epsilon \phi_{2 \eta}+O\left(\epsilon^{2}\right), \\
\Rightarrow \quad & \nabla^{2} \psi=\epsilon^{-1} \phi_{1 \eta \eta}+\psi_{0 x x}+\phi_{2 \eta \eta}+O(\epsilon) \\
& b=\mathcal{B}(x, \eta, \tau)+O(\epsilon)
\end{array}
$$

From above equations it is evident that leading order inner solution, $\psi_{0}(x, 0, t)$, matches the corresponding outer solution at $y=0$. As mentioned before the discontinuity in tangential velocity in the outer solution will be smoothed by the inner solution,

$$
2 A(x, t)=-\int_{-\infty}^{\infty} \mathcal{Z} d \eta
$$

where $\mathcal{Z}=\phi_{1 \eta \eta}$ is the leading order defect vorticity. The velocity jump equals the vorticity integrated across the defect.

Substituting the expansion 18 in equations 6 and 7 and collecting the leading order terms one obtains the 'defect' vorticity-buoyancy equations,

$$
\begin{aligned}
& \mathcal{Z}_{\tau}+\eta \mathcal{Z}_{x}+\Phi_{x} \mathcal{Z}_{\eta}=\mathcal{B}_{x}+\lambda Z_{\eta \eta}, \\
& \mathcal{B}_{\tau}+\eta \mathcal{B}_{x}+\Phi_{x} \mathcal{B}_{\eta}=\frac{\lambda}{S c} B_{\eta \eta}, \\
& \quad(\mathcal{Z}, \mathcal{B}) \rightarrow 0, \text { as }|\eta| \rightarrow \infty \\
& \tilde{\Phi}=-\frac{1}{2|k|} \int_{-\infty}^{\infty} \tilde{\mathcal{Z}} d \eta, \quad \Rightarrow \mathcal{H}(\Phi)_{x}=-\frac{1}{2 \pi} \int_{-\infty}^{\infty} \mathcal{Z} d \eta
\end{aligned}
$$

where, $\mathcal{H}($.$) denotes the Hilbert transform of a variable defined in terms of the following$ Cauchy principal value integral,

$$
\mathcal{H}(u)=\frac{1}{\pi} f \frac{u(\xi)}{\zeta-\xi} d \xi
$$

and $\lambda=\left(\epsilon^{3} R e\right)^{-1}$ is representative of the ratio of nonlinearity and viscosity. In critical layer theory there exists a similar quantity, Haberman parameter, which denotes competition of nonlinearity and viscosity.

Equations 23-25 are the 'defect' vorticity-buoyancy equations relevant for stratified shear layer studies. During the course of reduction we have retained the necessary physics due to unsteadiness, nonlinearity and dissipation. Though the obtained nonlinear integrodifferential equations appear more daunting than the governing equations we started off with it would become clear that the 'defect' equations are more amenable to analytical and numerical treatments. Similar equations have been derived in critical layer theories for stratified shear flows [14]. 


\section{Conservation laws}

The inviscid, nonlinear 'defect' equations (equation 23-24 for $\lambda=0$ ) contains certain conserved quantities, some deducible from evident symmetries in the problem and others not so obvious ones. Lets us define a spatial average,

$$
<.>=\iint . d x d \eta
$$

In absence of dissipation both average vorticity and buoyancy remains conserved,

$$
<\mathcal{Z}>_{\tau}=0, \quad<\mathcal{B}>_{\tau}=0
$$

momentum remains conserved,

$$
<\eta \mathcal{Z}>_{\tau}=0
$$

and energy also is conserved,

$$
<\frac{\eta^{2}-\Phi}{2} \mathcal{Z}-\eta \mathcal{B}>_{\tau}=0
$$

As always is the case with ideal fluid besides the above ones there exists an infinitude of conserved quantities - Casimirs. For 2D homogeneous fluid any functional of vorticity is a conserved quantity. Enstrophy - a representative of vortex stretching and tilting - is one such commonly used candidate. For stratified flows due to baroclinic generation of vorticity enstrophy is no longer conserved. For stratified 'defects' one instead has the any functional of buoyancy and product of vorticity with any buoyancy functional as the Casimirs,

$$
<G(\mathcal{B})>_{\tau}=0,<\mathcal{Z F}(\mathcal{B})>_{\tau}=0
$$

The conserved quantities thus obtained help in obtaining the stationary states for the system and also comment on its nonlinear stability. More importantly it also helps as a check in numerical computations to ensure that the numerical method indeed conserve the necessary invariants.

\section{Inviscid linear stability}

Earlier it was mentioned that previous studies on flow with slight distortions exploited the simplicity of the model in deriving explicit integral dispersion relations. Conventionally inviscid linear stability demands solution of Rayleigh equation or in the case of stratified shear flow Taylor Goldstein equation (TGE),

$$
\left[\frac{d^{2}}{d y^{2}}-k^{2}\right] \hat{\psi}=\frac{\bar{U}^{\prime \prime} \hat{\psi}}{(\bar{U}-c)}-\frac{N^{2} \hat{\psi}}{(\bar{U}-c)^{2}}
$$

where $\hat{\psi}$ is the disturbance streamfunction, $\bar{U}$ the background base-flow, $N=\sqrt{-g / \rho_{m} d \rho / d z}$ the BruntVäisälä (buoyancy time-scale), $k$ is the disturbance wave-number and $c$ is the wave-speed. The relative strength of stratification vis-a-vis inertia is denoted by Richardson 
number, $R i=\left(N L_{0} / U_{0}\right)^{2}$. Solution of the above boundary value problem, even numerically, is no easy task and analytical solutions can be obtained only for broken-line profiles. The 'defect' model circumvents this difficulty with ease.

Consider the 'defect' vorticity and buoyancy to be decomposed of a base-state contribution and a perturbation,

$$
\begin{array}{r}
\mathcal{Z}(x, \eta, \tau)=F(\eta)+\zeta(x, \eta, \tau) \\
\mathcal{B}(x, \eta, \tau)=\int^{\eta} N^{2}(y) d y+\beta(x, \eta, \tau)
\end{array}
$$

Substituting the above expressions in equations 23 and 24 and considering the linearised equations one has,

$$
\begin{aligned}
& \zeta_{\tau}+\eta \zeta_{x}+r_{x} F_{\eta}=\beta_{x}, \\
& \beta_{\tau}+\eta \beta_{x}+r_{x} N^{2}=0 \\
& 2 \hat{r}=-k^{-1} \int_{-\infty}^{\infty} \hat{\zeta} d \eta
\end{aligned}
$$

On assuming a normal-mode form, $\zeta(x, \eta, \tau)=\hat{\zeta}(\eta) e^{i k(x-c \tau)}$, the eigenfunctions can be expressed as,

$$
\begin{aligned}
& \hat{\beta}=-\frac{\hat{r} N^{2}}{(\eta-c)}, \\
& \hat{\zeta}=-\frac{\hat{r} F_{\eta}}{(\eta-c)}-\frac{\hat{r} N^{2}}{(\eta-c)^{2}}
\end{aligned}
$$

and the dispersion relation,

$$
\mathcal{D}(c, k ; F, N) \equiv 2 k-\int_{-\infty}^{\infty}\left[\frac{F_{\eta}}{(\eta-c)}+\frac{N^{2}}{(\eta-c)^{2}}\right] d \eta=0
$$

This is the stratified version of the integral dispersion relation obtained in [3]. $\mathcal{D}$ is an analytic function of $c$ but for the real-axis where it has a branch-cut. The branch-cut gives rise to the continuous spectrum, a common feature of inviscid shear flows. The presence of the continuous spectrum contributes to transient growth in flows which otherwise are modally stable - Orr mechanism being a well known example. Sometimes the dispersion relation instead of having zeros in the principal complex plane, vanishes at points on different Riemann sheets. While doing the initial value problem this leads to observation of a collective behaviour of the continuous spectrum as a single damped discrete mode - known as Landau pole or quasi-mode. This damping, eponymously known as Landau damping in plasma physics, is a purely inviscid phenomenon arising due to phase mixing. Quasi-modes have been probed to understand the mysterious connection between the inviscid and viscous initial value problem in the limit of vanishing viscosity. The present analysis will focus purely on unstable modes and hence no longer address the above intriguing phenomena. We will now compute dispersion relations for various stratified shear layer configurations. Before proceeding on to calculations for both broken and smooth profiles a note can be 
made borrowing from Nyquist theory. For a closed curve $\mathcal{C}$ in the complex $c$ plane, the winding number,

$$
\frac{1}{2 \pi i} \oint_{\mathcal{C}} \frac{\mathcal{D}^{\prime}(c)}{\mathcal{D}(c)} d c=\mathrm{N}-\mathrm{P}
$$

where $\mathrm{N}$ is the number of zeros and $\mathrm{P}$ gives the number of poles of $\mathcal{D}(c)$ inside $\mathcal{C}$. Considering $\mathcal{D}$ an analytic function of $c$ the winding number gives us the number of eigenmodes. Earlier works on 'defect' theory like [3] and [6] elucidates how Nyquist theory can be used effectively for the 'defect' model.

\subsection{Holmboe instability}

Holmboe instability occurs when there exists a density interface embedded in a mixing layer. It is a popular model to represent instabilities arising due to horizontal shearing of density interfaces. This classical instability, in the symmetrical case, exhibits a transition from the stationary Kelvin-Helmholtz instability to the propagating Holmboe waves. Though Holmboe waves are inherently difficult to visualise in nature due to their non-stationary behaviour they have been observed in laboratory experiments - exchange flow between two basins of different density being an example.

\subsubsection{Broken-line profile}

For a broken-line Holmboe profile denoted by the following defect vorticity and buoyancy profile,

$$
\begin{aligned}
& F_{\eta}=f\left[\delta\left(\eta-\eta_{0}\right)-\delta\left(\eta+\eta_{0}\right)\right], \\
& N^{2}=d \delta(\eta)
\end{aligned}
$$

one obtains the dispersion relation,

$$
c^{4}-\left(1-\frac{f}{k}+\frac{d}{2 k}\right) c^{2}+\frac{d}{2 k}=0, \quad \eta_{0}=1, c=c_{r}+i c_{i}
$$

It was previously mentioned that the 'defect' theory is suited to weak stratification calculations. Thus the results obtained are representative of small $R i$ cases in the complete problem. $d$ determines the strength of stratification and a qualitative comparison is made of contours of growth-rate in $d-k$ plane with the one obtained from the solution of TGE for a symmetric shear layer. The basic features are well captured by the present model (figure $5)$. One immediate observation is the differentiation of stationary $\left(c_{r}=0\right)$ and propagating instabilities $\left(c_{r} \neq 0\right)$.

\subsubsection{Smooth profile}

For smooth profiles the integral dispersion relation can be evaluated using Cauchy residue theorem. The simplest profile to consider would be a Lorentzian.

$$
\begin{aligned}
& F_{\eta}=\frac{\alpha f}{\pi}\left[\frac{1}{(\eta+1)^{2}+\alpha^{2}}-\frac{1}{(\eta-1)^{2}+\alpha^{2}}\right], \\
& N^{2}=\frac{\alpha d}{\pi} \frac{1}{\eta^{2}+\alpha^{2}}
\end{aligned}
$$




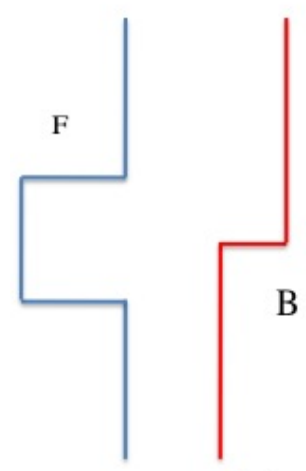

(a)

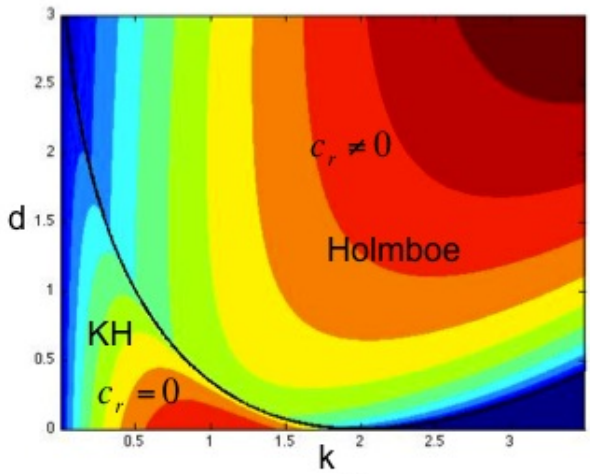

(b)

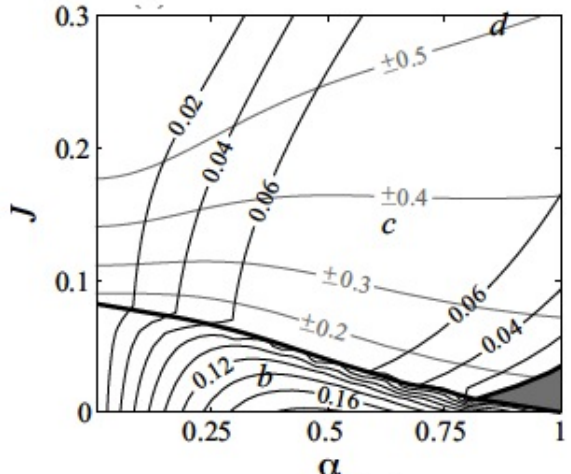

(c)

Figure 5: Holmboe instability. a) A broken-line profile, b) Contours of growth-rate $\left(c_{i}\right)$ in $d-k$ plane $(f=2), c)$ Growth-rate contours in $R i-k$ plane from a complete linear stability calculations (Carpenter et al.[8])

For the Lorentzian profile the dispersion relation is identical to equation 43 with $c$ in the quadratic expression no longer being the complex wave-speed but instead replaced by $c \rightarrow$ $c+i \alpha \operatorname{sgn}\left(c_{i}\right)$.

A smooth profile which would be used in the numerical calculations and represents sharper variations in flow quantities is a tanh profile.

$$
\begin{aligned}
& F=\frac{f}{2}\left[\tanh \left(\frac{\eta-\eta_{0}}{\Delta}\right)-\tanh \left(\frac{\eta+\eta_{0}}{\Delta}\right)\right] \\
& N^{2}=\frac{d}{2 \Delta} \operatorname{sech}^{2}\left(\frac{\eta}{\Delta}\right)
\end{aligned}
$$

One could note that the both the smooth profiles chosen above are functions which are nascent delta functions, i.e. as $\alpha, \Delta \rightarrow 0$ we obtain the generalised functions form as given in equation 42.

From residue calculus we can evaluate the following integrals,

$$
\begin{aligned}
& \mathcal{I}_{1}\left(\Delta, \eta_{0}, c\right)=\int_{-\infty}^{\infty} \frac{\operatorname{sech}^{2}\left(\frac{\eta-\eta_{0}}{\Delta}\right)}{(\eta-c)} d \eta=2 \pi i\left[\operatorname{sech}^{2}\left(\frac{c-\eta_{0}}{\Delta}\right) s-\frac{1}{\pi^{2}} \Psi^{(1)}(\alpha)\right] \\
& \mathcal{I}_{2}\left(\Delta, \eta_{0}, c\right)=\int_{-\infty}^{\infty} \frac{\operatorname{sech}^{2}\left(\frac{\eta-\eta_{0}}{\Delta}\right)}{(\eta-c)^{2}} d \eta=-\frac{2 \pi i}{\Delta}\left[2 \operatorname{sech}^{2}\left(\frac{c-\eta_{0}}{\Delta}\right) \tanh \left(\frac{c-\eta_{0}}{\Delta}\right) s+\frac{i}{\pi^{3}} \Psi^{(2)}(\alpha)\right] \\
& \alpha=\frac{1}{2}-\frac{i}{\pi \Delta}\left(\eta_{0}-c\right), \quad s=\frac{1}{2}\left(1+\operatorname{sgn}\left(c_{i}\right)\right)
\end{aligned}
$$

where $\Psi^{(1)}(\alpha)$ and $\Psi^{(2)}(\alpha)$ are polygamma functions - $\Psi^{(n)}(z)=d^{n+1}(\log \Gamma(z)) / d z^{n+1}$. Based on the above evaluated integrals the dispersion relation for the tanh Holmboe profile can be computed explicitly as,

$$
2 k-\frac{1}{2 \Delta}\left[f\left\{\mathcal{I}_{1}\left(\Delta, \eta_{0}, c\right)-\mathcal{I}_{1}\left(\Delta,-\eta_{0}, c\right)\right\}+d \mathcal{I}_{2}(\Delta, 0, c)\right]=0
$$




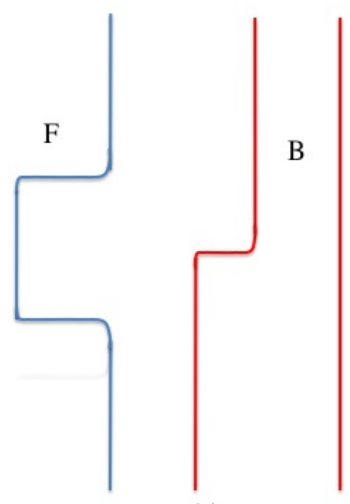

(a)

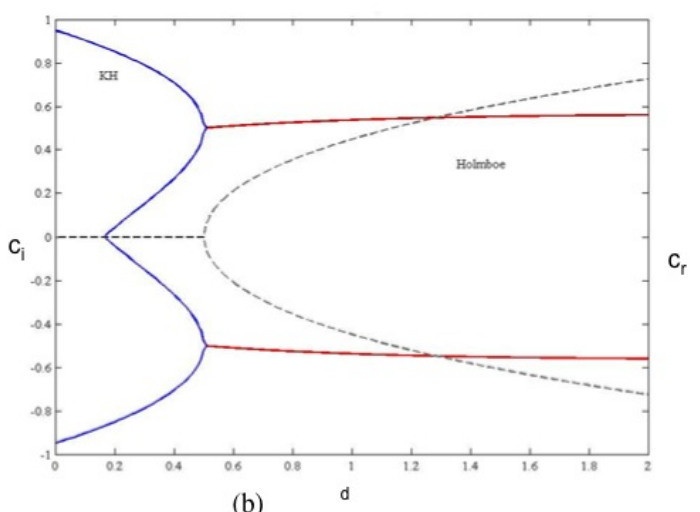

(b)

Figure 6: Holmboe instability. a) A Smooth profile, b) Variation of $c_{r}$ (dashed) and $c_{i}$ (continuous) with stratification (d) for $f=2, k=1, \Delta=1 / 3, \eta_{0}=1$

Though we have obtained an analytical dispersion relation for the tanh profile the numerical evaluation of the integrals is more efficient than working with the polygamma functions.

Figure 6 shows the stationary and propagating nature of the instabilities for a set of parameter values. Cases from the instability curves will be later visited during numerics. An important question that numerics will try to answer is how does the nonlinear states change as one increases stratification and transitions from a $\mathrm{KH}$ instability to Holmboe instability.

\subsection{Taylor-Caulfield instability}

Holmboe instability arose when gravity waves interacted with vorticity waves. Taylor[32] during the course of studying various broken line profiles observed that a stable stratification composed of two density jumps gets destabilised if there is an imposed shear. This initially surprising result can be understood as interaction of gravity waves facilitated by shear.

\subsubsection{Broken-line profile}

For Taylor-Caulfield instabilities there is no defect vorticity and instead one has variations of defect buoyancy,

$$
\left.N^{2}=d\left[\delta\left(\eta-\eta_{0}\right)+\delta\left(\eta+\eta_{0}\right)\right]\right)
$$

As seen in the case of Holmboe instability one obtains polynomial dispersion relations for broken-line profiles,

$$
c^{4}-\left(2+\frac{d}{k}\right) c^{2}+\left(1-\frac{d}{k}\right)=0, \quad \eta_{0}=1, c=c_{r}+i c_{i}
$$




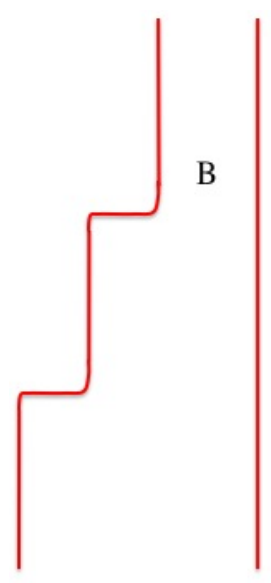

(a)

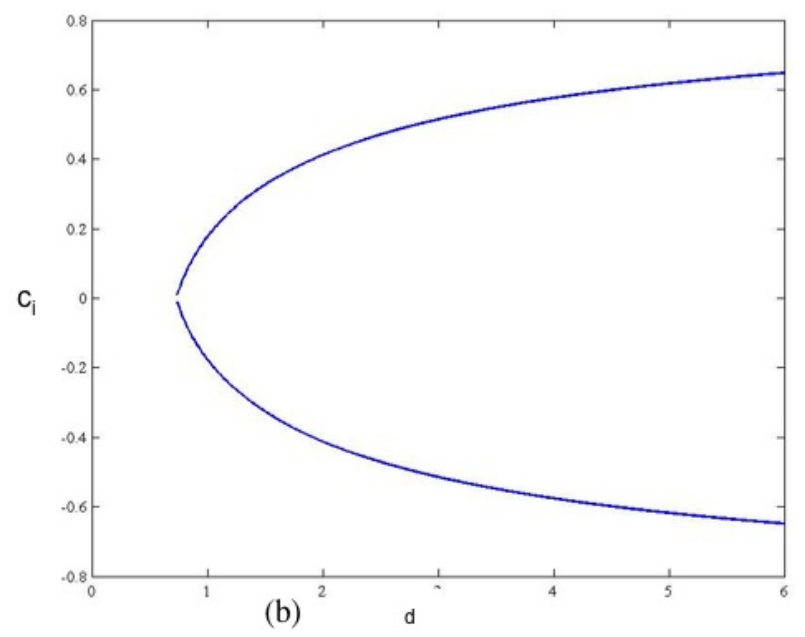

Figure 7: Taylor-Caulfield instability. (a) A Smooth profile, (b) Growth-rate $\left(c_{i}\right)$ variation with stratification (d) for $k=1, \eta_{0}=1$

\subsubsection{Smooth profile}

Similar to Holmboe case one can calculate stability characteristics for various smooth profiles. For a Lorentzian profile,

$$
N^{2}=\frac{\alpha d}{\pi}\left[\frac{1}{(\eta+1)^{2}+\alpha^{2}}+\frac{1}{(\eta-1)^{2}+\alpha^{2}}\right]
$$

The dispersion relation is identical to equation 50 with $c$ in the quadratic expression no longer being the complex wave-speed but instead replaced by $c \rightarrow c+i \alpha \operatorname{sgn}\left(c_{i}\right)$.

For a smooth tanh profile,

$$
N^{2}=\frac{d}{2 \Delta}\left[\operatorname{sech}^{2}\left(\frac{\eta-\eta_{0}}{\Delta}\right)+\operatorname{sech}^{2}\left(\frac{\eta+\eta_{0}}{\Delta}\right)\right]
$$

Once again the analytical form of the dispersion relation for can be written as,

$$
2 k-\frac{d}{2 \Delta}\left[\mathcal{I}_{2}\left(\Delta, \eta_{0}, c\right)+\mathcal{I}_{2}\left(\Delta,-\eta_{0}, c\right)\right]=0
$$

Figure 7 illustrates the stability characteristics for Taylor-Caulfield instability as obtained from 'defect' theory. The TC modes are purely stationary waves.

Though there exists some linear stability calculations and DNS calculations very little is known about the nonlinear states of Taylor-Caulfield instabilities. Next using numerical solution of 'defect' equations we will try to explore the details.

\section{$5 \quad$ Numerical solution}

A significant advantage that the 'defect' theory offers over the complete Bousinnessq NavierStokes equations is in numerical computation. As is commonly known that the notorious 
advective nonlinearity in Navier-Stokes serves as a severe bottleneck in full-fledged computations. The defect equations for $S c \rightarrow \infty$,

$$
\begin{aligned}
& \mathcal{Z}_{\tau}+\eta \mathcal{Z}_{x}+\Phi_{x} \mathcal{Z}_{\eta}=\mathcal{B}_{x}+\lambda Z_{\eta \eta}, \\
& \mathcal{B}_{\tau}+\eta \mathcal{B}_{x}+\Phi_{x} \mathcal{B}_{\eta}=0 \\
& \quad(\mathcal{Z}, \mathcal{B}) \rightarrow 0, \text { as }|\eta| \rightarrow \infty \\
& \tilde{\Phi}=-\frac{1}{2|k|} \int_{-\infty}^{\infty} \tilde{\mathcal{Z}} d \eta, \quad \Rightarrow \mathcal{H}(\Phi)_{x}=-\frac{1}{2} \int_{-\infty}^{\infty} \mathcal{Z} d \eta
\end{aligned}
$$

though nonlinear avoids the difficult nonlinearity $\partial\left(\phi_{1}, \phi_{1 \eta \eta}\right) / \partial(x, \eta)\left(\phi_{1 \eta \eta}=\mathcal{Z}\right)$. In the present form the 'defect' equation entails solving a set of advection-diffusion equations. The method adopted in the present work relies on exploiting the similarities of the 'defect' equations with the Vlasov-Poisson equation and using a operator-splitting technique.

\subsection{Operator splitting technique}

The operator splitting technique used is based on the method outlined in [13] for solution of Vlasov-Poisson equation and in [7] for studying critical layers in 2D vortices. This splitting technique is also known as Strang splitting[25].

The integration over time-step $[\tau, \tau+\delta \tau]$ is divided into three stages,

\subsubsection{Advect in $x$ for half time-step}

Consider the advection in $x$ in the buoyancy equation,

$$
\mathcal{B}_{\tau}+\eta \mathcal{B}_{x}=0
$$

for a time interval $\delta \tau / 2$. The exact solution is $\mathcal{B}(x, \eta, \tau+\delta \tau / 2)=\mathcal{B}(x-\eta \delta \tau / 2, \eta, \tau)$. Fourier interpolation is used to perform the $x$ advection $-\tilde{\mathcal{B}}(k, \eta, \tau+\delta \tau / 2)=\tilde{\mathcal{B}}(k, \eta, \tau) e^{-i k \eta \delta \tau / 2}$. For the vorticity equation,

$$
\mathcal{Z}_{\tau}+\eta \mathcal{Z}_{x}=\mathcal{B}_{x}
$$

the solution can be similarly written in Fourier space as -

$$
\tilde{\mathcal{Z}}(k, \eta, \tau+\delta \tau / 2)=\tilde{\mathcal{Z}}(k, \eta, \tau) e^{-i k \eta \delta \tau / 2}+i k \delta \tau / 2 \tilde{\mathcal{B}}(k, \eta, \tau+\delta \tau / 2)
$$

The above calculations in Fourier space is done using the FFT/IFFT routine in Matlab.

\subsubsection{Advect in $\eta$ for a complete time-step}

Both buoyancy and vorticity obeys identical $\eta$ advection equation,

$$
\begin{aligned}
& \mathcal{B}_{\tau}+\Phi_{x} \mathcal{B}_{\eta}=0 \\
& \tilde{\Phi}=-\frac{1}{2|k|} \int_{-\infty}^{\infty} \tilde{\mathcal{Z}} d \eta
\end{aligned}
$$

Before performing the advection $\Phi_{x}$ is computed by integrating $\mathcal{Z}$ in $\eta$, once again in Fourier space. The $\eta$ advection occurs with a velocity $\Phi_{x}-\mathcal{B}(x, \eta, \tau+\delta \tau)=\mathcal{B}\left(x, \eta-\Phi_{x} \delta \tau, \tau\right)$. Due 
to lack of periodicity we use linear/cubic spline interpolation in $\eta$ instead of Fourier. The same method is followed for advection of $\mathcal{Z}$. If advection happens into the domain from both above or below the computational domain then it is assigned the far-field equilibrium value.

\subsubsection{Advect in $x$ for another half time-step}

The first step is repeated for another half time-step, $\delta \tau / 2$. One can combine the first and third step and do two steps instead - advect in $\eta$ for $\delta \tau$ followed by advection in $x$ for another $\delta \tau$.

To account for diffusion of vorticity $(\lambda \neq 0)$ a forward in time Euler integration is carried out with diffusion operator in $\eta$ discretized using finite-difference technique.

The computational domain consists of $(x, \eta) \in[0,2 \pi] \times\left[-\eta_{\max }, \eta_{\max }\right]$. $\eta_{\max }$ is fixed at 10 so that the vertical extent is at least 5 times larger than the shear layer and the far-field is ensured to remain uninfluenced by the dynamics in the shear layer. The choice of timestep, $\delta \tau$, and horizontal discretization, $h=2 \pi / N,(N$ is the number of Fourier modes used $)$ is determined by the following stability condition,

$$
\left|\eta_{\max }\right| \Delta \tau \leqslant h
$$

For the present work $N$ has been chosen to either 256 or 512 and the time-step, $\Delta \tau=$ $1 \times 10^{-4}, 5 \times 10^{-4}$. The vertical discretization, $\Delta \eta$, is chosen so as to obey the von Neumann stability criteria,

$$
\frac{\lambda \Delta \tau}{(\Delta \eta)^{2}} \leqslant \frac{1}{2}
$$

$\Delta \eta=0.01,0.005$ is used in the calculations. $\lambda$, an estimate for momentum diffusion, has been widely varied from $0.05-10^{-4}$. All numerics reported hereafter are for $\lambda=10^{-3}$. To instigate instabilities in the flows the base-state is initially seeded with a disturbance of the form $e^{-\eta^{2}} \cos k x$ of magnitude $0.1 \%$ of the base-state maxima. The $\eta$ interpolation has been done using linear interpolation. The interp1 command in Matlab has been used for the purpose. For stratified KH (section 5.3.1) a grid resolution of $256 \times 2001$ has been used with a time-stepping of $5 \times 10^{-4}$. The remaining calculations have been done on a $512 \times 4001$ grid with $\Delta \tau=1 \times 10^{-4}$.

\subsection{Analytical comparisons}

To check the efficacy of the operator splitting technique a comparison is made between the numerical solution and analytical solution known for a passive scalar advection equation. The passive scalar advection equation,

$$
\chi_{\tau}+\eta \chi_{x}+2 \chi_{\eta} \cos 2 x=0
$$

was studied by Stewartson[31] in the context of critical layers. The above equation can be solved using method of characteristics and drawing analogy with motion of pendulum. For 


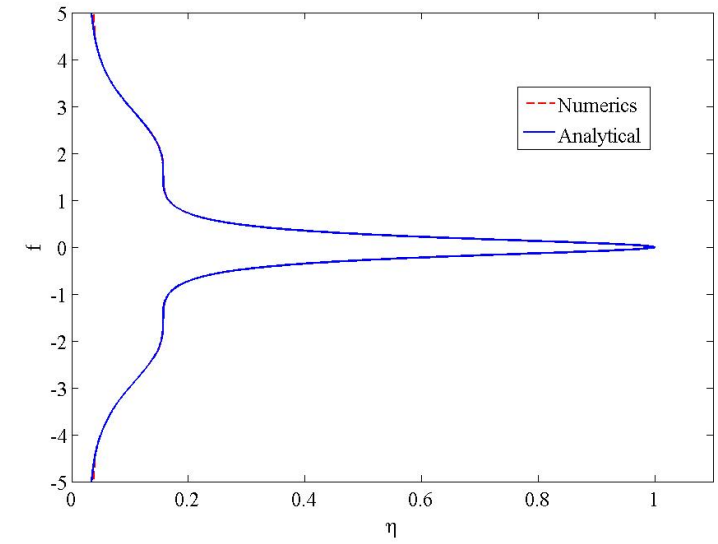

(a) $t=1$

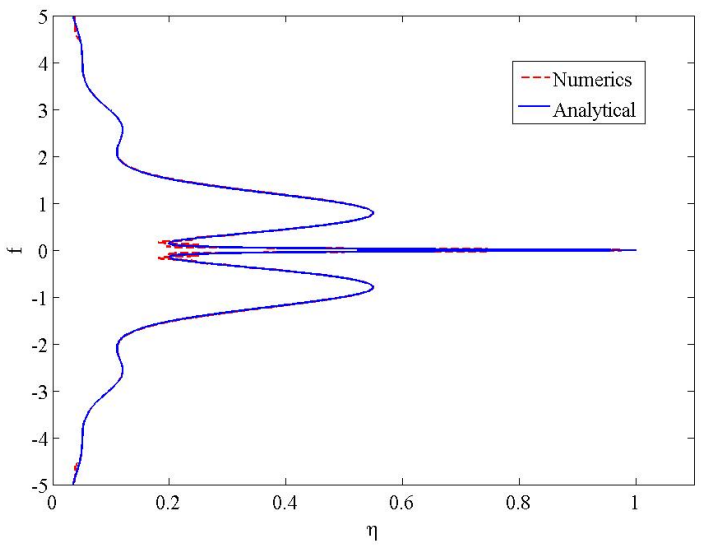

(b) $t=2$

Figure 8: Comparison of analytical and numerical results for the passive scalar problem at $x=3 \pi / 4 . \chi$ is $\chi(3 \pi / 4, \eta, 0)=1 /\left(1+\eta^{2}\right)$

$x=\pi / 4$ or $3 \pi / 4$ one can obtain explicit expressions for $\chi$. At $x=3 \pi / 4$ if initially the distribution of $\chi$ is $\chi(3 \pi / 4, \eta, 0)=f(\eta)$ then the solution at arbitrary times will be[7],

$$
\chi\left(\frac{3 \pi}{4}, \eta, \tau\right)=f\left(\frac{2 \operatorname{sgn}(\eta)}{\sqrt{m}} \operatorname{dn}\left(\mathrm{K}(m)-\frac{2 \tau}{\sqrt{m}}\right)\right), m=\frac{4}{4+\eta^{2}}
$$

where $\mathrm{dn}$ is the Jacobi elliptic function and $\mathrm{K}(m)$ the complete elliptic integral of first kind. Figure 8 shows the good agreement between numerical and analytical results for a Lorentzian initial condition. The agreement starts deteriorating with time due to generation of increasing fine-scales.

\subsection{Holmboe instabilities}

By increasing stratification we will try to explore various nonlinear dynamics for instability scenarios occurring in Holmboe instabilities starting from stratified $\mathrm{KH}$ and proceeding on to pure Holmboe waves. For the purpose of exploring various nonlinear states the tanh model discussed before is revisited.

$$
\begin{aligned}
& F=\frac{f}{2}\left[\tanh \left(\frac{\eta-\eta_{0}}{\Delta}\right)-\tanh \left(\frac{\eta+\eta_{0}}{\Delta}\right)\right] \\
& N^{2}=\frac{d}{2 \Delta} \operatorname{sech}^{2}\left(\frac{\eta}{\Delta}\right)
\end{aligned}
$$

$f=2, k=1, \Delta=1 / 3, \eta_{0}=1$ are the parameters maintained identical for all Holmboe simulations. We choose 3 values of $d$. $d=0.1,1.5 \& 8$. As visible from figure $6, d=0.1$ denotes stationary stratified $\mathrm{KH}$ waves in linear regime, $d=1.5,8$ denote linear propagating Holmboe waves ( $d=8$ is not shown in the figure). We will try to find how the nonlinear states behave for these cases. Does a linear Holmboe wave remain so always howsoever weak the stratification may be? 


\subsubsection{Stratified KH $(d=0.1)$}

Kelvin-Helmholtz in presence of weak stratification contains all the usual traits of the unstratified version - growth of instability waves, roll-up of the shear layer, vortex nutation (the horizontal rocking of partially form cat's eye) and finally culminating with the celebrated cat's eye pattern or the KH billow. Besides the weakened growth-rate due to the stabilising effect of stratification the stratified version exhibits intense stirring and mixing of the buoyancy field. 3D effects like elliptical instability of the billow and hyperbolic instability of the braid also emerge later in the evolution. Interested reader can find detailed discussions in the article by Caulfield and Peltier[12].

Figure 9 shows the evolution of buoyancy $(\mathcal{B})$ and vorticity $(\mathcal{Z})$ field at different instants of time. Figure 9(a)-(b) is a snapshot when the flow exhibits finite amplitude waves just prior to the roll-up. (c)-(d) is at a stage during the formation of the billow. The flow has departed from linear stability predictions and vortex nutations are observed at this stage. Finally in figure 9(e)-(f) the well homogenised KH billow is visible. Figure 10 shows the time-evolution of $\left\langle|\psi|^{2}\right\rangle^{1 / 2}$, a measure of disturbance amplification. Signatures of the features discussed above can be seen in the time-trace.

\subsubsection{Pure Holmboe $(d=8)$}

Next we explore a case where the flow is buoyancy dominant and the shear layer dynamics are very different from KH. For strongly stratified shear layers the flow exhibits propagating Holmboe instabilities. They are identified by oppositely travelling cusps which spews fluid from one region to other. Compared to a $\mathrm{KH}$ billow the Holmboe waves are highly inefficient in mixing. The dynamics is very often given by the beating phenomena of the two Holmboe waves. Laboratory experiments on Holmboe waves have been done by Lawrence et al. [22], Pouliquen et al.[26] and several researchers thereafter. Efforts towards using DNS to understand Holmboe wave dynamics was done by Smyth and co-workers ([28], [29] and [30]) amongst others. A recent work by Carpenter et al.[9] considers both experimental and numerical simulation aspects of the problem.

From figure 11 we observe that the 'defect' numerics are adept at capturing the well-known traits of Holmboe instability. Linear stability predicts presence of 2 unstable modes of same growth-rate but opposite phase-speed. As expected the numerical solutions do exhibit the beating phenomena between these 2 waves. As visible clearly from figure 11(c)-(d) onwards both the buoyancy and vorticity field has presence of elongated cusp-like structures and one can see both waves before moving past each other. Even at a much later time (figure 11(e)-(f)) the cusps refuse to disappear. If one takes a look at the evolution of disturbance amplitude, $\left\langle|\psi|^{2}\right\rangle^{1 / 2}$, (figure 12) the persistent beating pattern is immediately obvious. One cannot also help but notice in figure 11(e) the inefficient nature of mixing of the buoyancy field by Holmboe waves.

A qualitative comparison of the buoyancy field during Holmboe instability computed using 'defect' numerics with those obtained from experiments and $3 \mathrm{D} \mathrm{DNS}^{3}[9]$ can be seen in figure 13. Though the experiments and numerics are not in the exact parameter regime as the 'defect' simulation, it is reaffirming to observe the present calculations ability to

\footnotetext{
${ }^{3}$ Both experiments and numerics were done for Reynolds number, Re=630, Richardson number being 0.3 and Prandtl number (temperature equivalent to $S c$ ) kept at 700 for experiment and 25 for numerics.
} 


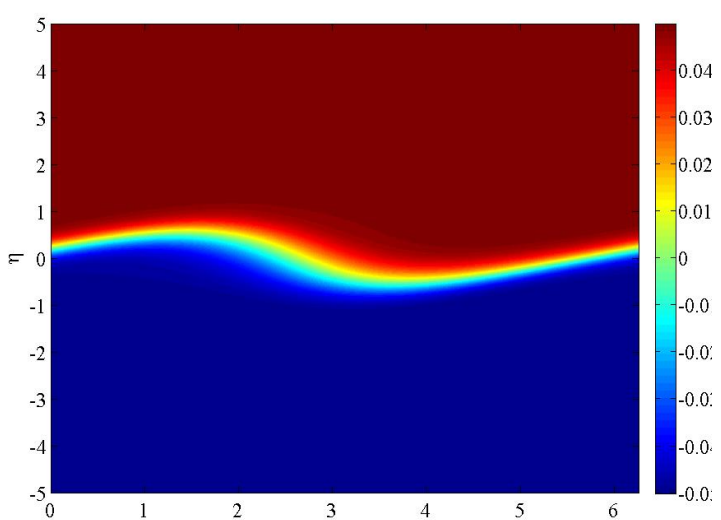

(a) $\mathcal{B}, t=7$

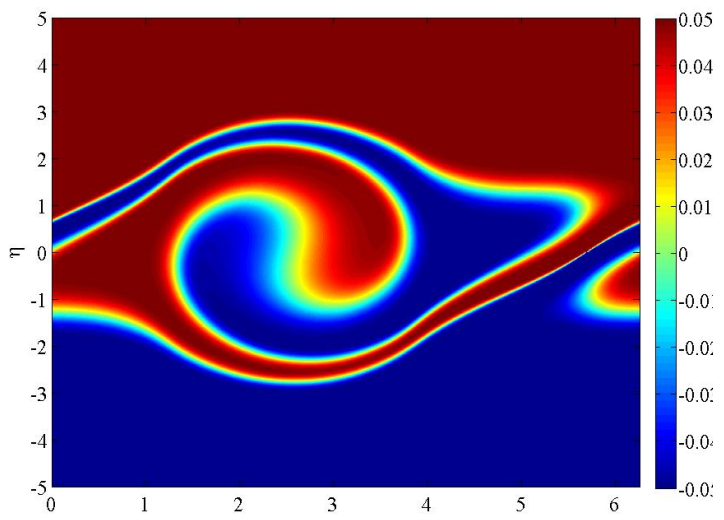

(c) $\mathcal{B}, t=12$

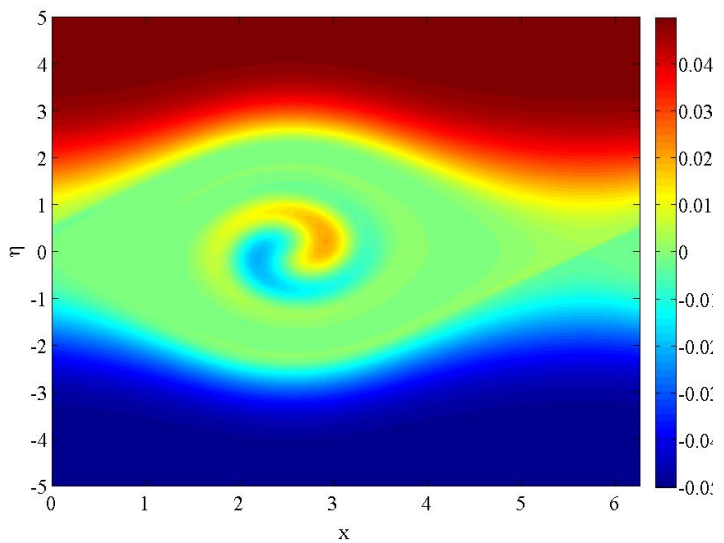

(e) $\mathcal{B}, t=80$

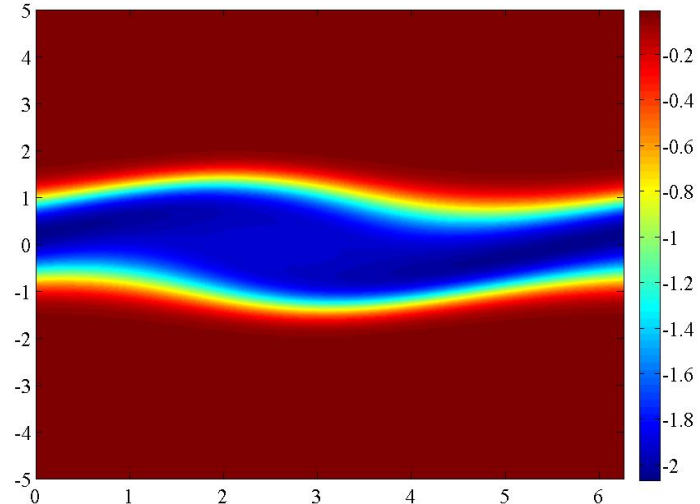

(b) $\mathcal{Z}, t=7$

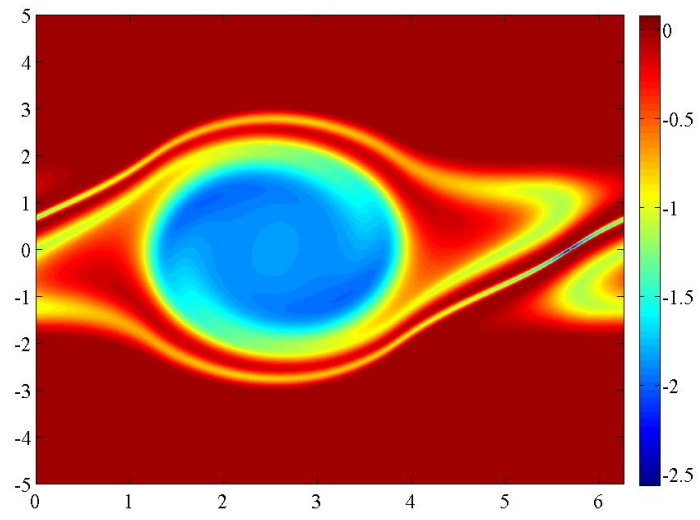

(d) $\mathcal{Z}, t=12$

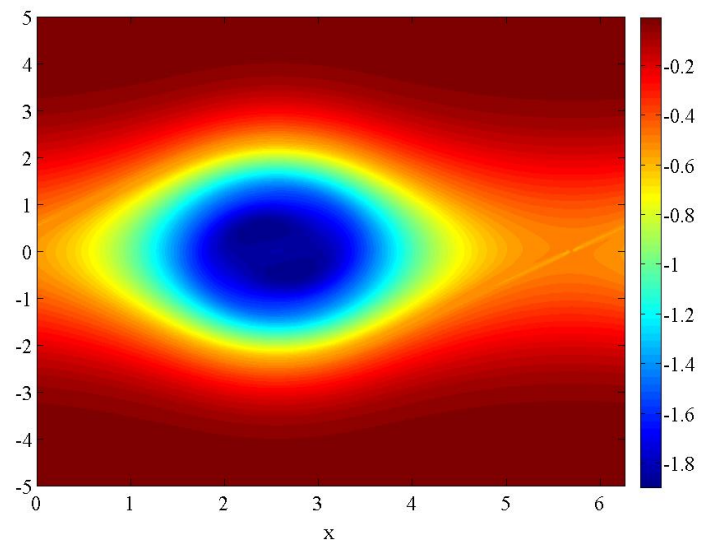

(f) $\mathcal{Z}, t=80$

Figure 9: Vorticity and buoyancy fields at different instants of time for stratified KH instability, $f=2, d=0.1, k=1, \Delta=1 / 3, \eta_{0}=1$. 


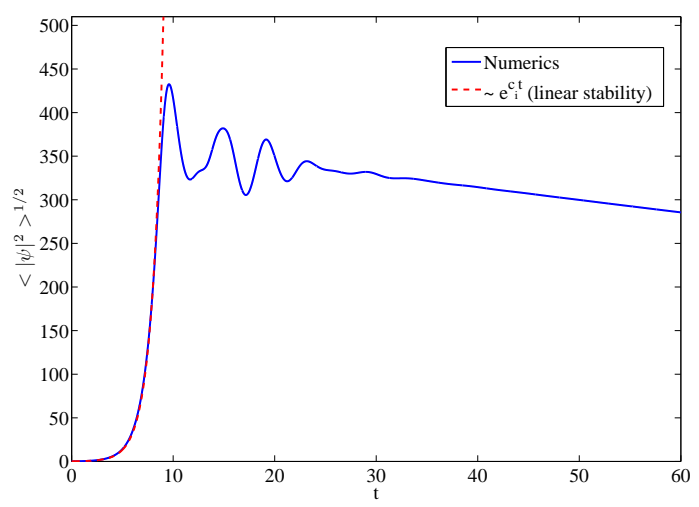

Figure 10: Evolution of disturbance amplitude for stratified KH. $c_{i}=0.9035$

reproduce the principal signature of the shear layer with its cuspy arms as visible in others.

\subsubsection{Holmboe-KH transition $(d=1.5)$}

Will the persistent counter-propagating cusps be present irrespective of how weak the stratification is as long as linear stability predicts them? Simulations for $d=1.5$ as seen in figure 14 answers in negative. $d=1.5$ is away from the critical stratification $\left(d_{\text {crit }}=0.5\right)$ required for transition from $\mathrm{KH}$ to Holmboe as predicted by linear stability. Cusps get formed as expected but as visible in figure 14(a)-(b) the cusps while moving past each other interact nonlinearly and lock each other. Later (figure 14(c)-(d)) these locked waves overturn leading to stirring of the buoyancy field leading towards a KH billow-like stage ((e)-(f)). One can find reference to such nonlinear locking and subsequent overturning/mixing in the experiments of Hogg and Ivey[19]. It is encouraging to see that the reductive model obtained can capture such intricate nonlinear dynamics at very low computational cost.

\subsection{Taylor-Caulfield (TC)}

Instability due to interaction of gravity waves via shear was one of the intriguing aspects of Taylor's (1931) analysis. It belied conventional belief that one needed an vorticity maxima in the flow or unstable stratification to induce instability in stratified shear flow. Moreover the observations of staircase formation in shear flows provides a strong motivation to understand how do multilayer density profiles interacts with shear. Though Taylor introduced the problem as a canonical stratified shear intability it wasn't until through a series of papers by Caulfield and co-workers (linear stability[10], experiments[11] and DNS[23]) that a great deal was known about the interaction of multilayer density profiles with unbounded shear and mixing layer. The present section will be focused on unbounded shear while mixing layer will be reserved for the next section.

The profile chosen for instability corresponds to the one already dealt before in the linear stability study -

$$
N^{2}=\frac{d}{2 \Delta}\left[\operatorname{sech}^{2}\left(\frac{\eta-\eta_{0}}{\Delta}\right)+\operatorname{sech}^{2}\left(\frac{\eta+\eta_{0}}{\Delta}\right)\right]
$$




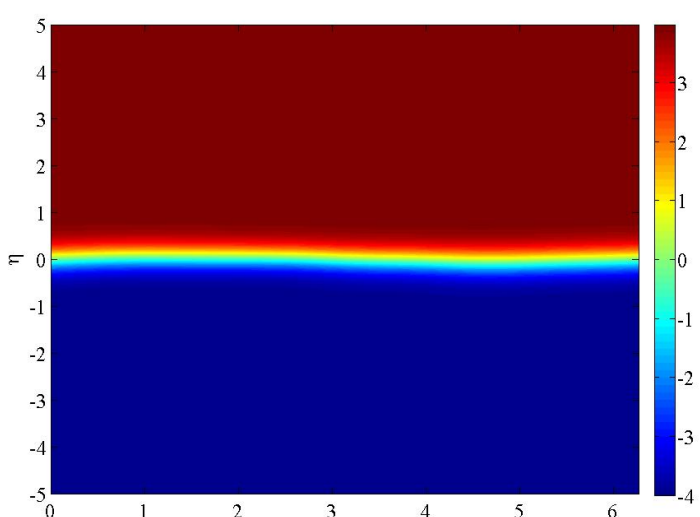

(a) $\mathcal{B}, t=13$

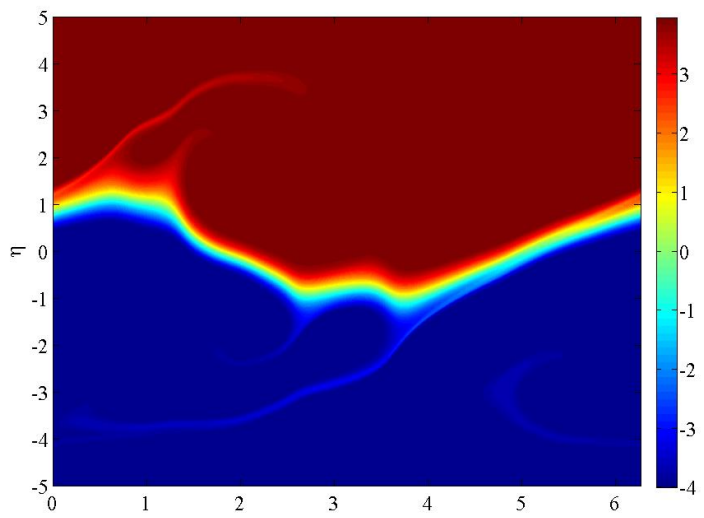

(c) $\mathcal{B}, t=21$

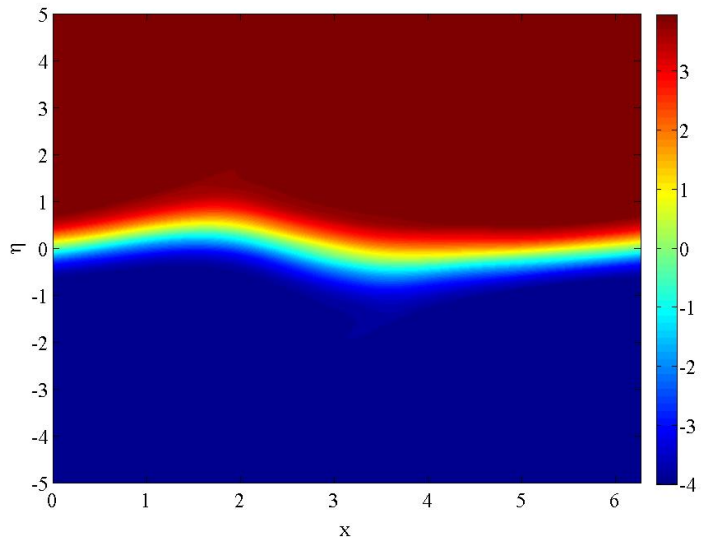

(e) $\mathcal{B}, t=75.5$

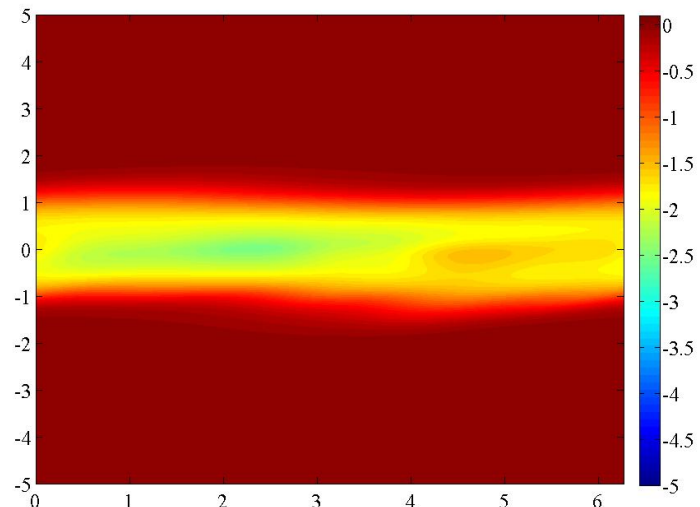

(b) $\mathcal{Z}, t=13$

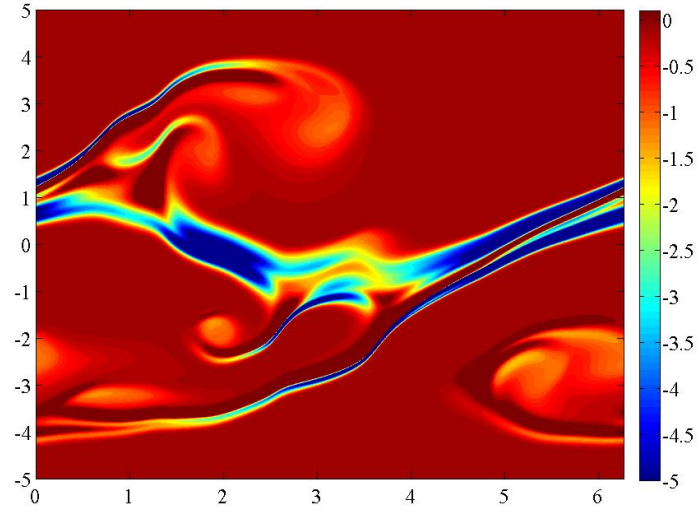

(d) $\mathcal{Z}, t=21$

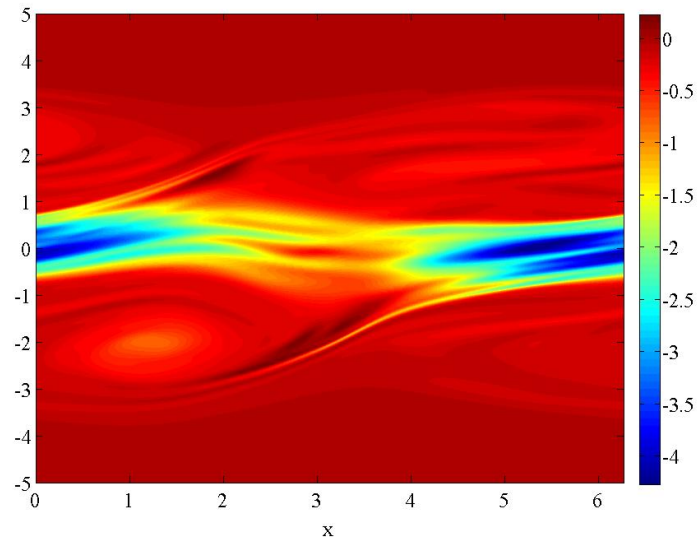

(f) $\mathcal{Z}, t=75.5$

Figure 11: Vorticity and buoyancy fields at different instants of time for pure Holmboe instability, $f=2, d=8, k=1, \Delta=1 / 3, \eta_{0}=1$. 


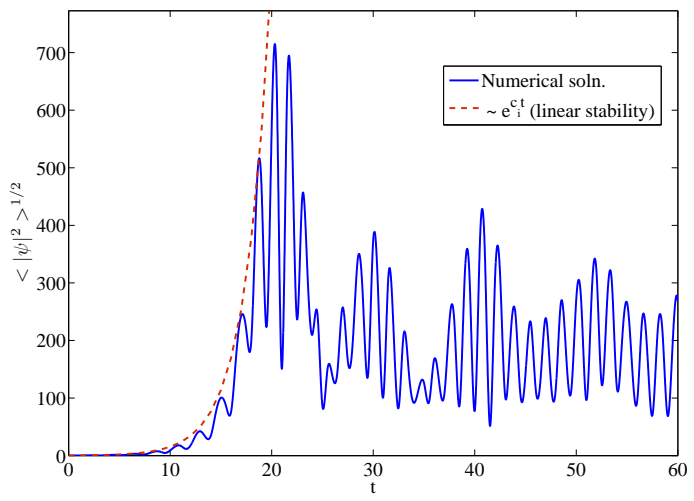

Figure 12: Evolution of disturbance amplitude for pure Holmboe instability. $c_{i}=0.4005$

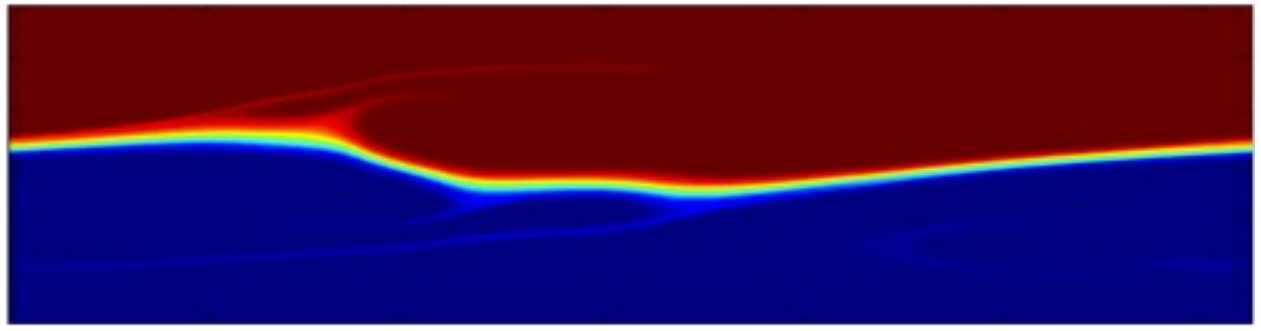

(a) 'Defect' theory

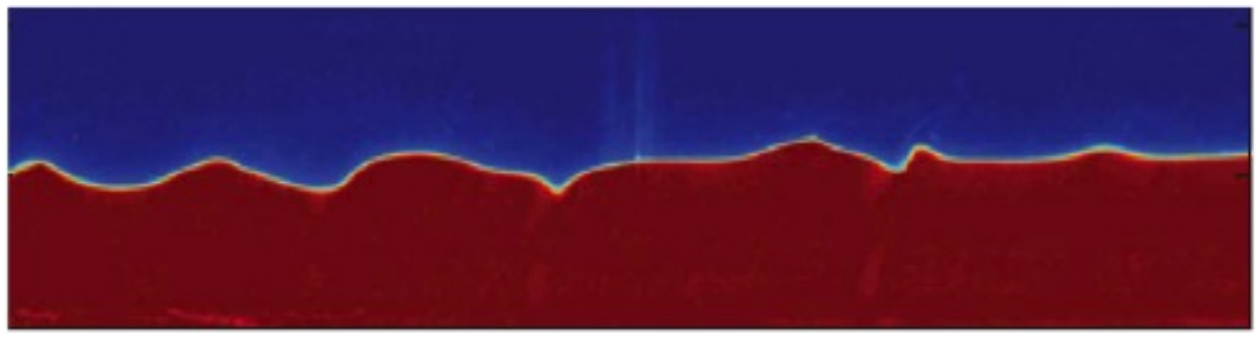

(b) Experimental results

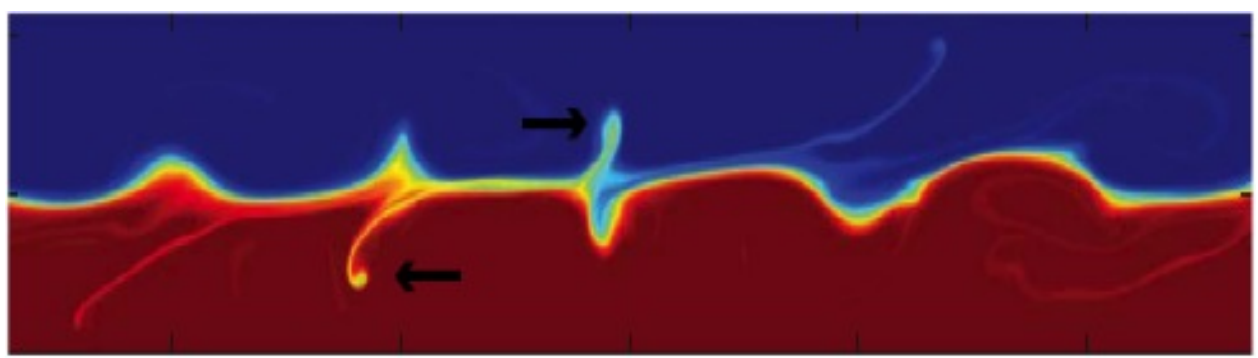

(c) 3D DNS

Figure 13: Qualitative comparison of buoyancy field as obatined from 'defect' theory and that of both experiments and DNS (Carpenter et al.[9]) 


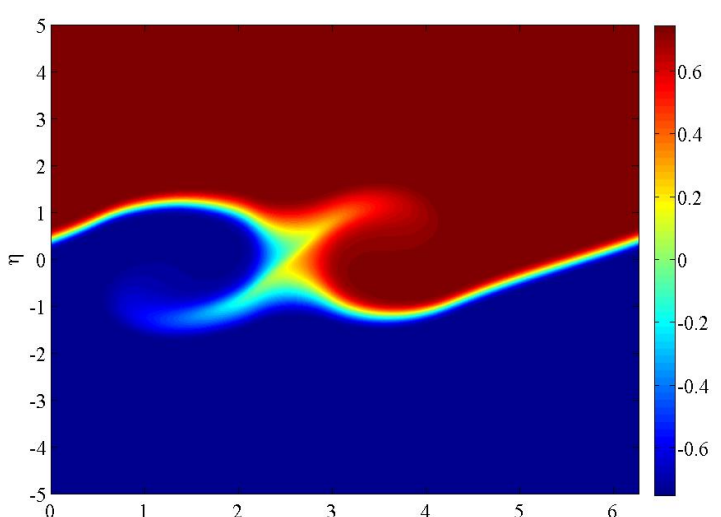

(a) $\mathcal{B}, t=13.5$

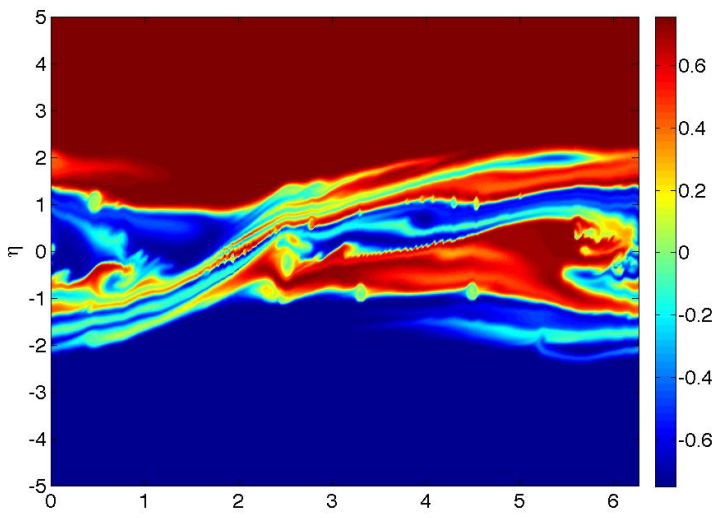

(c) $\mathcal{B}, t=21$

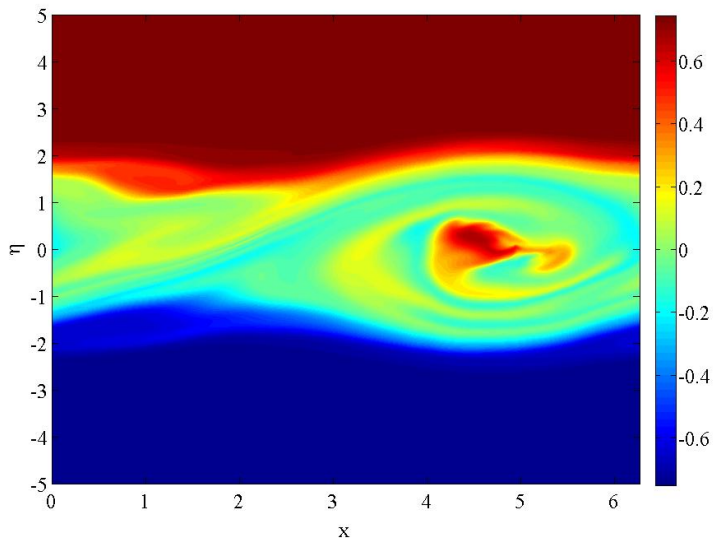

(e) $\mathcal{B}, t=62.5$

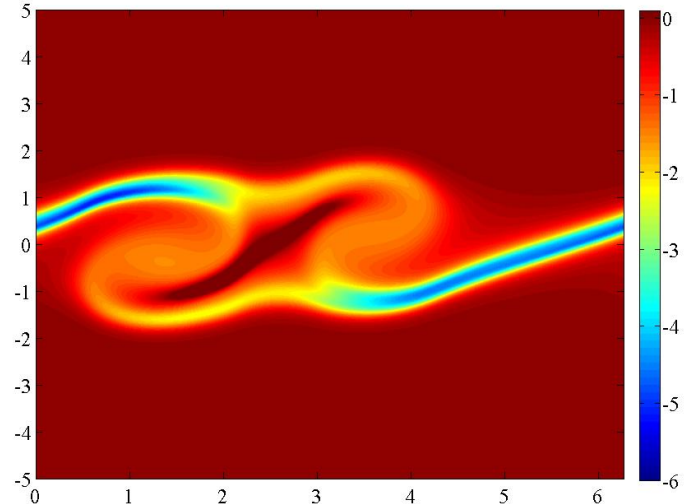

(b) $\mathcal{Z}, t=13.5$

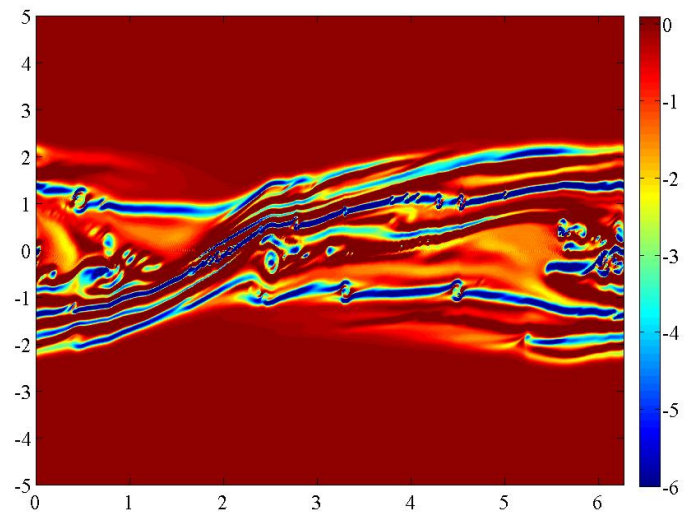

(d) $\mathcal{Z}, t=21$

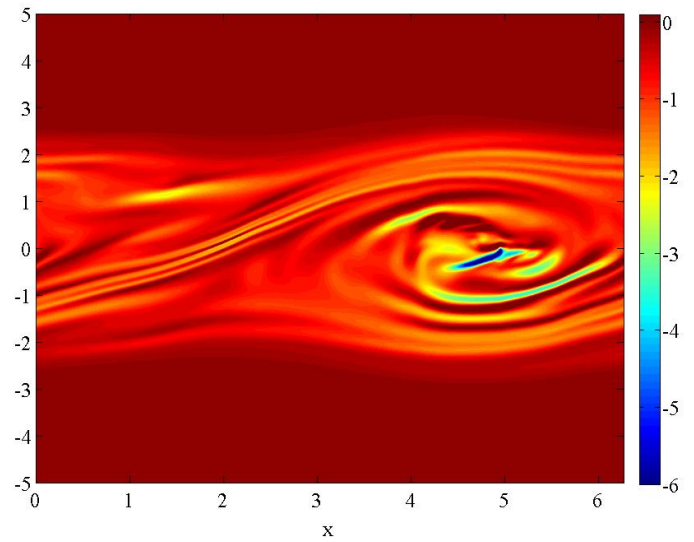

(f) $\mathcal{Z}, t=62.5$

Figure 14: Vorticity and buoyancy fields at different instants of time for Holmboe-KH transition, $f=2, d=1.5, k=1, \Delta=1 / 3, \eta_{0}=1$. 


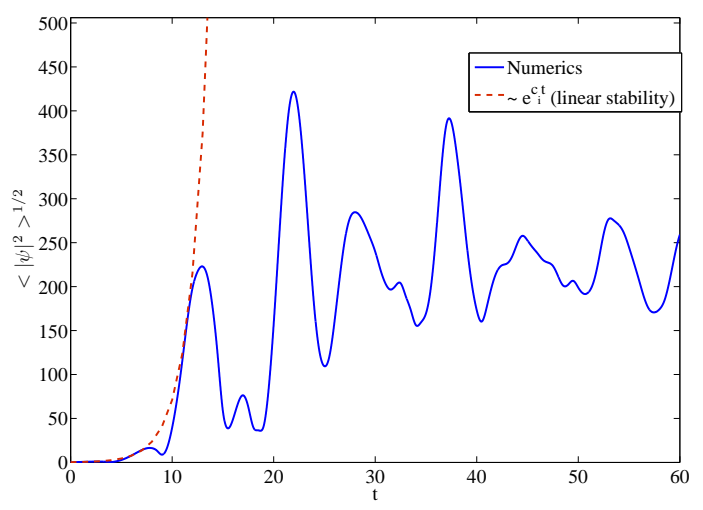

Figure 15: Evolution of disturbance amplitude for Holmboe-KH transition. $c_{i}=0.5537$

for the parameters $d=5, k=1, \Delta=1 / 3, \eta_{0}=2$. The base-state has been seeded with purely buoyancy perturbation and vorticity gets produced purely due to baroclinic effects. During the linear instability stage the baroclinically generated vorticity from the initial conditions gets accumulated at the density interfaces and amplifies (figure 16(a)-(b)). Once the stationary TC waves get nonlinearly saturated the buoyancy forms a billow which too exhibits nutation (figure 16(c), (d) and (f)). The billow formation in TC instability does not involve the extensive overturning as observed in stratified $\mathrm{KH}$ but is a more efficient mixing process than pure Holmboe waves (figure 16(e)).

\subsection{TC-Holmboe transition}

Finally let us consider a 3-layer density profile embedded inside a mixing layer. The extent of the density layer is taken to be smaller than that of the mixing layer (figure 17).

$$
\begin{aligned}
& F=\frac{f}{2}\left[\tanh \left(\frac{\eta-\eta_{2}}{\Delta}\right)-\tanh \left(\frac{\eta+\eta_{2}}{\Delta}\right)\right] \\
& N^{2}=\frac{d}{2 \Delta}\left[\operatorname{sech}^{2}\left(\frac{\eta-\eta_{1}}{\Delta}\right)+\operatorname{sech}^{2}\left(\frac{\eta+\eta_{1}}{\Delta}\right)\right]
\end{aligned}
$$

Simulation has been carried out for $f=2, d=4, k=1, \Delta=1 / 3, \eta_{1}=1, \eta_{2}=2$. Linear stability predicts 3 unstable modes - a faster growing stationary TC mode and a pair of slowly growing propagating Holmboe wave. Figure 18(a)-(b) shows the formation of a billow in the initial stages which starts spewing out cusps along its cusps ((c)-(d)). This cuspy billow persists and once again a typical Holmboe feature - beating amplitude pattern (figure 19) - can be seen. Thus if one were to do an experiment on such a profile, a propagating instability would be observed despite linear stability making predictions for a stationary billow. Exploration of such interesting scenarios with great ease was made possible by virtue of working with the 'defect' equations.

\section{$6 \quad$ Nonlinear stationary states}

For stratified KH and TC instabilities the time-trace for $\left\langle|\psi|^{2}\right\rangle^{1 / 2}$ seems attain a stationary state but for a viscous decay (figure 10 and 16(f)). A qualitative argument for a 


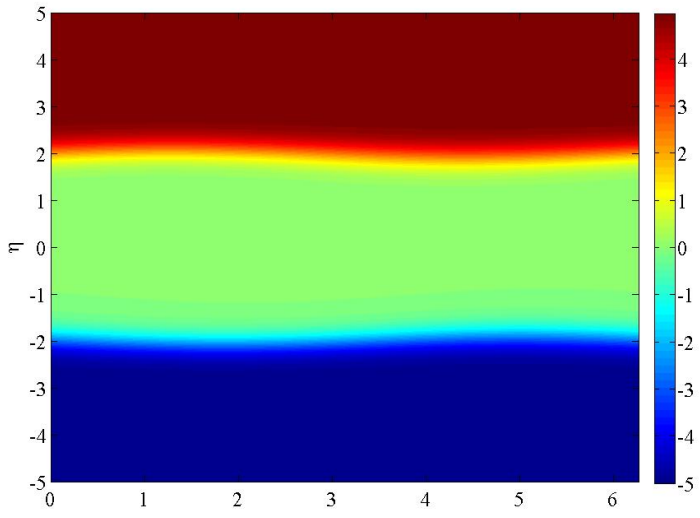

(a) $\mathcal{B}, t=9.5$

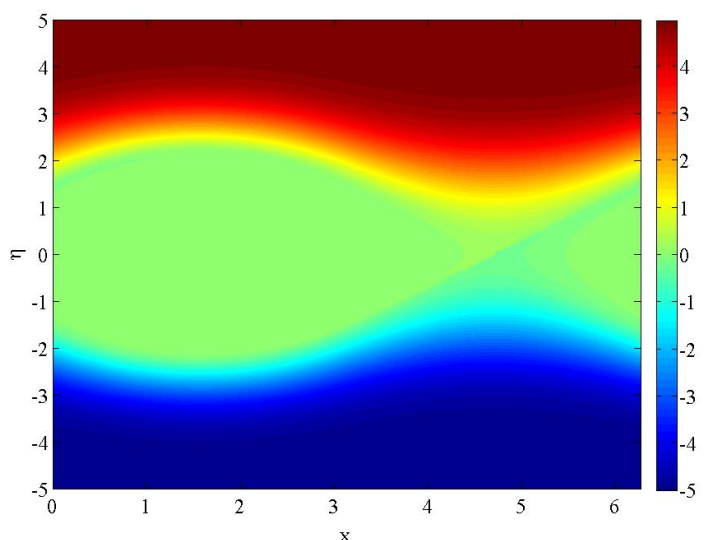

(c) $\mathcal{B}, t=72$

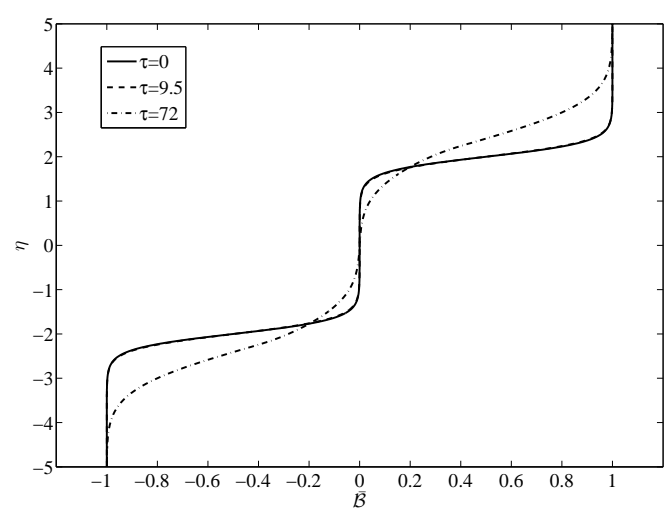

(e) Streamwise averaged instantaneous buoyancy profile $\left(\bar{\bullet}=\int \bullet d x\right)$ at different instants of time

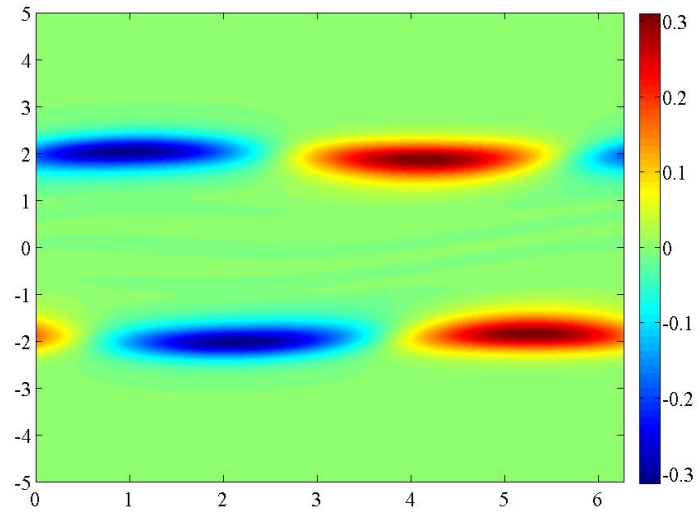

(b) $\mathcal{Z}, t=9.5$

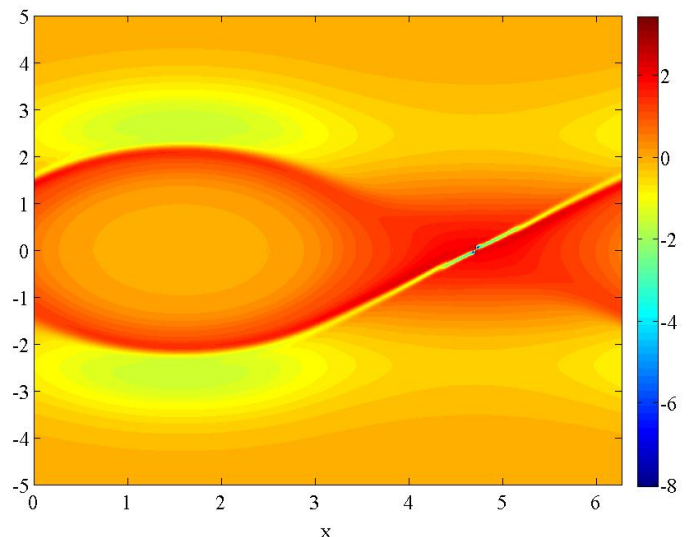

(d) $\mathcal{Z}, t=72$

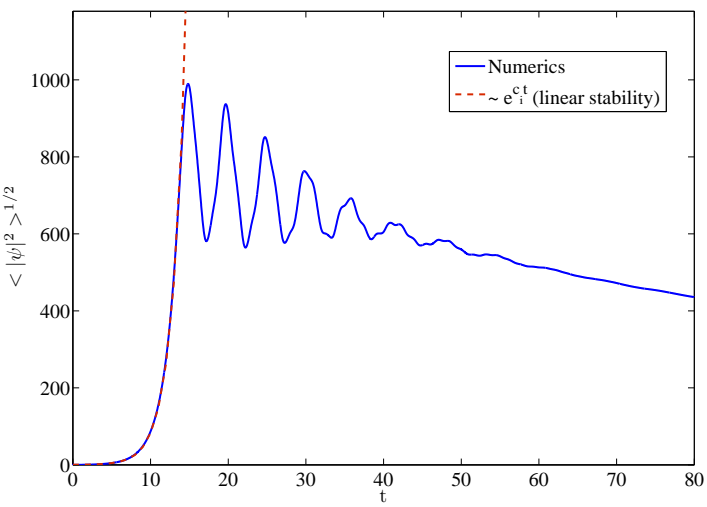

(f) Evolution of disturbance amplitude for TaylorCaulfield instability, $c_{i}=0.5804$

Figure 16: Vorticity and buoyancy fields at different instants of time for Taylor-Caulfield instability, $d=5, k=1, \Delta=1 / 3, \eta_{0}=2$. 


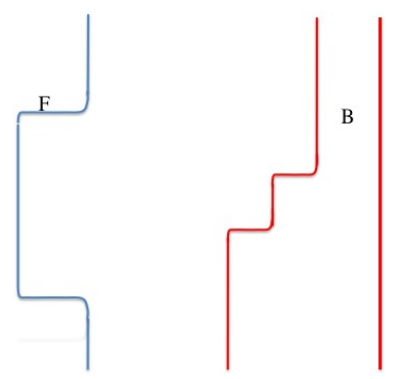

Figure 17: Density layer embedded in a mixing layer (TC-Holmboe transition)

stationary state also receives support when one observes the buoyancy and vorticity plots at the final time computationally attained (figure 9(f) and 16(c)). A time evolution of the plots show that they are remarkably static. Can one provide quantitative confirmations to such guesses?

Let us consider the vorticity-buoyancy defect equation under inviscid, steady ${ }^{4}$ assumptions,

$$
\begin{aligned}
& \eta \mathcal{Z}_{x}+\Phi_{x} \mathcal{Z}_{\eta}=\mathcal{B}_{x} \\
& \eta \mathcal{B}_{x}+\Phi_{x} \mathcal{B}_{\eta}=0
\end{aligned}
$$

The exact solutions of the above equations are,

$$
\begin{aligned}
\mathcal{B} & =\mathcal{P}(\mathcal{E}), \\
\mathcal{Z} & =\mathcal{Q}(\mathcal{E})+\eta \mathcal{P}^{\prime}(\mathcal{E})=\mathcal{Q}(\mathcal{E})-\mathcal{B}_{\eta}, \\
\text { where, } \quad \mathcal{E} & =-\frac{(\eta-c)^{2}}{2}+\Phi
\end{aligned}
$$

$c$ being the wave-speed of the state. $\mathcal{P}$ and $\mathcal{Q}$ are arbitrary functionals of the total streamfunction, $\mathcal{E}$. Thus any nonlinear stationary state needs to obey the above functional relationship. We go ahead and try applying this diagnostic on both stratified KH and TC instabilities ( $c=0$ in both cases).

At every instant of time besides $\mathcal{Z}$ and $\mathcal{B}$ the total streamfunction, $\mathcal{E}$, is also computed. Thus at every grid point $\left(x_{i}, \eta_{k}\right)$ values of $\mathcal{B}, \mathcal{Z} \& \mathcal{E}$ are known. We construct pair of points - $\left(\mathcal{E}_{i k}, \mathcal{B}_{i k}\right)$ and $\left(\mathcal{E}_{i k},\left(\mathcal{Z}+\mathcal{B}_{\eta}\right)_{i k}\right)$. Next all the $(\mathcal{E}, \mathcal{B})\left[\left(\mathcal{E},\left(\mathcal{Z}+\mathcal{B}_{\eta}\right)\right)\right]$ pairs are plotted in the $\mathcal{B}-\mathcal{E}\left[\left(\mathcal{Z}+\mathcal{B}_{\eta}\right)-\mathcal{E}\right]$ planes. If there indeed exists a functional relationship then the scatter of points should collapse on to a curve. That would be a confirmation that the obtained solution is indeed approaching a nonlinear stationary state.

First it is applied on stratified KH (section 5.3.1). As visible from figure 20 the initial functional dependence gets broken and there is a prominent scatter during an intermediate time - an instant of strong instabilities (figure 20(c)-(d)) - before approaching towards a curve (figure 20(e)-(f)). The near horizontal arm(s) in both the buoyancy and vorticity plots corresponds to the uniform region outside the shear layer. From the expression of the

\footnotetext{
${ }^{4}$ If the state is moving with a constant wave-speed $c$ then a co-moving reference frame is considered. Though not presented here such scenario was observed with simulations for asymmetric stratified shear layer. One of the Holmboe waves is then preferred and the system translates with a constant speed.
} 


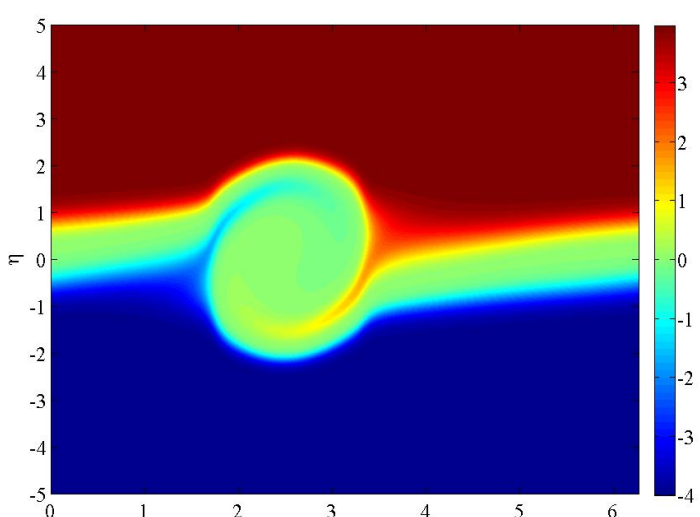

(a) $\mathcal{B}, t=10.5$

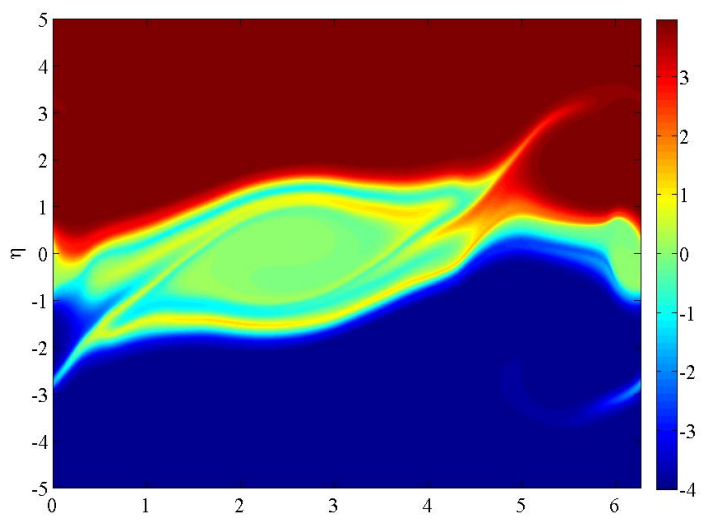

(c) $\mathcal{B}, t=14.5$

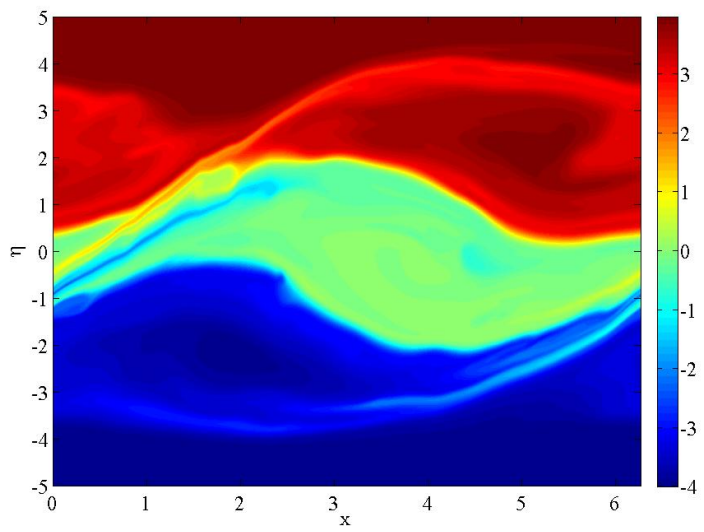

(e) $\mathcal{B}, t=65.5$

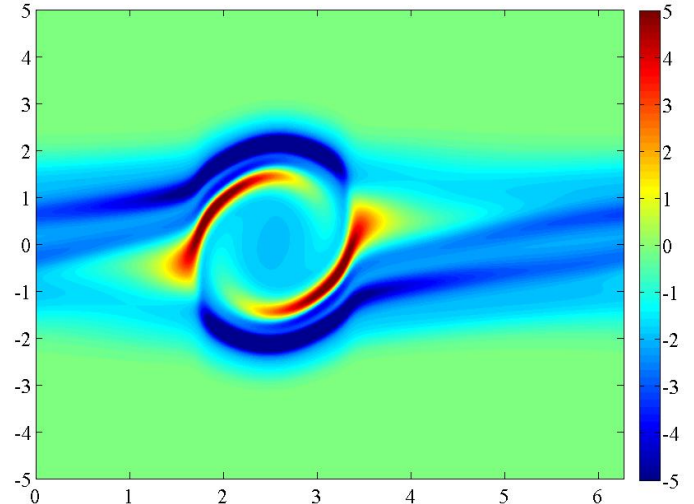

(b) $\mathcal{Z}, t=10.5$

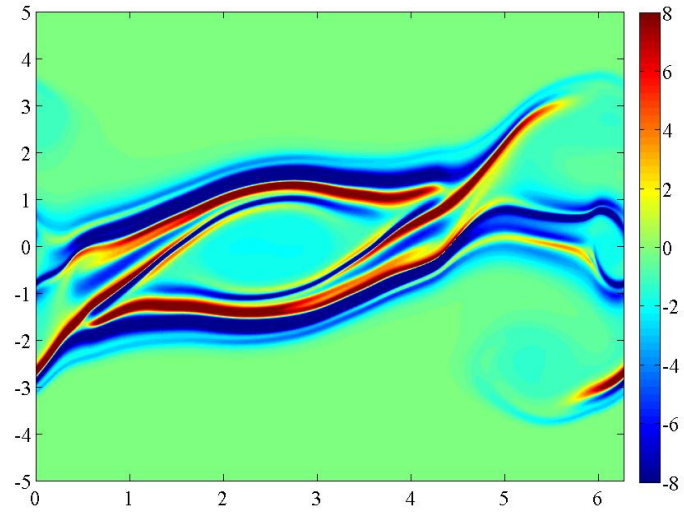

(d) $\mathcal{Z}, t=14.5$

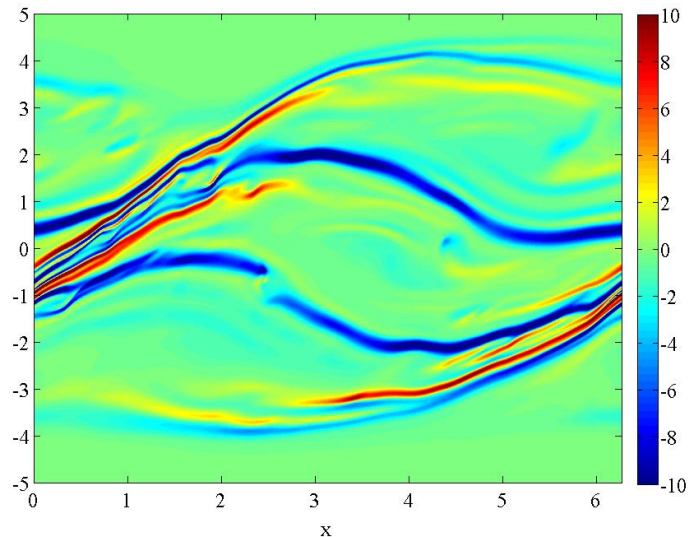

(f) $\mathcal{Z}, t=65.5$

Figure 18: Vorticity and buoyancy fields at different instants of time for TC-Holmboe transition, $f=2, d=4, k=1, \Delta=1 / 3, \eta_{1}=1, \eta_{2}=2$. 


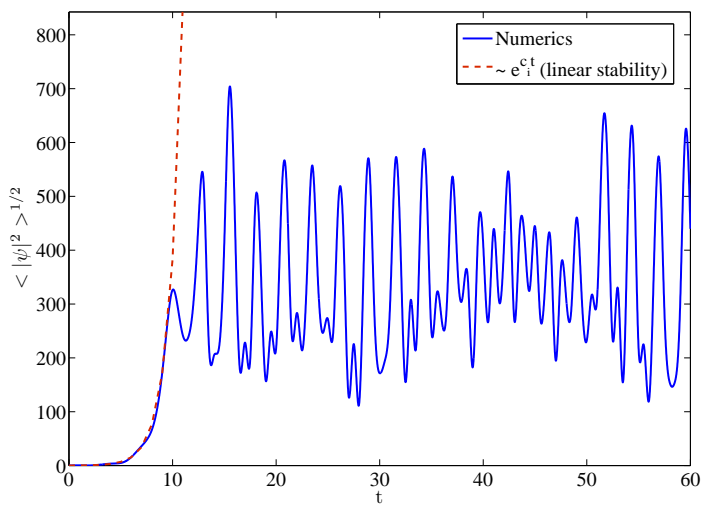

Figure 19: Evolution of disturbance amplitude for TC-Holmboe transition. $c_{i}=0.807$

total stream-function, $\mathcal{E}$, one can see why it should be large and negative outside the shearlayer. The vertical region, which hosts most of the dynamics corresponds to the defect. Also the locations from where points seem to spew out vertically, represent the hyperbolic points in the flow. In the last available buoyancy plot, figure 20(e), the bulbous head is representative of the cat's eye region of the flow. As visible from figure $9(\mathrm{e})$ the $\mathcal{B}$ field has not homogenised completely and there is signature of a dipole-like structure in the cat's eye, which contributes to the bulge.

As seen from figure 21 similar observations are also true for a Taylor-Caulfield case (section $5.4)$.

Having identified the nonlinear structures the nonlinear stability of the stationary states can be investigated. If we look for stationary points of the energy under the constraints that it has to obey - the conserved quantities determined previously (section 3) - we have the following 'Hamiltonian' (we defer from calling the system Hamiltonian since we have not checked for the Poisson bracket structure for it),

$$
\mathcal{H}=<\frac{\eta^{2}-\Phi}{2} \mathcal{Z}-\eta \mathcal{B}-c \eta \mathcal{Z}+F(\mathcal{B})+\frac{c^{2}}{2} \mathcal{Z}+\mathcal{Z} G(\mathcal{B})>
$$

where $<$. $>$ denotes average over entire space as defined before. The stationary points of the system are,

$$
\begin{aligned}
& \frac{\delta \mathcal{H}}{\delta \mathcal{Z}}=0, \Rightarrow \mathcal{E}=G(\mathcal{B}), \Rightarrow \mathcal{B}=\mathcal{P}(\mathcal{E}) \\
& \frac{\delta \mathcal{H}}{\delta \mathcal{B}}=0, \Rightarrow \mathcal{Z}=\frac{\eta-F^{\prime}(\mathcal{B})}{G^{\prime}(\mathcal{B})}=\mathcal{Q}(\mathcal{E})+\eta \mathcal{P}^{\prime}(\mathcal{E})
\end{aligned}
$$

confirming what was mentioned in the beginning of this section. Here $\delta \mathcal{H} / \delta \mathcal{Z}$ is the functional derivative of the functional $\mathcal{H}$. To check stability properties we need to check the second variation $\mathcal{H}$.

$$
\begin{aligned}
\delta^{2} \mathcal{H}= & <-\delta \psi \delta Z+\left\{\mathcal{F}^{\prime \prime}(B)+Z \mathcal{G}^{\prime \prime}(B)\right\}(\delta B)^{2}+2 \mathcal{G}^{\prime}(B) \delta Z \delta B> \\
= & <\left\{\delta Z+\mathcal{G}^{\prime}(B) \delta B\right\}^{2}+\left\{-\delta \psi \delta Z-(\delta Z)^{2}\right\}+\left\{\mathcal{F}^{\prime \prime}(B)+Z \mathcal{G}^{\prime \prime}(B)-\left(\mathcal{G}^{\prime}(B)\right)^{2}\right\}(\delta B)^{2}>
\end{aligned}
$$




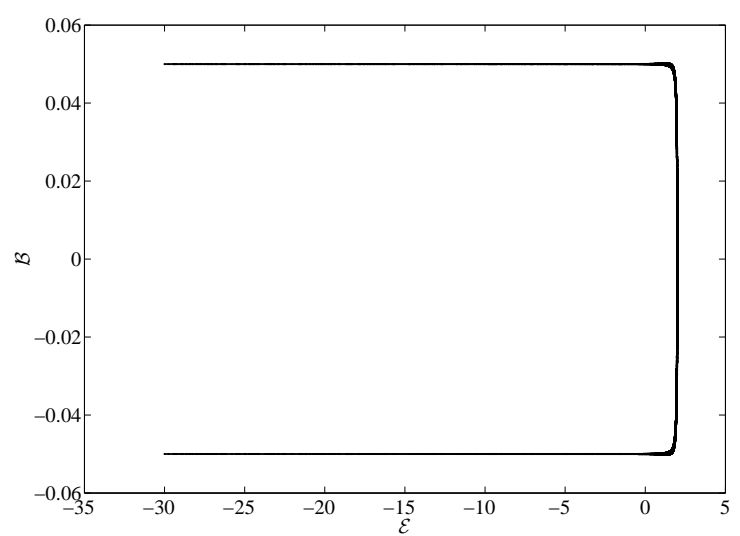

(a) $t=0$

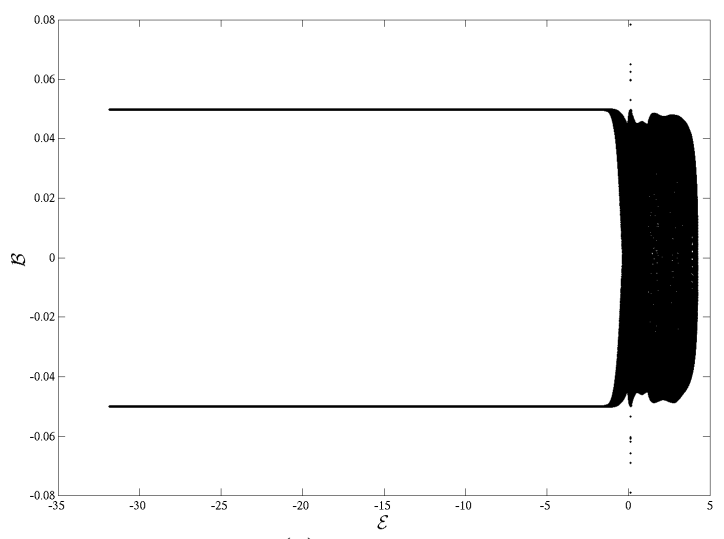

(c) $t=14.5$

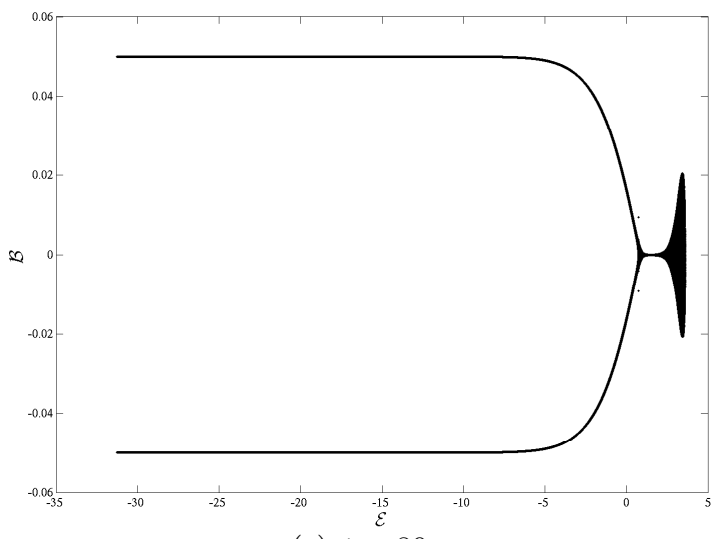

(e) $t=80$

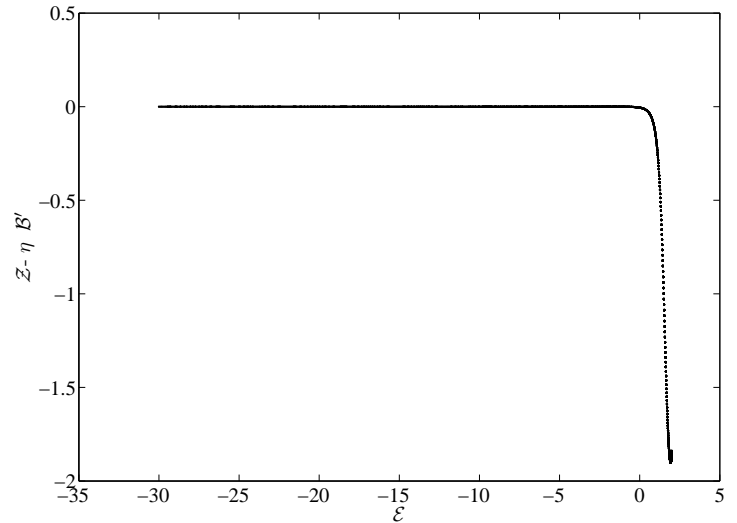

(b) $t=0$

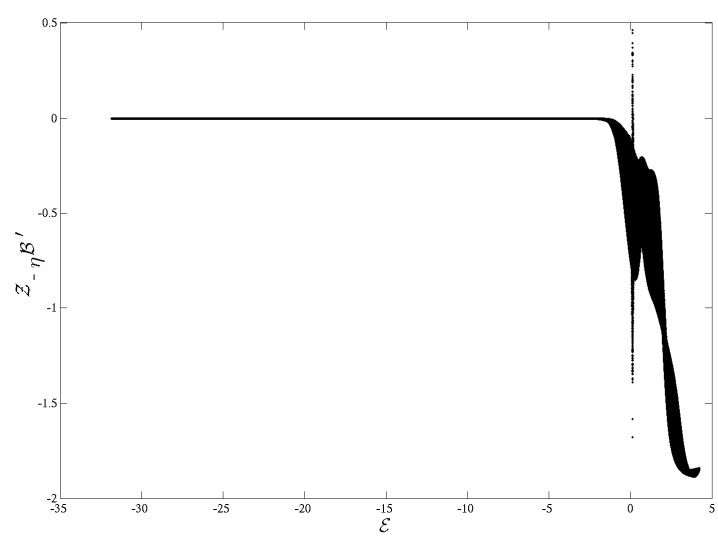

(d) $t=14.5$

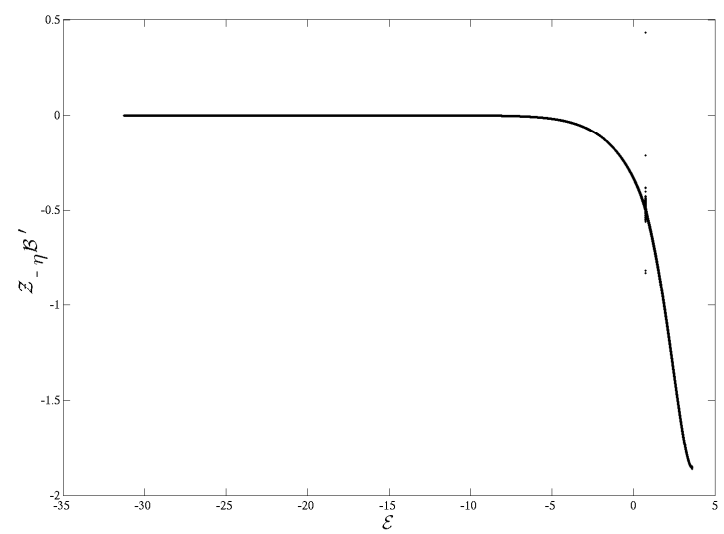

(f) $t=80$

Figure 20: Nonlinear stationary state diagnostics for stratified KH instability. Time evolution of the dependence of $\mathcal{B}$ with $\mathcal{E}$ is shown in (a), (c) and (e) and that of $\mathcal{Z}-\eta \mathcal{B}^{\prime}$ with $\mathcal{E}$ in (b), (d) and (f). 

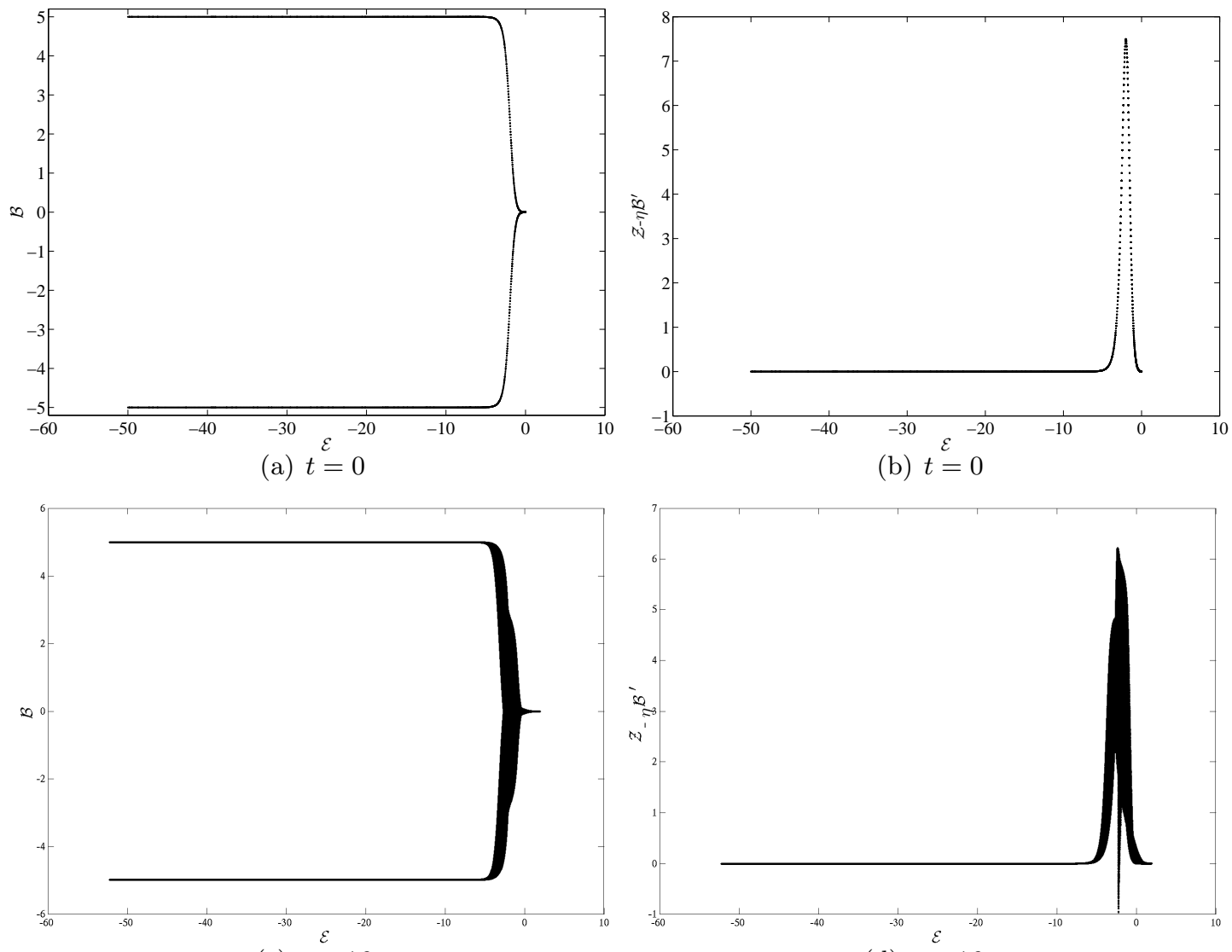

(c) $t=16$

(d) $t=16$
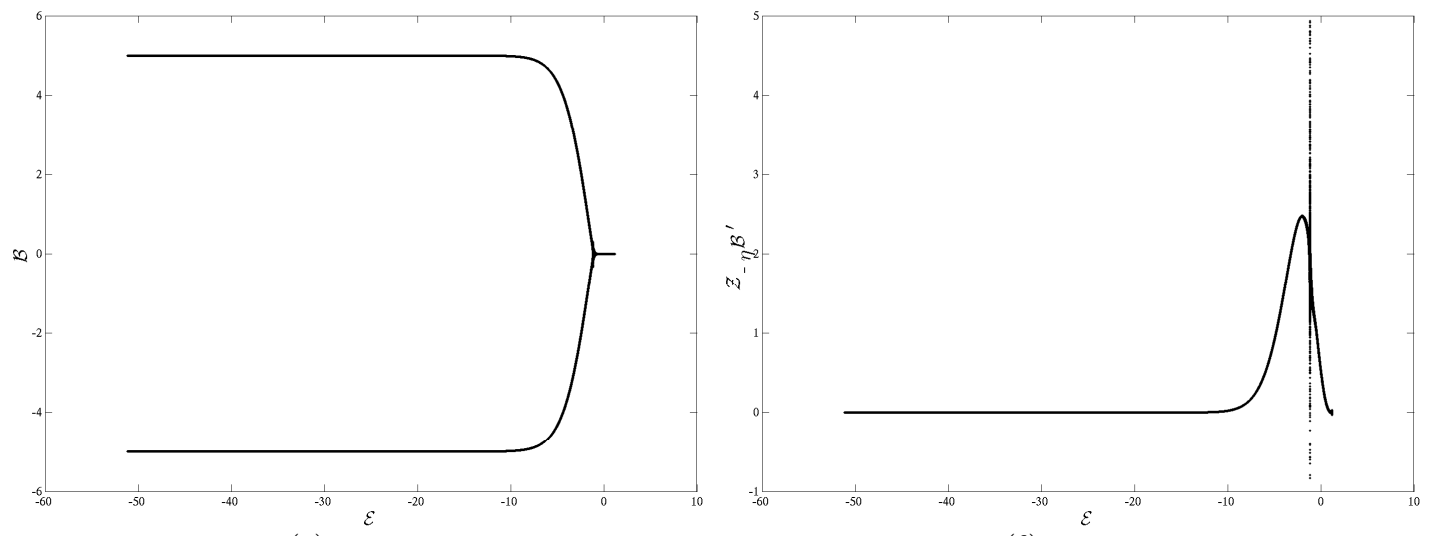

(e) $t=80$

(f) $t=80$

Figure 21: Nonlinear stationary state diagnostics for Taylor-Caulfield instability. Figure description same as figure 20 . 
In this form a comment cannot be made about nonlinear stability as $\delta^{2} \mathcal{H}$ is indefinite. But if $\int d x d \eta\left\{-\delta \psi \delta Z-(\delta Z)^{2}\right\}>0$ is positive definite then the condition for stability will be -

$$
\left\{\mathcal{F}^{\prime \prime}(B)+Z \mathcal{G}^{\prime \prime}(B)-\left(\mathcal{G}^{\prime}(B)\right)^{2}\right\}>0
$$

Now the question is under what cases is the assumption, $\int d x d \eta\left\{-\delta \psi \delta Z-(\delta Z)^{2}\right\}>0$, true. In 'defect' theory, for an unbounded domain, $\psi$ and $Z$ can be connected in Fourier space as,

$$
\hat{\psi}(k, t)=-\frac{1}{2 k} \int \hat{Z}(k, \eta, t) d \eta
$$

Thus using Parseval's theorem we have

$$
\begin{aligned}
& \int d x d \eta\left\{-\delta \psi \delta Z-(\delta Z)^{2}\right\}>0 \\
\Rightarrow & \int d k d \eta\left\{-\delta \hat{\psi} \delta \hat{Z}^{*}-|\delta \hat{Z}|^{2}\right\}>0 \\
\Rightarrow & \int d k\left\{\frac{1}{2 k}\left|\int d \eta \delta \hat{Z}\right|^{2}-\int d \eta|\delta \hat{Z}|^{2}\right\}>0
\end{aligned}
$$

Presently a detailed study in the above aspects is lacking but it is hypothesized that there would exist a band of wavenumbers in which a stratified shear flow, modelled by 'defect' theory, will be stable. For higher wavenumbers one can always find instability.

\section{Conclusion}

Exploiting the sharp variations present in stratified shear layers which occurs over small regions, 'defect' vorticity-buoyancy equations have been derived. This equation maintains all the necessary physics - unsteadiness, nonlinearity and viscosity. Linear stability calculations using the 'defect' equations becomes significantly convenient with the present equations. But the big strength of 'defect' equations was found to be in doing nonlinear calculations for various stratified shear layer profiles. Unlike conventional numerics done for Bousinessq Navier-Stokes equations the ones for 'defect' equations can be done with minimum computational effort.

We have been able to explore some interesting cases concerning nonlinear evolution of stratified shear layers. Though a great deal is known about the stratified KH instability, we were able to address some intriguing questions regarding the transition from $\mathrm{KH}$ instability to Holmboe waves and also whether a Holmboe instability persists nonlinearly with decreasing stratification. Very little is known about the nonlinear dynamics of Taylor-Caulfield instabilities. An effort has been made to probe TC instability in greater detail while working with 'defect' equations. Coexistence of a TC billow with a Holmboe cusp was also observed while studying density layers embedded in mixing layer.

A diagnostics was used with success to identify nonlinear stationary structures in stratified KH and TC instabilities. Motivated by visual arguments a check was done on the buoyancy, vorticity and total streamfunction field to determine if the flow is attaining an invariant form. Efforts to perform nonlinear stability have so far been inconclusive. 


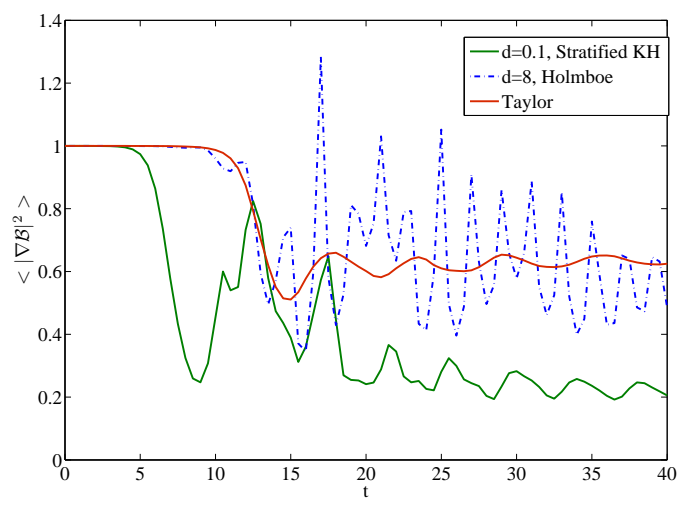

Figure 22: Time evolution of the scalar variance

Being built for flow with sharp interfaces or local distortions, the 'defect' theory has immense potential for stratified shear layer studies. TC instabilities is far from being well-understood and it will be very interesting to explore a wide range of parameter space armed with 'defect' theory. An aspect completely overlooked in the present analysis is the estimation of mixing in the considered flows. Since $S c \rightarrow \infty$ was the domain of interest an estimate of stirring was made based on the scalar variance, $\left\langle|\nabla \mathcal{B}|^{2}>\right.$. A low value of $\left\langle|\nabla \mathcal{B}|^{2}\right\rangle$ denote homegenisation of the scalar field. Figure 22 does indeed highlight an already discussed aspect, based on visual grounds, of mixing/stirring efficiency being maximum for stratified $\mathrm{KH}$, followed by TC and then Holmboe. A more careful analysis needs to be done in this regard and comparisons made with existing DNS and experimental results.

The 'defect' equations are amenable to analytical approaches more easily than the governing equations from which they have been derived. We haven't really used this to obtain the solutions in the nonlinear states in presence of dissipation. A quasi-steady critical layer theory approach is being investigated under the framework of 'defect' equations to answer the asymptotic form of the nonlinear stationary states. Finally a potential future work which has been left out from the present study is the study on the viscous spectrum, issue of quasimodes and transient growth.

\section{Acknowledgments}

I would like to thank Neil and Colm, without whose patience and constant encouragement this work would not have been possible; Phil Morrison for his help with calculating BGK modes and George for teaching softball lessons to a cricket fanatic. Finally thanks to all the fellows, the staff and the visitors to Walsh Cottage for a wonderful summer.

\section{References}

[1] G. K. Batchelor, Introduction to fluid dynamics, Cambridge University Press, 1967.

[2] N. J. Balmforth and P. J. Morrison, Singular eigenfunctions for shearing fluids I, (1995), Institute for Fusion studies, University of Texas, Austin, Report No. 692. 
[3] N. J. Balmforth, D. del-Castillo-Negrete, and W. R. Young, Dynamics of vorticity defects in shear. J. Fluid Mech., 333 (1997), pp. 197-230.

[4] N. J. Balmforth, and R. V. Craster, Dynamics of defects in visco-elastic shear. J. Non-Newtonian Fluids, 72 (1997), pp. 281-304.

[5] N. J. Balmforth, and W. R. Young, Long-Wave Instability in Marginally Stable Shear Flows, Phys. Rev. Lett., 79 (1997), pp. 4155-4158.

[6] N. J. Balmforth, Stability of vorticity defects in viscous shear, J. Fluid Mech., 357 (1998), pp. 199-224.

[7] N. J. Balmforth, S. G. Llewellyn Smith and W. R. Young, Disturbing vortices, J. Fluid Mech., 426 (2001), pp. 95-133.

[8] J. R. Carpenter, N. J. Balmforth, and G. A. Lawrence, Identifying unstable modes in stratified shear layers, Phys. Fluids, 22 (2010), p. 054104.

[9] J. R. Carpenter, E. W. Tedford, M. Rahmani and G. A. Lawrence, Holmboe wave fields in simulation and experiment, J. Fluid Mech., 648 (2010), pp. 205-223.

[10] C. P. Caulfield, Multiple linear instability of layered stratified shear flow, J. Fluid Mech., 258 (1994), pp. 255-285.

[11] C. P. Caulfield, W. R. Peltier, S. Yoshida and M. Ohtani, An experimental investigation of the instability of a shear flow with multilayered density stratification, Phys. Fluids, 7 (1995), p. 3028.

[12] C. P. Caulfield and W. R. Peltier, The anatomy of the mixing transition in homogenous and stratified free shear layers, J. Fluid Mech., 413 (2000), pp. 1-47.

[13] C. Z. Cheng and G. Knorr, The integration of the Vlasov equation in configuration space, J. Comput. Phys., 22 (1976), pp. 330-351.

[14] S. M. Churilov and I. G. Shukhman, The nonlinear critical layer resulting from the spatial or temporal evolution of weakly unstable disturbances in shear flows, J. Fluid Mech., 318 (1996), pp. 189-221.

[15] D. del-Castillo-Negrete, W. R. Young and N. J. Balmforth, Vorticity dynamics in shear flow, Proc. 1995 Summer Study Program on Geophysical Fluid Dynamics (ed. R. Salmon). Woods Hole Oceanographic Institution Technical Report.

[16] A. E. Gill, A mechanism for instability of plane Couette flow and of Poiseuille flow in a pipe, J. Fluid Mech., 21 (1965), pp. 503-511.

[17] S. Goldstein, On the stability of superposed streams of fluids of different densities, Proc. R. Soc. London, Ser. A, 132 (1931), pp. 524-548.

[18] H. Helmholtz, On discontinuous movements of fluids, Philos. Mag., 36 (1868), pp. 337-347. 
[19] A. McC. Hogg and G. N. Ivey, The KelvinHelmholtz to Holmboe instability transition in stratified exchange flows, J. Fluid Mech., 477 (2003), pp. 339-362.

[20] J. Holmboe, On the behavior of symmetric waves in stratified shear layers, Geofysiske Publikasjone, 24 (1962), pp. 67-113.

[21] Lord Kelvin, Hydrokinetic solutions and observations, Philos. Mag., 42 (1871), pp. 362-377.

[22] G. A. Lawrence, F. K. Browand and L. G. Redekopp, The stability of a sheared density interface, Phys. Fluids A, 3 (1991), p. 2360.

[23] V. Lee and C. P. Caufield, Nonlinear evolution of a layered stratified shear flow, Dyn. Atmos. Oceans, 34 (2001), pp. 103-124.

[24] J. Lerner and E. Knobloch, The long-wave instability of a defect in a uniform parallel shear, J. Fluid Mech., 189 (1988), pp. 117-134.

[25] R. J. LeVeque, Finite volume methods for hyperbolic problems, Cambridge University Press, 2002.

[26] O. Pouliquen, J. M. Chomaz and P. Huerre, Propagating Holmboe waves at the interface between two immiscible fluids, J. Fluid Mech., 266 (1994), pp. 277-302.

[27] J. W. S. Rayleigh, On the stability, or instability, of certain fluid motions, Proc. London Math. Soc., 12 (1880), pp. 57-72.

[28] W. D. Smyth, G. P. Klaassen and W. R. Peltier, Finite amplitude Holmboe waves, Geophys. Astrophys. Fluid Dyn., 43 (1988), pp. 181-222.

[29] W. D. Smyth and W. R. Peltier, Instability and transition in finite-amplitude KelvinHelmholtz and Holmboe waves, J. Fluid Mech., 228 (1991), pp. 387-415.

[30] W. D. Smyth and K. B. Winters, Turbulence and mixing in Holmboe Waves, J. Phys. Oceanogr., 33 (2003), pp. 694-711.

[31] K. Stewartson, The evolution of the critical layer of a Rossby wave, Geophys. Astrophys. Fluid Dyn., 9 (1978), pp. 185-200.

[32] G. I. Taylor, Effect of variation in density on the stability of superposed streams of fluid, Proc. R. Soc. London, Ser. A, 132 (1931), pp. 499-523.

[33] T. Warn and H. Warn, The evolution of a nonlinear critical level, Stud. Appl. Maths, 59 (1978), pp. 37-71. 
50272-101

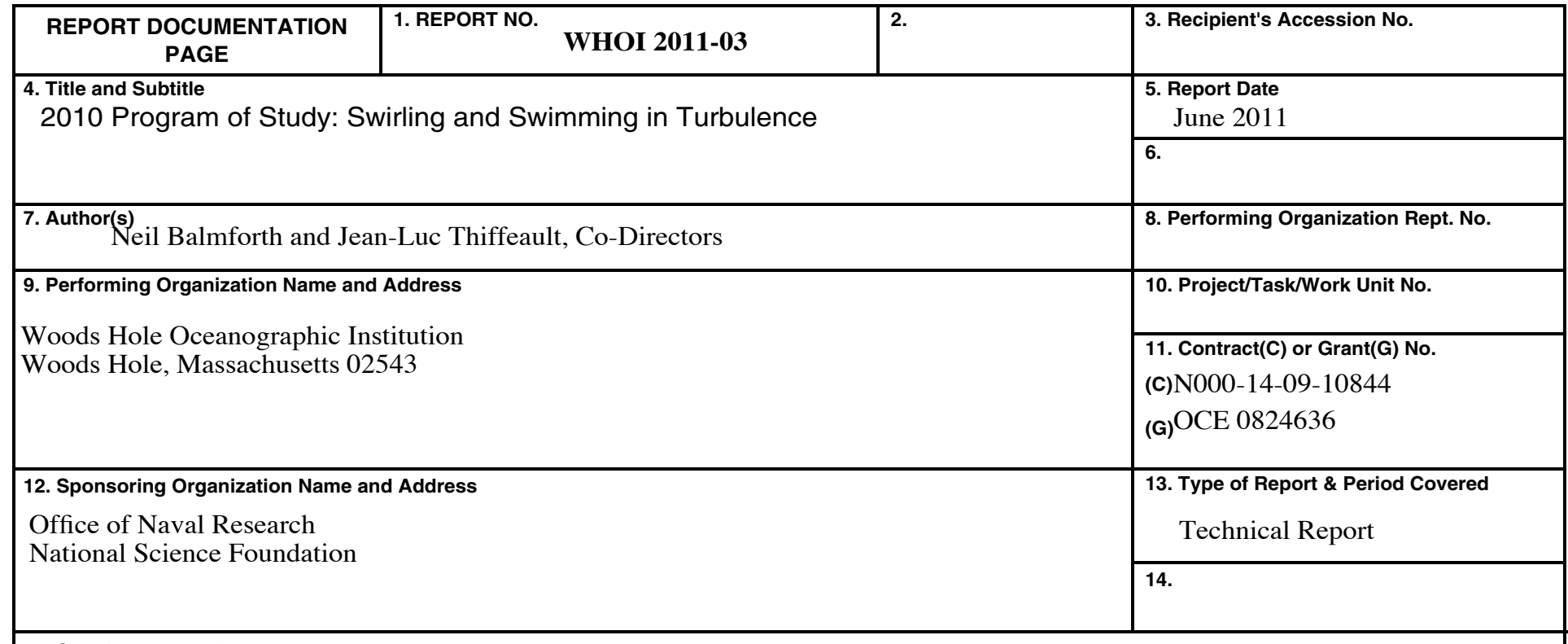

15. Supplementary Notes

This report should be cited as: Woods Hole Oceanographic Institution Technical Report, WHOI-2011-03.

16. Abstract (Limit: 200 words)

Swirling and Swimming in Turbulence was the theme at the 2010 GFD Program. Professors Glenn Flierl (M.I.T.), Antonello Provenzale (ISAC-CNR, Turin) and Jean-Luc Thiffeault (University of Wisconsin) were the principal lecturers. Together they navigated an elegant path through topics ranging from mixing protocols and efficiencies to ecological strategies, schooling and genetic development. The first ten chapters of this volume document these lectures, each prepared by pairs of this summer's GFD fellows. Following on are the written reports of the fellows' own research projects. This summer's fellows were: $\bullet$ Michael Allshouse, MIT • Emma Boland-Thompson, University of Cambridge • Renske Gelderloos, KNMI, Utrecht • David Goluskin, Columbia University • Georgy Manucharyan, Yale University • Woosok Moon, Yale University • Kiori Obuse, Kyoto University • Amanda O'Rourke, Princeton University • Sam Pegler, University of Cambridge • Anubhab Roy, Jawaharlal Nehru Centre for Advanced Scientific Research In 2010 the Sears Public Lecture was delivered by Professor Jim Murray, of Oxford University and the University of Washington. Jim challenged us to use mathematics in real world problems, citing examples from biology and sociology. Specifically, Jim argued how one could exploit mathematical modelling to assist in the prediction of tumour growth, and to gauge the successfulness of marriage partnership. Over a hundred listeners filed into Redfield for the occasion, and we enjoyed refreshments in the evening air afterwards at the back of Redfield. Neil Balmforth and Jean-Luc Thiffeault acted as the co-directors for the summer (once Jean-Luc's Lectures were over). New GFD Faculty member Colm Caulfield (University of Cambridge) nimbly acted as assistant, as well as putting in a stout performance on the Staff by supervising a good fraction of the fellows. Anders Jensen worked his usual magic in the Lab, and Janet Fields, Jeanne Fleming and Kathy Ponti smoothly ran the program behind the scenes.

\section{Document Analysis a. Descriptors}

stirring and mixing

ecological strategies

genetic development

b. Identifiers/Open-Ended Terms

c. COSATI Field/Group

18. Availability Statement

Approved for public release; distribution unlimited.

\begin{tabular}{|l|l|}
\hline $\begin{array}{c}\text { 19. Security Class (This Report) } \\
\text { UNCLASSIFIED }\end{array}$ & $\begin{array}{c}\text { 21. No. of Pages } \\
407\end{array}$ \\
\hline 20. Security Class (This Page) & 22. Price \\
\hline
\end{tabular}

